$\frac{54}{8-11}-92851$

DOE/ER-0701T

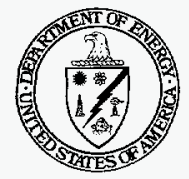

United States Department of Energy

June 1997

Otfice of Energy Research

Office of Biological and Environmental Research

Environmental Sciences Division

\title{
Environmental Sciences Division: Summaries of Research in FY 1996
}
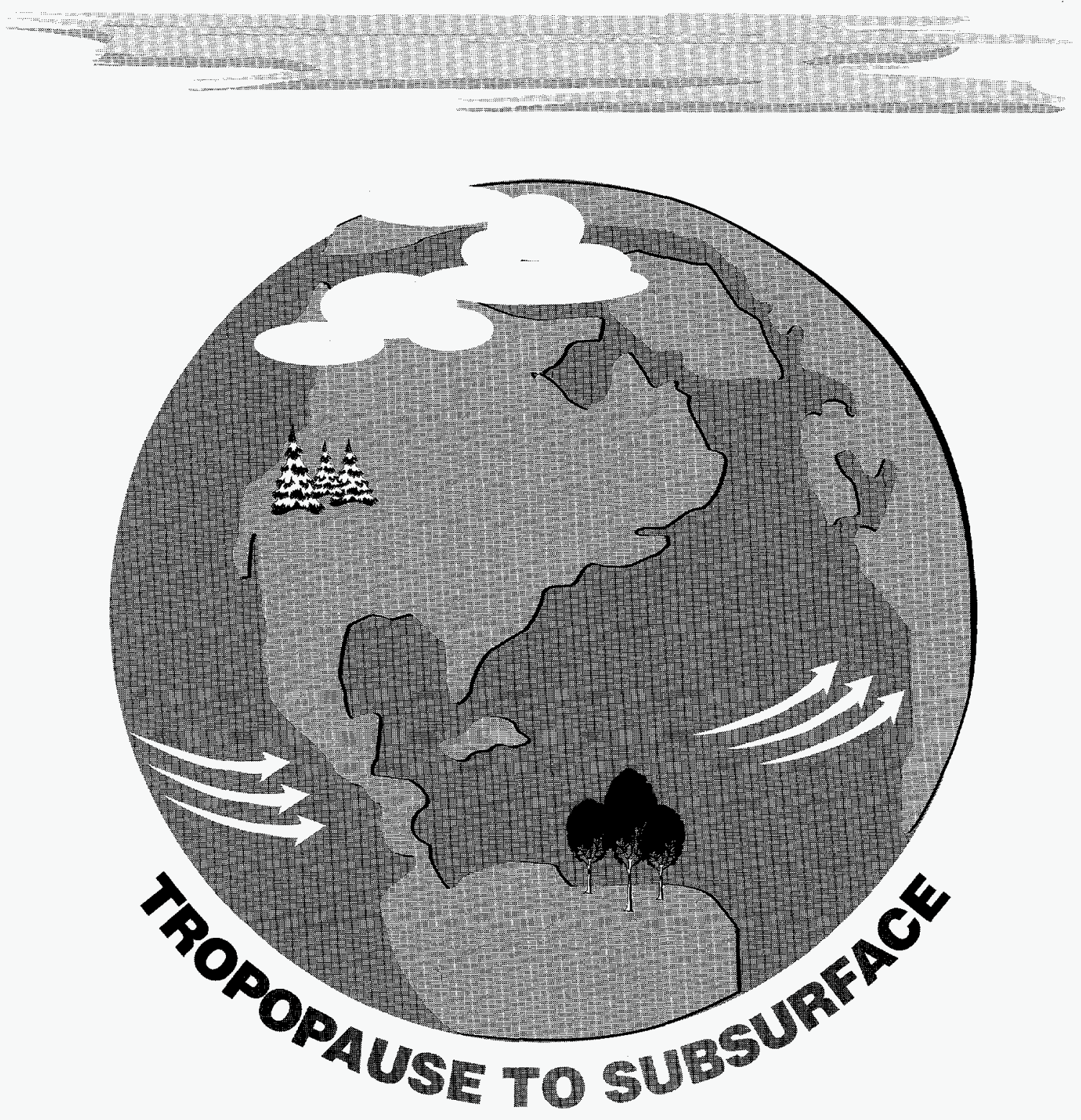
This report has been reproduced directly from the best available copy.

Available to DOE and DOE Contractors from the Office of Scientific and Technical Information, P.O. Box 62, Oak Ridge, TN 37831; prices available from (615) 576-8401.

Available to the public from the U.S. Department of Commerce, Technology Administration, National Technical Information Service, Springfield, VA 22161, (703) $487-4650$. 
Office of Energy Research

Office of Blological and Environmental Research

Environmental Sciences Division

Germantown, MD 20874-1290

\section{Environmental Sciences Division: Summaries of Research in FY 1996}





\section{DISCLAMMER}

Portions of this document may be illegible in electronic image products. Images are produced from the best available original document. 



\section{CONTENTS}

FOREWORD

PREFACE

vii

INTRODUCTION

GLOBAL-CHANGE RESEARCH

Atmospheric Radiation Measurement (ARM) Program

Unmanned Aerial Vehicles (UAV)

Atmospheric Sciences

Climate Modeling

Climate Research

Computer Hardware, Advanced Mathematics, and Model Physics (CHAMMP)

Program for Climate Model Diagnosis and Intercomparison (PCMDI)

Education

Information and Integration

Integrated Assessment

National Institute for Global Environmental Change (NIGEC)

Ocean Sciences Research

Program on Ecosystem Research

Terrestrial Carbon Processes

ENVIRONMENTAL-REMEDIATION RESEARCH

Environmental-Remediation Research Excluding Subsurface Science

Subsurface Science Program

APPENDIXES

A: Addresses of Principal Investigators

B: Acronyms

INDEXES

Principal Investigator Index

Institutional Index 



\section{FOREWORD}

I am pleased to provide you with the Fiscal Year 1996 book of abstracts of research supported by the Environmental Sciences Division, Office of Biological and Environmental Research. For those of you who have been annual recipients of these volumes, you may have noticed a change in our name. As many of you know, the scientific programs sponsored by the Office of Health and Environmental Research form the Biological and Environmental Research Program. In June 1997, the office name was changed to align with our program and to reflect better the totality of what this office sponsors, research that spans a broad range of biology and environment, with health issues included as part of the whole.

Within the Environmental Sciences Division (ESD), Fiscal Year 1996 was a year of continuation of decisions implemented in Fiscal Year 1995. The Atmospheric Studies in Complex Terrain program efforts were redirected to the planning and initial phases of the Aerosol and Visibility Evaluation Research Program in Mexico City, sponsored jointly by ESD and the Instituto Mexicano de Petroleo. The field campaign of the Ocean Margins Program reached a successful conclusion, as did the $\mathrm{CO}_{2}$ survey in the Indian Ocean. The Subsurface Science Program continued to wind down its activities while maintaining its tradition of transferring basic research results to the field in partnership with the Office of Environmental Management.

Among the many highlights of the Fiscal Year 1996 activities within ESD was the installation on Manus Island, Papua New Guinea, of the first suite of instruments to be stationed in the Tropical Western Pacific for the Atmospheric Radiation Measurement Program. The Unmanned Aerial Vehicle Program set a long-endurance science flight record, with the Altus flying and collecting data for just over 26 hours. The Natural and Accelerated Bioremediation Research (NABIR) program was initiated, including the launching of a very important component, the program element on Bioremediation and Its Societal Implications and Concerns. Interest continued to grow in the FreeAir- $\mathrm{CO}_{2}$-Exchange (FACE) experiments and in the $\mathrm{CO}_{2}$ flux measurement studies. The number of FACE experiments expanded to cover an increased range of ecosystems, and the AmeriFlux network now has $\mathrm{CO}_{2}$ measurement sites ranging from Alaska to Costa Rica.

For more information about the exciting science and the programs that we have supported, and continue to support, I urge you to peruse not only the following pages but also our World Wide Web site at http://www.er.doe.gov/production/oher/ESD_top.html. Included in the ESD web pages is a searchable database containing the abstracts found in this FY 1996 publication, as well as those in the FY 1994 and FY 1995 publications. In the future, this database will be the primary source for access to the abstracts of ESD supported research rather than hardcopy publications such as this.

Once again, I would like to thank the many scientists, both those active in research laboratories and the program managers in ESD, for their contributions to the research highlighted in this book. Special thanks go to Bobbi Parra, Karen Carlson, David Henderson, Jannean Elliott, Fred O'Hara, Eoin $\mathrm{O}^{\prime} \mathrm{Hara}$, and Linda $\mathrm{O}^{\prime} \mathrm{Hara}$ for organizing and preparing this document for publication.

In closing, happy reading!

Michelle S. Broido, Ph.D., Director

Environmental Sciences Division

Office of Biological and Environmental Research 



\section{PREFACE}

This document describes the Fiscal Year 1996 activities and products of the Environmental Sciences Division, Office of Biological and Environmental Research, Office of Energy Research. The report is organized into four main sections.

\section{SECTION}

Introduction

Research Areas and

Project Descriptions

\section{DESCRIPTION}

Identifies the basic program structure and describes the programs of the Environmental Sciences Division

Provides the level of effort for each program area

Give program contact information

Provide descriptions of individual research projects including:

Three-year funding history

Research objective and approach used in each project

Results to date

Appendixes

Provide postal and e-mail addresses for principal investigators

Define acronyms used in the text

Indexes

Provide indexes of principal investigators, research institutions, and keywords for easy reference

Questions concerning the Environmental Sciences Division's programs or specific projects may be addressed to the Environmental Sciences Division, U.S. Department of Energy, ER-74, 19901 Germantown Rd., Germantown, MD 20874-1290. The Division's telephone number is 301-903-3281. 


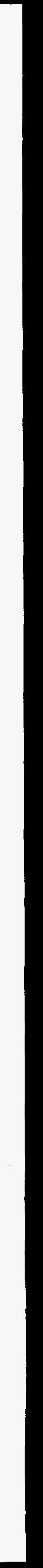




\section{INTRODUCTION}

In Fiscal Year 1996, the Environmental Sciences Division (ESD) supported research in the areas of global change and environmental remediation.

\section{GLOBAL-CHANGE RESEARCH}

The global-change activities include the process research and modeling efforts needed to (1) improve understanding of factors affecting the Earth's radiant-energy balance; (2) predict accurately any global and regional climate change induced by increasing atmospheric concentrations of greenhouse gases; (3) quantify sources and sinks of energy-related greenhouse gases, especially carbon dioxide; and (4) improve the scientific basis for assessing the potential consequences of climatic changes, including the potential ecological, social, and economic implications of human-induced climatic changes caused by increases in greenhouse gases in the atmosphere and the benefits and costs of alternative response options.

\section{Climate and Hydrology}

The Atmospheric Radiation Measurement (ARM) Program improves climate prediction by providing data and improved parameterization of clouds and their interactions with solar and terrestrial radiation through ground-based, airborne (manned and unmanned), and satellite platforms at or over three sites (U.S. Southern Great Plains, Tropical Western Pacific, and North Slope of Alaska).

The Unmanned Aerial Vehicle (UAV) Program uses remotely operated aircraft in support of the ARM Program to extend the ARM energy balance and cloud data to regional scales and to investigate other environmental processes via remote sensing. Through FY 1996, this program is a joint activity with the Department of Defense under the Strategic Environment Research and Development Program.

Climate modeling develops the tools necessary to predict accurately the global and regional climate change induced by increasing atmospheric concentrations of carbon dioxide and other greenhouse gases. The climate-modeling program has two key components: The Program for Climate Model Diagnosis and Intercomparison (PCMDI) promotes a coordinated and sustained effort that documents and determines the differences and agreements among climate simulations and determines why differences occur among model results and between models and observations. The Computer Hardware, Advanced Mathematics, and Model Physics (CHAMMP) Program encourages activities in four interrelated areas: theoretical studies of decade-to-century climate change and climate variability; development of massively parallel versions of general circulation models (GCMs), including the procurement of computer resources on leading-edge supercomputers; new numerical methods and model formulations; and better process parameterizations for GCMs.

\section{Atmospheric Chemistry and Carbon Cycle}

The Atmospheric Sciences Program improves understanding about and information on the atmospheric processes controlling ozone and UV-B trends; the heterogeneous chemistry, aerosol formation, measurement, and modeling of atmospheric flow over topographically complex terrain; and the transport, dispersion, and ultimate fate of energy-related emissions.

The Carbon Cycle Research Program measures, models, and estimates changes in the physical, chemical, and biological processes controlling the sources and sinks of atmospheric carbon dioxide; it emphasizes the carbon processes of terrestrial ecosystems.

Ocean Sciences Research seeks to understand ocean-atmosphere-climate interactions and the ocean carbon cycle. This activity includes a Global Survey of $\mathrm{CO}_{2}$ in the Oceans that is measuring the 
distribution of inorganic carbon throughout the world's oceans and the Ocean Margins Program, which is quantifying biogeochemical processes and fluxes of carbon at the land-ocean interface.

\section{Ecological Processes}

The Program for Ecosystem Research identifies the processes that allow terrestrial ecosystems to adjust to global environmental changes and uses this understanding to protect and restore ecosystems.

The Vegetation Program experiments on the response of terrestrial vegetation to excess $\mathrm{CO}_{2}$ and altered climate variables, augmenting studies of ecosystem function and response and terrestrial-carbon processes.

\section{Human Interactions}

The Global-Change Educational Program is designed to develop the next generation of scientists essential for the multidisciplinary research on global change.

The Information and Integration Program provides information for the evaluation of environmental issues associated with elevated atmospheric carbon dioxide and other atmospheric trace gases, including potential climate change.

The Integrated Assessment of Global Climate Change Research Program focuses on investigations that support future decision making on global-change issues (investigations into the critical economic analyses underlying decisions expected to be made two or more years from now). The program also emphasizes integrated analysis and the need for an economic framework that provides estimates of both benefits and costs of potential global change and of the actions contemplated to ameliorate it.

\section{National Institute for Global Environmental Change}

The National Institute for Global Environmental Change (NIGEC) was established by the U.S. Congress in the Energy and Water Act of 1989. The Institute is operated for the U.S. Department of Energy (DOE) by the University of California under a cooperative agreement.

\section{ENVIRONMENTAL-REMEDIATION RESEARCH}

The environmental-remediation research portfolio is focused on developing an understanding of the fundamental physical, chemical, geological, and biological processes that must be marshaled for the development and advancement of new, effective, and efficient processes for the remediation and restoration of the nation's nuclear-weapon-production sites.

\section{Bioremediation}

A primary activity of the environmental-remediation effort is a comprehensive research program in bioremediation that integrates the full range of fundamental scientific disciplines necessary to advance this emerging technology from one of hit-or-miss to one of sustained, cost-effective utility. The Natural and Accelerated Bioremediation Research (NABIR) Program promotes the use of living organisms to reduce or eliminate waste. The Microbial Genome Research Program provides genome sequence and mapping data on microorganisms of industrial importance and on those that live under extreme conditions.

\section{Cleanup Research}

The Environmental Technology Partnerships Program encourages partnership agreements among university, national laboratory, and industry researchers to address fundamental bioremediation and integrated-assessment research that is oriented toward reducing waste production and energy consumption in manufacturing processes. 


\section{Facility Operations}

The Environmental Sciences Division operates the Environmental Molecular Sciences Laboratory (EMSL), the only national collaborative-user facility focused on the Department's environmental missions.

\section{Subsurface Science}

The Subsurface Science Program seeks to understand the physical, chemical, and biological processes controlling the fate of complex chemical mixtures released to terrestrial subsurface environments; it also conducts research in the deep terrestrial biosphere.

\section{LEVEL OF EFFORT}

Funding for research in Fiscal Year 1996 (in millions of dollars) was distributed as follows:

\section{GLOBAL-CHANGE RESEARCH}

Atmospheric Radiation Measurement (including capital equipment)

Unmanned Aerial Vehicles

$\$ 40.1$

Atmospheric Science

Climate Research

Computer Hardware, Advanced Mathematics, and Model Physics

Program for Climate Model Diagnosis and Intercomparison

Education

Information and Integration

Integrated Assessment

National Institute for Global Environmental Change

Ocean Sciences Research

Program on Ecosystem Research

Terrestrial Carbon Processes 


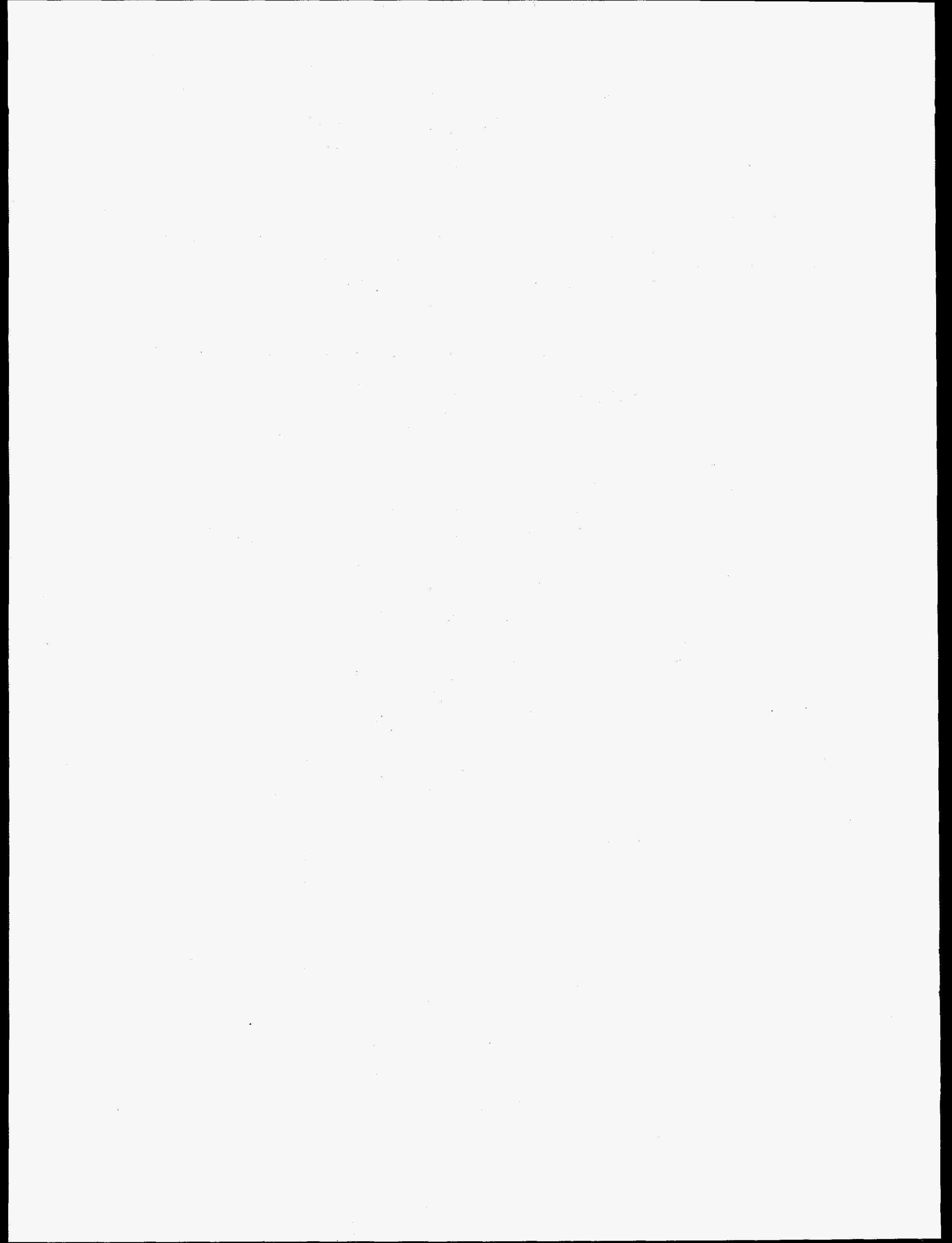


GLOBAL CHANGE RESEARCH 



\title{
ATMOSPHERIC RADIATION MEASUREMENT PROGRAM
}

The Atmospheric Radiation Measurement (ARM) Program is designed to improve climate prediction by providing data and improved parameterization of clouds and their interactions with solar and terrestrial radiation through ground-based, airborne (manned and unmanned), and satellite platforms at or over three sites (the U.S. Southern Great Plains, the Tropical Western Pacific, and the North Slope of Alaska).

\author{
Program Managers \\ Patrick A. Crowley \\ Environmental Sciences Division \\ Department of Energy, ER-74 \\ 19901 Germantown Road \\ Germantown, MD 20874-1290 \\ (301) 903-3069 \\ Internet: p.crowley@oer.doe.gov \\ Wanda R. Ferrell \\ Environmental Sciences Division \\ Department of Energy, ER-74 \\ 19901 Germantown Road \\ Germantown, MD 20874-1290 \\ (301) 903-0043 \\ Internet: wanda.ferrell@oer.doe.gov
}

Peter W. Lunn

Environmental Sciences Division

Department of Energy, ER-74

19901 Germantown Road

Germantown, MD 20874-1290

(301) 903-4819

Internet: peter.lunn@oer.doe.gov

\section{AN INTEGRATED CLOUD OBSERVATION AND MODELING INVESTIGATION ACKERMAN, THOMAS \\ THE PENNSYLVANIA STATE UNIVERSITY \\ FY 1996428 \\ FY 1995450 \\ FY 1994359}

Keywords: remote sensing, clouds, cloud-radiation parameterizations, cloud-base height, lidar, stratuscloud mean particle size, stratus-cloud mean particlenumber density, cirrus optical depth, cirrus size distribution

Objectives: To develop algorithms for ground-based remote sensing of clouds and to use acquired data to test cloud-radiation parameterizations.

Approach: Field data from multiple instruments will be used to develop algorithms for ground-based remote sensing of cloud properties. The core group of instruments are cloud radar, cloud lidar, microwave radiometer, and solar and infrared radiometers. The developed algorithms will be used to process the continuous data stream from the ARM Southern Great Plains
(SGP) Site in Oklahoma. The focus here is to produce simultaneous data streams of atmospheric environmental parameters, cloud properties, and surface radiative fluxes. A combination of the data streams and mesoscale-model results will be used to test cloud parameterizations currently used in GCMs.

Results to Date: New algorithms have been developed to retrieve automated cloud-base height from lidar, stratus-cloud mean particle size and number density; and cirrus optical depth and size distribution. A oneyear climatology of cloud-base height has been computed for the SGP site. An evaluation of the accuracy of mesoscale-mode upper-tropospheric relative humidity has been carried out with satellite data. And an initial evaluation of three cirrus parameterizations has been completed. 


\section{SCIENCE TEAM PARTICIPATION IN THE ARM PROGRAM \\ CESS, ROBERT D. \\ STATE UNIVERSITY OF NEW YORK AT STONY BROOK \\ FY 199664 \\ FY 199561 \\ FY 199456}

Keywords: absorption by clouds

Objectives: To better understand absorption of solar radiation by clouds.

Approach: Stacked-aircraft measurements made during ARESE will be analyzed.

Results to Date: A considerable effort has been devoted to participation in the ARM Enhanced Shortwave Experiment (ARESE) Science Team, organizing and participating in four planning meetings held at SUNY Stony Brook, NCAR, and Scripps Institution. Collocation of satellite and surface measurement of shortwave radiation has been performed to appraise absorption of shortwave radiation by clouds. Radiometric measurements from the Oklahoma site are being analyzed for the purpose of constructing a data set that can be used to test atmospheric GCMs. This test would be performed in conjunction with a related DOE project concerning the intercomparisons of GCMs. Excess cloud shortwave absorption exists in both the visible and near infrared.

\section{REPRESENTATION OF ANVIL CIRRUS IN GENERAL CIRCULATION MODELS: A NEW PARAMETERIZATION CHIN, HUNG-NENG

Keywords: anvil cirrus parameterization

Objectives: To develop a parameterization scheme that can accurately represent in GCMs the critical climate effects of convectively generated anvil cirrus clouds.

Approach: Cloud processes of mesoscale convective systems (MCSs) and their water mass budgets will be analyzed, and sensitivity studies will be conducted with cloud-resolving numerical models. ARM data from the Southern Great Plains Cloud and Radiation Testbed (CART) Site and the Tropical Western Pacific CART Site will be used with numerical models of deep convective clouds, cirrus clouds, and radiation transfer to determine which cirnus feedback processes must be represented in GCMs to adequately address key climate-change issues. The results of these studies will be used to develop an anvil-cirrus parameterization (ACP) that will predict the effects of anvil cirrus based on the GCM-resolvable atmospheric state. The ACP will be tested in at least one GCM.

Results to Date: In our development of the ACP, we used results from a sensitivity study that covers a wide range of environmental conditions. Because of computational constraints, this sensitivity study was conducted in the 2-D model. Results indicate that the bulk Richardson number (Ri) is a valuable index to categorize the mesoscale characteristics of MCSs. Therefore, it is feasible to parameterize the sub-GCM-grid process of interest.

The systematic change of the altitude and magnitude of horizontally averaged maximum stratiform ascent/descent with $\mathrm{Ri}$ allows us to parameterize a vertical profile of mesoscale vertical velocity with GCM-grid-resolved variables. This profile, along with the large-scale forcing, can be used to drive the prognostic microphysics scheme in GCMs. As a result of the stronger parameterized mesoscale vertical velocity than its large-scale counterpart, one would expect that the newly developed ACP can have a more prominent impact on the formation of anvil clouds and lead to stronger cloud-radiative feedback to large-scale climate.

The strong similarity of these mesoscale features between 2-D and 3-D simulations further suggests how to use the 2-D-based ACP in 3-D applications in GCMs. This ACP will be tested in a GCM to assess its impact on large-scale climate.

\section{RADIATIVE TRANSFER FOR CLEAR AND CLOUDY ATMOSPHERES: SPECTRAL MODELING AND VALIDATION CLOUGH, SHEPARD A. ATMOSPHERIC \& ENVIRONMENTAL RESEARCH, INC. \\ FY 1996207 \\ FY 1995227 \\ FY 1994136}

Keywords: radiative transfer models, model validation, spectral validation

Objectives: To develop a highly accurate radiativetransfer model for scattering and nonscattering atmospheres; to use calculations from this model for parameterizations required for GCM radiative-transport codes; and to validate this code.

Approach: A rapid radiative transfer model (RRTM) is being developed to achieve the accuracy and computational efficiency required for application to GCMs. The model LBLRTM is being used to calculate the effects of molecular absorption in both the short- and longwave spectral regions. Radiative transfer associated with scattering caused by clouds and aerosols is calculated at monochromatic spectral precision with the code CHARTS. The models will be frequently and consistently applied to the ARM spectral data to 
quantitatively assess the determination of the atmospheric state, the radiative-transfer modeling, and the measurement of spectral radiances of the ARM sites. The fluxes and cooling rates obtained from RRTM with these parameterizations together with approximations in the radiative transfer will be evaluated against the accurate calculations of the same quantities from LBLRTM. Technical support will be provided for the assessment and improvement of the necessary spectroscopic line parameters, the absorption cross-sections associated with the heavier molecules, and the watervapor continuum models.

Results to Date: The validations to the present time provide a clear indication that the determination of the water vapor profile for the radiating column, obtained from radiosondes, is the limiting factor in evaluating the model. The current level of validation is of the order of $4 \mathrm{~W} / \mathrm{m}^{2}$ from 550 to $3000 \mathrm{~cm}^{-1}$, limited principally by the water-vapor uncertainty. To address this problem, increased emphasis has been placed on the utilization of the microwave-radiance (MWR) measurements and the associated theory to obtain an improved value for the water-vapor column. An assessment of microwave radiative modeling capability has been initiated with LBLRTM to attain improvement in the water-vapor profiles by scaling the sonde or Raman lidar profiles. Improved and validated microwave modeling is also critical to use MWR measurements for column water vapor at the low water-vapor values of interest at the North Slope of Alaska (NSA) ARM site. A new model, MONORRTM, is being developed to provide radiativetransfer results at monochromatic frequencies; it includes the same physics as LBLRTM.

The radiative transfer model, RRTM, has been completed and validated for the longwave region. In addition to water vapor, carbon dioxide, and ozone, the model includes the radiative effects of methane, nitrous oxide, and the common halocarbons. Errors in the fluxes are less than $0.6 \mathrm{~W} / \mathrm{m}^{2}$ at all levels and in the cooling rates less than $0.06 \mathrm{~K} / \mathrm{d}$ in the lower atmosphere. RRTM includes the foreign and self watervapor continuum being developed under the present research effort. The model has a validation path through LBLRTM to spectral radiative measurements, particularly those at the Southern Great Plains ARM site. Preliminary evaluation suggests that the model is two times faster than the radiation module in CCM2. Extension of the model to the shortwave is $50 \%$ complete.

Detailed validations have been performed on selected cloud cases with the CHARTS multiple scattering model. Initial indications are that, for watervapor stratus clouds in which effective values for the mode radius, cloud liquid water, and cloud fraction provide agreement with AERII spectral measurements, results are as good as or better than those for the clear sky. Both the thermal component of channel 1 and the solar/thermal component of channel 2 are used in the analysis.

\author{
EXPLICIT SIMULATION AND \\ PARAMETERIZATION OF MESOSCALE \\ CONVECTIVE SYSTEMS \\ COTTON, WILLIAM \\ COLORADO STATE UNIVERSITY \\ FY 1996154 \\ FY 1995214 \\ FY 1994194
}

Keywords: mesoscale convective systems, parameterizations, cumulus convection, cloud parameterizations, general circulation models

Objectives: To develop a parameterization scheme of heating, moistening, and momentum transports of mesoscale convective systems derived, in part, from 3-D cloud-resolving simulations.

Approach: Cloud-resolving simulations will be performed, model-output data will be analyzed, a framework (scheme, calibrate, modify scheme, and test it against independent data sets, including ARMCART data) will be developed.

Results to Date: Three-dimensional cloud-resolving simulations have been performed; model output data have been statistically analyzed; and a scheme for heating, moistening, and momentum transports by mesocale stratiform anvils has been devised and tested against model-output data.

\section{AREALLY AVERAGED HEAT FLUX FOR ARM COULTER, RICHARD \\ ARGONNE NATIONAL LABORATORY \\ FY 1996120 \\ FY 1995150 \\ FY 1994145}

Keywords: energy balance, heat fluxes, remote sensing

Objectives: To develop methods for estimating surface sensible-heat fluxes representative of the land sites of the ARM program and to relate geographical variations of the heat fluxes to the measured variability of the clouds and radiative fluxes across the sites.

Approach: Research is conducted on methods to estimate surface heat fluxes representative of ARM land sites. Intensive field campaigns, often conducted in collaboration with other laboratories, focus on observations of air-surface interactions on scales ranging from individual boundaries of different surface types to circulations in the lower planetary boundary layer that result from discontinuities in surface energy balances. Data routinely obtained at the SGP CART Site are used extensively. Differences in the planetary- 
boundary-layer height inferred from balloon-borne sounding-system flights and from remote sensing provide important information on differences in horizontally integrated heat fluxes across the site. Models of the planetary boundary layer are used to help recover areally averaged estimates of heat flux from the planetary-boundary-layer structure. Differences in cloud cover, cloud base, temperature profiles, and moisture profiles across the site are used to interpret the surface flux data. Satellite data are used with an existing canopy model to include the effects of latent heat, moisture, and vegetation on the behavior of the planetary boundary layer across the site.

Results to Date: Data from intensive observation periods (IOPs) in October 1994 and June-July 1995 were used to investigate the relationship between coherent structures and areal averages of surface heat flux. Balloon-borne profiles of planetary-boundarylayer variables obtained at four sites provided data on the spatial variability of the planetary boundary layer across the CART site. An improved method was developed for estimating the convective velocity scale by using the vertical velocity field measured with the 915-MHz wind profiler. Estimates of areally averaged heat flux obtained by using the convective velocity scale and the capping inversion height compared favorably with site-wide averages of heat flux.

\section{ATMOSPHERIC RADIATION MEASUREMENT PROGRAM CRESS, TED $S$. \\ PACIFIC NORTHWEST NATIONAL LABORATORY \\ FY 199613676 \\ FY 199510144 \\ FY $1994 *$}

*In previous years, this entry was consolidated with others under the principal investigator Gerald $M$. Stokes.

Keywords: program management, site implementation, site operations, instrument selection, campaign planning, campaign coordination

Task I: Program Management. Much of the ARM Program Management is performed by a multilaboratory team, including Argonne, Brookhaven, National Renewable Energy Laboratory, Oak Ridge, Livermore, Los Alamos, Pacific Northwest, and Sandia. The Science Team coordination effort and the Data and Science Team Integration effort are funded separately.

Objectives: To improve the performance of GCMs of the atmosphere in predicting global and regional climate, focusing specifically on the treatment of radiative-transfer and related models in GCMs under clear, general-overcast, and broken-cloud conditions and the parameterization of cloud properties, cloud formation, and cloud maintenance in GCMs; to manage the ARM Program; to develop the experimental infrastructure of ARM to provide the basis for the testing and verification of representations of subgrid processes in GCMs; and to rapidly deploy experiments to the field.

Approach: The ARM Program is managed by the ARM Project Office, located at Pacific Northwest National Laboratory, on behalf of DOE. ARM consists of three distinct entities: The Cloud and Radiation Testbed (CART) and supporting infrastructure, the Data and Science Integration Team (DSIT), and the ARM Science Team. The Project Office provides central planning and management for the ARM infrastructure, support of DOE/OHER Environmental Sciences Division activities, and coordination with related efforts in other agencies and countries.

The Cloud and Radiation Testbed (CART) is the basic experimental infrastructure for the ARM Program and will consist of three permanent observing sites, an ability to support intensive observations and campaign activities, and a data system. The data system will acquire data from CART instruments as well as from outside sources, will process and archive appropriate data, and will deliver requested data to members and subgroups of the Science Team. The CART sites are established and managed by site program managers selected from within the DOE national laboratories. These teams address program needs relative to instruments, data processing and management, site operations, and campaign coordination. Funds to individual laboratories are managed by single points of contact at each laboratory, who are indicated in the individual task summaries that follow. Results to Date: The ARM Program now provides data on a continuous basis from two sites, with a third to be added early in FY 1998. A continuous stream of data is flowing from the first CART site in Oklahoma (and from external data sources) through the ARM data system to members of the Science Team and to the general scientific community. Special observational programs are being conducted on a regular basis, and a special emphasis is being placed on data quality and on data processing to meet the requirements of the Science Team. For deployment to the Tropical Western Pacific (TWP), a ruggedized, semiautonomous system, known as an Atmospheric and Radiation Cloud Station, was developed. The first system entered test and evaluation at the end of FY 1995 and was deployed in FY 1996 in accordance with the siting strategy for the TWP. Decisions on siting and instrument strategies focused on the deployment of the first Atmospheric and Radiation Cloud Station to Manus Province, Papua New Guinea. The command-and-control concepts for this remote site call for health-of-station data to be transmitted in near-real time with satellite-communi- 
cation systems; most data, however, will be recorded on tape and physically shipped to the ARM Experiment Center for processing and distribution to Science Team members and the Archive. The challenges of integrating instruments, data system, shelters, environmental controls, and communications for continuous remote operation were substantial. While successfully addressed, the effort required greater-than-anticipated time to complete and test, delaying deployment into FY 1996.

Planning is proceeding for transferring the integrated technology for the TWP semiautonomous observing system to the development of similar capabilities for the North Slope of Alaska and for supporting periodic interagency and interprogram collaborative field programs. Data from all three sites will be processed and transferred through the ARM data system and its various components.

Intensive operational periods are being conducted to meet data needs of the Science Team and to participate in collaborative measurements with other programs.

Task II: Site Implementation and Operations. This is a multilaboratory effort.

Objectives: To implement and operate the Southern Great Plains (SGP) and Tropical Western Pacific (TWP) sites and to plan site facilities and instruments for the North Slope of Alaska (NSA) Site.

Approach: Site program managers plan and direct the implementation of each CART site with support from the Site Operations Team. The Site Operations Team assists in the development of the initial site-operations plan, trains operations personnel as appropriate, and continues to assist the site operator and the site program manager in the initial stages of site operations. Site program managers for the SGP, TWP, and NSA are Douglas Sisterson, Argonne National Laboratory; William Clements, Los Alamos National Laboratory; and Bernard Zak, Sandia National Laboratory, respectively.

Results to Date: Southern Great Plains: While the SGP Site became operational in a phased deployment during FY 1992 and FY 1993, additional instrumentation and facilities continued to be implemented during FY 1994 and FY 1995. In FY 1995, the central facility at Lamont, Oklahoma, was complete with the exception of the radiometric calibration facility, which is now operational. The aerosol-measurement facility was made operational late in FY 1995. Several extended facility sites were delayed into FY 1996 because resources were concentrated on a major campaign late in FY 1995. A new class of remote sites was specific for planetary-boundary-layer measurements with 915-MHz wind-profiler/radioacoustic-sounding system instruments; three sites were selected, and instrument procurement was initiated.
Shelters for the four boundary facilities were acquired during FY 1996, and boundary-facility communications, data-acquisition, and data-processing capabilities were made permanent. Intended to support the data requirements for single-column-model cloudradiation prediction and evaluation, the boundary facilities rely upon balloon-borne sondes to provide vertical profiles of winds and thermodynamic variables.

Site mission plans continue to be prepared at 6-month intervals by the site scientist to guide site operations. The site scientist routinely examines the data streams being produced by the site to help ensure that data of known and reasonable quality is being provided to the Science Team. Intensive operational periods and campaigns are a significant part of the annual data-acquisition activity.

Tropical Western Pacific: The first Atmospheric Radiation and Cloud Station (ARCS) was successfully deployed to Manus Province, Papua New Guinea, late in FY 1996. Coordination with the government of Papua New Guinea proceeded successfully with additional agreements for cooperation and collaboration in the establishment and operation of the facility. Negotiations continue with the government of the Republic of Naunu for placing the second ARCS Station on that island in FY 1997. Collaboration with two programs in the area, the Schools of the Pacific Rainfall Climate Experiment and South Pacific Regional Environmental Program, continue. Each provides unique inroads with the various island governments. Data-transfer plans for the TWP continue to involve tapes being shipped to the Experiment Center on a regular basis. Small amounts of data to monitor the performance of the station and to schedule maintenance trips will be transmitted back to the site project office through low-data-rate satellite links. A collaboration with PEACESAT may be feasible wherein a larger amount of data, but not all, may be able to be transferred on a daily basis.

North Slope of Alaska: Site planning for the NSA Site at Barrow continues in coordination with local authorities. A measurement system specifically designed for the Arctic but drawing upon the systems integrated for the TWP ARCS will be deployed to one or more sites. They will be augmented with additional instrumentation, as required. Instrumentation is being tested in arctic conditions at the University of Alaska at Fairbanks. Planning for collaborative participation in the interagency SHEBA program continues, with the site program manager and several members of the ARM Science Team actively engaged in planning and coordination activity. The suite of instrumentation for SHEBA will be housed in small, portable modules and will ultimately comprise part of the second measurement facility on the North Slope, 
which will be in the village of Atqasuk, $50 \mathrm{~km}$ inland from Barrow.

Task III: Instrument Selection/Implementation. This is a multilaboratory effort.

Objectives: To manage and execute ARM-infrastructure activities concerning instrumentation and to identify, install, implement, and calibrate instrument systems.

Approach: The team lead for ARM's interlaboratory instrument team is from Argonne National Laboratory. The team is tasked to identify and evaluate instruments for use in the observational systems of CART, to provide technical specifications for instrument acquisition, to oversee instrument installation and the development of operational and calibration protocols, and to support quality control of data from the observational systems.

Results to Date: The ARM CART instrument team continued in the selection, acquisition, and implementation of instrument systems for the SGP CART Site and the ARCS for the Pacific and Alaskan locales. Instrument-team members assigned to mentor specific instrument systems worked with the Pacific Northwest National Laboratory to write technical specifications, to review proposals, to work with selected vendors to ensure meeting CART and ARCS requirements, to coordinate implementation, to participate in instrument-operation training, and to take responsibility for fully implementing CART and ARCS instruments for use by the site operators. In addition, the instrument mentors were required to provide data quality control by examining data acquired and to recommend when data could be released to the Science Team. Extensive coordination with the ARM Data and Science Integration Team was required.

Systems acquired and implemented at the SGP CART central facility included broadband solar and infrared radiometers for several extended facilities and a balloon-borne sounding system and a microwave radiometer for a fourth boundary facility. Several eddycorrelation stations were installed to test their reliability of operation. For these and previously installed instruments, considerable effort was devoted to the evaluation and improvement of data quality. Progress continued on implementation at the central facility of an atmospherically emitted-radiance interferometer, a micropulse lidar, a solar-radiance-transmission interferometer, and a whole-sky imager.

For the TWP, the following systems were acquired and integrated into the first ARCS at the test site in Albuquerque: a surface meteorological observation station, a set of sensors for measuring downwelling broadband-solar and infrared radiation, a set of sensors for measuring upwelling broadband-solar and infrared radiation, a cloud laser ceilometer, a microwave radiometer, a micropulse lidar, and a whole-sky imager. Components were ordered, and assembly was planned for a millimeter-wavelength cloud radar and an atmospherically emitted-radiance interferometer. For the NSA, activities proceeded on selection of instruments to meet scientific needs for measurements and on development of specifications necessary for instrument operation in the Arctic environment. Because several of the needed NSA observations are similar, if not identical, to those needed at the TWP, activities of acquisition proceeded simultaneously with those required for the second and third TWP ARCS stations.

Task IV: Campaign Planning and Coordination. Stephen Schwartz, Brookhaven National Laboratory. Objectives: To manage campaign identification, planning, and coordination activities, including the identification of potential relationships between ARM and other experimental programs.

Approach: Campaign planning and coordination is a centralized activity within the program and is embodied in a single focal point for ARM participation in possible campaigns with other programs. CART observational data will be reviewed and assessed to determine whether or not targeted campaigns will be necessary to meet specific ARM research objectives or to satisfy user-facility-type requests from other programs for data or for access to ARM sites to satisfy research objectives.

Results to Date: The ARM Enhanced Radiation Experiment (ARESE) was planned and coordinated with NASA, NOAA, and the ARM-UAV Program. The experiment involved the inclusion of new, more accurate broadband radiometers on the UAV and its chase aircraft (the high-altitude NASA ER-2) and at selected extended facilities under the flight trace of the UAV. The World Wide Web was used to share and disseminate coordination and planning information. Support and data were provided to GEWEX through routine coordination with site operations and the datamanagement facilities. Coordination with NASA's Earth Observing System program continues.

\section{UNDERSTANDING AND \\ PARAMETERIZATION OF HIGH-LATITUDE CLOUD AND RADIATION PROCESSES CURRY, J. A., and TILLEY, J. S. UNIVERSITY OF COLORADO FY 1996180 \\ FY 1995200 \\ FY 1994195}

Keywords: high-latitude clouds, cloud-climate interactions

Objectives: To understand the life-cycle of highlatitude clouds during different seasons and under different synoptic conditions and to develop and validate model parameterizations for models of high- 
latitude-cloud life-cycle and cloud-climate-feedback processes.

Approach: The hierarchical modeling approach will be used, with models ranging from 1-D to 3-D.

Results to Date: A case study of a polar low in the North Atlantic has been analyzed. An MM5 formulation of a strategy suitable for MM5 model initialization and validation was used to collect data from the BASE experiment. It incorporated a new surface-turbulence flux parameterization into MM5 and used SSM/I retrievals of clouds and precipitation to validate MM5 simulations in the North Atlantic. A parameterization of drop-size spectrum was developed for arctic stratus clouds that included stochastic condensation. The performance of a second-order turbulence-closure scheme was examined in a simulation of the arctic summertime cloudy boundary layer. Cloud-radiation interactions with the snow/ice surface were modeled. And modeling studies of atmospheric convection and clouds associated with leads and sea ice were continued.

\section{LOW-CLOUD OPTICAL-THICKNESS \\ FEEDBACK: ARM DATA ANALYSIS AND SINGLE-COLUMN MODELING DEL GENIO, ANTHONY \\ NASA GODDARD INSTITUTE FOR SPACE STUDIES \\ FY 1996162 \\ FY 1995180 \\ FY 1994180}

Keywords: low clouds, optical properties, cloud feedback, GISS GCM

Objectives: To determine whether the satellite-inferred transition with temperature from increasing to decreasing optical thickness of low clouds at warm temperatures is real, to diagnose the mechanisms responsible for the observed optical-thickness variability, and to test the ability of parameterizations in the GISS GCM to reproduce this behavior.

Approach: We combine MWR, Belfort ceilometer, BBSS, and satellite data from the SGP CART Site to isolate low-level clouds and to calculate their liquidwater content, cloud-base and -top heights, physical and optical thickness, and temperature. The temperature dependence of each cloud parameter is compared to that simulated in the GISS GCM to understand the nature of cloud-optics feedback in the GCM in climatechange simulations.

Results to Date: Data from all instruments have now been analyzed for 6 months, including several warm periods in which optical thickness decreases with temperature and one cold month in which it increases with temperature. This behavior is consistent with the satellite findings. In the warm months, MWR liquidwater path consistently decreases with temperature at most temperatures, and this is invariably explained by cloud physical thickness variation with temperature. Liquid-water content is subadiabatic and does not increase with temperature at all. In the one cold month, liquid-water path and cloud physical thickness increase with temperature, instead. There are hints that the cloud physical thickness depends on moist stability, but the synoptic dynamics appear to play a role as well. The observed temperature dependence is consistent with that in the GCM and is indicative of the cloudoptics feedback in a doubled- $\mathrm{CO}_{2}$ climate change. Simulations with and without low-cloud-optics feedback in the GCM show that its effect is to reduce the polar amplification of warming, which in turn alters climate changes in the general circulation.

\section{TREATMENT OF HETEROGENEOUS SURFACE FLUXES IN GLOBAL CLIMATE MODELS \\ DORAN, J. CHRISTOPHER \\ PACIFIC NORTHWEST NATIONAL LABORATORY \\ FY 1996378 \\ FY 1995420 \\ FY 1994420}

Keywords: global change, fluxes, surface boundary conditions, inhomogeneous fluxes, heterogeneous fluxes, climate modeling

Objectives: To develop methods to characterize accurately the surface heat and moisture fluxes over the SGP CART Site, and to determine the sensitivity of single-column models (SCMs) and GCMs to variations in those fluxes that are not explicitly resolved in a domain the size of the SGP Site.

Approach: The analysis of data collected routinely as part of the CART operations will be combined with the collection and analysis of additional data from supplementary intensive operation periods (IOPs) and the use of mesoscale and single-column models for numerical simulations and testing of parameterization schemes. Sensible- and latent-heat-flux data collected at the CART Site are combined with other meteorological data and incorporated into a surface-energy-exchange model to calculate the spatial distribution of fluxes over the CART Site. These flux data are used to provide lower boundary conditions in numerical models to test the sensitivity of the models' predictions to various formulations of the boundary conditions and to compare these predictions against observations. The results are then analyzed to determine methods for specifying surface fluxes that will allow models to incorporate any significant effects of spatial heterogeneity into their predictions.

Results to Date: Work was completed on the analysis of data collected near Boardman, Oregon, during two field experiments in 1991 and 1992. These experi- 
ments on the effects of inhomogeneous fluxes on boundary-layer properties were conducted before fluxmeasurement instrumentation was available at the SGP CART Site.

An empirical method for estimating sensible- and latent-heat-flux values over the CART Site was developed. Although the method is limited in its applicability, it provided an initial estimate of flux variations that was useful in modeling studies of the effects of flux differences on cloud formation. A modeling study of the sensitivity of boundary layer cloud formation to inhomogeneous surface fluxes was begun. Work continued on the application of the Simple Biosphere (SiB) model to obtain detailed flux maps of the CART Site. A number of difficulties in the application of the model and in the preparation of the interpolated data fields needed to drive the model were identified, and necessary corrections are being implemented.

An IOP was carried out in late June and early July at the CART Site. Multiple temperature and humidity soundings were taken at three sites distributed about the central facility within a radius of approximately $125 \mathrm{~km}$. The sounding clearly demonstrated the variations in boundary-layer depths, temperature, and humidity associated with spatially varying surface fluxes.

Work was begun to test the sensitivity of an SCM to parameterizations of surface conditions that determine flux. Initial efforts have been devoted to investigating the performance of the biosphere-atmosphere transfer scheme (BATS) used in the model to calculate the surface fluxes.

\section{REMOTE-SENSING TECHNIQUES FOR OBSERVING MORPHOLOGY, MICROPHYSICS, AND RADIATIVE PROPERTIES OF CLOUDS AND TESTS WITH A NEW, ROBUST CO 2 LIDAR \\ EBERHARD, WYNN; INTRIERI, JANET; and BREWER, ALAN \\ NOAA ENVIRONMENTAL TECHNOLOGY LABORATORY \\ FY 199662 \\ FY 1995119 \\ FY 1994220}

Keywords: lidar, clouds

Objectives: To develop new capabilities involving special attributes of infrared lidar for the simultaneous measurement of cloud geometrical, microphysical, and radiative properties.

Approach: A new type of infrared lidar will be developed and tested that will have a high pulse rate with an RF-excited laser as the transmitter, a higher frequency stability for improved Doppler accuracy, dual-wavelength capability, reduced maintenance and operator interaction (to evaluate potential for engineer- ing an infrared lidar for unattended operation), and a longer wavelength where greater absorption by ice makes theoretical prediction of lidar signals from nonspherical ice particles easier. New techniques will be analyzed and field evaluated to measure the mean or effective radius of nonprecipitating cloud drops averaged along a CO lidar path; to discriminate ice and water clouds with a $\mathrm{CO}$ dual-wavelength technique; to improve the retrieval of cirrus microphysics and emissivity from combined $\mathrm{CO}$ lidar, mm-wave radar, and infrared radiometer measurements and to investigate extending this technique to visible-wavelength lidar, and to explore what additional or improved microphysical and dynamic information becomes available by combining vertical Doppler lidar measurements with lidar backscatter or with vertical Doppler radar.

Results to Date: The laboratory development was completed of major components of the new lidar (so far, for a single wavelength), including discovery and solution of some significant technical problems. The construction of a field container was finished. Measurements were made with older lidar during spring 1994 for evaluating the technique for mean or effective drop radius; analysis of these lidar and infraredradiometer data was started, and aircraft in situ data were received for validation. Doppler simulations so far have investigated the potential information from using differential vertical velocity from Doppler lidar and Doppler radar. The differential velocity depends in a complicated way on particle habit and orientation and on median size and breadth of the size distribution. Only in some situations can the differential velocity provide additional information on particle microphysics or assist in separating air and particle motions. Therefore, differential velocity can be useful in case studies with intensive measurements but has minor value for general cloud sensing.

\footnotetext{
LONGWAVE RADIATION CODES FOR CLIMATE STUDIES: VALIDATION WITH ARM OBSERVATIONS AND TESTS IN GENERAL CIRCULATION MODELS ELLINGSON, ROBERT, and BAER, FERDINAND UNIVERSITY OF MARYLAND

FY 1996333

FY 1995350

FY 1994291

Keywords: longwave radiation models

Objectives: To develop an observationally calibrated, optimum longwave radiation model and to test it in general circulation models.

Approach: An optimum longwave radiation model will be developed for use in GCMs that has been calibrated with state-of-the-art observations; the impact of the longwave radiative forcing will be assessed in a
} 
GCM; the sensitivity of a GCM to the radiative model used in it will be determined; and the contribution of the longwave radiative forcing will be determined in comparison with shortwave radiative forcing, sensible heating, and thermal advection and expansion.

Results to Date: Our most recent analysis of model calculations with a subset of corrected AERI data show that clear-sky downward flux at the surface may be calculated to within about $5 \mathrm{~W} \mathrm{~m}-2$ for some selected case-study periods. This is a reduction of the uncertainty of model calculations by about a factor of 4 from previously accepted values based on pyrgeometer data. Uncertainties in the routine water-vapor observations provide the current limitation on these comparisons.

Our analysis of cloudiness and pyrgeometer shows that the general form of the variation of effective cloud amount $\left(\mathrm{N}^{*}\right)$ with bulk cloud parameters follows that predicted by our previous theoretical work. The results tend to favor the fractal distribution of spacings. This is the first validation of the form of the dependence of $\mathrm{N}^{*}$ on bulk cloud parameters with independently measured data at the surface.

We tested the effect of longwave radiative forcing in a GCM with all model features held fixed by running the CCM2 with two different heating rate algorithms, the NCAR version native to the model and the ECMWF algorithm implanted. To determine the effect of climatic variability, we ran ten realizations of the CCM2 with only slight variations in the initial states. The resulting mean heating rates show climate variability (deviation) on the order of $10 \%$ or more. When the realization means of the CCM2 with the NCAR algorithm are compared with the climate mean of the model with the ECMWF algorithm, the differences are three to four times as large as the individual climate variability. This highlights the importance of the algorithm in the model evolution.

CONTINUED DEVELOPMENT OF AN
INTEGRATED SOUNDING SYSTEM
GAGE, KENNETH; WESTWATER,
EDGEWORTH; HAN, YONG; SHAW, JOSEPH;
and CHURNSIDE, JAMES
NATIONAL OCEANIC AND ATMOSPHERIC
ADMINISTRATION and UNIVERSITY
OF COLORADO
FY 1996 295
FY 1995328
FY 1994200

Keywords: water-vapor profiles, microwave radiometers, radioacoustic sounding systems, lidars, Raman lidars

Objectives: To develop and apply techniques for deriving radiatively important meteorological parameters from remote-sensing data.
Approach: Water-vapor profiles will be derived from combined remote-sensor measurements from SGP and TWP CART sites, including those from microwave radiometers, radio-acoustic sounding systems, cloud lidars, and Raman lidars. We have developed a statistical-physical profile-retrieval algorithm for operational implementation. A Fourier-transform infrared radiometer (FTIR) has been modified for deployment on the NOAA Research Vessel Discover, which will sail in TWP waters.

Results to Date: We developed a method to derive water-vapor profiles during cloudy conditions that yields significant improvement over previous methods to derive profiles or column abundances of vapor or cloud liquid. We modified the FTIR to operate with or without liquid-nitrogen-based detectors and are currently comparing the data in the two modes.

We analyzed TWP data taken during the PROBE experiment in Kavieng, Papua New Guinea, in January and February 1993. We identified ten FTIR soundings during the time of radiosonde launches and delivered these to ARM Science Team investigators. We also analyzed observations of cloud liquid by wind profilers and microwave radiometers, radiometric observations of water vapor by radiometers, and comparisons of ground- and satellite-based measurements of water vapor. We participated in the ARM portion of the VORTEX experiment, conducted in the OklahomaTexas region during April and May 1995, by flying the NOAA Environmental Technology Laboratory's airborne microwave water substance radiometer on the NOAA P3 aircraft. Numerous research flights were conducted over several CART sites in the SGP. A unique data set was obtained on remote and in situ measurements of water vapor and cloud liquid water, as well as on solar irradiance.

Our FTIR measurements taken during PROBE showed that the modeling of the "water-vapor continuum" was inadequate, and new corrections to ARM radiation codes have been made, based on these data.

We have processed about $25 \%$ of the VORTEX data and verified that the AMWSR instrument was operating properly and was calibrated to an absolute accuracy of about $0.5 \mathrm{~K}$. Physically reasonable values of cloud-liquid path were measured as a function of aircraft altitude, and comparison with in situ data is in progress. 
SHIPBOARD MEASUREMENTS OF THE CLOUD-CAPPED MARINE BOUNDARY

LAYER DURING FIRE/ASTEX

FAIRALL, C. W., and KROPFLI, ROBERT A. NATIONAL OCEANIC AND ATMOSPHERIC ADMINISTRATION

FY 1996297

FY 1995290

FY 1994233

Keywords: marine boundary layer

Objectives: To develop and improve remote-sensing instruments and techniques for measuring cloud radiative and microphysical properties and to improve physical parameterizations in atmospheric models. Approach: The broad experience of the Environmental Technology Laboratory (ETL) in developing remote-sensing technologies and analysis techniques for cloud macrophysical, microphysical, and radiative properties will be exploited to deploy several new systems for the Atlantic Stratocumulus Transition EXperiment (ASTEX).

Results to Date: A new scanning Doppler lidar was deployed in the field for the first time (at Porto Santo). The ETL millimeter-wave cloud radar was also deployed at Porto Santo, the first use of such a radar in the marine environment. A unique, seagoing integrated-flux and remote-sensing system was deployed on the R/V Malcom Baldridge for operations in the ASTEX region. ASTEX has not only provided an opportunity to test and improve instruments and techniques for future CART Site efforts but has provided a new data set that is being used to improve cloud microphysical/radiation models and parameterizations.

\section{ANALYTICAL AND MODELING STUDY OF THE EFFECTS OF THE LOW-LEVEL JET ON CLOUDS AND RADIATION AT THE SGP CART SITE GHAN, STEVEN; ZHONG, SHARON; WHITEMAN, C. DAVID; BIAN, XINDI PACIFIC NORTHWEST NATIONAL LABORATORY \\ FY 1996144 \\ FY 1995180 \\ FY 1994174}

Keywords: clouds, low-level jet, general circulation models, diurnal cycles, thunderstorms

Objectives: To determine if or to what degree the lowlevel jet (LLJ) climatology and its impact on clouds and precipitation are being simulated by current GCMs, to improve the understanding of the interactions between the $L L J$ and the environment that lead to cloud formation, and to develop an improved cloudparameterization scheme for GCMs that incorporates the influence of the jet on clouds.
Approach: The ability of present GCMs to simulate the LLJ and its influence on clouds will first be assessed by comparing simulated and observed climatologies of LLJ frequency and the diurnal cycle of convective precipitation over the continental United States. Surface weather observations from the Oklahoma MESONET and wind observations from rawinsondes and from the NOAA Radar Profiler Demonstration Network will be analyzed to identify the mechanisms responsible for the initiation, maintenance, and decay of the LLJ; its characteristic structure; and its effects on clouds and radiation at the ARM sites. Numerical experiments with the Colorado State University Regional Atmospheric Modeling System (RAMS) will be used to improve understanding of the cloud forcing mechanisms related to the LLJ so that a parameterization can be developed.

Results to Date: Two GCM simulations have been analyzed for the presence of the LLJ. The simulated distributions of LLJ frequency are in remarkable agreement with the observed climatology, suggesting that GCMs can simulate the LLJ. However, neither GCM is able to simulate the observed nocturnal maximum in thunderstorm frequency associated with the diurnal cycle of the LLJ, suggesting that the relationship between clouds and the LLJ will have to be parameterized. Analysis of wind profiler measurements has revealed a strong LLJ on several days during the June 1993 IOP with important effects on moisture convergence and vertical motion at the ARM site in Oklahoma. Analysis of mesoscale-model simulations suggests that inertial oscillation resulting from the nocturnal relaxation of friction is the primary driving mechanism for at least one LLJ case. Sensitivity experiments with different soil moisture suggest that soil moisture influences the amplitude of the diurnal cycle of the LLJ through release of latent heat associated with precipitation related to the LLJ.

\section{DEVELOPMENT OF A STRATIFORM CLOUD PARAMETERIZATION \\ GHAN, STEVEN, and RUBY, LEUNG \\ PACIFIC NORTHWEST NATIONAL \\ LABORATORY \\ FY 1996171 \\ FY 1995190 \\ FY 1994184}

Keywords: cloud microphysics, GCMs

Objectives: To use surface and satellite observations to evaluate the parameterization of stratiform cloud properties and processes in a GCM and in a regional circulation model.

Approach: Four-dimensional data assimilation will be applied to global and regional circulation models and simulated cloud and radiation fields will be compared with surface and satellite observations. 
Results to Date: The climatological planetary radiation balance simulated by the global model is quite realistic. The outgoing longwave radiation simulated by the regional circulation model is too high, which indicates that insufficient high clouds are present. Both models simulate too little supercooled cloud water. Four-dimensional data assimilation in the global model yields daily agreement with observations. Simulated droplet-number concentrations are in good agreement with observations. Simulations with a single-column model driven by observations yielded poor agreement with observations.

\section{CLOUD EFFECTS ON RADIATION AT THE TOP OF THE ATMOSPHERE AND SURFACE GAUTIER, CATHERINE \\ UNIVERSITY OF CALIFORNIA, SANTA BARBARA}

$\begin{array}{lll}\text { FY } 1996 & 143 \\ \text { FY } 1995 & 190 \\ \text { FY } 1994 & 121\end{array}$

Keywords: inhomogeneous clouds, parameterization of cloud effects

Objectives: To study the effect of inhomogeneous clouds on radiation at the top of the atmosphere and at the suface and to contribute to the development of parameterization of cloud effects on radiation for climate modeling and remote-sensing data interpretation.

Approach: Observations and modeling studies will be used to analyze multisensor data with regards to cloud effects on surface radiation, and numerical simulations of 3-D cloud-radiation interaction will be used to understand the effects of clouds on radiation.

Results to Date: Three-dimensional-modeling results have helped us unravel the effects of 3-D clouds on atmospheric absorption. We found that different physical processes are at work (absorption by water vapor or liquid water) that depend on sun zenith angle and wavelengths. Their combined effect is complex and depends on cloud morphology.

\section{MEASUREMENTS AND MODELING OF THE EFFECT OF CONVECTIVE CLOUDS ON THE UPPER-TROPOSPHERIC MOISTURE BUDGET \\ GOLDSMITH, JOHN E. M.; BISSON, SCOTT E; and DEL GENIO, ANTHONY D. \\ SANDIA NATIONAL LABORATORIES and \\ NASA GODDARD INSTITUTE FOR SPACE STUDIES}

FY 1996200

FY 1995250

FY 1994243
Keywords: water vapor, Raman lidar

Objectives: To determine the upper-tropospheric moisture budget associated with convective events, and in particular, to extend process models to higher altitudes than have been achieved previously.

Approach: We are implementing a GATE (global atmospheric research program Atlantic tropical experiment)-type approach at the ARM CART Site, taking advantage of the existing measurement capabilities at the central and boundary sites to estimate the large-scale heat and moisture budgets and tendencies. The Sandia Raman lidar system, located at the central site, provides accurate water-vapor measurements to higher altitudes and much better temporal coverage than possible from conventional radiosondes. Our primary goal is to determine the moisture budget of the upper troposphere on a scale similar to that of a GCM gridbox. Ultimately we will synthesize our results by validating the cumulus parameterization of the GISS GCM against the lidar data and then improve the physics of the model to produce a more faithful representation of upper-tropospheric water-vapor feedback. Results to Date: Our measurement strategy requires a concentrated, campaign-style measurement at the ARM CART central facility, plus a higher frequency of radiosonde launches from the three boundary facilities. The multipurpose campaigns conducted at the CART Site during April in both 1994 and 1995 provided ideal measurement periods. In preparation for these campaigns, we made several modifications to the Sandia Raman lidar to enhance its long-range performance. During the two 3-week campaigns, we collected lidar data for 120 hours during 23 nights of operation, including a night that featured an ideal opportunity to study the passage of a cold front. We are statistically analyzing our data sets to investigate trends in the variability of upper-tropospheric moisture and comparing those trends with simulations performed with the GISS GCM. Preliminary analysis indicates a bimodal distribution of water vapor in the middle troposphere, giving way to a more unimodal distribution at higher altitudes; the GCM shows hints of a bimodal distribution in the lower troposphere, but not above.

\section{ROLE OF DAY vs NIGHT RADIATION DIFFERENCES ON THE DIURNAL VARIABILITY OF TROPOSPHERIC PARAMETERS \\ GRAY, WILLIAM M. \\ COLORADO STATE UNIVERSITY \\ FY 199698 \\ FY 1995130 \\ FY 1994116}

Keywords: cloud radiative processes, intense convection 
Objectives: To determine the nature and specific role of cloud radiative processes in modulating the diurnal cycle (morning maximum) of intense convection over the tropical oceans.

Approach: Broad-area tropical satellite (GMS and Polar Orbiter) cloud-temperature data (OLR) are acquired and analyzed in relation to surface-based atmospheric-profiler data ( $\mathrm{u}, \mathrm{v}$, and $\mathrm{w}$ components), island rainfall (hourly) data, regional rawinsonde soundings, regional (forecast) circulation analyses, and time-averaged GCM circulation data for the region. The GMS OLR data (eight images per day) are of much higher resolution than that of the OLR data used in most prior studies. Complementary diurnal variations of relatively warm (clear) and cold (cloudy) OLR areas, consistent with inferred broad-scale subsidence in clear areas adjacent to strong ascent and rainfall in convection, are examined. The vertical components of time-averaged (wind) profiler data at island sites are compared with this diurnal cycle with a relatively strong nocturnal subsidence maximum observed for clear regions within the equatorial dry zone. The inferred amplitudes of these circulations are compared with mean GCM data.

Results to Date: New results further confirm that the early morning maximum of intense convection in the tropical Pacific is largely the result of deep vertical circulations initiated by day-night differences of radiative cooling in convective areas vs cloud-free areas. Interannual differences of the strength and distribution diurnal variability over larger areas has been examined; this includes comparisons and syntheses of results for higher-resolution (level B1) GMS OLR data with surface-based wind-profiler data and with time-averaged GCM output. Analyses show:

- A stronger diurnal cycle occurs in very cold $\left(<65^{\circ} \mathrm{C}\right)$ cloud-top temperatures over ocean areas than has been reported previously for less detailed data.

- Cloud-free ocean areas adjacent to intense convection have a distinct early-morning peak of very warm $\left(+15\right.$ to $\left.+20^{\circ} \mathrm{C}\right)$ blackbody temperatures that are consistent with strong nocturnal subsidence in these areas.

- Collectively, these very warm and very cold areas continuously occupy as much as 20 to $30 \%$ of the tropical west Pacific.

- Inferred vertical and horizontal circulations are generally confirmed in profiler data.

- Both favorable and unfavorable comparisons are found in GCM output data.

\author{
IMPROVED CLOUD RADIATION \\ PARAMETERIZATION FOR GCMs \\ KIEHL, JEFFREY; HACK, JAMES; and \\ RAMASWAMY, $V$. \\ NATIONAL CENTER FOR ATMOSPHERIC \\ RESEARCH and GEOPHYSICS FLUID \\ DYNAMICS LABORATORY \\ FY 1996163 \\ FY 1995159 \\ FY 1994296
}

Keywords: parameterization of clouds, parameterization of radiation

Objectives: To use ARM data to improve the parameterization of clouds and radiation in the NCAR CCM and to validate line-by-line models.

Approach: A single-column model ( $\mathrm{SCM}$ ) of the NCAR CCM will be developed and forced with data from the ARM SGP Site. A multiclass-cloud-microphysics scheme will be implemented in the SCM to test the role of microphysics on the simulations.

Results to Date: We have identified problems with the SGP forcing data that need to be addressed for SCM research. We have shown that inclusion of microphysics does improve agreement between modeled cloud properties and observations. We have also carried out a detailed surface analysis in the Tropical Western Pacific to identify the issue of enhanced cloud absorption.

\section{DEVELOPMENT AND TESTING OF AN AEROSOL/STRATUS CLOUD PARAMETERIZATION SCHEME FOR MIDDLE AND HIGH LATITUDES KREIDENWEIS, SONIA, and COTTON, WLLLIAM COLORADO STATE UNIVERSITY FY 1996144 FY 1995160 FY 19940}

Keywords: Arctic stratus, cloud modeling, aerosol microphysics, cloud parameterization

Objectives: To develop an aerosol-cloud microphysics parameterization of mixed-phase stratus and boundarylayer clouds that responds to variations in cloud condensation nuclei (CCN) and ice nuclei (IN) concentrations for use in GCM simulations as a tool in understanding the role of $\mathrm{CCN}, \mathrm{IN}$, and Arctic clouds in radiation budgets.

Approach: Cloud-resolving, large-eddy simulations (LES) will be performed with explicit bin-resolving microphysics to provide guidance in the formulation of the parameterization scheme and calibration of coefficients. Several versions of the CSU Regional Atmospheric Modeling System (RAMS) will be used in this work, requiring development work to treat the stable Arctic atmosphere, radiation at high latitudes, and 
mixed-phase clouds. Both the LES/explicit-bin-resolving microphysics model and the final, parameterized model will be tested with data acquired at the SGP and NSA CART sites.

Results to Date: Our work has shown the inapplicability of standard bulk-microphysics schemes to summertime Arctic clouds; these schemes cannot predict the onset or magnitude of precipitation in such clouds. We have developed a new hybrid scheme that combines features of the bin-resolving model, which is able to better represent Arctic warm cloud microphysics, with the computational efficiency of the bulk scheme. Our present efforts are directed at extending the microphysics treatment to the ice phase, including representation of mixed-phase clouds. Our studies have also demonstrated the extreme sensitivity of Arctic clouds to the representation of microphysics and radiation; predicted cloud evolution varies widely with relatively small changes in these properties, confirming the importance of this research in developing improved understanding of Arctic cloud systems as elements in the Earth's radiation budget. In response to the results from exploratory modeling, we are developing new radiation schemes that accept predicted drop and ice size distributions and include their effects on radiative transfer. We have also examined the effects of choice of turbulence closure scheme on model results; most commonly used schemes are inappropriate for the stable Arctic atmosphere. Our ongoing LES modeling work will provide guidance on improving this model feature.

\section{PARAMETERIZATION OF THIN MIDLEVEL STRATIFORM CLOUDS \\ KRUEGER, STEVEN, and SASSEN, KENNETH UNIVERSITY OF UTAH \\ FY 199695 \\ FY 1995100 \\ FY 1994100}

Keywords: altocumulus, clouds, parameterization, cloud-resolving models, GCSS

Objectives: To increase our understanding of the physical processes that determine the distribution of altocumulus clouds and their effects on the atmosphere, and to develop and test a physically based parameterization for altocumulus layers for use in GCMs.

Approach: We are using a high-resolution cloud-scale numerical model, the University of Utah Cloud Resolving Model (CRM), and the ARM-CART measurements to: (1) Determine how large-scale and cloud-scale processes govern the structure of altocumulus layers; (2) Determine how altocumulus clouds modulate the radiation field; (3) Determine the extent to which altocumulus clouds are parameterizable in terms of large-scale processes; (4) Develop and test an elevated- mixed-layer model to diagnose vertically subgrid-scale altocumulus cloud layers within a GCM; and (5) Develop and test a parameterization of altocumuluscloud-layer optical properties (liquid-water path and effective mean radius) for use in climate models.

Results to Date: Our numerical simulations of altocumulus (Ac) clouds with a 2-D cloud-resolving model (CRM) that includes third-moment turbulence closure and an advanced radiative-transfer code suggest that Ac layers are well mixed. This motivated development of two simpler models for Ac layers: a 1-D turbulenceclosure model (TCM) and a mixed-layer model (MLM). The results of the TCM closely match those of the 2-D CRM, while the MLM results compare fairly well. We performed CRM simulations of an Ac layer observed over the SGP CART Site on April 24, 1994. Simulated cloud features agree reasonably well with in situ aircraft and ground-based remote-sensing measurements. We are also comparing calculated and measured downwelling broadband radiative fluxes at the surface for this case.

We are participating in the GEWEX Cloud System Study (GCSS). The goal of GCSS is to develop better parameterizations of cloud systems for GCMs through simulations with CRMs. As part of a GCSS project, we compiled the results of a model intercomparison based on simulations of the two Atlantic Stratocumulus Transition Experiment (ASTEX) Lagrangian experiments. We helped to design another GCSS project that will focus on convective cloud systems observed during TOGA COARE over the western tropical Pacific Ocean.

\section{ANALYSIS OF CLOUD RADIATIVE FORCING AND FEEDBACK IN A CLIMATE GCM LACIS, ANDREW; CARLSON, BARBARA; DEL GENIO, ANTHONY; CAIRNS, BRIAN; and MISHCHENKO, MICHAEL \\ NASA GODDARD INSTITUTE FOR SPACE STUDIES \\ FY 1996200 \\ FY 1995250 \\ FY 1994250}

Keywords: general circulation models, cloud parameterization, radiative fluxes

Objectives: To refine and validate the GISS GCM radiation code through model intercomparisons and comparison to ARM and satellite observations.

Approach: We are pursuing a broad range of rigorous theoretical studies to develop better models for computing scattering, absorption, and thermal emission by realistic distributions, sizes, and shapes of cloud and aerosol particles, including line-by-line absorption by atmospheric gases and the water-vapor continuum. This information then serves as the basis for develop- 
ing improved GCM parameterizations. We then compare GCM-generated solar and thermal fluxes with ISCCP, ERBE, and ARM measurements.

Results to Date: We have developed a GCM parameterization to account for enhanced absorption of solar radiation by cloud inhomogeneities. We have incorporated windblown desert dust and Mt. Pinatubo volcanic aerosols into the GISS GCM with explicit dependence on aerosol size and spatial distributions. We have performed detailed theoretical modeling of the radiative-scattering properties of nonspherical cloud and aerosol particles. We have made improvements on the theoretical model of water-vapor-continuum absorption.

\section{VARIATIONAL SINGLE-COLUMN DATA ASSIMILATION FOR ARM LOUIS, JEAN-FRANCOIS

Keywords: data assimilation, adjoint methods, data fusion

Objectives: To create a continuous analysis of the atmospheric structure over the ARM sites at a scale compatible with typical climate models and to compute the sensitivity of radiation computations to the various model parameters and input.

Approach: For the continuous analysis of the atmospheric structure, we use a variational data-assimilation method that adjusts a model simulation of the evolution of the atmosphere until the difference between simulated and observed variables is minimized. The adjoint of the model is used to compute the gradient of the forecast error with respect to nudging coefficients that are used to force the model closer to the observations. This adjustment is done iteratively with a minimum-search technique. Because the adjoint allows the computation of the gradient (or sensitivity) of any model output with respect to all the model variables and parameters, the second objective is a byproduct of the first.

Results to Date: We have developed the adjoint of the single-column model used for data assimilation, including the adjoint of the complete physical parameterizations of the model. We have explored several ways of defining the nudging coefficients, settling on a definition that includes a smooth variation in the vertical and a diurnal-cycle evolution. The data-assimilation system allows the use of radiosondes, surface observations, and surface-flux measurements. The convergence of the minimization experienced difficulties because of threshold processes related to clouds and convections; these problems are being investigated. Large errors in the observations of surface fluxes of latent and sensible heat also produced difficulties. In addition, the large-scale analyses that are needed to define the horizontal forcing for the model have not been available during the past year.

We also made a detailed study of the sensitivity of surface and tropopause temperature tendency to the optical characteristics of gases and clouds in the spectral domain.

\section{DEVELOPMENT OF CLOUD PARAMETER RETRIEVAL METHODS FOR CART MATROSOV, SERGEY Y., and KROPFLI, ROBERT A. \\ NATIONAL OCEANIC AND ATMOSPHERIC ADMINISTRATION \\ FY 199657 \\ FY 199557 \\ FY 19940}

Keywords: cloud microphysics, radar, IR-radiometer, microwave radiometers, remote sensing

Objectives: To enhance and verify the remote-sensing method to retrieve microphysical parameters of ice clouds from ground-based measurements taken by radar and radiometer.

Approach: The method will be improved by incorporating more-advanced schemes for the estimation of cloud optical thickness and considering radar reflectivity dependence on cloud particle shapes and densities. The method will be applied to ice-cloud data sets collected during recent field experiments. The results obtained remotely will be validated by comparing them with simultaneous data obtained by direct measurements and by other remote-sensing approaches.

Results to Date: The method to obtain ice-cloud parameters remotely from combined radar-radiometer measurements was applied to a number of data sets collected by the NOAA ETL radar and radiometers during FIRE-II, ASTEX, and the 1995 Arizona Program. The observed clouds were located in the upper troposphere. Their geometrical thickness varied from a few hundred meters to $3 \mathrm{~km}$. The variability of cloud parameters was assessed from the retrieval results. Characteristic cloud-particle sizes expressed in median mass diameters of equal-volume spheres varied from about $\mathbf{2 5}$ microns to about $\mathbf{4 0 0}$ microns. Typically, these sizes were larger in the lower part of the cloud, with the exception of the lowest-range gates, where particles were evaporating. The highest particle concentrations were usually observed near the cloud tops. The vertical variability of median sizes inside an individual cloud could reach (and even exceed) one order of magnitude; however, the standard deviations from the mean profile for a typical cloud were usually a factor of 2 or 3 smaller than the mean values of sizes. Typical values of retrieved cloud ice-mass content 
varied from about 1 to $100 \mathrm{mg}$ per cubic meter; however, individual variations were as high as 4 orders of magnitude. There was no consistent pattern in the vertical distribution of cloud ice-mass content. The power-law relations between retrieved cloud microphysical parameters and measured radar reflectivities were obtained. The parameters of these relations varied from cloud to cloud, the exponent generally exhibit ing lesser variability than the coefficient. The variability of these relations diminished only when stronger reflectivities (greater than $-20 \mathrm{dBZ}$ ) were considered.

\section{THE USE OF GROUND-BASED MILLIMETER-WAVE CLOUD PROFILING RADAR SYSTEM (CPRS) TO MEASURE CLOUD WATER AND ICE MCINTOSH, ROBERT UNIVERSITY OF MASSACHUSETTS FY 1996204 \\ FY 1995240 FY 1994221}

Keywords: cloud profiling radar system, radar, clouds Objectives: To determine the water and ice concentrations in clouds.

Approach: MIRSL has been gathering cloud data for a wide variety of cloud types at the Southern Great Plains CART Site and other locations in our efforts to develop model functions that allow scientists to interpret millimeter radar images of clouds. MIRSL will participate in several DOE-sponsored experiments with both its 33/95-GHz Cloud Profiling Radar System and $95-\mathrm{GHz}$ radar. We will collaborate with other researchers within ARM to improve existing radar cloud models and to develop new models for deriving cloud properties from dual-frequency radar.

Results to Date: The first dual-frequency measurements of Mie scattering in cirrus clouds were obtained as predicted by Matrosov, and a novel scheme for selfcalibrating measurements was developed. Dual-frequency full-spectrum Doppler (FFT) measurements were made of precipitating clouds and cirrus. Simultaneous measurements were made of the copolar correlation coefficients, $\mathrm{HV}$, and differential phase constants at the $\mathrm{Ka}$ and $\mathrm{W}$ bands. Neural nets were used for classification of cloud-particle phase with multifrequency millimeter-wave radar data. Millimeter cloud profiles were obtained concurrently with solar radiation measurements with radiometers mounted on an unmanned aerospace vehicle (UAV).

\section{SCANNING RAMAN LIDAR TO MEASURE \\ WATER VAPOR AND AEROSOLS FOR THE STUDY OF ATMOSPHERIC RADIATIVE TRANSFER}

MELFI, S. HARVEY; FERRARE, RICHARD; WHITEMAN, DAVID; ELLINGSON, ROBERT; and STARR, DAVID

UNIVERSITY OF MARYLAND, BALTIMORE

COUNTY, and HUGHES STX, NASA GODDARD SPACE FLIGHT CENTER

FY 1996200

FY 1995250

FY 1994250

Keywords: Raman lidars, water vapor, aerosols

Objectives: To acquire detailed measurements of water vapor and aerosols and to use these data to test radiation parameterizations with the suite of spectral and flux measurements at the CART site.

Approach: The GSFC scanning Raman lidar (SRL) will be used to supplement the CART radiation measurements with measurements of water vapor and aerosols. These lidar data will be used to determine the ability of radiation codes to model radiative transfer under both clear and hazy conditions. The Raman lidar aerosol measurements will also be compared with the CART surface aerosol measurements to assess the representativeness of the surface aerosol measurements and to investigate the relationships between the physical and optical properties of the aerosols and water vapor.

Results to Date: The SRL acquired water-vapor and aerosol-profile data during the first ARM Remote Cloud Sensing IOP held in April 1994 at the SGP CART Site. Our comparison of water-vapor measurements made by radiosondes, aircraft sensors, a microwave radiometer, and tower instrumentation revealed unexpectedly large differences in water-vapor amounts among these instruments. The lidar data have also been used to: (1) examine how changes in relative humidity affect aerosol extinction and backscattering and longwave atmospheric emission, (2) investigate the temporal and spatial scales of atmospheric variability, (3) study the structure and dynamics of a cold front in conjunction with the data provided by the Oklahoma surface mesonet, and (4) compare the aerosol optical properties measured by the lidar with those computed from aircraft aerosol-size-distribution measurements. 


\section{COMPLETION OF SPECTRAL ROTATING SHADOWBAND RADIOMETERS AND ANALYSIS OF ARM SPECTRAL SHORTWAVE DATA \\ MICHALSKY, JOSEPH, and HARRISON, LEE STATE UNIVERSITY OF NEW YORK AT ALBANY \\ FY 1996250 \\ FY 1995278 \\ FY 1994242}

Keywords: shortwave irradiance, spectral irradiance, multifilter rotating shadowband radiometer, rotating shadowband spectroradiometer, aerosols, ozone, water vapor, cloud optical depth

Objectives: To develop a high-quality data set of cloud- or aerosol-modified direct and diffuse solar irradiances that allow improved GCM parameterizations, to develop a charge-coupled-device (CCD) array rotating shadowband spectroradiometer (RSS), and to develop analytical techniques for the retrieval of cloud properties and atmospheric traces species that affect atmospheric radiation transfer.

Approach: Surface shortwave and longwave data collected at Albany, New York, is combined with National Weather Service data from the Albany County airport to provide data for the refinement of radiation codes in GCMs.

Results to Date: The RSS uses a CCD array to sense spectral radiation between 360 and $1050 \mathrm{~nm}$, simultaneously measuring 512 distinct wavelengths with 0.6-nm resolution in the ultraviolet and 8- $\mathrm{nm}$ resolution in the near-infrared. The prototype met our design goals for the RSS. A field-hardened version is being assembled for deployment at the SGP CART Site.

The current multifilter rotating shadowband radiometer (MFRSR) produces spectral direct and diffuse irradiance data at six wavelengths. Techniques for using these data to retrieve aerosol optical depth as a function of wavelength, total-column ozone, and total-column water vapor have been developed and are being validated. Additionally, cloud cover and cloud optical and microphysical properties are retrievable from MFRSR data.

Five years of radiation and meteorological data, including downwelling shortwave and longwave irradiance have been archived for Albany, New York. The clear-sky data have been used to test radiation codes in the GENESIS GCM. The infrared agreement appears satisfactory. The shortwave model produces higher irradiances than are measured, although some improvement has resulted after the incorporation of aerosol data. The RSS, which met our design criteria in the laboratory, showed instability in field use. We have determined the problems, redesigned to avoid these, and are assembling a new unit. The key to good retrievals of aerosol optical depth, total-column water vapor, total-column ozone, and cloud properties is careful calibration and characterization of the instrument and proper operation in the field. We believe that we can use our calibration facilities and our retrieval procedures to estimate aerosol optical depth to an accuracy of 0.005 or better, ozone column to $5 \%$ or better, and water vapor to $10 \%$ or better. Further, we have developed procedures for estimating cloud optical depth using the ratio of diffuse to direct irradiance and surface albedo at several MFRSR wavelengths based on a fast radiative-transfer calculation and a leastsquares fitting procedure. Optical depths of thick clouds appear underestimated by satellites in comparison to this procedure.

\section{IMPROVEMENTS IN MODELING THE MICROPHYSICAL AND RADIATIVE PROPERTIES OF CIRRUS CLOUDS WITH THE REGIONAL ATMOSPHERIC- MODELING SYSTEM \\ MITCHELL, DAVID L., and KORACIN, DARKO DESERT RESEARCH INSTITUTE \\ FY 1996113 \\ FY 1995150 \\ FY $1994 \quad 97$}

Keywords: microphysical properties of clouds, radiative properties of clouds, cirrus clouds, cloud-resolving models

Objectives: To improve the treatment of the microphysical and radiative properties of cirrus clouds in cloud-resolving models.

Approach: Model cirnis clouds are characterized by aircraft and remote measurements at the SGP CART Site with two regional-scale, cloud-resolving models (RAMS and MM5). A newly developed microphysical and radiation scheme is used in combination with the cloud-resolving models to predict the microphysical and radiative properties of these cirrus clouds. These predicted microphysical and radiative fields are compared with those predicted by RAMS and MM5 and with those observed. And an analytical approximation of Mie theory is being developed for treating extinction and absorption in water clouds; this should be significantly more accurate than current parameterizations and not be computationally prohibitive for large-scale models.

Results to Date: Resonance/surface-wave phenomena were found not to apply to ice particles. This finding has a significant impact on determining the absorption and extinction properties of ice clouds. A treatment for predicting the radiative properties of cirrus clouds was developed, which is applicable to two-stream radiationtransfer models. The absorption and extinction coefficients and the asymmetry parameter were formulated in terms of ice-particle shape, size-distribution parameters, and wavelength. The four main shapes found in 
cirrus were treated: hexagonal columns and plates, bullet rosettes, and planar polycrystals. The radiation scheme predicts that the 3-D crystal types reflect sunlight about twice as efficiently (per unit mass) as do the pristine hexagonal forms. The radiation scheme was validated against two cirrus case studies. In each case, the observed mean ice-particle size and ice-water path (IWP) were retrieved by the radiation scheme, based on the observed albedo, emittance, and iceparticle shape. This was the first time IWP retrievals have been successfully validated against in situ data. The cloud-resolving models RAMS and MM5 have been run for a SGP CART Site cirrus case study, which included aircraft microphysical measurements. A new microphysics module used ice mixing ratios from these models to predict microphysical fields. Based on observed particle shapes, predicted iceparticle sizes were similar to those observed. Concentrations were somewhat higher than measured by the 2D-C probe, but were considerably lower than measured by the FSSP. Predicted ice particle size and concentration were sensitive to the particle shape assumed. Through collaborative work, the cirrus radiation scheme was incorporated into NCAR's CCM2 along with a prognostic cloud-water scheme. Simulations show that just changing ice-crystal shapes from columns to bullet rosettes in the tropics may increase globally averaged albedo by $0.5 \%$. A new treatment for predicting the optical depth, absorptivity, and emissivity of water clouds was developed, yielding accuracies within about $2 \%$ relative to Mie theory. The treatment is analytical and computationally efficient. A new method for calculating ice-particle terminal velocities was developed, including ice-particle fall speeds in cirrus clouds. The method is very simple and efficient for use in large-scale models.

\section{DEVELOPMENT OF A SWIR SOLAR SPECTRAL RADIOMETER MURCRAY, FRANK UNIVERSITY OF DENVER

$\begin{array}{lr}\text { FY } 1996 & 105 \\ \text { FY } 1995 & 134 \\ \text { FY } 1994 & 0\end{array}$

Keywords: solar spectral radiometer

Objectives: To develop an accurate solar spectral radiometer for 1 to 5 microns.

Approach: Well-calibrated standard lamps have recently become available for the 1 - to 5 -micron region. However, these standards have about $1 \%$ of the intensity of the sun, making it difficult to transfer the calibration. We have begun construction of an instrument with demonstrated linearity over the entire dynamic range that can be operated in the field for accurate solar-radiance measurements. The instrument is integrated into a solar-tracking system, so the spectrometer is oriented in azimuth, and a small mirror tracks the elevation of the sun. The same mirror is used to view the calibration lamp, so the optics are identical in the sun and lamp measurements.

Results to Date: The spectrometer has been procured and tested. The linearity has been demonstrated, and the stability over one week is good. A calibration lamp has also been procured. Calibrated solar spectra with 2-wavenumber resolution have been obtained from Denver. The solar-tracking system is under construction. A few measurements were made from the ARM CART Site in Billings, Oklahoma, in September during the ARESE campaign with manual operation. These measurements included both clear-sky and thincirrus observations.

\section{DEVELOPMENT AND TESTING OF AN INTEGRATED DATA-ASSIMILATION AND SOUNDING SYSTEM (IDASS) \\ PARSONS, DAVID; DUDHIA, JM; COHN, STEPHEN; and KUO, YING-HWA (BILL) NATIONAL CENTER FOR ATMOSPHERIC RESEARCH \\ FY 1996540 \\ FY 1995600 \\ FY 1994580}

Keywords: integrated sounding systems, data assimilation, single-column models, nonhydrostatic models Objectives: To develop, test, and employ a highresolution data-assimilation system to derive accurate estimations of the boundary conditions to the ARM domain and high-resolution estimates of the 3-D conditions over the ARM domains of interest; to develop observational strategies for furthering this goal; and to use these measurements and the output from the data-assimilation scheme to investigate ARM science goals and to also make these measurements and assimilation products available to the ARM Science Team.

Approach: Measurements will include routine operational and ARM special measurements supplemented with observations taken by our integrated sounding systems (ISS). The data-assimilation experiments are being conducted with MM5, a nonhydrostatic version of the NCAR-Penn State Mesoscale Model. The assimilation tests include real-data work on a June 1993 IOP and Observation System Simulation Experiments (OSSEs). The accuracy of the assimilation scheme relative to objective analysis has been tested by both approaches.

Results to Date: The OSSEs show that objectiveanalysis schemes can produce significant errors that can render the products unacceptable for many SCM applications at sparse observational densities because of aliasing problems. Data assimilation is likely to outperform objective analysis in the presence of sharp 
spatial and temporal gradients, such as those that occur with clouds. Unfortunately, these conditions are of primary importance for ARM. The results from a tenday real-data experiment based on the June IOP shows that the mean errors from data assimilation over the ARM CART Site are typically on the order of $1^{\circ} \mathrm{C}$ and 1 to $2 \mathrm{~m} / \mathrm{s}$. A high-resolution simulation was conducted with a $2.2-\mathrm{km}$ version of the MM5 model nested as the inner grid for a $12-\mathrm{h}$ period within the June IOP experiment.

\section{CLOUD PARAMETERIZATIONS IN GLOBAL CLIMATE MODELS: THE ROLE OF AEROSOLS PENNER, JOYCE LAWRENCE LIVERMORE NATIONAL LABORATORY \\ FY 1996150 \\ FY 1995200 \\ FY 1994194}

Keywords: aerosol effects on clouds, radiative effects Objectives: To validate the LLNL coupled global aerosol/GCM model with ARM measurements and to assess the possible magnitude of the aerosol-induced cloud effects on climate.

Approach: The LLNL model GRANTOUR will be developed by compiling global emissions inventories for aerosols and for gas-phase species that form aerosols, by developing a module to treat gas-toparticle conversion processes for multicomponent aerosols and aqueous-phase transformation processes, and by coupling the improved aerosol model to a GCM for prediction of cloud optical properties and radiative interactions that result from changes to aerosols. A detailed microphysics model of aerosol/cloud interactions will be used to develop a parameterization suitable for use in a GCM of the effects of increasing emissions of aerosols on cloud microphysical properties. Data from the ARM sites will be used to evaluate the predictions of this model.

Results to Date: Global emissions inventories have been developed for (1) particulate matter (e.g., black carbon, organic carbon, and other minor species) produced from wood and dung fuel, charcoal burning and charcoal production, agricultural fires, and savannah and forest burning; (2) black carbon and organic matter produced from diesel and coal sources; and (3) emissions of natural organic matter. The accuracy of these new inventories was tested by a thorough comparison with observations. These aerosols presently form the pre-existing aerosol for a parameterization of the effects of anthropogenic sulfur emissions on cloud droplet concentrations. This has been applied in a global model to estimate the forcing by the indirect effect of anthropogenic sulfate emissions on cloud droplet concentrations. Pre-existing dust aerosols have also been modeled, and on a monthly average basis their effects have been shown to be small. Future work will aim to validate the parameterization by comparison with ARM data.

\section{USE OF LIDAR/RADIOMETER (LIRAD) TO OBTAIN OPTICAL PROPERTIES AND MICROPHYSICS OF HIGH AND MIDLEVEL CLOUDS \\ PLATT, CHARLES MARTIN RICHARD \\ COMMONWEALTH SCIENTIFIC AND \\ INDUSTRIAL RESEARCH ORGANISATION \\ FY 199680 \\ FY 199585 \\ FY 199474}

Keywords: clouds, atmospheric radiation, cloud ice formation, radiometry, IR radiometer, lidar

Objectives: To determine the key optical properties of clouds, particularly high clouds, by ground-based remote sounding for use in cloud/radiation models.

Approach: Cloud height, infrared and visible optical depth, cloud phase, and information on ice crystal habit and size will be determined with a combination of ground-based remote-sensing instruments. These include lidar/radiometer (LIRAD) techniques and, recently, additional information from millimeter radar and microwave radiometry. Information on crystal habit is inferred from lidar backscatter together with theoretical calculations from other workers. Additional detailed information on ice-crystal mean size is obtained from a comparison of lidar and millimeter-radar backscatter combined with infrared optical depth. Data are being analyzed from two recent field trips to tropical regions sponsored by ARM. Methods to automatically retrieve lidar- and radar-determined cloud bases and tops are being reviewed with the ultimate aim of choosing a suitable method for the ARM program.

Results to Date: Analysis of data from the ARM PROBE equatorial experiment is complete. Comparisons between deduced and theoretical values of backscatter phase function indicated that the experimental results fell within the range of theoretical computations. Small ice crystals, which were probably columns, were observed at the lower temperatures. Larger ice crystals, deduced as being hollow columns, rosettes, etc., were observed at higher temperatures. The clouds were primarily composed of ice.

Considerable progress is being made in the development of methods to obtain optical depths of clouds from combined lidar, radiometer, and radar observations. Some cases from the MCTEX experiment in Northern Australia have now been analyzed in detail. They show the distinct improvement in infrared data with the use of the new CSIRO/ARM infrared radiometer. With the high quality of lidar data and the 
availability of the water vapor path from continuous microwave measurements, excellent comparisons are being made between the lidar and infrared data. Cloudbackscatter coefficients at the lidar wavelength are at present being compared with millimeter-radar backscatter data taken by the University of Massachusetts. Theoretical calculations done recently indicate the great sensitivities in the ratios of the two sets of data to cloud particle size. At MCTEX, cloud types varied from deep storm anvils to very-low-density cirrus near the tropopause.

A new CSIRO/ARM radiometer has been completed with a Stirling-Cycle cooler for the infrared detector. Good performances have been obtained from this instrument. Currently, the cooler has been sent back to the U.S. manufacturers for maintenance.

\section{INTERACTION OF CLOUDS, RADIATION, AND DYNAMICS IN THE TROPICAL WESTERN PACIFIC \\ RAMANATHAN, V.; BARNETT, TIM P.; ZHANG, GUANG J;; and SCHNEIDER, NIKLAS \\ UNIVERSITY OF CALIFORNIA, SAN DIEGO \\ FY 1996540 \\ FY 1995620 \\ FY 1994146}

Keywords: western Pacific warm pool, sea-surface temperature

Objectives: To investigate the role of clouds, radiation, and atmospheric/ocean dynamics in maintaining the western Pacific warm pool.

Approach: We make extensive use of the available observational data, including ERBE, CEPEX, and TOGA COARE data, to address many important scientific issues in the warm pool, including the regulation of sea-surface temperature (SST) and the warm-pool-surface heat budget. We also employ stateof-the-art GCMs to help understand the observed results and the individual physical processes that contribute to the regulation of SST. In particular, we focus on the interaction of deep convection and its associated clouds and large-scale circulation with the surface-heating and-cooling processes and on the dynamics of the warm pool in a coupled GCM.

Results to Date: By analyzing surface, ship, buoy, and satellite data, our team found that clouds over the warm pool may be enhancing the atmospheric absorption of solar radiation, decreasing the solar energy reaching the sea surface by as much as $35 \mathrm{Wm}^{-2}$. If confirmed by carefully designed field experiments, this finding could fundamentally change our understanding on how the atmospheric circulation operates.

Analysis of data from the moored-buoy array in the equatorial Pacific and satellite observation identified a mechanism to explain the low surface evaporation observed in the warm pool involving the interac- tion of the ensemble of convection with the larger-scale atmospheric circulation.

In collaboration with NCAR scientists, we implemented a new convective parameterization scheme in the NCAR CCM leading to a vastly improved simulation of many climate parameters in the NCAR model: in particular, the simulated surface evaporation in the western Pacific warm, the simulation of the Asian monsoon precipitation, and the surface wind stress over the oceans.

Using satellite data, we found that, for convectivestratiform cloud systems in the tropical Pacific, GCMs with a horizontal resolution of $50 \mathrm{~km}$ can resolve $90 \%$ of the radiatively important clouds and that a GCM with a resolution of $250 \mathrm{~km}$ can resolve only $50 \%$. The low clouds that are unattached to convective-stratiform systems are mostly unresolvable by current GCMs. For convective-anvil clouds with areas larger than 100,000 $\mathrm{km}^{2}$, our statistics reveal that the area increases with the cloud lifetime, which suggests the need to explicitly account for the transport of cloud liquid/ice water in GCMs.

The coupling of the diurnal cycle of SST to longer time scales was investigated with 1-D models of the upper ocean and a single-column model for the atmosphere. Except for the well-known warm shift of the SST during times of large diurnal cycles, rectification of the diurnal cycle to longer time scales in the oceanic mixed layer is negligibly small and stems from the parameterization of turbulent dissipation during oceanic convection.

A detailed study was conducted with a coupled ocean-atmosphere model of the simulated Indonesian Through flow. Its transport of heat is an important (albeit not the central) heat sink for the western Pacific warm pool and a major heat gain for the Indian Ocean.

For incoming solar radiation during summer, temporal averaging of the central ARM site for a few hours makes data representative of GCM grid scales, the level of agreement being determined by choice of time-averaging interval and desired spatial scales. The radiation data has a shorter characteristic time scale than a few hours. This means the central-site data is limited in its ability to resolve independent samples of the radiation field at typical GCM space scales. 


\section{USE OF ARM DATA TO DEVELOP AND TEST IMPROVED CLOUD PARAMETERIZATION FOR USE IN CLIMATE MODELS RANDALL, DAVID COLORADO STATE UNIVERSITY \\ FY 1996184 \\ FY 1995200 \\ FY 1994171}

Keywords: cloud amount, cloud-water formation, cloud-ice formation

Objectives: To find and test a physically based parameterization for the subgrid cloud amount in order to construct a very simple theoretical model from which a parameterization can be derived analytically and to find and test a parameterization for the rate of cloud-water (and cloud-ice) formation by subgrid processes.

Approach: Explicit, phenomenologically specific mechanisms for cloud formation (i.e., condensation) will be introduced on the subgrid scale. The most obvious mechanism for subgrid-scale condensation, and probably the most important in a global sense, is cumulus convection. Other examples include boundary-layer turbulence and orographic waves. Our single-column model (SCM), cloud ensemble model (CEM), and general circulation model (GCM) will be combined with the ARM data to improve understanding of cloud formation and dissipation and the ability to simulate these important climate processes in climate models.

Results to Date: A wide variety of ARM data products have been put into a form suitable for use with models. The data have been made available to the ARM community through a Web site. The data have been used to drive both an SCM and a CEM. In addition, the CEM has been used in a variety of studies with other data sets.

\section{HIGH-SPECTRAL-RESOLUTION FTIR OBSERVATIONS FOR ARM REVERCOMB, HENRY E. UNIVERSITY OF WISCONSIN}

$$
\begin{array}{ll}
\text { FY } 1996 & 295 \\
\text { FY } 1995349 \\
\text { FY } 1994340
\end{array}
$$

Keywords: infrared radiation, Fourier-transform spectrometer, radiance

Objectives: To develop three types of instruments that provide highly accurate observations of emitted atmospheric radiation and of infrared atmospheric transmission and to develop techniques for using the data in spectroscopic and remote-sensing applications. Approach: State-of-the-art Fourier-transform-infrared (FTIR) radiometric instrumentation is being developed to acquire high-spectral-resolution, infrared, atmospheric radiance and absorption data, especially in atmospheric window regions where the water vapor continuum is important. Three instrument types are being developed:

- The standard AERI operates over the spectral range of 3.3 to 19 microns ( 520 to 3020 wavenumbers) at a resolution of 0.5 wavenumber. An extended-spectral-range version is also under development to extend the longwave limit to 24 microns for Arctic applications, where the low level of water vapor makes longer wavelengths important to the surface radiation budget.

- The AERI-X instrument provides improved spectral resolution ( 0.1 wavenumber, apodized) over the wavelength range from 6 to 14 microns (700 to 1500 wavenumbers). The higher spectral resolution of AERI-X will yield the best available emission observations for detailed evaluation of line-by-line calculations. It will also allow the potential advantages of still higher spectral resolution for atmospheric remote sensing to be explored.

- The SORTI tracks the sun to obtain absorption spectra at extremely high spectral resolution ( 0.0035 wavenumber, apodized), essentially at the full resolution of atmospheric absorption for one or more air masses. It will cover the range from 2.5 to 14 microns (700 to 4000 wavenumbers) to reveal deficiencies in the way model calculations characterize line strengths and line shapes. Also, it will provide remote sensing of the gaseous constituents of the upper atmosphere.

Results to Date: The AERI prototype was operated at the SGP CART Site from March 1993 to July 1995 in various configurations, with data being provided routinely to the ARM Science Team starting in December 1993. A refined data set extending from April 1994 to July 1995 was recently compiled to accurately remove the effect of a small sky-view obstruction discovered during April 1995, when the first operational AERI was brought to the site. The new AERI, incorporating a Stirling cooler for maintenance of cryogenic detector temperatures without liquid nitrogen, showed excellent agreement with the prototype after elimination of the prototype obstruction. AERI data are being used by many science teams for both spectroscopy and remote sensing of atmospheric temperature, water vapor, and cloud properties. Construction of multiple AERIs for SGP, TWP, and Alaska has begun.

The SORTI prototype has been operated successfully at the site on clear days since February 1994, and construction of the first operational SORTI is under way. The operational SORTI will incorporate several improvements to simplify operations and will make use of the automatic quality-control techniques developed 
with the prototype. The SORTI data stream, currently transferred to DU on the Internet and stored on optical disks, has been used to retrieve the total-column amounts of several gases $\mathrm{O}_{3}, \mathrm{~N}_{2} \mathrm{O}, \mathrm{CH}_{4}$, and $\mathrm{HNO}_{3}$ and the profiles of $\mathrm{O}_{3}, \mathrm{HCl}$, and $\mathrm{HF}$. The radiometric performance of the AERI-X was successfully demonstrated at the SGP in the spring of 1995.

\section{ARRHENIUS CONFERENCE PROCEEDINGS RODHE, HENNING \\ STOCKHOLM UNIVERSITY \\ FY 199625 \\ FY 19950 \\ FY $1994 \quad 0$}

Keywords: conference proceedings, workshops and conferences

Objectives: To facilitate the publication of the proceedings of the Ahrennius Conference.

Approach: The Swedish Academy will be assisted in publishing the Conference Proceedings. The Conference Proceedings will be published in FY 1997. The Conference honors a pioneer in science in may fields. His early prediction of the potential for global warming has been noted widely recently, and a conference honoring him and highlighting this particular observation is appropriate.

\section{CIRRUS CLOUD MEASUREMENT PROGRAM FOR IMPROVING SATELLITE AND GCM RESEARCH \\ SASSEN, KENNETH \\ UNIVERSITY OF UTAH \\ FY 1996120 \\ FY 1995162 \\ FY 1994340}

Keywords: remote sensing, cloud retrieval algorithms, lidar, radar

Objectives: To develop, test, and validate improved algorithms for identifying and characterizing clouds with ground-based and satellite remote sensors in order to derive cloud properties of importance to radiative transfer through the cloudy atmosphere.

Approach: We use a combination of complimentary approaches to improve the capabilities of various remote sensing instruments for probing clouds, including collection of advanced remote-sensing and in situ aircraft data sets at SGP CART Site field experiments, sophisticated model simulations of cloud contents that are converted to radiative properties with a variety of scattering theories, and comparison of our long-term (10-year) lidar cirrus-cloud data records to satellitederived cloud properties.

Results to Date: Using these approaches, we have analyzed several cloud case studies from the April 1994 Remote Cloud Sensing field experiment at the SGP CART Site by intercomparing remote sensing and in situ aircraft data from contrails, cirrus, and altocumulus clouds; a cold frontal passage; and rain from melting snow. A new discovery has been that the icecontaining raindrops at the bottom of the snowflake melting zone backscatter so little laser light that a conspicuous dark band is formed in the lidar signal displays. Cloud-model growth and scattering simulations have demonstrated the dependence of lidar multiple-scattering depolarization on cloud droplet size, and allowed the derivation of relations for estimating cumulus and cirrus cloud-mass contents (and even visible ice cloud optical depth) with radar-reflectivity factors. Intercomparisons of the 10-year FARS cirrus-cloud remote-sensing data set and ISCCPderived cloud properties are under way for the testing of satellite cloud-property algorithms.

\section{CLOUD-ALBEDO PERTURBATIONS ON CLIMATE SCHWARTZ, STEPHEN E. BROOKHAVEN NATIONAL LABORATORY FY 1996375 \\ FY 1995500 \\ FY 1994598}

Keywords: temperature anomaly trend, aerosols, sulfates, radiative forcing

Objectives: To determine empirically the temperaturesensitivity coefficient of climate response to perturbations in radiative forcing by comparison of the temperature anomaly trend to shortwave radiative forcing by anthropogenic aerosols.

Approach: Evidence for enhancement of cloud and clear-sky albedo by anthropogenic sulfate aerosol is examined as a function of time and location and systematically related to $\mathrm{SO}_{2}$ emissions with evaluated models for atmospheric sulfate driven by observationderived meteorological data. The direct and indirect shortwave radiative forcings by anthropogenic aerosols are determined with consideration of the historical patterns of emissions and the influence of biogenic and other natural aerosols on cloud and clear-sky albedo. These forcings are related to spatial and temporal patterns of temperature anomaly trend. If the perturbation in forcing can be reliably related to temperature anomaly trend, then from the magnitude of temperature anomaly response to this perturbation in forcing, the sensitivity of the Earth climate system to other perturbations in forcing can be empirically determined, most importantly the sensitivity to increases in concentrations of $\mathrm{CO}_{2}$ and other greenhouse gases.

Results to Date: Climate forcing by anthropogenic aerosols was found to be substantial in the context of global change during the industrial period. Estimated forcing by sulfate aerosols is about $-1 \mathrm{Wm}^{-2}$, global average, uncertain to somewhat more than a factor of 2. Secular, seasonal, and latitudinal patterns of temper- 
ature anomaly trend are coherent with those of anthropogenic sulfate loading, consistent with climate influence of anthropogenic sulfate. Global annualaverage temperature response to aerosol forcing evaluated with a GCM is the same as that for incremental greenhouse gases, but with regional, hemispheric, and seasonal differences indicative of substantial interhemispheric heat transfer. A method to retrieve total aerosol optical depth from satellite observations of cloud-free scenes over oceans with high spatial resolution (about 1 degree) and instantaneous temporal resolution has been developed. Random and systematic errors are 0.05 and 0.04 , respectively.

Fractional activation of submicrometer particles to form cloud droplets has been examined by aircraft measurements. Cloud-droplet-number concentration depends linearly on aerosol-particle concentrations at particle concentrations up to about $600 \mathrm{~cm}^{-3}$. At higher particle concentrations, sensitivity to updraft velocity is exhibited.

\section{SHORTWAVE RADIATIVE FORCING BY TROPOSPHERIC AEROSOL SCHWARTZ, STEPHEN E. BROOKHAVEN NATIONAL LABORATORY FY 1996173 \\ FY 1995230

FY $1994 \quad 0$

Keywords: aerosols, shortwave radiation, radiative forcing

Objectives: To test models relating shortwave radiative forcing of climate by aerosols to aerosol loading and microphysical and optical properties with CART data.

Approach: Models and parameterizations of shortwave radiative forcing by aerosols are being developed, and observations at the CART SGP Site are being used to determine their accuracy and uncertainty. Experiments consist of comparison of data streams representing measured and modeled quantities (for example, downwelling radiation measured vs modeled with measured aerosol properties). Data streams are produced by CART based on observations obtained with CART instruments.

Results to Date: Direct radiative forcing by sulfate aerosols has been calculated to determine the dependence of scattering efficiency, upscatter fraction, and normalized forcing on dry-particle size, composition, solar-zenith angle, latitude, and season. Forcing is strongly dependent on dry-particle size and relative humidity but relatively insensitive to composition. The present treatment, which uses a radiative-transfer code that accurately represents the angular scattering, gives substantially greater normalized forcing than is indicated in previous studies.
Aerosol properties pertinent to shortwave forcing depend sensitively on particle size. To obtain these properties for an aerosol, it is necessary to know the size distribution, to know the dependence of the property on particle size, to evaluate the property as a function of particle size, and to integrate over the size distribution. Often, the size distribution is not known because of measurement or modeling limitations. We have developed and tested a technique for calculating integral aerosol properties from the moments of the radial-size distribution without full knowledge of the distribution. This approach should permit accurate and efficient representation of aerosol properties in chemical-transport models and GCMs.

\section{SATURATED WATER SPECTROSCOPY SHARPE, STEVEN \\ PACIFIC NORTHWEST NATIONAL LABORATORY \\ FY 1996100 \\ FY 19950 \\ FY $1994 \quad 0$}

Keywords: spectra, water vapor, absorption of solar radiation by clouds

Objectives: To investigate the spectra of water vapor close to saturation to determine if absorption at relevant wavelengths increases enough to explain emerging results of data analysis on absorption of solar radiation by clouds.

Approach: Using state-of-the-art laboratory techniques, we will measure the absorption of water vapor near saturation as a function of wavelength.

Results to Date: Equipment has been designed. Construction of the laboratory instrumentations is nearly complete.

\section{CONTINUATION OF DATA-ANALYSIS- SOFTWARE DEVELOPMENT FOR THE ATMOSPHERIC EMITTED RADIANCE INTERFEROMETER (AERI) SMITH, WILLIAM UNIVERSITY OF WISCONSIN FY 1996152 \\ FY 1995190 \\ FY 1994195}

Keywords: radiative transfer, temperature profiles, moisture profiles

Objectives: To improve the modeling of radiative transfer for the molecular atmosphere, to improve the retrieval of temperature and moisture profiles of the planetary boundary layer, and to develop cloud-radiance parameterizations that relate climate-model variables to effective cloud radiances.

Approach: A long-term quality-measurement experiment continues to provide valuable insight into the size and character of uncertainties in clear-air radiative- 
transfer models. An improved planetary boundary layer retrieval algorithm has been implemented that combines data from a variety of sensors to best characterize the atmospheric state in the first $3 \mathrm{~km}$ of the atmosphere in both clear and cloudy conditions. Algorithms are being developed to obtain microphysical cloud properties from the infrared spectra.

Results to Date: Algorithms for providing temperature and moisture profile retrievals from AERI, micropulse lidar, and microwave total precipitable water data have been transferred to PNL. Software has been implemented at PNL for the routine production and archiving of planetary boundary layer thermodynamic structure data from the SGP CART Site. The retrievals possess good accuracy within the PBL; temperature and dewpoint temperature agreement with radiosondes is consistently better than 1 and $3^{\circ} \mathrm{K}$, respectively. Comparisons of daily vertical-time cross-sections with cross-sections of Raman-lidar water-vapor profiles show that the 10-min-interval AERI retrievals provide an important enhancement of the PBL water-vapor structure that is ill-defined by 3-h-interval radiosonde measurements conducted during ARM IOPs. The retrievals obtained for several IOPs have proved to be useful for defining important dynamic mesoscale meteorological features, including frontal structures, dryline characteristics, and diurnal variations in PBL structure. An improved version of the software to provide accurate profiles under low-cloud conditions is now under test at the University of Wisconsin and is planned to be transferred to the PNL as soon as the validation is completed.

\section{DIAGNOSTIC MODELING OF THE ARM EXPERIMENTAL CONFIGURATION SOMERVILLE, RICHARD C. J. UNIVERSITY OF CALIFORNIA, SAN DIEGO FY 1996171 \\ FY 1995184 \\ FY 1994155}

Keywords: cloud parameterizations, single-column models

Objectives: To test and develop cloud parameterizations with ARM data and a single-column model.

Approach: We have developed a single-column model (SCM) to validate $\mathrm{GCM}$ cloud-radiation parameterizations against ARM observational data. The parameterizations in the current version of the SCM are based on contemporary GCM practice. Our SCM is a computationally efficient 1-D representation of the atmospheric column overlying a single GCM grid cell. The model includes a variety of options for each of the key parameterizations of subgrid physical processes. We integrate this model in time from observed initial states, and we constrain it with observational estimates of horizontal-flux convergences.
Thus, the input to the model is the time-dependent advection of heat, water, and momentum at all layers specified from ARM observations. The model output is a complete atmospheric heat and water budget, including temperature and moisture profiles, clouds and their radiative properties, diabatic-heating terms, surfaceenergy-balance components, and hydrologic-cycle elements, all specified as functions of time. These model products can then be validated directly against ARM observations.

Results to Date: We have used ARM data diagnosed with our SCM, in conjunction with atmospheric-GCM experiments, to test a suite of alternative cloud-radiation parameterizations. We developed the GCM from a low-resolution (15-wave rhomboidal) workstation version of the NCAR CCM2. We have replaced most of the main NCAR parameterizations in CCM2 with our own, while retaining the adiabatic inviscid kernel and numerics of CCM2. The tested parameterizations include relative-humidity-based clouds and several prognostic cloud-water-budget schemes. We are now emphasizing testing stochastic approaches to cloudradiation parameterization. We have carried out sensitivity tests with these parameterizations, in which we examined the effect of varying tunable aspects of the schemes. Recently, we have applied the stochastic treatment to the problem of explaining anomalous absorption of solar radiation in a cloudy atmosphere. We find that the stochastic treatment of cloud-cloud interactions yields a sufficiently long mean photon path length to explain much of the discrepancy recently reported between observations and GCM results.

\section{CIRRUS AND AEROSOL PROFILOMETER DATA FOR ATMOSPHERIC RADIATION INTERPRETATION SPINHIRNE, JAMES \\ NASA GODDARD SPACE FLIGHT CENTER \\ FY 199660 \\ FY 199555 \\ FY 199490}

Keywords: micropulse lidar, cloud profilometers, aerosol profilometers

Objectives: To apply MPL lidar measurements in combination with radiometer and other data from the ARM site to derive and correlate aerosol and thincloud parameters of importance for atmospheric radiation.

Approach: Maintenance of data quality, instrument operation, and raw-data processing are supported. Processing algorithms are developed for second-order data products. A systematic set of code is in development for classifying atmospheric conditions, determining cloud locations, and also higher-level products of aerosol and cirrus backscatter cross-sections and optical thickness. Algorithms will be tested for mea- 
surement quality. Improved MPL instruments with greater resolution and higher signal-to-noise ratio and possibly with polarization measurements will be developed.

Results to Date: The first two upgraded MPL instruments, built by SESI of Burtonsville, Md., have been deployed with only minor after-pulsing problems. The new signals are at least 10 times greater than signals from the original instrument. A significant improvement in vertical resolution from $300 \mathrm{~m}$ to $75 \mathrm{~m}$ is in the final stages of development. An overlap correction has been applied to raw data from the two improved instruments, enhancing aerosol signals in the lowest $2 \mathrm{~km}$. Preliminary atmospheric aerosol profiles have been derived from the data from the two new instruments. Three cloud-classification algorithms are being compared for robustness and accuracy. The best will become the operational algorithm. Algorithms for corrected aerosol backscatter and optical thickness have been finalized but not implemented.

\section{MODELING AND REMOTE SENSING STUDY OF THE RADIATIVE HEATING OF CLOUDS STEPHENS, GRAEME; PLATT, MARTIN; and GABRIEL, PHILIP COLORADO STATE UNIVERSITY and COMMONWEALTH SCIENTIFIC AND INDUSTRIAL RESEARCH ORGANISATION FY 1996189 FY 1995210 FY 1994200}

Keywords: remote sensing, radiative heating, radiative transfer, lidar, cloud optical properties

Objectives: To contribute to the understanding and identification of the properties of clouds that govern the distribution of radiative heating in the atmosphere; to apply remote sensing as a way of deriving relevant optical properties, and to use multisensor data in models as a test and means for refining GCM parameterizations of atmospheric heating.

Approach: Research under this activity began as theoretical in nature, focusing on both understanding 3-D radiative transfer and parameterizing radiative transfer. Studies dealing with understanding the relative effects of cloud microphysics vs cloud morphology were carried out. While this research has considered two perspectives, namely effects relative to remote sensing and the effects relative to cloud radiative budgets, the latter has pointed to important new developments in radiative transfer and to ways of parameterizing this transfer.

Results to Date: The differences between the 3-D and plane-parallel results for solar fluxes were found to be partly caused by the shadowing of the direct solar radiation. A simple first-order closure to estimate fluxes and heating/cooling rates within or on the boundaries of the cloudy medium yielded domainaveraged fluxes that generally were more accurate than those predicted with the independent pixel approximation. Calculating the domain-averaged direct beam three-dimensionally and using this result in a planeparallel computation produced results with an accuracy that could exceed that of the independent pixel approximation, but most of the time lay between the former and standard plane parallel computations that use domain-averaged optical properties. In either case, cloud morphology is of fundamental importance.

A new parameterization scheme was developed that employs multisensor (lidar and millimeter radar) observations to prescribe the necessary cloud properties for the radiation scheme. During the ARM PROBE in Kavieng, Papua New Guinea, vertical lidar and passive radiometer observations on cirrus clouds were used to give values of cloud height, depth, infrared emittance and depolarization ratio. The cirrus was found to vary in altitude from 18 to $6 \mathrm{~km}$ and to vary widely in geometric and optical depth. Cirrus depths varied from more than $4 \mathrm{~km}$ to less than $100 \mathrm{~m}$, and infrared emittances from about 0.02 to near 1.0. A LIRAD analysis showed that, with temperatures less than $-35^{\circ} \mathrm{C}$, integrated backscatter increased with altitude. For temperatures above $-35^{\circ} \mathrm{C}$, the integrated backscatter exhibited anomalously enhanced values. The PROBE extinction fields derived from the LIRAD have been applied as input into the radiation parameterization. Similar data derived from millimeter-radar reflectivities have also been adopted to yield timeheight cross-sections of fluxes and heating rates. Unfortunately, measuring flux profiles with the same time-space resolution matching the profiles from active systems is not possible.

\section{ATMOSPHERIC RADIATION MEASUREMENT PROGRAM: SCIENCE TEAM COORDINATION STOKES, GERALD M. PACIFIC NORTHWEST NATIONAL LABORATORY \\ FY 19962025 \\ FY $1995 \quad 0$ \\ FY 1994 17976*}

*In previous years, this entry was a consolidation of this project with others under the principal investigators Ted S. Cress and Jimmy W. Voyles.

Keywords: radiative-transfer models, cloud parameterization, cloud properties, cloud formation, cloud maintenance

Objectives: To improve the performance of GCMs of the atmosphere in predicting global and regional climate, focusing specifically on the treatment of radiative-transfer and related models in GCMs under clear-sky, general-overcast, and broken-cloud condi- 
tions and the parameterization of cloud properties, cloud formation, and cloud maintenance in GCMs. Approach: The Science Team coordination is managed by the ARM Chief Scientist, located at Pacific Northwest National Laboratory, on behalf of DOE. The Chief Scientist provides management of specific Science Team efforts at DOE's request and coordination with related efforts in other agencies and countries.

The Science Team conducts approved research projects and provides basic guidance for site selection and implementation, for selection and deployment of instruments, and for creation of common data products. The Science Team participates in setting the scientific direction of the ARM Program. Science Team members are funded directly by DOE except in specific cases when DOE requests the Program Office to provide management oversight. Activities of the Science Team are coordinated through the Program Office. The site scientists for the CART sites are integral members of the Science Team and provide for onsite scientific expertise, manage a site-related educational program, and conduct their own ARM research program. The Science Team includes an adjunct status, wherein scientists funded from other sources can propose an ARM-related research program to DOE and, if the proposal is accepted, receive ARM data and participate as a member of the ARM Science Team.

Results to Date: Research results from the Science Team were presented at the Annual Meeting of the American Meteorological Society. The sixth ARM Science Team meeting was held in San Antonio, Texas, in March 1996 with more than 300 attendees. The meeting focused on the most significant measurement needs of the Science Team in light of recent findings and conclusions. New measurement concepts and plans were laid for future implementation.

In 1996, the JASONs reviewed the examination of the scientific aspects concerning shortwave absorption by the atmosphere and clouds by the ARM Enhanced Radiation Experiment (ARESE). Immediate feedback from the review indicated strong support for the program.

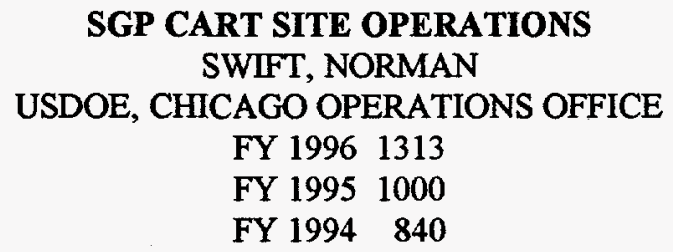

Keywords: site operations, project management Objectives: To oversee CART site operations. Approach: The Chicago Operations Office of the Department of Energy will manage the SGP CART Site through a subcontractor that will provide person- nel to staff, manage, operate, and maintain SGP CART Site systems and facilities. Subcontract activities are to provide site-implementation support; develop and provide operations and maintenance training; develop, validate, and implement site/system operations, maintenance, and repair schedules and procedures; develop, validate, and implement site/system certification, acceptance, and hand-off procedures; develop, validate, and implement site/system documentation and document maintenance; provide integrated logistic support, including staffing, budget, and inventory; and provide oversight of the site safety program, including the implementation of the Argonne National Laboratory Environmental Safety and Health (ES\&H) requirements.

Results to Date: The ARM site operations contract was officially transferred from NOAA to DOE-CH on February 11, 1994. The contract is fully funded and fully implemented.

\section{MODELING OF CLOUDS AND RADIATION FOR DEVELOPING PARAMETERIZATIONS OF CLOUDS IN GENERAL CIRCULATION MODELS TOON, O. B. \\ NASA-AMES \\ FY 1996160 \\ FY 1995200 \\ FY 1994200}

Keywords: cloud modeling, radiative-transfer modeling

Objectives: To model radiative transfer and cloud microphysics and test these models against other highaccuracy methods and against measurements under cloudy, partial-cloudy, and cloud-free conditions.

Approach: Recent measurements have suggested that clouds absorb more solar radiation than previously suspected; we are investigating this issue for marine stratus clouds and for horizontal inhomogeneity of clouds, which in general are overlooked in radiativetransfer models. We are comparing field observations and model results for cirrus clouds. We are developing new 3-D radiative-transfer codes, a Monte-Carlo code, and a time-efficient six-stream method to understand the effects of cloud inhomogeneity on (solar) radiative transfer.

We are continuing the development of a rapid radiative-transfer model (3-ARM) for use in climate simulations. The model combines an efficient twostream code, new k-distributions for gas-absorption from AER, and a new four-stream option. We are addressing concerns about the poor performance of solar radiative-transfer models under cloud-free conditions. We are also designing field programs to provide data to improve our understanding of cloud radiative properties. 
Results to Date: For clear-sky conditions, the 3-ARM infrared radiative-transfer code is in good agreement with accurate line-by-line codes and DOE AERI measurements (ICRCCM comparison). For cloudy conditions, increased cloud inhomogeneity increases the transmission and reduces the reflection and absorption; overlooked cloud inhomogeneity is unlikely to explain measurements of excess solar absorption; 3-D six-stream methods can be an excellent tool to quickly illustrate scattering patterns for inhomogeneous media; excess solar absorption in marine stratus must be negligible, otherwise these clouds would not exist; for cirrus, it is almost impossible to find unique relationships between two properties unless at least a third cirrus properties is considered.

\section{LABORATORY SPECTROSCOPY RELATED TO THE ARM PROGRAM VARANASI, PROSAD \\ STATE UNIVERSITY OF NEW YORK AT STONY BROOK \\ FY 1996162 \\ FY 1995180 \\ FY 19940}

Keywords: ground-based remote sensing, clouds, cloud-radiation parameterizations, cloud-base height, lidar, stratus-cloud mean particle size, stratus-cloud mean particle-number density, cirrus optical depth, cirrus size distribution

Objectives: To obtain laboratory spectroscopic data on greenhouse gases as applicable to the ARM Program. Approach: The data collection will be completed for carbon dioxide, nitrous oxide, and methane, and a detailed series of measurements will be made of the spectra of water vapor.

Results to Date: Extensive measurements have been performed, many of which are first of their kind, of the most accurate and reliable spectroscopic data on several important greenhouse gases using our state-ofthe-art Fourier Transform Spectrometer (FTS). Our previously reported measurements of the spectral absorption coefficients in the 9- and 11-micron bands of CFC-11 and CFC-12 and the 7.62-, 8.97-, and 12.3-micron bands of HCFC-22, have been extended to polar (both Arctic and Antarctic) atmospheric conditions. In addition, we have measured and catalogued the most extensive set of data ever obtained on the thermal infrared bands of both carbon tetrachloride and carbon tetrafluoride.

Numerous spectra have also been recorded for molecules that exhibit identifiable spectral lines by employing our FTS in the InSb region (2- to 5-micron region in the infrared) at various temperatures between 195 and $295 \mathrm{~K}$. The spectra were obtained for box-car apodization, which is the closest we can get to unapodized spectra. Studied in this initial set of experiments were the bands of $\mathrm{CO}, \mathrm{CO}_{2}, \mathrm{~N}_{2} \mathrm{O}$, and $\mathrm{CH}_{4}$ between 3 and 5 microns and the combination bands of $\mathrm{CO}_{2}$ at 2.7 microns.

\section{DATA AND SCIENCE INTEGRATION TEAM VOYLES, JIMMY W. PACIFIC NORTHWEST NATIONAL LABORATORY \\ FY 19968250 \\ FY 19955388 \\ FY 1994 *}

*In previous years, this entry was consolidated with others under the principal investigator Gerald $M$. Stokes.

Keywords: integrated data systems, project management, data

Objectives: To manage and operate the ARM infrastructure activities associated with the design, development, implementation, and deployment of an integrated data system for the acquisition, quality assessment, processing, distribution, and archiving of ARM data.

Approach: The data system will acquire data from CART instruments as well as from outside sources, will process and archive appropriate data, and will deliver requested data to members and subgroups of the Science Team. The archive will deliver requested data to non-ARM researchers. New or improved algorithms will be developed to produce value-added data products for the Science Team and to routinely compare, in the Experiment Center, two or more data streams to provide routine data-quality assessments.

Results to Date: The ARM Data System now provides data on a continuous basis from one site and has developed the data system for the Tropical Western Pacific (TWP) Site. The command-and-control concepts for the TWP Site call for health-of-station data to be transmitted in near real time with satellite-communication systems, with most data to be recorded on tape and physically shipped to the ARM Experiment Center for processing and distribution to the Science Team and the Archive. A WWW-interface for the Archive was developed to assist users in identifying and obtaining data. The interface and other Archive functions are being updated to provide better access.

TREATMENT OF CLOUD RADIATIVE
EFFECTS IN GENERAL CIRCULATION
MODELS
WANG, WEI-CHYUNG
STATE UNIVERSITY OF NEW YORK
AT ALBANY
FY 1996150
FY 1995310
FY 1994291


Keywords: general circulation models, radiation parameterization, cloud-radiation interaction

Objectives: To validate and improve the GCM longwave and solar radiation parameterizations; to simulate realistically radiation fluxes for clear sky with aerosols; to develop interactive-layer cloud optical properties and radiation; and to treat the subgrid scale variability of cloud-radiation interaction.

Approach: These studies are conducted with ground measurements from ARM sites together with satellite data and other meteorological data. Our modeling activities will be based on the use of the narrow- and total-band k-distribution function radiation models, the mesoscale model MM5, and the GCMs GENESIS and CCM3 developed at the National Center for Atmospheric Research.

Results to Date: For longwave radiation, the model calculations are in good agreement with measured values, especially for the Albany site (within $3 \mathrm{Wm}^{-2}$ ). However, the differences become larger for the SGP Site. Note that the Albany site covers a much longer period (10/86 to 9/92) and includes seasonal variations while SGP covers only April 1994. For shortwave radiation, the model systematically overestimates the downward flux, an indication of a smaller model atmospheric opacity. This feature is consistent with results from other modeling groups. The effect of aerosols is quite large, decreasing substantially the shortwave radiation reaching the surface. For example, the average values are $16 \mathrm{Wm}^{-2}$ at Albany and $10 \mathrm{Wm}^{-2}$ at SGP, thus highlighting the importance to include aerosols in GCMs.

Parameterizations for the shortwave and longwave radiative effects of high-level (ice) clouds for use in GCMs were developed. In the parameterizations, cloud particles are assumed to be composed of randomly oriented hexagonal crystals. For shortwave radiation, the broadband transmittance, reflectance, and absorptance are expressed as a function of single-scattering albedo, asymmetry factor, and optical depth, which in turn are functions of effective particle radius. For longwave radiation, the optical depth and emissivity are expressed in terms of cloud ice-water path. Both the effective particle radius and ice-water path are related to cloud temperature. Using this new parameterization with satellite-derived high-level clouds during the April 1994 IOP over SGP, we conducted a model-to-observation comparison of the downward flux at the surface and outgoing flux at the TOA for both shortwave and longwave radiation. The results suggest that the new parameterization with interactive microphysics and optical properties simulates observations better. For example, when compared with the old parameterization, the new parameterization reduces the rms difference in the TOA shortwave radiation flux by $50 \%$.
The vertical distribution of clouds significantly affects the radiative heating/cooling distributions. Consequently, the GCM-simulated climate can be sensitive to the treatment of cloud overlap, which is related to the subgrid-scale cloud vertical geometric association. Here, we developed and tested a mosaic treatment that can explicitly consider the cloud association in the GCM radiation parameterization. The effects of this treatment on radiative forcing and climate simulations were studied by comparing the results with those using the random overlap, which assumes clouds are independent and therefore yields a larger effective cloudiness.

The mosaic treatment calculates a significantly different atmospheric radiative heating/cooling distribution, which is caused mainly by the changes in infrared radiation. In the tropics, it yields a heating in the upper troposphere and a cooling in the lower troposphere, especially near the surface; opposite changes are calculated in the middle-to-high latitudes. Because of a smaller effective cloudiness, the mosaic treatment calculates less infrared downward radiation reaching the surface, which is partially compensated for by increased incident solar radiation. Differences in the climate responses are substantial, with several major model biases corrected by the mosaic treatment. For example, the middle-to-upper troposphere of the tropics and subtropics are warmed by more than $3^{\circ} \mathrm{C}$ throughout the year, and the polar-night northern stratosphere becomes much warmer, up to a maximum of $15^{\circ} \mathrm{C}$. A more realistic distribution of tropical precipitation is simulated, especially over the centralto-east Pacific Ocean. Other notable improvements include the decreased convective rainfall and surface evaporation, and the increased atmospheric moisture content. The mosaic treatment also enhances significantly the daily variability of surface air temperature and radiation fluxes in the low latitudes.

\section{IMPACT OF AIR-SEA INTERACTION ON TROPICAL CLOUD LIFE CYCLES \\ WEBSTER, P. J.; CURRY, J. A.; and EMERY, W. J. \\ UNIVERSITY OF COLORADO \\ FY 1996160 \\ FY 1995210 \\ FY 1994202}

Keywords: atmosphere-ocean interactions, clouds, cloud life cycles, turbulence, cloud microphysical processes

Objectives: To understand the ocean-atmosphere interface processes that affect cloud life cycles in the tropical ocean warm pools (TWP), the synoptic-scale processes that affect cloud life-cycles in the TWP, the role of clouds and radiation in maintaining the TWP ocean structure, the interactions among turbulence and 
radiation, and the cloud microphysical processes involved in the evolution of the cloudy atmospheric boundary layer over the TWP; and to determine the feedback mechanisms occurring between clouds and the upper ocean.

Approach: The current version of MM5 will be diagnosed in the tropics. Improved cloud and radiation parameterizations that are relevant to the TWP will be incorporated. Model results will be validated with TOGA COARE and ARM TWP data. MM5 will be coupled with an ocean model. And sensitivity studies and cloud life-cycle runs will be conducted.

Results to Date: A data set of atmospheric and surface characteristics that are needed for model initialization, boundary conditions, and validation has been constructed with data from the TOGA COARE intensive operational period. The effort has focused on atmospheric temperature and humidity; cloud characteristics (cloud fraction, ice and liquid-water contents, and vertical layering); sea-surface temperature; surface wind speed; and components of the surface fluxes of heat, fresh water, and momentum. In addition, a new surface-turbulence flux parameterization has been developed, the effects of precipitation on surface heat and momentum fluxes (in addition to freshwater flux) have been incorporated, an ocean mixed-layer model has been incorporated into the 3-D ocean model, a new skin SST parameterization has been incorporated into the ocean mixed-layer model, and the sensitivity of the ocean mixed-layer model to surface forcing has been investigated.

\section{MODELING OF CLOUD LIQUID WATER STRUCTURE AND THE RESULTANT RADIATION FIELD BASED ON SCALE-BY- SCALE STATISTICAL ANALYSES OF ARM AND OTHER DATA \\ WISCOMBE, WARREN; CAHALAN, ROBERT; MARSHAK, ALEXANDER; and DAVIS, ANTHONY \\ NASA GODDARD SPACE FLIGHT CENTER \\ FY 1996190 \\ FY 1995200 \\ FY $1994 \quad 0$}

Keywords: cloud structure, radiative transfer, scale dependence, scaling, cloud models, remote sensing, cloud lidar

Objectives: To develop a robust statistical characterization of cloud-liquid-water structure from data, with an emphasis on multifractal and wavelet statistics; a simple stochastic 2-D and 3-D cloud-liquid-water models that reproduce the observed statistics; and an analysis of radiative transfer in these cloud models, with an emphasis on the extent to which 1-D radiation models (used in GCMs) can be made to reproduce the 2-D and 3-D results.
Approach: State-of-the-art statistical analyses (spectral, multifractal, wavelets, etc.) will be applied to ARM and other cloud and cloud-radiation data to reveal much more about their true statistical, scaledependent nature than the customary means and variances. In the process, improvements and extensions to existing statistical methods will be developed. Based on those analyses, we will further develop our multifractal models of cloud-liquid-water fields to increase their verisimilitude. Based on those multifractal models, with model parameters determined by FIRE, ARM, and other cloud liquid data, 3-D Monte Carlo radiative-transfer calculations of the radiance and flux fields will be performed. The differences between simulated radiance fields and observed radiance fields from Landsat, from the AVIRIS and MODIS-Simulator aircraft radiometers, and from the ARM Whole Sky Imager will be statistically analyzed. Independent pixel approximation (IPA) methods, rooted in 1-D radiative transfer, will be generalized to better approximate the 3-D Monte Carlo results in a computationally cost-efficient way.

Results to Date: We empirically proved that horizontal liquid-water-content (LWC) fluctuations in stratocumulus are scale-invariant multifractal and nonstationary over at least three decades in scale. We used two scaling exponents as robust measures of nonstationarity and intermittency. We developed a simple spectral criterion for detecting nonstationarity in data on a scale-by-scale basis and a criterion for discriminating against multifractality caused by finite sampling effects. We applied Green function theory to the LANDSAT scale-break at 200 to $300 \mathrm{~m}$, the signature in spatial statistics of the net horizontal radiative fluxes excited by $L W C$ variability. We developed a cost-effective way of computing radiation fields for variable clouds by convolution of IPA fields with a smoothing kernel representing the "spot" of diffusely reflected light (spatial Green function) that results from steady illumination by an idealized laser beam. And we devised a new concept in cloud lidar based on remote measurements of the spatial and temporal Green functions for stratus layers; theoretically, cloud geometrical and optical thicknesses can be retrieved from this data at about $0.5-\mathrm{km}$ resolution.

\section{INTEGRATED CUMULUS ENSEMBLE/ TURBULENCE PARAMETERIZATION FOR GENERAL CIRCULATION MODELS}

YOUNG, G. S.; EVANS, J. L.; and FRANK, W. M. THE PENNSYLVANIA STATE UNIVERSITY FY $1996 \quad 84$
FY $1995 \quad 120$
FY $1994 \quad 116$

Keywords: parameterizations, general circulation models, mesoscale convection, cumulus clouds 
Objectives: To develop and test an integrated parameterization system for GCMs.

Approach: Missing physical links between the cumulus and boundary-layer components of the parameterization package will be developed and incorporated so they provide an accurate input to the radiative component of the package. The physical consistency of this integrated approach to GCM parameterization is expected to impact the regimes where boundary-layer turbulence and deep convective processes interact most strongly: mesoscale convection and shallow cumulus. In both of these regimes, failure to take these interactions into account in a physically realistic fashion can result in serious errors in the 4-D cloud distribution and hence in the 4-D radiation budgets.

Results to Date: The integrated parameterization package consists of a radiation parameterization, a boundary-layer scheme, a cumulus-ensemble parameterization, and a gust-front parameterization that simulates mesoscale effects of convection in the boundary layer. The host model is the CCM2. The radiation and boundary layer schemes are part of the primary CCM 2 code developed at NCAR. The cumulus-ensemble model has been developed and installed in CCM2 and is being tested in simulations of regimes typical of tropical maritime convection to determine the optimum cloud model properties. The individual cloud models that compose the ensemble are functioning realistically, producing heating profiles characteristic of deep convective regimes. Overall, the prototype cloud-ensemble parameterization appears to produce more realistic results than does the model's moisture-adjustment scheme. The gust-front parameterization has also been designed and installed in CCM2 and is currently being tested and coupled to the cloud-ensemble model.

A diverse selection of synoptic flow conditions (calm, approaching squall line, stratiform deck, cirrus situations) centered on the ARM CART Site were studied. Analyses of each of these situations were performed with data available from the ARM CART array of radiosondes, profilers, and ground-based observations. Analyses were vertical profiles of mass, temperature and moisture divergence, temperature and moisture advection, effective heat source, and effective moisture sink. Surface fluxes, averaged over the fourstation site, were also calculated. Quality-control and error estimations were performed, where possible, through the calculation of these analyzed fields by more than one technique. These data will now serve as input and validation fields for the single-column climate model. 


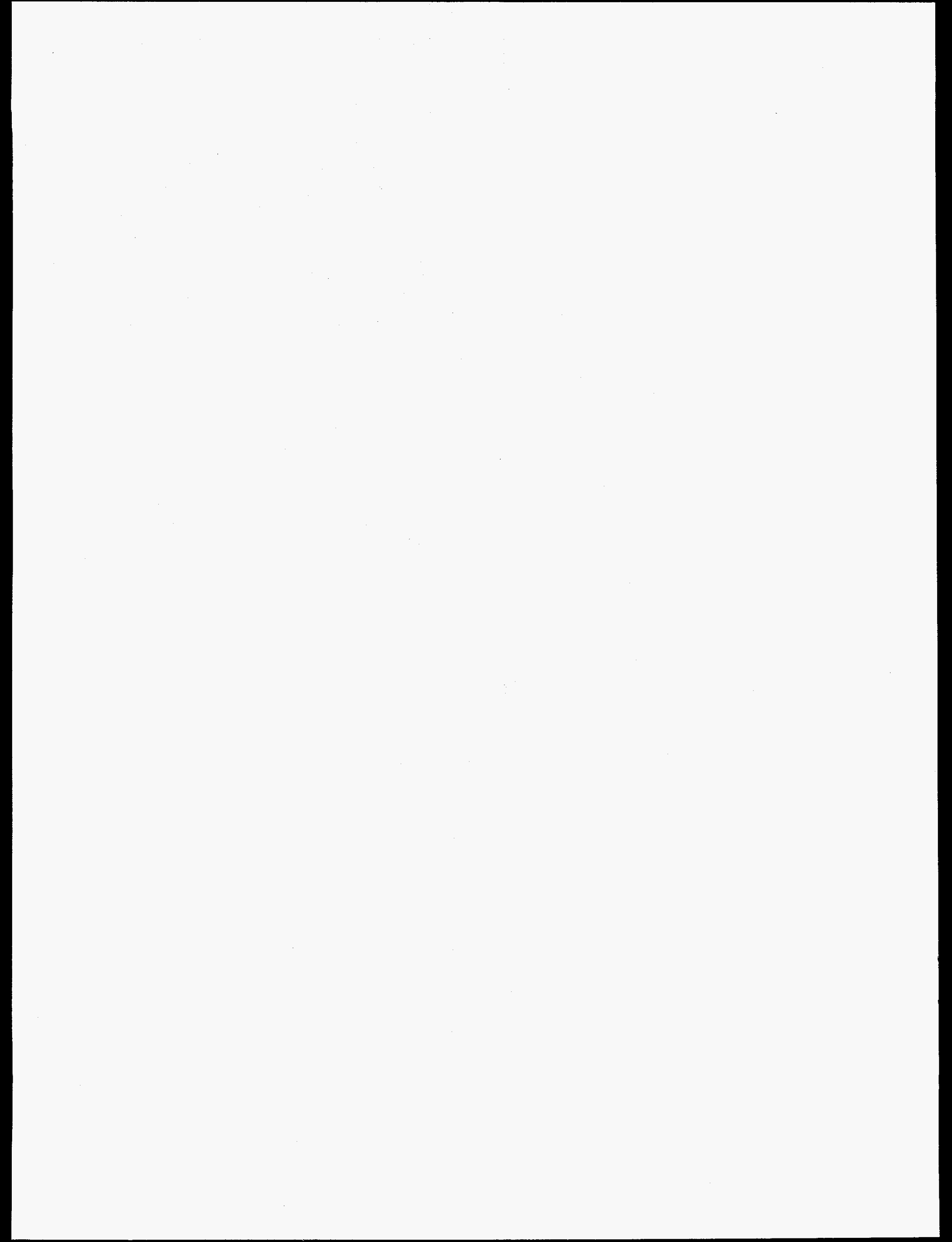




\section{UNMANNED AERIAL VEHICLES (UAV)}

Unmanned Aerial Vehicles (UAV) supports the ARM Program in its efforts to extend energy-balance and cloud data to regional scales and to investigate other environmental processes via remote sensing. Through FY 1996, this program was a joint activity with the Department of Defense under the Strategic Environment Research and Development Program (SERDP).

\section{Program Manager}

Patrick A. Crowley

Environmental Sciences Division

Department of Energy, ER-74

19901 Germantown Road

Germantown, MD 20874-1290

(301) 903-3069

Internet: p.crowley@oer.doe.gov

\begin{tabular}{|c|c|}
\hline $\begin{array}{r}\text { SAFETY AN } \\
\text { BARBER, RC }\end{array}$ & $\begin{array}{l}\text { ALYSIS } \\
\text { OBERT }\end{array}$ \\
\hline WORKER HEALTH & AND SAFETY \\
\hline FY 1996 & 150 \\
\hline FY 1995 & 65 \\
\hline FY 1994 & 0 \\
\hline
\end{tabular}

Abstract not available.

SAMPLING STUDIES FOR
ARM-UAV MISSIONS
CAHALAN, ROBERT F.
NASA
FY 199670
FY 19950
FY $1994 \quad 0$

Keywords: cloud structure, water-vapor structure Objectives: To explore the cloud and water-vapor structure observed in the UAV-flight research program.

Approach: The results of radiometric measurements from instruments on board the UAV will be compared with line-by-line spectral computations based on soundings and CAGEX data.

Results to Date: Several areas of discrepancies have been noted in clear-sky data. These are being pursued to see how far the observed temperature, moisture, and other vertical profiles must be perturbed to match the data. Data from cloudy-day missions will be analyzed next.

\section{CLOUDS AND SATELLITE-INSTRUMENT CALIBRATION FROM UAVS GAUTIER, CATHERINE UNIVERSITY OF CALIFORNIA, SANTA BARBARA}

FY 1996100

FY 19950

FY $1994 \quad 0$

Keywords: cloud properties

Objectives: To use high spatial and spectral resolution data from UAV instruments to perform studies of cloud and surface properties by developing techniques to calibrate operational sensors, performing bidirectional reflectance studies of clouds, and investigating causes of cloud radiative- and microphysical-property variations.

Approach: Sophisticated 1-D and 3-D Monte Carlo spectral radiative-transfer models will be used to guide interpretations of UAV observations.

Results to Date: Three-dimensional modeling studies suggest how we could analyze multispectral pushbroom imaging radiometer and SSP data to extract effects of cloud morphology on absorption in a cloudy atmosphere. 


\section{INTERPRETATION AND \\ PARAMETERIZATION OF THE RADIATIVE PROPERTIES OF CIRRUS CLOUDS FROM ARM/UAV MEASUREMENTS \\ LIOU, K. N. \\ UNIVERSITY OF UTAH \\ FY 199690 \\ FY 19950 \\ FY 19940}

Keywords: cloud parameterizations, radiative properties of clouds, cirrus clouds

Objectives: To analyze data from the UAV instrument suite to validate the radiation/cloud models for calculating spectral and broadband fluxes and radiances in clear and cirrus atmospheres for use in GCMs and climate models and to assist in the improvement and development of remote-sensing techniques for the retrieval of the cirrus ice-crystal size and optical depth with AVHRR data.

Approach: Comparison will be made of correlated k-coefficient spectra and line-by-line calculations with data from the UAV and AVHRR.

Results to Date: A new solar-radiation model with a spectral resolution of $20 \mathrm{~cm}^{-2}$ was developed. It features the solar-irradiance data compiled from LOWTRAN7 to drive the solar-radiation transfer, correlated $k$ coefficients for $\mathrm{H}_{2} \mathrm{O}$ from 2,000 to $21,000 \mathrm{~cm}^{-2}$, and the modification of previously developed radiativetransfer codes for specific application to the transfer of solar radiation in vertically inhomogeneous atmospheres ( $20 \mathrm{mb}$ per layer). This model has been used to understand and interpret UAV measurements in California and Oklahoma, particularly the downward solar and IR fluxes. Theoretical results appear to have slopes less steep than the measured values. Comparison of the correlated $k$-distribution model with line-byline data and observations show good agreement, validating the accuracy and reliability of this method for infrared-radiative-transfer calculations in inhomogeneous atmospheres.

\section{SATELLITE CALIBRATION AND VERIFICATION OF REMOTELY SENSED CLOUD AND RADIATION PROPERTIES WITH ARM UAV DATA MINNIS, PATRICK NASA \\ FY 199680 \\ FY 19950 \\ FY $1994 \quad 0$}

Keywords: cloud radiative properties, satellite data, meteorological satellite sensors, spectral radiance, radiative fluxes

Objectives: To develop and validate improved methods for remotely sensing cloud and radiation properties from satellites with data sets obtained during UAV field experiments and to calibrate operational meteorological satellite sensors for spectral radiances and broadband radiative fluxes.

Approach: GOES and POES data will be reduced and compared with radiance data obtained during the UAV flights. Radiation-transfer algorithms will be used to extrapolate measurements to the appropriate altitude. Results to Date: For longwave radiation, the calculation and the observations differ by approximately $10 \mathrm{Wm}^{-2}$.

$\begin{array}{cc}\text { UAV SUPPORT } \\ \text { SOWLE, DAVID H. } \\ \text { MISSION RESEARCH CORPORATION } \\ \text { FY } 1996 & 282 \\ \text { FY } 1995 & 0 \\ \text { FY } 1994 & 0\end{array}$

Keywords: data display

Objectives: To develop a system to support the UAV mission scientist with access to incoming data and aircraft status from the aircraft involved as well as data from other sources appropriate to the mission objectives.

Approach: In cooperation with the UAV mission scientist, UAV instrument scientists, PIs, and Sandia telemetry-operations personnel, techniques will be developed for reducing and displaying the data for use by the mission scientist and other PIs onsite when and where practical.

Results to Date: An impressive system has been developed that graphically shows the positions of the various aircraft, the orientation of the platforms, and the state of health of the instrument suite and incoming data. The input data is distributed to other PIs onsite and has led to a very strongly interactive effort to develop the best possible mission profiles and provided substantial improvement in research-team building through real-time participation in the research/analysis effort.

\section{UAV APPLICATIONS FOR STUDYING THE RADIATION AND OPTICAL PROPERTIES OF UPPER-TROPOSPHERIC CLOUDS STEPHENS, GRAEME L. COLORADO STATE UNIVERSITY FY 1996100 FY 19950 FY $1994 \quad 0$}

Keywords: radiation properties of upper-tropospheric clouds, cloud optical properties

Objectives: To participate in upcoming experiments over the ARM CART Site during Fall 96 in the measurement program, in the experimental design, and in the results analysis.

Approach: Clear-sky absorption and the relationship of this absorption to water-vapor path will be analyzed. 
These results, together with analyses of the new data, will be used as a direct test of climate-model radiation parameterization. Cloudy-sky radiometric data will be analyzed, and the relationship to cloud properties will be deduced from the lidar and radar and spectralradiance data.

Results to Date: The SSP instrument is now taking research-quality data. It has flown in the Gnat UAV, the Egret aircraft, and the Altus UAV. The instrument significantly enhances the UAV payload and is particularly promising for detection of optically thin clouds and aerosol and in conjunction with backscatter lidar.

\section{UAV RADIATION-MEASURING SYSTEM (UAV RAMS) VALERO, FRANCISCO

Keywords: clear-sky absorption, radiative fluxes Objectives: To investigate the clear-sky excess absorption of solar radiation by measuring shortwave flux profiles in the 0.3- to 4-micron and 0.7- to 2.8-micron spectral ranges; to analyze the data and compare them to model calculations to determine the magnitude of the apparent clear-sky "excess absorption" in the visible regions of the solar spectrum; and to determine the effect of observational strategies on the measurement of radiative fluxes.

Approach: With the RAMS instruments on board the UAV and other instruments (both airborne and ground-based), the radiation will be measured, and the resulting data will be analyzed to determine any absorption in the accessible spectral ranges.

Results to Date: RAMS instruments have been flown in the UAV program since the first DOE flights. Analysis of data from the RAMS has been published by various researchers. The Otter and the Egret aircrafts have been instrumented with suites including RAMS. These aircraft were used in the ARESE missions along with RAMS-equipped ER-2 aircraft. Data from these efforts are still being analyzed, but results indicate absorption in clouds higher than that predicted by radiation-transfer codes.

\section{VITKO, JOHN \\ FY 1996843 \\ FY 1995935 \\ FY 19940}

MISSIONS AND OPERATIONS

SANDIA NATIONAL LABORATORIES

Keywords: aerial observations, UAV, flying platforms Objectives: To develop platforms and instruments with which to collect data to complement the groundbased ARM Clouds and Radiation Testbed (CART) data streams, thus improving the performance of GCMs.

Approach: The ARM-UAV Program contributes to the ARM goals and objectives by providing data from instruments flown for long duration at the top of the tropopause over the ARM site. While involved in developing and proving instrument and operational feasibility, the program continually seeks to contribute unique and useful data throughout its development phase. DOE funding for this effort is solely in the area of safety assurance and operations. In the first years, the research, instrument development, ground data handling, and bulk of the operations cost was being funded by the DOD through its Strategic Environmental Research and Development Program (SERDP). DOE is funding operational cost only at this time. No improvements in instruments, new payload development, or PI research is included.

Results to Date: The program began instrument efforts with directed-development projects to build four devices: an imaging spectrometer, a cloud lidar, solar and longwave total radiometers, and a high-resolution interferometer. These are presently in various stages of development and operation. The imaging spectrometer is in the early operational phase. Named the multispectral pushbroom imaging radiometer (MPIR) and designed and built by Sandia, it flew with four of its nine imaging spectral bands operating in spring 1996. The cloud lidar, designed and built by Lawrence Livermore National Laboratory, operated very successfully, providing good data for all flights. Successful missions were flown in fall 1995, spring 1996, and fall 1996. These missions have provided data that are being analyzed to try and determine the absorption of short-wave radiation in clouds, which has surfaced as a major difference between predictions and measurements. Although temporarily overshadowed by the absorption question, data on the 3-D radiation field and diurnal radiation effects from these missions are also being examined with considerable enthusiasm. 



\title{
ATMOSPHERIC SCIENCES
}

Atmospheric Sciences improves the understanding of and information about atmospheric processes controlling ozone and $U V-B$ trends; the heterogeneous chemistry of Earth's atmosphere; aerosol formation; the measurement and modeling of atmospheric flow over topographically complex terrain; and the transport, dispersion, and ultimate fate of energy-related emissions.

\author{
Program Manager \\ Rickey C. Petty \\ Environmental Sciences Division \\ Department of Energy, ER-74 \\ 19901 Germantown Road \\ Germantown, MD 20874-1290 \\ (301) $903-5548$ \\ Internet: rick.petty@oer.doe.gov
}

\section{ATMOSPHERIC TRANSPORT OF CONTAMINANTS ADDIS, ROBERT P. \\ WESTINGHOUSE SAVANNAH RIVER COMPANY \\ FY 1996148 \\ FY $1995 \quad 0$ \\ FY 1994197}

Keywords: ASCOT, RAMS model, technology transfer, Mexico City Air-Quality Study Project

Objectives: To contribute to the Atmospheric Studies in Complex Terrain (ASCOT) program by way of advanced numerical mesoscale modeling.

Approach: The RAMS model will be developed for application to the ASCOT program, and information about the technology will be transferred to potential users.

Results to Date: A set of proposals were provided for consideration and use in the Mexico City Air-Quality Study Project. These proposals were "Optimizing the Intensive Field Monitoring Program for Mexico City," "Use of Mesoscale Model to Identify Meteorological Precursors to Severe Air-Quality Episodes in Mexico City," "Regional Transport of Mexico City Air Pollutants into the southwestern United States," "Use of a Mesoscale Atmospheric Model to Improve IR Remote Sensing and Boundary-Layer Modeling," and "The Impact of Aerosols on Thermally Driven Flows."

\section{THREE-DIMENSIONAL GLOBAL ATMOSPHERIC CHEMISTRY ATHERTON, CYNTHIA, and PENNER, JOYCE LAWRENCE LIVERMORE NATIONAL LABORATORY \\ FY 1996300 \\ FY 1995203 \\ FY 1994200}

Keywords: tropospheric chemistry, ozone, prediction, atmospheric chemistry

Objectives: To develop and apply a 3-D, global tropospheric model with realistic treatment of atmospheric chemistry.

Approach: We have improved our model treatment of the troposphere by developing and/or obtaining a number of state-of-the-art emission inventories for many key species. Additionally, we have extensively compared our model predictions with observations of many tropospheric species. We are coupling our model framework to a general circulation model that includes water as a prognostic species, which will allow us to treat heterogeneous and aqueous phase chemistry. We have also begun adding new techniques for simulating localized emissions more accurately.

Results to Date: We have shown that the summertime export of ozone and its precursors from North America occurs in events in which ozone concentrations over the North Atlantic Ocean increase by 5 to $25 \mathrm{ppbv}$. We have shown that decreasing North American fossil-fuel $\mathrm{NO}_{x}$ can decrease the mixed-layer ozone concentrations by 5 to $20 \mathrm{ppbv}$ over the North Atlantic Ocean. We have shown that the North Atlantic may be divided into four regions on the basis of the degree of impact of transport from North America: western, central, eastern, and an area of little impact. 


\section{RESEARCH AIRCRAFT OPERATIONS \\ BARCHET, W. RICHARD \\ PACIFIC NORTHWEST NATIONAL \\ LABORATORY \\ FY 1996738 \\ FY 1995630 \\ FY 1994361}

Keywords: airborne measurements, atmospheric chemistry, research facility

Objectives: To operate a research aircraft equipped for atmospheric-chemistry, aerosol, turbulence, and radiant-energy research by investigators funded by the U.S. Department of Energy.

Approach: A Gulfstream 1 twin turboprop aircraft is operated as a DOE research facility primarily for use by investigators in the Atmospheric Chemistry Program (ACP). Up to 50 hours of research flying is provided by the facility in support of research programs. Support includes assistance with the installation of investigator-provided equipment, instrument calibration and maintenance, off-site logistics, air and ground crews, and all aircraft maintenance and aircrew certification.

Results to Date: Data collected during the ACP/ Northeast field study that was conducted in August and September 1995 were provided to the participating ACP collaborators. A differential global position system (GPS) was installed, and flight tests were made to compare its performance to that of an inertial navigation system (INS). The new GPS system is significantly lighter in weight and consumes much less electrical power than the INS yet provides superior aircraft attitude data that are essential for measuring the turbulent and mean winds aloft. The unavailability of other investigators that measure NOy aloft required Brookhaven National Laboratory to cancel its planned comparison of $\mathrm{No}_{\mathrm{y}}$ inlets.

The principal investigator participated in the assembly of NARSTO/Northeast participants, the full international NARSTO assembly, and a planning meeting of the Analysis and Assessment Team. Planning is in progress regarding the coordination of ACP field studies with the NARSTO/Northeast field studies. The principal investigator participated in the annual meeting of the ACP and chaired a session on future measurement needs within the ACP and on the G-1.

\author{
FULL TURBULENCE SIMULATIONS OF \\ NEUTRALLY STRATIFIED FLOW IN \\ COMPLEX TERRAIN \\ BARNARD, JAMES, and RILEY, JAMES \\ PACIFIC NORTHWEST NATIONAL \\ LABORATORY and UNIVERSITY OF \\ WASHINGTON \\ FY 199666 \\ FY 199569 \\ FY 19940
}

Keywords: computational fluid dynamics, complex terrain, atmospheric diffusion

Objectives: To perform a direct numerical simulation (DNS) of turbulent flow over simple terrain shapes.

Approach: Direct numerical simulations of turbulent flow resolve all scales of turbulent flow. These simulations are restricted by computer size and speed to low Reynolds numbers. Despite this limitation, the DNS technique has proved to be an invaluable tool for investigating many aspects of turbulent flow. In recent years, boundary-layer flows have been simulated with the DNS methodology. These simulations have only examined flow over flat surfaces; the important case of boundary layer flow over complex, nonflat surfaces remains to be examined. This work will extend the DNS technique so that it may be used to simulate boundary-layer flow over simple terrain shapes. The resulting simulations will then be used to investigate a number of important aspects of flow in complex terrain. Specifically, this investigation will focus on the flow in the lee side of terrain obstacles where flow separation and turbulence are important features of the flow that are not well understood.

Results to Date: To date, this investigation has concentrated on solving the Navier-Stokes (NS) equations in complex geometry. Achieving this goal is a many-step process. Perhaps the most important step is the development of a scheme to quickly solve the Poisson equation (for pressure) in complex geometry. To this end, an orthogonal computational grid has been devised that allows the Poisson equation to be cast in a particularly simple form. When cast in this form, a solution for the pressure forcing term of the NS equations may be easily calculated. Computation of the other terms of the NS equations is then performed with a so-called compact method that possesses the virtues of high accuracy and low phase error. As a result:

- A DNS of boundary-layer flow over flat terrain has been completed. The results of these simulations compare favorably with simulations produced by Coleman et al.

- The compact numerical scheme has been investigated as a way of easily casting the DNS method into complex geometries. 
- An orthogonal grid has been developed that permits the rapid solution of the diagnostic pressure (Poisson) equation in complex geometries.

\section{MEXICO CITY AEROSOL RESEARCH PROGRAM}

BARR, SUMNER, and STREIT, GERALD

LOS ALAMOS NATIONAL LABORATORY

FY 1996100

FY 1995255

FY 19940

Keywords: ozone, aerosols, pollution, Mexico City Objectives: To design and propose a combination of field experiments and computational simulations to elucidate the aerosol-pollution problem in Mexico City.

Approach: Experts on air pollution science were invited from both the university and DOE-laboratory environments to attend a workshop-type discussion of program design. Following the workshop, the experts collectively wrote and edited a proposal to DOE OHER outlining an effective program. DOE OHER requested that Los Alamos National Laboratory evaluate the feasibility of conducting an aerosol-pollution research program in Mexico City. The project would be a new phase of the Mexico City Air-Quality Research Initiative (MARI), a program that had been funded at Los Alamos by the DOE Policy Office and that focused on the photochemical-ozone problem. As with MARI, the new aerosol study would involve collaboration with the Mexican Petroleum Institute and the Mexican National Oil Company, PEMEX.

Results to Date: No results were reported.

\section{SUPPORT TO DOE'S METEOROLOGICAL COORDINATING COMMITTEE (DMCC) BAYNE, STEWART \\ OAK RIDGE INSTITUTE FOR SCIENCE AND EDUCATION \\ FY 199610 \\ FY 19950 \\ FY 19940}

Keywords: Meteorological Coordinating Committee, meetings

Objectives: To aid DOE's Meteorological Coordinating Committee (DMCC) review and provide recommendations about meteorologically relevant activities within the DOE and to represent OHER in various activities of the interagency Office of the Federal Coordinator for Meteorology, which addresses various operational meteorological activities (such as emergency response) at the various $\mathrm{DOE}$ sites.

Approach: The DMCC focuses on identifying areas where resources and personnel can be shared or combined and also where meteorological assets can be used more effectively in emergency preparedness. The DMCC will continue to explore meteorological program interfaces and its crucial inputs regarding worker safety and threat assessments.

Results to Date: The DMCC has conducted two plenary-session meetings that have brought together atmospheric-science and/or site managers from the various DOE sites to address meteorological-information coordination and reporting efforts within the Department.

\section{OZONE PRODUCTION: A FIELD STUDY TO EVALUATE TROPOSPHERIC PHOTOCHEMICAL MECHANISMS BERKOWITZ, CARL M. PACIFIC NORTHWEST NATIONAL

$$
\begin{aligned}
& \text { LABORATORY } \\
& \text { FY } 1996451 \\
& \text { FY } 1995592 \\
& \text { FY } 1994400
\end{aligned}
$$

Keywords: airborne measurements, atmospheric chemistry, ozone

Objectives: To use airborne geophysical and chemical observations for the development and evaluation of hypotheses of the fate of tropospheric oxidants.

Approach: An interdisciplinary team consisting of boundary-layer meteorologists, atmospheric chemists and mathematicians work together in the planning, execution, and analysis of field programs related to oxidant levels in the atmosphere. The following activities were carried out in FY 1996:

- Participated in the annual meeting of the Atmospheric Chemistry Program,

- Conducted an ACP field study organized by PNNL on the measurement of the distribution of gaseous chlorine and bromine in the marine atmosphere,

- Conducted an ACP field study on the role of boundary-layer processes in urban and regional air quality being organized by PNNL and BNL as part of NARSTO-NE, and

- Carried out analysis and presentations of observations.

Results to Date: Data collected during the ACP/ Northeast field study that was conducted in August and September 1995 were provided to the participating ACP collaborators. ACP field studies were coordinated with the NARSTO/Northeast field studies for the summer of 1996 . The principal investigator participated in the annual meeting of the ACP and chaired a session on interdisciplinary studies and future research needs within the ACP. 
ATMOSPHERIC STUDIES IN COMPLEX TERRAIN

BOSSERT, JAMES

LOS ALAMOS NATIONAL LABORATORY

FY 1996164

FY 19950

FY $1994 \quad 0$

Keywords: Mexico City, data analysis, modeling, complex terrain, ASCOT

Objectives: To extend the observational and modeling tochniques developed previously for complex terrain to the study of local and regional meteorology in large mountain valleys and basins and to conduct meteorological research in support of DOE air-quality studies in Mexico City.

Approach: The research includes observations, data analysis, and modeling. It provides general data and modeling capabilities that are used to support emergency response and environmental management at various DOE facilities. These capabilities are also being used to provide support for regional air-quality studies in Mexico City.

Results to Date: In FY 1996, efforts have been undertaken to complete two ASCOT-funded projects: (1) The Influence of Complex Terrain and Scale Interactions on the Meteorology of North-Central New Mexico in the Vicinity of Los Alamos National Laboratory and (2) Thermally Generated Flows Along the Colorado Front Range Region and Their Response to External Forcing. The task of redirecting research to address the Mexico City Complex Terrain region began, as well. These efforts are being conducted to contribute to a field study that will be conducted within the Mexico City region.

\section{THERMALLY GENERATED FLOWS ALONG THE COLORADO FRONT RANGE AND THEIR RESPONSE TO EXTERNAL FORCING BOSSERT, JAMES \\ LOS ALAMOS NATIONAL LABORATORY \\ FY 199687 \\ FY 199535 \\ FY 19940}

Keywords: complex terrain meteorology, valley meteorology, ASCOT, dispersion

Objectives: To improve understanding of the variability of katabatic flows in complex terrain by investigating the interaction of this phenomenon with topographically forced gravity waves.

Approach: Observational analysis and mesoscale meteorological modeling will be used to study katabatic flows.

Results to Date: No results were reported.

\author{
GLOBAL 3-D MODELING OF \\ ATMOSPHERIC OZONE IN THE FREE \\ TROPOSPHERE AND THE STRATOSPHERE \\ WITH EMPHASIS ON MIDLATITUDE \\ REGIONS \\ BRASSEUR, GUY; TIE, XUEXI; ERICKSON, \\ DAVID; and STACY, WALTER \\ NATIONAL CENTER FOR ATMOSPHERIC \\ RESEARCH \\ FY 1996131 \\ FY 1995127 \\ FY 1994107
}

Keywords: 3-D chemical-transport model, stratosphere, troposphere

Objectives: To use global chemical-transport models to study the chemical and dynamical processes that affect midlatitude stratospheric ozone and to quantify the budget of tropospheric ozone.

Approach: Four models will be improved and used: (1) a new version of our 2-D chemical-radiativedynamical model with microphysical processes of sulfate aerosols and polar stratospheric clouds (PSCs) and heterogeneous conversions on the surfaces of sulfate aerosis and PSCs; (2) the stratospheric version of a 3-D off-line chemical-transport model (STARS) with a relatively high horizontal resolution (2.8 degrees in latitude) with a microphysical formation of PSCs; (3) the tropospheric version of a 3-D off-line chemical-transport model (MOZART) with a details in the surface emissions and hydro-carbon reactions to estimate the tropospheric ozone budget and perturbations; and (4) the intermediate model of the global and annual evolution of species (IMAGES) with detailed chemical reactions but relatively lower resolutions. Model results will be compared with available data.

Results to Date: We have implemented in our 2-D model a chemical scheme describing more accurately the methane oxidation chain in the troposphere and have estimated the change in the tropospheric-ozone abundance caused by a doubling in the methane concentration.

We have also completed the development of the IMAGES model, which describes the 3-D distribution of approximately 50 chemical compounds from the surface to the 50-mbar level. The model has been used to assess the impact of aircraft emissions on tropospheric ozone and to investigate potential causes for the recently observed decrease in $\mathrm{CO}$ abundances.

Most recently, we have completed the development of a global 3-D model (MOZART) that simulates the distribution of ozone and its precursors in the troposphere and lower stratosphere. The model, which includes approximately $\mathbf{4 0}$ chemical species and 120 chemical and photochemical reactions, is driven by winds and temperatures provided by the NCAR Com- 
munity Climate Model (CCM-2). The spatial resolution is 2.8 degrees in longitude and latitude, with 18 levels in the vertical direction from the surface to $1 \mathrm{mb}$. Boundary-layer exchanges, cloud convection, and aqueous-phase chemistry are included in the model. Surface emissions are based on preestablished inventories.

\author{
EVALUATION OF ULTRAVIOLET \\ RADIATION, OZONE, AND AEROSOL \\ INTERACTIONS WITH AUTOMATIC \\ DIFFERENTIATION \\ CARMICHAEL, GREGORY, and \\ POTRA, FLORIAN \\ UNIVERSITY OF IOWA \\ FY 1996121 \\ FY 1995119 \\ FY 1994110
}

Keywords: tropospheric ozone modeling, UV-B radiation, automatic differentiation, sensitivity analysis Objectives: To investigate the interactions among ozone, aerosols, and ultraviolet radiation in a combined radiation-atmospheric-chemistry model with automatic-differentiation software.

Approach: The interactions among ozone, aerosols, and ultraviolet radiation are studied with a detailed radiation model combined with a 3-D atmosphericchemistry model. These models are joined with automatic-differentiation software to allow desired sensitivities to be calculated online with the radiation-chemistry computations. Automatic differentiation is a newly emerging technology for computing derivatives. The combined method is used to investigate (1) UV-B radiation at the Earth's surface as a function of changes in ozone and aerosols in the stratosphere and troposphere and (2) effects of changes in solar actinic flux on the photochemical oxidant cycle of the troposphere.

Results to Date: The couplings between tropospheric chemistry and radiative transfer have been investigated with the STEM-II model, automatic differentiation, and numerical improvements. Our analysis of the effects of tropospheric ozone on radiative transfer for the eastern United States confirmed previous findings that increases in tropospheric ozone can reduce the surface flux of UV-B. Furthermore, reductions in total ozone were found to decrease ground-level ozone in the summertime. This finding is opposite to that found in box-model studies for high-NO ${ }_{x}$ conditions. The differences arise because of the importance of transport processes.

ADIFOR (automatic differentiation software) has been used to calculate the sensitivity of ozone to all initial conditions and chemical-reaction-rate constants for the IPCC chemical-mechanism test scenarios (six cases). ADIFOR has been combined with the STEM-II model, and the sensitivities of UV-B and troposphericozone levels to changes in total ozone, aerosols, and other key parameters are now being calculated. Finally, we have devoted some effort to the development of new and more efficient chemical integrators. We have developed some new implicit integrators that offer more accurate and faster chemistry calculations.

\section{USE OF ASCOT NETWORK AND SATELLITE DATA TO STUDY DRAINAGE FLOW IN THE FRONT RANGE \\ COULTER, RICHARD; GAO, WEIGANG; LEE, IN YOUNG; and WESELY, MARVIN ARGONNE NATIONAL LABORATORY \\ FY 199667 \\ FY 199570 \\ FY $1994 \quad 0$}

Keywords: atmospheric dispersion, complex terrain, katabatic flow, Mexico City, ASCOT

Objectives: To evaluate the processes that affect the transport and diffusion of energy-related trace chemicals above nonuniform terrain at locations determined by the Atmospheric Dynamics Program.

Approach: The transport and dispersive properties of the lower atmosphere above nonhomogeneous terrain will be studied. Satellite observations of visible and infrared radiation from the Earth will be used with data on near-surface profiles of wind and temperature to achieve a detailed understanding of the development of katabatic flow and its dependence on external forcing mechanisms. A detailed radiative-transfer model will be coupled with a mesoscale meteorological model to analyze the effects of varying surface topography and type on katabatic flow in complex terrain. Argonne will participate in large, multiorganizational field experiments to study atmospheric transport and mixing processes above complex or nonuniform terrain like that found in the Southwest and in the mountainous basin in which Mexico City is located.

Results to Date: Instruments and equipment that were part of the ASCOT observational network were removed from the Front Range of Colorado. The data collection and distribution center at Argonne for the Front Range data was maintained. One meeting on initiatives for transport and diffusion in the Southwest and one meeting on possible transport and dispersion measurements above Mexico City were held. Analyses were completed and published on the numerical simulation of nocturnal drainage-flow properties in a rugged canyon and on the dependence of canyon winds on surface cooling and external forcing in the Colorado Front Range. 
FIELD STUDIES IN ATMOSPHERIC CHEMISTRY

DAUM, PETER H;; KLEINMAN, LAWRENCE;
and NEWMAN, LEONARD
BROOKHAVEN NATIONAL LABORATORY
FY $1996 \quad 613$
FY $1995 \quad 747$
FY 19941324

Keywords: ozone formation, energy emissions, pollutant transport, tropospheric photochemistry

Objectives: To understand the chemical and physical processes that determine the fate of energy related pollutants emitted to the atmosphere.

Approach: Field campaigns and analysis activities are designed to obtain a mechanistic understanding of the photochemical reactions responsible for the formation of $\mathrm{O}_{3}$ and related pollutants as they occur in the ambient atmosphere. Current foci are the exceedance of federal air quality standards for $\mathrm{O}_{3}$ downwind of urban areas and the export of $\mathrm{O}_{3}$ and related pollutants from the North American continent to the North Atlantic. Field campaigns typically incorporate both aircraft- and surface-based measurements.

Measurements include primary photochemical species, such as $\mathrm{O}_{3}, \mathrm{NO}_{x}$, and light hydrocarbons, as well as product species, such as aldehydes, peroxides, and nitrates. The relationships among the concentrations of these species, in the context of their spatial and temporal distribution, are used to identify and quantify the importance of potential pathways for the chemical production of $\mathrm{O}_{3}$ in the atmosphere.

Results to Date: Analysis of the data from the North Atlantic Regional Experiment (NARE) conducted in Summer 1993 has been completed. The objective of this project was to examine the fate and potential effects of pollutants transported from eastern North America to the western North Atlantic Ocean. Principal findings are: (1) $\mathrm{O}_{3}$ episodes in the NARE project region during the summer are associated with the transport of air from the Southwest in advance of eastward-moving cyclonic systems. (2) Transport of $\mathrm{O}_{3}$ and related species from the major industrial areas on the east coast of the United States to the western North Atlantic generally occurs in well-defined layers between 0.3 and $2 \mathrm{~km}$ above the ocean surface. Losses from these layers appear to be minimal leading to the accumulation of unusually high concentrations of photochemical product species, such as $\mathrm{HNO}_{3}$ and peroxides. (3) Air masses more than 2 days transit time downwind of eastern North America appear to have little potential for additional $\mathrm{O}_{3}$ production because of depletion of $\mathrm{NO}_{\mathbf{x}}$-reactive hydrocarbons. The presence of high peroxide concentrations suggests that further $\mathrm{O}_{3}$ production is limited by the availability of $\mathrm{NO}_{x}$. (4) Comparison of measurements of $\mathrm{O}_{3}$ and related species made aloft to measurements at island and coastal surface sites showed that the surface measurements did not accurately represent concentrations in the lower troposphere. This result implies that measurements at islands and coastal sites in these regions cannot reliably be used to examine the impact of North American emissions on the western North Atlantic.

\section{MEASUREMENT OF GAS/WATER UPTAKE COEFFICIENTS FOR TRACE GASES ACTIVE IN THE MARINE ENVIRONMENT \\ DAVIDOVITS, PAUL; WORSNOP, DOUGLAS; JAYNE, JOHN; and KOLB, CHARLES BOSTON COLLEGE and AERODYNE RESEARCH, INC. \\ FY 1996110 \\ FY 1995111 \\ FY 1994108}

Keywords: marine atmosphere, air/water exchange coefficients

Objectives: To measure air/water exchange coefficients of specific gases that are of importance in the marine atmosphere.

Approach: Uptake coefficients on aqueous droplets will be performed for the reduced-sulfur species DMS, $\mathrm{H}_{2} \mathrm{~S}, \mathrm{CS}_{2}, \mathrm{OCS}, \mathrm{CH}_{3} \mathrm{CH}$, and $\mathrm{CS}_{2}$ and their oxidation products MSA, DMSO, and $\mathrm{DMSO}_{2}$. Measurements will also be performed for $\mathrm{NO}, \mathrm{NO}_{2}, \mathrm{NO}_{3}, \mathrm{I}_{2}$, and $\mathrm{Cl}_{2}$. Studies will also be conducted on (1) codeposition of reduced-sulfur species with the atmospheric oxidizers $\mathrm{H}_{2} \mathrm{O}_{2}, \mathrm{NO}_{2}, \mathrm{NO}_{3}$, and $\mathrm{O}_{3}$; (2) studies of liquid-phase and gas/liquid-surface photochemical free-radical production; and (3) the initial development of a laseror charge-induced droplet-ablation technique to monitor trace-gas uptake. Measurements will be made as functions of temperature, $\mathrm{pH}$, and ionic strength. Measurements will provide the mass-accommodation coefficient, the solubility parameters, and information on surface chemistry for these species.

Results to Date: We have completed detailed uptake studies for MSA, DMSO, DMSO, DMS, $\mathrm{H}_{2} \mathrm{~S}, \mathrm{CS}_{2}$, $\mathrm{CH}_{3} \mathrm{SH}, \mathrm{OCS}, \mathrm{O}_{3}, \mathrm{NO}_{2}, \mathrm{~N}_{2} \mathrm{O}$, and $\mathrm{NH}_{3}$. Experiments were performed as a function of $\mathrm{pH}$, ionic strength, and $\mathrm{H}_{2} \mathrm{O}_{2}$ concentration. The uptake of gas-phase $\mathrm{Cl}_{2}$ as a function of aqueous $\mathrm{Br}^{-}$and $\mathrm{I}^{-}$concentration and $\mathrm{O}_{3}$ and $\mathrm{Br}_{2}$ as a function of $\mathrm{I}^{-}$concentration was also studied. A new set of uptake and codeposition studies for $\mathrm{SO}_{2}$ and $\mathrm{NH}_{3}$ were completed. The results were used to obtain mass-accommodation coefficients and Henry's Law coefficients. 


\section{INVESTIGATING THE UMKEHR OZONE PROFILE RECORD DELUISI, JOHN \\ NATIONAL OCEANICAND ATMOSPHERIC ADMINISTRATION}

FY 1996147

FY 1995137

FY 1994108

Keywords: atmospheric ozone, ultraviolet-B radiation, aerosol research, Umkehr-retrieved ozone profiles Objectives: To provide new ancillary information on errors to the Umkehr-retrieved zone profiles caused by aerosols and to document the climatology of the past and current state of stratospheric aerosol and ozone profiles, evaluate the performance of the new Umkehr algorithm, evaluate the uncertainties of using Dave's scalar radiative-transfer code to calculate aerosol errors, and perform a trend analysis of the corrected Umkehr ozone-profile data, including an assessment of the uncertainties of the results.

Approach: Improvement of our understanding of the Umkehr effect and the uncertainties of the retrieved ozone profiles will lead to increased credibility of the long-term ozone-profile trends determined from Umkehr observations dating back to 1958. An aerosol database will be compiled consisting of satellite, lidar, aircraft, sunphotometer, and astronomical observations dating back to 1958. An empirical time-dependent model of stratospheric aerosol characteristics, including size-distribution, vertical profiles, and latitudinal distribution, will be created. These data will be used to provide the a priori information for estimating profile and size-distribution characteristics during the earlier period for which detailed information is increasingly scarce and only surface solar radiation data are available. Also, an ozone climatology database will be developed for calculating aerosol errors when such data are not available concurrently with aerosol profiles. The present Dave scalar radiative-transfer algorithm used to calculate aerosol errors by comparisons with other algorithms of comparable precision will be qualified. An extensive error analysis of the aerosol correction procedure will be performed that will include effects of size-distribution errors, verticalprofile errors, and errors that use climatological profiles of ozone and aerosols vs directly observed profiles, such as provided by SAGE. The time dependence of SAGE aerosol optical extinction data will be examined in terms of aerosol-size-distribution changes. A best estimate will be calculated of aerosol errors to Umkehr ozone profiles as far back in time as the aerosol data reliably allows (for the new as well as the old Umkehr algorithm).

Results to Date: To evaluate the accuracy of Dave's radiative-transfer algorithm, we performed a set of comparisons with results of complete-spherical radiative-transfer models, such as Herman's full spherical atmosphere (vector) algorithm and pseudospherical (scalar) code, Mateer's pseudospherical atmosphere (vector) code (molecular scattering only), Dave's pseudospherical atmosphere (vector) code, Monte Carlo code, and the Stamnes discrete ordinate algorithm. Standardized atmospheres including ozone and aerosol profiles were developed for the comparison. As expected, the magnitude of the aerosol error depends strongly on the interpolation routine, introducing up to $20 \%$ difference in the retrieved results. This finding can be of a great importance to the ozone network because the automated Dobsons and Brewers take measurements at fewer solar zenith angles than required by the Umkehr retrieval procedure. The absent information is found by an interpolation technique. Such algorithms should be used with caution.

A sensitivity study of the Umkehr-retrieved-ozone trends to the solar-activity index showed a nonnegligible correlation between maximum and minimum solar activity and lower-stratosphere ozone variations. Four modeled ozone-mixing-ratio altitude profiles and the corresponding pressure profiles were studied. The Mateer-Dave radiative-transfer code was used to calculate Umkehr $\mathrm{N}$-values for these four ozone profiles, and the $\mathrm{N}$-value profiles were used in old (1964) and new (1992) Umkehr ozone-retrieval algorithms to obtain four Umkehr ozone profiles. The old Umkehr algorithm results are in a fairly good agreement (better than $1 \%$ ) with observed trends in layers 2 to 8 while the new Umkehr struggles to keep the profile of the trends in place and overestimates trends in layer 3. One explanation lies in the differences in the smoothing constraints applied in the algorithms. The old algorithm employs a looser smoothing constraint, while the new one employs a tighter constraint. Different a priori information in the two algorithms also needs to be considered.

The validation of the new and old algorithmretrieved Umkehr ozone profiles against satellite SBUV data reveals the largest discrepancies in the lowest layers (up to $20 \%$ ). The algorithm's first-guess ozone profile tends to overestimate the ozone amount in the lower atmosphere. The low-level SBUV data also needs to be questioned.

The averaging kernels in the lower layers of the new Umkehr algorithm are not well separated in space and do not have strong maxima centered in these layers. Therefore, these layers are considered to contain the least reliable profile information.

The total ozone climatology contains seasonal and latitudinal behavior, and thus, its change translates into variations of the ozone amount in the lower layers. However, abnormal amounts of total ozone can introduce seasonal errors in the first-guess profile and, therefore, affect the retrievals. 
Usually, the combination of two contiguous Umkehr layers improves the accuracy of the ozone retrievals and may help solve the problem with the overestimated ozone amount in the troposphere and correct the upper layers' ozone content.

\section{ATMOSPHERIC TRACER DEVELOPMENT AND USE \\ DIETZ, RUSSELL N., and SENUM, GUNNAR I. BROOKHAVEN NATIONAL LABORATORY \\ FY 199690 \\ FY 1995190 \\ FY 1994213}

Keywords: perfluorocarbon tracers (PFTs), watersoluble tracers, atmospheric tracing, Mexico City, industrial-leak detection

Objectives: To maintain and advance atmospherictracer technology for direct application to specific DOE and other Atmospheric Chemistry Program (ACP) needs, including model verification, source apportionment, complex transport and dilution, and cloud/rain scavenging and to apply the technology to other government and industrial problems, including underground leak locating, building ventilation and performance, and petroleum-reservoir studies.

Approach: The use of the capillary-carbon-layer opentubular (CLOT) column for small-volume gas-chromatographic analyses will be standardized, making possible short-term, passive (1 to 3 day), ground-based air sampling and active (1 to 3 hour), vertically resolved, balloon-borne, air sampling for multiple tracers. These measurements are useful in model verification and source-apportionment field studies. $A$ new approach to a real-time multiple PFT analyzer will be designed that permits a three-fold reduction in analysis time (to $1 \mathrm{~min}$ ) with a ten-fold improvement in detectability. A CLOT-column approach to precutting will be developed to reduce analysis time. Analytical procedures will be developed for aerosol water-soluble perfluorocarbon salt tracers for use in cloud, rain, and dry-deposition long-range atmospheric-transport studies. Developments in users' applications will be tested and applied.

Results to Date: The CLOT column was successfully introduced in the European Tracer Experiment (ETEX). Results gave 5\% precision for ambient PFT analyses with 400 passive samplers exposed for 2 weeks at 170 sites. A preprototype real-time PFT analyzer for use in dielectric-fluid leak pinpointing was delivered. The ability to measure down to trace background levels with less than $10 \%$ precision was demonstrated. Underground-cable dielectric-fluid-leak detection was performed in New York City and the United Kingdom. A passive PFT system was used in building-ventilation tests. PFT-equipment used in hospital-isolation-room studies indicate that aerosolprotective masks should not be donned or removed within anterooms. Six PFTs were used to evaluate water-alternate gas-enhanced oil recovery; some PFTs were followed for 2-1/2 years in the oil reservoir. Preparations started for determining underground-tank barrier-integrity at Hanford.

\section{ASCOT PROGRAM SCIENTIFIC DIRECTOR DORAN, J. CHRISTOPHER PACIFIC NORTHWEST NATIONAL LABORATORY \\ FY $1996 \quad 12$ \\ FY 199535 \\ FY 1994160}

Keywords: ASCOT, program coordination, air quality, complex terrain, planning

Objectives: To promote and coordinate research activities among the groups participating in the DOE's Atmospheric Studies in Complex Terrain (ASCOT) program.

Approach: Areas will be identified in which boundary-layer meteorology can address air-quality issues being studied in the Atmospheric Chemistry Program and in other cooperative programs, such as the North American Regional Strategy for Tropospheric Ozone (NARSTO) and the Southern Oxidants Study (SOS). Planning and coordination will be provided for possible ASCOT research in the Mexico City area. A research plan and proposal for a boundary-layer research program focused on the southwestern United States will be developed.

Results to Date: A draft proposal was written, describing the meteorological research identified as necessary for an understanding of the local and regional circulation patterns in the Mexico City area that affect the air quality of that region. A workshop was organized for approximately 30 participants drawn from federal and state agencies, universities, and utilities to help identify critical meteorological research needs for airquality issues in the southwestern United States. A report describing the results of that workshop was prepared and has been distributed to nearly 100 individuals. An invited talk was prepared and delivered at the Seventh Conference on Mountain Meteorology, describing the advances in numerical modeling of complex-terrain meteorology that have taken place in the ASCOT program. 


\author{
BOUNDARY-LAYER STRUCTURE AND \\ EVOLUTION IN THE FRONT RANGE \\ REGION OF COLORADO \\ DORAN, J. CHRISTOPHER; WHITEMAN, \\ C. DAVID; and BADER, DAVID C. \\ PACIFIC NORTHWEST NATIONAL \\ LABORATORY \\ FY 1996320 \\ FY 1995239 \\ FY 19940
}

Keywords: boundary layer, meteorology, air quality, complex terrain

Objectives: To investigate the structure and dynamics of the atmospheric boundary layer with an emphasis on those features related to air-quality problems and how they are affected by terrain and surface properties. Approach: The diurnal variations in the thermally and topographically forced structure and dynamics of the planetary boundary layer in the Front Range region of Colorado and the modifications of these properties by synoptic influences will be studied. The influence of boundary-layer structure and dynamics in air-quality studies such as those undertaken for the North American Research Strategy for Tropospheric Ozone (NARSTO) and the Southern Oxidants Study (SOS) will be investigated. Research will be carried out on the role of terrain and thermally driven winds on air quality in the Mexico City region. Studies will be extended to regional boundary layer behavior in the southwestern United States with the goal of establishing a long-term boundary-layer research program on air quality in the region.

Results to Date: A study of the meteorological factors affecting winds in a local canyon and how those winds can, in turn, affect the flows over the Rocky Flats Plant was completed. While conducting research on mountain-plain circulations, we discovered poorly understood solar semidiurnal atmospheric tidal oscillations above the Rocky Mountains and, later, in radar profiler records from other parts of North America. Research was begun on regional-scale diurnal circulations in the Rocky Mountains focusing on the mountain-plain circulation and the existence of the upper return branch of the daytime and nighttime circulations. We began an effort to develop a new multilaboratory, multiagency research program on the meteorology and air quality of the Southwest. A cooperative effort was launched with scientists in DOE's Atmospheric Chemistry Program to examine meteorological factors affecting ozone profiles off the coast of New England.

\section{ATMOSPHERIC CHEMICALS' SOURCES AND FATES \\ DOSKEY, PAUL \\ ARGONNE NATIONAL LABORATORY \\ FY 1996196 \\ FY 1995197 \\ FY 1994194}

Keywords: atmospheric chemistry, organic compounds, long-range transport, emissions

Objectives: To measure nonmethane organic compounds (NMOCs) in collaborative field experiments on the effects of energy-related trace chemicals on the photochemistry of the atmosphere and to evaluate processes that affect the long-range transport of NMOCs and their transformation products over global scales.

Approach: Whole-air samples are obtained aboard an aircraft with stainless steel sample canisters and analyzed subsequently at Argonne. An on-board sampling and measurement system with a gas chromatograph is being developed to make measurements of NMOCs in near-real time on the aircraft. NMOC profiles are measured at several heights above a deciduous forest to investigate the emission rates of isoprene and related compounds. NMOC profiles are also measured in coastal regions to investigate the role of coastal atmospheric circulations in redistributing NMOCs and their transformation products vertically. The resulting data are used in numerical models that simulate atmospheric chemistry, transport, and turbulent mixing to evaluate emission rates and investigate long-range transport. The feasibility of using data from the ocean-color-monitoring satellite (SeaWiFS) to evaluate the spatial and temporal variation of dimethyl sulfide production in oceanic regions will be studied. Results to Date: A cryogenic-preconcentration, highresolution gas-chromatographic system with a flameionization detector (FID) was assembled for installation aboard the Battelle G-1 research aircraft to make in situ measurements of $\mathrm{C} 2$ to $\mathrm{C} 6$ nonmethane organic compounds (NMOCs). The Southern Oxidants Study (SOS) in middle Tennessee and the Atmospheric Chemistry Program's Northeast Study (ACP NE) off Long Island were participated in. A sampling system was developed to collect whole-air samples in stainless steel canisters; the method was used aboard the Battelle G-1 aircraft for SOS. Total NMOC concentrations during SOS were 25 to $180 \mathrm{ppb}$ of carbon; the concentrations of hydrocarbons (such as acetone, methanol, and isoprene) were determined separately. 


\section{GLOBAL AEROSOL MODEL EVALUATION EASTER, RICHARD \\ PACIFIC NORTHWEST NATIONAL LABORATORY \\ FY 1996238 \\ FY 1995242 \\ FY 19940}

Keywords: aerosols, optical depth, radiative forcing, sulfate, climate

Objectives: To perform a comprehensive evaluation of a detailed global aerosol model with satellite- and surface-based aerosol-optical-depth data and surface concentration and deposition data and to estimate the direct radiative forcing by anthropogenic aerosols and associated uncertainties with the aerosol model.

Approach: A comprehensive evaluation of a detailed global aerosol model will be performed with satelliteand surface-based aerosol-optical-depth data and surface concentration and deposition data. The global aerosol model, which is being developed under separate funding, is coupled to a GCM that assimilates observed winds and temperature and predicts cloud water and precipitation. The aerosol model treats both anthropogenic and natural aerosols, including soluble inorganic species (sulfate, nitrate, and ammonium), organic species, elemental carbon, and dust. It simulates the aerosol size distribution with the method of moments. Several 6-week simulations will be performed. The simulations will be evaluated against available observations with an evaluation protocol developed during the project. Direct radiative forcing by anthropogenic aerosols, and associated uncertainties, will then be quantified with the aerosol-model simulations and the results of the model evaluation. Results to Date: Activities focused on identifying and acquiring observed data for the model evaluation and on developing a protocol for evaluating the model. Surface-based aerosol-optical-depth data from the 1980s onwards have been identified; a significant increase in the number and quality of these data in the past several years makes this time frame favorable for performing the model evaluations. AVHRR satellite data for August 1994 have been acquired and are being analyzed to obtain aerosol optical depths. Surface measurements from networks in the United States, Europe, and the North Atlantic have been obtained. The initial model-evaluation protocol has been developed and includes operational and diagnostic-evaluation components. A nudging procedure has been implemented in the GCM so the meteorology follows actual historical weather patterns for the simulation periods. Nudging is applied to horizontal winds and temperature with analyses from the European Centre for Medium Range Weather Forecasting.
URBAN AIR QUALITY MODELING

ERMAK, DONALD, and GUDIKSEN, PAUL

LAWRENCE LIVERMORE NATIONAL LABORATORY

FY 199657

FY 19950

FY 19940

Keywords: forecast models, pollutant plumes, urban air sheds, emergency response

Objectives: To adapt and apply the U.S. Navy 3-D wind-field-forecast models to the transport of pollutant plumes over urban air sheds involving spatial scales of 50 to $200 \mathrm{~km}$.

Approach: The U.S. Navy regional-scale wind-field models will be adapted to urban-airshed scales of 50 to $200 \mathrm{~km}$ and coupled with a suitable pollutant-transport model to produce an advanced urban-air-quality modeling system.

Results to Date: The U.S. Navy wind-field models have been initially tested for the San Francisco Bay Area. Initial results appear promising.

\section{THE USE OF ASCOT FIELD STUDY DATA IN A MESOSCALE MODEL EMPLOYING A FOUR-DIMENSIONAL DATA-ASSIMILATION TECHNIQUE \\ FAST, JEROME \\ PACIFIC NORTHWEST NATIONAL LABORATORY FY 1996149 \\ FY 1995112 \\ FY 19940}

Keywords: 4-D data assimilation, mesoscale modeling, complex terrain

Objectives: To use a mesoscale model employing 4-D data assimilation coupled with a Lagrangian particle dispersion model to simulate the transport and diffusion of tracers released in areas of complex terrain, such as the Rocky Flats Plant (RFP).

Approach: A multi-scale 4-D data-assimilation (FDDA) technique, based on Newtonian relaxation, has been incorporated into a mesoscale model and evaluated with meteorological and tracer data collected during the Atmospheric Studies in Complex Terrain (ASCOT) field experiment in the winter of 1991. The mesoscale model is used to predict the synoptically driven flows and small-scale circulations influenced by terrain along the Front Range in Colorado in the vicinity of RFP for several nocturnal and daytime periods during the ASCOT field experiment. FDDA is used to create dynamically consistent analysis fields based on the mesoscale forecasts and the special asynoptic data taken during this experiment. Observations from surface stations, towers, minisodars, airsondes, tethersondes, rawinsondes, and profilers are incorporated into the high-resolution analysis fields. 
The wind and turbulence quantities produced by the mesoscale model are then used to determine the dispersion of tracers released from RFP. A subjective and statistical evaluation of the meteorological and dispersion results is performed to examine the effect of FDDA on the nocturnal and daytime circulations and tracer transport.

Results to Date: As expected, the FDDA technique reduced the overall errors in the atmospheric and dispersion calculations while the model produced realistic small-scale circulations not resolved by the data. The results of this study indicate that the dataassimilation technique can have a positive impact on the mesoscale flow fields; however, care must be taken in its application to grids of relatively fine horizontal resolution. Continuous FDDA is useful in producing high-resolution mesoscale analysis fields that can be used to evaluate the flows during the ASCOT field study, to create better initial conditions for mesoscale atmospheric models, and to drive transport models for dispersion studies. When the mesoscale model is initialized with the high-resolution analyses created by FDDA with only the wind observations, the forecasts are improved for 1 to $\mathbf{2 ~} \mathrm{h}$ after the initialization time. However, when temperature is included in the analyses created by FDDA, the forecasts are improved for up to $9 \mathrm{~h}$ after the initialization time. Small-scale recirculation zones are also predicted in the vicinity of RFP. The location of the peak concentrations are found to be better simulated by the mesocale-dispersion modeling system with FDDA when compared to results from a diagnostic emergency-response model.

\section{LABORATORY STUDIES OF THE SENSITIVITY OF TROPOSPHERIC OZONE TO THE CHEMISTRY OF SEA-SALT AEROSOL FINLAYSON-PITTS, BARBARA UNIVERSITY OF CALIFORNIA, IRVINE FY 1996168 FY 1995166 FY 1994160}

Keywords: sea salt, halogens, troposphere, sodium chloride, nitrogen oxides, ozone

Objectives: To assess the role of reactions of sea-salt particles in the chemistry of the troposphere by determining the kinetics and mechanisms of reactions of sea salt and its components that produce photochemically active halogen compounds and (in sunlight) chlorine, bromine, and perhaps iodine atoms and by determining the fate of halogen atoms in the troposphere and, in particular, their role in the formation and fate of ozone.
Approach: The formation of free-radical intermediates will be studied with electron paramagnetism in the reactions of $\mathrm{NaCl}$ and model sea salt with oxides of nitrogen. The uptake of gaseous oxides of nitrogen by the major components of sea salt will be measured with Knudsen cells. The formation of both surface nitrate and bulk nitrate produced in the reaction of oxides of nitrogen with sea salt and its components will be followed with diffuse reflectance infrared spectroscopy (DRIFTS). And the reactions of tropospheric gases with chlorine atoms will be studied with long-path Fourier transform infrared and gas chromatographymass spectrometry.

Results to Date: The major component of sea salt, $\mathrm{NaCl}$, has been shown to react with nitrogen dioxide, a major air pollutant, to form a relatively long-lived free-radical anion intermediate in the salt, likely $\left[\mathrm{Cl} . . . \mathrm{NO}_{2}-\right]$. This reaction may have health implications in that it could serve as an additional mechanism for delivering reactive species into the deep lung. Knudsen-cell studies of the reaction of $\mathrm{HNO}_{3}$ with $\mathrm{NaCl}$ have shown that strongly adsorbed surface water is a controlling factor in determining the uptake and reaction of $\mathrm{NO}_{3}$ with $\mathrm{NaCl}$. This observation has led to a new model for these surface reactions, in which the surface is best represented as a concentrated salt solution rather than a solid. DRIFTS studies of the reaction of $\mathrm{NO}_{2}$ and $\mathrm{HNO}_{3}$ with synthetic sea salt have shown that the hydrates that are minor components in sea salt play a major role in the reaction. Chlorine atoms were shown to react with isoprene to form $\mathrm{HCl}$, $\mathrm{HCOCl}$, and some as-yet-unidentified chlorine-containing unsaturated carbonyl compound(s) that may prove to be useful markers of chlorine atom chemistry in the marine boundary layer.

\section{MEXICO CITY AIR-QUALITY STUDY FORMENTO, JOHN \\ ARGONNE NATIONAL LABORATORY \\ FY 1996100 \\ FY 19950 \\ FY 19940}

Keywords: planning, project management Objectives: To provide resources to the Mexico City Aerosol Research Program field study.

Approach: Planning, instrumentation acquisition, and siting activities will be carried out in preparation for the Mexico City Aerosol Study filed study.

Results to Date: Various contract and acquisition activities have been carried out and coordinated to achieve the objective of this task. 


\section{LABORATORY AND FIELD STUDIES EXAMINING THE ROLE OF PEROXYACYL NITRATES AND ORGANIC PEROXIDES IN TROPOSPHERIC CHEMISTRY \\ GAFFNEY, JEFFREY, and MARLEY, NANCY ARGONNE NATIONAL LABORATORY FY 1996226 \\ FY 1995227 \\ FY 1994175}

Keywords: atmospheric chemistry, organic compounds, oxidants, peroxyacetyl nitrates, PANs

Objectives: To develop a better fundamental understanding of the chemical and physical properties of organic oxidants and aldehydes to improve field measurement and atmospheric modeling capabilities. Approach: A luminol-based peroxyacetyl nitrate (PAN) analysis system is being developed for use on the Battelle G-1 research aircraft in FY 1997 for field studiesconcerned with oxidant formation and transport. Measurement of nitrogen dioxide and PANs by the use of honeycomb capillary columns and helium as a carrier gas is being explored to improve the analysis times and the sensitivities of detection. Improved analysis methods are used in the laboratory to examine the heterogeneous reactions of PANs on surfaces. Basic laboratory studies are conducted on the physical and chemical properties of higher-analog PANs, organic peracids, and peroxides. The aqueous chemistries of the organic peroxides and peracids are examined by using cylindrical internal reflectance Fourier transform infrared spectroscopic techniques. The possibility of coupling a luminol-based detection system with a high-performance liquid chromatograph will be evaluated for the measurement of organic peracids, organic peroxides, and hydrogen peroxide in air and aqueous samples.

Results to Date: A luminol chemiluminescent system was obtained and modified for analysis of nitrogen dioxide and PANs. Methods were developed to synthesize organic peracids, peroxyacyl nitrates, and peracids; these methods are being used for testing PAN sensitivities on the luminol analyzer. Collection of gas-phase and liquid-phase spectra of these compounds began as a step in determining their ultraviolet and infrared absorption cross-sections. Preliminary studies of the photooxidation of acetaldehyde confirmed that peracetic acid is formed and that formaldehyde is an intermediate in the photochemical transformation leading to the hydroperoxyl radical.

\author{
THEORETICAL STUDIES OF GLOBAL \\ TROPOSPHERIC AND STRATOSPHERIC \\ CHANGES IN OZONE \\ GROSSMAN, ALLEN \\ LAWRENCE LIVERMORE NATIONAL \\ LABORATORY \\ FY 1996147 \\ FY 1995145 \\ FY 1994150
}

Keywords: ozone change, radiative forcing, stratosphere

Objectives: To perform theoretical studies of the effects of changes in the ozone distribution in the global atmosphere to improve understanding of the mechanisms responsible for these ozone changes.

Approach: We have used our 2-D chemical-radiative-transport model as well as our detailed radiative transport models to investigate radiative forcing effects caused by various trace-gas-change scenarios. We have begun the development of a 3-D chemical-radiative-transport model designed for parallel-architecture computers.

Results to Date: The tropospheric radiative forcing has been calculated for ozone and water-vapor perturbations caused by the emissions from a realistic highspeed civil transport (HSCT) aircraft. Atmospheric profiles of water vapor and ozone were obtained with the LLNL 2-D chemical-radiative-transport model (CRT) of the global troposphere and stratosphere. IRradiative-forcing ca'sulations were made with the LLNL correlated $k$-distribution radiative transfer. $U V$, visible, and near-IR radiative forcing calculations were made with the LLNL two-stream solar-radiation model. For the case of water vapor, the IR and near-IR radiative forcing was determined at five latitudes and then averaged to obtain the global average value. For ozone, the radiative forcing was calculated for the globally averaged atmosphere. For both ozone and water vapor, the radiative forcing was too small to be of major climatic consequence. We have also added the capability to include aerosol scattering and absorption effects in the radiative-transfer models used in connection with coupled chemistry-GCM studies of sulfateradiative-forcing effects. This capability will provide a more accurate determination of both shortwave surface-flux changes and radiative-forcing values caused by ozone-change scenarios.

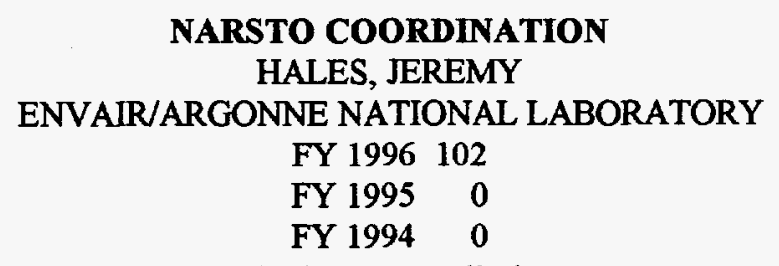

Keywords: NARSTO, ozone pollution, management, troposphere 
Objectives: To serve as Management Coordinator for NARSTO (an international, multiagency, private/ public partnership dedicated to the effective management of urban and regional ozone pollution), to serve as advisor to the DOE Atmospheric Chemistry program (ACP), and to conduct specific modeling activities within the ACP.

Approach: NARSTO's principal aim is to consolidate and coordinate tropospheric ozone research to develop the most effective strategies possible for management of regional and urban ozone pollution. DOE is a charter member of NARSTO. Its support of the management coordinator's office will provide coordination of the total program.

Results to Date: The NARSTO Management Coordination Office has been activated, and NARSTO's WWW home page has been implemented. An organizational structure has been set up, and a strategic execution plan has been drafted. A NARSTO annual meeting has been held and coordinated, and the NARSTO QA and data-management plans have been put in place. NARSTO's 1988 Ozone Assessment has been launched and coordinated. Two issues of the NARSTO News newsletter have been published, and the user's manual for the Pluvius II reactive-storm model has also been published. The $\mathrm{NO}_{x} \mathrm{NO}_{y}$ Asian/ Pacific pilot simulations have been completed for spring 1995.

\section{PARTIAL SUPPORT TO THE CONSORTIUM FOR ADVANCED MODELING OF REGIONAL AIR QUALITY \\ HANSEN, D. ALAN \\ ELECTRIC POWER RESEARCH INSTITUTE \\ FY 199630 \\ FY 199520 \\ FY 19940}

Keywords: air-quality modeling, air-quality research tools, air-quality management

Objectives: To develop, evaluate the performance of, and apply comprehensive modeling systems for the analysis of air-quality issues on regional and smaller scales.

Approach: Research will be coordinated internationally on air-quality modeling and decision support among public- and private-sector organizations that can materially contribute to the development of comprehensive modeling systems. A framework will be designed for developing and maintaining a comprehensive modeling system, and the design will be executed.

Results to Date: No results were reported.

\author{
PREFLIGHT AND POSTFLIGHT \\ SPECTRORADIOMETRIC CALIBRATION \\ ERRORS FOR SBUV- AND TOMS-TYPE \\ INSTRUMENTS \\ HEATH, DONALD; AHMAD, ZIA; and \\ WEI, ZONGYING \\ SCIENCE AND DATA SYSTEMS, INC., and \\ RESEARCH SUPPORT INSTRUMENTS, INC. \\ FY 199696 \\ FY 199594 \\ FY 199488
}

Keywords: calibration, ozone measurements, ultraviolet atmospheric radiance, ozone

Objectives: To determine satellite-instrument and surface-based-instrument biases in ozone total and profile amounts with surface-based comparison measurements.

Approach: Direct-sun Langley-type and zenith-sky Umkehr measurements of solar irradiance and atmosvheric radiances at Dobson, SBUV-2, and Brewer wavelengths will be analyzed to determine sources of bias in ozone total column and profile measurements between space-borne and surface-based spectroradiometric measurements. Space instruments will be calibrated to a common spectroradiometric scale based upon NIST standards of spectral irradiance and the internally illuminated spherical integrator technique. The Dave-Mateer radiative-transfer code will be used so calculate atmospheric radiances and solar irradiances at the surface with satellite-overpass measurements of ozone and to compare them with Dobson and SBUV-2 surface-based measurements.

Results to Date: The surface-based comparison of Langley-type direct-sun measurements between SBUV-2 and SSBUV flight models with the world standard Dobson instrument Nos. 83 and 61 at Dobson wavelengths have confirmed a $2 \%$ bias in the space measurements with the Dobson total-ozone amounts lower. Note the most recent TOMS version 6 and 7 data sets indicate a negligible bias. A sphere-derived spectral-radiance-calibration scale removed the ozone altitude-dependent profile bias with SAGE II measurements. Surface-based zenith-sky spectral-radiance measurements that used the satellite instruments have been inverted to derive total-column ozone amounts. The total-column ozone amounts, which were derived with the same double wavelength pairs as the directsun observations, were $1 \%$ higher than the direct-sun values from the world-standard Dobson instruments Nos. 83 and 61 . No significant solar-zenith-angle effect is seen in the total-column ozone measurements derived from either the direct-sun or zenith-sky measurements relative to the Dobson direct-sun observations. 


\author{
SUPPORT FOR OPERATIONS OF THE \\ WMO/GAW QUALITY-CONTROL AND \\ SCIENCE ACTIVITIES CENTER FOR THE \\ AMERICAS \\ HICKS, BRUCE, and VOLKER, MOHNEN \\ NOAA AIR RESOURCES LABORATORY and \\ STATE UNIVERSITY OF NEW YORK \\ AT ALBANY \\ FY 1996100 \\ FY 1995100 \\ FY 1994100
}

Keywords: ozone, carbon dioxide, greenhouse gases, chlorofluorocarbons, methane, optical depth, turbidity, precipitation chemistry, UV-B, radiation, radioactivity, global sites, regional sites, international sites, quality assurance

Objectives: To provide, through agency collaboration, a center of excellence in the L rited States that would impose quality-assurance techniques on data collected by national air- and precipitation-chemistry monitoring networks operating in the Americas (North, Central, and South).

Approach: The WMO has adopted a scheme in which three quality-assurance centers will deal with dataintercomparability problems in three major regions of the world, the Americas, Europe and Africa, and Asia and Oceania. The QA Center for the Americas has special focus on surface ozone, airborne radioactivity, aerosol optical depth, and precipitation chemistry. The QA Center for the Americas is funded jointly by DOE, EPA, and NOAA. The final products of this activity will be regional and global data sets created by combining observations made in different nations with a variety of sampling methods that previously did not share common data-quality guidelines or calibration procedures or benefit from periodic intercomparisons and other confidence-building steps.

Results to Date: A major intercomparison of ozone sondes was conducted as a joint activity with QA/SAC Europe/Africa. A redesigned precipitation-chemistry quality-assurance program was tested. Transfer of activity from the EPA to the QA/SAC Americas is nearly completed. A search for a new provider of benchmark chemical standards (derived from NIST sources) was initiated. The first steps were taken to organize quality assurance for the array of surface ozone instruments now being set up in South America under the CONE program. Plans for a revised approach to monitoring atmospheric aerosols have been refined and are currently under consideration before implementation.

\section{STATISTICAL ANALYSIS AND \\ INTERPRETATION OF STRATOSPHERIC OZONE AND TEMPERATURE TRENDS WITH SATELLITE DATA HOOD, LON L. \\ UNIVERSITY OF ARIZONA \\ FY 199686 \\ FY 199586 \\ FY 199486}

Keywords: ozone, stratosphere, troposphere

Objectives: To use global satellite measurements of ozone, temperature, and related quantities to study long-term variability, including trends, in the lower stratosphere and upper troposphere. The work will also allow a more detailed investigation of stratospheretroposphere exchange at midlatitudes and an evaluation of the utility of satellite data for the detection of tropospheric ozone anomalies.

Approach: Observational studies to be performed include (1) multiple regression statistical trend analyses of available measurements of ozone, temperature, and geopotential height for the period 1979 to the present; (2) detailed case studies of specific temporal events with daily total ozone, $100 \mathrm{hPa}$ temperature and height, and potential vorticity (PV) on selected isentropic surfaces; and (3) studies of the detectability of tropospheric ozone anomalies in the tropics. Data sets to be employed include the following: (1) Version 7 Nimbus 7 and Meteor 3 TOMS total ozone data; (2) combined Nimbus 7 and NOAA 9/11 SBUV and SBUV/2 ozone profile measurements; (3) NOAA satellite Microwave Sounding Unit (MSU) temperature measurements for the several upper tropospheric and lower stratospheric pressure layers; (4) Free University of Berlin geopotential height and temperature analyses of mainly radiosonde data at 100,50 , and $30 \mathrm{hPa} ;(5)$ U.S. National Centers for Environmental Prediction (NCEP) gridded temperature and height data for both the free troposphere and the lower stratosphere; and (6) UARS Microwave Limb Sounder ozone data for the 1 to $68 \mathrm{hPa}$ pressure range. Supporting calculations will be performed with both linear mechanistic models and, in collaboration with other groups, with 2-D and 3-D models of the stratosphere-troposphere system.

Results to Date: A study of the altitude dependence of stratospheric ozone trends with Nimbus 7 SBUV ozone profile data showed that the observed upper stratospheric trends are approximately consistent with those predicted by gas-pliase chemical models that account for the observed $\sim 0.1 \mathrm{ppbv}$ per year increase in tropospheric chlorine. The dominant contribution to column ozone trends was confirmed to occur in the lower stratosphere where significant negative trends are present at latitudes $>20^{\circ}$ in both hemispheres.

The observed dependence on season and latitude of total ozone trends derived from Nimbus 7 TOMS 
data was found to be qualitatively consistent with models that include chlorine-induced heterogeneous losses on lower stratospheric aerosols and polar stratospheric cloud particles. However, at northern midlatitudes in winter and spring, the observed trends are significantly larger than predicted by heterogeneous chemical models. The latitude dependence of the observed trends also differs substantially from model predictions.

A study of the longitude dependence of observed ozone trends in winter showed that this longitudinal asymmetry is a consequence of long-term changes in vertical and horizontal ozone transport by quasistationary planetary-scale waves. This was demonstrated with a linear, adiabatic transport model together with observed changes in the amplitudes and phases of waves 1 to 6 in the geopotential height fields. In support of the model calculations, $100 \mathrm{hPa}$ geopotential height differences or "anomalies" between groups of Januaries near the end of the 1980s and near the beginning of the 1980 s were found to be inversely correlated with the geographic distribution of ozone trends. An examination of available data indicated that the observed decadal changes in wave properties were most probably a consequence of decadal climate variability in the troposphere.

An empirical method was developed to estimate the possible contribution of long-term differences in advective transport to zonal mean total ozone trends at northern midlatitudes. This method simulates monthto-month total ozone variability that results from differences in advective transport as measured by $100 \mathrm{hPa}$ temperature and geopotential height fields. At $45^{\circ} \mathrm{N}$, a large part of the observed monthly zonal mean total ozone variability between 1979 and 1991 is simulated. Results indicate that long-term changes in wave forcing originating in the troposphere can potentially explain more than half of the midlatitude total ozone trend. The latitude dependence of the observed trends, peaking at midlatitudes and decreasing at higher latitudes, is also simulated by the empirical model. Subtracting the estimated wave forcing contribution from the observed trends yields a residual meridional trend profile that agrees more closely in latitude dependence and amplitude with twodimensional stratospheric model estimates. An investigation of total ozone decadal variability associated with the 11-year solar cycle was also completed. This variability is one natural component of ozone change that must be known for an accurate evaluation of quasi-linear trends. Multiple regression statistical analyses of Nimbus 7 SBUV and NOAA 11 SBUV/2 data for a 15-year period showed that most (about $85 \%$ ) of the 1.5 to $2 \%$ solar cycle variation of global mean total ozone occurs in the lower stratosphere (altitudes $<28 \mathrm{~km}$ ). Evidence was obtained for a related solar cycle variation of lower stratospheric temperature $(50$ to $150 \mathrm{hPa})$ and geopotential height $(30,50$, and $100 \mathrm{hPa}$ ) with geographic dependences similar to those of the solar cycle variation of total ozone.

\section{ATMOSPHERIC-TRANSPORT STUDIES WITH RADON \\ LARSEN, RICHARD J., and LEE, H. N. ENVIRONMENTAL MEASUREMENTS LABORATORY \\ FY 1996540 \\ FY 1995540 \\ FY 199499}

Keywords: boundary-layer parameterizations, atmospheric-chemistry models, measurements of radon, boundary-layer structure

Objectives: To calculate flight-specific, quantitative, boundary-layer parameterizations for use in atmospheric-chemistry models by making continuous measurements of radon with the Radgrabber during G-1 aircraft missions and to improve the boundarylayer structure in the Global Chemistry Model (GChM).

Approach: Flight-specific quantitative boundary layer parameterizations will be determined with EML's advanced numerical model for calculating vertical profiles of radon and vertical eddy diffusivity in the atmospheric boundary layer. Continuous measurements of radon will be provided on appropriate ACP/G-1 flights with EML's Radgrabber. Experiments will be conducted for continued calibration and optimization of the Radgrabber performance. The boundarylayer structure in the GChM model will be improved. Results to Date: The EML Radgrabber was successfully used during the 1995 ACP Northeast Field Study on Long Island to infer radon concentrations. The Radgrabber is the only analyzer in the world ce.pable of continuously measuring radon from an aircraft. The resulting data are currently being used to develop and evaluate EML's advanced numerical model for calculating vertical profiles of radon and vertical eddy diffusivity in the atmospheric boundary layer. A joint modeling effort was established between Lee (EML) and Hales (ENVAIR) for improving the boundary layer prediction in the GchM.

NEW UV-B SPECTRAL RADIOMETER
LAULAINEN, NELS; HARRISON, LEE; and
LALA, GAR
PACIFIC NORTHWEST NATIONAL
LABORATORY and STATE UNIVERSITY OF
NEW YORK AT ALBANY
FY 1996172
FY 1995176
FY 1994150


Keywords: ultraviolet-B radiation, spectroradiometer, field instrumentation

Objectives: To develop and test a new, low-cost ultraviolet-B (UV-B) rotating-shadowband spectroradiometer (UVB-RSS) with performance characteristics to detect trends and to provide status monitoring of surface UV-B radiation.

Approach: Experience gained from the development of the multifilter rotating-shadowband radiometer (MFRSR) and the rotating-shadowband spectroradiometer (RSS), developed under the DOE Quantitative Links and Atmospheric Radiation Measurement (ARM) programs, will be used to design, fabricate, and field test a UV version of the rotating-shadowband radiometer. To maximize throughput and minimize the effects of out-of-band light, the UVB-RSS uses allquartz optics and a detection system that rejects light outside the UVB region and is capable of selecting 3 to 5 spectral bandpasses between 300 and $330 \mathrm{~nm}$. The variant of choice uses a CCD array for detection. An alternate version uses a photomultiplier tube detector that is blind to solar radiation at wavelengths greater than $330 \mathrm{~nm}$ and a rotating slit-wheel for selecting 3 to 5 spectral bandpasses between $300 \mathrm{~nm}$ and $330 \mathrm{~nm}$. A prototype instrument is expected to undergo rigorous bench tests and may be evaluated in the NIST-NOAAUSDA-NSF Table Mountain intercomparison. After the initial field and bench tests are completed, shortcomings of the prototype unit will be corrected, and a final hardened version will be ready for extensive field testing. The final field tests to evaluate instrument performance against reference spectroradiometers will be conducted at a suitable site where reference Brewer and/or Dobson instruments are in operation, such as in Toronto, Ontario, or Poulder, Colorado.

Results to Date: Individu' optical modules have been acquired and/or fabricated, and initial proof-ofprinciple tests have been per.ormed. Light-throughput tests have been completed for the various modules in the optical train and a prototype version is being assembled for bench testing. It is expected that a working prototype will be ready to deploy to the field in June 1996, possibly as part of the NIST-NOAAUSDA-NSF Table Mountain intercomparison.

\section{INELUENCE OF COMPLEX TERRAIN AND SCALE INTERACTIONS ON THE METEOROLOGY OF NORTH-CENTRAL NEW MEXICO \\ LEE, J. T., and BOSSERT, JAMES \\ LOS ALAMOS NATIONAL LABORATORY \\ FY 1996216 \\ FY 1995240 \\ FY $1994 \quad 0$}

Keywords: complex-terrain meteorology, ASCOT, dispersion
Objectives: To perform meteorological observations, data analysis, and modeling to define and understand the meteorology of the Rio Grande Valley in the vicinity of Los Alamos National Laboratory.

Approach: Meteorological observations, data analysis, and modeling will be performed.

Results to Date: No results were reported.

\section{MULTIPHASE ATMOSPHERIC CHEMISTRY LEE, YIN-NAN \\ BROOKHAVEN NATIONAL LABORATORY FY 1996221 \\ FY 1995225 \\ FY 1994252}

Keywords: reaction kinetics, photo-oxidants, ozone, hydrocarbons, nitrogen oxides, carbonyl compounds, organic peroxides, organic nitrates, wet scavenging, dry deposition

Objectives: To determine the fundamental aqueous-phase kinetics and equilibrium properties of key atmospheric trace species and to assess the contributions of gas-liquid interactions to the atmospheric transport and transformations of these species.

Approach: A systematic and interactive approach is taken to quantitate the role gas-liquid reactions play in the distribution of atmospheric trace species and its influence on photooxidant formation. Laboratory studies are performed to determine the fundamental chemical and physical properties of selected atmospheric trace species. Field measurements with analytical techniques developed based on laboratory chemical data are conducted to characterize temporal and spatial distributions of these compounds. The laboratory and field data thus derived are incorporated into numerical models to identify and to assess the contributions of relevant atmospheric processes. Insights obtained from model calculations are then used to guide additional laboratory investigations.

Results to Date: We determined that the Henry's Law solubility of hydroxymethylhydroperoxide is sufficiently high for this organic peroxide to be efficiently removed by dry deposition as well as by wet-scavenging processes. By measuring the aqueous kinetics of oxidation of dimethylsulfide (DMS) by ozone, we estimated that this aqueous reaction is responsible for up to $10 \%$ of DMS oxidation in remote marine boundary layer air. We developed ground and aircraft instrumentation for sensitive detection (20 parts-per-trillion) of a group of carbonyl compounds (formaldehyde, glycolaldehyde, glyoxal, methylglyoxal, glyoxylic acid, and pyruvic acid). Field measurements have been performed to determine the distribution of these compounds as a measure of photochemical reactivity under widely different environments including: pristine (Mauna Loa, Hawaii), urban center (metropolitan Nashville), forested (Metter, Georgia), and a remote 
but downwind area (Nova Scotia). The results suggest that photooxidant formation is dominated by isoprene in areas that are vegetated and away from population centers and is dominated by methane in the remote free troposphere. We have also found for urban areas that high levels of man-made hydrocarbons can be the limiting reagent in ozone production because oxides of nitrogen $\left(\mathrm{NO}_{\mathrm{x}}\right)$ are abundant there. Downwind remote regions, on the other hand, are often impacted by pollutants transported from source regions and exhibit a $\mathrm{NO}_{\mathrm{x}}$-limited behavior in ozone production. In addition, we have found that formaldehyde contributes in a major way to the free radical budget ( 30 to $40 \%$ ). A formaldehyde intercomparison study involving five techniques showed that the performance of the technique developed in this laboratory is comparable to a tunable diode laser absorption spectroscopy technique.

EML-SAMPLE ARCHIVES
LEIFER, ROBERT
ENVIRONMENTAL MEASUREMENTS
LABORATORY
FY 1996250
FY 1995344
FY 1994299

Keywords: databases, radioactivity, environmental sampling, stratosphere

Objectives: To provide a computerized database of the analytical results from samples obtained by the Environmental Measurements Laboratory (EML) during programs supported by OHER and its predecessors and to provide a detailed listing of the availability of archived samples for use by the scientific community. Approach: Important and historic environmental samples were obtained by EML during the following programs:

- Project ASHCAN (balloon): radioactivity measurements from 1957 to 1983

- Project STARDUST (aircraft): radioactivity measurements from 1957 to 1967

- Project AIRSTREAM (aircraft): radioactivity measurements from 1967 to 1983

- Project SASP (surface air): radioactivity measurements from 1957 to 1993

- Global Deposition Program: strontium-90 in deposition from 1954 to 1993

- Radioactivity in Soils and Lake Sediments: collected 1953 to 1991

- Radioactivity in Human Diet and Bone Samples: collected 1969 to 1982

A complete computerized database of all of EML's stratospheric and upper tropospheric radioactivity measurements will be developed. Most of the data is in the form of reports and will be entered into computer files. Previously published trace gas data from Project AIRSTREAM, for the years 1973-1983, will also be included in this phase of the project. EML is also the repository of most of the actual filters collected during these atmospheric sampling programs. We intend to carefully inventory these samples and create a detailed computerized database of their availability for possible future experimental use by the DOE sponsored scientific community. Samples and data from EML's Diet and Bone Program, Radioactivity in Soils Program, Surface Air Sampling Program (SASP), Strontium-90 in Global Deposition Program, and CombustionRelated Pollutants in Lake Sediments Project will be incorporated into EML's computer database in the last phase of the project.

Results to Date: The compilation of the stratospheric radionuclide database (RANDAB) has been completed. The RANDAB represents the worlds largest collection of stratospheric and upper tropospheric radionuclide data ever compiled for computer analysis. These data represent measurements obtained from Projects ASHCAN, STARDUST, AIRSTREAM, and the HighAltitude Sampling Program (HASP) for the years 1957 through 1983. More than 20,000 filters were collected during this period and analyzed for up to 40 radionuclides. All the available data associated with each filter are included in the database. Because of the lack of filter identification numbers, a separate database of plutonium isotopic ratio data for the years 1959 thru 1970 was formed. This database contains more than 500 samples.

In addition to the radionuclide database, EML's stratospheric database of trace gases, collected during Project AIRSTREAM has been completed and is presently available through EML. This database contains information on more than 1000 samples. Each sample was analyzed for one or more of the following gases $\mathrm{CCl}_{3} \mathrm{~F}, \mathrm{CCl}_{2} \mathrm{~F}_{2}, \mathrm{CCl}_{4}, \mathrm{~N}_{2} \mathrm{O}, \mathrm{SF}_{6}, \mathrm{CO}_{2}$, $\mathrm{CH}_{4}, \mathrm{CH}_{3} \mathrm{CCl}_{3}$, and $\mathrm{COS}$.

\section{MARINE AEROSOL CHARACTERIZATION WITH EML'S G-1 AIRCRAFT AEROSOL SAMPLING SYSTEM AND SCANNING ELECTRON MICROSCOPE \\ LEIFER, ROBERT; KROMIDAS, LAMBROS; ALBERT, BRIAN; KNUTH, RONALD; and ROIZ, PETER \\ ENVIRONMENTAL MEASUREMENTS LABORATORY \\ FY 1996300 \\ FY 1995300 \\ FY 1994399}

Keywords: aerosol characterization, aircraft sampling, aerosol scattering coefficient

Objectives: To design and operate a sampling package for the physical and chemical characterization of aerosol in and above the marine boundary layer during 
flights of the G-1 aircraft for use in modeling chemical changes in the aerosol.

Approach: The Environmental Measurements Laboratory (EML) has built an aerosol-sampling package containing a newly designed impactor to characterize the aerosol composition and size distribution in and above the marine boundary layer during flights of the G-1 aircraft. In addition, we evaluated a newly designed shrouded aircraft aerosol probe to improve aerosol collection from the ambient air at sampling speeds of $100 \mathrm{~m} \mathrm{sec}^{-1}$. The resultant data will be used for characterizing chemical changes in the marine aerosol. Specifically, losses of chlorine and increases in the sulfur of sea salt particles collected for different size ranges will be looked at and compared to changes in gaseous chlorine measurements independently measured by other researchers on the G-1 aircraft.

Among the major concerns when sampling aerosol on a moving platform, such as the G-1 aircraft, are whether a sampled aerosol is representative of the true ambient aerosol and is the aerosol modified as it passes through the aircraft sampling probe. To evaluate the performance of our shrouded probe, we installed a size distribution instrument [Met One, Optical Particle Counter (OPC)] in the aircraft to isokinetically sample the airstream from the probe. A second size distribution instrument (Particle Measurements Systems, PCASP), operated by the Pacific Northwest National Laboratory is mounted in the free airstream to provide a reference for comparison.

An EML-designed rotating drum impactor, known as the "Four-Parallel-Impactor" or "4" was used for aerosol sampling. This impactor has the capability of collecting four simultaneous aerosol samples for multiple chemical analyses. The impactor weighs about $0.5 \mathrm{~kg}$ and is controlled by a small computer. The impactor can sequentially collect more than $\mathbf{5 0}$ samples on a single drum for scanning-electronmicroscope (SEM) analysis. Sampling can be preset for collections at specific times or can be directed by an independent instrument, such as a pressure transducer (altitude), OPC, or relative humidity probe for sampling in and out of the marine boundary layer. The individual jets of the 4 are replaceable and allow for the selection of different cut sizes $(0.18 \mathrm{~m}, 1.0 \mathrm{~m})$. The sampling times to collect sufficient "giant" sea salt particles for SEM/X-ray analyses can be adjusted with the on-board computer with the $1.0 \mathrm{~m}$ channel of the OPC. The collection cylinder from this impactor is easily removed and can be directly placed in the SEM chamber, coated or uncoated, for observation and chemical analyses. An integrating nephelometer, massflow controller, vacuum system, and computer complete the aerosol sampling package.

Results to Date: Our aerosol sampling package performed extremely well. The OPC was successfully used to determine the sampling time for the 4 so that sample deposition was optimized. Our shrouded probe worked to specifications, qualitatively following the PCASP concentration time series. Absolute differences in the concentrations for specific size ranges were less than a factor of two and in some cases less than $20 \%$. These differences are presently under investigation. The limited number of flights for the June 1996 summer marine study did not allow SEM/X-ray analysis for sea salt aerosol. We are focusing our efforts on the previous summer's experiments, where we had many successful flights.

\section{MODELING OF REGIONAL CLIMATE OVER CHINA}

LEUNG, L. RUBY; GHAN, STEVEN; and WIGMOSTA, MARK

PACIFIC NORTHWEST NATIONAL

$$
\begin{array}{cc}
\multicolumn{2}{c}{\text { LABORATORY }} \\
\text { FY } 1996 & 300 \\
\text { FY } 1995 & 0 \\
\text { FY } 1994 & 0
\end{array}
$$

Keywords: regional climate model, East Asian monsoon, model intercomparison

Objectives: To provide an objective assessment of the skills of regional climate models driven by observed lateral boundary conditions and GCMs for simulating the intraseasonal and interannual regional climate variabilities over China.

Approach: A model intercomparison of several regional climate models will be designed and conducted to simulate the summer monsoon conditions over China for a normal, a drought, and a flood year. Realistic lateral boundary conditions will be used to drive the models. The model simulations will be used to drive a detailed surface hydrology model to test the usefulness of regional climate models for addressing water resource management issues. The nesting of regional climate models within GCMs will then be tested for long-term climate simulations.

Results to Date: A model intercomparison experiment is being set up with participation from the Pacific Northwest National Laboratory (PNNL), the China Meteorological Administration (CMA), and the State University of New York at Albany. The flood of the 1991 summer has been selected as the first case study. Each model simulation is being made under standard conditions, common diagnostics are being archived in standard format, and each model is being validated against a common data set. 


\section{ULTRAVIOLET RADIATION \\ CLIMATOLOGY OF THE EARTH'S \\ SURFACE AND LOWER ATMOSPHERE \\ MADRONICH, SASHA, and STAMNES, KNUT \\ NATIONAL CENTER FOR ATMOSPHERIC \\ RESEARCH and UNIVERSITY OF ALASKA \\ FY 199697 \\ FY 199599 \\ FY 199488}

Keywords: ultraviolet radiation, photolysis rates, photodissociation, atmospheric chemistry, UV monitoring

Objectives: To develop a climatology of tropospheric ultraviolet (UV) radiation (averages and variability) as functions of wavelength, altitude, geographical location, time of the year, and secular change (e.g., due to stratospheric ozone depletion) and to develop fast but accurate methods for calculating tropospheric photodissociation coefficients (J-values) and surface biologically weighted UV doses.

Approach: We are currently updating the Tropospheric Ultraviolet-Visible (TUV) model with critically assessed input data (extraterrestrial flux, absorption cross-sections, and molecular quantum yields). A number of radiative-scheme improvements are being tested, including a fast pseudospherical method for more accurate calculation of twilight, and we are examining alternate two-stream formulations to increase the accuracy of actinic-flux calculations. We are also beginning the coding framework of the global UV model, in which the 3-D distributions and seasonality of the atmosphere are derived from satellite and atmospheric model data. Anticipated impacts include the availability of accurate but fast photolysis calculations for atmospheric-chemistry models, and the development of a surface UV climatology for comparison with UV monitoring networks.

Results to Date: The TUV model input data have been updated based on recent reevaluation. By comparing TUV model results to actinometric measurements, we have contributed to a new recommendation for the quantum yield of the reaction $\mathrm{O}_{3}+\mathrm{hv} \rightarrow \mathrm{O}(1 \mathrm{D})+\mathrm{O}_{2}$. This reaction controls the oxidizing capacity of the global troposphere, and the new values have a significant impact on our understanding. A theoretical study clarified how the scaling transformations affect the calculation of the actinic flux in two-stream models. We have also developed a new pseudospherical method for more accurate calculation of radiation for low-sun conditions. Preliminary tests show that this method is nearly an order of magnitude faster than earlier methods while retaining good accuracy in the UV and visible wavelength ranges. We have also begun coupling the UV model to a global framework that assimilates atmospheric data from satellite and global climate/chemistry model output. This coupling uses an interactive postprocessor developed for visualization of atmospheric chemistry model results and produces global maps of UV radiation as a function of altitude, season, etc. Preliminary runs at $2.5 \times 2.5$ latitudelongitude resolution show that the global radiation field can be calculated with a two-stream model in a reasonable time on a local workstation, and the planned further optimization of the code will lead to further reductions in computational needs.

\section{EFFECT OF NATURAL PHENOMENA AND INDUSTRIAL ACTIVITY ON STRATOSPHERIC OZONE TRENDS MCELROY, MICHAEL B.; SCHNEIDER, HANS R; and LOGAN, JENNIFER HARVARD UNIVERSITY FY 1996176 \\ FY 1995171 \\ FY 1994164}

Keywords: ozone trends, stratospheric transport, stratosphere-troposphere exchange, stratospheric $\mathrm{CO}_{2}$ concentrations

Objectives: To improve our understanding of the effects of natural variability vs man-made perturbations on ozone.

Approach: The region of the atmosphere between the tropopause and about $20 \mathrm{~km}$ contains a significant fraction of the total ozone column. To assess the longterm effect of pollutants on total ozone, the natural variability of the lower stratosphere must be better understood. In particular, the description of transport in this region needs to be validated and improved. Our approach consists of a critical analysis of ozone data and the development and validation of a hierarchy of models. Ozonesondes provide the only information on trends in tropospheric ozone above the surface and on trends in the stratosphere below 90 mbar; they also provide unique information on stratospheric trends below 10 mbar prior to 1979 , when satellite measurements commenced. Model studies are performed with versions of a coupled 2-D model of the dynamics, chemistry, and radiation of the stratosphere to analyse transport in an average year and to explore interannual variability induced by the Quasibiennial Oscillation (QBO). We are developing a 3-D mechanistic dynamics module that will enable us to examine the effect of the variability of the tropospheric wave driving on column ozone.

Results to Date: We have completed an analysis of trends in ozonesonde data. There are significant spatial variations in the trends in the troposphere. Decreases in stratospheric ozone are found from about $24 \mathrm{~km}$ to near the tropopause. Ozone losses below $17 \mathrm{~km}$ appear to be responsible for the $20 \%$ difference between trends in column ozone derived from SAGE and TOMS. Losses vary with season, consistent with patterns 
reported by Stolarski et al. using TOMS data. We performed a study of transport between the tropics and midlatitudes of the stratosphere, using our 2-D model. Using a minimum of assumptions, we obtain good agreement with tracer data throughout the stratosphere. The seasonal changes of the mass flux are important in understanding the propagation of $\mathrm{CO}_{2}$ into the stratosphere. However, given available data, overturning rates in the stratosphere cannot be constrained to better than about $30 \%$. We reproduced the QBO in our model and studied the response of tracer fields in the tropics and subtropics. We found that the subtropical anomaly in ozone is synchronized with the seasonal cycle because of a seasonal asymmetry in wave forcing across the equator. We were able to account for distinct responses in upper and lower regimes as observed by Hitchman et al. In the easterly phase of the QBO, transport out of the tropics in the lower stratosphere is enhanced, while in the middle stratosphere, it is reduced. The opposite is true for the westerly phase.

\section{COMPOSITION OF FRESHLY NUCLEATED ULTRAFINE PARTICLES MCMURRY, PETER H. UNIVERSITY OF MINNESOTA \\ FY 199697 \\ FY 1995100 \\ FY 199492}

Keywords: homogeneous nucleation, ultrafine aerosols, sulfuric acid, pulse-height analysis, size distribution of aerosols

Objectives: To develop instrumentation to measure size distributions of freshly nucleated ultrafine atmospheric aerosols and to use this instrumentation to develop an experimentally verified theory for nucleation in the atmosphere.

Approach: We have developed the pulse-heightanalysis (PHA) technique to measure size distributions of aerosols in the 3- to $\sim 10$-nm-diam range. This technique involves the use of an ultrafine condensation nucleus counter (UCNC) in which very small particles are grown by butanol condensation until they can be detected optically. For particles smaller than $\sim 15 \mathrm{~nm}$, the condensed droplet size decreases markedly with initial particle size. Therefore, initial particle sizes can be inferred from the pulse heights produced by the UCNC photodetector. We investigated the sensitivity of the PHA response to particle material and sampling pressure $(0.25 \mathrm{~atm}$ to $1.0 \mathrm{~atm})$ and developed an inversion method to obtain size distributions from measured pulse-height distibutions.

We have also worked in collaboration with Dr. Fred Eisele of NCAR to study atmospheric nucleation phenomena. Dr. Eisele uses chemical ionization mass spectrometry to measure sulfuric acid vapor concentra- tions. We conducted measurements of sulfuric acid vapor, ultrafine aerosols, and aerosol size distributions in the 15- to 500-nm-diameter range on Mauna Loa, Hawaii.

Results to Date: We found that the PHA method is a simple and sensitive method for measuring ultrafine size distributions. The method works well at pressures down to $0.25 \mathrm{~atm}$, and therefore can be used with aircraft sampling at altitudes up to about 30,000 feet. We found that the commercial UCNC (the TSI 3025) is not suitable for PHA measurements because droplets are detected with monochromatic light that is scattered by $90^{\circ}$, which leads to a nonmonotonic relationship between particle size and pulse-height signal. Our laboratory prototype instrument uses forward scattering from a white light source.

We found that particle production rates at Mauna Loa were much higher than can be explained by binary nucleation of sulfuric acid and water. Recent measurements in Colorado and elsewhere with funding from another source produced a similar result. We believe that other species (possibly ammonia) may participate in the nucleation process, and we have been conducting experiments to investigate this possibility.

\section{PARTIAL SUPPORT FOR THE INTERNATIONAL GLOBAL ATMOSPHERIC CHEMISTRY CORE PROJECT OFFICE MOYERS, JARVIS \\ NATIONAL SCIENCE FOUNDATION

$\begin{array}{lr}\text { FY } 1996 & 19 \\ \text { FY } 1995 & 0 \\ \text { FY } 1994 & 0\end{array}$

Keywords: International Global Atmospheric Chemistry, administrative support

Objective: To promote a fuller understanding of the processes that determine the chemical composition of the atmosphere and the interaction among atmospheric, biospheric, and chemical processes, including predictions of impacts of natural and anthropogenic forcings on the chemical composition of the atmosphere.

Approach: The International Global Atmospheric Chemistry (IGAC) Core Program Office will coordinate day-to-day operations of projects, develop and maintain IGAC activities in conjunction with other core projects (i.e., Global Change and Terrestrial Ecosystems and Biospheric Aspects of the Hydrologic Cycle). Additionally, it will be responsible for the general administration, such as maintaining records of various meetings and establishing and promoting communications between the IGAC and other member core project offices under the International GeosphereBiosphere Program (IGBP).

Results to Date: The IGAC has initiated a new focus in cooperation with the Commission on Atmospheric 
Chemistry and Global Pollution of the International Association of Meteorology and Atmospheric Sciences. Because of the uncertainty associated with the role of anthropogenic aerosols in radiative forcing and climate, the IGAC will address aerosol characterization and process studies, direct aerosol radiative forcing, aerosol-cloud interactions, and stratospheric and upper-tropospheric aerosols.

\section{THERMALLY FORCED CIRCULATIONS IN THE AIRSHEDS SURROUNDING THE ROCKY FLATS PLANT AND THE INFLUENCE OF WEAK SYNOPTIC AND MESOSCALE WEATHER SYSTEMS \\ NEFF, WILLIAM D., and BANTA, ROBERT M. NOAA, ENVIRONMENTAL RESEARCH LABORATORIES \\ FY 1996130 \\ FY 1995130 \\ FY 19940}

Keywords: thermally forced flows, synoptic influences, ozone, lidar

Objectives: To document synoptic influence on thermally forced circulations and develop advanced remote sensing techniques in support of the DOE ASCOT program.

Approach: Data from previous field programs will be analyzed, and new data collected in tests will be examined prior to Mexico City experiments in 1997. Results to Date: Along the Front Range of Colorado, the orientation of a valley with respect to the prevailing flows will have a dominant effect on the structure and frequency of thermally forced circulations within that valley. Downstream of the Continental Divide, turbulent mixing, induced by strong flows alof, reduces temperature gradients and thus pressure gradients driving the thermally forced circulations. This allows dynamic pressure gradients and ambient winds to dominate. Upstream of the Divide, regions of stable air above the thermally forced circulations insulate these flows from the ambient winds aloft. The boundary layer structure above Middle Park, west of the Divide; exerts a strong influence on the strength of the prevailing flows as they cross the Continental Divide. These speed variations, in turn, exert a strong influence on the boundary-layer structure downstream.

A new tool, a DIAL system, was used to monitor vertical flux profiles for trace gases in general and for $\mathrm{O}_{3}$ in particular, providing measurements previously very difficult or impossible to obtain. We now have a data set over relatively homogeneous topography that we are using to assess this device. Once this has been done for $\mathrm{O}_{3}$, DIAL systems for other species can be designed and built. We have also explored (under separate funding) equipping these systems with scanners for some applications, enabling researchers to map out the 2-D (and eventually 3-D) spatial distribution of contaminant species. Meanwhile, an important data set over flat topography has been amassed to test and validate the ability of the $\mathrm{O}_{3}$ DIAL to measure profiles and fluxes. Experience with the temporal behavior of these fluxes over flat terrain will allow us to more reliably interpret the behavior over more complex terrain in future experiments.

\section{AEROSOL OPTICAL PROPERTIES NOVAKOV, TIHOMIR \\ LAWRENCE BERKELEY NATIONAL \\ LABORATORY \\ FY 1996197 \\ FY 1995150 \\ FY 1994150}

Keywords: optical properties of aerosols, cloud condensation nuclei

Objectives: To obtain experimental data on optical and nucleation properties of aerosols.

Approach: Physical and chemical properties of laboratory and ambient aerosols will be measured with special emphasis on their carbonaceous component.

Results to Date: The absorption cross-section of aerosol black carbon was found to increase from $9.2 \mathrm{~m}^{2} / \mathrm{g}$ at $700 \mathrm{~nm}$ to $22.3 \mathrm{~m} 2 / \mathrm{g}$ at $250 \mathrm{~nm}$. Aerosol mass in Atlantic tropical trade winds is dominated by water-soluble, CCN active organic material. Laboratory results obtained with pure water-soluble organic compounds show that these are efficient $\mathrm{CCN}$.

\section{TRENDS AND EVOLUTION OF MINIMAL OZONE AMOUNTS, 1979-1992 RECK, RUTH \\ ARGONNE NATIONAL LABORATORY \\ FY 1996137 \\ FY 1995137 \\ FY 1994140}

Keywords: atmospheric chemistry, ozone depletion, TOMS data

Objectives: To identify and explain the day-to-day variability of total ozone over various regions of the globe.

Approach: The daily temporal and spatial variability in column ozone over portions of the Northern and Southern Hemispheres will be studied for the years 1979 to 1992. The reasons for the differences in the total ozone seen in Total Ozone Mapping Spectrometer (TOMS) data and the variability of ozone amounts in different regions of the Northern and Southern Hemispheres are to be identified and explained. Wavelet analysis is used to identify the conditions under which episodes of strong fluctuations of total ozone occur in the midlatitudes. An approach similar to that of Hood and Zaff (Lunar and Planetary Laboratory, University of Arizona, Tucson) will be used to analyze monthly 
averages of ozone in winter, and a mechanistic ozone model will be developed to quantitatively resolve the components of dynamic, radiative, and photochemical forcing in the day-to-day variability of ozone over various regions.

Results to Date: The ozone variability and its influence in a region ( 7 deg latitude by 6.25 deg longitude) of the eastern central United States were explored for all seasons of the years 1979 to 1991. A day-to-day variability range as large as $50 \%$ was found in the data for the region; in contrast, the decadal trend is reported to be about $-3 \%$. The range of total ozone variability was explored across the globe, and wavelet analysis was used to spectrally decompose the 13 years of data for each day of the record for a few locations. Trends in the frequency of daily global and hemispheric ozone were also evaluated.

\section{CONFIRMATORY ANALYSIS OF SAGE-I AND -II DATA \\ RUSCH, DAVID; RANDALL, CORA; and SOLOMON, STANLEY \\ UNIVERSITY OF COLORADO and LASP, UNIVERSITY OF COLORADO}

FY 1996218

FY 1995212

FY 1994217

Keywords: ozone, stratosphere, SAGE satellite Objectives: To confirm and/or modify the analysis technique and the ozone densities and trends measured by the Stratosphere Aerosol and Gas Experiments-I and -II (SAGE-I and -II) and to develop a new inversion method that will produce realistic uncertainties in the computed ozone densities and trends.

Approach: Our approach involves the full understanding of the SAGE instruments, the data-reduction and -inversion techniques, and error analysis now being used for the SAGE data. We are developing an analytic approach that is distinct from the current SAGE philosophy and involves a new inversion technique, error analysis, and a new method of linking SAGE-I to SAGE-II data for the purpose of defining long-term ozone changes with the combined data sets. We have developed a forward model that simulates the SAGE data and that includes SAGE instrument parameters. The simulated data are used to compare to the SAGE data, to test inversion codes with noiseless data and with artificial noise imposed, and to simulate trends in the data. The inversion method will return the errors as determined explicitly in the inversion analysis. For example, the correlation between ozone and aerosols is being used to investigate the influence of errors in the derived ozone and aerosol densities.

Results to Date: The forward model is fully developed and operational. The inversion algorithm is running and being tested on forward model simulations and
SAGE transmission data. The inversion uses six of the seven SAGE channels. A square $70 \times 70$ system of equations representing a spherical shell model of the atmosphere is solved with singular-value decomposition. To invert from optical depth to densities, we solve a 6 x 4 (six wavelengths and four species: ozone, aerosol, neutral atmosphere, and nitrogen dioxide) over-determined system of equations at each altitude point. For a given choice of aerosol parameters, the four species are determined at each altitude by minimizing residuals between the model and the data. The minimum thus becomes a function of the aerosol parameters chosen. For an assumed log-normal aerosol distribution, the parameters are the average particle radius and the standard deviation (or broadness) of the distribution. These parameters are varied over broad limits, and a family of acceptable solutions is found. The true answer is derived from the calculated minimum in this family of solutions. In this way, the aerosol parameters that best fit the data can be derived along with the ozone density from the best-fit solution. The results show that for low aerosol concentrations, our results agree quite well with the SAGE results. We are now looking at times of high aerosol loading where large differences between the standard SAGE analysis and our analysis are expected to arise.

CHEMICAL AND MICROPHYSICAL AEROSOL MODEL

SCHWARTZ, STEPHEN E., and BENKOVITZ, CARMEN $M$.

BROOKHAVEN NATIONAL LABORATORY

FY 1996246

FY 1995250

FY 19940

Keywords: clouds, albedo, aerosols, microphysical models

Objectives: To develop, evaluate, and apply models describing the chemical and microphysical properties of atmospheric aerosols resulting from energy-related and other activities on a hemispheric scale.

Approach: A physical simulation model will be developed and evaluated that represents the geographical distribution of the loading and the chemical and microphysical properties of atmospheric aerosols, especially anthropogenic aerosols resulting from energy-related activities. The model will be extended and modified on the basis of a process-level understanding of the phenomena being modeled derived from laboratory studies and field measurements. The model will be evaluated by comparison of model results with field measurements of the geographical and vertical distribution of aerosol loading and properties. These models will be applied and tested in conjunction with field projects by ACP and others and by comparison with satellite observations. A key feature 
of the modeling conducted here is that the model is driven by observation-derived meteorology so that comparison can be made at specific locations and times of the measurements.

Results to Date: A subhemispheric Eulerian model for tropospheric sulfate driven by operational meteorological data has been developed and applied to the North Atlantic and adjacent continental regions. Dailyaverage modeled concentrations accurately represent the magnitudes and spatial and temporal variability of measured surface concentrations. Examination of scavenging of nitrate and $\mathrm{HNO}_{3}$ during cloud formation shows significant differences versus the sulfate/ $\mathrm{SO}_{2}$ system because of different solubilities and oxidation kinetics (e.g., nitrate being in larger drops than sulfate). Nonetheless, the main solute mass is present in the main water mass, as it is for sulfate.

Calculations of the rate of binary nucleation in mixtures of sulfuric acid and water indicate substantial flux across the free-energy ridge at high supersaturations, rather than through the free-energy minimum as previously assumed, substantially increasing nucleation rates. An examination of nucleation with an exact expression for the evaporation rate of individual clusters rather than the customary canonical average over an equilibrium ensemble shows considerable reduction in evaporation rates, with a consequent increase in the nucleation rate.

A global, seasonal inventory of anthropogenic $\mathrm{So}_{\mathrm{x}}$ and $\mathrm{NO}_{\mathrm{x}}$ emissions has been compiled on a 1-degree grid.

\section{OBSERVATION, ANALYSIS, AND \\ PREDICTION OF COMPLEX-TERRAIN CIRCULATIONS OVER THE HANFORD SITE SHAW, WILLIAM J., and SKYLLINGSTAD, ERIC PACIFIC NORTHWEST NATIONAL LABORATORY \\ FY 1996139 \\ FY 1995142 \\ FY 19940}

Keywords: boundary-layer chemistry, atmospheric turbulence, RASS, wind-profiling radar, SOS, mesoscale circulations

Objectives: To develop new techniques that can be readily used by operational forecasters for predicting patterns of boundary-layer circulations over the Hanford site and to perform general observational studies of the influences of boundary-layer structure and circulations on atmospheric chemistry.

Approach: The observational component of this work focuses on the use of surface-based remote-sensing systems, particularly wind-profiling radars, to collect data from which the mesoscale temporal and spatial structure of the atmospheric boundary layer can be inferred. These measurements are collected in collabo- ration with investigators studying atmospheric chemistry to investigate the role that boundary-layer structure plays in the chemistry of air-pollution episodes. The analytical component of this work will (1) develop a general description of the mesoscale variation of boundary-layer structure from data collected from arrays of surface-based remote-sensing systems and other supporting instrumentation, accounting for the influence of local topography, and (2) simulate the observations with the RAMS (Regional Atmospheric Modeling System) model to further examine the physics of characteristic observed circulations and to understand, where applicable, variations in surface concentrations of atmospheric pollutants.

Results to Date: No results were reported.

\section{APPLICATION OF SENSITIVITY ANALYSIS TO REDUCE UNCERTAINTIES IN STRATOSPHERIC-OZONE CHEMICAL-DYNAMICS MODELS \\ SHORTER, JEFFREY, and RABITZ, HERSCH \\ MISSION RESEARCH CORPORATION and PRINCETON UNIVERSITY \\ FY 1996179 \\ FY 1995151 \\ FY 1994151}

Keywords: ozone, sensitivity analysis, uncertainty analysis

Objectives: To define key mechanistic pathways in models; to determine the importance of reactions with large uncertainties; to provide a better understanding of the ozone chemical dynamics models; to provide a quantitative assessment of the uncertainties in the model predictions; and to develop fast efficient analysis techniques for application to large, complex multidimensional assessment models.

Approach: State-of-the-science, systematic, and rigorous sensitivity analysis techniques are being developed and applied to stratospheric/tropospheric ozone chemical-dynamics models. A well-established gradient-based Green's function technique is being applied to calculate the model prediction sensitivities to each of the model input parameters. A rapid, steadystate, sensitivity-analysis technique is being developed to calculate prediction sensitivities based on a steadystate assumption. And an intelligent Guided Monte Carlo technique is being developed and applied that uses the information gained from the previously calculated model prediction sensitivities for statistical analyses of model predictions (c.g., the accurate calculation of model output uncertainties and model risk assessments).

Results to Date: A new statistical analysis tool, the Guided Monte Carlo, was developed. It reduces the computational cost of Monte Carlo uncertainty analysis by a factor of 3 to 5 , while still calculating an accurate 
result. With the Guided Monte Carlo technique, the uncertainty of the model-predicted ozone concentrations caused by the chemistry were calculated for $45^{\circ} \mathrm{N}$ latitude between 20 and $40 \mathrm{~km}$ altitude both with and without pseudotransport terms. The ozone concentration uncertainty is 25 to $30 \%$. A sensitivity analysis was also performed in the same region, providing a rank ordering of reactions that contribute the most to the model-output uncertainty. This sensitivity analysis also quantifies the affect of transport on the chemical system, allowing the identification of reactions that are enhanced, muted, or unaffected by the addition of transport processes. A prototype sensitivity-analysis tool to rapidly evaluate model prediction sensitivities based on the assumption of steady state has also been developed.

\section{ATMOSPHERIC-SCIENCES TRANSFER BETWEEN RESEARCH ADVANCES AND ENERGY-POLICY ASSESSMENTS SLINN, GEORGE, and BARCHET, W. RICHARD CASCADE SCIENTIFIC RESEARCH CORPORATION and PACIFIC NORTHWEST NATIONAL LABORATORY \\ FY 1996167 \\ FY 1995200 \\ FY $1994 \quad 0$}

Keywords: project management, data interpretation, information dissemination

Objectives: To help the nation receive optimum return on investments in atmospheric-chemistry research.

Approach: Assistance will be provided to DOE's Atmospheric Chemistry Program and DOE program managers in research design, data interpretations, and broad dissemination of research results and by service on panels of the U.S. National Academy of Sciences. Results to Date: No results were reported.

\section{SENSITIVITY AND UNCERTAINTY ANALYSIS OF ATMOSPHERIC-OZONE PHOTOCHEMISTRY MODELS \\ SMITH, GREGORY P.; KINNISON, DOUGLAS E.; and CONNELL, PETER $S$. \\ SRI INTERNATIONAL and LAWRENCE \\ LIVERMORE NATIONAL LABORATORY \\ FY 1996198 \\ FY 1995200 \\ FY 1994194}

Keywords: ozone, sensitivity analysis

Objectives: To determine the sensitivity coefficients of ozone to local photochemical reactions in global models and to assess the resulting uncertainties.

Approach: The Sandia Chemkin/Senkin codes are used to compute sensitivity coefficients for localized boxes from the LLNL 2-D atmospheric photochemistry model output. Appropriate methods are required to prevent concentration drifts during the long integration times required for convergence. A correction procedure based on net family deviations from the local photostationary state was developed to consistently apply the technique to steady solutions from the 2-D model. The results from a wide sample of conditions are used to propagate our evaluation of rate-parameter error limits to estimate uncertainty limits for model ozone values. This method also identifies key sources of model control and uncertainty for improvement and is being applied to assessment scenarios and to other observable atmospheric species.

Results to Date: A global survey of ozone-sensitivity computations has been completed for model parcels throughout the stratosphere and into the lower troposphere for a range of latitudes up to $62^{\circ} \mathrm{N}$ at various seasons. Sensitivities quantitatively show the relative control exerted on stratospheric ozone by the $\mathrm{HO}_{x}$, $\mathrm{NO}_{x}, \mathrm{ClO}_{x}$, and $\mathrm{BrO}_{x}$ catalytic-loss cycles, reactions coupling these families. The transition from oxygen photolysis to $\mathrm{NO}_{\mathrm{x}}$-hydrocarbon chemistry as the source of ozone is evident near the tropopause. Propagation of the rate-constant uncertainties with the sensitivities shows sizable uncertainties in predicted ozone, absent any other constraints. Net ozone uncertainties are about equally divided between kinetics and photolysis and are largest at low altitude and high latitude. Our computations also furnish sensitivity coefficients for all other species, identifying observational tests and constraints to various parts of the kinetic mechanism.

The sensitivity-uncertainty analysis technique was also applied to predicted ozone changes for a proposed high-altitude aircraft fleet, examining 2-D model results at 47 degrees $\mathrm{N}$. Considerable cancellation of sensitivities occurs in the assessment comparison, but 11 key reactions involving nitrogen chemistry were identified as major sources of uncertainty.

Selected 2-D model reruns indicate the current approach may overestimate modeling uncertainties by up to $50 \%$ and that kinetic confidence in the assessment predictions should be better than $4 \%$.

\section{DEVELOPMENT OF INSTRUMENTATION FOR FIELD PROGRAMS \\ SPRINGSTON, STEPHEN R.; LEE, JAI HOON; and NEWMAN, LEONARD \\ BROOKHAVEN NATIONAL LABORATORY \\ FY 1996246 \\ FY 1995250 \\ FY $1994 \quad 0$}

Keywords: instrumentation, nitrogen oxides, carbon monoxide, sulfur dioxide, peroxides

Objectives: To provide enhanced instrumentation for measuring trace atmospheric species as required for 
planned Atmospheric Chemistry Program field measurement campaigns.

Approach: These development activities are driven by the objectives of measurement programs scheduled or anticipated by the Atmospheric Chemistry Program. Several crucial measurement systems currently aboard the research aircraft facility are inappropriate for the scheduled programs because of limited sensitivity, slow time response, or both. The capabilities of these existing instruments must be significantly enhanced if the field programs are to be successful. Enhancements to measurement methods for nitrogen oxides (NO, $\mathrm{NO}_{x}, \mathrm{NO}_{y}$, and peroxyacetyl nitrate), peroxides, carbon monoxide, and sulfur dioxide are being conducted. Also under development are sensitive fluorimetric procedures for nitrous acid and peroxy radicals. Nitrous acid will be determined based on its reaction with a diamino compound to form highly fluorescent triazole. Peroxy radicals will be determined by their fast reaction with $\mathrm{Fe}$ (III) to form $\mathrm{Fe}$ (II) to produce, in the presence of hydrogen peroxide and benzoic acid, the highly fluorescing hydroxy benzoic acid. Additional new methods with improved sensitivity and specificity are being investigated, one for measuring trace concentrations of $\mathrm{NO}_{2}$ and $\mathrm{HNO}_{3}$ and a second for measuring $\mathrm{SO}_{2}$. The resulting instruments are specifically for deployment on the DOE research aircraft facility but will have utility for ground-based operations.

Results to Date: A compact instrument for simultaneous measurement of $\mathrm{NO}, \mathrm{NO}_{2}$, and $\mathrm{NO}_{\mathrm{y}}$ was designed and constructed. Detection limits during aircraft operations are $10 \mathrm{pptv}(\mathrm{NO})$ and $\sim 50 \mathrm{pptv}$ $\left(\mathrm{NO}_{2}\right.$ and $\left.\mathrm{NO}_{y}\right)$ with a 10-s time response. The redesigned molybdenum converter used to detect $\mathrm{NO}_{\mathrm{y}}$ species in this instrument is simple and dependable, and it provides performance comparable to the alternative gold/carbon monoxide converter. A three-channel peroxide analyzer capable of speciating inorganic peroxide, hydroxymethylhydroperoxide, and methylhydroperoxide was constructed and evaluated. Detection limits are less than $100 \mathrm{pptv}$ with a $1-\mathrm{min}$ response. A compact instrument for airborne calibration of analyzers with standard additions of $\mathrm{NO}, \mathrm{NO}_{2}$, $\mathrm{HNO}_{3}, \mathrm{CO}, \mathrm{SO}_{2}$, and other species in ambient air was designed and constructed. A prototype instrument for measuring $\mathrm{SO}_{2}$ at high sensitivities (less than $10 \mathrm{pptv}$ with a 1-min response) was constructed on the basis of an aqueous fluorescence method developed at Brookhaven. The sensitivity, stability, and time response of a commercial $\mathrm{CO}$ analyzer was improved, increased, and shortened (respectively). The time response of a commercial $\mathrm{SO}_{2}$ analyzer was shortened from $2 \mathrm{~min}$ to $\sim 10 \mathrm{~s}$ with minimal change in detection limit (less than $200 \mathrm{pptv}$ ).
MEXICO CITY AIR-QUALITY STUDY

STOOKSBURY, BILLIE

OAK RIDGE INSTITUTE FOR

SCIENCE AND EDUCATION

FY 199650

FY 19950

FY 19940

Keywords: planning, Mexico City

Objectives: To provide travel resources for planning and siting activities for the FY 1997 Mexico City Aerosol Research Program field study.

Approach: Provide travel resources to contractor and field personnel on an as-needed basis to identify various tasks and operations that need to be performed during the field study.

Results to Date: Have provided assistance for travel to Mexico City for various field personnel identified to perform siting and planning activities.

\section{ATMOSPHERIC-AEROSOL CHEMISTRY AND DYNAMICS \\ TANG, IGNATIUS N. \\ BROOKHAVEN NATIONAL LABORATORY \\ FY 1996236 \\ FY 1995240 \\ FY 1994252}

Keywords: aerosols, thermodynamics, sulfates, nitrates, visibility, radiative forcing

Objectives: To elucidate the fundamental processes governing the chemical and dynamic behavior of atmospheric aerosols.

Approach: A unique single-particle levitation technique developed at BNL is being used for determining the thermodynamic and optical properties of microparticles that are otherwise inaccessible to measurement with bulk samples. These data should find application in mathematical models for predicting the dynamic behavior, visibility degradation, and radiative effects of atmospheric aerosols. Experimental studies are being carried out to investigate with laser Raman and Mie resonance spectroscopic teclniques the phase transformation and metastable states in hygroscopic microparticles.

Results to Date: Light-scattering coefficients as a function of percent relative humidity have been calculated for the sulfate and nitrate aerosols of atmospheric importance, with the extensive optical and thermodynamic properties recently determined at BNL. For given aerosol size distributions, the chemical effect on light scattering is outweighed by the size effect of the aerosol. Also, contributions by external and internal mixtures differ only slightly. At least for the common sulfate- and nitrate-salt aerosols, external mixtures may be used as an aerosol surrogate in model computations to predict light-scattering properties. This technique has a definite advantage for either visibility- 
degradation or climatic-impact modeling studies because relevant data are now available for pure components but are very scarce for multicomponent mixtures. The hydration and crystallization of inorganic salt particles are investigated in the singleparticle levitation apparatus. Laser-Raman- and Miescattering techniques are used to probe the chemical and physical state of the microparticle. We have obtained the first spectroscopic evidence that new metastable solid states form in hygroscopic aerosol particles. The formation of such states is not predicted from bulk-phase thermodynamics, and in some cases, the resulting metastable state is entirely unknown heretofore. More work is in progress.

\section{ECOLOGICAL EFFECT OF UV-B RADIATION}

TUSKAN, GERALD; HILL, WALTER; SHUGART, LEE; COOPER, LEE; and ANDERSON, SUSAN OAK RIDGE NATIONAL LABORATORY and LAWRENCE BERKELEY LABORATORY

FY 1996246

FY 199520

FY 1994250

Keywords: UV-B radiation, ecosystem health, genetic damage, reproduction

Objectives: To evaluate the effect of UV-B radiation on ecosystem health, both at a genetic level and an ecological level.

Approach: Organisms used in each component of this project have been selected on the basis of their economic importance and ecological relevance, and represent terrestrial, freshwater, and marine ecosystems. Pollen from ponderosa pine (a wind-pollinated species) and yellow-poplar (an insect-pollinated species) is being exposed to artificially elevated levels of UV-B. Genetic damage will be quantified with the alkalinegel assay and the alkaline-unwinding assay.

The vulnerability of freshwater periphyton and grazing snails to current and elevated UV-B levels is being tested in natural streams and mesocosms. Mortality, growth, and potential DNA damage to sensitive stages will be assessed. Sea urchin eggs and developing embryos are being exposed to ambient UV-B radiation in a marine environment. DNA damage is being determined via agarose-gel electrophoresis, number of unhatched eggs, and number of abnormally developing embryos. Integration of results from the three ecosystems will provide a general overview of the ecological effects of elevated UV-B radiation.

Results to Date: Preliminary results indicate that increased UV-B inhibits germination and causes DNA damage to ponderosa pine pollen. Pollen sources from higher elevations and lower latitudes generally have higher percent germination, suggesting an inherent tolerance to UV-B radiation associated with geographic origin.

Experiments in natural streams on the current effects of ambient fluxes of UV-B radiation showed no significant effect on periphyton primary production or adult-grazer densities. Mesocosm studies also showed no significant effect of current and elevated UV-B on snail eggs, juveniles, and adult fecundity. However, exposure regimes in the mesocosms were affected by vigorous growth of benthic algae, which probably absorbed significant proportions of the UV-B that the ecosystem was exposed to.

In field work at Kasitsna Bay, Alaska, progress was made in establishing a UV damage model for sea urchin embryos in Arctic environments. DNA-damage assay results have shown increased DNA damage in the presence of UV-B radiation. The number of abnormal sea urchin embryos in each treatment was significantly correlated with the quantitative measure of DNA damage attributed to UV radiation.

\section{ATMOSPHERIC OZONE AS A CLIMATE GAS WANG, WEI-CHYUNG \\ STATE UNIVERSITY OF NEW YORK \\ AT ALBANY \\ FY 1996105 \\ FY 1995107 \\ FY 1994111}

Keywords: atmospheric ozone, general circulation models

Objectives: To improve understanding of the physical, chemical, and dynamical processes that control midlatitude $\mathrm{O}_{3}$ in the lower stratosphere and free troposphere and to develop improved predictions of future $\mathrm{O}_{3}$ changes in these regions and their influence on (and response to) future climate changes.

Approach: The observed $\mathrm{O}_{3}$ climatology together with radiation model will be used to evaluate the importance of atmospheric $\mathrm{O}_{3}$ to radiation balance and to study how the observed $\mathrm{O}_{3}$ changes during the 1980 s and 1990s affect the total radiation and UV fluxes as well as their comparison with increasing other greenhouse gases. The climate-chemistry interactions will be studied first with the NCAR global climate model and the University of Oslo's 2- and 3-D photochemicaltransport model, with a focus on the importance of the physical and chemical mechanisms. Exploratory studies to incorporate the chemical code into the climate model with focus on computational efficiency and consistency will then be conducted.

Results to Date: During FY 1996, we completed two studies. The first was on the $\mathrm{CH}_{4}$ indirect effect on radiative forcing. The results showed that tropospheric $\mathrm{O}_{3}$ can be substantially increased in response to $\mathrm{CH}_{4}$ increase, which is, however, in sharp contrast to the $\mathrm{O}_{3}$ decrease that occurs when increases in moisture and 
temperature are included. In addition, the magnitude of atmospheric $\mathrm{CH}_{4}$ increase can be substantially reduced when enhanced $\mathrm{OH}$ associated with global warming is taken into account. The decreases in $\mathrm{O}_{3}$ and $\mathrm{CH}_{4}$ thus reduce the total radiative forcing by about $12 \%$. The potential effect of increasing stratospheric water vapor by $\mathrm{CH}_{4}$ oxidation is calculated to be small, about $3 \%$ of the total effect associated with $\mathrm{CH}_{4}$ increase.

The second was on the effect of changes in surface emissions of $\mathrm{NO}_{x}$ in different parts of the world on tropospheric $\mathrm{O}_{3}$ distribution and its subsequent climate implications. The $\mathrm{O}_{3}$ changes simulated from the University of Oslo's 3-D chemical-transport model (CTM) were used to calculate the perturbation to radiative forcing. The results suggest that the effect of $\mathrm{O}_{3}$ changes on radiative forcing because of a $20 \%$ reduction of surface $\mathrm{NO}_{\mathrm{x}}$ emissions is small on the global-mean basis but can be a few tenths of a watt per square meter on the regional basis.

\author{
ANALYSES OF OZONE AND \\ ULTRAVIOLET-B RADIATION DATA \\ WEATHERHEAD, ELIZABETH; FREDERICK, \\ JOHN; TIAO, GEORGE; and \\ REINSEL, GREGORY \\ CIRES, UNIVERSITY OF COLORADO; \\ UNIVERSITY OF CHICAGO; and UNIVERSITY \\ OF WISCONSIN \\ FY 19960 \\ FY 1995181 \\ FY 1994194
}

Keywords: UV-B, ozone, ultraviolet radiation

Objectives: To examine available ozone and UV data to assess trends and long-term changes.

Approach: Ozone and UV data are examined together with radiative-transfer calculations and other available data, such as cloudiness, aerosols, and pollutants to understand the observed changes in both ozone and UV radiation.

Results to Date: A comprehensive reanalysis of the UV data collected by Robertson-Berger meters from 1974 to 1995 was performed, revealing that the data are not appropriate for reliable trend analysis. This result helps to explain the previously disturbing results published by Scotto et al. in 1996 showing a decrease in UV. The reanalysis shows that it is likely an increase has occurred in UV reaching the ground throughout the midlatitudes.

\author{
ATMOSPHERIC PEROXIDES \\ WEINSTEIN-LLOYD, JUDITH \\ STATE UNIVERSITY OF NEW YORK/ \\ OLD WESTBURY \\ FY 199687 \\ FY 199590 \\ FY 199484
}

Keywords: peroxide, free radicals

Objectives: To study free radicals of atmospheric importance and their reaction products in the gaseous and aqueous phases and to develop detection methods for these species.

Approach: In the laboratory, hydroperoxy and peroxy radicals are produced in aqueous solution with pulse and continuous radiolysis methods, and their reactions are studied by fast spectroscopic techniques and product analysis. Hydrogen peroxide and organic peroxides, the end products of peroxy radical reactions, are determined in the field by measuring the fluorescence of hydroxybenzoic acid isomers formed when peroxides react with $\mathrm{Fe}$ (II) in the presence of benzoic acid.

Results to Date: A new reagent for the analysis of hydroperoxy radicals was evaluated by pulse radiolysis. It was shown to react rapidly and specifically with these radicals to produce chemiluminescence. This reagent is being pursued for potential use in the field. A three-channel continuous-flow instrument was developed for airborne peroxide analysis. This instrument has a detection limit of $200 \mathrm{pptv}$ with a 1 -min response and was deployed for the first time during the 1995 Southern Oxidant Study in Nashville, Tennessee.

COORDINATION OF ATMOSPHERIC
CHEMISTRY PROGRAM
WESELY, MARVIN
ARGONNE NATIONAL LABORATORY
FY 1996333
FY 1995192
FY 1994184

Keywords: atmospheric chemistry, program management, tropospheric ozone

Objectives: To provide scientific coordination and enhance overall scientific progress of DOE/OHER's Atmospheric Chemistry Program (ACP) and to provide support for activities of the management coordinator of the North American Research Strategy for Ozone (NARSTO) program.

Approach: This project provides scientific guidance for the ACP and the NARSTO program on issues dealing with the emission, transport, transformation, and fate of energy-related trace chemicals in the atmosphere. The ACP scientific coordinator works with $\mathrm{ACP}$ researchers at $8 \mathrm{DOE}$ laboratories and at approximately 20 universities and non-DOE laboratories to enhance overall scientific progress. Objectives 
of the ACP include (1) understanding the atmospheric chemistry processes of energy-related air pollutants, (2) developing predictive models for these processes and acquiring the data to validate the models, and (3) developing an understanding of the trends in midlatitude stratospheric ozone changes and the heterogeneous chemistry and dynamics controlling ozone in this region. The NARSTO program is an international effort involving Canada, Mexico, and the United States that is devoted to providing the scientific and engineering basis for dealing with tropospheric ozone pollution above the North American continent. A 1998 scientific assessment of NARSTO research results on modeling, observations, and emissions will influence plans for subsequent research activities supported by and in cooperation with NARSTO. This ACP project supports the activities of the NARSTO management coordinator, who also devotes $10 \%$ of the effort to research on global atmospheric chemistry modeling for the ACP. Results to Date: The annual ACP meeting held in December 1995 focused on progress on and plans for (1) studies of stratospheric ozone and ultraviolet-B radiation, (2) numerical modeling and theoretical studies, (3) cooperative field experiment activities, (4) aircraft instrumentation, and (5) aerosol studies. An informal meeting report including descriptions of successes and potential new directions for research was produced. A technical advisory group was established, consisting of Dr. Jeremy M. Hales at Envair, Dr. Gregory Carmichael at the University of Iowa, and Dr. Barbara Finlayson-Pitts at the University of California at Riverside. The subcontract for the NARSTO management coordinator was finalized in February 1996. Nearly monthly publication of the ACP Monthly Update continued.

DRY-AIR-SURFACE EXCHANGE
WESELY, MARVIN; DOSKEY, PAUL;
and GAO, WEIGANG
ARGONNE NATIONAL LABORATORY
FY 1996286
FY 1995286
FY 1994170

Keywords: atmospheric chemistry, dry deposition, micrometeorology, natural emissions

Objectives: To develop methods to improve estimates of the air-surface exchange rates of energy-related sulfur and nitrogen compounds and atmospheric oxidants over North America and surrounding oceanic areas.

Approach: Field measurements, theoretical studies, parameterization of exchange rates, and numerical modeling are carried out. Vertical fluxes of $\mathrm{NO}, \mathrm{NO}_{2}$, and $\mathrm{O}_{3}$ fluxes are measured by the micrometeorological technique of eddy correlation, PAN dry-deposition rates are evaluated by a micrometeorological-gradient approach, and emission and uptake of organic compounds that contribute to the formation of atmospheric oxidants are observed with the use of environmental enclosures placed on the ground. A new version of the Argonne dry-deposition module is being developed for use in regional-scale and large-scale atmosphericchemistry models. Optical remote-sensing data from satellites are meshed with land-use maps from Argonne's dry-deposition module to provide smoothly varying changes in surface-resistance components. Argonne's turbulence-chemistry model is used to develop parameterizations of changes of $\mathrm{NO}, \mathrm{NO}_{2}$, and $\mathrm{O}_{3}$ fluxes with height and to generate efficient algorithms for use in the atmospheric chemistry models. Methods to estimate emissions of NO and possibly other nitrogen oxides from soils over regional scales are developed for incorporation into the Argonne dry deposition module.

Results to Date: Methods were developed, tested, and documented to use surface spectral reflectances remotely sensed by environmental satellites to improve parameterization of surface processes in modeling dry deposition over large geographic regions. Progress was made on experimental evaluation of the rates of NO emission from soils. The resulting data were used to infer that fast chemical reactions involving $\mathrm{NO}_{x}$ and $\mathrm{O}_{3}$ caused approximately $50 \%$ of the NO flux originating from soil emissions to be converted to $\mathrm{NO}_{2}$ at a height of $5 \mathrm{~m}$ during the daytime. Experiments were conducted on, and techniques were improved for, the measurement of the dry-deposition rate of peroxyacetyl nitrate (PAN). Articles were prepared on the methodology and results of measuring the air- surface exchange of volatile organic compounds in a prairie ecosystem.

\section{BOUNDARY-LAYER EVOLUTION AND THERMALLY DRIVEN WIND SYSTEMS IN THE COMPLEX TERRAIN OF THE SOUTHWESTERN UNITED STATES WHITEMAN, C. DAVID \\ PACIFIC NORTHWEST NATIONAL LABORATORY \\ FY 1996122 \\ FY 1995125 \\ FY 19940}

Keywords: complex-terrain meteorology, boundarylayer meteorology, air quality, thermally driven wind systems, temperature inversions

Objectives: To investigate thermally driven and terrain-channeled complex-terrain circulations and the related boundary-layer structure that affect air-pollution transport and dispersion in the U.S. Southwest.

Approach: Data analyses and model simulations are being used to meet the project objectives. The structure 
and evolution of wintertime boundary layers and thermally driven circulations are being investigated in the Grand Canyon area of Arizona with an extensive high-quality meteorology and air-quality data set collected by private utilities in 1990 at a cost of $\$ 14 \mathrm{M}$. Collaborations with other scientists are used to extend the scope of the research results and to evaluate the generality of the findings obtained in the Southwest. Results to Date: Wintertime soundings inside the Grand Canyon show a persistent near-neutral temperature structure within the canyon and the frequent occurrence of sharp temperature jumps or temperature inversions just above the canyon rims. The temperature jumps appear to represent episodes of warm air advection above the canyon associated with the movement of large-scale pressure systems. Analyses are under way to investigate several hypotheses concerning the observed temperature structure evolution.

Mass, moisture, and heat-budget analyses were performed for a southwest basin with observations and a numerical model. The nighttime flow through the narrow basin outlet was stronger than expected, and the subsidence of air into the top of the basin and the downward turbulent heat fluxes on the sidewalls played important roles in the basin heat budget. Heat budget measurements on the basin floor were shown to be nonrepresentative of the basin as a whole. The observations and simulations were compared, and a general method was developed for classifying basins and valleys relative to their energetics.

The effects of topography on turbulence were evaluated with measurements of horizontal wind variance at different tower sites in the Grand Canyon region. Day/night and terrain-related turbulence variations were found, and a means of classifying the effects of stability and terrain type was developed with statistical parameters.

Collaborations were begun with Swiss researchers to characterize regional-scale wind systems in the Grand Canyon region with cluster analysis and to investigate the effects of coupling between valley and above-mountain circulations. Significant modifications had to be made to a Swiss wind-field cluster-analysis scheme to obtain useful results for the Southwest. New schemes were developed for making pressure-field interpolations from stations surrounding major mountain barriers.

Plans were initiated with other national laboratory investigators to begin a study of the role of terrain and thermally driven wind systems on the air quality of Mexico City.

\section{HETEROGENEOUS CHEMISTRY \\ AFFECTING UPPER-TROPOSPHERIC AND STRATOSPHERIC OZONE \\ WORSNOP, DOUGLAS R; JAYNE, JOHN T.; KOLB, CHARLES E.; and DAVIDOVITS, PAUL AERODYNE RESEARCH, INC. and BOSTON COLLEGE \\ FY 1996151 \\ FY 1995150 \\ FY 1994140}

Keywords: heterogeneous chemistry

Objectives: To investigation the heterogeneous kinetics involved in gas-liquid interactions related to ozone formation and destruction in the upper atmosphere.

Approach: The rates of gas uptake into aqueous solution will be measured in the laboratory with waterdroplet and bubbler techniques for fast and slow gas/liquid mass transfer, respectively. Heterogeneous reaction rates of sulfur- and nitrogen-containing gases will be evaluated in aqueous solution, including dilute and strong acids, for inclusion in kinetic models of upper-tropospheric and lower-stratospheric aerosol and ozone photochemical cycles.

Results to Date: Initial work implemented improvements in our experimental techniques, including enhancements of mass-spectrometric detection sensitivity and the development of a modified bubble-train flow reactor to improve precision and sensitivity to slow gas/liquid heterogeneous processes. Droplet and bubble flow reactors in our laboratory can measure uptake rates corresponding to uptake coefficients ranging from 10:1 to 7:1. Results to date have focused on the temperature-dependent uptake of gas-phase formaldehyde $\left(\mathrm{CH}_{2} \mathrm{O}\right)$ into aqueous solution, ranging from basic conditions to concentrated sulfuric and nitric acids. Results have been analyzed in terms of solubility (including protonation in concentrated-acid solution) and hydrolysis reaction rates. The dissolution and reaction of $\mathrm{CH}_{2} \mathrm{O}$ in atmospheric sulfate aerosols may play a key role in nitrogen cycles that control ozone chemical reactivity. In particular, the reaction of $\mathrm{CH}_{2} \mathrm{O}$ with $\mathrm{HNO}_{3}$ in aqueous solution may perturb nitric oxide speciation in the upper atmosphere. Gasuptake results have been evaluated in terms of kinetic models appropriate for inclusion of heterogenous processes in general atmospheric photochemical models. 
THERMALLY DRIVEN REGIONALSCALE CIRCULATION SYSTEMS PRODUCED BY

BASIN AND PLATEAU LAND FORMS

ZHONG, SHARON, and WHITEMAN, C. DAVID

PACIFIC NORTHWEST NATIONAL

LABORATORY

FY 199631

FY 199534

FY 1994 0

Keywords: complex terrain meteorology, mesoscale circulation, local wind systems, thermally induced circulation, basin-plain/plateau circulation

Objectives: To develop an improved understanding of the basic physics of thermally driven plain-basin and plain-plateau circulations and the key role that topography plays in their development.

Approach: Data analyses from previous field experiments conducted over basin or plateau topography will be combined with numerical simulations with a 3-D mesocale numerical model.

Results to Date: Data from the Colorado's Sinbad Basin have been analyzed, and a state-of-art mesoscale numerical model has been used to provide an improved understanding of the flow and energy balance in the basin. The thermal forcing of the local circulation system and the sources of air that support the circulation have been determined. Individual terms of the basin atmospheric heat budget have been calculated from the model results and compared with limited observations. The results show that the forcing by advection and turbulent diffusion is significantly larger on the sidewalls than that over the basin floor; therefore, measurements made over the floor would not be representative of the basin as a whole. The cooling on the basin floor is a result of local radiative-flux divergence and advection of cold air from the sidewalls, and the cooling above the basin sidewalls is caused primarily by turbulent sensible-heat-flux divergence. Sensitivity tests show that the thermal structure, circulations, and rate of cooling and heating can be significantly affected by the ambient wind direction and, to a lesser extent, by vegetation coverage on the basin floor and sidewalls. 
The Climate Modeling Program develops the tools necessary to predict accurately the global and regional climate change induced by increasing atmospheric concentrations of carbon dioxide and other greenhouse gases. In addition to basic research on climate, two key components of the climate-modeling program are the Computer Hardware, Advanced Mathematics, and Model Physics Program (CHAMMP) and the Program for Climate Model Diagnosis and Intercomparison (PCMDI). CHAMMP encourages activities in four interrelated areas: theoretical studies of decade-to-century climate change and climate variability; development of massively parallel versions of general circulation models (GCMs), including the procurement of computer resources on leading-edge supercomputers; new numerical methods and model formulations; and better process parameterizations for GCMs. And PCMDI promotes a coordinated effort to document the differences and agreements among climate simulations, determine the sources of any differences among model results, and diagnose differences that occur between model results and observations.

\section{Program Managers}

David C. Bader

Environmental Sciences Division

Department of Energy, ER-74

19901 Germantown Road

Germantown, MD 20874-1290

(301) 903-4328

dc_bader@pnl.gov

Michelle S. Broido

Environmental Sciences Division

Department of Energy, ER-74

19901 Germantown Road

Germantown, MD 20874-1290

(301) 903-3281

Internet: michelle.broido@oer.doe.gov

Patrick A. Crowley

Environmental Sciences Division

Department of Energy, ER-74

19901 Germantown Road

Germantown, MD 20874-1290

(301) 903-3069

Internet: p.crowley@oer.doe.gov

\author{
Wanda R. Ferrell \\ Environmental Sciences Division \\ Department of Energy, ER-74 \\ 19901 Germantown Road \\ Germantown, MD 20874-1290 \\ (301) 903-0043 \\ Internet: wanda.ferrell@oer.doe.gov \\ Bobbi M. Parra \\ Environmental Sciences Division \\ Department of Energy, ER-74 \\ 19901 Germantown Road \\ Germantown, MD 20874-1290 \\ (301) 903-3316 \\ Internet: bobbi.parra@oer.doe.gov \\ Rickey C. Petty \\ Environmental Sciences Division \\ Department of Energy, ER-74 \\ 19901 Germantown Road \\ Germantown, MD 20874-1290 \\ (301) 903-5548 \\ Internet: rick.petty@oer.doe.gov
}

Michael R. Riches

Environmental Sciences Division

Department of Energy, ER-74

19901 Germantown Road

Germantown, MD 20874-1290

(301) 903-3264

Internet: mike.riches@oer.doe.gov 


\section{Climate Research}

\author{
FORUM ON SCIENCE, TECHNOLOGY, AND \\ THE GLOBAL SOCIETY \\ AHEARNE, JOHN F. \\ SIGMA XI \\ FY 199630 \\ FY 19950 \\ FY 19940
}

No abstract available for Sigma Xi 1996 Forum on Science, Technology, and the Global Society, held March 7 and 8, 1996.

\section{PARTIAL SUPPORT OF THE FY 1996 COMMITTEE ON ENVIRONMENT AND NATURAL RESOURCES' WORKING GROUP ON GLOBAL CHANGE \\ BAERWALD, THOMAS J. \\ COMMITTEE ON ENVIRONMENT AND NATURAL RESOURCES \\ FY 1996112 \\ FY 199592 \\ FY $1994 \quad 51$}

Keywords: program management, U.S. Global Change Research Program

Objectives: To provide partial support for activities of the Committee on Environment and Natural Resources (CENR) Subcommittee on Global Change Research (SGCR).

Approach: The National Science Foundation has assumed the task of collecting funds from all agencies participating in the U.S. Global Change Research Program to pay for the distributed-agency costs of activities of the CENR Subcommittee on Global Change Research (SGCR). These costs are shared by all of the participating agencies in the U.S. Global Change Research Program that support focused global-change research. The activities supported include the National Academy of Sciences Board on Global Change, which combines the activities of five previous NAS boards and committees that dealt with aspects of global-change research; the Snowmass Global-Change Summer Institutes Program, organized by the University Corporation for Atmospheric Research; printing costs for the CENR global-change documents; and operating expenses of the CENR SGCR Secretariat.

Results to Date: No results were reported.

\section{CLIMATE SYSTEM RESEARCH: STUDIES OF GLOBAL AND \\ REGIONAL INSTRUMENTAL DATA}

BRADLEY, RAYMOND S., and DIAZ, HENRY F. UNIVERSITY OF MASSACHUSETTS and NOAA

FY 1996187

FY 1995200

FY 1994237

Keywords: climate record, paleo data, temperature record

Objectives: To place the modern record of climate variability in a longer perspective and to characterize the spatial and temporal dimensions of climate variations in the 20th century.

Approach: The instrumental records of climate, GCM simulations, and proxy records in the preinstrumental period will be analyzed.

Results to Date: Our reconstruction of Northern Hemisphere summer temperatures back into the 15th century suggests that the record may be interpreted in two quite different ways: Either there was unprecedented recent (20th century) warming, or there were simply minor variations around an underlying lowfrequency oscillation, with nothing particularly unusual occurring in the 20th century. Extending the paleo temperature record further back in time may help to resolve these contrasting interpretations. We used long GCM simulations to assess the optimum distribution of paleo data to be used in reconstructing largescale paleo temperatures, finding that a mix of lowlatitude oceanic and higher-latitude continental sites provide the optimum network. We also examined characteristic scales of climate variability from the local to the global scale.

$$
\begin{aligned}
& \text { CO }_{2} \text {-INDUCED CLIMATE CHANGE } \\
& \text { CESS, ROBERT D., and HAMEED, SULTAN } \\
& \text { STATE UNIVERSITY OF NEW YORK AT } \\
& \text { STONY BROOK } \\
& \text { FY } 1996488 \\
& \text { FY } 1995467 \\
& \text { FY } 1994434
\end{aligned}
$$

Keywords: general circulation models

Objectives: To improve cloud-climate interactions in general-circulation models. The results of models will be compared to satellite radiometric data.

Approach: A major portion of the research effort has been devoted to understanding cloud-climate interactions in 18 general circulation models (GCMs) with the goal of improving their representations of cloud feedback associated with climate change. Although no surrogate is available for long-term climate change that will address this issue, if a perfect GCM were 
available, it would produce proper cloud feedback as well as correct seasonal changes in cloud radiative forcing. The latter constitutes one test of cloud-climate interactions in GCMs, and we have tested the 18 GCMs in this way with data from the Earth Radiation Budget Experiment.

Results to Date: The models need considerable improvement. The four centers of action, the Azores High, the Icelandic Low, the Hawaiian High, and the Aleutian Low control climate over a large part of the Northern Hemisphere. We have performed diagnostic studies that can help in the prediction of the intensities and the locations of these centers of action. We have found that statistically significant precursors of the intensity and location of the wintertime and summertime Azores High and the wintertime Icelandic Low occur in (1) preseason sea-surface temperatures in different sectors of the North Atlantic and (2) preseason information on the intensities and locations of the four centers of action. We have used these precursors to formulate linear multiple-regression equations; good agreement between the regression series and observations from 1900 to 1994 suggests that not only are the four centers of action coupled to one another by teleconnection patterns but also conditions of one may be predictable by antecedent conditions in the others. In a separate study, the locations of the Aleutian Low and the Hawaiian High were diagnosed to be influenced by the solar cycle. The Aleutian Low migrates eastward during minimum solar activity, and the Hawaiian High migrates southward. Effects on the intensities of these centers of action are also significant and are in general agreement with recent GCM results of Haigh. Because shifts in the centers of action change storm trajectories, large anomalies in regional climatic conditions can be produced in the extreme phases of the solar cycle.

\section{ACCELERATED LINE-BY-LINE CALCULATIONS FOR THE RADIATIVE TRANSFER OF TRACE GASES RELATED TO CLIMATE STUDIES \\ CLOUGH, SHEPARD A. ATMOSPHERIC AND ENVIRONMENTAL RESEARCH, INC. \\ FY 199669 \\ FY 199569 \\ FY 199469}

Keywords: radiative transfer, line-by-line calculations, fluxes, cooling rates, radiativetransfer model

Objectives: To provide detailed, line-by-line (LBL) radiative codes for quantitatively studying spectral outgoing radiances, to extend detailed LBL codes to the visible and ultraviolet spectral regions, and to test the effects of improved parameterizations in dynamical models, including GCMs.
Approach: Model calculations with the LBLRTM code provide radiative-forcing results for greenhouse gases (including water vapor, carbon dioxide, methane, nitrous oxide, and the halocarbons) that must be properly represented by the radiation codes used in dynamical models. A key result is the archived spectral information obtained from the LBLRTM that can be used for validating and improving rapid-radiation models. An important element of this research effort is that the LBLRTM model is undergoing continuous validation with spectral radiative measurements obtained under the ARM program.

Flux and cooling-rate results are obtained for the full range of climatologic atmospheres, for selected changes in the climatologic profiles to study the implications of radiative forcing as a function of trace gas profile change, and for real atmospheres of importance to specific dynamical model studies (e.g., singlecolumn-model experiments).

Results to Date: A longwave rapid radiation model, RRTM, which provides flux and cooling-rate results consistent with the LBL calculations, has been developed and is being implemented in the single-column ALFA model and the 3-D CCM2 to evaluate the feedback effects of specific aspects of radiation modeling. Of particular interest are the separate effects of the foreign- and self-water-vapor-continuum and ozoneprofile changes. This effort will ultimately be extended to the shortwave region and to improved parameterizations for cloud radiative transfer. GCM results will be diagnosed with quantities and model runs that are consistent with the Atmospheric Model Intercomparison Project (AMIP).

LBLRTM has been used to calculate clear-sky spectral fluxes and cooling rates for $\mathrm{CO}_{2}, \mathrm{O}_{3}, \mathrm{CH}_{4}$, $\mathrm{N}_{2} \mathrm{O}$, and the most important halocarbons. Spectral values for longwave fluxes and cooling rates have been established for three atmospheres as a reference for climate models, and the sensitivity of these quantities to anticipated 10-year (1990 to 2000) trace-gas-profile changes has been assessed. The results are presented spectrally as a function of $\log$ pressure to emphasize the pressure regimes of particular processes. Doubling $\mathrm{CO}_{2}$ increases the stratopause cooling by $25 \%$ but lowers the tropospheric cooling by $5 \%$ for the midlatitude summer atmosphere.

A rapid radiative transfer model, RRTM, is being implemented into two dynamical models: the singlecolumn ALFA model and the 3-D CCM2. The model will also be implemented in the recently available CCM3. RRTM provides results for fluxes and cooling rates that are effectively identical to those from LBLRTM and thus have a validation with ARM data through LBLRTM. Initial indications are that RRTM is a factor of two faster than the CCM2 radiation model. 


\section{DATABASE SUPPORT TO ASSIST IN \\ QUANTIFYING THE LINK BETWEEN CHANGES IN ATMOSPHERIC \\ COMPOSITION AND CLIMATE CHANGE \\ CUSHMAN, ROBERT, and \\ BODEN, THOMAS \\ OAK RIDGE NATIONAL LABORATORY

$\begin{array}{lr}\text { FY } 1996 & 0 \\ \text { FY } 1995 & 455 \\ \text { FY } 1994 & 485\end{array}$

Keywords: atmospheric chemistry, radiative balance, climate, aerosols, clouds, atmospheric temperature

Objectives: To provide quality-assured data sets and other information needed for quantifying the link between atmospheric chemistry, the Earth's radiative balance, and climate.

Approach: The first task was to compile, document, and distribute a data set suitable for long-term climate analyses. The second task was to acquire, inspect, and document climate-related data sets from the former Soviet Union. The third task was to develop a priority list, based on the results of a survey of climate researchers, of the most important extant climate-related data sets needed for quantifying the link between radiative balance and atmospheric temperature. The fourth task was to pro-duce a series of technical reports based on the trans-lation into English of Russianlanguage literature important to the Quantitative Links Program.

Results to Date: During FY 1996, the third report in the Russian-English bibliography series on climate change was published: Selected Translated Abstracts of Russian-Language Climate-Change Publications: III. Aerosols (CDIAC-88, Proceedings of RIHMIWDC, Number 164).

\section{ENVIRONMENTAL SCIENCES DATABASE DUNLAP, KELLY, and HENDERSON, DAVID DOE/OSTI \\ FY 199610 \\ FY 199514 \\ FY $1994 \quad 0$}

Keywords: information systems

Objectives: To compile an annual database of research projects that are sponsored by DOE's Environmental Sciences Division (ESD).

Approach: This project is being accomplished with WAIS software (compatible with Z39.50 Version 2) that has a World Wide Web interface. Online authorities are being used to ensure data standardization, and hyperlinks have been embedded to connect related entries.

Results to Date: FY 1994 and FY 1995 research summaries are complete and online. Work on the FY 1996 research summaries is under way.

\section{DATA PREPARATION AND ANALYSIS FOR ANNEX III, USAPRC COOPERATION IN ATMOSPHERIC TRACE GASES \\ EASTERLING, DAVID, and KARL, THOMAS \\ NOAA/NESDIS/NATIONAL CLIMATIC DATA CENTER \\ FY 1996225 \\ FY 1995258 \\ FY 1994258}

Keywords: climate data, trend analysis, homogeneity of data

Objectives: To develop global and regional climate data sets suitable for climate-change research and to analyze these data sets for evidence of climate change. Approach: Climate data sets, such as monthly maximum and minimum temperatures, will be compiled, and complex quality-control procedures will be performed on them. The time series will be examined and adjusted for homogeneity. Perform trend analyses and extreme-event analyses will be performed on these data, and downscaling methods will be developed to produce regional climate-change scenarios for use in climate-impact models.

Results to Date: A new method has been developed for detecting and adjusting for inhomogeneities in climatic time series. We analyzed global and regional time series of maximum and minimum temperature for changes in the diurnal temperature range and found that urban effects are insignificant in global and regional analyses. Using the downscaling method, we developed regional climate-change scenarios for use in crop models to examine the transient effects of climate change on corn production in the central United States.

\section{TEMPORARY ASSIGNMENT TO THE COMMITTEE ON ENVIRONMENT AND NATURAL RESOURCES (CENR) EDGERTON, SYLVIA

Keywords: administrative support

Objectives: To provide technical assistance on airquality issues to the Director of Energy Research and the CENR Subcommittee on Air Quality Research.

Approach: Analysis, integration, and coordination efforts are provided for the CENR Subcommittee on Air Quality Research. Communication linkages are developed with the White House Office of Science and Technology Policy. Assistance is given in developing research priorities and recommendations for future airquality research programs to be undertaken by federal agencies.

Results to Date: Priorities for federally supported research on air quality have been established and are 
continually being updated. Long-term strategic plans and communication strategies are under way. International collaborations and workshops are also held periodically.

\section{PARTIAL SUPPORT FOR THE NATIONAL ACADEMY OF SCIENCES BOARD ON ATMOSPHERIC SCIENCES AND CLIMATE FEIN, JAY $S$.}

NATIONAL SCIENCE FOUNDATION FY 199650

FY 19950

FY 199450

Keywords: administrative support

Objectives: To study problems of cost efficiency and recommend solutions; to understand conflicting views of characterizing rainfall; and to examine the opportunities, challenges, and imperatives for the atmospheric sciences.

Approach: The health of atmospheric sciences as affected by administrative, managerial, and funding decisions will be studied. The Federal Energy Regulatory Commission will be aided in understanding the conflicting views of characterizing rainfall. And the priorities in atmospheric sciences for the 21 st century will be assessed.

Results to Date: No results were reported.

\section{$\mathrm{CO}_{2}$-EFFECTS RESEARCH: TRACE GASES IN THE GLOBAL ATMOSPHERE GROSSMAN, ALLEN S. LAWRENCE LIVERMORE NATIONAL LABORATORY FY 1996298 FY 1995298

FY 19940

Keywords: trace gases, radiative forcing, carbon dioxide

Objectives: To assist DOE in determining the potential effects on climate and the global environment from increasing atmospheric trace-gas concentrations.

Approach: Our 2-D chemical-radiative-transport model and our detailed radiative-transport models are used to investigate radiative-forcing effects caused by various trace-gas-change scenarios. A 3-D chemicalradiative-transport model designed for parallel architecture computers is being developed. Information on past trends and the potential for future trends of the various trace species will be developed. Budgets of relevant trace gases will be examined to better establish which uncertainties require consideration in our development of scenarios of future emissions. Existing capabilities for modeling global atmospheric chemical, radiative, and physical processes will be examined. And model-sensitivity analyses will be performed to better define the mechanisms, key parameters, and overall uncertainties of potential trace-gas influences on the atmosphere. These studies should lead to an improved understanding of the impact that $\mathrm{CO}_{2}$ and other trace gases may have in determining the time rate of climatic change. In addition, the effects of $\mathrm{CO}_{2}$-induced changes in atmospheric chemistry will be evaluated.

Results to Date: We have developed a new IR radiative-transfer model based on the correlated k-distribution method for band absorption. It uses a parallel-processing-computer architecture. The model is significantly faster and more computationally efficient than our previous model for the calculation of trace-gas IR and near-IR absorption coefficients.

We have considered the efficiency of various radiative-transfer parameterizations for combined absorption and scattering effects in the calculation of IR radiative forcing for typical GCM applications. A combined delta two-four stream model has been developed that provides high accuracy as well as computational economy for these applications.

\section{DETECTION OF GREENHOUSE- GAS-INDUCED CLIMATE CHANGE \\ JONES, PHILIP D., and WIGLEY, TOM M. L. UNIVERSITY OF EAST ANGLIA and \\ UCAR/NCAR \\ FY 1996198 \\ FY 1995192 \\ FY 1994178}

Keywords: aerosols, climate-change detection, instrumental climate data

Objectives: To assemble and analyze instrumental climate data and to develop and apply climate models as a basis for detecting and projecting anthropogenic climatic change and for validating GCMs.

Approach: Global surface climate databases will be expanded and updated with the data available to the Climatic Research Unit from a variety of sources. Data analyses will focus on the use and development of appropriate statistical techniques for signal detection and pattern recognition. Interpretation will be guided by appropriate results from GCMs and energy-balance models (EBMs).

Results to Date: We continue to improve our landonly temperature dataset, incorporating many additional temperature records. The new analyses use a base of 1961 to 1990 , and the station temperatureanomaly values are averaged into a dataset with a grid box of 5 degrees by 5 degrees. A method for estimating errors on the hemispheric and global scales has been developed. For annual global averages since 1950, the error is $+1-0.04$ degrees $C$. Temperatures during 1994 and 1995 increased dramatically from the Pinatuboinduced cooler years of 1992 and 1993. 1995 is the warmest year on record. 
Further applications have been made of our pattern-correlation-detection technique. For horizontal temperature patterns, we find a significant upward trend in the correlation between observed and modeled temperature-change patterns for model simulations that include the effect of sulfate aerosols. For $\mathrm{CO}_{2}$ alone, there is no significant trend. We now obtain similar results for changes in zonal-mean vertical temperature profiles. In this case, both $\mathrm{CO}_{2}$-alone and $\mathrm{CO}_{2}$-plus-aerosol results are significant, with the latter being more significant. While these results must be viewed cautiously because of signal and noise uncertainties, they have been highly influential in recent IPCC work. They form the basis for the statement in the IPCC Summary for Policy Makers that the balance of evidence suggests a discernible human influence on global climate.

\section{METHANE FLUXES FROM RICE FIELDS: THE EFFECTS OF CLIMATE AND AGRICULTURAL FACTORS KHALIL, M. A. K., and RASMUSSEN, R. A. PORTLAND STATE UNIVERSITY and OREGON GRADUATE INSTITUTE \\ FY 1996426 \\ FY 1995408 \\ FY 1994381}

Keywords: methane, rice agriculture, climate Objectives: To determine emissions of methane from rice fields in China and to estimate the contribution of rice fields to the global meth:ine budget.

Approach: Methane is a potent greenhouse gas that is increasing in the atmosphere and may cause global warming. Rice fields are a major source of methane, and rice agriculture has contributed significantly to the increase of methane during the past century. We have conducted extensive measurements of methane emissions from rice fields in China. The data provide the longest available time series of emissions from the same location. It shows interannual variability of the flux and its relation to climatic variables and agricultural practices. The foundation of the project is the direct measurement of methane emissions from rice fields in China. A chamber is placed on top of the soil and rice plants. Air samples are drawn from the chamber about 3 minutes apart. The measured buildup of methane in the chamber is used to calculate the emission rate. A field gas chromatograph with a flameionization detector (FID) is used to measure the concentration in the samples collected.

Results to Date: Methane emissions from rice fields in various locations have been measured between 1988 and 1995. The data from Tuzu, near Leshan City in Sichuan Province (China), constitute the longest existing record of systematic measurements of methane flux from rice fields. This record shows remarkable constancy of methane emission rates at an average of about $31 \mathrm{mg} / \mathrm{m}^{2}-\mathrm{h}$. Shorter records from Guangzhou, in Guangdong Province (China), show comparable emissions rates at about $35 \mathrm{mg} / \mathrm{m} 2$-hr. At other locations, lower emissions were recorded: in Indonesia, $17 \mathrm{mg} / \mathrm{m} 2-\mathrm{hr}$ and at Beijing, $9 \mathrm{mg} / \mathrm{m}^{2}-\mathrm{hr}$. The reasons for the lower concentrations in Beijing are likely to be the periodic loss of water from the fields and the use of mineral fertilizers instead of the conditions at Tuzu where the fields are continuously flooded and organic fertilizers are used.

\section{RECENT CHANGES OF WEATHER PATTERNS IN NORTH AMERICA KUKLA, GEORGE \\ LAMONT-DOHERTY EARTH OBSERVATORY \\ FY 1996265 \\ FY 1995248 \\ FY 1994220}

Keywords: climate change, North America, weather variability, aerosols

Objectives: To empirically study recent time-related changes of weather extremes, tropospheric aerosols, and transport of atmospheric water vapor in North America.

Approach: Statistical analysis will be performed of daily and monthly observations over intervals of variable length limited by the accuracy and completeness of historical weather records.

Results to Date: The trends of maximum and minimum temperature in North America between 1950 and 1990 were inversely correlated with industrial emissions of $\mathrm{SO}_{2}$. With a cause-and-effect relationship assumed, the 1990 emissions are estimated to have decreased the mean surface air temperature by about $1.0^{\circ} \mathrm{C}$. The transition from caal power plants to nuclear energy in the 1970 s in France resulted in a sharp decrease of $\mathrm{SO}_{2}$ emissions. We found maximum daily temperatures to be relatively low at times of high sulfur emissions. Preliminary results from an ongoing study in which an index of weather variability is defined as the combined frequency of occurrence of cold and warm extremes in daily temperature minima and maxima generally show a decrease in summer and no change in winter in the U.S. Midwest from earlier this century to the mid-1980s.

A JASON STUDY OF SELECTED TOPICS
MAHEN, EDWARD C., Jr.
ADVANCED RESEARCH PROJECTS AGENCY
FY 1996149
FY 199575
FY 19940

No Abstract Available 


\section{GLACIAL CYCLES AND ORBITAL INCLINATION \\ MULLER, RICHARD A. \\ LAWRENCE BERKELEY NATIONAL \\ LABORATORY \\ FY 199633 \\ FY 19950 \\ FY 19940}

Keywords: tephra layers, orbital inclination

Objectives: To study the hypothesis that orbital inclination plays a role in the Earth's climate.

Approach: Experimental investigation of tephra layers in Italy allow argon-argon determination of ages, which can then be studied to match orbital calcuations. In addition, theoretical work will be carried on to understand better the physics of the extraterrestrial influences on the atmosphere, land, and ocean system, both in present-day climates and paleoclimates.

Results to Date: Argon-argon ages have been successfully obtained at a series of tephra layers, and their relationship to orbital parameters is being investigated. A claimed linkage between climate and precession is disputed.

$$
\begin{aligned}
& \text { DETECTION AND ATTRIBUTION OF } \\
& \text { CLIMATE VARIATIONS AND CHANGES } \\
& \text { MURRAY, WILLIAM L. } \\
& \text { NATIONAL OCEANIC AND ATMOSPHERIC } \\
& \text { ADMINISTRATION } \\
& \text { FY } 1996500 \\
& \text { FY } 19950 \\
& \text { FY } 1994 \quad 0
\end{aligned}
$$

Keywords: climate-variation detection

Objectives: To focus on the detection and attribution of climate variations and changes.

Approach: The results of 17 projects designed to provide information to improve the understanding of climate variability on decadal to century time scales will be analyzed to assess their contribution to the detection and attribution of climate variations and changes.

Results to Date: New project.

\section{CLOUDS AND OCEAN-ATMOSPHERE INTERACTIONS IN THE PACIFIC BASIN RANDALL, DAVID COLORADO STATE UNIVERSITY FY 1996166 \\ FY 1995157 \\ FY 1994190}

Keywords: coupled atmosphere-ocean models, FANGIO, AMIP, clouds, ocean-atmosphere interactions

Objective: To lead research activities in both FANGIO and AMIP and a coupled ocean-atmosphere modeling project.
Approach: We plan to use our coupled ocean-atmosphere models to study the effects of clouds on the coupled system, with emphasis on two types of tropical clouds: the cloud systems associated with deep convection in the tropical Western Pacific and the shallow stratocumulus clouds that dominate the tropical and subtropical Eastern Pacific.

Results to Date: A FANGIO meeting is planned for fall 1996. The principal investigator has become a member of the AMIP Panel and is actively involved in the planning of AMIP 2.

\section{RESEARCH ON GREENHOUSE- GAS-INDUCED CLIMATE CHANGE SCHLESINGER, MICHAEL UNIVERSITY OF ILLINOIS FY 1996150 FY 1995150 FY 1994146}

Keywords: models

Objectives: To simulate and understand greenhouse-gas-induced climate change, both future and past.

Approach: Models of the Earth's climate system will be developed and tested and then used to simulate past, present, and possible future climates. Methods to analyze both simulated and observed climates will be developed, tested, and employed to examine alternative global-warming policies.

\section{GLOSSARY OF METEOROLOGY STEPHENS, PAMELA NATIONAL SCIENCE FOUNDATION \\ FY 199633 \\ FY 199533 \\ FY 199433}

Keywords: glossaries, meteorology

Objectives: To provide partial support for the preparation of a glossary of meteorology.

Approach: The American Meteorological Society (AMS) is preparing and will publish a new Glossary of Meteorology. The AMS is managing the selection of authors, editing, and publication. Both hard copy and electronic copy will be produced. Support for this effort is being provided by several federal agencies, the AMS, and the scientific community at large.

Results to Date: The AMS Glossary project is now into its third year. Each of the 39 subject-area editors has tasked writers and reviewers to prepare updates to terms from the first edition as well as definitions for new terms that will be added to the new edition. Five editors have completed their work and returned their material to AMS Headquarters for technical and copy editing. The remainder of the submissions are expected to arrive on a rolling basis through March 1997. 
The AMS has selected Allen Press to provide publication services for the printed and electronic forms of the glossary. Publication is expected by the fall of 1997.

\section{CARBON DIOXIDE EFFECTS RESEARCH \\ TAYLOR, KARL, and PENNER, JOYCE \\ LAWRENCE LIVERMORE NATIONAL LABORATORY \\ FY 1996496 \\ FY 1995499 \\ FY 1994600}

Keywords: climate change, chemistry-climate interactions, anthropogenic aerosols, paleoclimate, climatechange detection

Objectives: To sharpen understanding of climate change by determining the response to prescribed changes in atmospheric composition and surface boundary conditions, by increasing understanding of complicating factors (such as the interactions between climate and chemistry), by analyzing paleoclimate simulations and climate records, and by comparing modeled and historically observed climate changes.

Approach: Theoretically based global climate models are being used to simulate past and future climatic conditions. The simulation of paleoclimates (with prescribed, realistic changes in glacial-ice distribution, greenhouse-gas concentrations, and insolation) are being compared with paleoclimate data and with other models run under the same conditions (as part of the Paleoclimate Modeling Intercomparison Project, PMIP) to help evaluate model skill in predicting known climate changes. One of the principal investigators co-coordinates PMIP, which is endorsed by the International Geosphere/Biosphere Program and by the Working Group on Numerical Experimentation of the World Climate Research Program. About 18 climatemodeling groups from nearly a dozen countries participate in this project.

Results to Date: The same models are being used to predict patterns of climate change resulting from recent increases in greenhouse gases and aerosols to determine whether these predicted patterns are consistent with the observational record. A major aim is to continue development of a coupled chemistry/climate model that can make improved estimates of climatic effects of sulfate and other important tropospheric aerosols. The pattern of climate change predicted by this model will be used to determine the extent to which human influences can be identified in the recent historical climate record.

Simulations of the climate of the most recent glacial maximum ( 21,000 years ago) have been carried out, and model output has been contributed to the PMIP data archive. A preliminary analysis indicates that the tropical SSTs predicted by the model are not as low as in some earlier simulations and may be more consistent with paleoclimate reconstructions. Further work is needed to verify this conclusion and to understand the differences between various models.

Simulations of climate change caused by human activities have indicated that aerosols can have significant impacts on the pattern of climate change. The climate changes simulated by the coupled chemistryclimate model in response to historical increases in carbon dioxide and/or sulfur emissions have been compared with the observed climate record. That comparison has provided some of the first evidence that human activities have already altered climate on a global scale. The increasing correspondence found between modeled and observed patterns of change is unlikely to be explainable in terms of natural variability unless recent coupled-atmosphere-ocean GCMs are underestimating internal climate variability on decadal to century time scales.

\section{STUDY OF REGIONAL CLIMATE CHANGE WANG, WEI-CHYUNG STATE UNIVERSITY OF NEW YORK AT ALBANY \\ FY 1996357 \\ FY 1995342 \\ FY 1994349}

Keywords: regional climate, teleconnection, contemporary and historical climate in China, general-circulation models

Objectives: To diagnose and improve GCM simulations of the regional climates in the United States and east Asia, to understand their teleconnections, and to conduct historical climate reconstruction for China.

Approach: The research program is organized into two major scientific tasks. The first consists of GCM diagnosis and improvement with a major effort on the climates in the United States and east Asia and a minor effort on climate feedbacks. These studies will be carried out by conducting and analyzing NCAR (GENESIS and CCM3) simulations together with observational analysis as a participant in the two DOEsponsored GCM intercomparison programs, AMIP and FANGIO. The second major task involves collaboration with the Institute of Geography of the Chinese Academy of Sciences on historical climate reconstruction in China.

Results to Date: For the GCM study, both conceptual and diagnostic frameworks were developed to describe the key characteristics of the east Asian summer monsoon, including both the large- and regional-scale features as well as their evolution (e.g., pre-onset, onset, and post-onset during mid-April to mid-June; and monsoon movement in July to September). These frameworks were then used to conduct GCMto-observation and GCM-to-GCM comparisons with a 
focus on systematic errors of climatological mean characteristics of east Asian climate.

We have analyzed the monthly mean (1) geographic distribution of the surface air temperature and precipitation; the $200-$ and $850-\mathrm{hPa}$, velocity potential, and stream function; the $500-\mathrm{hPa}$ height; and the outgoing longwave radiation; (2) zonal mean altitude distribution of wind $(u, v)$ and temperature; and (3) geographical distribution of surface heat-flux components.

The comparisons were conducted for 30 AMIP models and the CCM3. In addition to the climatological means, we have also examined the association between the $200-\mathrm{mb}$ jet, the precipitation, and other relevant parameters over east China. Preliminary results indicate that the jet has a dominant influence and can potentially serve as a predictor for regional climate.

The GCM representation of subgrid-scale variability of cloud-radiation interaction continues to be improved. Simulation results show that a mosaic treatment to account for cloud physical association in the vertical direction within a grid can significantly affect climate simulations and correct many model biases, especially in the tropical regions.

The study of historical climate reconstruction in China has continued in two aspects, the documentation and some preliminary analyses. The documentation has focused on the description of the available historical writings and on the procedure and consideration of preparing and validating the proxy data sets: (1) the 63-station data set from $137 \mathrm{BC}$ to $\mathrm{AD} 1470$ for flood, drought, and good yields derived from historical books; (2) the 82-station data set from 1471 to 1949 for famine, good yields, flood, drought, frost, snow, hail, and ice conditions derived from gazettes, personal diaries, and newspapers of recent years; and (3) the 87-station data set from 1730 to 1949 for summer and autumn harvest and the 100-station data set for 1736 to 1911 for yu-xue-fun-cun (rain and snow depth) derived from the Memos to the Emperors. Analyses of the variability of the spatial patterns of the flood/drought on the interannual and decadal time scales were conducted.

The principal investigator continues to serve as the Chief Scientist to two DOE collaborative agreements with China: the US/DOE-PRC/CAS joint project on the greenhouse effect and the US/DOE-PRC/CMA joint study on regional climate. In addition to coordinating the scientific tasks among the U.S. and Chinese participants, the Eighth Science Team meeting of the DOE-CAS agreement and the Second Science Team meeting of the DOE-CMA agreement in Beijing have been organized.
ENHANCED RESEARCH PROGRAM ON

THE LONG-RANGE CLIMATIC EFFECTS

OF INCREASING ATMOSPHERIC

CARBON DIOXIDE

WASHINGTON, WARREN M., and MEEHL, GERALD A.

NATIONAL CENTER FOR ATMOSPHERIC

RESEARCH

FY 1996507

FY 1995450

FY 1994433

Keywords: climate modeling, climate change, CHAMMP

Objectives: To couple an enhanced version of an atmospheric GCM to a 1-degree-by-1-degree dynamical ocean GCM with a dynamic/thermodynamic sea-ice model, which is used to perform experiments on the sensitivity of climate change to anthropogenic carbon dioxide and sulfate aerosols.

Approach: Our enhanced atmospheric model will be coupled to a dynamical ocean model that is a 1-degree-by-1-degree version of the Semtner-Chervin 1/2-degree-by-1/2-degree ocean model with 20 vertical levels. The version used explicitly resolves boundary currents and some aspects of the mesoscale eddies as does the parent model, but research has shown that observed ocean features sufficient for climate experiments can be simulated without such a detailed, explicit eddy calculation. The sea-ice component involves a version of the Flato-Hibler ice-dynamics formulation and the three-layer Semtner thermodynamic scheme.

The coupling scheme is synchronous rather than asynchronous. In the DOE/NCAR scheme, the atmospheric model provides wind stress, precipitation minus evaporation, and the sum of the surface energy balance to the ocean. The ocean provides surface temperature and sea-ice distributions to the atmosphere. The coupled experiment was started from separate nuns of the new models, each forced with the appropriate observed climate conditions [e.g., the observed sea-surface temperature (SST) for the spinup of the atmospheric model]. This procedure allows separate diagnosis of any model problems before coupling. Sensitivity experiments with a $1 \%$ per year (compounded) increase in carbon dioxide and sulfate aerosols are being performed.

Results to Date: We performed coupled oceanatmosphere-sea-ice GCM experiments to assess the combined sensitivity of the Earth's climate system to increased $\mathrm{CO}_{2}$ and sulfate aerosols. We examined changes to the atmospheric and oceanic circulations and changes of sea-ice distribution. We showed that the North Atlantic Ocean became warmer and fresher, sea-ice margins retreated, and the thermohaline circulation in the ocean weakened. We also saw an 
interesting warming of the midocean water in the far North Atlantic. The continents warmed faster than the oceans did, and enhanced dry conditions occurred during summer in the increased- $\mathrm{CO}_{2}$ environment. In the tropical Pacific, the SSTs in the eastern equatorial Pacific warmed faster than the SSTs in the western equatorial Pacific partly because of cloud-albedo feedback. This warming produced an El-Niño-like signature for $\mathrm{CO}_{2}$ climate change in the Pacific region not unlike some aspects of recently observed climate anomalies in the Pacific. With sulfate aerosols, we found much smaller global warming, especially in high latitudes. Also, a cooling or minimum warming that was closer to observed was found over the southeast United States, Europe, and Southeast Asia.

\author{
SENSITIVITY OF CLIMATE MODELS: \\ COMPARISON OF SIMULATED AND \\ OBSERVED PATTERNS FOR PAST \\ CLIMATES \\ WEBB, THOMPSON, and PRELL, WARREN \\ BROWN UNIVERSITY \\ FY 1996521 \\ FY 1995450 \\ FY 1994388
}

Keywords: climate model testing, past climates, paleoclimatic sensitivity modeling studies, PMIP, CCM

Objective: To use paleoclimatic data to test the ability of atmospheric general circulation models to simulate past climates and thus contribute to PMIP (the Paleoclimate Modeling Intercomparison Project).

Approach: The climates of the past 20,000 years will be simulated, paleoclimatic data will be used to test how well the simulations match the data, and intercomparisons will be performed with other model results for 6,000 and 21,000 years ago.

Results to Date: The NCAR CCM1 was used to simulate the climates at $21,000,16,000,14,000$, 11,000 , and 6,000 years ago. Tests with global and continental data sets show improved simulation of 21,000 years ago (the last glacial maximum) over the previous simulation with CCM0 but not as good simulation of climates at $16,000,14,000$, and 11,000 years ago. Sensitivity tests with the NCAR CCM1 showed its response to varying levels of carbon dioxide, insolation, and ice sheets. These tests helped in evaluating the model results. Other sensitivity tests showed the importance of vegetation feedbacks to climate at 6000 years ago.

\section{Computer Hardware, Advanced Mathematics, and Model Physics (CHAMMP)}

\section{DEVELOPMENT OF AN ADVANCED FINITE- DIFFERENCE ATMOSPHERIC GENERAL CIRCULATION MODEL ARAKAWA, AKIO, and MECHOSO, CARLOS R. UNIVERSITY OF CALIFORNIA, LOS ANGELES \\ FY 1996187 \\ FY $1995 \quad 0$ \\ FY 1994179}

Keywords: GCMs, cloud parameterizations, cumulus clouds, stratocumulus clouds, generalized vertical coordinate model

Objectives: To further develop the physical and computational aspects of the UCLA GCM for use in CHAMMP.

Approach: The parameterization of shallow cumulus and stratocumulus clouds in the advanced finitedifference atmospheric general circulation model will be revised, and the revised model will be coupled to the GFDL/Princeton University Modular Ocean Model (MOM) to evaluate the impact of model revisions on the simulation of the seasonal cycle and interannual variability of the atmosphere-ocean system. Decade- long simulations will be run to compare distributions of the ozone mixing ratio that are prescribed to those that are predicted. We will also develop a model based on a generalized vertical coordinate.

Results to Date: The parameterization of shallow cumulus and stratocumulus clouds has been revised. Recent experiments with the UCLA coupled GCM show that simulation of sea-surface temperature (SST) is extremely sensitive to formulations of physical processes in the model, especially in its atmospheric component (AGCM).

Earlier experiments with the standard version of the AGCM indicate that the simulated seasonal cycle is reasonably realistic. The incidence of subtropical marine stratocumulus, however, is underpredicted. In view of this model deficiency, we have revised and improved (1) the formulation of the properties of air entraining into the PBL, (2) the processes after the stratus cloud layer becomes unstable, (3) the effects of stratus clouds on shortwave radiation, (4) the largescale condensation processes in the PBL, and (5) the "drizzle" effect in the PBL. With these revisions, the 
simulation of precipitation, surface evaporation, and stratocumulus incidence is far more realistic than that with the standard version of the AGCM.

We contrasted decade-long simulations obtained with distributions of the ozone mixing ratio that are either prescribed (according to an observed climatology) or predicted (according to a highly simplified photochemistry). Our results show that errors in predicted ozone in the upper troposphere/lower stratosphere can result in large cold biases at these levels and unrealistically strong stratospheric jets. With ozone predicted, the simulated zonal mean circulation is much improved. We updated the calculation of absorption cross-sections for water vapor and ozone Again, the results of several simulations for the Northern Hemisphere winter show significant improvements. We also incorporated the effects of vertical mixing of momentum by dry-convective adjustment processes and allowed for smaller scales to be retained in the depth of the PBL predicted by the AGCM. The results of several simulations for the northern winter show that these model changes have a relatively minor impact on the troposphere, but a substantial effect on the stratosphere.

We have made significant progress in the development of a model based on a generalized vertical coordinate, which can be nearly identical to the Charney-Phillips grid sigma-coordinate model near the lower boundary and the standard theta-coordinate model away from the boundary with a smooth transition between the two regions. Two versions of the model are available. One uses a mid-latitude channel on the beta-plane; the other has a global domain. We are using the beta-plane version with high horizontal and vertical resolutions to simulate the details of the nonlinear evolution of extratropical cyclones with particular emphasis on the associated frontogenesis. Our results show that the model captures the details of extratropical cyclone development without serious computational difficulties. Similar simulations have been performed with the spherical version of the model, also with highly encouraging results.

\section{CHAMMP PROGRAM DIRECTION BADER, DAVID

Keywords: program administration

Objectives: To build a state-of-the-science global climate prediction program that makes use of the best available computing technology.

Approach: A distributed model development and climate research program has been developed that makes the best use of the talent and capabilities found in the national laboratories, the academic community, and other research institutions. The end goal is to use the potential offered technologically by the next generation of massively parallel scientific supercomputing platforms and the breakthroughs in scientific understanding to produce accurate, verifiable predictions of climate variability and climate change on 10to 100 -year time scales.

Results to Date: No results were reported.

\section{EXPLOITATION OF PARALLELISM IN CLIMATE MODELS \\ BAER, FERDINAND \\ UNIVERSITY OF MARYLAND \\ FY 1996299 \\ FY 1995275 \\ FY 19940}

Keywords: massively parallel computing, subgridscale parameterizations, atmospheric models

Objectives: To reconfigure atmospheric-model prediction equations to run on massively parallel (MPP) computer systems.

Approach: Atmospheric-model prediction equations will be reconfigured to compress the time-iteration procedure for massively parallel computer systems; to develop scale selective subgrid-scale parameterizations for atmospheric models; and to explore the possibilities of doing an ensemble of climate-prediction runs at one time on a massively parallel computer system.

Results to Date: No results were reported.

\section{STUDIES OF OCEAN PREDICTABILITY AT DECADE TO CENTURY TIME SCALES WITH GLOBAL OCEAN GENERAL CIRCULATION MODELS \\ BARNETT, TIM \\ SCRIPPS INSTITUTION OF OCEANOGRAPHY FY 1996149 \\ FY 1995144 \\ FY 1994150}

Keywords: climate variability, ocean general circulation modeling

Objectives: To determine the space and time scales of natural oceanic variability; characterize the physical mochanisms responsible for the variability; determine the relative importance of heat, fresh water, and momentum fluxes on the variability; and explore the predictability of North Pacific decadal variability and its effect on North American climate.

Approach: Complete, high-resolution, ocean general circulation models (OGCMs) coupled to simplified physical and statistical atmospheric models will be used to explore issues affecting the predictability of the oceanic El Niño/Southern Oscillation and the North Pacific climate variability. These oceanic predictions 
will be applied as boundary conditions to high-resolution atmospheric GCMs to determine the effect of this variability on the climate of North America.

Results to Date: A complete coupled ocean/atmosphere GCM produced variability in the North Pacific in much the same way as is found in observations and with similar effects on wintertime temperature and precipitation over the United States. We constructed a simplified, statistical, atmospheric model based on this full coupled run, and it appears to capture important aspects of this decadal-scale variability in the North Pacific. We are currently attempting a series of "hindcast" runs to see if the model would have been able to predict the shift in North Pacific climate observed during the late $70 \mathrm{~s}$ and the corresponding effects on U.S. climate.

\section{PARALLEL METHODS FOR CLIMATE MODELS BARNETTE, DANIEL SANDIA NATIONAL LABORATORIES \\ FY 1996149 \\ FY 1995150 \\ FY 199450}

Keywords: computational fluid dynamics, ocean models

Objectives: To evaluate methods of computational fluid dynamics developed for other fields for their usefulness in ocean modeling.

Results to Date: No results were reported.

\section{DEVELOPMENT AND EVALUATION OF A GLOBAL VERSION OF THE MIAMI ISOPYCNIC-COORDINATE OCEAN MODEL BLECK, RAINER, and ROOTH, CLAES UNIVERSITY OF MIAMI \\ FY 1996156 \\ FY $1995 \quad 0$ \\ FY 1994151}

Keywords: global ocean modeling, isopycnic coordinates, climate dynamics

Objectives: To test the ability of the Miami Isopycnic-Coordinate Ocean Model (MICOM) to simulate the global ocean circulation, setting the stage for the model's incorporation into coupled global climate models.

Approach: An existing basin-scale model will be expanded to global domain; suitable atmospheric forcing fields, including precipitation and river runoff, will be selected; the modeling of abyssal flow will be improved by incorporating compressibility and particularly thermobaric effects; a sea-ice model will be added; parameterization options will be explored for subgrid-scale deep convection; parallel coarse- and fine-mesh simulations will be carried out to investigate the impact of grid resolution; the sensitivity of the model's solution to magnitude of vertical (diapycnal) exchange coefficient will be studied; and long-term trends in meridional heat transport and water-mass properties in model solutions will be documented and interpreted.

Results to Date: Preparations for global runs were completed in late summer 1995. Parallel eddy-resolving and coarse-mesh simulations (mesh sizes $\mathbf{0 . 3 5}$ degree and 1.4 degree longitude on a Mercator map, respectively) with identical forcing functions were carried out for $>50$ and $>400$ years, respectively. These runs were succeeded by coarse-mesh twin experiments with different values for the vertical-exchange coefficient. Despite the fact that the North Atlantic is truncated (the grid domain ends at $65^{\circ} \mathrm{N}$ ), all four experiments carried out to date maintain the Atlantic overturning circulation ("conveyor belt") that makes the Atlantic up to $5^{\circ} \mathrm{C}$ warmer than the Pacific. With the atmosphere locked into a fixed annual cycle, there is no indication of the interannual to multidecadal oscillations often observed in model simulations that use restoration boundary conditions at the sea surface. Overall, MICOM performs as expected. If outfitted with a sea-ice component, MICOM would now be ready for use in coupled climate simulations.

\section{VERIFICATION OF THE GENT- MCWILLIAMS MESOSCALE EDDY PARAMETERIZATION BRYAN, KIRK PRINCETON UNIVERSITY \\ FY 199681 \\ FY 199577 \\ FY 199488}

Keywords: climate, ocean modeling, mesoscale eddies Objectives: To improve ocean climate models.

Approach: The results of the high-resolution Los Alamos ocean-circulation model will be used to determine methods for improving low-resolution ocean climate models through simple parameterization of the effects of mesoscale eddies.

Results to Date: Because of the very great technical and logistical difficulties of making long-term field measurements in the ocean, few data are available on the details of the role of the mesoscale eddies in maintaining the mean density structure and determining the distribution of tracers in the ocean. The high-resolution ocean-circulation model developed at Los Alamos National Laboratory offers a special opportunity to test theories on the interaction of time-dependent flow with the time-averaged ocean circulation. The approach of this collaborative study has been to examine proposed closure schemes for representing eddies in terms of time-averaged variables. The motivation of the closure schemes is to represent the effect of mesoscale eddies in low-resolution ocean climate models. The time- 
averaged model data indicate that there are real difficulties with most of the mesoscale eddies proposed in the oceanographic literature. A recent proposal based on the Andrews-McIntyre equations, which attempts to extend the Gent-McWilliams formulation, requires variations of the vertical density gradient in the thermocline to be small compared to the mean vertical density gradient. The Los Alamos POP solutions show that this assumption does not hold in energetic-mesoscale-eddy areas. The Gent-McWilliams formulation appears to be incomplete in several respects: (1) It is a purely local theory, and nonlocal effects appear to be quite important in transporting mesoscale energy from generation regions to decay regions. (2) Its representation of tracer transport is limited to advection by a bolus velocity down the thickness gradient or a diffusion along isopycnal surfaces. The POP-model results show that there is also a vigorous bolus component along isopycnal surfaces perpendicular to the thickness gradient. This component could be very important for tracers, whose fields are not alligned with density.

Comparison with the POP results indicate that the most consistent approach is that of DeSoeke-Bennett, in which all variables are averaged on instantaneous isopycnals with thickness weighting. This approach gives a uniform treatment of the density equation, the tracer equations, and the equations of motion. A closure approximation must then be inserted in the equations of motion to ensure that the bolus velocities both parallel and perpendicular to the thickness gradient are represented. The analysis suggests both a local closure and a possible nonlocal closure in which eddy-energy source and sink regions do not completely coincide.

\section{A HIERARCHY OF THERMOHALINE CIRCULATION MODELS \\ CESSI, PAOLA, and YOUNG, WILLIAM SCRIPPS INSTITUTION OF OCEANOGRAPHY FY 19960 \\ FY 1995121 \\ FY 1994111}

Keywords: thermohaline circulation, convection, mixed-layer dynamics

Objectives: To understand the variability caused by the competitive roles of salt and heat in the ocean circulation and to improve the parametrization of convection in models of the ocean circulation.

Approach: A hierarchy of models will be developed that isolate essential physical mechanisms. One level is box models, which are suited to study the statistical properties of stochastically forced thermohaline flows. The second level is a line of diffusively coupled grid points, which are suited to study the spatial interaction of temporal oscillations. The third level is asymptoti- cally reduced equations, valid in a distinguished range of the controlling parameters.

Results to Date: The characteristics of the quasiperiodic centennial and millenial oscillations found in OGCMs, associated with alternating suppression and activation of high-latitude convection, are extremely sensitive to the salinity flux and specific choice of convective adjustment scheme. In particular, the period of the oscillation depends crucially on the salinity fluxes and can be arbitrarily long.

The convection-adjustment schemes currently used in OGCMs lead to grid-scale instabilities that affect the large-scale distribution of properties. The gridscale instability is not a "computational mode," and convective-adjustment algorithms achieve the desired parametrization (i.e., suppress the grid-scale instability) only when coupled with poor accuracy, which is a step in the wrong direction, given the ongoing progress in computational technology.

A systematic relation between the strength and thickness of thermohaline fronts leads to temperature and salinity correlations such that buoyancy gradients are partially compensated for by the antagonistic contributions of heat and salt gradients.

SCIENTIFIC DEVELOPMENT OF A
MASSIVELY PARALLEL OCEAN
CLIMATE MODEL
CHERVIN, ROBERT
NATIONAL CENTER FOR ATMOSPHERIC
RESEARCH
FY 1996205
FY 19950
FY 1994202

Keywords: ocean modeling, parallel computing, climate simulation

Objectives: To understand the influence of wind forcing on global ocean circulation and on the transport of heat and fresh water.

Approach: The archived data from a series of four decadal experiments completed with the 1/2-degree version of the Parallel Ocean Climate Model (POCM) are being comprehensively analyzed to understand the influence of wind forcing on global ocean circulation and its associated climatically important transports of heat and fresh water.

A detailed analysis of the freon tracer experiments with the Parallel Ocean Program (POP) is being. Additional experiments are being planned by Chervin, Craig, and their PMEL collaborators.

Results to Date: The 512 PE Cray T3D at the Pittsburgh Supercomputing Center was used to investigate the issue of scalability of POP with respect to both number of processors and model resolution. Several performance bottlenecks were identified and corrected. We were then able to consider doing integrations with 
a 2/3-degree (on average), displaced-pole, global version of POP instead of only the 4/3-degree (on average) version. The standard 2/3-degree grid ( $384 \times 256 \times 32)$ was modified (i.e., to $384 \times 288 \times 32$ ) to include increased latitudinal resolution near the equator to resolve the strong tropical current systems. Also, because of the displaced pole, there is high horizontal resolution in the eastern North Pacific, in the Arctic Straits near northern Canada and Greenland, and in the Gulf Stream area. This modified version allowed for a very realistic representation of the continents and bottom topography to obtain correct volume transport flow in many important regions of the global ocean. We also implemented a highly scale-selective biharmonic (i.e., del fourth) spatially varying horizontal viscosity and diffusivity instead of the more traditional Laplacian (i.e., del squared) formulation which permitted a doubling of the time step. This version of POP has been integrated for approximately 25 years, and the results are quite promising. Further experimentation with this $2 / 3$-degree version of POP is ongoing with the expectation of its eventual inclusion in a new CHAMMP-based coupled model. The uncoupled performance of this component model is being evaluated, improved, and optimized.

The various versions of POP that have evolved over the past several years are being collected and organized. A consolidated version of the model, featuring all the latest and tested developments, should result from this effort.

The interpolation schemes being developed by Phillip Jones at LANL are being carefully evaluated. These schemes are critical for communication of component-model information through the flux coupler and also for the analysis of the performance of all versions of POP that feature generalized curvilinear coordinates and a displaced pole.

\section{CLIMATE-SYSTEM MODELING \\ DANNEVIK, WILLIAM, and MIRIN, ARTHUR LAWRENCE LIVERMORE NATIONAL LABORATORY \\ FY 1996447 \\ FY 1995450 \\ FY 1994436}

Keywords: general circulation models, massively parallel computing, coupled climate models

Objectives: To develop and apply an advanced generation of climate models for high-performance computing systems.

Approach: Parallelism is achieved through both domain decomposition and model concurrency. Explicit message-passing is used to accomplish both data transfer and process synchronization. Both machine transportability and architecture-dependent optimization are achieved by way of libraries and conditional compilation. Our primary computer platforms are the Cray T3D machines at LLNL and LANL, but we have also been using the IBM SP2, Meiko CS2, and Intel Paragon. We are evaluating how well our numerical procedures and algorithms scale to systems having very large numbers of processors and how well the computational load is distributed among the processing elements.

Results to Date: Multiple 10-year Atmospheric Model Intercomparison Program (AMIP) integrations of the atmospheric code have been completed with 64 processors of the Cray T3D. An ensemble of 20 realizations has been carried out, and the results have been analyzed.

Finite-width approximations to the global highlatitude filters in the AGCM have been developed, implemented, and validated; the workload of the filters has been repartitioned among all the processors of a massively parallel computing system.

The atmospheric code has been optimized to the extent that it executes on both the Cray T3D (256 PE) and the IBM-SP2 (170 PE) as least as fast as an efficient multitasked code would on a 16-processor Cray C90.

A free-surface model has been installed and validated in the ocean code with an explicit finitedifference approach.

The atmosphere, oceans, and sea ice have been coupled within our parallel climate-system-modeling framework. Our framework design is sufficiently flexible that other component models of the atmosphere and oceans can be substituted. We have initiated exploratory coupled calculations designed to characterize climate drift.

\section{PARAMETERIZATION OF THE EFFECTS OF UNRESOLVED MESOSCALE EDDIES IN GLOBAL OCEAN-CIRCULATION MODELS DANNEVIK, WILLIAM LAWRENCE LIVERMORE NATIONAL LABORATORY \\ FY 1996149 \\ FY 1995135 \\ FY 19940}

Keywords: ocean turbulence, subgrid-scale parameterization, mesoscale eddies, direct numerical simulation, ocean general circulation models

Objectives: To develop physics-based subgrid-scale parameterizations of mesoscale-eddy effects for use in ocean general circulation models.

Approach: Statistical two-point closure approximations of turbulence are being used in conjunction with eddy-resolving direct numerical simulations to develop physics-based subgrid-scale parameterizations of mesoscale-eddy effects that will improve the fidelity of global-ocean-circulation simulations with horizontal 
resolutions of order 1 degree $x 1$ degree. The statistical closures, based on the direct interaction approximation (DIA) and the Lagrangian renormalized approximation (LRA), provide prognostic evolution equations for the two-point, two-time correlations of the subgridscale fluctuations. The solution of these equations yields scaling relations for the spectra and transport properties as a function of the resolved dynamic and thermodynamic fields. Analytical expressions for effective eddy-damping and stochastic backscatter terms will be derived and tested with the numerical simulations. Subsequently, the scaling relations obtained from the solutions of the closure equations and the analytical expressions for the damping and backscatter terms will be used to formulate subgridscale parameterizations, which will be implemented in full ocean general circulation models used by CHAMMP for coupled-ocean-atmosphere climate simulations. The performance of the new parameterization will be tested against the anticipated-potentialvorticity method proposed by Basdevant and Sadourny and the Gent-McWilliams tracer-mixing parameterization .

Results to Date: Modular codes have been developed and tested to solve the DIA equation corresponding to a set of quadratically nonlinear primitive evolution equations with arbitrary forcing and dissipation and to execute direct numerical simulations with the same primitive equations.

Diagnostics have been developed to assess the predictions of the closure code compared to the direct simulations, and studies have been completed to ensure that each code is consistent with fundamental properties of the evolution equations (e.g., energy and entropy conservation by nonlinear terms, relaxation to inviscid equilibrium states, etc.).

Preliminary experiments have been performed on a prototypical two-layer quasigeostrophic problem in which mesoscale eddies are driven by a prescribed resolved-scale field. (This problem embodies the salient features of mesoscale-eddy generation, baroclinic-barotropic energy conversion, and dissipation.)

To economize the DIA and LRA calculations for cases in which baroclinicity introduces extended equilibration time scales, an option has been added to the closure code to implement an approximatecumulant-update procedure; preliminary experiments have been completed to assess the fidelity of this approximation.

\section{DEVELOPMENT AND EVALUATION OF A GLOBAL VERSION OF THE MLAMI \\ ISOPYCNIC-COORDINATE OCEAN MODEL DEAN, SUMNER, and BLECK, RAINER \\ LOS ALAMOS NATIONAL LABORATORY and UNIVERSITY OF MIAMI \\ FY 1996149 \\ FY 1995149 \\ FY 19940}

Keywords: isopycnic ocean modeling, MICOM, parallel computing, climate simulation

Objectives: To develop a practical, high-resolution version of the University of the Miami Isopycnic Coordinate Ocean Model (MICOM) that is suitable for global climate studies.

Approach: The model will be improved by creating a global version of the University of Miami Isopycnic Coordinate Ocean Model (MICOM), improving the numerical algorithms, and implementing new physical parameterizations. Specific tasks dealing with model numerics are globalization, improvement of the barotropic/baroclinic mode decomposition, improvement of the time-step restriction, improved efficiency $r$ massively parallel computers, change to the B grid, and testing of a two-time-level time-integration scheme. Specific tasks dealing with model physics are optimization of the potential-density coordinate for deep ocean basins, subgrid-scale parameterization of penetrative convection, implementation of mixed boundary conditions, improvement of the mixed-layer model, and incorporation of an ice model.

The model will be used to assess the effectiveness of this isopycnic model on Thinking Machine's CM-5 and other massively parallel platforms for the purpose of running long-term and high-resolution simulations of the world ocean circulation. Specific tasks include comparing MICOM with other (z-coordinate) models, making high-resolution runs to study the effect of eddies, comparing explicit and implicit integrations of the barotropic processes in the model, and exploring the sensitivity of the meridional heat transport to the parameterization of deep convection.

Results to Date: A data-parallel global version of MICOM is nunning on the CM-5 at Los Alamos. Two simulations that use identical forcing functions but different mesh sizes $\left(0.35^{\circ} \mathrm{vs} 1.4^{\circ}\right)$ are being run. They have completed ocean circulation simulations of more than 30 and 300 years, respectively. The first 15 years of the $0.35^{\circ}$ and $1.4^{\circ}$ simulations show that the poleward heat transport is 5 to $10 \%$ higher in the finemesh simulation. This figure agrees with results obtained in previous single-basin experiments.

The MiCOM model has been ported to several parallel computer architectures, improvements have been made to the treatment of mixed-layer detrainment, and the diapycnic mixing was totally rewritten. 


\section{FINE-MESH TREATMENT OF THE LAND COMPONENT OF A GLOBAL CLIMATE MODEL \\ DICKINSON, ROBERT E. \\ UNIVERSITY OF ARIZONA \\ FY 1996192 \\ FY 1995195 \\ FY 1994181}

Keywords: GCMs, land-surface model, BATS, Biosphere-Atmosphere Transfer Scheme, fine-mesh atmospheric parameterizations, precipitation statistics Objectives: To refine an approach to spatial heterogeneity of land surfaces in a global climate model by dividing each CCM (Community Climate Model) mesh square over land into a submesh, providing a fine-enough division to allow representation of the effects of the most important heterogeneities on regional climate.

Approach: Fine-mesh data structures will be developed in the VBATS [Vectorized Biosphere Atmosphere Transfer Scheme] model and its link to CCM. The BATS model parameterization will be improved. The fine-mesh atmospheric parameterizations will be completed and refined. And sensitivity studies will be conducted. A version of the Climate System Model named CCM3 has been released by NCAR and has replaced CCM2 in our research. A vectorized version of BATS (VBATS) incorporating the parameterization modifications described above and combined with CCM3 will be used to evaluate improvements. In addition, a semi-Lagrangian version of CCM that should provide gains in computational efficiency is being jointly developed at the University of Arizona and NCAR.

Results to Date: BATS Version le has been upgraded to current programming standards and made available to users along with supporting documentation and data via anonymous FTP and the Web. A vectorized version of BATS (VBATS) has also been made available along with a user's guide. VBATS has been linked with CCM3 and is running on an eight-processor SGI machine at the University of Arizona. We have put together surface-boundary data sets (soil color, texture, and vegetation) for use with BATS at high resolution. A mapping of a high-resolution mesh over a T42 CCM3 grid resolution is more than halfway complete.

Hourly (area-averaged) precipitation intensity, frequency, and amount from the NCAR CCM2/BATS has been compared with (locally) observed rain-gauge data over the continental United States to demonstrate that the use of GCM output to directly represent local values of intensities and frequencies may lead to large errors in air-land coupling. A simple regression equation based on model output and observations has been developed to adjust GCM precipitation intensity for hydrologic studies. A consistent treatment of fractional precipitation cover has been incorporated into BATS, and sensitivity tests have been performed with various field experimental data and climate simulations.

\section{NETWORK INTERFACE FOR CHAMMP DRAKE, JOHN, and NACHTIGAL, NOEL OAK RIDGE NATIONAL LABORATORY FY 199698 FY $1995 \quad 50$ FY 1994124}

Keywords: World Wide Web, home pages Objectives: To maintain and improve an electronic, World Wide Web network interface supporting the exchange of CHAMMP computer codes and providing an electronic repository for online technical documents and program information.

Approach: Taking advantage of the emerging network information technologies, ORNL has installed and will maintain a WWW server containing CHAMMP program data, computer codes, and electronic documents. A home page will be accessible with Netscapeclass web browsers. Preparation of documents for online viewing will be supported on a limited basis.

Results to Date: The CHAMMP program home page has been established and updated periodically during the past year. News items and interesting research results from the CHAMMP Science Team have been highlighted. Codes developed by the CHAMMP Development Effort have also been made available with the web interface. FTP services have served for the transferring of large files between sites supporting CHAMMP collaborations. Usage statistics indicate that several hundred visits are made to the site each month.

\section{PARALLEL ATMOSPHERIC CLIMATE MODEL \\ DRAKE, JOHN B.; WORLEY, PAT; and SEMERARO, DAVE \\ OAK RIDGE NATIONAL LABORATORY \\ FY 1996500 \\ FY 1995500 \\ FY 1994776}

Keywords: climate modeling, par ' computing, parallel algorithms

Objectives: To provide state-of-the climate models on massively paritlei computing platforms; to produce the required algorithm research for efficient use of parallel computers by future highresolution global general circulation models; and to develop advanced climate models that couple atmospheric and other components of a climate-simulation system.

Approach: An interdisciplinary, interagency collaboration involving researchers from the National Center for Atmospheric Research, Oak Ridge, and Argonne National Laboratories is actively working toward the 
stated objectives. The parallel atmospheric-GCM model, PCCM2, based on the NCAR Community Climate Model, has been implemented on the target systems for use in parallel climate simulations. Improvements to the physics, resulting in an NCAR CCM3 and CCM4, will be implemented on the parallel platforms. Extending the models for coupled oceanatmosphere-ice simulations will be accomplished in conjunction with the CHAMMP Model Development Team based on the LANL POP code and the NCAR Climate System Model.

Results to Date: New numerical methods for dynamics and novel parallel algorithms for climate computations are being tested as candidates for inclusion in the climate model with the nonlinear shallow-water equations on the sphere. A variety of numerical methods are being investigated with regard to numerical accuracy and efficiency for dynamics calculations as well as parallel performance and suitability for massively parallel computation.

The performance of the parallel codes on a variety of supercomputing platforms has been summarized in a recent special issue of the journal Parallel Computing. On the Intel Paragon XPS150 at ORNL, a peak sustained computational rate of 5 Gflops has been achieved. The model has been used for a study of highresolution (T170) model climate with the ORNL Paragon. This work was performed in collaboration with Argonne and NCAR.

With researchers at NCAR, a new method for discretizing partial differential equations on the sphere has been developed. The method solves the "pole problem" by working with 3-D Cartesian coordinates and projected differential operators on a set of spherical harmonic basis functions. The method was applied to the shallow-water-equations test suite and presented at the Breckenridge PDE on the Sphere meeting.

In support of the development of coupled atmospheric and oceanic models, the LANL/GFDL Parallel Ocean Program has been optimized for MIMDmessage-passing computers. It is being used in the implementation of a coupled-climate-system model in support of CHAMMP simulations.

\section{VARIATIONAL OPTIMIZATION OF SUBGRID-SCALE CONVECTION EMANUEL, KERRY

Keywords: cumulus convection, water vapor, singlecolumn models, subgrid-scale convection

Objectives: To optimize a representation of cumulus convection with field experimental data.
Approach: The adjoint of the linear tangent of a single-column model and, independently, a standard optimization code will be used to find the best set of parameters in a convection scheme.

Results to Date: The single-column model was written and tested. Both the standard and the linear-adjoint optimization codes were written and tested. A preliminary optimum parameter set has been found with 120 days of Inner Flux Array data from the TOGA COARE data. The single-column-model prediction of relative humidity, as a function of time and altitude, is excellent.

\section{ROLE OF BAROCLINIC-WAVE AMPLITUDE AND TRANSPORT VARIATION IN CLIMATE CHANGE \\ FARRELL, BRIAN \\ HARVARD UNIVERSITY \\ FY 199652 \\ FY 199553 \\ FY 199453}

Keywords: baroclinic waves

Objectives: To understand the statistical sensitivity to climate perturbation of atmospheric-baroclinic-wave variance to properly assess the radiatively mediated feedbacks and to obtain an understanding of the physical factors controlling the strength and distribution of synoptic and planetary-scale baroclinic waves in the atmosphere.

Results to Date: No results were reported.

PARALLEL CLIMATE MODELING FOSTER, IAN, and ANDERSON, JOHN R. ARGONNE NATIONAL LABORATORY and UNIVERSITY OF WISCONSIN

FY 1996546

FY 1995534

FY 1994545

Keywords: parallel computing, spectral transport, semi-Lagrangian transport, coupled climate model, low-frequency variability

Objectives: (1) To improve parallel algorithms used in climate models by developing parallel algorithms for reduced-grid and semi-Lagrangian transport methods, developing testbed codes, and analyzing performance and scalability; (2) to develop PCCM2, a parallel community climate model based on the NCAR Community Climate Model, thus providing one component for coupled advanced climate models; and (3) to develop and demonstrate a dynamic, coupled, oceanatmosphere model and use the model to perform very long $(10,000$-year) simulations of the low-frequency variability of the climate system.

Approach: We are combining theoretical and empirical investigations of parallel algorithms with development activities designed to transfer promising results 
to PCCM2. New versions of the shallow-water testbed are being developed to explore decomposition, performance, and scaling of semi-Lagrangian transport and of reduced-grid spectral transport. The latter has the potential to reduce the number of points in physical space by $35 \%$, compared with a nonisotropic grid, providing computational savings in the Fourier transforms and also in physics computations (once physics is added in a full-climate code). Taking advantage of isotropism, reduced-physical grid transport schemes will also provide advantages for domain-decomposed parallel semi-Lagrangian transport schemes because trajectories will not span as many grid cells near the poles.

Results to Date: The coupled ocean-atmosphere project combines the results of two previous CHAMMP-funded projects aimed at very long climate integrations. One is a collaborative effort among Argonne National Laboratory, Oak Ridge National Laboratory, and NCAR to develop a scalable parallel implementation of PCCM2 for the Intel Paragon, IBM SP2, and other large, distributed-memory, parallel platforms. PCCM2 is a 2-D domain-decomposed version of the spectral/semi-Lagrangian NCAR Community Climate Model. The other is a project at the University of Wisconsin to implement an ocean model for similar platforms that provides for highly efficient ocean-dynamics integration. These two models have been incorporated into a single system, with simple models for sea-ice formation, land-surface hydrology, and river runoff being added to form a closed hydrologic cycle. Model stability for simulations with durations in excess of a modeled century has been verified. The model will be run for long durations, and statistical properties of the resulting system will be examined. An immediate goal is to identify physically plausible mechanisms of natural climate variability on time scales of decades to centuries. These statistics will be used as baseline calculations to assist in the implementation of ocean models forced by statistical representations of atmospheric dynamics, allowing for integrations of ocean climate of still longer durations.

A range of new spectral-transform algorithms have been designed, and a comprehensive comparison of the best algorithms has been completed, indicating regimes in which each was superior. A testbed code, PSTSWM, that incorporates these algorithms has been made available to the community.

PCCM2 is operational on Intel Paragon and IBM SP multicomputers. The PCCM2 code and also the parallel shallow-water testbed, PSTSWM, are available from http://www.epm.ornl.gov/chammp.

The initial development of the coupled oceanatmosphere model system has been completed. An initial integration of 150 years duration has been performed and analyzed. On the basis of the results of this calculation, a more complete representation and a new parameterization of vertical mixing in the ocean have been implemented and included. Preliminary results with the modified model show fidelity to the real-world climate comparable to that obtained from pure atmospheric models of comparable resolution. The resulting model is believed to be suitable for very long integrations of the climate system, which will begin shortly.

MODELING THE PAN-ARCTIC
TERRESTRIAL AND ATMOSPHERIC
WATER CYCLE AND POTENTIAL
CONNECTIONS TO SEA-ICE AND
DEEP-WATER FORMATION
GUTOWSKI, WILLIAM; VOROSMARTY,
CHARLES; and PERSON, MARK
IOWA STATE UNIVERSITY,
UNIVERSITY OF NEW HAMPSHIRE, and
UNIVERSITY OF MINNESOTA
FY 1996150
FY 19950
FY $1994 \quad 0$

Keywords: hydrologic cycle, land-surface characterization

Objectives: To model the coupled terrestrial-atmospheric hydrologic cycle of Arctic and neighboring land masses, including their discharge of fresh water into the Arctic Ocean, for the purposes of assessing the impact of pan-Arctic hydrology on global climate. Approach: We will build on existing modeling and collaborative experience to construct a regional model of the Arctic hydrologic cycle. Initially, the model will contain the terrestrial and atmospheric branches of the cycle, and it will feed its output to an Arctic sea-ice and ocean-circulation model being nun by other CHAMMP investigators. Eventually, we will work toward coupling the land, atmosphere, and ocean models into one unit for a more complete assessment of Arctic-hydrologic-cycle feedbacks that can affect global-climate variability.

Results to Date: This project began late in FY96; there are no results as yet.

\section{PARALLEL CCM DEVELOPMENT \\ HACK, JAMES; WILLIAMSON, DAVID; KIEHL, JEFF; and ALBRECHT, BRUCE \\ NATIONAL CENTER FOR ATMOSPHERIC RESEARCH and THE PENNSYLVANIA STATE UNIVERSITY \\ FY 1996271 \\ FY 1995475 \\ FY 1994172}

Keywords: CCM, parameterizations, radiative transfer Objectives: To improve computational and numerical aspects of the NCAR CCM for execution on massively 
parallel scientific supercomputers in collaboration with other CHAMMP institutions and to improve CCM parameterizations for clouds and atmospheric radiative transfer in collaboration with ARM investigators.

Results to Date: No results were reported.

$$
\begin{aligned}
& \text { INTERNATIONAL FORUM ON } \\
& \text { FORECASTING EL NIÑO } \\
& \text { HALL, MICHAEL J. } \\
& \text { NATIONAL OCEANIC AND ATMOSPHERIC } \\
& \text { ADMINISTRATION } \\
& \text { FY } 199650 \\
& \text { FY } 19950 \\
& \text { FY } 19940
\end{aligned}
$$

Keywords: workshops

Objectives: To hold a workshop on developing seasonal-to-interannual operational climate predictions and the potential benefits of climate forecasts for health, agriculture, planning, and other needs.

Approach: An international forum will be held to discuss the opportunities to develop seasonal-tointerannual operational climate predictions and the potential benefits of climate forecasts for health, agriculture, planning, and other needs.

Results to Date: The international forum was held in November 1995 in Washington, D.C. It was hosted by the agencies participating in the U.S. Global Change Research Program.

$$
\begin{aligned}
& \text { MIXED-LAYER PROCESSES AND } \\
& \text { PARAMETERIZATIONS IN } \\
& \text { HIGH-RESOLUTION OCEAN MODELS } \\
& \text { JENSEN, TOMMY, and RANDALL, DAVID } \\
& \text { COLORADO STATE UNIVERSITY } \\
& \text { FY } 199680 \\
& \text { FY } 19950 \\
& \text { FY } 19940
\end{aligned}
$$

Keywords: oceanic mixed layer, ocean models, parameterizations

Objectives: To investigate various mixed-layer formulations in an arbitrary Lagrangian-Eulerian ocean model and implement a bulk-mixed-layer formulation in the Los Alamos POP ocean model.

Approach: A general arbitrary Lagrangian-Eulerian ocean model is used to investigate bulk-mixed-layer formulations and their impact on sea-surface temperature (SST). This is done with basin models of a tropical ocean and a polar ocean. The most successful mixed-layer formulation will be implemented as a module for the Los Alamos Parallel Ocean Program (POP) ocean model.

Results to Date: No results were reported.

\author{
MODELING OF HYDROLOGIC AND \\ TRANSPORT PROCESSES IN RELATION TO \\ CLIMATE CHANGE \\ JOHNSON, DONALD \\ UNIVERSITY OF WISCONSIN \\ FY 1996149 \\ FY $1995 \quad 0$ \\ FY 1994109
}

Keywords: isentropic-sigma coordinate model, numerical model, climate model, long-range transport, hydrologic cycle, water vapor, clouds

Objectives: To advance the modeling and understanding of atmospheric processes involving water substances and trace constituents in relation to climate change and to examine diagnostically the limits of global and regional climate predictability involved with the simulation of transport processes and the hydrologic cycle.

Approach: The University of Wisconsin (UW) global hybrid isentropic-sigma (I-S) coordinate model has been developed to examine the attributes of isentropic coordinates in simulating long-range transport and the atmosphere's hydrologic cycle. Apart from a sigmacoordinate planetary boundary layer (PBL) $150-\mathrm{mb}$ thick, the model uses isentropic (entropy) coordinates to represent the free atmosphere, including the troposphere and stratosphere. Emphasis is placed on validating the accuracy of the long-range transport of water vapor, clouds, and trace constituents. Because long-range transport occurs primarily through isentropic processes, the large-scale exchange of water vapor and trace constituents within baroclinic phenomena remains two-dimensional when modeled in isentropic coordinates as opposed to 3-D coordinates in sigma coordinates. Relative accuracies of predicted long-range transport have been determined from comparisons of simulations among the UW I-S and sigma models and the National Center for Atmospheric Research (NCAR) Community Climate Model 2 (CCM2).

Results to Date: Four validation experiments testing conservation of trace constituents and cloud production were completed. The first examined the models' abilities to transport an inert trace constituent and conserve the initial maxima. The second examined predictability by a comparison of the joint distributions of isentropic potential vorticity (IPV) and a trace constituent related to the initial IPV. The third compared equivalent potential temperature and a trace constituent related to the initial equivalent potential temperature. The fourth examined the ability of the models to generate and transport clouds. In all of these experiments, the UW I-S model simulations were superior to the other tested models: trace-constituent conservation was higher in the first experiment, higher joint distribution correlations occurred in the second 
and third experiments, and the UW I-S model produced more clouds and improved their spatial pattern in the fourth experiment. A final set of experiments initiated was a series of 4.5-month-long integrations with the UW I-S model. Seasonal December-JanuaryFebruary and June-July-August diagnostics of synoptic variables, atmospheric heating, precipitable water, and precipitation minus evaporation all compared well to the National Center for Environmental Prediction/ NCAR reanalysis.

\section{COMPUTATIONAL SUPPORT FOR PARALLEL CLIMATE MODELING KLIEWER, KENNETH

Keywords: climate modeling, PCCM2, Center for Computational Sciences, Intel Paragon

Objectives: To make accurate, high-resolution, multiyear climate projections with a parallel version of the CCM2 code running on the 3072-processor Intel Paragon XP/S 150 located in the Center for Computational Sciences at ORNL.

Approach: A proper characterization of the climate requires complex and sophisticated mathematical models of the Earth's atmosphere and oceans, computer codes incorporating these models, and computers of sufficient power to run these codes. To achieve the desired temporal and spatial accuracy in climate descriptions and predictions requires the most powerful parallel computers now in existence. One such computer is the 3072-processor Intel Paragon XP/S 150 , the centerpiece of the Center for Computational Sciences (CCS) at ORNL, rated at $150 \mathrm{Gflops,} \mathrm{or} 150$ billion mathematical operations per second. CCS staff members have developed a parallel version of the NCAR-developed climate code named Community Climate Model 2 (CCM2) for the Paragon. This new code, PCCM2, is now being used in CHAMMP studies.

Results to Date: PCCM2 on the Paragon has been used to perform multiyear calculations of the Earth's climate at high resolution. Current efforts are directed toward producing a parallel version of the next-generation atmosphere code (CCM3) and then employing this code to investigate climate predictions for even longer periods. Another major effort is to achieve improved climate descriptions through tightly coupling PCCM2 (or PCCM3) to a code that models the oceans with comparable sophistication. This coupled system will be run on the huge Paragons at ORNL and Sandia National Laboratories linked via a very-high-speed network.

\author{
DYNAMIC HEAT AND MOISTURE \\ TRANSPORT AND BAROCLINIC \\ ADJUSTMENT \\ LINDZEN, RICHARD \\ MASSACHUSETTS INSTITUTE OF \\ TECHNOLOGY \\ FY 199643 \\ FY 199549 \\ FY 199444
}

Keywords: atmospheric-potential-vorticity mixing, climate feedbacks, water vapor

Objectives: To quantify the climatically significant interactions between the tropical and extratropical components of the atmospheric general circulation, to understand the implications of atmospheric-potentialvorticity mixing for climate, and to determine aspects of the climate feedbacks associated with atmospheric water vapor.

Results to Date: No results were reported.

CHAMMP MODEL DEVELOPMENT
MALONE, ROBERT
LOS ALAMOS NATIONAL LABORATORY
FY $1996 \quad 923$
FY $1995 \quad 1066$
FY $1994 \quad 973$

Keywords: atmospheric modeling, oceanic modeling, parallel computing, climate simulation

Objectives: To develop, optimize, validate, and couple atmospheric and oceanic general circulation models on massively parallel computers.

Approach: Work will continue on the development of climate models for massively parallel computers, in particular for the 1024-node CM5 and 256-processor T3D and future clustered-SMP computers at Los Alamos. The main focus is a collaborative effort between Los Alamos and the National Center for Atmospheric Research to couple the NCAR Community Climate Model (CCM3), an atmospheric model, to the Los Alamos Parallel Ocean Program (POP). Improvements in the representation of physical processes in POP will continue; a new sea-ice model is being developed for use with the coupled model. Our collaboration will continue with the University of Miami on development, optimization and application of the Miami Isopycnal-Coordinate Ocean Model (MICOM). MICOM will also be coupled with CCM3, to allow comparisons of Eulerian and isopycnic ocean models when coupled to atmospheric and sea-ice models. Oceanographic-research studies with POP and MICOM will continue, emphasizing high spatial resolution.

Results to Date: High-resolution simulations made with POP on the CM5 have been very successful. Remarkable agreement has been found between seasurface height variations simulated with POP and 
those observed by the TOPEX/Poseidon radar altimeter; comparisons with hydrographic data, such as data on the flow through the Drake Passage, have also been good. These agreements provide important validations of the model. The Gent-McWilliams isopycnic transport parameterization has been installed and tested with POP's general curvilinear coordinate system. A new, fully explicit, "elastic-plastic" sea-ice model that is much faster than previous formulations has been developed and is being validated. The MICOM ocean model has been further optimized on the CM5, and long high-resolution global simulations have been completed and are being analyzed. Output is also being analyzed from a very-high-resolution simulation recently completed on the CM5 with the parallel version of GFDL's SKYHI atmospheric model, jointly developed by GFDL and Los Alamos.

\section{ASSESSING PREDICTABILITY IN GLOBAL OCEAN MODELING MARGOLIN, LEN G. LOS ALAMOS NATIONAL LABORATORY FY 1996298 \\ FY 1995248 \\ FY 1994242}

Keywords: ocean modeling, predictability, climate simulation

Objectives: To assess the limits of predictability of ocean models, based on analysis of the equations that they approximate, and to help formulate more predictive models and algorithms.

Approach: We will apply the methods of nonlinear dynamics, including scale analysis and asymptotics, to analyze the principal balances of the governing equations at various levels of truncation. We will use nonlinear enslavement to build numerical algorithms that preserve these balances.

Results to Date: We have used our shallow-water models, one based on nonhydrostatic (Green-Naghdi) equations and the other on hydrostatic equations, to simulate an idealized ocean-basin circulation. The problem is the 1.5-layer wind-driven basin, with forcing chosen to develop a double-gyre flow. We ran each of the models for 100 years. We produced a time series of the basin-averaged potential and kinetic energies and used the maximum-entropy method and singular-spectrum analysis to compare them. We found systematic differences in the spectra at the lowfrequency end (i.e., for periods on the order of 10 years and more). These differences are comparable to those found when making large changes in the Rayleighfriction or Laplacian-viscosity coefficients.

During the past few years, we have developed a technique (which we call nonlinear enslavement) to improve the accuracy and efficiency of finite-difference simulations of partial differential equations (PDEs).
We demonstrated this technique in the context of various nonlinear dissipative PDEs, in one- and twospace dimensions. This year, we have extended the technique to problems closer to ocean modeling. In particular, we applied the technique to a shallow-swat s, model simulating the double-gyre flow desc:

above. This model used leapfrog time-differencing, which is commonly used in ocean models. The interesting new feature here is that dissipation is not part of the principal balance in these PDEs, and so we enslaved the small scales in terms of the rotation, instead. We were able to demonstrate that our enslaved model has the accuracy of the original model nun on a twicefiner mesh. The enslaved model further captures the dynamics of the twice-finer mesh that are not resolved on the coarse mesh of the original model. Details are reported in available publications.

\section{FORWARD-IN-TIME METHODS FOR GLOBAL CLIMATE MODELS MARGOLIN, LEN G. \\ LOS ALAMOS NATIONAL LABORATORY \\ FY 1996238 \\ FY 199573 \\ FY 1994252}

Keywords: atmospheric modeling, numerical methods, climate simulation

Objectives: To develop physically realistic and computationally efficient algorithms for atmospheric applications on climate time scales.

Approach: Representative atmospheric models based on nonoscillatory forward-in-time methods will be developed, tested, and validated in regimes of interest to climate simulation.

Results to Date: We began by constructing explicit models of shallow water on the sphere, one based on Eulerian techniques and the other on semi-Lagrangian. We applied these models to the standardized set of shallow-water test problems published by Williamson et al. and showed that our nonoscillatory, forward-intime models achieved comparable accuracy to more traditional leapfrog methods. We further developed semi-implicit versions of the shallow-water models in which the fastest waves of the system (the gravity waves) are treated implicitly, thus allowing a much larger computational time step. These implicit systems require the solution of a nonsymmetric matrix equation on the mesh, and the ultimate saving of CPU resources depends strongly on the efficiency of the elliptic solver. We constructed a solver based on conjugate residuals, which has proved satisfactory.

We have extended our techniques to 3-D models and implemented and tested a reduced-grid capability to further improve the computational efficiency. We have ported several of our models to the massively parallel CM-5. We have begun to implement water 
vapor processes, including a novel procedure for treating cloud boundaries. Finally, in collaboration with another CHAMMP project and an HPCCI project, we have generalized one of our shallow-water models to simulate the Green-Naghdi equations, a dispersive shallow-water theory.

We have also constructed a fully nonhydrostatic grid-point model, in Cartesian coordinates and on the sphere, and are testing its performance. The model consistently incorporates either Eulerian or semiLagrangian differencing, at the user's option. We have used this model as a vehicle to compare the advantages of Eulerian and semi-Lagrangian methods. At the present, it appears that each method has advantages for certain classes of problems and that neither is uniformly superior.

\section{STUDIES IN LONG-TERM NOISE STATISTICS, REGIONAL CLIMATE SENSITIVITY, AND PREDICTABILITY NORTH, GERALD, and KIM, K.-Y. TEXAS A\&M UNIVERSITY FY 1996171 \\ FY 19950 \\ FY 1994164}

Keywords: empirical orthogonal functions, energybalance models

Objectives: To derive EOFs from model control runs for use in climate-change estimation and detection formalisms.

Approach: Empirical orthogonal functions (EOFs) are an essential ingredient in the estimation and detection formalisms we developed. Unfortunately, we cannot estimate EOFs accurately because observational records are not long enough and are also contaminated by unwanted forced signals, such as the greenhouse warming trend and volcanic imprints. A logical alternative is to use EOFs derived from model control runs. But present coupled climate models cannot accurately simulate the second-moment statistics of the surface temperature field. Thus, it is important to support any detection and estimation statements with appropriate confidence statements.

Results to Date: We have derived a rigorous description of eigenvalue and eigenfunction errors, allowing us to examine the sensitivity of the EOF-based detection algorithms to the inaccuracy of eigenvalues and EOFs in terms of the signal-to-noise ratio. Specifically, the utility of the technique has been demonstrated in detecting a greenhouse warming signature.

We continued using our simple energy-balance model (EBM) to address some of climatic problems of concern to us. The sunspot-detection problem was revisited with an improved EBM. The results indicate that the discrimination of noisy and quiet months in background fluctuations helps to enhance the detectability of such a feeble signal. Different sources of error of the detection example were also examined.

The estimation problem was also revisited with the so-called cyclostationary EOFs, which allow one to account for monthly variation of the statistics of natural variability. It turned out that the cyclostationary estimator is more accurate than that based on the stationarity assumption, especially when the number of sampling points is small. The new estimator is also beneficial for temporal interpolation.

The role and significance of forced variability, specifically the insolation variation, were studied. It seems that the surface temperature variability caused by the insolation variation comprises a significant portion of the so-called "natural variability" in the decadal-to-centennial-scale band. Therefore, forced variability may have an important implication for climatic sensitivity as well as in detecting anthropogenic-greenhouse-warming signatures. Other forced variability produced by such forcings as volcanoes and aerosols are being examined.

\section{OCEAN-CONVECTION PARAMETERIZATION \\ PALUSZKIEWICZ, THERESA; SKYLLINGSTAD, ERIC; and HIBLER, LYLE PACIFIC NORTHWEST NATIONAL LABORATORY \\ FY 1996198 \\ FY 1995200 \\ FY 1994194}

Keywords: ocean physics, convective mixing, parameterizations for ocean GCMs

Objectives: To develop an ocean-convective-mixing parameterization for ocean general circulation models that incorporates realistic ocean physics.

Approach: A set of parameterizations for mixed-layer dynamics, sea-ice formation, penetrative convection, and deep overflow mixing will be developed and tested. The parameterizations will follow a nonlocal mixing algorithm based on a parcel approach. Parcel energy will be determined from buoyant potential energy and kinetic energy derived from surface heat flux, wind, and wave forcing. Tests of the parameterization will be made by comparing results from a 1-D column, representing a single ocean general circulation model (OGCM) grid point with average results from turbulence models and by performing comparisons between OGCM simulations with existing mixing parameterizations and those using the improved algorithm. OGCM experiments will use the Coupled Arctic Program (CAP) developed by W. Maslowski, Y. Zhang, and $A$. Semtner in both vector-parallel and data-parallel configurations.

Results to Date: Results from tests of the convective parameterization in an early version of the Parallel 
Ocean Circulation Model indicate that the convective parameterization can have a significant impact on the temperature and salinity profiles in Arctic seas. The upcoming tests will verify these results in a more realistic setting with high-resolution bathymetry and sea ice.

The convective parameterization has been successfully integrated into CAP and is ready for extensive testing over a multiple-year integration. Analysis will focus primarily on the western Greenland Sea, where previous modeling and observational studies indicate active deep convection along the edge of the coastal ice shelf. These experiments will determine the importance of thermobaric effects in controlling deep convection in the presence of sea ice.

Improvements to the convective parameterization are the addition of wind- and wave-generated turbulent energy to the parcel-grid-column energy budget. These additions are implemented by the use of well-known scalings for boundary-layer turbulence based on similarity theory and by the use of new scaling arguments taken from observations and modeling of turbulence under surface waves. Evidence from largeeddy simulations suggests that mixing from surface wind effects can cause up to a doubling of the surface mixed-layer depth in typical fall Greenland Sea conditions. Inclusion of these effects in OGCMs is critical for the correct representation of convective mixing in polar regions.

\section{COUPLED ICE-OCEAN MODELS FOR CLIMATE RESEARCH

Keywords: ice models, ocean models, Arctic ice, remote sensing

Objectives: To simulate Arctic ice-ocean variability for the past decade with coupled ice-ocean models on parallel computers and to compare the results with SSMI satellite observations.

Approach: Atmospheric forcing and SSMI microwave satellite data on ice concentration in the Arctic will be collected for the past 10 years. The current numerical schemes and coding algorithms will be adapted or replaced with ones optimal for massively parallel computers. The model sensitivity to physical parameters and grid resolution will be studied. The feasibility of short-term (decadal) climate simulation will be evaluated, and model results will be verified for 1986 to 1995 with satellite data. Any discrepancies will be related to model deficiencies and/or forcing errors.

Results to Date: The ice and ocean models have been converted to the CM5. The ice-rheology Helmholtz equation has been changed to a four-color overrelaxation scheme. The ice-cover variability of the Arctic Basin and its marginal seas has been simulated for 1986 to 1994. We have found significant increases of ice area and volume from 1986 to 1988 and large decreases in the summers of 1990,1991 , and 1993. The 1991 anomaly seems to be mainly caused by some deficiencies in atmospheric forcing, but the 1990 and 1993 decreases correspond to similar behavior in the SSMI satellite data. The simulated polar-ice thickness agrees well with submarine observations from 1986 to 1992. Model resolutions of $25 \mathrm{~km}$ or better were needed to transport sufficient oceanic heat for realistic ice-edge behavior in the Greenland and Barents seas. Watermass-census and mixed-layer-depth analyses in the Greenland Sea have indicated that the model mixing and thermodynamics components function very well.

\section{FURTHER DEVELOPMENT OF AN ADVANCED FINITE-DIFFERENCE ATMOSPHERIC GENERAL CIRCULATION MODEL RANDALL, DAVID COLORADO STATE UNIVERSITY FY 1996137 FY 1995130 FY 1994157}

Keywords: general circulation models, parameterizations, radiation, planetary boundary layer, gravity waves

Objectives: To develop improved physical parameterizations for use in the CSU GCM, including cloudiness, radiation, the planetary boundary layer, the land surface, gravity-wave, and convective downdrafts and to develop improved numerical methods for the simulation of climate.

Approach: An improved cloud parameterization will be developed, and an improved boundary-layer parameterization will be developed and tested. A gravitywave-drag parameterization developed at UCLA will be introduced to improve the simulation of the zonal wind, and a 3-D version of the geodesic grid model will be created with an isentropic vertical coordinate. Results to Date: We have completed preliminary tests of an aerosol parameterization and also a new radiation parameterization developed by G. Stephens of CSU. We have constructed a higher-order closure model that can be combined with the stretched vertical coordinate used in our earlier boundary-layer parameterization. We have tested the gravity-wavedrag parameterization. We are testing a very radical 3-D global dynamical model based on an icosahedral grid with an isentropic vertical coordinate. 


\section{SCIENTIFIC DEVELOPMENT OF A MASSIVELY PARALLEL OCEAN CLIMATE MODEL \\ SEMTNER, ALBERT \\ NAVAL POSTGRADUATE SCHOOL \\ FY 1996294 \\ FY 1995252 \\ FY 1994195}

Keywords: ocean modeling, parallel computing, climate simulation

Objectives: To develop physically realistic, computationally efficient global ocean models for climate applications.

Approach: Physical components will be implemented into global ocean models on parallel computers, the simulated results will be validated against the best available observations, and these models will be coupled to atmospheric ones for simulations of climate variability and climate change.

Results to Date: A $1 / 4^{\circ}$ (avg.) global ocean circulation model was run through 1995 with smooth daily wind forcing, history-tape averaging, and ECMWF (European Centre for Medium-Range Weather Forecasts) heat fluxes. Tokmakian's comparison of model output with in situ sea-level observations shows an impressive median correlation of 0.59 . With new wind data, CM-5 integrations were run to 1996 . A coupled $1 / 6^{\circ}$ Arctic ocean-ice model was developed by Maslowski of NPS and NOAA postdoc Zhang in both vector- and massively-parallel forms (the latter with NCAR). Dynamic sea ice was added to the $1 / 4^{\circ}$ global model by Zhang and Semtner. The two coupled models have run for many simulated years with forcing fields of 1990 to 1994, and analysis is in progress; also, convective parameterizations are being tested with PNL.

\section{MASSIVELY PARALLEL GLOBAL OCEAN MODELING \\ SMITH, RICHARD D., and DUKOWICZ, JOHN K. LOS ALAMOS NATIONAL LABORATORY \\ FY 1996298}

FY 1995297

FY 1994213

Keywords: global ocean model, parallel computing, sea-ice model

Objectives: To develop a global ocean general circulation model suitable for use on parallel computers and to conduct and analyze global ocean simulations to validate and improve the model so it will be suitable for use in studies of global climate change.

Approach: Our current research is in three main areas: A series of eddy-resolving simulations (about $1 / 6^{\circ}$, so far the highest-resolution global simulations ever performed with a primitive-equation model) will be conducted and analyzed to study the role of mesoscale eddies and other features of the general circula- tion not accessible to coarser-resolution models and to validate the model by comparing its results with observations. New parameterizations of subgrid-scale phenomena, especially the effects of unresolved mesoscale eddies, will be implemented and tested. A sea-ice model that can be used in the coupled oceanatmosphere-ice simulations that are a central focus of the CHAMMP model-development effort will be developed and implemented.

Results to Date: We previously developed an optimized code called the Parallel Ocean Program (POP). We have now completed three 10-year global simulations at about 1/6 with POP on the Los Alamos CM-5. These simulations were driven by realistic winds from the ECMWF atmospheric weather-prediction model and used climatological fields for surface-buoyancy forcing. We are currently undertaking different analyses of the model output. The simulations reproduce the upper-ocean wind-driven circulation quite well. For example, this is the first eddy-resolving model to simulate the transport of the Antarctic Circumpolar Current within the observational limits. We have also undertaken detailed comparison of the sea-surfaceheight variability with the recent, very accurate dataset from the TOPEX/Posiedon satellite mission. Comparisons of the mean circulation, the mesoscale variability, the amplitude and phase of the annual cycle, as well as intraseasonal and interannual changes show that the simulations and observations agree remarkably well over a broad range of space and time scales. In addition, both the model and the observations revealed an unexpected new class of large-scale, barotropic, intraseasonal variability.

We have implemented the Gent-McWilliams parameterization of the effects of mesoscale eddies on the tracer distributions and are currently undertaking a detailed analysis of the eddy-resolving simulations to test the validity of this parameterization, which has proven very successful in improving the fidelity of coarse-resolution models. The development of the seaice model for POP (but not the validation) is nearly complete. The new formulation for the dynamics contains an explicit elastic-plastic adjustment process. This formulation has the great advantage of solving the standard Hibler visco-plastic dynamics at slow time scales but with a far more efficient explicit elastic mechanism. The new technique is orders of magnitude faster than Hibler's original method, while giving essentially the same answers at the time scale associated with wind forcing. It is also fully explicit, which is important for parallelization in POP. 


\section{COUPLED ATMOSPHERE-OCEAN MODELING \\ STONE, PETER, and MAROTZKE, JOACHIM MASSACHUSETTS INSTITUTE OF TECHNOLOGY \\ FY 199665 \\ FY 199552 \\ FY 199449}

Keywords: ocean-atmosphere models

Objectives: To develop simple, but realistic, models of the coupled ocean-atmosphere climate system and employ them in the study of climate dynamics.

Results to Date: No results were reported.

$$
\begin{gathered}
\text { CHAMMP CM-5 AND T3D } \\
\text { COMPUTER SUPPORT } \\
\text { WHITE, ANDREW } \\
\text { LOS ALAMOS NATIONAL LABORATORY } \\
\text { FY } 19961185 \\
\text { FY } 19952820 \\
\text { FY } 1994 \quad 1769
\end{gathered}
$$

Keywords: computer resources, massively parallel computing

Objectives: To provide computer resources on the Thinking Machines CM-5 and Cray T3D massively parallel scientific supercomputers.

Results to Date: No results were reported.

\author{
VARIATIONAL OPTIMIZATION OF \\ SUBGRID-SCALE CONVECTION \\ PARAMETERS \\ ZIVKOVIC, MARINA \\ ATMOSPHERIC AND ENVIRONMENTAL \\ RESEARCH, INC. \\ FY 199631 \\ FY 199530 \\ FY 199429
}

Keywords: cumulus convection, water vapor, singlecolumn models, subgrid-scale convection

Objectives: To optimize a representation of cumulus convection with field experimental data.

Approach: The adjoint of the linear tangent of a single-column model and, independently, a standard optimization code will be used to find the best set of parameters in a convection scheme.

Results to Date: The single-column model was written and tested. Both the standard and the linear-adjoint optimization codes were written and tested. A preliminary optimum parameter set has been found with 120 days of Inner Flux Array data from the TOGA COARE data. The single-column-model prediction of relative humidity, as a function of time and altitude, is excellent.

\section{PROGRAM FOR CLIMATE MODEL DIAGNOSIS AND INTERCOMPARISON (PCMDI)}

\author{
PROGRAM FOR CLIMATE MODEL \\ DIAGNOSIS AND INTERCOMPARISON \\ GATES, LAWRENCE, and POTTER, GERALD \\ LAWRENCE LIVERMORE NATIONAL \\ LABORATORY \\ FY 19963874 \\ FY 19953881 \\ FY 19943894
}

Keywords: climate modeling, intercomparison, climate data, AMIP

Objectives: To develop and implement improved methods and tools for the diagnosis, validation, and intercomparison of global climate models (GCMs) to facilitate model improvements necessary for climatechange studies.

Approach: Support will be provided to the Atmospheric Model Intercomparison Project (AMIP) and to selected other model intercomparison and diagnostic projects focused on specific aspects of model performance. In addition to the support of model diagnosis, a comprehensive observational database will be pre- pared to provide the community up-to-date reanalysis products as well as standard observational data from the surface, atmosphere, and space. Diagnostic software will be developed for model analysis and visualization, including an advanced computational library to support model diagnosis and the detection of climate change.

Results to Date: The AMIP original suite of simulations have been completed, and the results have been quality controlled, archived, and made available to the modeling and diagnostic communities. The Visualization and Computation System (VCS) software has been distribute to collaborators, and observational data have been made available to AMIP participants. Results from selected diagnostic studies include several significant findings: (1) Models must be improved substantially to successfully simulate the observed climate variability. (2) Models with the most realistic intraseasonal oscillations have precipitation distributions that are better correlated with sea-surface temperatures, and these models predominantly employ convec- 
tive parameterizations that are closed on buoyancy rather than moisture convergence. (3) None of the models produces a realistic seasonal cycle of precipitation for the AMIP period, and all simulations show too strong a seasonal cycle for some regions. (4) Ensembles of simulations must be performed to determine predictability. (5) Analysis of coupled ocean/atmosphere model simulations has provided evidence of detectable human influences on the large-scale climate because of increased $\mathrm{CO}_{2}$ and aerosols. (6) The tropical short-wave cloud radiative forcing is systematically too strong when compared to satellite observations, while the short-wave forcing is too weak in the summerhemisphere extratropics.

\section{PCMDI CLIMATE-DATA-MANAGEMENT SYSTEM AND DATA-DIMENSION INTERFACE POTTER, GERALD \\ LAWRENCE LIVERMORE NATIONAL LABORATORY \\ FY 1996173 \\ FY 1995190 \\ FY 1994174}

Keywords: climate modeling, climate data, data management

Objectives: To provide an interface to the variety of data formats in use at PCMDI and other institutions and to provide a uniform view of data for PCMDI and other climate researchers.

Approach: A library, cdunif, will be developed that provides uniform access to data and metadata stored in a variety of standard self-describing file formats.

Results to Date: The cdunif interface is modeled closely on the netCDF interface and supports a somewhat wider variety of data models, including netCDF (UCAR), GRIB (WMO), DRS (PCMDI), and the format(s) supported by the GrADS (Gridded Analysis Data System). The logical model of data is that a file contains a set of variables, dimensions, and attributes. Dimensions have associated coordinate vectors and may be global (applying to all variables in a file) or local (applying only to a given variable). The cdunif library has been designed so the addition of further formats requires minimal effort. In addition to extending the range of formats that can be supported in CDMS, the cdunif package has become the basic $I / O$ library for a number of other PCMDI-developed utilities. In particular, it has been integrated with PCMDI's Visualization and Computation System (VCS). An emulation library (cddrs) has been developed for the DRS library, allowing existing Fortran or C DRS applications to be easily integrated with CDMS. 
The purpose of the Global Change Educational Program is to develop the next generation of scientists essential for the multidisciplinary research conducted on global change by DOE and other governmental and nongovernmental agencies.

\author{
Program Manager \\ Curtis R. Olsen \\ Environmental Sciences Division \\ Department of Energy, ER-74 \\ 19901 Germantown Road \\ Germantown, MD 20874-1290 \\ (301) 903-5329 \\ Internet: curtis.olsen@oer.doe.gov
}

\section{GRADUATE FELLOWSHIPS FOR \\ GLOBAL CHANGE \\ CONSTANTIN, MILTON J. \\ OAK RIDGE INSTITUTE FOR SCIENCE

\begin{tabular}{cc}
\multicolumn{3}{c}{ AND EDUCATION } \\
FY 1996 & 531 \\
FY 1995 & 511 \\
FY 1994 & 1603
\end{tabular}

Keywords: fellowships, graduate education

Objectives: To encourage and support cross-disciplinary graduate education and research in global-change sciences; increase the number of professionals with graduate degrees doing research in global-change sciences; and strengthen links among the academic community, DOE facilities, and other federal agency facilities in all aspects of global-change research.

Approach: The program solicits applications that are evaluated by a panel of faculty and scientists, and recommended applicants are appointed as Fellows enrolled at designated participating universities.

Results to Date: Sixty-five applicants were appointed to the program, 47 of them have completed either M.S. or Ph.D. degrees; and Fellows have a list of thesis, dissertations, reports, abstracts, and articles in scientific journals of more than 500 entries.

\section{MINORITY UNIVERSITIES AND COLLEGES FACULTY/STUDENT

Keywords: minority universities and colleges, education, faculty and student grants

Objectives: To strengthen the capabilities of academic institutions that historically have had a large percentage of minority students; to increase the participation of those academic institutions in the education and training of scientists and engineers in disciplines leading to careers in global-change research; and to establish new and to strengthen existing global-change research links between those academic institutions and the DOE facilities.

Approach: The program is advertised nationally, research/education proposals are received, applications are evaluated, recommendations for awards are made, and awards are made to faculty/student teams for research and education in global-change sciences. Results to Date: Six faculty/student teams are conducting research and education projects at five minority academic institutions.

\section{GLOBAL-CHANGE DISTINGUISHED POSTDOCTORAL FELLOWSHIPS SPEJEWSKI, EUGENE \\ OAK RIDGE INSTITUTE FOR SCIENCE AND EDUCATION \\ FY 19961100 \\ FY 19951100 \\ FY 19941115}

Keywords: education, postdoctoral fellowships

Objectives: To increase the involvement of scientists and engineers in areas supportive of the U.S. Global Change Research Program.

Approach: Recent doctoral-degree recipients conduct research in support of the USGCRP as it relates to the specific mission of DOE and the Subcommittee on Global Change Research of the Committee on the 
Environment and Natural Resources. Fellows spend up to two years at approved laboratories (DOE, university, or other), conducting research in technical areas related to the strategic priorities of the USGCRP. Fellows gain access to a valuable combination of outstanding professional staff, scientific equipment and facilities, and ongoing global change research. These fellowships help promote the influx of new ideas and skills into the laboratories, complement and extend the traditional academic research and training programs, and enhance interactions between the academic community and federal researchers.

Results to Date: The last application cycle for this program was held in FY 1995. Six of the eight fellows who were appointed during this cycle renewed their appointments in FY 1996. One terminated during FY 1996, and the other will renew in the first quarter of FY 1997. Nine of the ten fellows appointed in FY 1994 continued their appointments. Five of these completed their appointments in FY 1996; the remaining four will finish in the first quarter of FY 1997. The remaining 5 of the 12 fellows who were appointed in FY 1993 finished their appointments in FY 1996. In all, 22 fellows participated in the program during FY 1996 at five DOE, six university, four CENR, and three private laboratories. All fellows will terminate by the end of FY 1997. 


\title{
INFORMATION AND INTEGRATION
}

The Information and Integration effort provides information to support the reasoned evaluation of environmental issues associated with elevated atmospheric carbon dioxide and other atmospheric trace gases, including potential climate change.

\author{
Program Manager \\ Bobbi M. Parra \\ Environmental Sciences Division \\ Department of Energy, ER-74 \\ 19901 Germantown Road
}

Germantown, MD 20874-1290

(301) 903-3316

Internet: bobbi.parra@oer.doe.gov

\section{CARBON DIOXIDE INFORMATION ANALYSIS CENTER \\ CUSHMAN, ROBERT; BODEN, THOMAS; JONES, SONJA; KAISER, DALE; and NELSON, TOMMY \\ OAK RIDGE NATIONAL LABORATORY \\ FY 19962079 \\ FY 19951928 \\ FY 19942039}

Keywords: newsletters, data center, information analysis center, information, data sets, databases Objectives: To acquire or compile, quality-assure, document, archive, and distribute data and other information concerning $\mathrm{CO}_{2}$, other greenhouse gases, and climate change in support of DOE's Global Change Research Program (GCRP) and to operate the World Data Center-A for Atmospheric Trace Gases (a component of the World Data Center System of the International Council of Scientific Unions).

Approach: The Carbon Dioxide Information Analysis Center (CDIAC) will identify users' needs by working closely with the research community, the GCRP, policymakers, and others by attending workshops, reviewing the literature, and maintaining personal contacts. Data, models, and other products and services will be evaluated for usefulness and acquired if available or compiled at CDIAC if not in a usable form. These products will undergo extensive quality assurance and documentation in full coordination with the original supplier of the information. They will then be made available to requesters. In addition to data packages, CDIAC will also produce and distribute newsletters, research summaries, and glossaries. CDIAC will work with other data centers and individual researchers to promote the compilation and exchange of data. CDIAC's activities and approaches will evolve with changing research needs and technology, and future emphasis will be placed on exploring new methods of communication, new media for archiving and distribution, and new data products.

Results to Date: During FY 1996, eight new or updated numeric data packages were published (topics included oceanic carbon chemistry, carbon emissions from fossil-fuel combustion and cement production, global cloud coverage, long-term U.S. monthly temperature and precipitation, optical depth, carbon in tropical African vegetation, and atmospheric carbon dioxide) and were made available online; also, four other databases were put online.

Two issues in the DOE Research Summary series and two issues of the newsletter CDIAC Communications were published. CDIAC published Selected Translated Abstracts of Russian-Language ClimateChange Publications. III: Aerosols and a brochure for the U.S. Global Change Data and Information System. CDIAC also maintained home pages for itself and GCDIS.

In FY 1996, CDIAC filled 25,297 requests for data and information; 4,782 of these requests were "traditional" (i.e., via letter, fax, or email) from 2,052 individuals in 75 countries, and 20,515 were directly from CDIAC's anonymous ftp area or World Wide Web site. This brings CDIAC's total to 99,271 requests from users since FY 1985. 
TECHNICAL REVIEW OF CARBON DIOXIDE RESEARCH INITIATIVE PROPOSALS SPEJEWSKI, EUGENE; ROBERTS, DOROTHY; COULTER, BRYAN; and STOOKSBURY, BILLIE OAK RIDGE INSTITUTE FOR SCIENCE AND EDUCATION

FY 1996150

FY 1995300

FY 1994367

Keywords: technical reviews, administrative support Objectives: To provide the technical review of proposals submitted in response to Special Research Grant Program notices.

Approach: Program documents are provided to potential applicants upon request; proposals are received from applicants via ESD/OHER and administratively screened; the proposals are processed and reviewed for completeness; acknowledgment letters are prepared; application, reviewer, and scoring databases are established and maintained; custom computer reports are produced; custom software is designed and developed with state-of-the-art programming languages and hardware technologies; program-specific review guidelines are prepared; postal reviews are coordinated and conducted; travel support and information are provided to reviewers; a meeting site is identified; technical-review meetings are arranged and coordinated; all contractual matters are negotiated; on-site scoring and evaluations are prepared; reviewer comments and recommendations of the review panels are documented; letters of appreciation, award, and declination are prepared; reimbursement of travel and payment of honoraria to reviewers are prepared; workshops, conferences, and site visits are coordinated as required; documentation of program activities is provided; overheads and presentation materials are prepared; and an annual program report is provided. ORISE also coordinates and contracts any special personal and professional meeting requirements (such as stenography, audio/visual, copying/reproduction administrative, and support) and generally acts as a liaison to resolve all meeting-related difficulties.

Results to Date: The technical assistance provided by ORISE helps to ensure that the handling of requests and the merit and peer review process operates smoothly. Staff members are trained in all aspects of federal review and meeting planning and implementation. In addition, ORISE staff tailors the level of support to meet each program sponsor's needs. ORISE guarantees that all merit and peer review meetings and evaluations adhere to all federal statutory rules, regulations, and guidelines. 
The Integrated Assessment of Global Climate Change Research Program focuses on investigations that support future decision making on global-change issues, and investigations into the critical economic analyses underlying decisions expected to be made two or more years from now. The program also emphasizes integrated analysis and the need for an economic framework that provides estimates of both benefits and costs of potential global change and of the actions contemplated to ameliorate it.

\section{Program Manager}

John C. Houghton

Environmental Sciences Division

Department of Energy, ER-74

19901 Germantown Road

Germantown, MD 20874-1290

(301) 903-8288

Internet: john.houghton@oer.doe.gov

\section{INVESTMENT IN NEW TECHNOLOGIES: AN EXPERIMENTAL APPROACH BJORNSTAD, DAVID, and CUMMINGS, RONALD \\ OAK RIDGE NATIONAL LABORATORY and GEORGIA STATE UNIVERSITY \\ FY 199615 \\ FY 199585 \\ FY 199415}

Keywords: integrated assessment, social science, investment theory, technological change

Objectives: To investigate the behavioral foundations for a newly developed body of investment theory, irreversible investment theory, and to extend the implications of that theory to policies intended to bring about technological change by influencing investment foundations.

Approach: Our approach is to conduct a "horse race" between classical investment theory and irreversible investment theory. The outcome of this test is primarily important because a major implication of irreversible investment theory is the bad-news principle. Basically, this principle holds that agents confronting uncertain investment returns, an inability to recover sunk costs, and an ability to wait for more information without incurring strategic disadvantage will focus on worst-case returns and ignore best-case returns when making an investment decision. This contrasts with classical investment theory, which suggests that agents consider expected values, basically averaging probable high and low returns. The implications for policy are significant. If the bad-news principle holds, policies should focus on worst-case outcomes, a posture quite different than current practices.

Results to Date: Our work provides strong behavioral evidence for the existence of a bad-news principle.

\section{THE VALUE OF THE GLOBAL ENVIRONMENT \\ BJORNSTAD, DAVID, and \\ CUMMINGS, RONALD}

OAK RIDGE NATIONAL LABORATORY and GEORGIA STATE UNIVERSITY

FY 199670

FY 199580

FY 19940

Keywords: integrated assessment, social science, contingent valuation

Objectives: To develop a contingent-valuation instrument to address the issue of willingness to pay for policies to mitigate global climate change and to demonstrate the validity of this instrument in a crosscultural context.

Approach: We will develop a survey instrument, test it with focus groups, and apply it with laboratory experimental methods. This instrument makes use of a series of double-auction exercises. Following the double auctions, we hold a referendum from one of several formats. The double auctions are intended to allow subjects to earn money that they may or may not choose to expend in the referendum, a procedure intended to remove bias attributable to "found money." Our approach is to develop a set of experiments, test them in the United States with focus groups, test them 
in Mexico and the United States with the referendum, and modify the instrument, followed by further retesting. The research posits the existence of similar utility functions among Mexican and U.S. subjects. Debriefing questions are used to control for other demographic or experiential variation.

Results to Date: Our initial work indicates that among matched subjects in Mexico and the United States it is possible to develop a single survey instrument that yields compatible results. We are now studying these results.

\section{DATA SUPPORT FOR INTEGRATED- ASSESSMENT RESEARCH CUSHMAN, ROBERT; BODEN, THOMAS; MCCORD, RAYMOND; OLSON, RICHARD; and TURNER, ROBERT \\ OAK RIDGE NATIONAL LABORATORY FY 1996198 \\ FY 199550 \\ FY 19940}

Keywords: integrated assessment, databases, sulfur, emissions, aerosols, climate

Objectives: To provide data support to global-changeassessment research by identifying important databases and improving their usefulness to the development of integrated-assessment models.

Approach: A database whose unavailability has been identified by integrated-assessment researchers as limiting the development of integrated-assessment models (global gridded emissions of sulfur, a precursor to aerosols) will be obtained, quality-assured, documented, archived, and made available to the integrated-assessment research community and others. A thorough survey will be made of integrated-assessment researchers to assess the need for additional data support, in terms of both subject matter and methods of documentation, format, and distribution. A leading candidate data set to be enhanced in this project is long-term climate data expressed on a fine latitudelongitude grid and on the basis of political units. Additional selected databases identified in the survey may also be enhanced as part of this project.

Results to Date: Arrangements were made with Brookhaven National Laboratory to provide data on sulfur emissions, and initial information was delivered to ORNL. Data quality-assurance and documentation began. A survey of data needs was distributed to hundreds of integrated-assessment researchers in the United States and abroad.

\section{CORE SUPPORT FOR COMPREHENSIVE INTEGRATED ASSESSMENT OF CLIMATE CHANGE \\ DOWLATABADI, HADI \\ CARNEGIE MELLON UNIVERSITY \\ FY 19960 \\ FY 1995324 \\ FY 1994250}

Keywords: integrated assessment

Objectives: To further our understanding of the precursors to, processes of, and consequences of climate change to a point where our integrated knowledge can be usefully employed in the design of interventions and in shaping the agenda for applied climate-change research.

Approach: Fundamental research will be conducted into processes of climate change and into the development of integrated-assessment tools. Integrated-assessment models will be developed.

Results to Date: Many implicit assumptions in current generation analyses appear to be suitable to local, short-term changes and unsuitable for application in long-term global change. In particular: Assumptions about tochnical change have been shown to be inaccurate and leading to biased estimates of future BAU emissions and efficacy of abatement policies. Globalwarming potentials have been shown to be inaccurate and misleading. We have developed global land-cover models capable of reproducing current land cover with Kappa factors better than 0.95 for a $0.5 \times 0.5$ grid. We have found that microsimulation of market adaptation to sea-level rise is far less efficient than hitherto assumed. We have shown that storms play a significant role in determining the coastal impacts of sea-level rise.

\section{CORE SUPPORT FOR GLOBAL-CLIMATE- CHANGE MODELING EDMONDS, JAMES E. PACIFIC NORTHWEST NATIONAL LABORATORY \\ FY 1996500 \\ FY 1995250 \\ FY 1994242}

Keywords: integrated assessment

Objectives: To increase understanding of the relationship between human activities that produce greenhouse-gas emissions and the consequences of changes in the atmosphere/climate system.

Approach: GCAM is a modeling system within which two integrated-assessment models are under development. The first system is simpler and more parameterized, while the other incorporates significant process detail into the framework. We refer to the simpler paramaterized system as the MiniCAM and to the other as PGCAM. Both models within the GCAM 
framework are being implemented with a collaborative strategy.

The U.S. Department of Energy already supports the development of the second-generation model (SGM), the principal GCAM tool to model human activities (module A). The support provided in this project will be used to strengthen the development of any or all of the GCAM's modules. This project, therefore, focuses on the development of a working version of the entire GCAM and not simply the SGM. Results to Date: Research quantified the emissionsmitigation requirements for stabilizing the concentration of atmospheric $\mathrm{CO}_{2}$ at levels varying between 350 and $750 \mathrm{ppmv}$. This work showed that the cost of stabilizing the concentration of $\mathrm{CO}_{2}$ could be cheaper than anticipated if a long-term, dynamic, geographically diverse policy could be formulated that emphasized technology development in the early years of the stabilization program and an increasingly stringent program of emissions mitigation over time. The time in which technologies could be developed in preparation for global-emissions reductions was shorter the lower the ceiling of $\mathrm{CO}_{2}$ concentrations, and for very low ceilings (below 450 ppmv) was not even an option.

Gains to "where" and "when" flexibility in implementing international agreements to control fossil-fuel carbon emissions were quantified. "Where" flexibility is the capability to reduce emissions wherever in the world it is cheapest to do so. "When" flexibility is the capability to reduce emissions whenever it is cheapest to do so. Building both types of flexibility into agreements can reduce the cost of implementation by up to $85 \%$. Building in "where" flexibility alone can reduce costs by $70 \%$, while building in "when" flexibility alone can reduce costs by $30 \%$ relative to a program in which emissions paths are specified explicitly for each nation.

\section{GLOBAL CLIMATE CHANGE AND THE SOCIAL SCIENCES: AN INTERNATIONAL ASSESSMENT OF TECHNOLOGY EDMONDS, JAMES E.

Keywords: integrated assessment, social science Objectives: To assess the human processes associated with technology and to increase our understanding of global climate change and the responses and policy issues that are related to global climate change Approach: A state-of-the-art report (SOAR) on the role of social sciences and climate change will be produced in cooperation with the International Social Science Council, Human Dimensions of Global Envi- ronmental Change Programme, and multiple international sponsors. The report is scheduled for release in late 1995 with the preliminary title Human Choice and Climate. The assessment is being undertaken by a set of international researchers with emphasis on broad international participation by leading researchers in all of the relevant fields.

Results to Date: The first general SOAR meeting occurred at the Aspen Institutes' Wye Plantation, Md., Nov. 3-6, 1993, and the second was held Mar. 17-19, 1994, in Laxenberg, Austria. Preliminary drafts of all 12 chapters have been received and are undergoing review by the Editorial Working Group. The final product will be a commercially published book of approximately 1000 pages. Work has begun on a set of preliminary findings to be released in Berlin on the occasion of the Conference of the Parties to the United Nations Framework Convention on Climate Change. SOAR is counseled by an international advisory board.

\section{POPULATION-ENVIRONMENT- TECHNOLOGY INTERACTIONS GOULDER, LAWRENCE H.; KENNEDY, DONALD; SCHNEIDER, STEPHEN; and EHRLICH, PAUL R. STANFORD UNIVERSITY \\ FY $1996 \quad 0$ \\ FY 1995196 \\ FY 19940}

Keywords: integrated modeling, population impacts, technological change

Objectives: To assess implications of induced technological change for the design and potential gains from climate-change policy and to understand interactions between population growth and the design of climate policy.

Approach: We construct two models for evaluating population-environment-technology interactions. The first model examines the significance of induced technological change for climate policy. The model considers connections between (1) R\&D investments and technological change and (2) ways in which expanded R\&D investments in one industry affect $R \& D$ efforts in other industries.

The second model examines interactions between population growth, industrial output, emissions of greenhouse gases, and damages from climate change. This is a two-region global model that considers the implications of changes in population growth for greenhouse-gas emissions under different specifications for technological change. In the first part of the project, we develop and employ an intertemporal general equilibrium model of the United States in which policy changes, incentives to invest in $R \& D$, and technological progress are connected. We explore how the presence of induced technological change 
affects the attractiveness of climate policies. We consider these issues under differing specifications as to preexisting subsidies to $R \& D$ and to spillovers in knowledge. In the second part of the project, we develop and employ a two-region global general equilibrium model to examine the implications of population growth for emissions of greenhouse gases. In addition, we consider how changes population growth affect the costs of policies needed to obtain given emissions or concentration targets. These issues are explored under alternative scenarios of the nature of technological change.

Results to Date: We found that the presence of induced technological change usually implies that a given carbon tax will have higher gross costs to the economy, but higher net benefits (environmental benefits minus abatement costs) as well. We show that carbon taxes are likely to reduce the economy-wide rate of investment in R\&D but will allow for less costly abatement by redirecting R\&D toward production of alternative energy. We also show that the presence of induced technological change itself does not justify subsidies to $R \& D$; such subsidies are justified only to the extent that there are spillovers in knowledge. In the absence of such spillovers, abatement targets are reached most cheaply through carbon taxes alone. Finally, we show the importance in integrated models of accounting for the costs of inducing technological change. Models that treat induced technological change without attention to these costs are likely to understate significantly the gross costs of carbon abatement policies.

\section{UNCERTAINTY AND INTEGRATED- CLIMATE-ASSESSMENT MODELS HAMMITT, JAMES; WUEBBLES, DONALD; and JAIN, ATUL \\ HARVARD UNIVERSITY and UNIVERSITY OF ILLINOIS \\ FY $1996 \quad 0$ \\ FY 1995166 \\ FY $1994 \quad 0$}

Keywords: uncertainty, integrated assessment

Objectives: To improve understanding of current uncertainties about factors affecting global change and their implications for policy choice.

Approach: Development of a pair of integrated assessment models, the Integrated Science Assessment Model (ISAM) and the Harvard Center for Risk Analysis model (HCRA), will be continued; the probability distributions will be developed to represent key model parameters; and parameter uncertainties will be propagated through the models.

ISAM includes coupled state-of-the art modules for representing the carbon cycle, effects of greenhouse gas emissions on atmospheric composition, effects on global temperatures with an energy-balance/upwellingdiffusion climate model, and processes affecting sea level change. The HCRA model is a recently developed, simplified integrated-assessment model for rapid analysis of alternative emission policy choices. It includes a reduced-form abatement-cost component, response-function representation of the carbon cycle, two-box climate module, and damage function components. Subjective probability distributions to represent current uncertainties about key model parameters will be developed, relying on summary reports (e.g., IPCC, Stanford Energy Modeling Forum), recent elicitation studies (e.g., Morgan and Keith and surveys by Manne and Richels and Nordhaus), and analysis of errors in historical projections of population, energy-sector, and other statistics. Effects of these uncertainties on future projections and on the merits of alternative policies will be assessed using Monte Carlo analysis to propagate parameter uncertainties through models.

Results to Date: The ISAM model has been updated to improve the treatment of methane, the distributions of ozone and hydroxyl, and the effects of changes in aerosol content. The carbon cycle model was used to estimate the past trends of $\mathrm{CO}_{2}$ and its isotopes; we found that model-estimated isotopic variation in the atmosphere and oceans are consistent with the observational data. Bayesian posterior estimates of carboncycle model parameters have been developed with a quantitative reconstruction of the past carbon budget. Prior distributions of model parameters (e.g., fractionation coefficients and land-use emissions) were updated with observational data on $\mathrm{CO}_{2},{ }^{14} \mathrm{C}$, and ${ }^{13} \mathrm{C}$ (such as the ocean inventory of bomb- ${ }^{14} \mathrm{C}$ ). This analysis reveals that ocean uptake of carbon is $2.1 \pm$ $0.6 \mathrm{GtC} / \mathrm{yr}$, a smaller range than previous estimates.

The HCRA model was used to determine the least-cost emission paths associated with stabilization of atmospheric $\mathrm{CO}_{2}$ at specified levels and to compare these with emission paths that minimize the sum of abatement costs and damages. This comparison showed that policies based on a benefit-cost perspective are likely to require greater abatement in the near-term (the next few decades) than those based on an atmospheric-stabilization goal as envisioned in the Framework Convention on Climate Change.

A probability distribution to represent current uncertainties about climate sensitivity is being developed with the expert-elicitation data obtained by Morgan and Keith. The individual-expert data have been recoded as probability distributions and alternative approaches to incorporate possible overconfidence bias and to combine the experts' judgments into a single distribution are being evaluated. 


\section{MODELS, LINKAGES, AND FEEDBACKS IN INTEGRATED ASSESSMENTS OF \\ GLOBAL CHANGE \\ HOFFERT, MARTIN I. \\ NEW YORK UNIVERSITY \\ FY 19960 \\ FY 199585 \\ FY 19940}

Keywords: energy policy, carbon cycle, integrated assessment

Objectives: To develop parameterized models of temperature and precipitation change, sea-level change, and biospheric impacts from anthropogenic greenhouse gases and aerosols for application to integrated assessments for policy analysis.

Approach: Global mean temperature and carbon-cycle changes will be computed from reduced-form climate/ ocean models. Impacts on the carbon cycle of agricultural and natural ecosystems from paleoscenarios will be assessed and compared with scenarios of regional climate change derived from global climate models. The realism of assuming nearly universal patterns of temperature and precipitation changes for highly nonuniform aerosol forcing, transient heat uptake by the oceans, and biosphere/climate feedbacks from changes in vegetation and soils will be assessed. In addition, reduced-form carbon cycle and ocean/ climate models will be used to assess IPCC stabilization scenarios called for in the Rio climate convention and their implications for carbon-free energy sources in the twenty-first century.

Results to Date: Stabilization of atmospheric $\mathrm{CO}_{2}$ at twice preindustrial levels $(550 \mathrm{ppm})$ by the year 2050 will require new carbon-free energy sources at least equal to the present global energy consumption of about $10 \mathrm{TW}$ if carbon-to-energy emission factors evolve according the IPCC business as usual scenario. Policies fostering carbon dioxide stabilization at the $550 \mathrm{ppm}$ level include (1) early research, development, and demonstration of sustainable carbon-free energy sources suitable for tropical developing nations where most of the new demand is projected and (2) technological and cost breakthroughs in the infrastructure of renewable-energy transmission and storage.

\section{SUSTAINABILITY, DISCOUNTING, AND INTEGRATED ASSESSMENT HOWARTH, RICHARD \\ UNIVERSITY OF CALIFORNIA, SANTA CRUZ \\ FY 199640 \\ FY 199560 \\ FY $1994 \quad 0$}

Keywords: climate change, integrated assessment, overlapping-generations models, discount rates
Objectives: To explore methods for linking concepts of intergenerational fairness and discounting procedures in integrated-assessment models.

Approach: The project is developing a computationally tractable overlapping-generation model of climate-economy interactions based on the assumptions of Nordhaus's DICE model concerning technology, population growth, and climate dynamics. The project also examines the aggregation assumptions implicit in DICE and related models that maximize the discounted utility of an infinitely lived representative agent.

Results to Date: Efficient rates of greenhouse-gasemissions control are strongly sensitive to the distribution of welfare between present and future generations. Under status quo conditions, preliminary model runs find that greenhouse-gas-emissions taxes rise from 21 to 74 \$/tonne-carbon during the next century, while mean global temperature increases by $5.1^{\circ} \mathrm{C}$ over the long run. Under undiscounted utilitarianism, in contrast, emissions taxes rise from 126 to 420 \$/tonnecarbon between 2000 and 2105 , limiting the long-run temperature increase to $2.7^{\circ} \mathrm{C}$. Either of these outcomes may be supported as a Pareto efficient competitive equilibrium, given appropriate transfers of capital from present to future generations. Infinitely lived agent models with a constant rate of pure time preference may be understood as reduced forms of more realistic overlapping generations models if individual preferences are time-neutral and logarithmic in consumption and the social rate of time preference reflects the relative weight decision makers attach to the welfare of successive-age cohorts.

\section{INTEGRATED FRAMEWORK FOR CLIMATE-CHANGE ASSESSMENT \\ JACOBY, HENRY, and PRINN, RONALD MASSACHUSETTS INSTITUTE OF TECHNOLOGY \\ FY 1996545 \\ FY 1995248 \\ FY 1994248}

Keywords: integrated assessment

Objectives: To develop analytical methods capable of comprehensive, global analysis of climate change, with the detail required for scientific credibility and the computational efficiency needed to study the sensitivities and uncertainties crucial to policy evaluation.

Approach: Existing models of component processes, chosen to achieve a balance between scientific adequacy and computational efficiency, have been adapted for use in an integrated framework for study of climate change and associated policy choice. Also, new component models have been designed and implemented. 
The main components of the resulting MIT Integrated Global System Model now include (1) an economic model for analysis of emissions of greenhouse gases and aerosol precursors, (2) a coupled model of the biogeochemistry of climatically important species and the climate dynamics of the atmosphere and oceans, (3) a transient model of the response of terrestrial ecosystems to climate change, (4) a model of the natural emissions of $\mathrm{CH}_{4}$ and $\mathrm{N}_{2} \mathrm{O}$, and (5) a model of the ocean absorption of $\mathrm{CO}_{2}$. Each of these submodels is supervised by a specialist in the particular disciplinary area.

Results to Date: The MIT Emissions Prediction and Policy Analysis (EPPA) model has been modified and improved to include an revised representation of backstop technologies, improvements in the representation of the transportation and electric power sectors, and upgraded underlying databases and parameters.

The 2-D model of atmospheric chemistry has been coupled as an integral component of a combined chemistry-climate model, and this coupled model has been linked with a transient version of the Terrestrial Ecosystems Model (TEM). The resulting system is capable of full transient analysis of climate phenomena. A model of the natural emissions of $\mathrm{N}_{2} \mathrm{O}$ and $\mathrm{CH}_{4}$ has been completed and coupled into the system, and the combined EPPA-chemistry/TEM-natural emissions system has been applied to a study of the importance of feedbacks of climate change (including terrestrial ecosystem response) on these natural emissions. They are found to be significant and worthy of inclusion in integrated-assessment models of climate change.

Models of components of the carbon cycle have been added to the system. The land sink is quantified with TEM results, and ocean sequestration is analyzed by means of a new submodel of the coupled chemistry/ climate model. Initial results are consistent with observations for recent years, and the combined system shows promise of improved understanding of the response of the carbon cycle to climate change.

The Integrated Global System Model has been applied to several studies during this period. Highlights of this work include (1) a set of reference calculations used in documenting the model structure; (2) tests of the sensitivity of system results to variation in key uncertain input parameters; (3) analysis of the distribution of burdens, and the climate effects, of policies proposed within the Framework Convention on Climate Change; (4) studies of alternative methods for calculation of multigas indices; and (5) studies of the relationship between local air-pollution control (specifically SOx reduction) and climate change.

\section{INCORPORATING ECOLOGICAL- ECONOMIC THRESHOLDS INTO THE INTEGRATED ASSESSMENT OF GLOBAL CLIMATE CHANGE KAHN, JAMES R. \\ OAK RIDGE NATIONAL LABORATORY \\ FY 199630 \\ FY 19950 \\ FY 19940}

Keywords: integrated assessment, social welfare, human society

Objectives: To valuate environmental feedbacks so that social-welfare impacts can be incorporated into integrated assessments.

Approach: First, we will consider the potential for climate change to force environmental systems across critical thresholds. We will use scarcity rent and insurance models to valuate the risk of these major environmental impacts. Second, we will select a suite of environmental endpoints based on (1) availability of data, (2) potential for change from climate change, and (3) potential for estimating the socioeconomic value of the change. Third, we will use Compensating Wage Differential models to link the endpoints to quality of life. Past studies with these models have focused on air pollutants and proximity to toxic sites but have not considered endpoints sensitive to climate change. Finally, county-level data on industrial outputs and inputs will be spatially linked to the endpoints through total-factor-productivity models. Past studies have used this approach to examine the economic impact of environmental regulations.

Results to Date: New project.

\section{LEARNING AND ENHANCED CLIMATE REPRESENTATION IN INTEGRATED ASSESSMENT MODELS KOLSTAD, CHARLES \\ UNIVERSITY OF CALIFORNIA, SANTA BARBARA \\ FY 1996225 \\ FY 1995202 \\ FY 199430}

Keywords: learning, integrated-assessment model, dynamic programming, uncertainty, irreversibilities Objective: To improve the way in which economic models deal with learning (endogenous and/or exogenous) within an economy in the context of a standard Ramsey growth model.

Approach: Learning (information acquisition) will be incorporated into an optimal-growth model. Previous work has focused on exogenous learning, the starshaped spreading of beliefs. This task involves extending this approach and developing several additional representations of learning, particularly Bayesian learning about climate sensitivity. The enhanced 
model's pollution-control and -damage representations will be refined, and it will be used to perform analysis. Results to Date: We have successfully incorporated Bayesian learning about climate sensitivity into a DICE-like integrated-assessment model. The standard optimization-type integrated-assessment, optimalgrowth model has been represented as a stochastic dynamic program. This allows the state of knowledge about climate sensitivity to be represented as a state variable that evolves with time according to Bayesian rule as learning occurs regarding climate sensitivity. The empirical implications of our examination of climate sensitivity is that it may take 50 to 100 years to resolve uncertainty, based purely on the stochastic temperature record. Learning about climate damage (which we are currently examining) appears to take an even longer period of time.

A new algorithm that uses neural networks to approximate the value function in the dynamic program has been developed for solving dynamic programs of the complexity of an integrated-assessment model. As an ancillary result, the use of dynamic programming in solving integrated assessment models has been successfully demonstrated. Also, the underlying distribution on climate shocks has been deduced with a GCM and the instrumental temperature record. This work is important not only for integrated-assessment activities but also in and of itself. We have backed sulfate aerosols and tropospheric ozone out of the temperature record, leaving an induced distribution on climate sensitivity to greenhouse-gas forcing.

\section{IMPACTS OF NEW TECHNOLOGY ON THE NEAR-TERM CLIMATE-CHANGE POLICY CHOICES: AN EXPLORATORY MODELING AND ADAPTIVE-STRATEGIES APPROACH LEMPERT, ROBERT J. RAND CORPORATION \\ FY 199690 \\ FY 19950 \\ FY 19940}

No abstract provided.

\section{ECONOMIC AND ENERGY DEVELOPMENT IN CHINA: POLICY OPTIONS AND IMPLICATIONS FOR CLIMATE CHANGE MCELROY, MICHAEL HARVARD UNIVERSITY \\ FY 199625 \\ FY 1995311 \\ FY 19940}

Keywords: China, economic growth, energy use, taxes, emission controls, air pollution

Objectives: To analyze the impacts of alternative environmental policies on economic growth in the
People's Republic of China, to provide a detailed analysis of the technological options available for China to reduce pollution and improve efficiency in the energy sector, and to support efforts to link this research to a broad, interdisciplinary research program on energy and environment in China.

Approach: The growth of the Chinese economy will be modeled on the basis of the best information that is now available. This includes the benchmark interindustry table for 1992, recently completed by the State Statistical Bureau, and a social accounting matrix prepared by the Development Research Center. Optimizing models will be developed, and specific innovative technologies will be investigated. The models being developed are based upon elaborate existing mathematical programming models developed; they will be extended to include uncertainty in both the demand estimates and also in the performance of technology that is innovative and that is being slowly taken up in China and elsewhere.

Results to Date: The data required for the analysis of environmental policies in China is being assembled. We have also assembled a prototype model for simulating the impact of these policies on Chinese economic growth. We are now extending this model to incorporate important institutional features of the Chinese economy, such as the continuing role of socialist planning in the pricing and allocation of energy and the allocation of saving and investment among industrial sectors. We have examined seven different capacity-expansion scenarios for the Chinese electric power sector. These scenarios range from a base case of meeting all the future demand until the year 2000 by traditional coal-fired steam turbines, with increasing stringency on local and regional environmental controls, all the way up to strict local and regional pollution controls with selective catalytic reduction for carbon dioxide control on all new plants. We have found that the cost of carbon dioxide control could amount to as much as an additional $\$ 50$ billion over the next 5 years compared to not controlling it.

\section{COMPARISON OF ALTERNATIVE METHODS TO MEASURE THE ECONOMIC IMPACT OF CLIMATE CHANGE ON RECREATION \\ MENDELSOHN, ROBERT, and PENDLETON, LINWOOD \\ YALE UNIVERSITY and UNIVERSITY OF SOUTHERN CALIFORNIA FY 19960 \\ FY 199532 \\ FY 19940}

Keywords: integrated assessment, recreation, valuation of recreational resources 
Objectives: To explore methods to value the changes in recreation from climate change.

Approach: A general theory is developed that incorporates two methods for valuing the quality of the environment for recreation: hedonic travel cost and random utility models. The two models are shown to be based on the same underlying theoretical structure. Applications of these two techniques often make different assumptions about the utility function. Practitioners of the random utility model often assume a constant marginal value for quality, whereas the practitioners of the hedonic-travel-cost method assume that the marginal value of quality declines with additional quality. Linking GCM climate predictions with a biological model of individual species, the impact of warming on fish populations is predicted. These changes in fish populations are then valued by the recreation model. Results to Date: The GCM climate predictions for a doubling are linked with biological model of freshwater fisheries in New England. The models predict that warming will cause some cold-water fish populations to be largely wiped out but that there will be increases of warm-water fish populations. These changes in fish populations are valued with both the hedonic-travel-cost and random-utility-recreational models. Both recreational models find that the warmwater fishery has more value than the cold-water fishery. The value of the increases in the warm-water populations outweigh the value of the losses in the cold-water populations on average over the region. However, some areas that depended largely on the cold-water fishery are made worse off.

\section{INTEGRATING ECOLOGY AND ECONOMICS TO MEASURE THE IMPACT OF CLIMATE CHANGE ON GLOBAL TIMBER MARKETS \\ MENDELSOHN, ROBERT, and SOHNGEN, BRENT YALE UNIVERSITY and OHIO STATE UNIVERSITY FY 19960 FY 199592 FY 19940}

Keywords: integrated assessment, timber Objectives: To measure the economic impact of climate change on global timber markets.

Approach: A GCM is used to predict changes in global temperature and precipitation throughout the world. These climate predictions are then used to drive Biome 3, an ecological model that incorporates both biogeographical and biogeochemical processes. These projections of equilibrium changes in biomes and productivity are then used to predict a set of changes in the yield functions of timber types grown around the world. These physical changes, which are assumed to gradually increase with temperature, are then evaluated with a dynamic economic model.

Results to Date: The timber model of the world has been calibrated for a baseline run over the next 150 years. Using the results of Biome 3, productivity and biome changes have been predicted over that time period for a doubling scenario. The Biome 3 model predicts a widespread increase in forest productivity throughout the world. The timber model consequently predicts that supply will increase because of climate change, resulting in increased production and lower prices. Alternative scenarios are currently under examination.

CONFERENCES AND OTHER
MISCELLANEOUS SUPPORT
MOSS, RICHARD
PACIFIC NORTHWEST NATIONAL
LABORATORY
FY 1996210
FY 1995210
FY 1994210

Keywords: workshops and conferences, administrative support

Objectives: To provide support for miscellaneous integrated-assessment activities.

Approach: Funding and administrative support are provided for integrated-assessment-related workshops, support of authors for the Working Groups II and III of the Intergovernmental Panel on Climate Change, advice and assistance to the Integrated Assessment Program in proposal and research evaluation, seed money for new model approaches, salary for administrators and staff of the Intergovernmental Panel on Climate Change and the U.S. Global Change Research Program Office.

Results to Date: No results were reported.

\section{INDUCED TECHNOLOGICAL CHANGE WITH APPLICATIONS TO MODELING OF CLIMATE-CHANGE POLICIES NORDHAUS, WILLIAM D. YALE UNIVERSITY \\ FY 1996106 \\ FY 19950 \\ FY $1994 \quad 0$}

Keywords: integrated assessment, social sciences, technological change, DICE

Objectives: To examine induced innovation in the energy sector and the implications of induced innovation for climate change and climate-change policy.

Approach: The first part of the research investigates the impact of energy prices on inventive activity focusing on the energy sector to improve our understanding of the determinants of inventive activity and to examine a number of hypotheses and specifications 
of the relationship. The theoretical specifications and empirical results obtained will then be incorporated into the DICE or RICE integrated-assessment models of climate change. Upon completion, the research will address the question of the qualitative and quantitative importance of policies to enhance induced innovation as part of the strategy to combat global warming. Results to Date: New project.

\section{ASSESSMENT STRATEGIES FOR GLOBAL ENVIRONMENTAL CHANGE PARSON, EDWARD A., and CLARK, WILLIAM C. HARVARD UNIVERSITY FY 19960 FY 1995256 FY 19940}

Keywords: integrated assessment

Objectives: To investigate empirically how assessments of global environmental change have been designed, implemented, and used and to investigate what strategies for assessment have been most useful in informing policy and decision.

Approach: A review of cumulative assessment experience will be conducted that is more detailed, thorough, and consistent than has been hitherto undertaken. Empirical regularities will be identified in relationships between the issue context in which assessments are undertaken; their management, methods, and processes; and their subsequent use in informing policy-making. Relevant theoretical literatures will be reviewed to identify a small set of testable hypotheses about determinants of the effectiveness of assessments. And preliminary testing of the hypotheses identified will be conducted.

Results to Date: A conceptual scheme was developed for relating assessment characteristics to effects. A research protocol was developed that presents a set of specific, answerable empirical questions to pose of each assessment studied and that spans our conceptual and policy-advisory concerns about assessment design, management, mandate, process, methods, and effects. A preliminary review was conducted of approximately 30 assessments for climate change and 30 for stratospheric ozone to identify salient clusters of assessment design and management. From this preliminary investigation, we developed a set of five common "assessment strategies," linked choices of design, scope, management, participation, and assessment methods that recur repeatedly among assessments that attain at least moderate prominence.

\author{
BEYOND AEEI: TECHNICAL CHANGE IN \\ INTEGRATED ASSESSMENT \\ RICHARDS, KENNETH \\ INDIANA UNIVERSITY \\ FY 199684 \\ FY 19950 \\ FY 19940
}

No abstract provided.

\section{TECHNOLOGY ADOPTION AND ENERGY EFFICIENCY IN MANUFACTURING ROOP, JOSEPH $M$. \\ PACIFIC NORTHWEST NATIONAL LABORATORY \\ FY 199633 \\ FY 19950 \\ FY $1994 \quad 0$}

Keywords: industrial sector, integrated assessment, impact of technology adoption

Objectives: To distinguish the influence of new manufacturing technologies from incremental process improvements in the projection of industrial energy consumption.

Approach: A simple theoretical model of the impact of technology adoption in the industrial sector serves as a framework for examining this issue. Plant-level data from the 1991 and 1994 Manufacturing Energy Consumption Surveys conducted by the Energy Information Administration will be used to quantify the impacts of key industrial technologies. The changes in industry energy consumption will then be decomposed into key components (including technology adoption and process change), improved controls and operational procedures, and changes in plant-level market shares.

Results to Date: New project.

\section{COMPACT STRUCTURAL MODEL OF RESIDENTIAL AND COMMERCIAL BUILDINGS FOR INTEGRATED ASSESSMENT \\ SANDS, RONALD; BELZER, DAVID; SCOTT, MICHAEL; and PRATT, ROBERT PACIFIC NORTHWEST NATIONAL LABORATORY FY 199692 FY 199520 FY 19940}

Keywords: buildings, integrated assessment Objectives: To construct a model that simulates the potential impact of climate change on energy consumption in buildings over time and simulates policies that reduce energy consumption in buildings.

Approach: This project focuses on the major energy end uses in residential and commercial buildings. The level of end-use detail is selected to include weather- 
sensitive end uses and end uses that might be targets of conservation or load-management programs and to simulate major thermal interactions between end uses. Major simplifications must be made to limit the model's data requirements and keep its structure transparent. Buildings energy consumption in the United States is modeled with a representative residence and a representative commercial building, with some regional disaggregation to capture differences in climate. Energy consumption in buildings is simulated for electricity, natural gas, and fuel oil. Climate sensitivity is captured through a response to heatingand cooling-degree days, with the balance temperature a function of internal gains.

Results to Date: A prototype static model is operating that simulates the effect of changes in climate and energy efficiency on the current U.S. building stock. The model simulates space heating, space cooling, lighting, refrigeration, and other end uses combined. The model chooses an optimal level of insulation and energy-using capital given climate, energy prices, and the cost of energy-efficient equipment.

\section{EVALUATION OF TECHNOLOGICAL CHANGE FOR INTEGRATED-ASSESSMENT MODELS: INNOVATION AND DIFFUSION IN ENERGY-INTENSIVE INDUSTRIES IN INDIA SATHAYE, JAYANT \\ LAWRENCE BERKELEY NATIONAL

$$
\begin{array}{ll}
\text { FY } 1996 & 70 \\
\text { FY } 1995 & 0 \\
\text { FY } 1994 & 0
\end{array}
$$$$
\text { LABORATORY }
$$

Keywords: integrated assessment, social sciences, technological change, India

Objectives: To consider the differential impacts on output growth of technological innovation through R\&D within a country and the effects of technology diffusion in lesser-developed countries through international trade.

Approach: Six energy-intensive manufacturing subsectors from India will be formally described with industrial organization techniques followed by empirical analysis of total factor productivity (TFP) for each of these subsectors; partial equilibrium models for each subsector will be estimated to test the robustness of the specification of TFP. The analysis for India will be compared with parallel analysis performed on the same subsectors in the United States. Output of this analysis will enhance several of the current general equilibrium modeling efforts and provide a better understanding of the effects on growth of technological innovation and diffusion.

Results to Date: New project.
STRUCTURAL MODELS OF LONG-TERM ENERGY DEMAND: UNDERSTANDING THE LONG-TERM PROSPECTS FOR CARBON DIOXIDE EMISSIONS

SCHIPPER, LEE; GREENING, LORNA; and WILCOXEN, PETER

LAWRENCE BERKELEY NATIONAL LABORATORY and UNIVERSITY OF TEXAS, AUSTIN

FY 1996118

FY 1995128

FY $1994 \quad 0$

Keywords: integrated assessment, energy demand Objectives: To pursue the reconciliation of top-down and bottom-up modeling approaches to energy- and carbon-conservation policies within the context of a computable general equilibrium (CGE) framework. Approach: Energy-consumption behaviors by both firms (production side) and consumers (demand side) will be explicitly incorporated into the Jorgenson/ Wilcoxen general equilibrium model for the United States. The overall goal of the project is to pursue the reconciliation of top-down and bottom-up modeling approaches to energy and carbon conservation policies, within the context of a computable general equilibrium (CGE) framework. Three points need to be remembered in the process of reconciliation: (1) Energy demand is a derived demand for firms and for consumers, if viewed in a household production framework, and the true commodity demanded is energy service. (2) The introduction of energy-efficiency-enhancing technological change requires the tradeoff or substitution between the various factors that provide energy services, most specifically energy and capital. (3) Particularly for capital, the mix and types of inputs may change with time. To address these points, the current structure of the Jorgenson/Wilcoxen model is being respecified to reflect the demand for energy services by both firms and consumers.

Results to Date: Six methods of aggregate-energyintensity decomposition were applied to the same set of data for the manufacturing sector in ten OECD countries from 1970 to 1992 . The choice of decomposition methods was found to be crucial to the accurate identification of the various effects that determine changes in aggregate energy intensity. Declines in aggregate carbon intensity for these countries range from less than $30 \%$ to more than $70 \%$ and may be primarily attributed to increases in the average product of energy. However, these declines in aggregate carbon intensity appear to have a complex linkage to increases in energy prices as well as to changes in other determinants of gross industrial output. 


\section{UNCERTAINTY IN MINICAM: INVESTIGA- TION OF UNCERTAINTY ISSUES IN AN INTEGRATED-ASSESSMENT FRAMEWORK SCOTT, MICHAEL; SANDS, RONALD; EDMONDS, JAE; and LIEBETRAU, ALBERT PACIFIC NORTHWEST NATIONAL LABORATORY \\ FY 199699 \\ FY 199520 \\ FY $1994 \quad 0$}

Keywords: integrated assessment, uncertainty, emissions

Objectives: To develop and incorporate a Monte Carlo preprocessor component and postprocessor component for the MiniCAM model and to test the relative importance of emission and climate uncertainties in the development of mitigation and adaptation policies of the control of greenhouse gases.

Approach: The new code is a simple, interactive FORTRAN shell that calls a random-number generator and up to 15 statistical distributions for values of model parameters selected by the user. The model then calls a Latin Hypercube sampling code, runs it, produces values for the parameters of the MiniCAM model, runs MiniCAM, and saves selected output. Results to Date: No results were reported.

\section{GLOBAL CHANGE AND THE VALUE OF BIODIVERSITY FOR NEW-PRODUCT RESEARCH \\ SIMPSON, DAVID, and SEDJO, ROGER RESOURCES FOR THE FUTURE

$\begin{array}{lr}\text { FY } 1996 & 5 \\ \text { FY } 1995 & 86 \\ \text { FY } 1994 & 0\end{array}$

Keywords: biodiversity, genetic resources, agriculture, pharmaceuticals

Objectives: To estimate the economic value of biological diversity as a source of new products and to estimate the effects that global change will have on these biodiversity values.

Approach: Economic search models are being constructed to estimate the value of species and genetic diversity as sources of new agricultural and pharmaceutical products. Incremental values ("values at the margin") are calculated for changes in the number of species or genotypes available for new-product research. Data from the pharmaceutical industry and from agriculture are used to calibrate the theoretical models for applications in drug development and crop improvement, respectively. Two basic approaches are being emphasized in the current phase of the research. First, models are being developed in which sequential search takes place over time, and either investment in capacity or intensity of search determines the values likely to be realized in subsequent searches. Second, models are being developed in which the social marginal value of biodiversity is calculated (as distinct from simply private gains to those engaged in newproduct research).

Results to Date: Results to date suggest that the economic values produced by biodiversity as a source of new products may not be very important in modeling the aggregate economic effects of climate change. This is not to say that biodiversity is not important; it may be important for any number of commercial, esthetic, ethical, and ecological reasons. With respect to new-product development leads, however, it would appear that there is a sort of "diamonds and water paradox" at work. Biodiversity is simply so plentiful with respect to researchers' needs that it is not very valuable on the margin. The value of the "marginal species" to a pharmaceutical researcher would appear to be on the order of a thousand dollars or less. Social values may be greater, because private values do not reflect consumer surplus, but preliminary investigations suggest that these values may also not be great.

\section{INNOVATIONS IN ENERGY-EFFICIENCY TECHNOLOGIES AND THE ECONOMIC AND REGULATORY ENVIRONMENT \\ STAVINS, ROBERT, and JAFFE, ADAM HARVARD UNIVERSITY and BRANDEIS UNIVERSITY \\ FY 19960 \\ FY 1995215 \\ FY 19940}

Keywords: technology innovation, energy efficiency Objectives: To understand the degree to which innovation of energy efficiency of the capital stock is endogenous to relative price changes in the economy and to regulations.

Approach: We will collect cross-sectional and timeseries data on various energy-using durable goods, ranging from household water heaters to industrial motors, and then will estimate econometrically the relationship between innovation and the economic and regulatory environment.

Results to Date: We analyzed the effects of energy prices and energy efficiency regulations on the menu of air conditioner and water heater models available on the market for approximately a 30-year period. By adapting the induced-innovation model to a productcharacteristics framework, we developed econometric estimates that indicate that a large portion of the improvement in energy efficiency in these technologies has taken the form of neutral (equiproportional) improvements in all product characteristics and that this neutral innovation does not appear to be responsive to energy prices or regulations. A significant nonneutral component of innovation, which was tilted away from energy efficiency before 1970, later shifted 
to favor energy efficiency. This nonneutral component of innovation was substantially and significantly influenced by energy prices, by product-labeling requirements, and by mandatory efficiency standards. Looking forward, we estimate that if energy prices remain at current levels, declining rates of neutral innovation combined with a return to nonneutral innovation tilted away from energy efficiency will result in little further improvement in energy efficiency of new models. Energy taxes of 10 to $30 \%$ of retail prices could significantly change this prediction. We predict that such taxes would lead to further energyefficiency increases of 10 to $50 \%$ for air conditioners by the year 2025 .

\section{DEVELOPMENT AND APPLICATION OF A TAXONOMY OF GLOBAL-CLIMATE- CHANGE SURPRISES \\ STREETS, DAVID, and GLANTZ, MICHAEL ARGONNE NATIONAL LABORATORY and NATIONAL CENTER FOR ATMOSPHERIC RESEARCH \\ FY 199661 \\ FY 199530 \\ FY 19940}

Keywords: climate surprises, extreme events

Objectives: To develop a taxonomy of climate change surprises that have occurred in the past, together with information on their effects and policy responses, to develop recommendations on how surprises might be treated within integrated-assessment models and frameworks.

Approach: The Earth's climate is in many ways an unpredictable physical system. It is the surprises that cause the greatest disruption to society in terms of loss of capital, natural resources, and human life. The current generation of integrated assessment models is not well equipped to provide help in this area. The current task will begin an organized scientific discussion among physical scientists, social scientists, and economists, aimed at including climate-related surprises in the suite of tools available to decision makers. Using the technique of forecasting by analogy, a review will be undertaken of historical climate surprises, their repercussions, and the policy actions that were taken at the time. From this information, a taxonomy of climate-related surprises will be developed. Finally, recommendations will be made to guide future model-development efforts.

Results to Date: No results were reported.
SUMMER WORKSHOPS ON CLIMATECHANGE IMPACTS AND INTEGRATED ASSESSMENT OF CLIMATE CHANGE WEYANT, JOHN P. STANFORD UNIVERSITY

FY 199630

FY 1995175

FY $1994 \quad 0$

Keywords: workshops and conferences Objectives: No abstract provided.

Approach: The 1996 workshop was held July 25 to August 1, 1996.

Results to Date: No results were reported.

\section{SUPPORT FOR THE STANFORD ENERGY MODELING FORUM WEYANT, JOHN P. STANFORD UNIVERSITY \\ FY 199675 \\ FY 199565 \\ FY 19940}

Keywords: workshops and conferences

Objectives: To provide a structured framework in which experts on energy, environment, and natural resources from government, industry, universities, and other research organizations could meet to study important energy and environmental issues of common interest.

Approach: "Integrated Assessment of Climate Change" is the topic of the EMF 14 study. It is built around a working group of about 100 international interdisciplinary experts involved in integrated assessment modeling, the disciplines upon which it draws, and the policy analysis and development communities. Results to Date: No results were reported.

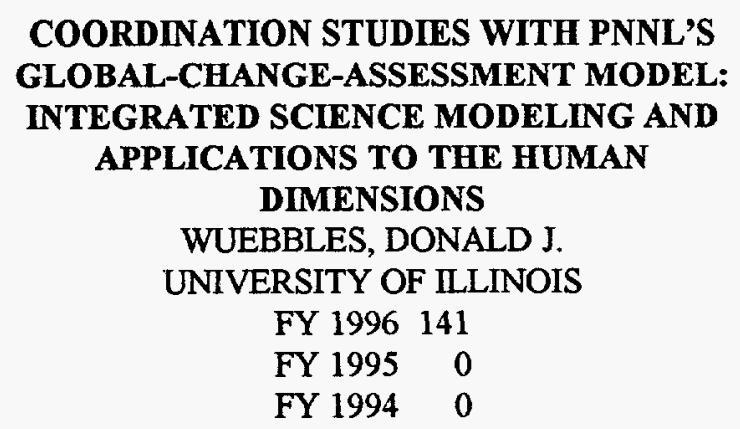

No abstract provided. 


\title{
NATIONAL INSTITUTE FOR GLOBAL ENVIRONMENTAL CHANGE (NIGEC)
}

The National Institute for Global Environmental Change (NIGEC) was established by the U.S. Congress in the Energy and Water Act of 1989. The Institute is operated for the U.S. Department of Energy (DOE) by the University of California under a cooperative agreement. Because NIGEC operates on a different calendar from other programs, funding amounts are reported for only two fiscal years. These entries are not indexed.

\author{
Program Manager \\ Jerry W. Elwood \\ Environmental Sciences Division \\ Department of Energy, ER-74 \\ 19901 Germantown Road \\ Germantown, MD 20874-1290 \\ (301) 903-4583 \\ Internet: jerry.elwood@oer.doe.gov
}

\section{Northeast Regional Center}

\author{
RESPONSES OF TEMPERATE FOREST \\ ECOSYSTEMS TO ELEVATED \\ ATMOSPHERIC $\mathrm{CO}_{2}$ CONCENTRATIONS \\ AND OTHER GLOBAL-CHANGE \\ CONDITIONS \\ BAZZAZ, FAKHRI A. \\ HARVARD UNIVERSITY \\ FY 1995119 \\ FY 1994122
}

Objectives: To scale leaf-level gas exchange of seedlings to that of mature trees.

Approach:We are looking at the effects of growth in an elevated $\mathrm{CO}_{2}$ atmosphere in a controlled environment in the glasshouse. With these data we construct scaling models that allow us to make inferences of mature-tree leaf-level responses to climate change based on seedling responses to simulated globalchange scenarios. There are significant differences between species and between individuals within the species. However, the differences between species account for a greater proportion of the variance than differences between conspecific individuals. Furthermore, there is more variation explained by the differences within an individual canopy than differences between individual trees. The differences in the scaling factors among the species suggest that scaling is more accurate for some species than for others. But for modeling purposes, it is feasible and appears to be a promising approach.
We are also comparing seedlings, saplings, and mature-tree foliage in terms of carbon gain to assess their contribution to total carbon flux in the forest. We use red oak (the most dominant species in the research site). We examine the changes in drought response with ontogeny and ask whether juveniles cope with stress differently than do adults.

Results to Date: Light-saturated photosynthetic rate and nitrogen content, both calculated on either an area or a weight basis, and the leaf mass to area ratio were all significantly different between species as well as between individuals within species $(p<0.0001)$. Differences between species accounted for a greater proportion of variance in the light-saturated photosynthetic rates and the nitrogen content than the differences between individuals within a species $(58.51$ to $78.82 \%$ of the total variance for the measured parameters was attributed to species-level differences versus 5.47 to $17.43 \%$ of the variance attributable to differences among individual trees of a given species). Furthermore, more variation is accounted for by differences among leaves in a single individual tree, than by differences among individual trees of a given species ( 10.74 to $30.40 \%$ vs 5.47 to $17.43 \%$ ). This result allows us to compare species-level photosynthesis, even if the sample size of the number of trees is low. This finding is helpful because studies of canopy-level photosynthesis are often limited by the difficulty of canopy access. 


\section{THE COMPOSITION OF AIR IN THE FIRN \\ OF POLAR ICE SHEETS \\ BENDER, MICHAEL \\ UNIVERSITY OF RHODE ISLAND \\ FY 199592 \\ FY 1994105}

Objectives: To sample and analyze air in the firn (roughly, the upper $100 \mathrm{~m}$ of unconsolidated snow) of polar ice sheets to research this natural archive of air dating back as far as 100 years and to understand the how the changing composition of the past atmosphere is recorded in bubbles in polar ice sampled in ice cores. Approach: The composition of air in the firn is sampled by drilling a short hole, inserting an inflatable rubber sleeve, inflating it to seal the bottom of the hole from surface air, and pumping air from below the sleeve through a tube to the surface. Sampling is extended to deeper depths by drilling more deeply and repeating the sampling procedure. We have adopted this method and modified the pumping to flush out contamination at the point of sampling, thereby providing a clean airstream. Using this method, we have sampled air from Vostok (twice), Taylor Dome (Transantarctic mountains), GISP2 (Summit, Greenland), and Tunu (northeastern Greenland). Vostok and Tunu were sampled during the past year. The firn-air samples are then analyzed for

- The $\delta^{15} \mathrm{~N}$ of $\mathrm{N}_{2}, \delta^{36} \mathrm{Ar}$, and $\mathrm{Kr} / \mathrm{Ar}$ elemental ratio

- The concentration and isotopic composition of biogenic greenhouse gases

- The $\mathrm{O}_{2} / \mathrm{N} 2$ ratio of air

- Halocarbons and $\mathrm{SF}_{6}$

- Hydrocarbons

Results to Date: We have had two recent field programs: Vostok in January 1996 and Tunu (northeastern Greenland) in May/June 1996. One profile was collected at Vostok, and a full profile and a shallow one were collected at Tunu. $\delta^{15} \mathrm{~N}$ analyses of the Vostok samples showed that these are compromised by flow at subsurface depths of 30 to $60 \mathrm{~m}$. The deepest two samples have the expected $\delta^{15} \mathrm{~N}$. Also, $\mathrm{CH}_{4}$ and $\mathrm{CO}_{2}$ concentrations in these samples plot on the curve of covariation observed for the South Pole samples. These deepest samples thus maintained their integrity and can be used for atmospheric reconstructions. Although it is unlikely that century-old air can be sampled anywhere in Greenland, results at Tunu were very encouraging. The deepest sample had a $\mathrm{CO}_{2}$ concentration of about $308 \mathrm{ppm}$, which corresponds to the atmospheric concentration in about 1930. Tunu thus gives us a site for extending the atmospheric histories of gases over Greenland back more than 60 years.

Our interpretation of $\mathrm{CO}_{2}, \mathrm{CH}_{4}$, and $\mathrm{N}_{2} \mathrm{O}$ concentrations, and $\mathrm{O}_{2} / \mathrm{N}_{2}$ ratios in South Pole firn air show that the $\mathrm{CO}_{2}$ and $\mathrm{CH}_{4}$ concentration-time histories measured by Etheridge and colleagues $(1992,1995)$ in Antarctic ice cores successfully predict the covariation of these gases in South Pole firn air. They also show that the $\mathrm{N}_{2} \mathrm{O}$ concentration profile supports the concentration-time histories for these gases measured in the Mizuho ice core. We have reconstructed the $\mathrm{O}_{2}$ budget during the decade around 1980 to constrain the partitioning of fossil $\mathrm{CO}_{2}$ uptake between ocean and the land biosphere. We calculate that the land biosphere was nearly in balance, with an uncertainty of about $1 \mathrm{Gt} \mathrm{C/yr}$. Our result is in good agreement with a number of studies of the anthropogenic carbon balance during the eighties that generally indicate that anthropogenic $\mathrm{CO}_{2}$ emissions were balanced by accumulation in the atmosphere and ocean uptake.

Like $\delta^{15} \mathrm{~N}, \delta^{36} \mathrm{Ar}$ and $\mathrm{Kr} / \mathrm{Ar}$ ratios in South Pole firn air change with depth very nearly as predicted for gravitational fractionation. Expressed in units of per mil mass unit, $\delta^{36} \mathrm{Ar}$ was depleted (because ${ }^{36} \mathrm{Ar}$ is lighter than ${ }^{40} \mathrm{Ar}$ ) and $\mathrm{Kr} / \mathrm{Ar}$ was enriched relative to $\delta^{15} \mathrm{~N}$ by about 0.01 to 0.02 per mil, which is in general agreement with earlier work. The $\delta^{13} \mathrm{C}$ of $\mathrm{CH}_{4}$ in samples from firn air collected at Vostok indicates that the $\delta^{13} \mathrm{C}$ was about $1.5 \%$ less than at present. This result confirms that the burning of biomass, which contributes isotopically very heavy $\mathrm{CH}_{4}$ to the atmosphere, has become a more important $\mathrm{CH}_{4}$ source during the past 40 years. Halocarbon concentrations in Vostok and Tunu samples are mostly compatible with known atmospheric histories of these gases and the histories prior to direct measurements inferred from emission data. The Tunu data clearly shows a subsurface maximum, reflecting the phaseout of certain CFCs and their decreasing emission rate.

\section{AN EMPIRICAL EVALUATION OF THE EFFECTIVENESS OF POSSIBLE STRATEGIES FOR THE ASSESSMENT OF GLOBAL ENVIRONMENTAL CHANGE \\ CLARK, WILLIAM C., and DICKSON, NANCY $M$. HARVARD UNIVERSITY \\ FY 199557 \\ FY 19940}

Objectives: To determine what strategies for the conduct and integration of global environmentalchange assessments will create the most useful bridges between policymakers and scientists in the United States.

Approach: We use three parallel approaches. The empirical approach involves locating, characterizing, and classifying the major assessments of global climate change, stratospheric-ozone depletion, and acid rain that have involved United States scientists and assessors during the period from the Stockholm Conference (1972) to the Rio Conference (1992). The theoretical 
approach considers four broad propositions identified by prior research:

- Science assessments are more used and useful the more "global" (e.g., international) the assessment process; policy assessments, in contrast, are more used and useful the more the process is focused upon the choices faced by "local" (e.g., corporate or state) decision makers.

- Assessments are more used and useful when they expand the range of options available to policymakers, rather than focusing on a few detailed option comparisons.

- Multiple partial assessments (e.g., of the relationship between emissions and their first order environmental impacts) have turned out to be more used and useful than single comprehensive (e.g., end-to-end) assessments.

- Repeated assessments conducted over several years by the same group or institutions are more used and useful that one-time assessments by ad hoc teams.

These propositions are transformed into specific measures of effectiveness and hypotheses that can be tested with empirical data. Finally, the institutional approach expands the project's intellectual and resource base through development of complementary research programs.

Results to Date: We have completed our initial inventory and analysis of American experience in global environmental assessment, including works by government agencies, academics, nongovernmental organizations, the private sector, and the international community. And a descriptive typology of assessments and an analytical characterization of the assessments in our inventory has been completed, allowing us to classify all assessments according to their point of focus.

We found that America has an exceptional appetite for formal assessments linking scientific research to policy problems. Their purpose is to provide a comprehensive, integrated evaluation of the causes, impacts, costs, and response options to support the development of appropriate policy with the best available expertise. American assessments, however, have been partial, disintegrated, and (when conducted by the federal government) politicized. They have also been one-directional, conveying science into the policy debate but not translating the needs of policymakers back into modifications of research agendas. Moreover, crude political intervention has frequently undermined the credibility of government assessments through the truncation of research agendas, the suppression of policy analysis, and the editing of executive summaries. Three forces underlie these patterns: a basic tension between comprehensive and limited assessment, American attitudes that put an enormously heavy premium on consensus, and the general separation of policy assessments from science assessments. Its assessments, however, have actually served America well in dealing with global environmental. The pluralistic and open character of the country's research and policy processes has meant that a great variety of groups have launched assessments of global environmental risks. As a result, the American debate on global environmental risks seems to have moved beyond a need to evaluate specific impacts, adopting instead a strategic perspective that treats major modifications of the global environment as something to be avoided.

\section{SOURCES OF ATMOSPHERIC METHANE IN THE EASTERN UNITED STATES: AN INTEGRATED ASSESSMENT CRILL, PATRICK M., and HARRISS, ROBERT C. UNIVERSITY OF NEW HAMPSHIRE FY 199541 \\ FY 199444}

Objectives: To establish a long-term, low-budget program that can acquire the decade, or longer, databases required for a quantitative integrated assessment of variations in the climatology of $\mathrm{CH}_{4}$ at the Harvard Forest.

Approach: An analytical measurement system for measuring ambient $\mathrm{CH}_{4}$ will be designed and implemented at the air chemistry tower in the Harvard Forest (HF), Petersham, Mass., for quasicontinuous, high-precision measurement of $\mathrm{CH}_{4}$. An emissions inventory of both natural and anthropogenic sources of $\mathrm{CH}_{4}$ will be compiled for New England and New York. Ambient $\mathrm{CH}_{4}$ concentration variations and emission maps will be used to elucidate the detection capabilities of the tower for specific local and regional sources of $\mathrm{CH}_{4}$. The data from the Harvard Forest tower will be used to test the potential for using continuous $\mathrm{CH}_{4}$ measurements in a continental industrial/urban setting to detect changes in source emissions.

Results to Date: From a database of roughly 125,000 observations from 1992 to 1995 , we have defined a subset of the data that is consistent with global background concentrations. These data exhibit both seasonal and diurnal cycles and show a long-term increasing trend in tropospheric mixing ratios of $5 \pm 0.5 \mathrm{ppbv} / \mathrm{year}$ for the entire period, which is similar to that reported for other sites monitoring background levels.

The variability in ambient $\mathrm{CH}_{4}$ concentrations is correlated wind direction, with the highest concentrations coming from the south and southwest, consistent with a hypothesis that a significant part of the measured variability is influenced by landfill emissions within $200 \mathrm{~km}$ of the tower. Enhanced $\mathrm{CH}_{4}$ correlates 
relatively well with $\mathrm{CO}, \mathrm{NO}_{x} / \mathrm{NO}_{y}$, and other anthropogenic nonmethane hydrocarbons (NMHCs), probably because the major landfills are collocated with areas of higher industrial activity and fossil-fuel combustion.

The top $10 \%$ of monthly concentrations occur in late summer, fall, and early winter probably because of enhanced emissions during summer and fall, combined with more frequent and persistent boundary-layer inversions during late fall and winter.

\section{CONTROLS ON SOIL CARBON STORAGE AND SOIL $\mathrm{CO}_{2}$ FLUXES AT THE HARVARD FOREST CORE EXPERIMENT DAVIDSON, ERIC A., and TRUMBORE, SUSAN E. \\ THE WOODS HOLE RESEARCH CENTER and UNIVERSITY OF CALIFORNIA, IRVINE FY 1995128 FY 19940}

Objectives: To determine the spatial heterogeneity and temporal dynamics of soil carbon cycling, to estimate rates of accumulation of carbon in the soil, and to complete below-ground carbon budgets for study areas within the research site.

Approach: Six study sites are arrayed along northwest and southwest transects that include a range of soildrainage classes. Two well-drained sites were added to address differences in previous land use as well as drainage class. Efflux of $\mathrm{CO}_{2}$ from the soil is measured weekly during the summer and biweekly during the autumn and spring. Soil temperature and water content are also measured.

Soil pits were dug and quantitatively sampled for ${ }^{14} \mathrm{C}$ analysis of organic matter throughout the soil profiles. Samples of soil gases were also collected for analysis of ${ }^{14} \mathrm{C}$ in the soil $\mathrm{CO}_{2}$. Together with the soilrespiration data and the litter and root biomass data, the ${ }^{14} \mathrm{C}$ data will allow us to model the turnover times and accumulation rates of carbon within the soil profiles. Samples from the soil pits have been saved for sorting for dead and live root biomass. All samples will be analyzed for ${ }^{14} \mathrm{C}$. Litterfall will be measured biweekly throughout the autumn and monthly thereafter.

Results to Date: Preliminary results from ${ }^{14} \mathrm{C}$ analyses show that living and relatively undecomposed fine roots $(>63 \mathrm{~m}$ but $<2 \mathrm{~mm}$ ) have mean ages of 3 to 9 years. Low-density material $<63 \mathrm{~m}$ in size (including pollen, and plant litter broken down into small pieces) had lower ${ }^{14} \mathrm{C}$ content, though still containing $>20 \%$ postbomb ${ }^{14} \mathrm{C}$ (carbon fixed since 1963). The ${ }^{14} \mathrm{C}$ content of $\mathrm{CO}_{2}$ respired by the soil in early May (before complete leafout) was significantly greater than that of atmospheric $\mathrm{CO}_{2}$ in 1996. This suggests a major contribution to soil respiration from decomposition of soil organic matter with decadal turnover times.
Soil respiration increased from June to July 1995 , but declined rapidly after the onset of drought. Soil water contents and soil respiration rates increased following mid-September rain, but not to pre-drought levels. An exponential model based on soil temperature, with a Q10 value of 3.9 , accounts for $80 \%$ of the variation in $\mathrm{CO}_{2}$ fluxes across all sites and dates. At high water contents, soil water content covaried with soil temperature. The mean annual estimate of soil $\mathrm{CO}_{2}$ efflux was $7.2 \mathrm{Mg}$ ha-1, but ranged from 5.3 in the swamp site to 8.5 in a well-drained site, indicating that spatial heterogeneity of soil $\mathrm{CO}_{2}$ efflux within the forest is related to soil drainage class. The complete soil carbon budgets and models that we are developing for each of these sites will differentiate between root and microbial processes and will include the effects of carbon inputs, temperature, water content, and gaseous transport.

\section{ROLE OF BAROCLINIC-WAVE AMPLITUDE AND TRANSPORT VARIATION IN CLIMATE CHANGE \\ FARRELL, BRIAN F. HARVARD UNIVERSITY \\ FY 199548 \\ FY 199452}

Objectives: To obtain a comprehensive theory for the statistical dynamics of synoptic and planetary-scale transient waves.

Approach: In physical terms, normal systems lack the essential processes of baroclinic/barotropic growth resulting from exchange of energy between the mean flow and the wave field. We have constructed a theory that takes into account the pivotal nonnormality of the underlying dynamics. The maintained variance in this nonnormal system is not determined by a balance between the energy input by stochastic forcing and the energy lost to dissipation, as is the case in familiar normal systems. Rather, the primary balance is between dissipation and energy transferred from the mean flow by the perturbations, with the energy input by the stochastic forcing playing a relatively minor role. This source of energy from the mean to the perturbation field leads to greatly increased variance compared to what would have been anticipated from modal resonance alone. These methods indicate that excitation at a level of $\mathrm{Wm}^{-2}$ is capable of maintaining the observed transient energy of the midlatitude atmosphere and of producing the observed transient heat flux. This excitation, in turn, is traced in part to the scattering of waves because of nonlinearity in the underlying dynamical system.

Results to Date: We have found that stochastic analysis of the observed zonal mean jet produces a distribution of variances and fluxes in remarkable agreement with observations. One implication of this work is that 
models must not only be sufficiently resolved that they include the functions predominantly responsible for the variance at large scales but also resolve the generally smaller-scale disturbances responsible for producing this variance. The theory explicitly finds these functions so that adequacy of resolution can be assessed. Another implication is that parameterized dissipation mechanisms are important in determining the spectrum and fluxes, and the theory explicitly reveals the effect of changes in parameterizations of dissipation. A third implication, which is presently being investigated, arises from the observation that certain relatively small perturbations induce large changes in variances and fluxes. This observation allows us to construct a theory for the sensitivity of variances and fluxes to changes in the system.

\section{OBSERVATION, ANALYSIS, AND PARAMETERIZATION OF FOREST- ATMOSPHERE EXCHANGE MECHANISMS FITZJARRALD, DAVID R., and MOORE, KATHLEEN E. STATE UNIVERSITY OF NEW YORK AT ALBANY FY 199589 FY 199496}

Objectives: To describe the turbulent mechanisms that lead to energy and mass exchange between northeastern forests and the atmosphere, to relate the long-term flux data record at Harvard Forest to radiative parameters measured in situ and by satellite, and to assess the effect of the forest on regional climate. Approach: We measure radiative parameters above a red oak forest in central Massachusetts, turbulent fluxes of heat, water vapor and (with collaborators) $\mathrm{CO}_{2}$ above and within the canopy. We also measure soil heat flux. Global solar albedo and PAR albedo measured at the site both are good quantitative indicators of the spring leaf emergence and autumnal defoliation of the canopy. Appearance of the leaves in spring partially decouples the subcanopy space from the lower atmosphere; soil evaporation dominates total-forest vapor output in later spring. Large eddies have an important role in effecting the transport of $\mathrm{H}_{2} \mathrm{O}$ away from the canopy and $\mathrm{CO}_{2}$ toward it. To define the size of these eddies, pressure fluctuations at the forest floor are being measured with a prototype microbarometer. This device is the first of several that will be deployed in a spatial array at the research site.

Results to Date: In many forests, carbon uptake may be larger on cloudy rather than clear days because growth of the convective boundary layer during the day can lead to forced cumulus clouds that alter the surface energy balance and carbon uptake by modulating the surface light environment. We have previously reported that such clouds appear at Harvard Forest in spring, about the time of leaf emergence. This feature was not prominent during 1995. A prolonged dry spell occurred from approximately day 150 through day 190. During this period, $20 \mathrm{~mm}$ of rain fell, compared to the climatological norm of about $100 \mathrm{~mm}$. Just after leaf emergence, increased boundary-layer vapor density was accompanied by an increase in the frequency of boundary-layer forced cumulus, as inferred by the radiation statistics. As the dry spell continued, boundary-layer warming and subsidence led to a near absence of clouds during days 170 to 185 and unseasonably low values of $q$. During the latter part of this period, there was a significant increase in canopy resistance, reversing the normal trend.

We are currently studying how the observations at Harvard Forest compare with those made by conventional weather and climate stations. For example, the National Weather Service automatic weather station at Orange ( $7 \mathrm{~km} \mathrm{NW}$ ) lies along an airport runway in a large clearing. Our tower sits within a mixed-deciduous forest. Daytime maximum (minimum) 2-m temperatures at Orange can be as much as 5 carbon greater (smaller) than those at Harvard Forest as measured just above the canopy. Specific humidity is comparable at each site.

\section{REGIONALIZING THE HARVARD FOREST LONG-TERM FLUX MEASUREMENTS: COMPLEMENTARY STUDIES FROM HOWLAND, MAINE \\ GOLTZ, STEWART M.; HOLLINGER, DAVID Y.; and DAVIDSON, ERIC \\ UNIVERSITY OF MAINE, USDA FOREST \\ SERVICE, and WOODS HOLE RESEARCH CENTER \\ FY 199577 \\ FY 19940}

Objectives: To understand the mechanisms responsible for regulating forest carbon exchange and storage. Approach: Carbon dioxide, water vapor, heat, and momentum fluxes are measured by the eddy-correlation technique. The present system is based on a three-axis sonic anemometer and a fast-response $\mathrm{CO}_{2} / \mathrm{H}_{2} \mathrm{O}$ infrared gas analyzer. Stored data are transported to Durham and archived. Our prototype system has been improved, increasing reliability and data capture. The system operated during $84 \%$ of the total $1 / 2$ hour periods in the first half of 1996 , collecting useful data for $66 \%$ of those periods. We expect the system to function for more than $90 \%$ of all time periods in the future and to provide useful flux data for more than $70 \%$ of them. Unsuitable meteorological conditions will likely limit useful $\mathrm{CO}_{2}$ flux data to below $80 \%$ of all possible time periods.

Results to Date: Flux data through August 23, 1996, have been edited for quality, but spectral corrections, 
which should increase flux rates by 10 to $15 \%$, have yet to be made. Preliminary analysis indicates that, during the winter months (Julian days 1 to 90), net ecosystem exchange (carbon uptake by the forest is positive) averaged $-0.17 \mathrm{mmol}-\mathrm{m}^{-2}-\mathrm{s}^{-1}$. Day and nighttime rates of carbon efflux were similar. Air and $10-\mathrm{cm}$ soil temperatures averaged -6.0 and $-1.2^{\circ} \mathrm{C}$, respectively. With the thawing of soils about JD 90 , daytime carbon uptake rapidly increased. However, nighttime respiration did not increase substantially for another 20 days as soils remained cold. By summer (JD 150 to 235), daytime ecosystem carbon uptake rates had increased to an average of $6.3 \mathrm{mmol}-\mathrm{m}^{-2}-\mathrm{s}^{-1}$, and the nocturnal efflux rates had increased to $-3.5 \mathrm{mmol}-\mathrm{m}^{-2}-\mathrm{s}^{-1}$. The average daily net ecosystem carbon uptake during the summer was about $2 \mathrm{gc}^{-\mathrm{m}^{-2} \text {-day }}{ }^{-1}$.

When examined as a function of air temperature, midday carbon exchange was very low below $0^{\circ} \mathrm{C}$, but increased to a maximum around $22^{\circ} \mathrm{C}$. Above $25^{\circ} \mathrm{C}$, net ecosystem exchange decreased rapidly, presumably because of reduced photosynthetic uptake and enhanced soil respiration. Nighttime carbon efflux increased with temperature. Growing-season daytime $\mathrm{CO}_{2}$ uptake saturated at a photosynthetically active photon flux density above about $700 \mathrm{mmol}-\mathrm{m}^{-2}-\mathrm{s}^{-1}$. The ecosystem was, on average, a net sink for $\mathrm{CO}_{2}$ when the photosynthetically active photon flux density exceeded about $100 \mathrm{mmol}-\mathrm{m}^{-2}-\mathrm{s}^{-1}$.

\section{INTEGRATING AN ECOSYSTEM MODEL INTO A GLOBAL CHANGE IMPACT ASSESSMENT \\ JACOBY, HENRY D., and PRINN, RONALD G. MASSACHUSETTS INSTITUTE OF TECHNOLOGY \\ FY 199584 \\ FY 199470}

Objectives: To couple a terrestrial ecosystem model (TEM, constructed by the Ecosystems Center of the Marine Biological Laboratory at Woods Hole, Mass.) into the MIT Integrated Global System Model (constructed by the MIT Joint Program on the Science and Policy of Global Change), which includes an economic model for analysis of greenhouse gas emissions and mitigation proposals, a model of the biogeochemistry of the climatically important species, and a global climate model.

Approach: To determine the relative contribution of changes in atmospheric $\mathrm{CO}_{2}$ concentration, temperature, precipitation and cloud cover to the overall equilibrium response of NPP and carbon storage, we ran an equilibrium version of the Terrestial Ecosystem Model (TEM, version 4.0) in a partial factorial experiment. We used two levels of atmospheric $\mathrm{CO}_{2}$ concentration (315 and $522 \mathrm{ppmv} \mathrm{CO}_{2}$ ), contemporary climate, and changes in temperature, precipitation, and cloudiness as estimated by the 3-D GFDL GCM and the 2-D MIT L-O climate model. The TEM results for contemporary climate with $315 \mathrm{ppmv} \mathrm{CO}_{2}$ were used as the baseline for the analysis. The TEM results for complete climate change at $522 \mathrm{ppmv} \mathrm{CO}_{2}$ represent the full-response model. Four other TEM simulations were run, each with one of the four driving variables $\left(\mathrm{CO}_{2}\right.$, temperature, precipitation, and cloudiness) set at the baseline value while the other three variables used values from the climate-change predictions or elevated $\mathrm{CO}_{2}$.

For sensitivity analysis of NPP and carbon storage to transient changes in atmospheric $\mathrm{CO}_{2}$ and climate, we ran a transient version of TEM (version 4.1) for 1977 to 2100 using outputs of three scenarios out of seven generated by an economic-emission model and atmospheric chemistry-climate model. We also ran TEM (4.1) to estimate the effect of $\mathrm{CO}_{2}$ fertilization on the terrestrial biosphere using data on the global atmospheric $\mathrm{CO}_{2}$ concentration from 1765 to 2100 . Results to Date: Elevated $\mathrm{CO}_{2}$ and projected increases in temperature account for most of the overall equilibrium responses of NPP and carbon storage of the terrestrial biosphere to changes in climate and $\mathrm{CO}_{2}$, while the projected changes in precipitation and cloudiness contribute little. This result is attributable in part to the magnitude of changes in $\mathrm{CO}_{2}$ and climate variables as projected by the climate models. NPP has a bimodal distribution for complete climate change at 522 ppmv $\mathrm{CO}_{2}$ for both the GFDL climate and the MIT L-O climate. There are large increases in both tropical regions and the midlatitude in the Northern Hemisphere. For the scenarios with no change in $\mathrm{CO}_{2}, \mathrm{NPP}$ decreases in the tropical regions but increases in the midlatitude temperate zones. For the scenarios with no temperature increase, NPP response is large in tropical regions, but small in mid to high latitudes.

The study also shows that the interactions among changes in $\mathrm{CO}_{2}$ and climate variables play a significant role in the equilibrium responses of NPP and carbon storage to changes in $\mathrm{CO}_{2}$ and climate. The interaction between a change in $\mathrm{CO}_{2}$ and a change in temperature is the most significant. The sensitivity analysis showed that the rate of global NPP increase is high in the HHL case, moderate in the reference case, and low in the LLH case. A modeled increase of atmospheric $\mathrm{CO}_{2}$ concentration for 1765 to 2100 increases both NPP and heterotrophic respiration (Rh). The NPP increase is larger than the $\mathrm{Rh}$ increase, so the difference (NEP) is positive, and the terrestrial biosphere is a net carbon sink in the global carbon budget. 


\author{
DYNAMIC HEAT AND MOISTURE \\ TRANSPORT AND BAROCLINIC \\ ADJUSTMENT \\ LINDZEN, RICHARD S., and \\ CHANG, EDMUND K. M. \\ MASSACHUSETTS INSTITUTE \\ OF TECHNOLOGY \\ FY 199539 \\ FY 199443
}

Objectives: To relate the intensity of the Hadley circulation to heat transport in middle and high latitudes; to determine the effect of mixing of potential vorticity on the stability of the atmosphere, on the thermal structure of the atmosphere, and on the atmospheric response of the atmosphere to the stationary forcing which determines storm paths and regional climate; and to determine the main processes determining the level of water vapor in the atmosphere and how these processes are affected by climate change.

Approach: Our approach is to use

- idealized general circulation models of the atmosphere as well as theoretical studies to investigate dynamical issues concerning the interface between the tropics and the midlatitude atmosphere,

- relatively modest calculations using DEC Alpha workstations and the analysis of data to check and evaluate connections between tropical forcing and extratropical eddies, and

- simple theoretical insights together with analyses of data and GCM behavior.

Results to Date: A study of the role of eddies in communicating changes in the zonal mean state between the tropics and extratropics showed that an oscillatory heating perturbation centered about the equator produced a poleward-propagating zonal-wind anomaly emanating from the tropics into the midlatitudes when the heat source oscillates with a period of 25 to 100 days. At higher frequency, most of the zonal-wind perturbation is confined within the tropics; while at lower frequency, the main signal occurs in the midlatitudes. Examination of the angular-momentum budget and Eliassen-Palm cross-sections suggests that eddies communicate changes in the tropics into the midlatitudes by a shift in the eddy angular momentum divergence pattern and by changes in the amplitude of eddy fluxes and, hence, eddy momentum divergence.

We have finally developed a self-consistent way of using the mixing of PV subject to the constraint of radiative balance to realistically calculate the atmosphere's temperature distribution. We have determined that, at least, 2-level models display baroclinic neutralization. In addition, we have developed a 2-D hemispheric energy balance climate model based on the assumption that PV mixing occurs on isentropes. This model seems like a promising way of predicting some basic aspects of the observed climate, such as the pole-to-equator temperature difference, the height of the tropopause height, and the temperature structure of troposphere, using only readily comprehensible principles of fluid mechanics.

Global climate sensitivity to a doubling of the $\mathrm{CO}_{2}$ concentration is significantly reduced when sea surface temperatures in the eastern equatorial Pacific are held at their control simulation values. Sensitivity of the global mean temperature is reduced from $2.4^{\circ} \mathrm{C}$ in the unconstrained case to $1.9^{\circ} \mathrm{C}$ when the sea surface temperature constraint is applied. The results emphasize the importance of correctly modeling the dynamical processes in the ocean and atmosphere that help determine the SST.

\section{EVALUATION OF SOURCES AND SINKS FOR GREENHOUSE AND OZONE-DEPLETING GASES IN RURAL NEW ENGLAND: A PRELUDE FOR MITIGATION McELROY, MICHAEL B., and ELKINS, JAMES W. \\ HARVARD UNIVERSITY and NATIONAL OCEANIC AND ATMOSPHERIC ADMINISTRATION \\ FY 1995117 \\ FY 1994121}

Objectives: To quantify and characterize the sources and sinks of chemically and/or radiatively active trace gases in the atmosphere in the northeastern United States.

Approach: Direct measurements of $\mathrm{H}_{2} \mathrm{O}, \mathrm{CO}_{2}, \mathrm{CH}_{4}$, $\mathrm{N}_{2} \mathrm{O}, \mathrm{NO}_{x}, \mathrm{NO}_{y}, \mathrm{NMHC}$ (C2 to $\left.\mathrm{C} 6\right), \mathrm{CO}, \mathrm{O}_{3}, \mathrm{H}_{2}$, CFC-11, CFC-12, CFC-113, $\mathrm{CHCl}_{3}, \mathrm{CH}_{3} \mathrm{CCl}_{3}, \mathrm{C}_{2} \mathrm{HCl} 3$, $\mathrm{C}_{2} \mathrm{Cl}_{4}$, Halon-1211, $\mathrm{CH}_{3} \mathrm{Cl}$, and $\mathrm{SF}_{6}$ are currently being obtained continuously throughout the year. The nonmethane hydrocarbon (NMHC) measurements both above and below the forest canopy are carried out every 45 minutes with an automated gas chromatograph equipped with dual flame-ionization detectors. Detection of the 14 additional gases is performed at the same heights every 24 minutes by a second gas chromatograph equipped with four channels coupled to electroncapture detectors. Measurements for this array of species will allow us to identify and quantify their emissions for the northeastern United States. Harvard Forest is ideally located near landfills, sewage-treatment plants, and agriculture and is downwind of a major industrial center (New York City); we will be able to distinguish between the many anthropogenic and natural processes that release greenhouse gases, including those of the forest itself.

Results to Date: Data collection with the four-channel gas chromatograph began in January 1996 and has continued with occasional interruptions to the present. Measurements of 14 atmospheric species at two tower 
heights ( $29 \mathrm{~m}$ and $24.5 \mathrm{~m}$ ) alternate with calibration runs every 12 minutes. As expected, in the early spring, $\mathrm{N}_{2} \mathrm{O}$ emissions do not reflect the variability found in other compounds. Such intercomparisons of $\mathrm{N}_{2} \mathrm{O}$ concentrations with the other thirteen species opens new possibilities for the identification of sources of $\mathrm{N}_{2} \mathrm{O}$.

Whole-ecosystem emission rates for ethene, propene, and 1-butene from Harvard Forest were determined for January through December 1993. Comparison of our measurements with a model of anthropogenic sources suggests that emissions of propene and 1-butene by vegetation during summer exceed anthropogenic emissions in the northeastern United States and emissions of ethene by vegetation contribute about $50 \%$ of anthropogenic sources. During winter, anthropogenic sources dominate.

The seasonality and annual mean concentration of $\mathrm{OH}$ in northern midlatitudes were elucidated from two years of nonmethane hydrocarbon measurements (August 1992 through July 1994) and comparison with model results. The seasonal cycle of background concentrations for anthropogenic alkanes could be cleanly extracted from the roughly 900 measurements each month. Background concentrations exhibited seasonal maxima in winter and minima in summer, reflecting the rate of reaction of of each species with $\mathrm{OH}$.

Whole-ecosystem isoprene emissions have been measured since June 1995. Leaf-level isoprene emissions were measured in July 1995 to intercompare leaflevel measurement techniques, to understand how leaf level emissions can be scaled and extrapolated to whole-ecosystem emissions, and to develop a database to test isoprene emission models.

\section{ATMOSPHERE-BIOSPHERE FEEDBACK MECHANISMS IN FOREST ECOSYSTEMS MELILLO, JERRY M;; NADELHOFFER, KNUTE J.; and STEUDLER, PAUL A. WOODS HOLE MARINE BIOLOGICAL LABORATORY$$
\text { FY } 1995155
$$$$
\text { FY } 1994138
$$

Objectives: To increase our understanding about how midlatitude forest soils respond to changes in the climate and the chemistry of the environment and what these responses imply for net-ecosystem carbon storage.

Approach: In year two, we continued a combination of field studies at the Harvard Forest and modeling, including measurements of carbon and nitrogen processes on the 18 plots set up in the original soilwarming study, the measurement of $\mathrm{CO}_{2}$ emissions from the new trenched-plot study that includes a warming treatment, and the application of four exist- ing models to the Harvard Forest and the northeastern United States.

Results to Date: The increases in $\mathrm{CO}_{2}$ flux caused by warming were between 30 and $40 \%$ in years 1 and 3 and 20 and $24 \%$ in years 4 and 5 . In year 2 , the increase was just under $15 \%$. The interannual differences could be related to year-to-year variations in ambient temperature.

Cumulative soil respiration in the untrenched plots and trenched plots was $224 \mathrm{gC} / \mathrm{m}^{2}$ and $158 \mathrm{gC} / \mathrm{m}^{2}$ during the 1996 growing season. Heating increased total respiration by $19 \%$. Microbial respiration was estimated to be about $70 \%$ of the total and was only slightly greater $(76 \%)$ in the heated plots. Heating decreased root respiration a by about $6 \%$ compared to the unheated plots.

Nitrogen mineralization rates increased in the surface soil-organic layer plus the upper to $10 \mathrm{~cm}$ layer of mineral soil over the first four years of the soilwarming study. Increases from warming were about $140 \%$ in the fourth year in combined soil layers. However in 1995 , we observed only a $36 \%$ increase in response to warming.

A ${ }^{15} \mathrm{~N}$ pulse-chase study examines the effects of soil warming on soil and root nitrogen dynamics by injecting ${ }^{15} \mathrm{~N}$-ammonium and ${ }^{15} \mathrm{~N}$-nitrate (separately) into split plots located in 3 disturbed control and 3 heated plots and following the movements of the tracers through soil, dissolved-inorganic, dissolvedorganic, fine-root, and microbial nitrogen pools. Soilmicrobial, soil-solution, and fine-root pools showed increased gross nitrogen mineralization (total nitrogen flux from organic to mineral nitrogen pools) and decreased $\mathrm{NH}_{4}$ consumption (assimilation by soil organisms: roots and microbes) in heated versus disturbed control plots.

We have used the data from the soil-warming studies to modify our whole-ecosystem model, TEM, which we have used to evaluate the potential impacts of climate change at the regional and global scales. Currently, we are comparing TEM to PnET; preliminary results indicate that they yield similar estimates of regional NPP for contemporary climate (PnET = $145 \mathrm{Tg} \mathrm{C} /$ year; $\mathrm{TEM}=140 \mathrm{Tg}$ C/year).

\section{VARIABILITY IN OBSERVED LARGE-SCALE ATMOSPHERIC MOISTURE FLUXES ROSEN, RICHARD D., and SALSTEIN, DAVID A. ATMOSPHERIC AND ENVIRONMENTAL RESEARCH, INC. \\ FY 199572 \\ FY 19940}

Objectives: To produce a more thorough assessment than heretofore possible of the range in variability 
observed in water vapor and its dynamic transport across the globe.

Approach: Initially, our diagnoses are being based on global analyses of radiosonde data prepared by Oort for each month of the 30 years since 1958. Fields of quantities related to water vapor and its transport are available on a $2.5^{\circ}$ lat. $\times 2.5^{\circ}$ lon. grid at up to 11 pressure levels. One of the tasks involved in our effort is to produce consistent column-integrated integrals of water-vapor and vapor-transport fields from these pressure-level data.

Recognizing that a number of well-known shortcomings exist in the radiosonde-based analyses, we also plan to compare results from these analyses with those we will derive from products of the reanalysis projects of NCEP/NCAR and NASA/GSFC. We will also examine the tropospheric humidity fields being obtained from a separate reduction of the radiosonde station data for 1973-93 being performed by NOAA's Air Resources Laboratory. By comparing statistics from these different, independently derived observational data sets, we can assess the confidence to be attached to these statistics. We view such an assessment of the errors present in analyses of the atmospheric water cycle to be an important aspect of our study.

Results to Date: The Oort radiosonde-based analyses were obtained and an additional data set has been obtained from Oort containing circulation statistics at individual stations for each month of the 30-year data record. These station values formed the basis for Oort's monthly-mean analyses. As such, they will prove useful in determining the extent to which interannual variability in the analyzed fields has resulted from changes in station distribution. NCEP/NCAR Reanalyses data set is also being processed during this early part of the project.

The global values of $\mathrm{W}$ derived from the radiosonde-based analyses of Oort were used to help assess the simulations of humidity from $28 \mathrm{GCMs}$ that participated in the Atmospheric Model Intercomparison Project (AMIP). Our results indicate that, although the models tend to be drier than observed, they do seem to reproduce the observed seasonal cycle in global precipitable water quite well and they reveal the presence of an El Niño signal in their interannualanomaly time series. A comparison between profiles of the net meridional transport of water vapor for the AMIP models with one derived from the radiosondebased analyses indicates a clear tendency for the models systematically to overestimate the poleward flux of moisture (and latent heat) over much of the globe.
COUPLED ATMOSPHERE-OCEAN

CLIMATE MODELS

STONE, PETER H., and MAROTZKE, JOCHEM

MASSACHUSETTS INSTITUTE

OF TECHNOLOGY

FY 199549

FY 199448

Objectives: To ascertain the importance of various factors in determining the atmosphere-ocean interaction and to investigate how the simulation of these factors affects climate sensitivity.

Approach: Our approach is to develop a series of coupled atmosphere-ocean models, each of which simulates some of the potentially important climate processes realistically but is otherwise simplified so that it is efficient enough to carry out many experiments. These "process" models are then used to address the project's objectives.

Results to Date: Several simple models have been developed and studied. The most important results were: (1) Atmospheric eddy meridional transports of both heat and moisture supply positive feedbacks to the ocean's thermohaline circulation (THC), thereby tending to destabilize it. (2) If a model has errors that require flux adjustments of the order of $100 \%$ to be added to the surface fluxes of heat and moisture to reproduce the current climate and if these additive adjustments are held fixed in climate change experiments, then in general there will also be errors on the order of $100 \%$ in the stability of the simulated THC. (3) Heat transports associated with western boundary currents like the Gulf Stream are simulated poorly in coarse-resolution model ( e.g., at $4^{\circ}$ resolution the heat transport is underestimated by about 50\%). A resolution of at least $1^{\circ}$ is necessary to obtain a reasonable simulation of the heat transport. (4) The equilibrium strength of the THC is independent of the atmospheric meridional moisture flux in the Northern Hemisphere and increases as the atmospheric meridional moisture flux increases in the Southern Hemisphere. (5) The stability of the THC depends on the ratio of the atmospheric meridional moisture flux in the Northern Hemisphere to that in the Southern Hemisphere. As this ratio increases, the stability decreases; and the THC collapses when the ratio is sufficiently large.

$$
\begin{aligned}
& \text { FIELD, LABORATORY, AND MODEL } \\
& \text { COMPARISONS FOR SOIL-GAS-FLUX } \\
& \text { MEASUREMENTS } \\
& \text { SUNDQUIST, ERIC T., and } \\
& \text { WINSTON, GREGORY C. } \\
& \text { U.S. GEOLOGICAL SURVEY } \\
& \text { FY } 199557 \\
& \text { FY } 19940
\end{aligned}
$$

Objectives: To assess the accuracy and precision of $\mathrm{CO}_{2}$ flux estimates based on inverted-chamber mea- 
surements to develop a mechanistic understanding of relationships between chamber measurements and the processes that control gas exchange at the soil surface. Approach: The core of our field work will be a series of comparative field measurements with an initial emphasis on $\mathrm{CO}_{2}$ measurements. The first set of comparisons will be with the results of other investigators. We will place our chambers on exactly the same "footprint" monitored by the other investigators and perform our chamber measurements in a few minutes, allowing direct comparison with others' results with minimal natural temporal variations. For the second set of comparisons, we have established our own study site to provide a common point of reference for all comparisons and to assure that each chamber method is assessed at least once on a site where below-ground soil properties are thoroughly characterized.

We haved initiated a series of laboratory experiments using the controlled-flux-column methods. One column will contain chemically inert silicate sand and the other inert silicate clay or silt to provide information about physical effects that cannot easily be isolated from chemical and biological processes in the field.

Modeling is used as an integral diagnostic and self-teaching tool. Thus our objective is not to create a better soil-transport or carbon model but to use modeling in planning our experiments and understanding our results. We will attempt to use our models, in conjunction with our $\mathrm{CO}_{2}$ flux measurements and detailed soil $\mathrm{CO}_{2}$ concentration profiles to estimate effective soil diffusivities.

Results to Date: We tested various chamber designs over an experimental soil column with a known flux. Closed-chamber measurements systematically underestimate true fluxes, but our results suggest that careful chamber design and flux calculation can significantly reduce systematic errors. Using our standard chamber configuration, estimated $\mathrm{CO}_{2}$ fluxes were consistently within $10 \%$ of independently measured steady-state fluxes. Three-dimensional numerical model results were very consistent with our experiments. The error was greater when the chamber was shorter and concentrations were fit over longer intervals. Estimates of $\mathrm{CH}_{4}$ uptake were significantly less accurate because of the longer time required for detection and because of methane's higher diffusivity.

We have established a study site for comparisons of soil surface chamber flux. Two trenches were excavated approximately $10 \mathrm{~m}$ apart, normal to the local slope. Soil samples were kept for analysis. Gassampling tubes, thermistors, and TDR probes were inserted; each trench was backfilled; and measurements have commenced.

Using our standard chamber configuration, we observed diel variations in soil-surface $\mathrm{CO}_{2}$ fluxes that appear to be spatially coherent over scales of tens of meters. This coherence is apparent in normalized fluxes even though absolute fluxes may vary significantly on the same spatial scale. We suggest that observation of diel variations might be a good test of scale- and method-dependent differences between flux estimates based on chamber measurements and those based on less direct techniques, such as below-canopy eddy correlation.

\section{THE ATMOSPHERE-BIOSPHERE EXCHANGE OF NITRIC ACID IN A FOREST SETTING}

TALBOT, ROBERT W., and LEFER, BARRY L. UNIVERSITY OF NEW HAMPSHIRE

FY 199545

FY 199449

Objectives: To quantify the atmospheric inputs of gas phase ammonia $\left(\mathrm{NH}_{3}\right)$ and particulate ammonium $\left(\mathrm{NH}_{4}{ }^{+}\right)$and nitrate $\left(\mathrm{NO}_{3}\right)^{-}$to the Harvard Forest in Petersham, Massachusetts.

Approach: We are determining the dry deposition fluxes of $\mathrm{HNO}_{3}$ and $\mathrm{NH}_{3}$ to the Harvard Forest ecosystem using the flux-gradient-profile and resistanceanalogy approaches. With the flux-gradient method, the flux of $\mathrm{HNO}_{3}$ or $\mathrm{NH}_{3}$ is described by the product of the eddy diffusivity of the gases $\left(\mathrm{KHNO}_{3}\right.$ or $\left.\mathrm{KNH}_{3}\right)$ and the vertical gradient in atmospheric concentration above the deposition surface $\left(\mathrm{dHNO}_{3} / \mathrm{dz}\right.$ or $\left.\mathrm{dNH}_{3} / \mathrm{dz}\right)$. The challenge of this technique is to find a suitable method to estimate the eddy-diffusivity component. We are using both the diffusivity of sensible heat $(\mathrm{KH})$ and momentum (KM) at certain times in our calculations.

The resistance analogy approach describes deposition to the forest ecosystem with three serial resistances: a measure of the stability of the atmosphere above the deposition surface [the bulk aero-dynamic resistance $(\mathrm{Ra})]$; the boundary layer resistance $(\mathrm{Rb})$; and the surface uptake resistance (Rc), a function of biological parameters and the ability of the vegetation surface to absorb the gas molecule.

To estimate the dry deposition of particulate nitrogen to the Harvard Forest, we are modifying models obtained from the literature to calculate the flux as a function of particle size as measured with 10-stage cascade-impactor sampling. These size distributions will then be applied to our bulkparticulate-gradient measurements to estimate particulate fluxes summed from 0.1 to $25 \mu \mathrm{m}$.

Results to Date: It appears that $\mathrm{HNO}_{3}$ deposition accounts for the majority of the $\mathrm{NO}_{\mathrm{y}}$ dry deposition flux and is of similar magnitude to the nitrogen inputs from wet deposition. Recent results indicate that the dry deposition of particulate nitrogen may be a significant additional input of fixed nitrogen to these ecosystems. 
The 10-stage cascade impactor revealed that 50 to $90 \%$ of the aerosol $\mathrm{NO}_{3}{ }^{-}$is found in the coarse ( $>2 \mu \mathrm{m}$ diam) size fraction at Harvard Forest. In addition, this coarse aerosol $\mathrm{NO}_{3}^{-}$appears to be associated with particulate $\mathrm{Ca}^{2+}$, suggesting that $\mathrm{HNO}_{3}$ vapor is absorbed onto the surface of crustal soil material. Given that these supermicron particles have large deposition velocities (a few $\mathrm{cm} \mathrm{s}^{-1}$ ), this could significantly accentuate the deposition of $\mathrm{HNO}_{3}$ vapor.

During a June sampling intensive, two impactors were run simultaneously, one at the top of the canopy (19 m) and another below the canopy

$(7 \mathrm{~m})$, to measure the gradient in aerosol $\mathrm{NO}_{3}^{-}$concentration as a function of particle diameter. While the concentration of small particle $\mathrm{NO}_{3}^{-}(<2 \mu \mathrm{m})$ had identical concentrations at both levels, coarse (2 to $25 \mu \mathrm{m}$ ) aerosol $\mathrm{NO}_{3}^{-}$showed significantly higher concentrations above the canopy than below the canopy.

The monomodal particle size distribution of $\mathrm{NO}_{3}^{-}$ in the June sample is notable, indicating that bulk aerosol sampling is not sufficient to characterize $\mathrm{NO}_{3}{ }^{-}$ at this site. Our measurements suggest that the majority of the aerosol $\mathrm{NO}_{3}^{-}$resides in the coarse fraction and can account for 5 to $20 \%$ of the total (gas + aerosol) atmospheric $\mathrm{NO}_{3}{ }^{-}$loading.

\section{LONG-TERM, WHOLE-ECOSYSTEM OBSERVATIONS OF ATMOSPHERE- BIOSPHERE EXCHANGE IN A MIDLATITUDE FOREST: RATES OF CARBON STORAGE AND INPUTS OF NITROGEN OXIDES \\ WOFSY, STEVEN C.; GOULDEN, MICHAEL L.; MUNGER, J. W.; and DAUBE, BRUCE C. HARVARD UNIVERSITY FY 1995148$$
\text { FY } 1994 \quad 0
$$

Objectives: To define the response of northern forests to climate variations on seasonal and annual time scales to help predict future shifts in carbon storage in northern forests associated with variations in climate. Approach: We will continue ongoing measurements of net $\mathrm{CO}_{2}$ exchange at Harvard Forest for three years, continue development of long-term eddy correlation flux measurements as a technique applicable to major issues in the DOE global-change program, and analyze long-term flux measurements to help define the role of the terrestrial biosphere in the global carbon cycle, at present and in changing climates of the future. We will also continue measuring net exchanges of $\mathrm{NO}_{y}$ and $\mathrm{O}_{3}$ and performing comprehensive analyses of the long data records to define the magnitude of nutrient and pollutant inputs and to elucidate effects on carbon uptake.

Results to Date: We have used eddy covariance throughout the past six years to monitor the net exchanges of $\mathrm{CO}_{2}$ and $\mathrm{H}_{2} \mathrm{O}$ above the Harvard Forest. Net ecosystem exchange at Harvard Forest varied significantly over the period. We found that phenology (dates for canopy development in spring and senescence in fall) and the snow cover in winter had the most important effects on seasonal and annual net carbon uptake. They tend to make carbon net uptake increase during warm climatic anomalies, such as warm spring or fall periods which lengthen the growing season, and snow-free winter conditions, which promote desiccation and deep freezing of soils, an unexpected result. We also found that climatic anomalies over the land mass of the northern hemisphere could explain many of the variations observed in the long-term growth of atmospheric $\mathrm{CO}_{2}$, principally because of effects on deciduous forests.

Eddy fluxes of $\mathrm{NO}_{\mathrm{y}}$ to forest canopies were observed to be controlled by three environmental processes: advection from source regions, chemical reaction, and vertical mixing. Deposition of $\mathrm{NO}_{\mathrm{y}}$ was insensitive to changes in either leaf area or stomatal conductance, although these were significant for $\mathrm{O}_{3}$. At both Harvard Forest and Schefferville, Ont., the net $\mathrm{NO}_{\mathrm{y}}$ flux was from the atmosphere to the canopy; soil emissions of NO were measurable but relatively small.

Seasonal changes in $\mathrm{OH}$ concentrations induce seasonal changes of about a factor of 10 in $\mathrm{NO}_{x}$ oxidation rates because of homogeneous reactions. However, we observed similar rates of $\mathrm{NO}_{\mathrm{y}}$ eddy fluxes in summer and winter and little seasonal variation of background levels of $\mathrm{NO}_{y}$, implying that the supply of $\mathrm{HNO}_{3}$ or other depositing species does not decline proportionally with $\mathrm{OH}$ concentrations. Evidently heterogeneous reactions must be important in winter.

The results provide a picture of the average monthly deposition of nitrogen oxides. Deposition appears to remove 75 to $90 \%$ of the $\mathrm{NO}_{\mathrm{x}}$ emitted in the northeast, spreading the nutrients/pollutants over wider areas in winter than in summer. The unexpected efficiency of removal in winter is caused by heterogeneous conversion of $\mathrm{NO}_{x}$ to depositing species, which takes over from homogeneous oxidation of $\mathrm{NO}_{\mathrm{x}}$ during winter as well as on summer nights. 


\section{Southeast Regional Center}

\author{
SPATIAL MODELING OF THE BIOLOGICAL \\ AND ECONOMIC CONSEQUENCES OF \\ CLIMATE CHANGE ON FORESTED \\ ECOSYSTEMS OF THE SOUTHEASTERN U.S. \\ ABT, ROBERT C.; DESTEIGUER, ED; \\ and MCNULTY, STEVE \\ NORTH CAROLINA STATE UNIVERSITY \\ and USDA FOREST SERVICE \\ FY 199583 \\ FY $1994 \quad 0$
}

Objectives: To integrate existing economic and biological models and data related to the forest resource in the South and investigate the spatial distribution of the economic and ecological effects of climate change and to determine the potential economic impact of global climate change on recreational coldwater angling in the southern Appalachian Mountains. Approach: The project integrates these ongoing modeling efforts to assess the economic and policy consequences of region-wide changes. The key premise is that the diversity of ecosystems, the active market, and the nature of resource policy in the South requires integration of spatial aspects of ecological and economic modeling to be relevant for policy analysis. Specifically, this project models physiological, market, and nonmarket impacts at the USDA Forest Service's Forest Inventory and Analysis surveý unit level.

The possible change in trout population caused by changes in the regional climate will be estimated by studying global-climate-change scenarios under a doubling of $\mathrm{CO}_{2}$ to determine projected temperature and precipitation changes. These estimated temperature and precipitation changes will be placed in coldwater-fishery models of trout habitat to determine the changes in fish population under a doubling of $\mathrm{CO}_{2}$. To serve as a check on the determination of changes in trout population by means of modeling, a Delphi survey of trout-fisheries experts will also be conducted to obtain estimates of changes in fish population. The changes in angler welfare caused by changes in fish population will be estimated by conducting a survey of trout anglers to estimate a random utility model (RUM) for use in an economic assessment. The RUM model will estimate the degree of preference by anglers for trout fishing based upon trout population. The estimates will be combined with the changes in trout habitat/population to determine the total change in trout anglers' welfare.

Results to Date: The inventory database has been compiled and the inventory statistics have been used to aggregate the physiological data into broad management types and regions consistent with the economic model.
Climate-change scenarios have been obtained for four GCMs: the Goddard Institute for Space Studies, Oregon State University, United Kingdom Meteorological Office, and General Fluid Dynamics laboratory models. Estimates of temperature changes and precipitation changes have been obtained for each model. Some models of cold-water-fishery habitat have been examined, but none has yet been selected. An initial draft of the Delphi-survey questionnaire has been prepared and a list of experts has been obtained. The mathematical specification of the RUM model has been completed. The questionnaire to be used to estimate the RUM model has been completed and pretested. Selection of a sample group of trout anglers in now under way and will result in sample of about 2000 persons with North Carolina trout licences.

\section{NONHYDRAULIC SIGNALING OF SOIL DRYING AND STOMATAL REGULATION IN A FOREST ECOSYSTEM AUGÉ, ROBERT M. \\ THE UNIVERSITY OF TENNESSEE \\ FY 199573 \\ FY 19940}

Objectives: To determine if nonhydraulic signals inhibit stomatal opening and gas exchange of forest trees before soil drying is sufficient to affect leaf water status, to investigate whether stomatal control during varied forest-soil-moisture conditions is better explained in terms of both hydraulic and nonhydraulic factors, and to determine if nonhydraulic inhibition of leaf behavior is allied with other mechanisms that define particular plant-drought-resistance profiles. Approach: Native, in situ forest trees in the DOEsponsored Throughfall Displacement Experiment (TDE) being conducted on the Walker Branch Watershed near Oak Ridge, Tenn., will be used for demonstrating nonhydraulic signals of soil drying in forest trees and for linking nonhydraulic and hydraulic factors in control of stomatal behavior. Potted saplings grown outdoors in a shade house will be used to study species sensitivity to nonhydraulic signaling of soil drying and drought-resistance profiles of forest trees.

The wet, ambient and dry plots of the TDE allow us to track stomatal behavior, abscisic acid transport, and leaf and soil hydration throughout the growing season in trees that cycle through drought episodes of varying duration and intensity. Here, we are determining whether stomatal conductance is better correlated with leaf or soil factors or with the movement of abscisic acid from roots to leaves in three species: Nyssa sylvatica, Cornus florida, and Acer rubrum, important understory species that show varying de- 
grees of drought resistance and dehydration tolerance. In a shade house, Cornus florida, Acer rubrum, Liriodendron tulipifera, Oxydendrum arboreum, Halesia carolina, and Chionanthus virginicus have been grown with roots divided between two pots. Stomatal behavior of plants having roughly half their roots dried is being compared to trees with both halves of the root system kept moistened as well as with trees that have had half of their roots removed. This allows us to measure the influence of the drought-induced signal independently of any hydraulic limitation caused by soil drying. The moist roots of "half-dried" trees provide sufficient water to leaves to keep them as hydrated as nondroughted control trees, while drying roots of the other half of the root system produce elevated amounts of the drought signal and move it to leaves, and to stomates, in the transpiration stream. We will examine whether trees that tend to use drought avoidance mechanisms when faced with water deficits also have a relatively more highly developed root-to-shoot signaling mechanism.

Results to Date: $N$. sylvatica and L. tulipifera were the most sensitive to dehydration, having lethal leaf water potential of -2.04 and $-2.38 \mathrm{MPa}$, respectively; C. virginicus, $Q$. prinus, $A$. saccharum, and $Q$. acutissima withstood the most dehydration, with leaves not dying until the leaf water potential dropped to -5.63 MPa or below. Declining lethal leaf water potential was significantly correlated with increasing capacity for osmotic adjustment. C. virginicus and $Q$. acutissima showed the most osmotic adjustment during the lethal soil drying episode, with the osmotic potential at full turgor declining by 1.73 and $1.44 \mathrm{MPa}$, respectively. Lethal leaf water potential was loosely correlated with lethal soil water contents and not correlated with lethal leaf-relative water contents.

Leaves of $A$. saccharum were able to withstand more desiccation than either $Q$. alba or $Q$. rubra, despite the fact that soil ultimately became as dry $(Q . a l b a)$ or drier $(Q$. rubra $)$ in the oaks than in $A$. saccharum. In nature, flowering dogwood usually exhibits relatively little drought tolerance, but its leaves withstood as much or more dehydration than any of the native oaks or maples we studied. This suggests that $C$. florida probably has very poorly developed drought-avoidance mechanisms, such as deep rooting. Obviously, red and white oak possess other mechanisms than leaf-dehydration tolerance that allow them to grow on more xeric sites than sugar maple.

\author{
CARBON BALANCE AND GROWTH \\ ADAPTATION OF CONTRASTING C3 AND C4 \\ PERENNIAL FORAGE SPECIES TO \\ INCREASED $\mathrm{CO}_{2}$ AND TEMPERATURE \\ BOOTE, K. J.; SINCLAIR, T. R.; ALLEN, L. H.; \\ and SOLLENBERGER, L. E. \\ UNIVERSITY OF FLORIDA and USDA-ARS \\ FY 199577 \\ FY 199489
}

Objectives: To quantify climate change effects on Cand $\mathrm{N}$-balance processes of two perennial warm-season forage species differing in photosynthetic pathway and to quantify the long-term effects of elevated temperature at ambient and elevated $\mathrm{CO}_{2}$ on photosynthesis, respiration, growth, nutritive value, assimilate partitioning, rhizome growth, and carbohydrate-storage dynamics in rhizomes/stolons in two perennial warmseason species.

Approach: This study is evaluating the growth and carbon balance dynamics of bahiagrass and perennial peanut for 3 years at 350 or $700 \mathrm{vpm} \mathrm{CO}_{2}$ and at temperatures from ambient to $+4.5^{\circ} \mathrm{C}$ in temperature-gradient greenhouses (TGGs). The experiment has two "replicate" TGGs at each $\mathrm{CO}_{2}$ level, with four temperature sectors $(0,1.5,3.0$, and $4.5 \mathrm{C}$ above ambient) within each TGG. The forages are fertilized with $80 \mathrm{~kg} \mathrm{~N}$ ha-1 in 11 split applications and irrigated optimally. Air and soil temperature, $\mathrm{CO}_{2}$, and growth dynamics are recorded at $\mathbf{4}$ sites in each temperature segment and at one outside site. Measured plant growth responses include rate of establishment, leaf area, shoot growth, rhizome/stolon growth, root growth, photosynthesis, respiration, forage quality, and composition of rhizomes/stolons, roots, and shoots. Small growth samples are taken in triplicate from each plot on five dates. Samples include belowground components to $20-\mathrm{cm}$ depth. Samples are washed and separated into roots, stolon-stems (bahiagrass), rhizomes (Arachis), stems (Arachis), and leaves. Leafarea index, numbers of shoots/tillers, leaf number per tiller, and number of rhizomes are determined. Plant components are dried, weighed, and analyzed for nitrogen, carbohydrates, and nutritive quality. Leaf and canopy carbon exchange rates are measured at midday. Establishment-year herbage yield is estimated with a single harvest ( $218 \mathrm{~d}$ after planting) from a harvested plot 1.8 by $4.7 \mathrm{~m}$.

Results to Date: The TGG-computer system controlled well the temperature gradients over successive sectors within each TGG. The enriching $\mathrm{CO}_{2}$ concentration was maintained near $700 \mathrm{vpm}$, with short-term variability of $70 \mathrm{vpm}$ about the mean.

The biomass production of both species increased with elevated $\mathrm{CO}_{2}$ concentration and increased temperature. Averaged over five sampling dates, elevated $\mathrm{CO}_{2}$ increased total biomass accumulation by $52 \%$ for 
rhizoma peanut and $14 \%$ for bahiagrass. Both species increased biomass production as temperature increased, although the effect was not significant; in both species the aboveground to belowground ratio increased with temperature.

Herbage production of bahiagrass showed a consistent increase with temperature increase; however, there was no temperature effect on herbage production of rhizoma peanut. Elevated $\mathrm{CO}_{2}$ increased harvested herbage of bahiagrass from 321 to $376 \mathrm{~g} \mathrm{~m}^{-2}$ and rhizoma peanut from 318 to $385 \mathrm{~g} \mathrm{~m}^{-2}$, increases of $17 \%$ and $21 \%$, respectively. Separate small sampling indicated that doubled $\mathrm{CO}_{2}$ increased stolon mass of bahiagrass by $13 \%$ and rhizome mass (Arachis) by $51 \%$.

There was no significant temperature-treatment effect on leaf or canopy photosynthesis in either species. Doubling $\mathrm{CO}_{2}$ increased leaf photosynthesis by $41 \%$ for rhizoma peanut and $18 \%$ for bahiagrass. For both species, elevated $\mathrm{CO}_{2}$ caused a 6 to $9 \%$ decrease in nitrogen concentration of leaves collected throughout the season and of final harvested herbage. Increasing temperature decreased nitrogen concentration of final herbage samples of both species.

\section{ISOTOPIC SIGNATURES OF ATMOSPHERIC METHANE AT NIGEC TOWER SITES AND OF ANTHROPOGENIC SOURCES OF METHANE OF THE ATMOSPHERE: SOUTHEAST LANDFILLS, BIOMASS BURNING, AND WASTEWATER TREATMENT CHANTON, JEFFREY P. FLORIDA STATE UNIVERSITY FY 199568$$
\text { FY } 19940
$$

Objectives: To determine the stable isotopic composition of anthropogenic $\mathrm{CH}_{4}$ sources (coal mining, landfills, biomass burning and waste water treatment) to identify sources of atmospheric $\mathrm{CH}_{4}$ at regional and global scales.

Approach: We are conducting measurements of the stable-isotopic composition of anthropogenic $\mathbf{C H}_{4}$ sources (landfills, biomass burning, and wastewater treatment) to identify sources of atmospheric $\mathrm{CH}_{4}$ at regional and global scales. Stable-isotope mass-balance models have been very useful in constraining estimates of global sources and sinks of atmospheric methane. While source-term isotopic-composition values for methane emitted from wetlands, rice fields, and cattle are fairly well documented, values for anthropogenic inputs from landfills, coal mining, and sewage-treatment plants are constrained by only a few scattered measurements. In a joint effort with the University of New Hampshire (UNH), we are quantifying the isotopic composition of anthropogenic methane sources and identifying which sources are causing excess methane levels at tower sites. We are also determining the isotopic composition of methane released from anthropogenic sources: landfills, biomass-burning, and wastewater treatment facilities. The results will enable development of "chemical fingerprints" of particular sources of emissions.

Results to Date: A method was developed to analyze methane isotopic ratios in samples of air that contain less than $2 \mathrm{ppm} \mathrm{CH}_{4}$. Results from one sample at the Florida tower are consistent with the oxidation of atmospheric methane on the forest floor, those from a second sampling appear to reflect a plume of air from a nearby pulp mill.

Methane concentrations in smoke samples from biomass burning range from 4 to $3915 \mathrm{ppmv}$ with a mean of 349 ppmv. The samples also contain significant quantities of the $\mathrm{C} 2$ hydrocarbons acetylene (mean, $49 \mathrm{ppm}$ ), ethane (24 ppm), and ethylene (122 $\mathrm{ppm})$. Methane ${ }^{13} \mathrm{C}$ values range from -16 to $-31 \%$. Variations appear to be associated with combustion efficiency and the relative abundance of $\mathrm{C}-3$ or $\mathrm{C}-4$ vegetation.

Methane emitted from landfills is significantly enriched in ${ }^{13} \mathrm{C}$ (values range from -36 to $-50 \%$ ) relative to methane within landfills $(-54$ to $-58 \%$ ) because methane is oxidized to a great degree as it diffuses through the soil cap on landfills. We are developing models to calculate the quantity of methane oxidized as it diffuses through the soil cover of landfills. The quantity of methane oxidized in this manner is the greatest uncertainty in landfill methane emission estimates.

Methane from sewage-treatment plants is very ${ }^{13} \mathrm{C}$ enriched, with ${ }^{13} \mathrm{C}$ values of $-46.0 \pm 5.0 \%$ and $-296.4 \% \pm 16$ for $D$ of hydrogen on methane.

\section{ANALYSIS OF CLIMATE VARIABILITY AND ITS RELATIONSHIP TO ATMOSPHERIC CARBON CONCENTRATIONS CHRISTY, JOHN R, and MCNIDER, RICHARD T. UNIVERSITY OF ALABAMA, HUNTSVILLE FY 199571 FY 19940}

Objectives: To examine the natural variability of the climate of the southeastern United States and its reproducibility in several GCMs, to determine the relationship between temperature/winds and $\mathrm{COx}\left(\mathrm{CO}_{2}\right.$ and $\mathrm{CO}$ ) concentrations for use in assessing global and regional variations, and to investigate the sensitivity of the terrestrial model of carbon production to our satellite-temperature input by comparing the model's results with observations.

Approach: The output from GCMs, integrated from 1979 to 1988 , will be directly compared with the satellite temperatures that began in 1979 as well as 
with other in situ data (e.g., surface hydrological variables). In addition, we will secure the output of at least two GCMs that have been executed for 100 years of increasing $\mathrm{CO}_{2}$ and examine their parameters of variability. Air-mass trajectories will be examined in terms of their pathways over COx sources and sinks with the objective of determining whether subsequent COx loading may differ during ENSO years. As an exploratory research effort, we will attempt to determine the annual variation in $\mathrm{CO}_{2}$ caused by anomalous circulations. We will prescribe the surface temperature of the ORNL Terrestrial Carbon Model from monthly satellite temperature anomalies since 1979 as a means to further constrain the system and compare model production rates with observations.

Results to Date: Initial examination of GCM output has revealed that simulated climate-parameter fluctuations (e.g., temperature) are greater in magnitude and of longer persistence than observed in the real world. Such behavior may be interpreted as significant droughts and/or heat and cold waves. We suspect that the factors that cause the actual climate to return to "normal" or "average" are not sufficiently represented in current GCMs.

We have accessed the Oak Ridge database for measurements of gas concentrations at various sites around the world and are updating this data set past 1991. The global temperature variations as measured by tropospheric temperature appear to be related more to the rate of change of $\mathrm{CO}_{2}$ (for example) than the absolute concentration of the gas. The Mauna $\mathrm{Laa} \mathrm{CO}_{2}$ growth rate anomalies tend to lead the global temperature anomalies by two months $(r=0.49)$, and growthrate anomalies at the South Pole lead Southern Hemispheric temperatures by one month $(r=0.46)$. It is too early to say whether these "leads" are significantly different from zero lag. In regional comparisons, several midlatitude temperature anomalies lead the Mauna Loa growth rate anomalies by 3 months.

\section{EXCHANGES OF ENERGY AND RADIATIVELY ACTIVE GASES BETWEEN SLASH PINE AND CYPRESS ECOSYSTEMS AND THE ATMOSPHERE IN THE SOUTHEASTERN UNITED STATES GHOLZ, HENRY L.

Objectives: To measure net ecosystem exchanges of energy, $\mathrm{H}_{2} \mathrm{O}, \mathrm{CO}_{2}, \mathrm{CH}_{4}$, and nonmethane hydrocarbons (NMHC) from a slash pine forest site (SP) over a range of phenological and environmental conditions with eddy covariance and conditional sampling; to compare energy, $\mathrm{H}_{2} \mathrm{O}$, and $\mathrm{CO}_{2}$ fluxes to those measured at an adjacent cypress-pond site (CP); to modify and validate existing models and develop new models to scale up process-level information obtained from chamber and cuvette measurements of vegetation and soil to estimate net ecosystem fluxes of $\mathrm{H}_{2} \mathrm{O}, \mathrm{CO}_{2}, \mathrm{CH}_{4}$, and NMHC; and to evaluate model predictions with net ecosystem exchange estimates.

Approach: Net ecosystem exchanges of energy, $\mathrm{H}_{2} \mathrm{O}$, and $\mathrm{CO}_{2}$ are measured with eddy covariance and those of $\mathrm{CH}_{4}$ and $\mathrm{NMHC}$ with relaxed eddy accumulation ("conditional sampling") at the SP and CP sites. Turbulence and energy exchange are measured with standard meteorological instruments and a sonic anemometer mounted above the canopy on towers; $\mathrm{H}_{2} \mathrm{O}$ and $\mathrm{CO}_{2}$ are measured; $\mathrm{CH}_{4}$ is measured using Tedlar bags or syringes and GC-FID; and NMHCs are adsorbed on TENAX adsorption tubes and analyzed with a GC-MS/GC-FID system. The SPM and a multilayer hydrology model (ETM) are being modified to incorporate chamber and cuvette flux measurements of $\mathrm{H}_{2} \mathrm{O}$, $\mathrm{CO}_{2}, \mathrm{CH}_{4}$, and NMHCs from vegetation, forest floor, and/or soil. The SPM and ETM are driven by meteorological and environmental measurements from the tower and soil layers. Model output will be validated with subsets of above-canopy measurements.

Results to Date: We have measured net ecosystem exchanges of energy, $\mathrm{H}_{2} \mathrm{O}$, and $\mathrm{CO}_{2}$ at the $\mathrm{SP}$ and $\mathrm{CP}$ sites over a range of meteorological and phenological conditions since May 1995. Net radiation (Rnet) balanced sensible and latent heat fluxes within $20 \%$. Mean evapotranspiration (ET) rates during dry-canopy conditions at the SP site were a nearly linear function of Rnet. Summer vs winter rates were strongly dependent on leaf area; ET rates were ca. $1.7 \pm 0.3 \mathrm{~mm} \mathrm{H}_{2} \mathrm{O}$ day-1 (mean $\pm 1 \mathrm{SD}$ ) in the summer and $1.1 \pm 0.3 \mathrm{~mm}$ $\mathrm{H}_{2} \mathrm{O}$ day-1 in the winter. Little effect of vapor pressure deficit (VPD) of the atmosphere on ET rates was noted. The ETM was used to predict the exchanges of energy and $\mathrm{H}_{2} \mathrm{O}$ from the $\mathrm{SP}$ site during the summer, and comparison with measured ET values indicated good agreement. Net $\mathrm{CO}_{2}$ exchange was strongly controlled by PAR during both summer and winter at the SP site, but rates were very low at the $\mathrm{CP}$ in the winter (leafless) period. Nighttime net $\mathrm{CO}_{2}$ exchange rates were a Q10 of about 2, but half-hour exchange rates were variable and reflected increased turbulence and periodic "ventilation" of the air space below the canopy. The SPM underestimated net ecosystem $\mathrm{CO}_{2}$ exchanges at night and also underestimated net $\mathrm{CO}_{2}$ exchange at the highest PAR values. These initial results are consistent with the facts that the biomass of live, respiring tissue was underestimated in the model, and that LAI values were underestimated because the understory was not explicitly modeled (summer LAI = 1.5). We have modified the SPM to incorporate these factors. NMHC-flux measurements have been made above the canopy throughout spring and summer 
of 1996. Mean midday net emission rates of pinene, isoprene, and total NMHCs were $34 \pm 3,53 \pm 3$, and $108 \pm 12 \mathrm{~g} \mathrm{~m}-2 \mathrm{hr}-1$ in the summer at the SP site and dropped to nearly zero at night.

\section{IMPACT OF ELECTRIC VEHICLES ON ELECTRIC POWER GENERATION AND GLOBAL ENVIRONMENTAL CHANGE HASKEW, TIM A., and LINDLY, JAY K. UNIVERSITY OF ALABAMA FY 199534 \\ FY 19940}

Objectives: To develop a computer-based model to accurately predict the net emissions (hydrocarbons, $\mathrm{CO}, \mathrm{NO}_{\mathrm{x}}, \mathrm{CO}_{2}$, and $\mathrm{SOx}$ ) impact of replacing major portions of internal-combustion-engine fleets with electric vehicles.

Approach: A database including vehicle miles per day, regional temperature ranges, average regional elevation, breakdown of vehicle types, and breakdown of vehicle age will be compiled from data of the Alabama Department of Transportation. The EPA software MOBILE5a will be used to provide vehicle emissions excluding carbon dioxide. $\mathrm{CO}_{2}$ emissions will be calculated by other empirical relationships. Based on vehicle energy output, average vehicle efficiency, and average electric vehicle efficiency, a geographic distribution of electric-vehicle-charging load will be determined (called the incremental load curve). This load curve will be superimposed onto the nominal utility loading curve to provide a net geographic load curve.

With utility system data from the Alabama Power Company, the load will be allocated to the various transmission system buses. With a complete system description and load profile, typical generation dispatch algorithms, such as load flow (LF), unit commitment (UC), economic dispatch (ED), and optimal power flow (OPF), will be employed to allocate the increased loading to generation facilities. Note that this load allocation considers economic, security, and environmental constraints. Hence, powerplant outputs can be computed, which is sufficient for computing generator emissions.

Results to Date: The transportation-fleet model is complete with all necessary data and emissions calculation software. For uniform electric vehicle penetration, the provided emissions will be prorated appropriately. For studies where specific vehicle fleets are replaced with electric vehicles, the emissions-model software must be re-executed.

Survey data for the City of Tuscaloosa, Alabama, indicates that case studies replacing specific vehicle fleets (like police, fire, etc.) may not warrant investigation because of the relatively low number of vehicle miles per day for these fleets compared to privatevehicle miles per day.

The Alabama Power Company system electrical and geographic data has been obtained and is being formatted. Software for economic dispatch is complete, and commercially available software is available for verification. Commercially available packages will be used prior to custom packages for other power system operation solutions, such as unit commitment. Much effort is presently being spent on determining the best manner to adequately represent neighboring utilities and regulated tie lines during case studies, a matter that is crucial to developing a realistic model.

\section{RESPONSES OF SOUTHEASTERN FORESTS TO INCREASED $\mathrm{CO}_{2}$ AND CLIMATIC CHANGE: EXPERIMENTAL TEST OF AN ECOSYSTEM MODEL AND ITS USE FOR IMPACT ANALYSIS HUNT, E. RAYMOND UNIVERSITY OF WYOMING FY 199522 FY $1994 \quad 0$}

Objectives: To assess the ecosystem simulation model BIOME-BGC for a deciduous forest ecosystem in conjunction with the Walker Branch Throughfall Displacement Experiment to see how well it predicts soil volumetric water contents over time, stable carbon isotope ratios $\left({ }^{13} \mathrm{C} /{ }^{12} \mathrm{C}\right)$, soil and stem respiration, and leaf nitrogen contents.

Approach: Qualitative tests will compare predictions with measured data for the ambient, wet, and dry plots. Quantitative tests will compare predictions with measured data over the seasonal cycle for each plot. With the IPCC "business-as-usual" scenario of increasing atmospheric $\mathrm{CO}_{2}$, the positive benefits of increased water-use-efficiency will be combined with various frequencies of drought to assess the impacts of changing climate in the southeastern United States. Then, a simple model of the interactions of atmospheric $\mathrm{CO}_{2}$, drought frequency, and the nitrogen cycle will be developed for use in integrated-assessment models.

Results to Date: The trend among the plots is the same for both measured and simulated results. Interestingly, red maple had the opposite measured trend in leaf nitrogen contents compared to the plot-wide data. Whereas this comparison suggests the model is adequate, the trends in the qualitative predictions may be correct for the wrong reasons. More data on the nitrogen cycle are necessary. Similar comparisons for the wet and dry plots had somewhat greater discrepancies, assuming a constant $30 \%$ of the precipitation was collected from the dry plot and routed to the wet plot. The simplest explanation is that the efficiency of precipitation collection and transport varied with each storm. In conclusion, the agreement between model 
predictions and data from the Throughfall Displacement Experiment is promising but not conclusive for determining the interactions of the carbon and nitrogen cycles during chronic drought.

\section{INTERACTIVE EFFECTS OF ELEVATED $\mathrm{CO}_{2}$ AND TEMPERATURE ON INSECT CONSUMERS OF FOREST TREES LINCOLN, DAVID E. UNIVERSITY OF SOUTH CAROLINA FY 199563$$
\text { FY } 19940
$$

Objectives: To study the direct effects of higher temperature and elevated $\mathrm{CO}_{2}$ on two widespread, polyphagous troe-feeding insects and their host trees in terms of the insects' patterns of consumption, growth, and development.

Approach: Sugar and red maple are being grown in 3.5-m-diam open-topped chambers at the Oak Ridge National Laboratory Field Research Site. Seedlings were planted in native soil in 1994 and exposed to one of four temperature/ $\mathrm{CO}_{2}$ treatments: ambient temperature/ambient $\mathrm{CO}_{2}$, ambient temperature/elevated $\mathrm{CO}_{2}$, elevated temperature/ambient $\mathrm{CO}_{2}$, or elevated temperature/elevated $\mathrm{CO}_{2}$. Two widespread lepidoptera (eastern tent caterpillar and gypsy moth) are being used to study whether long-term exposure of eastern tent caterpillar egg masses to elevated temperature in the open-topped chambers alter the critical timing between plant and insect phenology and whether potential $\mathrm{CO}_{2}$ and temperature effects on maple leaf phytochemistry affect the growth and consumption of gypsy larvae of different ages. These two approaches address different aspects of the global climate-change scenarios in the experimental design. Egg masses of the eastern tent caterpillar are collected from indigenous populations and placed within the chambers over winter. The timing of egg hatch with host-tree development is recorded. Gypsy-moth larvae are bagged directly onto plants within chambers, and the growth of and consumption by caterpillars are assessed. Levels of leaf nitrogen, nonstructural carbohydrates, and carbon-based phenolics will be related to insect performance.

Results to Date: Eastern caterpillar egg masses maintained in open-topped chambers throughout the dormant winter period at $+3.5 \mathrm{C}$ above ambient hatched 2.5 weeks earlier than those placed in ambient temperature chambers. Subsequent exposure to seasonally low temperatures resulted in very high mortality of early hatching larvae (i.e., those experiencing expected higher temperatures). The timing between egg hatch within the ambient temperature chambers and egg hatch of indigenous masses on native host trees was indistinguishable. Results from the gypsy-moth feeding experiments demonstrate that responses to the $\mathrm{CO}_{2}$ and temperature treatments are tree-species dependent. Third-instar larvae feeding on red maple grew significantly slower when reared on high- $\mathrm{CO}_{2}$-grown leaves $(\mathrm{P}<0.02)$, while temperature had no effect. A marginally significant interaction between $\mathrm{CO}_{2}$ and temperature $(\mathrm{P}<0.09)$ was the result of a large decrease in the growth of larvae feeding on elevated- $\mathrm{CO}_{2}$-grown leaves at ambient temperature. Leaf consumption was unrelated to either temperature or plant $\mathrm{CO}_{2}$ treatment, although a large increase in the consumption of leaf biomass by larvae feeding on elevated- $\mathrm{CO}_{2}$-grown leaves at elevated temperature resulted in a significant $\mathrm{CO}_{2}$-temperature interaction $(\mathrm{P}<0.05)$. Fourth instar larvae feeding on sugar maple had increased growth at elevated temperatures $(\mathrm{P}<0.05)$, but did not respond to plant $\mathrm{CO}_{2}$ growth condition. Consumption of leaf biomass followed a similar trend as growth.

\section{CARBON FLUXES IN A MANAGED PINE FOREST UNDER AMBIENT AND ELEVATED $\mathrm{CO}_{2}$ OREN, RAM DUKE UNIVERSITY \\ FY 1995101 \\ FY $1994 \quad 0$}

Objectives: To estimate sources, sinks, and fluxes of $\mathrm{CO}_{2}(\mathrm{Fc})$ under ambient and elevated atmospheric $\mathrm{CO}_{2}$ conditions.

Approach: The study site is a 15-year-old managed plantation of loblolly pine in Duke Forest, Durham, N.C. A 30-m-diam circular plot within the stand was arbitrarily selected to be exposed to elevated $\mathrm{CO}_{2}$ under otherwise ambient conditions with a free-air $\mathrm{CO}_{2}$ enrichment (FACE) tochnique. The $\mathrm{CO}_{2}$ concentration target of the operational volume of the plot was set at $550 \mathrm{ppm}$ as a conceivable point that atmospheric $\mathrm{CO}_{2}$ will reach by the middle of the 21 st century. From porometry measurements, A-Ci curves will be developed for different levels in the canopy and under a wide range of photosynthetically active radiation (PAR) and soil moisture content. From eddy-correlation water vapor $(\mathrm{Fq})$ and $\mathrm{Fc}$ flux measurements and the $\mathrm{CO}_{2}$ and water vapor concentrations, the ratio of water vapor flux to $\mathrm{CO}_{2}$ flux will be determined under ambient $\mathrm{CO}_{2}$ concentrations $(\mathrm{Ca})$ and a wide range of environmental conditions. From eddy-correlation Fq measurements and branch/stem Granier heat flux measurements, a relation between the Granier measurements and the stand-level water vapor flux will be established. From the above considerations and parameterization of the plant physiology and biophysical processes, the $\mathrm{CO}_{2}$ flux for elevated $\mathrm{CO}_{2}$ conditions can be estimated.

Results to Date: The Localized Near-Field Theory was developed, and preliminary testing was carried out with a two-day data set. Canopy-water-vapor-conduc- 
tance measurements for natural and elevated $\mathrm{CO}_{2}$ conditions were performed. Our measurements indicated that for a wide range of climatic and hydrologic conditions, elevated $\mathrm{CO}_{2}$ had no significant impact on stomatal conductance. However, canopy stomatal conductance was reduced by elevated $\mathrm{CO}_{2}$ to $80 \%$ of a reference stand. Leaf-level measurements (A-Ci curves) over a limited range of environmental conditions suggest that elevated atmospheric $\mathrm{CO}_{2}$ did not alter the functional relationship between $\mathrm{A}$ and $\mathrm{Ci}$.

\section{SOIL CARBON DYNAMICS IN A TEMPERATE FOREST AND ITS CULTIVATED COUNTERPART \\ POST, WILFRED M., and HARRISON, KEVIN G. THE UNIVERSITY OF TENNESSEE and DUKE UNIVERSITY \\ FY 199526 \\ FY 19940}

Objectives: To make measurements on soil samples and fit models to the measurements in order to understand soil carbon dynamics.

Approach:We are measuring the radiocarbon content in each horizon for two sets of soil samples, one from an uncultivated plot, the other from a cultivated field. Samples were collected in 1950, 1984, and 1994, a critical time sequence that allows the use of nuclearweapons-produced radiocarbon to determine carbon incorporation and turnover in soil. In addition, we are performing long-term (50-week) laboratory incubations of samples collected in 1994 to measure the carbon mineralization potential and associated firstorder rate coefficient, measures of the size and turnover of the most active soil carbon components, respectively. This study will result in measurements of active soil carbon turnover times and inventories for native temperate forests and cultivated temperate sites. An application of this result will be to examine how cultivation alters soil carbon dynamics for a native forest.

Results to Date: The field collections have been completed. There was apparently a problem with radiocarbon contamination of the sample in the laboratory during the processing of the samples. We will reprocess the samples in a different, radiocarbon-clean laboratory and rerun the radiocarbon measurements.

Additional samples were obtained from the soil inside open-topped chambers used in a 4-year exposure of white oak seedlings to ambient and elevated $(+150$, and $+300 \mathrm{ppm}) \mathrm{CO}_{2}$ concentration. Whole-plant mass was $58 \%$ higher $(+150)$ and $135 \%$ higher $(+300)$. Lateral woody roots were the most responsive. The purpose of this study was to see if the elevated carbon dioxide levels increased soil carbon storage. Since the experiment was optimized to study above-ground responses, leaves were not allowed to decompose and form soil organic material. This implies that the observed soil carbon $\mathrm{CO}_{2}$ fertilization factor represents a lower limit. We expect the soil carbon turnover time at this site to be faster than the 25-year time found from native soil because the plowing destroyed the soil's macrostructure. A similar recovering site in South Carolina that had been cultivated less recently had a 12-year turnover time. This faster soil carbon turnover allows the soil carbon to respond quicker to $\mathrm{CO}_{2}$ fertilization. If the time is faster than predicted, the measured $\mathrm{CO}_{2}$ fertilization factor will be smaller and vice versa. There was $5.2 \%$ more carbon in the soil surrounding +300 trees. This difference is significant at the $85 \%$ confidence level. The active soil carbon pool increased by $9.0 \%$. Using a $\mathrm{CO}_{2}$ fertilization model incorporating the above assumptions and observations, we calculate a soil-carbon $\mathrm{CO}_{2}$ fertilization factor of 0.30 for the period of the experiment. This means that $30 \%$ more carbon was retained by the active soil carbon pool in the +300 treatment chambers than in the control chambers.

\section{RESPONSES OF SOUTHEASTERN FOREST SPECIES TO ELEVATED ATMOSPHERIC $\mathrm{CO}_{2}:$ PATHOLOGY AND RELATED BIOTIC INTERACTIONS ABOVE AND BELOW THE GROUND \\ ROGERS, HUGO H.; RUNION, G. BRETT; MITCHELL, ROBERT J.; PRIOR, STEPHEN A.; AMTHOR, JEFFREY S.; and WILSON, MARK AUBURN UNIVERSITY and LAWRENCE LIVERMORE NATIONAL LABORATORY FY 199579 \\ FY 19940}

Objectives: To determine the effects of $\mathrm{CO}_{2}$ and nitrogen $(\mathrm{N})$ treatments on growth, nitrogen content, C:N ratio, carbohydrate content, and allelochemical content of plant tissues and to determine the effects of these changes on incidence and severity of diseases induced by obligate and facultative pathogens on beneficial microorganisms (i.e., mycorrhizal fungi and plant-growth-promoting rhizobacteria), and on phyllosphere epiphytic microbial communities and their interactions with foliar fungal pathogens.

Approach: This work, building on a three-year NIGEC effort that assessed the physiological responses of longleaf pine to elevated atmospheric $\mathrm{CO}_{2}$, will investigate community-level interactions (i.e., biotrophic interactions of microbes with host plants). This research will test the hypothesis that atmospheric $\mathrm{CO}_{2}$ enrichment will decrease the nitrogen content and increase the $\mathrm{C}: \mathrm{N}$ ratio of plant tissues resulting in increased production of plant allelochemicals which will differentially alter incidence and severity of diseases caused by obligate compared to facultative pathogens. 
Plant material (i.e., longleaf and loblolly pine seedlings, several species of oaks, wiregrass, and others) are being exposed to ambient ( $360 \mu \mathrm{mol} \mathrm{CO}$ mol-1) or elevated ( $720 \mu \mathrm{mol} \mathrm{CO} 2 \mathrm{~mol}-1$ ) $\mathrm{CO}_{2}$ within open-topped chambers. Nitrogen treatments ( 1 or $7 \mathrm{mM} \mathrm{NH_{4 }} \mathrm{NO}_{3}$ ) are being applied to plants within each $\mathrm{CO}_{2}$ treatment. The trees will be exposed to several pathogens (singly and in combination). Effects of $\mathrm{CO}_{2}$ and nitrogen on disease development will be assessed by monitoring time to symptom development, latent period (time to sporulation), sporulation (quantity of spores produced), sporulation period, and spore viability. For each disease system, we will assess changes in tissue quantity (biomass production and allocation) and quality (N, C:N, lignin, carbohydrates, and allelochemicals) resulting from treatment effects. Plant-tissue dry weights will be taken as a measure of photosynthate partitioning and analyzed for total carbon and $\mathrm{N}$. Carbon partitioning and nitrogen uptake will be calculated from plant carbon and nitrogen concentration/biomass weight relationships. Secondary metabolites will be assessed from fresh plant material. Total phenols, hydrolyzable tannins, condensed tannins, volatile leaf mono- and sesqui-terpene content, and lignin content of tissue will be quantified according to standard methods. Assessment of host plant physiological processes will also be conducted to determine physical $\left(\mathrm{CO}_{2}\right.$ and $\left.\mathrm{N}\right)$ and biological (microbe) treatment effects; photosynthesis and respiration will be measured using $\mathrm{LiCor} \mathrm{CO}_{2}$ gas-exchange systems. The effects of elevated $\mathrm{CO}_{2}$ and nitrogen on response of pines to plant-growth-promoting rhizobacteria (PGPR). Mycorrhizal colonization of fine roots will also be examined on plants within the disease and PGPR studies. We will examine ectomycorrhizae on pines and oaks, vesicular-arbuscular endomycorrhizae on wiregrass, and ericoid mycorrhizae on Vaccinium sp. to compare effects of increased $\mathrm{CO}_{2}$ on these various types of symbiotic microbial associations.

Results to Date: Both loblolly and longleaf pines were inoculated with the pitch canker fungus. Although this test was prematurely terminated because of an early freeze, no differences in disease incidence or severity were observed for either host. This test will be repeated.

Northern red oak seedlings were inoculated in the Spring of 1996 with the fusiform rust fungus. Preliminary data indicate reduced disease severity and increased developmental time because of elevated $\mathrm{CO}_{2}$. Basidiospores from infected oak leaves will be used to inoculate loblolly pine seedlings growing under test conditions.

Loblolly pine seedlings, inoculated with the fusiform rust fungus are currently being grown under ambient and twice-ambient $\mathrm{CO}_{2}$. Disease incidence, severity, and progression will be monitored during the coming year. A preliminary test with clonally propagated slash pine seedlings inoculated with the fusiform rust fungus and a study examining the interacting effects of elevated $\mathrm{CO}_{2}$ with three levels of nitrogen on loblolly pine seedlings inoculated with plant-growthpromoting rhizobacteria are also in progress.

\section{CONSEQUENCES OF ANTICIPATED GREENHOUSE WARMING IN THE SOUTHEAST}

\section{SAXENA, V. K.; YU, S. C.; MENON, S.; ANDERSON, J.; WENNY, B. N.; and BAHRMANN, $C$. \\ NORTH CAROLINA STATE UNIVERSITY \\ FY 199584 \\ FY 19940}

Objectives: To investigate the aerosol direct shortwave and longwave forcings by analyzing the climate data at $\mathbf{4 7}$ stations in the southeastern United States during from 1949 to 1994 and the stratospheric volcanic aerosol microphysical characteristics over the area for 1985 to 1994 and to investigate the role of sulfate aerosol and carbonaceous aerosol in altering the cloud albedo on the basis of experiments at a remote, regionally representative site in the free troposphere, namely Mt. Mitchell.

Approach: Monthly, seasonal, and yearly climate data for 47 stations in the southeast were obtained from the Office of the N.C. State Climatologist and the trends of climatological variables during the past 46 years were determined. The stratospheric volcanic aerosol microphysical characteristics over the area for 1985 to 1994 were inferred from the Stratospheric Aerosol and Gases Experiment (SAGE) II satellite extinction measurement. The aerosol radiative forcing is based on the fact that increased aerosol loading would decrease maximum daytime temperature from incoming radiation (shortwave forcing) and would enhance absorption and backscattering of outgoing radiation (longwave forcing), resulting in an increase of minimum nighttime temperature.

During the 1995 field experiment in Mt. Mitchell State Park, cloud-droplet sizes were measured with the Forward Scattering Spectrometer Probe (FSSP). The $\mathrm{pH}$ of the cloud water was immediately measured after collection, and cloud-water samples were subsequently prepared for later ionic analysis via ion chromatography. The $\mathrm{CCN}$ concentrations and the light-scattering extinction coefficient (bscat) were measured. The cloud albedo was inferred from the Advanced Very High Resolution Radiometer (AVHRR) data.

Results to Date: The arithmetic average of annual mean daily temperature for 47 stations in the Southeast has decreased by $0.09 \mathrm{C}$ during the past 46 years. The results are consistent with the expected shortwave and 
longwave radiative forcing caused by aerosols. According to theoretical analysis, the diurnal temperature range (DTR), which is defined as the difference between the maximum and minimum temperature, will decrease as the aerosol radiative forcing increases because of the decrease of maximum temperature and the increase of minimum temperature. It was found that there were dominant decreasing trends of mean DTR for annual and all seasons over the periods 1985-87 and 1992-94 compared to that of the period 1988-90. The aerosol forcing can have an effect on the daily temperature and precipitation depending on the season and regional environment.

A study of the sensitivity of cloud microphysical and optical properties to sulfate aerosols showed a positive nonlinear relationship between the cloud albedo and cloud water sulfate concentration in both cases. These results indicated that anthropogenic pollution over the continent could change the reflectivity of thin, nonprecipitating clouds.

A study of the CCN concentration at $1 \%$ supersaturation indicated that not all of the $\mathrm{CCN}$ were sulfate derived. As pointed out by Novakov and Penner, organic aerosols could make an important contribution to both total aerosol number concentration and the $\mathrm{CCN}$ fraction, even at a marine site.

\section{PLANT-CONSUMER ADJUSTMENTS TO CLIMATE-INDUCED RAINFALL MODIFICATION SHURE, DONALD J. EMORY UNIVERSITY FY 199554 FY 19940}

Objectives: To evaluate the changes in plant resource allocation to foliar nutrients and phenolic defenses following rainfall manipulation and to quantify the extent of insect herbivory from tree species experiencing wet, ambient, or drought conditions as part of the Throughfall Displacement Experiment.

Approach: The experiment was initiated on the Walker Branch Watershed at Oak Ridge in July 1993 using a gravity-driven transfer of $33 \%$ of total throughfall precipitation to create dry, ambient, and wet plots ( 0.64 ha each) within the watershed. We have been using seedlings, saplings and mature trees of white oak (Quercus alba), chestnut oak ( $Q$. prinus), and red maple (Acer rubrum). Twenty seven trees of each species were selected from within each 0.64-ha experimental plot (243 total trees). Extension pole cutters were used to obtain leaves from each tree in late August 1992 and in early July and late August of 1993, 1994, and 1995. The leaf samples are photocopied and then processed for chemical analysis. The photocopy of each leaf is digitized to determine total leaf area and the extent of damage by each major insect feeding guild, including strip feeders, pit feeders, leaf skeletonizers, leaf miners, leaf galls, and leaf rollers. Leaf chemical analyses include foliar nitrogen, total phenolics, hydrolyzable tannins, and condensed tannins. Total phenolic content is assayed using the FolinDenis technique, while condensed and hydrolyzable tannin assays are performed according to Bate-Smith procedures.

Results to Date: Treatment results in 1994 and 1995 have enabled us to test the degree of fit between weather-related (1992 to 1994) and treatment effects on plant-herbivore processes. The general absence of treatment differences in 1994 probably reflected the especially wet summer that prevented soil-moisture reductions on the dry plot from affecting plant foliar chemistry. Nevertheless, insect herbivore damage from all three tree species was lower in dry than wet plots because of reduced feeding by skeletonizers (oaks) and strip feeders (maples) across this treatment gradient.

Treatment effects in 1995 closely fit the patterns developed during our initial three-year study. Maple trees contained lower foliar nitrogen concentrations in dry than wet plots during the early stages of drought in July 1995. Total phenol and condensed tannin concentrations in all species were also reduced on the dry treatment plot and somewhat elevated on the wet plot in early July. Soil-moisture deficits on the dry plot in early summer were apparently sufficient to restrict overall carbon allocation to plant phenolics or limit the synthesis of phenolic precursors. No consistent treatment effects were evident for hydrolyzable tannins. Interestingly, the early treatment differences in 1995 were partially offset as further drought produced severe soil water deficits on all treatment plots during August. However, condensed tannin levels in oaks and maples and foliar nitrogen concentrations in maples remained somewhat lower in dry than wet plots by the end of August. These findings thus indicate that forest tree spocies exhibit complex but predictable adjustments in their foliar chemistry at varying intensities of soil moisture deficits.

\section{EFFECTS OF GLOBAL ENVIRONMENTAL CHANGE ON NATIVE AND EXOTIC NITROGEN-FIXING TREES OF SOUTHERN FLORIDA \\ THOMAS, RICHARD B., and SASEK, THOMAS WEST VIRGINIA UNIVERSITY and NORTHEAST LOUISIANA UNIVERSITY FY 199598 \\ FY 19940}

Objectives: To determine the responses of two exotic and two native nitrogen-fixing trees from southern Florida to $\mathrm{CO}_{2}$ enrichment and temperature increase, particularly in regard to carbon fixation, respiration, nitrogen fixation (using $15 \mathrm{~N}$ isotope analysis), nutrient 
uptake, and allocation patterns of carbon and nitrogen and to determine how these responses may be influenced by resource availability (nutrients, water, and light).

Approach: This year, the interactive effects of $\mathrm{CO}_{2}$ enrichment, temperature, and nutrient availability $(\mathrm{N}$ and $\mathrm{P}$ ) are being evaluated. In the second year, the interactive effects of $\mathrm{CO}_{2}$, temperature, and water stress will be examined. In the third year the interactive effects of $\mathrm{CO}_{2}$, temperature, and light availability will be examined. Each year, tree seedlings are grown for approximately 4 months from June through September in the Duke University Phytotron in a $2 \times 2 \times 5$ factorial design $\left(\mathrm{CO}_{2} \times\right.$ temperature $\times \mathrm{N}: \mathrm{P}$ availability) using disinfected coarse sand as the soil media. Phytotron growth chambers are being used to accommodate the four combinations of $\mathrm{CO}_{2}$ and temperature. Carbon dioxide is being maintained at 350 or $700 \mathrm{ppm}$. Temperature treatments represent current ambient from May to September, adjusting mean day/night temperature every 2 weeks, and ambient $+4 \mathrm{C}$. Within each chamber, subplots are used to vary resource-availability treatment combinations. Five ratios of soil nitrogen to phosphorus fertilizer are being used in all $\mathrm{CO}_{2}$ and temperature combinations, varying either nitrogen or phosphorus from zero to nonlimiting levels. Once plant nitrogen is partitioned between $\mathrm{N}_{2}$-fixation and root uptake, these measurements will allow us to calculate specific absorption rates (SAR) of nitrogen from the soil and nitrogenfixation rates will be calculated from stable-isotope analyses.

Results to Date: New project.

\section{IMPLEMENTATION OF A REGIONAL SYSTEM FOR THE DIFFUSION OF GLOBAL ENVIRONMENTAL CHANGE THROUGH IN-SERVICE TRAINING OF GRADE SCHOOL TEACHERS \\ WEAVER, DAVID , and SHANKMAN, DAVID UNIVERSITY OF ALABAMA FY 199528 \\ FY 1994 0}

Objectives: To implement an effective regional system for the diffusion of global-environmental-change education that is efficient (low cost) relative to the benefits achieved through the diffusion process.

Approach: The program consists of a two-week summer institute for in-service teachers of social studies and science grades 4 through 12 . The institute on Issues in Global Environmental Change is planned according to a standard formula developed by the National Geographic Society for its National Geographic Alliance Network of a two-week residential experience involving a mix of content lectures, classroom-activity demonstrations, field trips, and resource demonstrations. Participants are drawn from a broad area of the southeastern United States.

Results to Date: Two institutes have been held to date, one in June 1994 and the second in June 1996. In 1994, 21 teachers from eight states were trained. In 1996, 23 teachers attended the institute, also from eight states. In addition to a series of content lectures on global-change topics, each teacher received a substantial package of suggested teaching activities as well as supporting resource materials. The primary new text and resource materials for 1996 were "Science for Understanding Tomorrow's World: Global Change" produced by the Education in Global Change Project of the International Council of Scientific Unions, and the Global Systems Science Series developed by Lawrence Hall of Science.

\section{INCREASING ACCURACY OF CLIMATE- CHANGE MODELS BY APPLICATION OF LIPID-MOLECULAR TECHNIQUES WHITE, DAVID C. THE UNIVERSITY OF TENNESSEE FY 199580 FY 199493}

Objectives: To define characteristics of the belowground microbiota in terms of viable biomass, community composition, and physiological status resulting from exposures to elevated atmospheric $\mathrm{CO}_{2}$; these characteristics are to be measured in situ independent of the necessity for culture and isolation of the organisms.

Approach: A variety of plant rhizospheres will be analyzed so a comprehensive database of belowground microbial (both prokaryotic and eukaryotic) characteristics can be established. In addition, individual species will be examined under a variety of conditions (i.e., nutrient supplements, moisture content of the soil, $\mathrm{pH}$ differences, temperature differences, and growth stage) to gain an understanding of how these parameters interact with exposures to elevated atmospheric $\mathrm{CO}_{2}$.

The ability to quantitatively measure microbial communities in situ requires that a chemical approach be taken. To date, the most quantitative and comprehensive assay for the assessment of microbial communities in situ entails the use of lipid biomarkers. By applying this technology to the rhizospheres and bulk soils of specific plants, we are able to describe microbial communities in terms of both abundance and community composition. In addition, we are able to quantitatively describe the impacts of different treatments, nutrients, water stress, growth stage, and $\mathrm{CO}_{2}$ level, on belowground bacterial and mycorrhizal communities.

Results to Date: In white oak, rhizosphere-associated microbiota increased in cell numbers (per unit area) as levels of atmospheric $\mathrm{CO}_{2}$ increased, coinciding with 
an increase in fine-root mass density. There was, however, no significant increase in prokaryotic biomass per gram of (white oak) fine-root material. The prokaryotic communities in rhizosphere and nonrhizosphere soils showed shifts in composition as a result of exposure to elevated atmospheric $\mathrm{CO}_{2}$, but the specific natures of the shifts are still unknown.

Nitrogen and moisture content both have significant impacts on belowground microbial abundance (about a threefold increase) and composition (shift towards increased Gram-negative abundance) in association with long leaf pine. These observations coincided with microbial-respiration measurements. An impact of elevated atmospheric $\mathrm{CO}_{2}$ on the belowground populations was also identified (as a shift in community composition), again pointing towards an increase in the relative percentage of a signature biomarker descriptive of the Actinomycete sp.

Increased incubation temperatures effected changes in microbial community composition (of aspen rhizospheres), providing an explanation for the discrepancy between modeled rates of microbial respiration and actual measured rates. High- and loworganic carbon soils were also shown to contain differing extant microbial communities.

The application of signature-lipid biomarkers (PLFA and sterols) to the identification of mycorrhizal species corresponds to identifications based on classical taxonomy. It was also shown that a single mycorrhizal species inoculated into sorghum mats and sterile soils could be monitored by assessing changes in the overall patterns of PLFA and sterols.

\section{Midwest Regional Center}

\section{MAXIMUM AND MINIMUM TEMPERATURE VARIATIONS IN A PERTURBED CLIMATE AND IMPLICATIONS FOR SPECIALIZED AGRICULTURE IN THE GREAT LAKES REGION \\ ANDRESON, JEFF, and WINKLER, JULIE MICHIGAN STATE UNIVERSITY \\ FY 199570 \\ FY 199474}

Objectives: To develop and evaluate empirical transfer functions for generating local scenarios of daily maximum and minimum temperature for six representative stations in the Great Lakes region; to apply the transfer functions to simulations of free-atmosphere variables from a specific GCM to develop a set of plausible local climate change scenarios; and to evaluate, using the local scenarios, the potential impacts on specialized agriculture in the Great Lakes region, including changes in the length of the growing season, annual and seasonal growing-degree-day accumulation, and the frequency of extreme warm and cold temperatures.

Appraach: The empirical transfer function methodology used here is a modification of that of Karl et al., whereby daily series of free-atmosphere variables are related, via regression techniques, to local temperature. The underlying assumption is that GCMs better simulate free-atmosphere variables than surface variables. For each station, transfer functions are the 1970 to 1989 daily series of 500 -mb-height and sealevel pressure (i.e., the predictor variables), interpolated to the station location, and local maximum and minimum temperatures (i.e., the predictands). The functions are then applied to 10-year $1 \times \mathrm{CO}_{2}$ and $2 \mathrm{x}$ $\mathrm{CO}_{2}$ simulations of the predictor variables from the second-generation Canadian Climate Centre Model. This model was selected because it includes a full diurnal cycle, and the accuracy of the $1 \times \mathrm{CO}_{2}$ simulations of the predictor variables has been documented. Agriculturally relevant temperature indices, including the dates of first and last frost, growing season length, frequency of extreme temperatures, and base $5^{\circ} \mathrm{C}$ and $10^{\circ} \mathrm{C}$ growing-degree-day units, are calculated from the temperature scenarios. Differences between the perturbed and control scenarios in the mean and median values of these indices are used to assess the possible impacts of climate change on specialized agriculture.

Results to Date: At all stations, the transfer functions (TFs) explained between $61 \%$ and $86 \%$ of the temperature variation and accurately reproduced the daily temperature series for the validation period (1960 to 1969). When the TFs were applied to the CCC GCM $1 \times \mathrm{CO}_{2}$ simulations of the predictor variables, the ensuing temperature series more closely corresponded with present-day climatology than did the raw GCM temperature series. These results indicate that the TF approach is capable of incorporating local effects, including the impact of the Great Lakes, on temperature.

Downscaling CCC GCM simulations to locations within the Great Lakes regions with the TFs suggests that:

- The median date of last spring freeze will occur approximately 17 to 34 days earlier than at present. A similar shift to a later date (18 to 39 days) is projected for the first fall freeze. As a result, a 
substantial increase ( 45 to 67 days) is projected in the median growing-season length.

- A modest increase (0 to 21 days) in the number of days with potentially damaging high temperatures (i.e., $>35^{\circ} \mathrm{C}$ ) is projocted, whereas a large decrease ( 37 to 73 days) in the frequency of $<0^{\circ} \mathrm{C}$ temperatures is suggested.

- Base $5^{\circ} \mathrm{C}$ annual growing-degree-day (GDD) accumulations increase by 948 to 1547 units, with the largest increases occurring in the southern Great Lakes region. Increases in base $10^{\circ} \mathrm{C}$ GDD accumulation range from 716 to 1244 GDDs.

\section{EFFECT OF CLIMATE CHANGE ON SPECIES DISTRIBUTION, DIVERSITY, AND PRODUCTIVITY IN GRASSLAND FOREST HABITATS: A REGIONAL PERSPECTIVE CAMERON, GUY N. UNIVERSITY OF HOUSTON \\ FY 199572 \\ FY 199481}

Objectives: To obtain a qualitative sense of the direction of change in a number of different ecosystem components, allowing us to better elucidate the net effoct that climatic change is likely to have on ecosystems as a whole.

Approach: We use GIS to assess plant productivity and animal distributions in Texas under doubled $\mathrm{CO}_{2}$ conditions predicted by the Canadian Climate Centre and Geophysical Fluid Dynamics Laboratory R30 models. Current vegetation patterns are determined from the Holdridge classification scheme and longterm climatic data from 354 weather stations. Digitized maps of mammalian distributions are overlaid on the vegetation maps and corrected for the ecological requirements of each species. Spatial patterns of habitats are determined for current climate, for the wetter GFDLR30 scenario, and for the drier CCC scenario. Habitats are analyzed separately, after grouping into broad community types (e.g., forest and grassland) and after grouping by environmental categories (e.g., tropical and temperate). Productivity is estimated by relating patterns in NDVI (normalized differential vegetation indices, determined from EROS satellite imagery) to productivity calculations (with conversions from the weather station data).

Community composition is analyzed for 57 rodent species because rodents represent a diverse taxon whose vagility and size promote associations with habitats. Our primary metric is the proportion and area of communities of a given species diversity occupied by a particular species. Occurrences are then analyzed under each individual scenario, and changes in species composition resulting from climate change are identified. We also test for the effects of life-history variables on these distributions.
Results to Date: Although different climates changed the distribution and composition of habitats substantially relative to current conditions, no commensurate differences in fragmentation or relative isolation were observed. Our analyses demonstrated that, at some scales, changing environments may produce turnover in the constituent communities of landscapes yet leave spatial patterns unaffected. Thus, the impact of climate change on a species may depend on whether its distribution is influenced primarily by spatial pattern or by composition of habitats. The way species are distributed across a spatial gradient in species diversity is dependent on life-history characteristics of the species. However, the specific relationship between distribution and life history depends on both climate and the way in which a distribution is measured. The importance of particular species to the communities they compose are generally similar under divergent climate scenarios, while the importance of communities of different diversities for particular species are much more sensitive to environment. The pattern of species' responses to environmental change is not governed strictly by habitat associations, however. Many species that occupy different habitats exhibit similar spatial trajectories under different environments. However, at this scale community-level patterns were more variable, since community-weighted areas exhibited much more variable spatial trajectories across the climate scenarios. Thus, the impact of climate change will depend on the scale of interest, and characteristics other than habitat associations may be strong predictors of species responses to climate change.

We have created raster images of the NDVI data from our study area based on three years and from two seasons. These data will allow us to estimate the stability of our predictions as well as the sensitivity of the different ecoregions to environmental change.

\section{CLIMATE-CHANGE EFFECTS ON MIDWESTERN AGRICULTURE: \\ EVALUATION OF FIRM-LEVEL IMPACTS AND POTENTIAL ADAPTATION AND MITIGATION STRATEGIES \\ DOERING, OTTO; MAZZOCCO, MIKE; RANDOLPH, J.C.; LOWENBERG-DEBOER, JESS; and PFEIFER, REBECCA \\ PURDUE UNIVERSITY, UNIVERSITY OF \\ ILLINOIS, and INDIANA UNIVERSITY \\ FY 1995121 \\ FY 19940}

Objectives: To produce crop-production and ecosystem models for representative upper-midwest farms; to evaluate optimal farm decisions regarding cropping patterns, irrigation, drainage, nitrogen fertilization, seed technology, and tillage practices in response to climate change; to evaluate the effects of alternative 
policy constraints and incentives on selected farm practices and their effects; and to evaluate farm-firm adaptation strategies regarding climate change, including crop insurance, crop subsidies, and subsidization of new technology.

Approach: Farm firms representing likely future (2050) agricultural production units will be modeled for each region. CENTURY will be used as the initial climate/production modeling tool, and the Purdue crop/livestock linear-programming farm-management model will be used for the farm-level decision analysis. Output from these crop-production and ecosystem models will be used to produce economic-production models based on climate, management decisions, and other inputs. Production and decision models will be altered to incorporate decision constraints, and the economic performance of the representative farms will be compared to their unconstrained performance. The optimal decision set for each farm firm will be evaluated for its environmental impacts with the ecosystem models. Basic environmental concerns will be checked to ensure that adopted practices or different cropping systems under climate-change characteristics still meet basic agricultural environmental guidelines.

Results to Date: Ten unique/representative Great Lakes cropping regions have been identified and GIS mapped. Data for counties within the study area have been collected and developed. CENTURY 4.0 has been applied at the county level to these regions to calculate preliminary crop yields under climate change. These crop-yield outputs have been studied to see how the management strategies used in the crop/livestock models will be incorporated into future Century 4.0 model runs. Representative farm sites have been identified for all the regional locations. The resource/structure bases of representative, existing Illinois commercial farms have been analyzed and used to construct farms with characteristics for 2050; preliminary runs have been made on economicdecision models for these sites to identify potential resource constraints. The projection has begun of critical climate-based factors (e.g., number and timing of good field days and early and late frost dates) that are critical to farm decisions and cropping systems.

\section{CONTRASTING SUCCESSIONAL RATES AND CARBON CYCLING IN EUTROPHIC AND OLIGOTROPHIC ECOSYSTEMS OF AMAZONIA \\ MORAN, EMILIO, and RANDOLPH, J. C. INDIANA UNIVERSITY \\ FY 1995155 \\ FY 1994169}

Objectives: To compare deforestation and ecological succession in the Brazilian Amazon and to elucidate the role of these processes in the global carbon cycle.
Approach: Biomass sampling was carried out in $\mathbf{4 8}$ plots in 12 successional forest stands representing early, intermediate, and late successional ages at our two oligotrophic study sites. An additional 150 plots in 15 successional forest sites in the same regions were sampled during summer 1995. From these samples, we have determined standing-crop biomass and carbon content (and are well along in determining nitrogen, $P$, $\mathrm{K}, \mathrm{Ca}$, and $\mathrm{Mg}$ content) for the major vegetation compartments, aggregated by morphology, by size class, by litter, and by roots at three soil depths. With a portable photosynthesis analyzer, photosynthetic rates of 20 dominant successional tree species were measured under a variety of conditions. Related measurements include diurnal characterization of total solar radiation, photosynthetic photon flux density, air and soil temperatures, atmospheric carbon dioxide concentrations, vapor pressure, soil moisture, and soil respiration.

Results to Date: Leaf biomass is the dominant aboveground compartment at younger sites, whereas bole biomass becomes the dominant above-ground compartment with increasing site age. Biomass and carbon content of understory vegetation and small trees is significant in early successional sites. After about 5 years, the understory biomass and carbon pool is minor. With increasing site age, the proportion of carbon sequestered in trees increases, although even in relatively old sites it remains less than the carbon content of the soils. There is a slight increasing trend in the total carbon pool with increasing site age. Carbon sequestration of early successional forest is significant in amounts and may in some cases exceed the sequestration of older secondary forests and even mature forests.

Landsat Thematic Mapper satellite data show significant regrowth after deforestation even in nutrient-poor areas and even under conditions of one century of land use. Soil fertility is a key discriminant between rates of regrowth in Amazonia. Deforestation in nutrient-rich areas such as those where eutrophic alfisols may be found, while regrettable, may result in rapid sequestration of carbon, without loss of the belowground carbon stocks which are significant in these areas as well. Deforestation in nutrient-poor oxisols has a middling impact mediated a great deal by the forms of land use implemented after deforestation. Deforestation in extremely nutrient-poor spodosols leads to the slowest rates of regrowth, but even here the rates are surprisingly robust, even after floristic impoverishment due to a century of land use. These differences need to be taken into account in recalculating GCMs. 


\author{
COMPARATIVE ANALYSIS OF CARBON \\ BUDGETS IN SUCCESSIONAL FORESTS \\ RANDOLPH, J. C. \\ INDIANA UNIVERSITY \\ FY 199561 \\ FY 19940
}

Objectives: To improve understanding of the global carbon cycle by conducting a gradient analysis of carbon budgets in deciduous broadleaf forests in early stages of secondary succession under current climate conditions.

Approach: The project quantifies pool sizes and flux rates of carbon in vegetative, litter, and soil compartments in similarly aged successional forests across a latitudinal gradient ranging from northern Michigan to eastern Brazil. A preliminary analysis is being conducted of hardwood forests in southern Indiana. Carbon content of vegetation is determined by compartment (leaves, stems, boles, and roots) for woody and herbaceous plants, standing dead and litter, and soils; and soil nutrients (N, P, K, Ca, and $\mathrm{Mg}$ ) are determined. Plot data are collected in identically aged stands at locations differing in slope aspect, slope steepness, and slope position and also among stand groups, each composed of a number of identically aged stands. To ensure constant stand age, only clear-cut regrowth with a known date of origin was sampled. A randomly located, nested plot design was used: larger trees $(\mathrm{dbh}>7 \mathrm{~cm})$ were sampled in $150 \mathrm{~m}^{2}$ plots and saplings (dbh 1 to $7 \mathrm{~cm}$ ) in $10 \mathrm{~m}^{2}$ subplots. Within the subplots, $1-\mathrm{m}^{2}$ areas were selected randomly for complete harvest, including litter and soil compartments. Forty-eight litterfall trays $\left(0.5-\mathrm{m}^{2}\right.$ catchment) were placed randomly along transects stratified by slope aspect and aligned to coincide with established plots. Wherever possible, litterfall trays were positioned at the center of large plots.

Results to Date: Eighty pairs of large $\left(150 \mathrm{~m}^{2}\right)$ and medium $\left(10 \mathrm{~m}^{2}\right)$ plots were established in four groups of identically aged younger stands $(13,24,28$, and 54 years old). Consumptive sampling was performed at 16 small plots; each small plot required approximately 15 person-hours in the field. For eight of these, a full suite of physical and chemical analyses were performed in the lab. A global positioning system (GPS) receiver integrated with a geographical information system (GIS) was employed to identify real-world coordinates of all plot and litter-tray locations. To facilitate analysis, all data are stored in a relational database. Standard statistical techniques (ANOVA, regression) are being used to examine the significance of environmental variables.

\section{TRACE GAS FLUXES $\left(\mathrm{N}_{2} \mathrm{O}, \mathrm{CH}_{4}\right.$, AND $\left.\mathrm{CO}_{2}\right)$ IN AGRICULTURAL ECOSYSTEMS: BIOSPHERIC FEEDBACKS \\ ROBERTSON, G. P., and PAUSTIAN, $K$. MICHIGAN STATE UNIVERSITY \\ FY 1995115 \\ FY 1994131}

Objectives: To provide a more complete understanding of the role that row-crop agriculture plays in the production of radiatively active trace gases.

Approach: Extensive measurements will be made of fluxes in a series of crop and native communities representative of major row-crop systems (cornsoybean-wheat rotations in conventional tillage, no-tillage, and low-chemical-input tillage systems) vs fluxes in adjacent never-tilled communities in native vegetation. Intensive measurements will be made of fluxes in row-crop communities. Existing biogeochemical models will be parameterized to allow prediction of fluxes in response to long-term management. Extensive measurements will be based on weekly to biweekly samplings in large replicated field plots and native communities within the KBS Long-Term Ecological Research site. Intensive measurements will be based on a near-continuous in situ trace flux measurement system developed for this study.

Results to Date: Results from the past 3 years suggest a large historical effect of cultivation on both $\mathrm{N}_{2} \mathrm{O}$ and $\mathrm{CH}_{4}$ fluxes. Methane uptake continues to be severely depressed in cultivated treatments relative to rates in forested, never-tilled sites on the same soil series. This flux change appears to be independent of fertilizer- $\mathrm{N}$ application and instead to be related to a historical effect of cultivation itself. In contrast, $\mathrm{N}_{2} \mathrm{O}$ fluxes appear to increase along this gradient, again more in response to cultivation itself than in response to fertilizer application, suggesting that global budgets for $\mathrm{N}_{2} \mathrm{O}-\mathrm{N}$, usually constructed on the basis of some fraction of fertilizer- $\mathrm{N}$ converted to $\mathrm{N}_{2} \mathrm{O}$, are underrepresenting the impact of agriculture on global $\mathrm{N}_{2} \mathrm{O}$ fluxes. Measurements of fluxes in replicated fields suggest that $\mathrm{N}_{2} \mathrm{O}$ production is 50 to $100 \%$ higher in cultivated systems than in adjacent native vegetation.

Laboratory experiments show that $\mathrm{CH}_{4}$ uptake is sensitive to soil moisture but, within the agricultural communities, relatively insensitive to soil nitrogen. Our intensive flux-measurement system shows high temporal variability for $\mathrm{N}_{2} \mathrm{O}$ and $\mathrm{CH}_{4}$ fluxes, with fluxes varying by up to $100 \%$ within the same day. Such variability appears to be an important missing consideration in the construction of local $\mathrm{N}_{2} \mathrm{O}$ flux budgets for agricultural systems. 


\section{CLIMATE FORCING BY AEROSOL PARTICLES IN THE MIDWESTERN UNITED STATES \\ ROOD, MARK J. \\ UNIVERSITY OF ILLINOIS \\ FY 199580 \\ FY 1994130}

Objectives: To provide reliable measurements of ambient aerosol particle properties to reduce uncertainties in the estimated radiative forcing by ambient aerosol particles and to compare the measurements with appropriate models to validate the measurements and the models and quantify the uncertainties.

Approach: Aerosol size and particle composition, total light-scattering and backscattering coefficients, and light-absorption coefficient will be measured at a site near Bondville, Ill., an anthropogenically perturbed continental site. Ambient aerosol is sampled through a 10-m-high sampling stack. A small flow of $1 \mathrm{Lpm}$ of the aerosol passes through a condensation nuclei counter (CNC). In the first path, the aerosol stream flows through (1) impactors that characterize properties of submicrometer and coarse-diameter aerosol particles and (2) an integrating nephelometer with a backscatter shutter that measures total light-scattering and backscattering coefficients as a function of controlled RH. A second aerosol stream passes through a $1-\mu \mathrm{m}$ cutpoint impactor and Teflon filters. These filters are measured gravimetrically and analyzed chemically with ion chromatography to determine soluble $\mathrm{Na}^{+}, \mathrm{K}^{+}, \mathrm{Mg}^{2+}, \mathrm{Ca}^{2+}, \mathrm{NH}_{4}^{+}, \mathrm{SO}_{4}^{2-}, \mathrm{Cl}^{-}, \mathrm{Br}^{-}$, and $\mathrm{NO}_{3}{ }^{-}$. A third sample stream passes through a continuous light absorption photometer to measure light absorption coefficients. And a fourth stream passes through a low-pressure seven-stage impactor, which is used during intensive sampling periods to measure ambient aerosol mass size distributions. A thermodynamic equilibrium model, SEQUILIB, was modified to accommodate the experimental input from the monitoring station.

Results to Date: For the dry (RH 40\%) aerosol, the mean total mass concentration and standard deviation is $12.6 \pm 8.0 \mu \mathrm{g} / \mathrm{m}^{3} . \mathrm{NH}_{4}^{+}$and $\mathrm{SO}_{4}^{2-}$ typically constitute about $95 \%$ of the identifiable mass. The inorganic mass is $52 \pm 14 \%$ of the total dry gravimetric mass. The daily geometric average of the reference lightscattering coefficient for the green wavelength of is $4.27 \mathrm{e}^{-5} \mathrm{~m}^{-1} \pm 3.55 \mathrm{e}^{-5} \mathrm{~m}^{-1}$, and the arithmetic mean condensation nuclei count is $6470 \mathrm{~cm}^{-3} \pm 2820$ particles $/ \mathrm{cm}-3$. The mass size distributions are typically bimodal with mean diameters of $0.3 \mathrm{~mm}$ and $2 \mu \mathrm{m}$. $\mathrm{SO}_{4}{ }^{2-}$, and $\mathrm{NH}_{4}^{+}$were typically found in the smaller mode. $\mathrm{Na}^{+}, \mathrm{K}^{+}, \mathrm{Ca}^{2+}, \mathrm{Mg}^{2+}, \mathrm{Br}$, and $\mathrm{Cl}^{-}$were observed in the larger.

For wet aerosol particles, the arithmetic mean $\mathrm{f}(\mathrm{RH})$ values for the 80 to $85 \% \mathrm{RH}$ range and the green wavelength of light that correspond to eight days of sampling and 126 humidograms are $2.19 \pm 0.87$ and $1.18 \pm 0.34$ for light scattering and backscattering, respectively. Mean arithmetic $b$ values as a function of $\mathrm{RH}$, for the same time period are $0.15 \pm 0.03$ and $0.08 \pm 0.01$ for $\mathrm{RH}<40 \%$ and $80 \%<\mathrm{RH}<85 \%$, respectively. Overall, b decreases as a function of $\mathrm{RH}$, as predicted with Mie light-scattering theory. The part of the gravimetric mass that is not characterized by IC appears to contribute to light scattering by the ambient aerosol. Therefore, accurate quantification of its properties is expected to bring better agreement between the measurements and the models.

\section{AN INTEGRATED ASSESSMENT OF THE ECONOMIC, ECOLOGIC, AND ENERGY DEMAND IMPACTS OF GLOBAL CLIMATE CHANGE ON THE GREAT LAKES BASIN RUBIN, BARRY, and OSTER, CLINTON INDIANA UNIVERSITY}

FY 1995124

FY 1994129

Objectives: To develop and test a method for undertaking an integrated assessment of the regional economic impacts of global climate change; to identify the specific economic and energy-demand impacts of and potential adaptations to global climate change for the Great Lakes region; and to provide a framework for developing and evaluating public policy designed to mitigate the negative effects of regional climate change.

Approach: This research evaluates the regional socioeconomic and energy demand consequences of global climate change in terms of a large number of conomic activity and resource consumption measures. Such indicators include gross regional product, agricultural and industrial output, employment, unemployment, wages, income, population growth, and energyresource usage with an econometric model. Once it provides accurate estimates of endogenous variables over the sample period, the model will be used to produce a baseline scenario and a climate-change scenario. In the baseline scenario, the exogenous climate variables will remain relatively constant over the forecast period. In the climate-change scenario, exogenous climate variable values will be derived from GCM forecasts under doubled atmospheric carbon dioxide levels. Comparison of the endogenous-variable output produced by each scenario will reveal the anticipated socioeconomic effects of climate change, given prevailing economic relationships.

Results to Date: A working forecast model has been produced. The model indicates that the variables that tend to be economically and politically most critical (total employment, total average wages, annual unemployment rate, per capita income, gross state product, 
returns to capital resources, returns to labor resources, farming employment, and manufacturing employment) are changed by no more than $3.7 \%$ by 2010 under the climate-change scenario. The model also projects that several sectors will experience significant gains in economic activity at a relatively small expense to numerous other sectors. State and local government employment is expected to increase by $6.7 \%$, and total government employment is predicted to increase by $6.0 \%$ under the climate-change scenario. Agriculture, forestry, and fisheries employment is projected to grow by $11.2 \%$ under the climate-change scenario. Relatively smaller decreases in economic activity are projected in most other sectors.

Policy analysis with the model indicated that a $15 \%$ tax levied on aggregate energy prices and then on fuel oil would substantially impact farming, mining, transportation, public utilities, and total energy consumption; broader measures, such as gross state product, returns to labor, and returns to capital would not be substantially affected by the policy.

\section{ABOVEGROUND AND BELOWGROUND ECOSYSTEM RESPONSES TO ELEVATED ATMOSPHERIC $\mathrm{CO}_{2}$ TEERI, JAMES

Objectives: To understand the extent to which forest ecosystems will store more or less carbon as atmospheric $\mathrm{CO}_{2}$ increases and whether soil nitrogen availability is the primary factor regulating changes in net primary productivity in response to increased atmospheric $\mathrm{CO}_{2}$.

Approach: An array of 20 large open-topped chambers ( $2.4 \mathrm{~m}$ tall and $3 \mathrm{~m}$ in diameter) at the University of Michigan Biological Station is used to investigate the interactive effects of atmospheric $\mathrm{CO}_{2}$ and soil nitrogen availability on the cycling of carbon and nitrogen between plants and soil microorganisms. Factorial treatments of atmospheric $\mathrm{CO}_{2}$ (ambient and twice-ambient) and soil nitrogen availability (low and high) are arranged in a randomized complete block design with each treatment combination replicated five times.

Each open-topped chamber/root box contain 12 saplings propagated from root cuttings of 6 local $P$. tremuloides genotypes (two individuals of each genotype). In the summer of 1996, the trees were in their third growing season. The following measurements were performed prior to final harvest: leaf area, stem volume, leaf gas exchange, leaf tissue secondary chemistry, root growth and demography, soil and root respiration, and responses of soil biota to the treatments.
Results to Date: After two years of growth in the treatments, the trees in the high-nitrogen availability soil exhibited significantly greater leaf area $(+23 \%)$, stem volume $(+31 \%)$, and leaf-litter dry weight $(+20 \%)$. There were no significant differences in these traits in the low-nitrogen availability soil. Net nitrogen mineralization in the two treatments, assayed by a 9-week laboratory incubation, was $348 \mu \mathrm{g} \mathrm{N} \mathrm{g}^{-1} \mathrm{~d}^{-1}$ in the high-nitrogen treatment, and $45 \mu \mathrm{N} \mathrm{g}^{-1} \mathrm{~d}^{-1}$ in the low-nitrogen treatment. These rates bracket those occurring across a range of forest ecosystems in northern lower Michigan.

Leaf-condensed tannin content increased with elevated $\mathrm{CO}_{2}$ and low soil fertility as predicted by the carbon nutrient balance hypotheses. Elevated atmospheric $\mathrm{CO}_{2}$ depressed leaf litter $\mathrm{C} / \mathrm{N}$ ratios at both high and low levels of soil nitrogen availability. At high soil nitrogen availability, leaf-litter $\mathrm{C} / \mathrm{N}$ ratios averaged 42 at ambient $\mathrm{CO}_{2}$ and 62 at elevated $\mathrm{CO}_{2}$. At low soil nitrogen availability leaf-litter $\mathrm{C} / \mathrm{N}$ ratios averaged 56 at ambient $\mathrm{CO}_{2}$ and 68 at elevated $\mathrm{CO}_{2}$. The concentration of atmospheric $\mathrm{CO}_{2}$ had little influence on the $\mathrm{C} / \mathrm{N}$ ratio in roots. $\mathrm{C} / \mathrm{N}$ ratios of fine roots ranged from 15 to 20 .

From our preliminary analyses, it appears that actively growing plant roots contribute the majority of $\mathrm{CO}_{2}$ to soil respiration in the experimental chambers.

\section{EFFECT OF VARIABILITY IN MICROCLIMATE AND REGIONAL CLIMATE ON THE PRODUCTIVITY OF NEOTROPICAL MIGRANT LANDBIRDS IN MIDWESTERN FORESTS WHITEHEAD, DONALD; \\ GRIMMOND, C. SUSAN; and ROBESON, SCOTT INDIANA UNIVERSITY \\ FY 1995153 \\ FY 199490}

Objectives: To determine the effects of (1) within-site variation in microclimate, (2) within- and between-year climatic variation, and (3) projected climatechange scenarios on the productivity of four abundant neotropical migrant breeding birds, acadian flycatcher (Empidonax virescens), wood thrush (Hylocichla mustelina), worm-eating warbler (Helmitheros vermivorus), and the ovenbird (Seiurus aurocapillus) in forest contexts in south-central Indiana.

Approach: We are (1) obtaining demographic data from intensive nest monitoring, (2) gathering comprehensive micrometeorological data from each forest context and individual nest site, (3) monitoring insect abundance and recording food items brought to nestlings, (4) analyzing the effects of both landscape pattern and micrometeorological parameters on productivity, (5) developing models relating productivity 
to climatic parameters, and (6) determining the effect of regional weather variables on productivity.

Results to Date: 2938 nests have been monitored (1377 acadian flycatcher, 300 wood thrush, 133 ovenbird, and 223 worm-eating warbler). There is between-year variation in reproductive success for all species. Analysis of regional climate data indicates patterns that may control the observed variations. Between-year variation in reproductive success is not consistent among species.

We have quantified arthropods in the interior ravines with both Malaise traps and leaf counts. There are subtle differences between years and significant changes within each breeding season.

Differences in temperature and vapor pressure with elevation and aspect were analyzed on a diurnal basis. The largest differences between ravine bottom and higher locations occur in midmorning and late afternoon. The maximum difference between stations at different elevations occurs in the late afternoon/early evening. Seasonal variations are present in the elevation comparisons. Earlier periods have cooler nighttime temperatures at the lower elevation, whereas later in the season the higher elevation is cooler. Differences in vapor pressure because of elevation are not as distinct as those for air temperature. The ravine-bottom location is more humid (throughout the entire day). Aspect appears to produce more consistent temperature differences than does elevation. In late afternoon, the northwest-facing slope becomes warmer than the southeast-facing slope.

\section{South Central Regional Center}

\author{
REGIONAL ATMOSPHERE/FOREST \\ EXCHANGE AND CONCENTRATIONS \\ OF CARBON DIOXIDE \\ BAKWIN, PETER S., and DAVIS, KENNETH J. \\ UNIVERSITY OF COLORADO and \\ UNIVERSITY OF MINNESOTA \\ FY 199562 \\ FY 1994100
}

Objectives: To determine, by direct measurements (eddy correlation), the net ecosystem exchange of $\mathrm{CO}_{2}$ between the atmosphere and a mature mixed forest dominated by hardwoods in northern Wisconsin over several annual cycles and on a regional scale.

Approach: We measure by eddy correlation the vertical fluxes of $\mathrm{CO}_{2}, \mathrm{H}_{2} \mathrm{O}$, heat, and momentum at 30,122 , and $396 \mathrm{~m}$ on the WLEF-TV transmitter tower, located in the Chequamegon National Forest in northern Wisconsin. The region is in a heavily forested zone of low relief. We also measure $\mathrm{CO}_{2}$ mixing ratios at $11,30,76,122,244$, and $396 \mathrm{~m}$. With these measurements, we can continuously monitor, at the largest possible spatial scale, the surface fluxes of $\mathrm{CO}_{2}$ and $\mathrm{H}_{2} \mathrm{O}$ and assess the vertical flux divergence within the planetary boundary layer. We also measure a suite of environmental variables that have relevance to the surface exchange of $\mathrm{CO}_{2}$ and $\mathrm{H}_{2} \mathrm{O}$ : soil temperature, soil moisture, within-canopy air temperature, incoming and transmitted photosynthetically active radiation, leaf-area index, total hemispherical radiative flux (up and down), and rainfall. In addition, analysis of remotely sensed data on the regional distribution of vegetation-cover type and temporal dynamics is being carried out to assess the correlation between vegetation type and status on $\mathrm{CO}_{2}$ and $\mathrm{H}_{2} \mathrm{O}$ fluxes observed at the tower.
Results to Date: Measurement of $\mathrm{CO}_{2}$ mixing ratios at $11,30,76,122,244$, and $396 \mathrm{~m}$ were begun in October 1994. Flux measurements $\left(\mathrm{CO}_{2}, \mathrm{H}_{2} \mathrm{O}\right.$, heat, momentum), at 76 and $396 \mathrm{~m}$, and observations of net radiation at the surface were begun in late April 1995. Flux data were obtained intermittently during the summer of 1995 because of frequent instrument failures. The main problem that we encountered was that the electronics boxes were not weatherproof. After several attempts to seal the boxes, we substituted better boxes, and the sonics have worked well since then. In June 1996, we changed the eddy-correlation measurement levels to 30,122 , and $396 \mathrm{~m}$ to improve observations of the shallow nocturnal layer $(30 \mathrm{~m})$ and of daytime vertical flux divergence (122 and $396 \mathrm{~m}$ ). Preliminary analyses have confirmed the viability of the experiment design. Carbon dioxide flux data show the start of significant photosynthetic activity by the forest around the tower site during May and June. Analysis for flux data for the summer of 1996 is ongoing.

\section{USING PALEOCLIMATE DATA \\ TO CONSTRAIN SYSTEM RESPONSE TO \\ CARBON DIOXIDE INCREASES CROWLEY, THOMAS J. TEXAS A\&M UNIVERSITY FY 199563 \\ FY $1994 \quad 0$}

Objectives: To address the problem of an ostensible first order disagreement between tropical SSTs predicted by models and tropical SSTs inferred from various proxy data for time periods globally warmer than the present by: (1) compiling existing paleoclimate data from time intervals warmer than the present; (2) performing an error analysis of that 
paleoclimate data; and (3) performing ocean-model simulations to test a new hypothesis about how the ocean responds to changed boundary conditions during such warm periods.

Approach: Information about meridional SST profiles will be updated and acquired for five time intervals warmer than the present [i.e., the Pliocene ( $3 \mathrm{Ma}$ ), the Eocene (55 Ma), the Maastrichtian (70 Ma), the Santonian (85 Ma), and the Cenomanian (90 Ma)]. The uncertainties in estimates of SSTs from proxy data will be quantified.

The ocean circulation in the North Atlantic will be simulated with the Miami Isopycnic Ocean Model (MICOM), which, via its use of isopycnic coordinates, allows the straightforward simulation and discernment of the evolution of the thickness of the mixed and underlying isopycnic layers as well as possible changes in the latitudinal extent of the gyre structures. Published results indicate that MICOM reproduces the features of the wind-driven North Atlantic circulation well.

Results to Date: The Pliocene database has been expanded to encompass the entire Atlantic Ocean with newly available information from published sources. Data from the Maastrichtian and Santonian periods has been obtained from studies in progress by Huber et al, and S. D'Hondt. The full and completely analyzed Holocene core-top $\delta^{18} \mathrm{O}$ database has been obtained from James Zachos. These acquisitions have allowed the development of a set of comparison curves with both a better Holocene comparison curve and a larger range of SST profiles from warm periods against which to compare it.

The hypothesized changes in the circulation of the North Atlantic subtropical gyre were investigated with MICOM after extensively modifying it to facilitate ease of usage. The input, output, and graphics portions of the package were rewritten and extensively documented to allow quick and easy changes in forcing functions, choices of output fields, and choices of graph types to be plotted. Additionally, the sketchy model documentation was expanded and formatted to develop a user's guide containing information about the variables, parameters, and fields used within the model.

A spun-up North Atlantic gyre circulation was first obtained as a baseline case by integrating the model for $\mathbf{3 0}$ model years using monthly and zonally averaged climatological forcing fields (e.g., latent and sensible heat flux, wind stress, and precipitation) derived from the COADS and Levitus data sets. After it was ascertained that the mixed and seasonally vented layers had reached a reasonably steady state, a further 10-year integration was performed wherein the wind effects (both thermodynamic and dynamic) were reduced to zero. Preliminary results show a gradual reduction of the latitudinal heat flux at all latitudes and a weakening of the western boundary current, although the hypothesized northward movement of the northward limb of the subtropical gyre has not been clearly identified. Temperature does increase at all latitudes in reduced-wind-forcing simulations, caused mostly by the reduction of the cooling effects of the latent heat flux resulting from the reduced wind forcing.

\section{DOCUMENTING CHANGES IN EASTERN PACIFIC CLIMATE WITH CORALS: BASELINE DATA FOR VALIDATION OF CLIMATE MODELS DUNBAR, ROBERT B., and LINSLEY, BRADDOCK K. \\ RICE UNIVERSITY and STATE UNIVERSITY OF NEW YORK, ALBANY \\ FY 1995108 \\ FY 1994118}

Objectives: To develop the use of corals at two sites in the tropical eastern Pacific for paleoclimatic reconstruction of ocean temperature and rainfall in the eastern Pacific for the past 200 years.

Approach: This project is developing paleoclimatic reconstructions of the eastern tropical Pacific by examining massive corals from open-ocean sites at Clipperton Atoll and Cocos Island. This work has primarily focused on reconstructions of sea-surface temperature for the past 200 years with the goal of developing techniques to separate the effects of salinity and water temperature on the oxygen isotopic composition of coral skeletons.

Results to Date: We have analyzed the oxygen isotopic $\left(\delta^{18} \mathrm{O}\right)$ composition of cores from three colonies of the massive coral Porites lobata from Clipperton Atoll. Seasonal changes in the oxygen isotopic composition of coral skeletal aragonite at Clipperton are shown to be primarily a function of changes in sea-surface temperature, with changes in sea-surface salinity playing only a small role in controlling coral $\delta^{18} \mathrm{O}$. Annually averaged $\delta^{18} \mathrm{O}$ analyses of the three coral cores have generally coherent $-0.4 \%$ upward trends and are also consistent with warming observed in shorter instrumental sea-surface temperatures spanning the past 30 years. Only sparse temperature measurements are available from this area before the 1970s; this reconstruction extends the record back to the $1880 \mathrm{~s}$ and demonstrates the relative thermal stability of the eastern Pacific warm tongue over this time period.

We employed a multitracer approach to calibrate to the recent climatic history of Cocos Island and then reconstructed conditions over the past 200 years. The period since 1890 was analyzed at bimonthly (or finer) resolution. Annual average analyses extend the record to about $\mathrm{AD} 1760 . \delta^{18} \mathrm{O}$ decreases by about $0.4 \%$ since 
the late 1800 s. If interpreted solely in terms of temperature, $\delta^{18} \mathrm{O}$ indicates a warming of the surface ocean in the vicinity of Cocos Island of about 1 to $2^{\circ} \mathrm{C}$. However, $\mathrm{U} / \mathrm{Ca}$ ratios at $\mathrm{Cocos}$ Island also show a longterm trend, increasing by about $15 \%$ since the late $1800 \mathrm{~s}$, indicating a cooling of $\sim 1.5^{\circ} \mathrm{C}$. We are currently exploring the probability that $\delta^{18} \mathrm{O}$ is responding to changes in regional seawater isotopic composition.

\section{MILLENNIA-SCALE CORAL RECORDS OF SEA-SURFACE TEMPERATURE AND AN EVALUATION OF SCLEROCHRONOLOGIC TECHNIQUES \\ GILL, IVAN P., and HUBBARD, DENNIS K. UNIVERSITY OF PUERTO RICO and UNIVERSITY OF NEW ORLEANS FY 1995100 \\ FY $1994 \quad 0$}

Objectives: To establish a coral-based record of sea-surface temperature and climate over the past several thousand years, to establish the accuracy and precision of sclerochronologic techniques, and to investigate the feasibility of using Acroporid corals for paleoclimatic work.

Approach: This project will attempt to establish a millenia-scale climatic record using isotopic and trace elemental techniques on long-lived corals. Laboratory techniques will be devised to avoid potential laboratory artifacts that could result in significant inaccuracies. Samples will be derived from extensive cores taken by the principal investigators through the Holocene shelf sections in several parts of the northeastern Caribbean as well as cores taken through individual growing corals.

Results to Date: We have developed a sampling technique that produces high-resolution samples with adequate mass for reliable isotopic analysis. The technique avoids problems of mineralogic inversion and potential isotopic artifacts. Initial experiments have also found the technique suitable for trace elemental work. Blanks run at the University of Cambridge showed no contamination or interference effects, and the samples produced reliable signals for mass spectroscopic analysis.

The nine coral pieces selected for the milleniascale record ranged in age from $660 \mathrm{ybp}$ to $5830 \mathrm{ybp}$. These are conventional carbon-14 ages with a standard deviation (1-sigma) of about $70 \mathrm{y}$ for the youngest age to about $100 \mathrm{y}$ for the oldest. Converted to calendar dates, the slabs range in age from approximately $A D$ 1660 to $4310 \mathrm{BC}$. The two youngest slabs, ca. 660 and 730 years bp $\left({ }^{14} \mathrm{C}\right)$, respectively, have been sampled between 12 and 20 times per annual growth band. Both show strong annual isotopic periodicity in both ${ }^{18} \mathrm{O}$ and ${ }^{13} \mathrm{C}$.

\section{PREDICTING LARGE-SCALE PATTERNS IN VEGETATED-SURFACE CONDUCTANCE FOR $\mathrm{CO}_{2}$ AND WATER VAPOR: PHYSIOLOGICAL AND ECOLOGICAL REGULARITIES AND THEIR CONSEQUENCES GUTSCHICK, VINCENT P., and CHOUDHURY, BHASKAR J. \\ NEW MEXICO STATE UNIVERSITY and NASA - GODDARD SPACE FLIGHT CENTER}

\section{FY 199591 \\ FY 1994101}

Objectives: To improve models of vegetation conductances for $\mathrm{CO}_{2}$ and water vapor and to test such models on many spatial scales, from leaf to multikilometer region.

Approach: To determine if there is a universal behavior of stomatal conductance among species and sites, we measure leaf-level gas exchange on four dominant or locally dominant $\mathrm{C} 3$ shrub species and two warmseason C4 grass species on the Jornada Range in New Mexico, soybean at the Evapotranspiration Laboratory site at Kansas State University, and red maple and red oak at the Harvard Forest in Petersham, Mass.

Canopy and regional conductance is being modeled at several levels with particular attention to models of micro-environment on the plant scale. Experimental tests of scaling of evapotranspiration to larger spatial scales is tested with long-term retrospective data on evapo-transpiration at our site and the Harvard Forest and with our data from intensive field campaigns. In the latter, we use gas exchange at the leaf level, sapflow gauges at the branch or plant level, Bowen ratio at the $100-\mathrm{m}$ scale, and the residual method at the kilometer and greater scales. All measurements are coupled with determinations of microenvironment at the respective spatial scale and of leaf area by image analysis. To explain regularities in community-average conductances at larger scales despite diversity in plant community structure, we are developing models of competitive plant water use and drought tolerance.

Results to Date: Our analysis of 56 day-long runs of gas exchange over three years on five arid-land woody perennials, soybeans, and the two deciduous Eastern trees supports the hypothesis that plants follow a universal stomatal control program. The fundamental scaling of stomatal conductance is by total leaf capacity for $\mathrm{CO}_{2}$ assimilation. To be able to predict stomatal conductance and transpiration at all leaves, we deployed 50 leaf-mounted photodiodes to measure leaf-received irradiances on tree-seedling leaves. The data are being compared with calculations of geometric optics and selected statistical models of plant canopies. We also measured leaf and surface temperatures with a thermal infrared sensor, finding large differences 
that track surface cover according to a semiempirical model of surface energy balance.

We are extending the study of stomatal control programs and microenvironmental patterns to several forest sites around the country. A scaling-up campaign was performed in Arizona during August 1995 with concurrent measurements of leaf-gas exchange, Bowen ratio, sensible heat flux, and evapotranspiration. The area was in a prolonged drought, so evapotranspiration was not significant. The campaign did allow testing of all our equipment. It also revealed that the variability in gas exchange and (future) sapflow was too extensive to sample in a one-day campaign.

\section{IMPACT OF CLIMATE CHANGE ON FLOWER AND FRUIT PRODUCTION IN COTTON \\ HODGES, H. F.; REDDY, K. R; and MCKINION, J. M. \\ MISSISSIPPI STATE UNIVERSITY \\ FY 1995104 \\ FY 199439}

Objectives: To determine the effects of temperature and carbon dioxide enrichment on cotton fruit production and abscission and to incorporate these effects into the cotton model, to determine the effects of season-long variable sinusoidal temperatures and $\mathrm{CO}_{2}$ enrichments projected by GCMs on cotton growth and yield, to simulate cotton crops based on weather hypothesized in global circulation models, and to simulate alternative management strategies to avoid the damaging effects of high-temperature environments.

Approach: A cotton-crop model that was developed to aid cotton producers make long-term planning and in-season management decisions will be used to predict crop growth, development, and yield in response to soil, weather, and crop cultural practice inputs.

The cotton-crop model will be tested with 30 years of Stoneville, Miss., weather data to determine its sensitivity to different management practices and how successfully cotton might be produced in high-temperature environments. The model will be run with real Stoneville weather or with Stoneville weather modified daily by varying amounts. Superimposed on the weather will be alternative simulated crop-management practices to determine if the adverse effects of high-temperature conditions could be avoided.

Results to Date: Controlled-environment studies with varying temperatures added or subtracted from ambient temperatures showed that 1995 ambient temperature plus $5^{\circ} \mathrm{C}$ caused 6 to 8 times more growth of cotton seedlings. Boll weights produced at the end of the experiment in the various temperatures declined to nearly zero or zero at ambient plus $5^{\circ} \mathrm{C}$ and plus $7^{\circ} \mathrm{C}$, respectively. Nearly equal number of bolls were produced at all temperatures tested, but bolls retained to maturity declined to zero on plants grown at ambient plus $5^{\circ} \mathrm{C}$ or higher. Most bolls abscised within $5 \mathrm{~d}$ after flowering at these high temperatures. Vegetative growth at high temperature was not reduced.

Plants grown at ambient temperatures plus 5 or $7^{\circ} \mathrm{C}$ reached fruiting much earlier than plants grown at lower temperatures. Atmospheric $\mathrm{CO}_{2}$ concentration did not affect maturation rate or ameliorate hightemperature injury. The cotton simulations of Stoneville weather without high-temperature modification showed progressively lower yields as daily temperatures increased from ambient. The model was more sensitive to planting dates at ambient and lower temperatures.

\section{NITROGEN AND PHOSPHORUS DEPOSITION AND TERRESTRIAL CARBON STORAGE \\ HOLLAND, ELISABETH A.; BRASWELL, BOBBY $H$; ; and TOWNSEND, ALAN \\ NCAR, UNIVERSITY OF NEW HAMPSHIRE, and UNIVERSITY OF COLORADO \\ FY 199587 \\ FY $1994 \quad 0$}

Objectives: To quantify the global terrestrial carbon sink resulting from the widespread deposition of nitrogen.

Approach: Previous work with NDEP, a perturbation model developed to quantify the terrestrial carbon storage resulting from human-induced nitrogen deposition, has shown that carbon uptake is sensitive to the spatial distribution and quantity of deposited nitrogen. To further examine the role of atmospheric chemistry and transport, which can differ among chemical transport models, $\mathrm{NO}_{\mathrm{y}}$ deposition fields $\mathrm{NO}_{\mathrm{y}}=\mathrm{NO}_{\mathbf{x}}+$ $\mathrm{HNO}_{2}+\mathrm{HNO}_{3}+\mathrm{HO}_{2} \mathrm{NO}_{2}+\mathrm{NO}_{3}+2 \mathrm{~N}_{2} \mathrm{O}_{5}+\mathrm{PAN}$ (peroxyacetyl nitrate)] from five 3-D chemical models (GCTM, GRANTOUR, IMAGES, MOGUNTIA, and ECHAM) are used to drive NDEP in simulations to evaluate the importance of the spatial distribution of nitrogen deposition for carbon uptake and to better quantify its magnitude and uncertainty.

Results to Date: Because of differences in atmospheric sources of $\mathrm{NO}_{x}$, transport, resolution, and representation of chemistry, each of these models predicts distinct spatial patterns of nitrogen deposition on the global land surface. These difference lead to distinct patterns of carbon uptake that vary between 0.7 and $1.3 \mathrm{Gt} \mathrm{C}$ globally. Addition of $\mathrm{NHx}\left(\mathrm{NH}_{3}{ }^{+}\right.$and $\left.\mathrm{NH}_{4}{ }^{+}\right)$ to $\mathrm{NO}_{\mathrm{y}}$ deposition increased carbon storage to between 1.5 and $2.0 \mathrm{Gt} \mathrm{C}$ of total terrestrial carbon storage annually. Less than $10 \%$ of the nitrogen was deposited on forests, which are most able to respond with increased carbon storage because of the wide $\mathrm{C}: \mathrm{N}$ ratio 
of wood as well as its long lifetime. However, the ability of terrestrial ecosystems to fix and store carbon may be compromised by the deleterious effects of nitrogen saturation and by feedbacks operating through atmospheric chemistry, especially $\mathrm{O}_{3}$ damage. We estimate that nitrogen stimulation of terrestrial carbon uptake could result in a global carbon sink of 1.4 to $2.0 \mathrm{Gt} \mathrm{C} \mathrm{y}{ }^{-1}$, while the "missing terrestrial sink" is quite similar in magnitude. Thus, global air pollution appears to be an important influence on the global carbon cycle.

\section{BELOWGROUND RESPONSES TO ELEVATED $\mathrm{CO}_{2}$ IN A C3-C4 GRASSLAND JACKSON, ROBERT B.

Objectives: To test the role of grassland ecosystems as potential carbon sinks and whether they may in fact already be acting as net carbon sinks.

Approach: This study, done in collaboration with the USDA/ARS, is the first to examine the effect of $\mathrm{CO}_{2}$ from glacial to elevated $\mathrm{CO}_{2}$ concentrations (200 to $500 \mathrm{ppm}$ ). The experiment is in a natural grassland near Temple, Texas, and provides a continuous gradient of $\mathrm{CO}_{2}$ from glacial to elevated $\mathrm{CO}_{2}$ concentrations. Results to Date: We have purchased, tested, and installed a TDR system for measuring soil moisture along the tunnel. We have made and installed sleeves for soil-respiration measurements and are regularly monitoring carbon efflux. We built and installed 50 root-ingrowth cores to estimate root production. We have installed tubes for minirhizotron monitoring of fine root growth and will the use the same tubes for neutron-probe measurements of deep soil moisture. Finally, we have begun to parameterize the soil-water model for the Temple, Texas, system, including information on the soils and climate record.

\section{EFFECTS OF CLIMATE CHANGE ON HYPOXIA IN COASTAL WATERS and RABALAIS, NANCY N. \\ LOUISIANA STATE UNIVERSITY and LOUISIANA UNIVERSITIES MARINE CONSORTIUM \\ FY 199585 \\ FY 19940}

Objectives: To describe functional relationship between the Mississippi River freshwater and nutrient inputs, net productivity, and hypoxia in the northern Gulf of Mexico; to provide reliable estimates of global oxygen fluxes across the two main interfaces, air-seawater and pycnocline, for the present-day climate and for a doubled $\mathrm{CO}_{2}$ climate; to predict probable changes in seasonal dynamics, areal extent, and severity of hypoxia if $\mathrm{CO}_{2}$ concentration doubles; and based on global sensitivity analysis, to discuss how climate change may affect the ecosystem of the northern Gulf of Mexico.

Approach: Our project integrates state-of-the-art modeling and a sophisticated sampling program. In this study, we adopted a 1-D modeling scheme. We will use three synchronized rapid-pulse oxygen meters at our mooring located offshore Cocodrie, Louisiana, enabling simultaneous measurements of dissolved oxygen, temperature, and salinity in the upper and lower water column. A third meter will be deployed at the approximate depth of the pycnocline. This design is expected to provide valuable information for computation of vertical $\mathrm{O}_{2}$ fluxes and parameterization of a 1-D mathematical model.

Results to Date: We largely completed the source code and the calibration of a coupled physical/biological 2-box oxygen model, and we are now working on a 1-D modeling scheme. Also, we acquired instrumentation for continuous measurements of dissolved oxygen, temperature, and salinity. The instruments were deployed in June 1996, and data collection is now in progress.

\section{THE INFLUENCE OF ELEVATED $\mathrm{CO}_{2}$ AND CLIMATE WARMING ON FOREST NON- METHANE HYDROCARBON EMISSIONS AND THE LIFETIME OF METHANE MONSON, RUSSELL K. UNIVERSITY OF COLORADO \\ FY 199597 \\ FY 19940}

Objectives: To study the influence of elevated $\mathrm{CO}_{2}$ and concomitant climate change on the emission of nonmethane hydrocarbons (NMHCs) from forest trees and the resultant perturbations to the atmospheric lifetime of methane $\left(\mathrm{CH}_{4}\right)$.

Approach: Several feedbacks important to climateforcing scenarios are driven by atmospheric chemistry. We plan to quantify the gain of one such feedback, $\mathrm{TCH}_{4} / \mathrm{NMHCe}$, where $\mathrm{TCH}_{4}$ represents the tropospheric lifetime of $\mathrm{CH}_{4}$ and $\mathrm{NMHCe}$ represents the emission rate of nonmethane hydrocarbons. We will focus on coniferous and broadleaf trees that compose major forested ecosystems in the continental United States. The research will include empirical measurements of nonmethane hydrocarbon (NMHC) emissions in response to elevated $\mathrm{CO}_{2}$ concentrations. These measurements, along with past studies of the temperature dependence of NMHC emissions, will be used to parameterize models of NMHC emissions, formulate chemistry based feedbacks between climate change and $\mathrm{CH}_{4}$ lifetime, and assess implications of elevated $\mathrm{CO}_{2}$ on forest hydrocarbon emissions and climate change. 
Results to Date: Gas-exchange techniques were developed for measuring the emissions of monoterpenes and isoprene from trees growing at elevated $\mathrm{CO}_{2}$. Monoterpene emissions from Ponderosa pine trees in Placerville, Calif., and Douglas fir trees in Corvallis, Ore., were measured. At both sites the trees are growing in open-topped chambers and are exposed to conditions of elevated $\mathrm{CO}_{2}$ and normal $\mathrm{CO}_{2}$. At Placerville, nitrogen fertilization is another factorial variable; at Corvallis, temperature is another variable.

At these sites, measurements were made of monoterpene emissions from intact needle tissues in each treatment. Ten replicate measurements were taken from each treatment, resulting in more than 200 samples, which have now been analyzed in the laboratory. Data trends are currently being analyzed. The influence of simulated herbivory on the monoterpene emission rate for trees grown in all $\mathrm{CO}_{2}$, temperature, and fertilization treatments was measured. To date, we have determined that natural and simulated herbivory have similar effects on the monoterpene emission rate. More than 1200 tissue samples were taken for cyclaseactivity analysis; we are in the process of conducting these assays. We also collected tissues from each of the plants to determine needle nitrogen concentrations and needle starch concentrations.

\section{ENERGY-USE IMPLICATIONS OF CLIMATE CHANGE \\ SAILOR, DAVID J. TULANE UNIVERSITY \\ FY 199554 \\ FY 199476}

Objectives: To determine the impact of climate variability and climatic change on the energy sector and on energy demand in particular.

Approach: We will use statistical analysis of GCM simulations and measured surface and upper-atmosphere parameters to relate the coarse grid predictions of GCMs to regional scales. We will then use statistical analysis of historical energy use and climate data to establish statistical models relating energy demand (for gas and electricity) to climate parameters, such as temperature, humidity, and wind speed. The output of these two objectives will then be combined to provide reliable estimates of how climatic change will impact the electric utilities in eight high-energy-use states in diverse regions.

Results to Date: A preliminary energy-climate analysis has been completed. The regional climate modeling results show a strong correlation between upperatmosphere model predictors and surface climate predictands. The implication is that through suitable statistical analysis we should be able to generate regional climate change predictions. The more interesting result is that while our regional climate change predictions are similar to those taken directly from the GCM grid-cell output for most of the year (for New Orleans), we predict a much more significant warming in the fall months. The same analysis applied to other regions in the states of Washington, New York, California, and Texas indicate some instances where the raw GCM output appears to overpredict regional warming and other cases where it underpredicts regional warming. Combining the raw GCM output from several models and the energy analysis results we have produced a preliminary energy-impact assessment.

\section{USE OF GCM PREDICTIONS OF CLIMATE CHANGE TO ASSESS IMPACT ON WATER RESOURCES SYSTEMS \\ THOMPSON, DAVID B., and PETERSEN, RICHARD

Objectives: To develop a method for transferring climate change as predicted by a GCM to a river basin water-resources system, to apply the method to the Brazos River Basin to assess likely changes to the hydrology of the watershed, and to assess the likely impact of changes on the plant regime and hydrologic cycle of the Brazos River watershed.

Approach: Hydrologic and climatologic databases will be developed. The Brazos River Basin model will be calibrated. The GCM output will be analyzed, and a climate model will be constructed. Climate variables under historical and climate-change conditions will be specified. The river-basin model will be operated with the specified climate variables. The modeling results will be analyzed, interpreted, and reported.

Results to Date: A literature review of articles pertinent to both meteorologic and hydrologic modeling of climate change impacts on river basin meteorology and hydrology has been completed. Delineation of the watershed and subbasins on 1:1,000,000 scale map and verification on USGS $71 \frac{1}{2}$-minute quadrangle maps is complete. Collection and review of meteorologic data is near completion. All existing hydrologic data from the USGS has been collected and reviewed. Soil classification data for the watershed has been collected and analyzed, and ET calculations with the FAO-24 Penman method have begun. Model evaluation began with a literature review of models used in similar projects. The STORM model was examined for applicability to the study watershed. Problems with a maximum run length of one year and the fundamental algorithm make STORM less appropriate to semiarid regions. The USGS MMS (modular modeling system) has also been reviewed. The system may work for the study project, but the lack of hydrograph-routing 
algorithms makes it less appropriate for large watersheds. Preliminary review of the National Weather Service-River Forecast System indicates this model is designed for problems similar to that under study. A request for the model has been made to the NWS, and completion of the review is pending receipt of program documentation.

\section{SOIL MOISTURE AND AGRICULTURAL WATER IN A CHANGING CLIMATE: COUPLING A REGIONAL HYDROLOGIC MODEL WITH GCM ESTIMATES \\ VALDES, JUAN B., and BOWMAN, KENNETH P. TEXAS A\&M UNIVERSITY \\ FY 199569 \\ FY 19940}

Objectives: To help clarify specifically how GCM land-surface-hydrology parameterizations might beimproved for better simulation of precipitation, evaporation, soil moisture, and other surface-hydrology quantities.

Approach: The amount of land surface moisture 'recycled' into local precipitation was quantified in two climate simulations using the Community Climate Model (CCM), a general circulation model of the atmosphere (GCM). The quantities are being compared to earlier estimates derived from an observation-based model of continental moisture recycling. Total precipitation over a given continental area is made up of moisture carried into the region by advection plus moisture carried upwards from within the region by evapotranspiration. The extent to which moisture that is stored on the surface or in the soil gets recycled back into local precipitation is an important aspect of regional hydrology and may also contribute to globalscale climatic patterns.

Simulations of the global climate with GCMs may or may not adequately account for local recycling mechanisms. Or, the recycling proportions may be accurate but the lack of interannual soil moisture carryover in the models may be artificially interrupting the moisture recycling mechanism. In either case, these model mechanisms could be partially responsible for problems often observed in GCM simulation of continental precipitation, particularly in the summer months.

The results of these studies will help clarify specifically how GCM land-surface-hydrology parameterizations might be improved for better simulation of precipitation, evaporation, soil moisture, and other surface-hydrology quantities. They will also provide a basis for off-line testing of specific GCM hydrologic parameterizations, including adjusted precipitation quantities. A point hydrologic model calibrated to a nine-state region in the midwestern United States is being used offline to test variations in the recycling process and their effects on soil moisture and evaporation in both the present and the changed climates.

Results to Date: New project.

\section{Great Plains Regional Center}

\section{EFFECTS OF ALTERED SOIL MOISTURE AND TEMPERATURE ON SOIL COMMUNITIES, PRIMARY PRODUCERS, AND ECOLOGICAL PROCESSES IN GRASSLAND ECOSYSTEMS \\ BLAIR, JOHN M.; KNAPP, ALAN K.; TODD, TIMOTHY C.; and RICE, CHARLES W. KANSAS STATE UNIVERSITY FY 199559 \\ FY 199482}

Objectives: To determine the responses of prairie grasses, soil organisms, and key soil and plant processes to different precipitation and soil water regimes and also to identify the potential consequences of these responses for ecosystem function in light of the predictions of global-climate-change models.

Approach: We use naturally occurring gradients of soil-water availability as experimental treatments to address the constraints that water availability imposes on ecological processes in grasslands. We are studying a mesic tallgrass prairie located at the Konza Prairie Long-Term Ecological Research (LTER) site and a more arid mixed-grass site at the Fort Hayes Agricultural Experiment Station. Average annual precipitation at Konza is $835 \mathrm{~mm}$, while average annual precipitation at the Hays site is only $580 \mathrm{~mm}$. Large, intact soil cores dominated by Andropogon gerardii (big bluestem) with associated plants are transplanted between these sites. Half of the 70 cores are placed back into holes at the site from which they were taken, and half were transplanted into holes at the other site. The end result is a grid of 35 paired tallgrass cores and "mixedgrass cores" arranged in a randomized block design at each of the two sites. A set of 10 cores ( 5 Konza and 5 Hays cores) is collected from each site and destructively sampled in the spring and autumn. Aboveground biomass is analyzed for carbon and nitrogen content. Cores are analyzed for total soil carbon and nitrogen, inorganic soil nitrogen, soil microbial biomass carbon and nitrogen, and soil-invertebrate numbers (nema- 
todes and microarthropods). Plant roots are analyzed for $\mathrm{C} / \mathrm{N}$ content. Plant ecophysiological responses are measured for A. gerardii tillers at both sites. At Konza, we are also using field sites with different average soilwater availability to examine the long-term responses of tallgrass ecosystems to different soil-moisture conditions. Response variables measured include ANPP, root-tissue carbon and nitrogen content, litter decomposition rates, soil-invertebrate numbers and composition, and nitrogen availability.

Results to Date: Leaf-level photosynthesis and aboveground productivity are lower at the more arid site, regardless of core origin. Root biomass and root:shoot ratios are increased in cores transplanted to the arid site, and root biomass is decreased in cores transplanted to the more mesic site. Mineralizable soil carbon and nitrogen rapidly declines in cores transplanted to the more mesic site; this occurrence is accompanied by concurrent increases in inorganic soil nitrogen. Soil-nematode communities change. These results demonstrate some important functional responses of grassland ecosystems (e.g., altered patterns of plant carbon allocation and soil nitrogen availability) to changes in water availability. Comparisons of the responses in transplanted cores with the extant differences in "native" cores from the two sites suggests that some of these responses are transitory, and that short-term responses may not parallel long-term changes associated with altered precipitation regimes.

\section{SPACE-TIME LOCAL HYDROLOGY INFLUENCED BY CHANGING CLIMATOLOGY: DISAGGREGATION, PREDICTION, AND COMPARISON BOGARDI, ISTVAN \\ UNIVERSITY OF NE \\ FY 199585 \\ FY 1994120}

Objectives: To relate the arrival rate of atmospheric circulation patterns of a given type to the probability of space-time hydrological variables, to provide a tool for using existing synoptic outputs of GCMs for prediction of space-time hydrological variables at a regional or watershed level, and to compare and evaluate the climate change impact on hydrological variables over several regions of the United States and Europe.

Approach: Three types of daily circulation pattern data are used: historical data for the central USA from January 1948 to June 1989 from the National Meteorological Center (NMC), a 10-year-long data series for the same pressure level from the output of the MPI (Max Planck Institute) and CCC (Canadian Climate Centre) GCMs corresponding to the $1 \times \mathrm{CO}_{2}$ scenario, and an analogous series for the $2 \times \mathrm{CO}_{2}$ scenario. These data are used to compile circulation types with principal component analysis (PCA) coupled with k-means. Autoregressive processes modified by a transformation are used to establish a relationship between the distribution of a local climatic factor and a normal distribution are used to relate local climate to $\mathrm{CP}$ types. With the $2 \times \mathrm{CO}_{2}$ data, we use the geopotential height of a pressure level as an indicator of the atmospheric pressure and temperature. Probability distributions are then estimated corresponding to the $1 \times \mathrm{CO}_{2}$ and $2 \times \mathrm{CO}_{2}$ monthly mean heights of the $500 \mathrm{hPa}$ pressure field, and the probability distribution calculated from the whole historical data set is adjusted according to the difference between the two above mentioned distributions $\left(\mathrm{CO}_{2}\right.$ case) .

Results to Date: Automated, manual, fuzzy-rulebased, and artificial-neural-net-based classifications of the 500-hPa CPs above the western USA have been constructed, and a method to select the most appropriate classification scheme in view of describing local hydrology has been developed. Daily precipitation values have been conditioned and produced stochastically on the basis of the time series of types and average heights of $500-\mathrm{hPa} \mathrm{CPs}$. The 10 -year time series of daily CPs obtained from the output of the $\mathrm{CCC}$ and MPI GCMs were analyzed in the cases of $1 \times \mathrm{CO}_{2}$ and $2 \times \mathrm{CO}_{2}$ scenarios using the same techniques that were used for historical data. No significant difference in either typology (by PCA) or Markov properties (transition probabilities from one type to another) could be found for either summer or winter. The average height of the 500-hPa pressure field was then taken as a third physically meaningful characteristic of CPs under climate change, leading to a clear differentiation between the $1 \times \mathrm{CO}_{2}$ case and the $2 \times$ $\mathrm{CO}_{2}$ case. Precipitation over the Nebraska sites appears to exhibit different site-specific behavior in the $2 \times$ $\mathrm{CO}_{2}$ vs $1 \times \mathrm{CO}_{2}$ cases. Overall, there is only a slight increase in mean precipitation but a substantial increase in variability. Daily wind speed was estimated under climate change in several locations in Nebraska where climate change may lead to considerable decrease of the within-the-year variability of wind speed. The stochastic climatological model outlined above has been adapted to estimate daily solar radiation to assess the impact of climate change on solar radiation.

\section{ASSESSMENT OF CLIMATE CHANGE ON A MIXED AGRICULTURAL LANDSCAPE ON THE NORTH AMERICAN GREAT PLAINS BRANDLE, J. R. UNIVERSITY OF NEBRASKA FY 199594 FY 1994102}

Objectives: To examine the potential interaction of climate change, trees, and Great Plains agroecosystems by quantifying the influence of a shelterbelt on its pressure field, measuring the impact of a shelterbelt on 
the surface energy budget in the sheltered zone, developing an individual-based model to predict treespecies diversity in a narrow linear riparian corridor, and refining an economic input/output model to reflect changes in the input requirements related to developing and evaluating a long-term shelterbelt-planting program.

Approach: The project will assess the value of linear forests in the Great Plains agroecosystem. Three models will be developed to provide tools to assist in our evaluation effort. As the models continue to improve, we anticipate being able to consider and evaluate a variety of scenarios for adapting agricultural production in the northern Great Plains region to potential climate change.

Results to Date: To assess the impact of the pressure differences in the wind's flow field leeward of a shelterbelt, pressure measurements were made along several transects leeward, windward, and across a two-row, green ash, Austrian pine, eastern red cedar shelterbelt. The shelter created a narrow region of high pressure windward of the shelter and a broad region of low pressure in the lee that delayed the windspeed recovery and created an elongated sheltered region.

Simulations were performed to determine changes in evaporation (latent heat flux) in the lee of a shelter. By reducing the wind speed and breaking down the large turbulent length scales, the shelterbelt reduces the vertical transport of water vapor and therefore reduces evaporation from the surface. The simulations indicated that daytime latent heat flux is decreased $20 \%$ to $50 \%$ in the 4 to $8 \mathrm{H}$ behind the shelter, and the total water preserved by the surface is substantial. The reduction in evaporation is balanced through the surface energy budget by an increase in sensible heat loss, which is increased by a factor of 4 in the same 4 to $8 \mathrm{H}$.

Linear forest gap model: The narrow, linear forests scattered throughout the Great Plains region are not represented well by forest-gap models. We have developed a gap model called Seedscape that can accommodate these Great Plains forests by modifying the existing single-plot model JABOWA-II. The model was run for five iterations for each of three forestcorridor widths. Output of the model [the species, diameter at $1.37 \mathrm{~m}$ (dbh), height, and biomass for each tree on all plots] was compared to vegetation sampled in 1995 near Mead, Nebr. The number of stems and $\mathrm{dbh}$ were recorded for all woody species occurring on five $10 \times 10-\mathrm{m}$ plots within the corridor. Ten woody species were present at the field site; the model correctly identified all ten as being present. Only Q. macrocarpa was predicted to occur in significant numbers. According to the simulations, wider corridors have greater stem density and basal area. The number of stems per plot was overestimated by the model. The basal-area predictions were more in line with field data.

\section{ECONOMIC AND ENVIRONMENTAL IMPACT OF MAJOR SHIFTS IN GREAT PLAINS LAND USE INTO ENERGY BIOMASS PRODUCTION \\ DYKE, PAUL}

\author{
TEXAS A\&M UNIVERSITY \\ FY 199572 \\ FY 199470
}

Objectives: To determine if woody and herbaceous biomass compete economically with traditional agriculture at the firm or farm level for the use of land and water resources under the existing climate, to identify the environmental impacts of large-scale biomass production compared with those of traditional agricultural production, to investigate how $\mathrm{CO}_{2}$-induced climate change might affect the assessment of the economic and environmental feasibility of biomass since the process of substituting biomass for fossil fuels would occur gradually over several decades, and to identify the impacts of large-scale biomass production on the regional economy.

Approach: The study will analyze (1) the economic and environmental characteristics of biomass production at the farm level relative to those of conventional agricultural practices in the region; (2) the aggregate environmental characteristics when biomass production is expanded to the regional scale; (3) the comparative regional impacts of the MINK (Missouri-IowaNebraska-Kansas) economy as a whole; and (4) the impact of climate change at the farm level, at the regional-environment level, and at the regional-economy level.

The farm-level analysis employs the EPIC biophysical-process model in concert with climatechange data produced by the nested regional GCM model to examine changes in yield and subsequent profitability under climate change. Baseline data sets, initially developed for use in the MINK study (30 original farms), serve as the base data with changes made for the expected cropping pattern change under climate change. The regional analysis employs the HUMUS (Hydrologic Model of the United States) to analyze the collective environmental impacts on the MTNK region in both climate scenarios. Regional and national agriculture-based economic impacts, including the comparative shifts in crop production and prices induced by climate change and the introduction of the biomass crop alternative, will be estimated via the ASM (Agricultural Sector Model). A subportion of the analysis will also address how alternative grid sizes within the Nested Regional GCM affect the magnitude of the estimated environmental and economic impacts. This latter step will begin to attempt to answer the 
question of how fine a grid for reflecting weather is needed for regional analyses.

Results to Date: Nebraska-based personnel, in cooperation with NCAR, are nearing completion for determining the regional climate-change scenarios driver for the MINK region under two alternative grid sizes.

New crop-enterprise budgets for the MINK region have been obtained for use with EPIC and HUMUS and are currently being matched to the historical MINK representive farms and soils.

EPIC (calibrated for switchgrass as well as the traditional crops grown in the MINK region) was run with mean annual temperature that varied from current values by -2 to $+6^{\circ} \mathrm{C}$ and with precipitation that varied from current by $-30 \%$ to $+30 \%$. The simulations indicate that switchgrass can produce substantial yields at a variety of sites within the MINK region. Climatechange results indicate that switchgrass is less sensitive to changes in precipitation than are corn, sorghum, and soybeans. Switchgrass is favored by a warming climate while the traditional crops suffer reductions in yield. In fact, switchgrass may prove much more suitable than other crops for the MINK region if the climate of that region warms and dries as many general circulation models predict.

\section{REGIONAL PROJECTIONS OF CARBON DYNAMICS WITH GLOBAL CHANGE IN THE CENTRAL U.S.: INTERACTIVE EFFECTS OF MANAGEMENT, CLIMATE, AND ELEVATED $\mathrm{CO}_{2}$ ELLIOTT, EDWARD T.; PAUSTIAN, KEITH; PETERSON, GARY A.; COLE, C. VERNON; and KILLIAN, KENDRICK \\ COLORADO STATE UNIVERSITY \\ FY 199568 \\ FY 1994100}

Objectives: To separate the interactive effects of climate, elevated $\mathrm{CO}_{2}$, and management on soil organic matter (SOM) dynamics in the central U.S. wheat- and corn-growing regions.

Approach: We are using the agroecosystem version (V4) of the Century model to simulate carbon and nitrogen dynamics of cropping systems in the central United States, focusing on the Great Plains region. We use a site-level approach that uses long-term experimental sites located throughout the region that represent a diverse sampling of climate, soil, and management histories of the region. The rationale behind the use of the long-term experimental sites is that it affords us a unique opportunity to test, evaluate, and validate the model predictions of SOM dynamics and crop-soil interactions against a high-quality, detailed set of observational data.

Results to Date: We have recently completed an analysis of six sites. Swift Current, Saskatchewan;
Sidney, Nebr.; and Stratton and Walsh, Colo., represent the western extent of the Great Plains, arrayed along a potential evapotranspiration gradient resulting primarily from changing temperature. The other two sites at Arlington, Wisc., and Manhattan, Kans., represent significantly higher rainfall areas along the northern and western edges, respectively, of the corn belt.

The model proved itself useful for making future projections of changes in soil carbon as a result of changes in climate and other production factors, such as atmospheric $\mathrm{CO}_{2}$ levels and management systems. Simulations that included two management variables (cropping intensity and tillage) and variations in climate and $\mathrm{CO}_{2}$ were run for 60 years (1995 to 2055) with climate-change scenarios obtained from the VEMAP (Vegetation/Ecosystem Modeling and Analysis Project) database and rerun for the historical period (pre-1995). For displaying the effects of climate and $\mathrm{CO}_{2}$ on the different management systems at each of the sites, we chose the CCC model. The general pattern exhibited for most of the site/management combinations was a decrease in soil carbon with climate change alone, an increase in soil carbon with enriched $\mathrm{CO}_{2}$ alone and an intermediate response to the combination of increased $\mathrm{CO}_{2}$ with climate change. Of all the factors included in the simulation experiments, cropping intensity had by far the greatest influence on soil carbon levels.

\section{CARBON, WATER, AND ENERGY FLUXES FROM A TALLGRASS PRAIRIE: A LONG-TERM INVESTIGATION OF ENVIRONMENTAL, BIOLOGICAL, AND LAND-MANAGEMENT FACTORS HAM, JAY M., and KNAPP, A. K. KANSAS STATE UNIVERSITY FY 199581 \\ FY 199430}

Objectives: To evaluate the flux of carbon, water, and energy from a tallgrass prairie over an extended period and to determine the effect of environmental, biologi$\mathrm{cal}$, and land-management factors on these fluxes.

Approach: Meteorological stations will be established on the Konza Prairie Research Natural Area (KPRNA) that will provide continuous long-term (i.e., 10-year) measurements of $\mathrm{CO}_{2}$, water vapor, and energy exchange. Measurement stations will be operated on three watersheds with differing land management regimes: (1) burned annually, (2) burned every 4 years, and (3) burned and grazed annually. Each measurement facility will consist of a 9-m-tall meteorological tower, a small supplemental tower, and supporting equipment. The main tower will support a sonic anemometer, gas-sampling intakes for conditional sampling and gradient methods, and other basic 
meteorological sensors. The supplemental mast will support a net radiometer and infrared transducer, so that the sensors will have an unobstructed view of the surface. Measurement of several ancillary site attributes will provide supporting biotic and abiotic information on the underlying biophysical mechanisms affecting mass flux from the prairie. Measurements of leaf photosynthesis, leaf water potential, and sap flow will be used to explore the biophysical effect of vegetation on trace gas fluxes. Other measurements will include leaf-area indexes, leaf nitrogen content, aboveground biomass, species composition, soil fertility, and soil water content.

Results to Date: Two flux monitoring systems were deployed on an annually burned watershed on the KNPRA, one on an upland and the other on a lowland, $0.7 \mathrm{~km}$ apart. The purpose of these measurements was to test the feasibility of the conditional sampling approach and determine how convoluted terrain might impact the results. If the measurements proved robust, the potential impact of landscape position (upland vs. lowland) could be discerned. Preliminary results suggest that the conditional sampling may, indeed, be a legitimate method for measuring mass flux on a continuous basis.

Data not yet subjected to post-processing indicate that soil water was not limiting and the leaf area index of the canopy was approximately 4.0. NCE was strongly correlated with PAR during clear (DOY 226) and cloudy (DOY 227) conditions. The magnitudes of the fluxes were similar to results obtained previously in large open-top chambers. Nighttime ecosystem respiration was similar to soil-surface $\mathrm{CO}_{2}$ fluxes that were measured with a soil chamber on a nearby plot. When skies were clear, the ecosystem had a daily (24-h) net carbon gain of $25.8 \mathrm{~g} \mathrm{CO}_{2} \mathrm{~m}^{-2}$; however, under cloudy conditions the carbon gain dropped to $5.2 \mathrm{~g} \mathrm{CO}_{2} \mathrm{~m}^{-2}$. Obtaining ET from the site in conjunction with NCE will be critical over long periods because previous research suggests that the water and carbon budget are strongly coupled. Latent-heat flux was measured with the conditional sampler and simultaneously and independently with the Bowen ratio energy balance method. Good agreement was observed between the two techniques. While only a small amount of data is available, results show that NCE from upland lowland sites are similar when water is not limiting. As drought develops, we expect NCE from the upland to be less than that in the lowland because the soil depth in the upland is less than half of that observed in the lowland.
THE EFFECT OF ECOSYSTEMS ON CLOUD MICROPHYSICS AND AEROSOL DISTRIBUTION

HAN, QINGYUAN Y., and WELCH, RONALD M. SOUTH DAKOTA SCHOOL OF MINES AND TECHNOLOGY

FY 199555

FY 199463

Objectives: To examine changes of ecosystems of regional, national, and global scale in a changing climate and to evaluate the relationship between ecosystem and cloud cover and the relationship between ecosystem, cloud droplet sizes, and total cloud liquid water content.

Approach: "Clear" pixels of the ISCCP cloud products retrieved from 5 years of AVHRR data will be used to calculate the NDVI. The volume of this dataset is equivalent to six 6250-bpi data tapes per satellite per month, requiring about 9 months to process 5 years of data. However, once we develop the retrieval and analysis method, we will add our processing software to the other programs running on the CX dataset, which may mean that the actual pace of analysis will be slower. This approach allows us to use the automated data-handling facilities and software-management system at the ISCCP Global Processing Center at NASA Goddard Institute for Space Studies, which reduces the expense of the analysis to the marginal cost of some staff time and additional data tapes.

Results to Date: We have constructed two years of data, which have simultaneous retrieval of NDVI, cloud effective radius, and cloud cover. The developed retrieval scheme has been sent to the ISCCP processing center to retrieve cloud properties and NDVI for the other three years. We have made efforts to validate the results, and to apply the dataset in research related to climate-change studies.

We validated the cloud effective radii (re) and the cloud liquid water path (LWP) derived from this study with ground-based pyranometer and microwave radiometer measurements taken on San Nicolas Island during the 1987 FIRE IFO. Values of re derived from this study were also compared to values retrieved by a hybrid method that used the combination of LWP derived from microwave measurement and optical thickness derived from GOES data. The results show that there is significant variability in cloud properties over a $100 \times 80-\mathrm{km}$ area and that the values at San Nicolas Island are not necessarily representative of the surrounding cloud field. On the other hand, the re values remained relatively constant (within 2 to $3 \mu \mathrm{m}$ in most cases) in the marine stratocumulus.

It has been proposed that an increasing aerosol number density by human activities may lead to smaller cloud droplet sizes and thus make clouds brighter, but most of the cloud-albedo variations 
examined here do not exhibit such a negative correlation between cloud droplet size and albedo.

We did find a positive correlation between NDVI and effective radius of water cloud for most of the regions. As an extension of this finding, we have started to investigate the possible effect of ecosystems on microphysics of high cloud.

We investigated the cloud temperature-cirrus crystal size relationship based on the cirrus-particlesize data retrieved from ISCCP CX data. We found that for thick clouds, most of the regions over the globe show positive relationships between cloud temperature and cirrus ice crystal sizes. However, if relatively thin clouds are included, this correlation becomes negative for tropical areas while remaining mostly positive for midlatitudes. Optical thickness and effective ice crystal sizes have been retrieved over ocean areas on a nearglobal scale $\left(50^{\circ} \mathrm{N}-50^{\circ} \mathrm{S}\right)$ by using the ISCCP CX data and a radiative-transfer model. Generally, ice-crystal size increases for increasing cirrus optical thickness.

\section{WATER RESOURCES AND CLIMATE CHANGE: REGIONALLY INTEGRATED ASSESSMENT OF CONSEQUENCES HOTCHKISS, ROLLIN H. UNIVERSITY OF NEBRASKA FY 199590 \\ FY 19940}

Objectives: To develop a hydrologic model of the Missouri River Basin that simulates the impacts of climate change by using results from climate-change modeling, hydrologic modeling, and ecosystem process modeling and to predict the hydrologic and economic impacts of climate change in the Missouri River basin. Approach: An existing, climate-change-prepared watershed model (SWAT) will be applied to the Missouri River basin and subbasins. The model will be tested for sensitivity to scale, process-based algorithms, and uncertainty considerations. The model will be modified to incorporate (1) ecosystem-process-based modeling results, after learning what scales and variables most affect model output; (2) algorithms to more adequately represent the Missouri main-stem reservoir operations as they impact energy and economic factors; (3) suggestions for making output more amenable to resource and policy analysis; and (4) the ability to provide feedback to climate models. The impact of climate change on the water resources of the Missouri River basin will be simulated, including firstorder economic impacts as they affect adaptation and mitigation strategies.

Results to Date: The model of the Missouri River is being adapted from work done on the basin by the Agricultural Resource Service for the HUMUS project. All relevant digital datasets to be used as input have been acquired. The soil and land-use data were com- bined effectively with STATSGO, STATSGO I, USGS Land Use/Land Cover, and the Census of Agriculture according to rules developed by the USDA-NRCS and Texas A\&M University that assure that appropriate crops are grown on appropriate soils. The topography of the basins was defined with USGS Digital Elevation Model databases. The calibration of the model is being performed by subbasins and by reaches, with a reach defined as a main stream that reaches the outlet of an 8-digit subbasin.

Three subbasins of the Missouri River basin have been chosen for detailed modeling efforts to investigate scale issues as well as improving certain algorithms: the headwaters region of Montana (snowmelt dominated), the Black Hills region of South Dakota (forest-dominated), and the plains region of Kansas (crop-dominated). The data for the Spring Creek watershed in the Black Hills of South Dakota have all been obtained and implemented in the model, and simulations are now being run. The model is being calibrated against USGS gage readings, then climate change scenarios will be investigated along with the scale and algorithm issues discussed previously.

We are also in the process of implementing routines to better model the five reservoirs operated by the Corps of Engineers on the main stem of the Missouri River. These reservoirs control almost half of the drainage area of the Missouri River, and thus have great potential for adapting water use to different conditions under global climate change. The reservoirs are currently modeled in a very simplistic way that does not approach the actual operations done by the Corps.

\section{IMPACT OF GLOBAL CLIMATE CHANGE ON GRASSLAND INSECT PEST POPULATIONS JOERN, ANTHONY \\ UNIVERSITY OF NEBRASKA FY 199536 \\ FY 19940}

Objectives: To characterize biophysically imposed constraints on time and space utilization in target grasshopper species, to establish the impact of biophysical constraints on activity-time budgets to energymass budgets in environments with variable food quality, and to evaluate the impact of variable energy-mass budgets on demographic parameters to be used for population projections at local and regional levels in response to climate change scenarios.

Approach: The distribution of microclimates that respond to climate shifts from six habitats arrayed along an incoming radiation gradient will be examined. Preferred temperatures in the field will be estimated by evaluating actual use of microhabitats by 
grasshoppers in the field and in controlled thermal gradients.

Demographic responses (survival, growth rate, developmental rate and possibly fecundity) of two grasshopper species (Melanoplus sanguinipes and Phoetaliotes nebrascensis) will be assessed at the six sites in response to the incoming solar energy gradient and food quality. A two-by-two-by-two factorial, randomized complete block design includes: nitrogen fertilization, water, and additional shading (with cloth) at each of the six blocks.

Ongoing lab experiments also investigate the interactive roles of thermal environments and food quality. Factorial experiments that vary protein content (expressed as \%N) and \% sucrose in artificial diets are presented to grasshoppers in controlled environmental chambers that vary in the number of hours per day that the grasshoppers can maintain body temperatures at preferred temperatures (ca. 35 to $36^{\circ} \mathrm{C}$ ). Chamber temperatures are otherwise maintained at $28^{\circ} \mathrm{C}$, a satisfactory but nonoptimal temperature.

Results to Date: Microclimatic data could not be obtained in the 1995 field season because dataloggers were not obtained until early-September. Late spring and especially summer months are the critical periods for obtaining this information. Six sites situated along a gradient of solar input are in place and operating for the 1996 field season.

Lab-based experiments are under way that illustrate the importance of thermal input, food quality (C:N) and their interaction for the common range grasshopper, Phoetaliotes nebrascensis. Nitrogen content of the food and the thermal environment significantly impacted food processing. Both nitrogen and sucrose levels in the food as well as thermal conditions significantly influenced demographic responses. Thus, anticipated responses of climate change could influence insect herbivore populations both directly through food-processing capabilities and indirectly through effects on food quality.

\section{INFORMATION IN A SPATIALLY EXPLICIT MODEL OF ECOLOGICAL RESPONSE TO GLOBAL CHANGE \\ MALANSON, GEORGE P., and ARMSTRONG, MARC $P$. \\ UNIVERSITY OF IOWA \\ FY 199537 \\ FY 199420}

Objectives: To investigate how ecologically relevant information from fine-scale analyses can be maintained in models of ecological response to climatic change at coarser scales.

Approach: Our modification of the JABOWA FORET simulation model, the Module Of Spatially Explicit Landscapes (MOSEL), is used for simulation runs.
Simulations are run for periods of 400 to 1000 years. The cells are linked by dispersal. To examine scaling a simulation up to a larger area, the environmental gradient represented by the cells is changed so that different environmental steps between cells are represented; then we impose a long climatic gradient $(5000$ GDD), a short climatic gradient with the same center as the long gradient (2000 GDD), and a uniform climate similarly centered.

The consequences of coarsening the scale is examined by reducing the number of cells for a given climatic gradient. The scale is coarsened by holding the number of cells constant and then grouping them into contiguous blocks so that environmental steps remain. We included 30 hypothetical species for which we defined response functions and species-specific growth terms in the simulation runs. These 30 hypothetical species were defined so that the effects of different numbers of species and their responses over a given portion of a landscape to be simulated can be controlled; we can then examine the spatial representation of the landscape and the array of species over it independently.

To examine the effects of spatial pattern and processes at an ecotone, the positive feedback hypothesis, seed rain, and the steepness of the environmental gradient are examined as potential controls on the pattern of treeline.

Differences in the two response functions, amount of niche overlap, disturbance, and removal and invasion of species are examined in simulation experiments. Following analysis of the effects of dispersal probability, generation time, seed-crop probability, and varying proportions and patterns of landscape fragmentation on migration rate, we will examine the consequences of changing the scale of the grid cells on the rates.

Results to Date: The diversity predicted in the simulation model increases asymptotically with the number of species simulated. The number of cells has a positive relation with species diversity. The between-cell step has a negative relation with diversity. For differences in the length of the climatic gradient, the effects are primarily caused by how well the species responses are matched with the simulated climate. The composition responds strongly to the climatic gradient simulated. For different climates, this is a trivial result of input to the model. For differences in the length of the climatic gradient, the effects are primarily caused by how well the species responses are matched with the simulated climate. The number of cells and the between-cell step have small effects on composition. These effects could lead to misinterpretation of simulation results, but probably only in cases where the range of the environment to be simulated is relatively small and spatial representation in the model is at a fine 
resolution. With a larger environmental range and coarser resolution, the effects of the simulated environment will probably outweigh the effects of spatial representation on composition.

The steepness of the abiotic gradient determines the general location of the ecotone, while the strength of feedback determines how abrupt it is. Increased seed rain and seedling survival modify the dominant patterns by creating patches or small seedlings. The feedbacks are spatially autocorrelated and so create waves of mortality and regeneration on the simulated slopes comparable to dynamics observed on some mountains. A consequence for the dynamics and functions at treeline is that interpreting the pattern at any one place and time for an actual ecotone may be too short a view to understand where in the wave the dynamics actually are.

Without disturbance, the realized-niche responses reveal abrupt boundaries between species in all cases, and thus the responses are generally platykurtic to rectangular with little overlap. In some cases, overlap through the extension of abundance of weaker competitors into the area of greater resources creates skewed distributions. When species are removed or invade, the abrupt boundaries and the lack of difference in final response shape make past removals difficult to detect.

\section{A NESTED REGIONAL MODEL FOR THE CONTERMINOUS U.S. AND \\ HIGH-RESOLUTION CLIMATE-CHANGE SCENARIOS WITH AN APPLICATION TO CROP CLIMATE MODELS \\ MEARNS, LINDA O.; GIORGI, FILIPPO; EASTERLING, WILLIAM; and WEISS, ALBERT NATIONAL CENTER FOR ATMOSPHERIC \\ RESEARCH and UNIVERSITY OF NEBRASKA FY 199589 \\ FY 1994118}

Objectives: To conduct a multiyear control run over the central Great Plains with the RegCM2 regional climate model, to use control-run climate output in a crop model, to produce a multiyear $2 \times \mathrm{CO}_{2}$ run over the central Great Plains nested within the CSIRO model, to perform a detailed analysis of the control and $2 \times \mathrm{CO}_{2}$ runs, and to produce climate-impact scenarios. Approach: Control and doubled- $\mathrm{CO}_{2}$ runs are performed with the RegCM2 driven by large-scale fields produced by GCM runs. The climatology of the control-nested run is validated against available observations to assess the model accuracy in presentday conditions. The statistics package of $L$. Mearns and colleagues was used for the daily analysis of temperature and precipitation. This assessment will help in the interpretation of the results from the doubled- $-\mathrm{CO}_{2}$ run.
The optimum spatial resolution of observed climate and soils data for simulating major crops grown in the central Great Plains will be determined, beginning at the scale of the European Centre for Medium-Range Forecasting (ECMWF) GCM grid cell and progressively disaggregating climate and soils data to finer spatial scales. With the Erosion Productivity Impact Calculator (EPIC) crop model, observed crop yields for the period 1984 to 1992 are compared with yields simulated with observed climate for the same period for a hierarchical distribution of grid cells nested within the ECMWF GCM grid network overlain on the MINK portion of the Great Plains. Climate and soils data are progressively disaggregated for each hierarchical level of resolution within a given GCM grid cell. The goal is to identify the level of resolution of data that minimizes statistical error between observed and modeled yields.

To simulate crop yields with the differences between current and doubled $\mathrm{CO}_{2}$ climates, the CSIRO model is applied to historical climate data aggregated to a $1 \times 1$ grid network in the study area. Then crop yields are simulated with high-resolution climatechange estimates from the NCAR RegCM driven by the CSIRO GCM. Statistical techniques are then used to compare the two methods of simulating crop yields. Results to Date: Two 5-year simulations with the NCAR RegCM were completed, one for present day conditions and one for doubled $\mathrm{CO}_{2}$ concentration. A preliminary analysis of these runs indicates that the main deficiency in the control run is a cold bias of about 3 degrees during the spring; simulated temperature is within $0.5^{\circ} \mathrm{C}$ of the observed temperature for the other seasons. Overall, the model tends to somewhat underestimate precipitation. Both the temperature and precipitation data indicate a reasonably good model performance in simulating average temperature and precipitation. A more detailed analysis showed that daily variance of temperature is well reproduced in January and April but is overestimated in July and underestimated in October. Diurnal temperature range in January is somewhat low but is well-reproduced in July. The frequency of precipitation is too high in all months, an error found in other runs of the RegCM. In the winter, the doubled- $\mathrm{CO}_{2}$-induced surface warming shows a strong meridional gradient from the Central Plains to the Great Lakes Region. Over the MINK region, the simulated surface warming varies from about 3.5 to $7.5^{\circ} \mathrm{C}$. During the summer, that warming is in the range of 3.5 to $5^{\circ} \mathrm{C}$. The model simulates an increase in precipitation under doubled- $\mathrm{CO}_{2}$ conditions for most of the MINK region in all seasons.

The aggregation of climate data at the GCM grid scale gave poor agreement between observed and modeled crop yields for all crops (dryland corn and wheat and meadow hay) that were modeled. 


\section{MODELING THE EFFECT OF GLOBAL CHANGE ON GRASSLAND DISTRIBUTION AND PRODUCTIVITY AT LANDSCAPE TO REGIONAL SCALES}

NEILSON, RONALD; LENIHAN, JAMES; DALY, CHRISTOPHER; and OJIMA, DENNIS OREGON STATE UNIVERSITY and COLORADO STATE UNIVERSITY FY 199564

FY 199440

Objectives: To investigate the transient vegetation response to climate change, the scale-dependence of vegetation processes, and the interactions of climate and management scenarios.

Approach: Wind Cave National Park is the primary test site for this modeling project because of the diversity of plant communities and the availability of research data from ongoing and past research studies. The distribution of forest and grassland communities appears to be determined by soil depth and moisture conditions. Fire and grazing regimes also play an important role in determining the establishment and composition of the plant communities.

MAPSS and CENTURY are being linked within a newly developed transient MAPSS modeling framework (T-MAPSS) to provide a flexible modeling system for a variety of developmental and model linkage efforts. The main components of T-MAPSS are: (1) a biogeographical kernel (MAPSS) that operates on a single grid point for a single time step; (2) a linked biogeochemical kernel (CENTURY) that also operates at a single point for a time step; and (3) a succession module that guides the progression of vegetation development and classification. The T-MAPSS model will be applied to Wind Cave National Park at 50-m resolution to assess its effectiveness in reproducing observed vegetation distribution patterns and dynamics at the landscape scale. T-MAPSS will also be applied to a series of fire, grazing, and climate scenarios, separately and in combination, at Wind Cave (landscape scale) and across the U.S. VEMAP grid (continental scale) to assess the sensitivity of vegetation to their direct and interactive effects on vegetation dynamics and carbon flux.

Results to Date: The environmental datasets for Wind Cave National Park are largely complete for water years 1993 and 1994. Included are monthly minimum and maximum temperature, precipitation, vapor pressure, wind speed, and solar radiation at 50-m resolution. The equilibrium MAPSS-CENTURY model was run successfully for the VEMAP U.S. region, with VEMAP long-term climate data. The simulation was found to be very CPU-intensive. Use of a parallel-processing system cut the run time by almost $\mathbf{9 0 \%}$. We are attempting to port the linked model to a massively parallel machine.

\author{
THE DETECTION OF CLIMATE CHANGE \\ WITH LONG-TERM DAILY CLIMATE \\ RECORDS OVER GRASSLAND REGIONS OF \\ THE NORTHERN HEMISPHERE \\ PALECKI, MICHAEL A.; DEWEY, KENNETH F.; \\ ROBINSON, DAVID A.; and \\ LEATHERS, DANIEL J. \\ UNIVERSITY OF NEBRASKA, \\ RUTGERS UNIVERSITY, and \\ UNIVERSITY OF DELAWARE \\ FY $1995 \quad 89$ \\ FY 199486
}

Objectives: To use the "calendar shift" climate change scenario production method to produce climate-change scenarios that minimize reliance on local-scale GCM outputs and incorporate natural interannual variability. Approach: The procedure for calendar shifting is relatively simple. First, the monthly temperature change from a control run to a 20-year segment of a transient increasing greenhouse gas run of a GCM is calculated for a large-scale region (in this case, the Great Plains). These monthly changes are then added to the observed daily regional averages in the Great Plains. A running mean of 21 days is then created for both the monthly temperature shifted and observed daily annual cycles. The observed record will provide the calendar shifts, while the monthly temperature shifted record provides the target for the calendar shift. The observed days are then matched with their closest target day, and the difference in calendar days becomes the calendar shift for the target day. Once the calendar shifts are calculated, then any and all synoptic variables can be resampled to the new seasonal cycle, except, of course, for direct measures of radiation. However, using shifted variables like temperature, humidity and clouds, one could estimate radiation parameters for a given calendar date, if needed. During each scenario, a no-analog situation will occur in the central summer period. During this time, a simple temperature shift is created by subtracting the smoothed observed average temperature from the smoothed target temperature for a given date. This change is then applied to the observational records for that date, with no attempt made to alter any other variables. Given the substantial uncertainties regarding hydrologic cycle feedbacks to increasing temperatures in mid-continental regions, we feel it is acceptable to retain present precipitation records for future scenarios during the no-analog periods.

Results to Date: The calendar-shift-scenario data exist as 60-year daily time series of calendar- or temperature-shifted observations resampled to the new climate state representing years 61 through 80 in the GFDL transient run. Changes in mean, variance, and extreme statistics during the calendar-shifted portion of the year reflect the statistics from a warmer part of the 
year. Therefore, precipitation during fall, winter, and spring increases in the calendar-shift scenario, reflecting the likely impact of warmer conditions in a region with a summer precipitation peak. The growing season in the Great Plains is projected to be longer, also, because the mean dates of last spring frost occur earlier and the mean dates of first autumn frost occur later.

With a longer growing season, soils are anticipated to be drier during midsummer. The no-analog period synoptic variables, such as precipitation, are not altered, so the calendar-shift scenario displays a temperature-increase-induced drying of the soil. The primary advantage of creating scenarios with 60 years of observations is to incorporate natural variability into climate-change scenarios. While the summer results are hardly surprising, it is interesting to note that the early growing season, through May, does not become noticeably drier during the climate-change scenario. However, increased soil-water deficits in midsummer may be sufficient to overwhelm any vegetative advantages derived from a longer and wetter spring.

\section{SEQUESTRATION OF CARBON DIOXIDE INTO SOIL ORGANIC CARBON POOLS UNDER AN ELEVATED CARBON DIOXIDE ENVIRONMENT RICE, CHARLES W., and OWENSBY, CLENTON E. KANSAS STATE UNIVERSITY FY 199522 FY $1994 \quad 0$}

Objectives: To determine the effect of increased carbon stored in the soil under elevated $\mathrm{CO}_{2}$ and the resulting impact on microbial activity and nitrogen availability.

Approach: The experimental site is located in pristine tallgrass prairie north of Manhattan, Kans. Vegetation on the site is a mixture of $\mathrm{C} 3$ and $\mathrm{C} 4$ species, dominated by big bluestem (Andropogon gerardii Vitman) and indiangrass [Sorghastrum nutans (L.) Nash]. Soils in the area are transitional from Ustolls to Udolls (Tully series: fine, mixed, mesic, montmorillonitic, Pachic Argiustolls).

Potentially mineralizable carbon and rate constant, $\mathrm{k}$, are determined by laboratory incubations, and the $\mathrm{CO}_{2}-\mathrm{C}$ evolved from soil cores is determined. Soil gas is collected weekly with gas-tight syringe and analyzed for ${ }^{13} \mathrm{C}$ expressed as parts per thousand $\left(\% \%_{00}\right)$ and the change, or delta. Measurements of soil microbial biomass carbon and ${ }^{13} \mathrm{C}$. Soil-microbial-biomass carbon is determined with the choloroform fumigation-incubation technique. The ${ }^{13} \mathrm{C}$ of the microbial biomass $\left({ }^{13} \mathrm{CMB}\right)$ is estimated as the ${ }^{13} \mathrm{C}$ of the carbon in the $\mathrm{CO}_{2}$ released from the fumigated soil $\left({ }^{13} \mathrm{CF}\right)$ in excess of that in the $\mathrm{CO}_{2}$ released from unfumigated soil $\left({ }^{13} \mathrm{CNF}\right)$.
Results to Date: After 3 years of exposure to elevated $\mathrm{CO}_{2}$, soil organic carbon increased in the $5-$ to $15-\mathrm{cm}$ soil layer but not in the surface $5 \mathrm{~cm}$ of the soil. Because the carbon did not increase in the surface $5 \mathrm{~cm}$, the increase in soil carbon storage is apparently caused by root inputs rather than aboveground plant biomass. To determine the amount of carbon storage and rate of incorporation, the soil organic matter was fractionated into three pools: microbial biomass, active, and passive. The microbial biomass has a turnover time of years, the active fraction on the order of decades, and the passive fraction on the order of centuries.

The carbon contained in the microbial biomass sampled in November of 1995 was not significantly affected by elevated $\mathrm{CO}_{2}$. The concentration of microbial biomass carbon in the surface $5 \mathrm{~cm}$ of soil was $50 \%$ higher than the 5- to $15-\mathrm{cm}$ soil layer. However, the ${ }^{13} \mathrm{C}$ content of the microbial biomass was significantly lower, indicating that the $\mathrm{CO}_{2}$ fixed by the plant was being transferred into the microbial pool. Preliminary calculations indicate $50 \%$ of the microbial carbon was newly fixed carbon produced under the elevated $\mathrm{CO}_{2}$ over the previous five 5 years. Early results of the ${ }^{13} \mathrm{C}-\mathrm{CO}_{2}$ released from the long-term incubation indicate the isotopic composition of the carbon is similar to that of the microbial biomass, as expected. As time proceeds, we would expect the ${ }^{13} \mathrm{C}$ content to be similar to that of the ambient conditions.

NATURAL RESPONSES OF SHALLOW
LAKES AND WETLANDS FOR MEASURING
AND ANALYZING IMPACTS OF CLIMATIC
AND ENVIRONMENTAL CHANGE
RUNDQUIST, DONALD C.; GOSSELIN,
DAVID C.; NARAYANAN, RAM M.; GOODIN,
DOUGLAS G.; and PEAKE, JEFFREY S.
UNIVERSITY OF NEBRASKA,
KANSAS STATE UNIVERSITY, and
UNIVERSITY OF NEBRASKA, OMAHA
FY 199551
FY 199475

Objectives: To perform historical analyses of hydrological, biological, and climatological signals and fluxes relating to selected lake/wetland systems in the Nebraska Sand Hills; to evaluate the relationships between/among hydrological, biological, and climatological signals and fluxes as they relate to lake/wetland processes; to investigate the variability over time in the flows of energy and carbon production; and to measure and map the spatial distributions of conditions related to the natural production of trace gases.

Approach: Procedures include extensive image analyses of remotely acquired data from (principally) the LANDSAT Multispectral Scanner (MSS), although some remotely sensed data from other systems such as 
the LANDSAT Thematic Mapper (TM) and the European Space Agency's ERS-1/-2 synthetic-aperture radar instrument are being examined. Detailed analyses of historical climatological and groundwater-level data have been conducted. Some attention is also being given to the recording of field data at selected sites (e.g., lake elevation, water temperature, spectral reflectance, and vegetative biomass).

Results to Date: A long-term analysis of "broad-band" lake-spectral reflectances is in progress. To date, mean per-lake reflectivities for 130 lakes on 30 scenes over a 15-year period, with associated variability, have been completed. We have determined that green, red, and near-infrared reflectivities for lakes in the region range from less than $1 \%$ to nearly $20 \%$. We have compiled the first "hyperspectral" datasets (252 narrow spectral bands) describing each of approximately forty lakes in the study area, indicative, in general, of the organic and inorganic components of the water column. Simulations with MODFLOW plus the lake package indicate a direct relationship between the seasonal persistence and decline of groundwater mounds between lakes and changes in the direction and magnitude of groundwater-lake seepage components.

We are digitally classifying and measuring wetland communities in an effort to highlight changes in spatial extent and/or reflectivity. We are also attempting to calibrate both satellite and spectroradiometer measures of aboveground biomass with in situ measures. The image analysis of LANDSAT-MSS data for wetlands interpretation is at a preliminary stage. However, in a cursory examination of 69 scenes acquired over the CLNWR between 1972 and 1987, we found that the most-variable areas (in vegetative greenness, or NDVI), are the marshes along the lake margins, followed by the adjacent wet meadows, remaining lowlands, and lowland-to-upland transition zones. We have processed ERS-1 data obtained in June, July, August, October, and November of 1995 to produce a multitemporal composite image that highlights the seasonal characteristics of the wetland vegetation. A surprising finding was that detection of aquatic vegetation, which is at or very close to the surface of the lakes, seems possible with ERS data.

One outcome is the creation of an archive of digital data for the study area. For example, 85 LANDSAT-MSS scenes covering the Western Sand Hills have been obtained, georeferenced, and processed to correct for changing solar-illumination angles and sensor flux. This archive is being used to measure surface areas of 130 lakes between 1972 and 1993 and search for "climatic signals" in the optical reflectivities and spatial extents of wetland canopies.

\section{ASSESSMENT OF CLIMATE- AND MANAGEMENT-INDUCED DIRECTIONAL CHANGES IN GREAT PLAINS VEGETATION WITH NDVI AND STABLE CARBON ISOTOPES \\ TIESZEN, LARRY L. AUGUSTANA COLLEGE \\ FY 199559 \\ FY 199480}

Objectives: To determine if today's native grassland systems are in a long-term steady state with climate or the degree to which they depart and the direction in which change is proceeding and to establish biophysical attributes of grassland canopies and relate these to vegetation indices as we evaluate ecosystem performance and the roles of the dominant $\mathrm{C} 3$ and $\mathrm{C} 4$ systems in grasslands.

Approach: We use seemingly diverse but integrating approaches to study the response of grasslands and land cover in the Great Plains to climate change. Stable isotopes of carbon are used to quantify fluxes from various pools of carbon, mainly atmosphere, plant, and SOM, and to allow us to interpret successional changes in vegetation as well as steady-state relationships between SOM isotopes and climate. This method requires the analytical capabilities of an isotope ratio mass spectrometer and the SOM fractionation techniques worked out with Cambardella.

Remote sensing, mainly AVHRR data, allows us to monitor grassland land-cover classes across the Great Plains and at specific sites where biomass and biophysical relationships can be derived. We have concentrated on a site at the Niobrara Prairie Preserve, Nebr., but have developed a Great Plains wide network of sites at Tallgrass Prairie Preserve, Okla.; Konza Prairie, Kans.; and Grasslands National Park, Canada. Our AVHRR analysis is supplemented by the availability of TM and SPOT data. We believe we can soon simulate daily fluxes of carbon and water largely based on remotely sensed data.

Results to Date: We have just completed a refined land cover classification of the Great Plains. The validation of the classification is under way at our Niobrara and Tallgrass sites as is an analysis of subpixel variation based on the SPOT imagery. The contribution by $\mathrm{C} 4$ species varies from $94 \%$ in the south to $29 \%$ in the north. The classes possess distinct patterns of NDVI with a consistent relative performance across 5 years of quite variable weather. Production seems largely independent of composition by photosynthetic type but shows a strong east-to-west gradient. Detailed ground radiometric work at Niobrara has allowed us to estimate biomass, LAI, and related canopy parameters. We have now been able to extend some of these generalizations across the Great Plains and across various grassland land cover classes. 
This and our associated PPAR estimates from remotely sensed data should allow us to simulate carbon uptake from remotely sensed reflectance data supplemented with minimal spatially explicit ground data. A general model for all grassland classes across 5 years accounted for $51 \%$ of the variance in TiNDVI. The most significant seasonal variables are: potential evapotranspiration, spring precipitation, accumulated growing degree days, and summer precipitation. Predictions were stronger within individual classes where $80 \%$ of the variation in TiNDVI was accounted for by growing-season rainfall alone.

STATSGO and SOM isotope data provided complementary data sets to evaluate the effect of climate on the distribution of proportional $\mathrm{C} 4$ production. That production is related positively to high temperature during the growing season and precipitation in July and negatively to the low temperature in April. High temperature during early spring, however, may favor $\mathrm{C} 3$ species.

Most of the ${ }^{14} \mathrm{C}$ dating has now been done, and mean residence times confirm our interpretations based on stable isotopes and should allow us to derive good estimates of turnover. We have supplemented that project with a detailed analysis of SOM as related to land cover and land use in the Niobrara site. Actual data show good agreement with STATSGO estimates on some sites and significant departures on other sites (e.g., forested areas).

\section{INTEGRATED INVESTIGATION OF METHANE AND CARBON DIOXIDE FLUXES IN MIDLATITUDE PRAIRIE WETLANDS VERMA, SHASHI B. UNIVERSITY OF NEBRASKA FY 1995168 FY 1994300}

Objectives: To expand the body of knowledge on surface fluxes of carbon dioxide and methane in midlatitude prairie wetlands.

Approach: Ballards Marsh in the Sandhills region near Valentine, Nebr., will be subjected to intensive measurements of fluxes of carbon dioxide, methane, and energy. It provides an excellent upwind fetch for the micrometeorological measurements and is characterized by three well-defined plant community types: 2- to 3-m-tall common reed grass (Phragmites austra lis); 1- to 2-m-tall bulrush (Scirpus acutus); and deeper, open water, where wild rice (Zizania aquatica) is the major emergent species and a submergent pondweed species (Potamogeton) is very common.

Results to Date: Until Phragmites developed green leaves in mid-May, the marsh was a net source of atmospheric $\mathrm{CO}_{2}$. In late May, the marsh quickly changed from a source to a sink for $\mathrm{CO}_{2}$. In September, both daytime and nighttime $\mathrm{CO}_{2}$ fluxes decreased rapidly as the vegetation senesced; the $\mathrm{CO}_{2}$ exchange between the marsh and the atmosphere approached zero in October. From early June to early July, the methane flux increased from 250 to $600 \mathrm{mg} \mathrm{m}^{-2} \mathrm{~d}^{-1}$. Midday methane flux peaked during August and early September, varying from 600 to $1200 \mathrm{mg} \mathrm{m}^{-2} \mathrm{~d}^{-1}$. From mid-September to mid-October, with senescing vegetation and falling temperatures, methane flux decreased to 100 to $350 \mathrm{mg} \mathrm{m}^{-2} \mathrm{~d}^{-1}$. Surface $\mathrm{CO}_{2}$ fluxes showed that both Phragmites and Scirpus had similar seasonal trends in surface $\mathrm{CO}_{2}$ flux: small early in the season, peaking in the middle of August, and decreasing thereafter. Single-leaf gas-exchange data in combination with LAI and canopy-structure measurements indicate that peak rates of canopy photosynthesis were near $17 \mu \mathrm{mol} \mathrm{m} \mathrm{m}^{-2}$ for Phragmites and about $12 \mu \mathrm{mol}$ $\mathrm{m}^{-2} \mathrm{~s}^{-1}$ for Scirpus. Peak values of canopy conductance, on a ground area basis, ranged from 0.5 to $0.6 \mathrm{~mol} \mathrm{~m}^{-2}$ $\mathrm{s}^{-1}$ for both species. Light intensity has a profound effect on these rates. Chamber-derived $\mathrm{CH}_{4}$ and $\mathrm{CO}_{2}$ flux rates were highly variable. Differences in flux rates between Phragmites- and Scirpus-dominated areas were slight. Between 75 and $90 \%$ of emitted $\mathrm{CH}_{4}$ leaves the system via plant transport during daylight hours. Adding carbon (as wheat straw) to the marsh had no significant effect on $\mathrm{CH}_{4}$ emissions by itself; but when added with nitrogen (as urea), it doubled $\mathrm{CH}_{4}$ emissions, indicating that anaerobic decomposition is nitrogen-limited.

We developed the Wetland Biogeochemistry Model to simulate $\mathrm{CH}_{4}$ generation and efflux; it models anaerobic and aerobic decomposition of organic matter; methanogenesis; methane oxidation; and storage and flux of $\mathrm{CO}_{2}, \mathrm{CH}_{4}$, and $\mathrm{O}_{2}$ via diffusion, bubbling, and plant-mediated transport. The model results to date indicate that the relative magnitude of $\mathrm{CH}_{4}$ efflux is highly sensitive to plant-mediated transport and diffusion within the sediment profile. 


\section{West Regional Center}

\section{CLIMATE AND LAND-USE CONTROLS ON $\mathrm{CO}_{2}$ EXCHANGE AND CARBON TURNOVER RATES IN SOILS OF THE SIERRA NEVADA, CALIFORNIA \\ AMUNDSON, RONALD \\ UNIVERSITY OF CALIFORNIA, BERKELEY \\ FY 199572 \\ FY 199490}

Objectives: To determine the effect of climate and land use on the carbon-turnover rates of soil organic matter in the Sierra Nevada range of California and to develop a thorough understanding of the oxygenisotope systematics of soil $\mathrm{CO}_{2}$ as a tool in quantifying rates of terrestrial ecosystem $\mathrm{CO}_{2}$ exchange with the atmosphere.

Approach: The research involves a combination of intensive field-based time-series measurements of soil respiration rates, soil temperature and moisture contents, and the ${ }^{14} \mathrm{C}$ content of soil-respired $\mathrm{CO}_{2}$. Accompanying the field-based observations are laboratory incubations to determine the ${ }^{14} \mathrm{C}$ content of humusderived $\mathrm{CO}_{2}$ in these same soils. Using this laboratory information, we will then use the ${ }^{14} \mathrm{C}$ content of soilrespired $\mathrm{CO}_{2}$ to partition total soil respiration (as measured in the field) into root- and humus-derived fractions and, ultimately, to calculate humus turnover rates. These turnover rates provide an additional, independent experimental approach that we will compare to our recent estimates of turnover times based on pre- and post-atomic bomb soil-organicmatter ${ }^{14} \mathrm{C}$ comparisons.

Results to Date: We have completed field-based measurements of total respiration and isotopic measurements of respiration and are now focusing on the laboratory incubations to determine the isotopic composition of humus-derived $\mathrm{CO}_{2}$. Field measurements show that the ${ }^{14} \mathrm{C}$ content of respired $\mathrm{CO}_{2}$ from soils in different climates and land managements are slightly different, but all contain a significant quantity of modern carbon. Laboratory incubations reveal that the humus carbon sources available to microbes (i.e., the ${ }^{14} \mathrm{C}$ content of humus) varies greatly with temperature and moisture conditions.

We have also partially completed our investigation of the processes controlling the ${ }^{18} \mathrm{O}$ content of soilrespired $\mathrm{CO}_{2}$. Recent field measurements, coupled with a year-long time series study, reveal that the $\delta^{18} O$ value of soil-respired $\mathrm{CO}_{2}$ can be up to $16 \%$ greater than previously reported model-based estimates. We are following our field observations with controlled laboratory experiments to more precisely understand the processes, and kinetics, of $\mathrm{CO}_{2}$ isotopic exchange with soil $\mathrm{H}_{2} \mathrm{O}$. Field measurements of the $\delta^{18} \mathrm{O}$ values of soil $\mathrm{CO}_{2}$ and $\mathrm{H}_{2} \mathrm{O}$ suggest that the $\delta^{18} \mathrm{O}$ values of soil-respired $\mathrm{CO}_{2}$ reflect the $\mathrm{O}$ isotope composition of the upper few centimeters of the soil water: water it reequilbrates with prior to diffusing into the overlying atmosphere.

\section{EFFECTS OF TREELINE MOVEMENT IN THE CIRCUMPOLAR ARCTIC ON GLOBAL CLIMATE CHANGE CHAPIN, F. STUART \\ UNIVERSITY OF CALIFORNIA, BERKELEY FY 199573 \\ FY 19940}

Objectives: To develop and use a dynamic model of vegetational transitions to predict the regional movement of treeline in the circumpolar arctic under various scenarios of climate change and to examine the consequences of predicted changes in vegetation for the rate of global change through their feedbacks to climate via effects on energy balance, carbon storage, and precipitation over a regional scale.

Approach: Models of vegetation response to climate change will be developed and combined with databases of carbon and energy budgets.

Results to Date: Forestation of tundra areas under the influence of global warming were simulated with computer models. The three major conclusions of our simulation modeling are that (1) there will be substantial time lags in forestation, regardless of warming rate; (2) warmer, drier climates will probably lead to substantial areas of low-statured grassland-steppe rather than forest; and (3) increased climatic variability can have the same effect as climatic warming in promoting forestation following time lags. The first two conclusions suggest that the positive feedbacks to climatic warming caused by the lower albedo of forest compared to tundra or grassland-steppe will be delayed relative to the assumption of equilibrium between climate and vegetation that is used in current GCMs. This provides a window of opportunity to regulate fossil-fuel emissions that currently drive climatic warming before the biological positive feedbacks associated with northward movement of treeline exacerbate climatic warming. 


\section{ASSESSING THE IMPACTS OF GLOBAL WARMING ON CALIFORNIA WATER RESOURCES \\ FEDDEMA, JOHANNES J. \\ UNIVERSITY OF CALIFORNIA, LOS ANGELES FY 199539 \\ FY 19940}

Objectives: To develop a regional-scale water balance model and a methodology for creating climate change scenarios that would help to evaluate the impacts of global warming on regional water resources.

Approach: The model will use a standard water budget as its core. In addition, a runoff model and sediment-transport model will be linked to the water budget to be able to evaluate overland runoff, water surplus and deficit conditions, and sediment flows in California river systems. The expected impact of global warming on California water resources will be compared to changes in water resources historically observed during relatively wet, dry, and normal climate conditions. A combined runoff, sediment, and waterbalance model will be used to estimate the annual water deficit and surplus conditions at $1 / 4$ by $1 / 4$ grid locations in California. Base input to the model will come from interpolated precipitation and temperature measurements during specific dry, wet, and climatically normal conditions. Other required inputs are related to topography from a 5-minute USGS Digital Elevation Model, soil properties from USGS soil surveys, soil-field-capacity estimates of each location, land use, and vegetation characteristics of each location. Changes in the water balance for these conditions will then be compared to the changes from predicted global-warming conditions. Global-warming scenarios will be created with the same three base-climate scenarios modified by GCM-predicted average changes in temperature and precipitation at each grid cell. Dividing the entire region into separate drainage basins will allow evaluating the impacts of global warming on individual watersheds. It is intended that this study will provide specific information on expected changes the water yield of specific drainage basins and on the amount of water available for human consumption. Furthermore, the water-balance approach is particularly appropriate for evaluating potential changes in the typically drought-limited ecosystems.

Results to Date: A climatological database has been created for California, including interpolated maps of precipitation and temperature for the normal, drought, and wet conditions in the instrumental climate record. A statewide land-use dataset has been compiled for inclusion in the model input. Daily precipitation data have been compiled, and representative regions have been identified for using the SCS runoff model in estimating monthly percentages of overland runoff.
Doubled- $\mathrm{CO}_{2}$ estimates of climate change have been collected. A variety of elevation, soil, and land-use data are being collected to serve as input to the sediment-flow model.

\section{LINKING THE CASA AND SIB GLOBAL TERRESTRIAL MODELS: IMPROVED ESTIMATES OF GLOBAL CARBON STOCKS AND FLUXES AND $\mathrm{CO}_{2}$ RESPONSES FIELD, CHRISTOPHER B., and BERRY, JOSEPH A. \\ CARNEGIE INSTITUTION OF WASHINGTON FY 199560 \\ FY 19940}

Objectives: To assess (1) patterns of global production and decomposition in relation to patterns of climate and disturbance during the satellite era, (2) possible changes in global production and decomposition in a world with doubled atmospheric $\mathrm{CO}_{2}$, and (3) implications of increased $\mathrm{CO}_{2}$ for surface climate through direct effects of $\mathrm{CO}_{2}$ on canopy conductance.

Approach: Our approach is to develop algorithms that capture as many as possible of the physiological, ecological, and biogeochemical mechanisms that control the terrestrial biosphere. We test these as extensively as possible against observations at the ecosystem and regional scale. We gather or develop the data sets necessary to run the models at the global scale and conduct experiments designed to find an acceptable compromise between the questions that most need answering and the questions the models are best suited to address.

Results to Date: CASA simulations indicate that, during the past decade and a half, global NPP has increased steadily at a rate between 0.5 and $1 \%$ per year, which is substantially larger than that required to sustain a current terrestrial sink of approximately 1.6 gigatons per year. Our simulations also indicate that the atmospheric amplitude at Mauna Loa is dominated by far-northern biomes. When the residence time of carbon in live woody tissue as well as in soil organic matter is accounted for, we calculate a globally averaged isodisequilibrium between the atmosphere and the terrestrial biosphere of 0.3 to $0.4 \%$ and that the current carbon sink is largely terrestrial. If a substantial fraction of the sink is in areas with $\mathrm{C} 4$ photosynthesis, the sink is overwhelmingly terrestrial.

We have developed a number of new model algorithms. The new algorithm for nitrogen control of NPP in CASA allows a wide variety in control mechanisms, reflecting observations from contrasting ecosystems. The new algorithm for soil respiration in CASA allows for the additional seasonality introduced by substrate limitation. Our algorithms for plant respiration build on well-established concepts of growth and maintenance respiration, with a new implementation 
that takes advantage of the satellite data. We have initiated the task of model integration with studies focused on specific sites, beginning with the site of the FIFE studies in Kansas. Using a very complete data set on climate, soil moisture, and surface exchanges, we are running $\mathrm{SiB} 2$ and $\mathrm{CASA}$ together, using $\mathrm{SiB} 2 \mathrm{GPP}$ to drive CASA. A new CASA version that runs on any time step facilitates the integration.

\section{ADJUSTING TO GLOBAL CLIMATE CHANGE: MANAGING INCREASED VARIABILITY IN ANADROMOUS FISHERIES IN THE PACIFIC NORTHWEST FISHER, ANTHONY, and HANEMANN, W. MICHAEL UNIVERSITY OF CALIFORNIA, BERKELEY FY 199550 FY 199460}

Objectives: To develop a model of investment in water resources under climate-change uncertainty.

Approach: An important question that arises is, how would such a change in the stochastic process that generates flows affect the optimal level of investment in water reserves or storage? We developed a model of investment in water reserves and storage capacity, subject to constraints on the accumulation of reserves and capacity, and a stochastic differential equation that describes the evolution of water flows over time. The authority's problem is to maximize storage capacity and the generation of water flows, the net present value of water use, the cost of adjusting the stock of water reserves, and the environmental cost of holding reserves.

Results to Date: We have gathered data on various aspects of water use by agricultural and urban users in California and critiqued recent Department of Water Resources projections of water demand in California to 2020. We have also assembled and reviewed various existing agency models of the major water systems and water-using sectors in California that cover both groundwater and surface-water resources. Modeling results indicate that, if storage and water flows are substitutes, then there exists a necessary and sufficient condition for a unique optimal storage capacity as a function of the variance of the flow. Under this condition, an increase in the variance implies an increase in optimal capacity. Building storage is like insuring the region against uncertain future damages. Because an increase in the variance of flow implies an increase in the probability of occurrence of extreme events, such as droughts, more reserves are required to reduce the potential damages. If there exists an asymmetry in adjustment costs of storage, then changes in the variance do not affect the optimal capacity within a certain range. Given an increase in the variance, for example, it will not be optimal to invest in more storage until the increase has gone above a critical value that can be calculated. If disinvestment is costly (e.g., if the cost entailed in restoring the natural environment is very large), then changes in variance again do not affect the optimal storage capacity. In this case, the range of inaction is greater than it is in the case of asymmetric purchase and sale prices. Irreversibility can be considered an economic phenomenon, related to the cost of disinvestment; the range of inaction is increasing with the cost of disinvestment.

\section{CLIMATE CHANGE AND THE DYNAMICS OF MARINE ECOSYSTEMS: THE INTEGRITY OF BIOGEOGRAPHIC BOUNDARIES GAINES, STEVEN D. UNIVERSITY OF CALIFORNIA, SANTA BARBARA \\ FY 199575 \\ FY 19940}

Objectives: To test between competing hypotheses for the causes of the abrupt biogeographic boundaries commonly found in marine communities: the mortality hypothesis that posits that species range limits are clustered because abrupt physical changes (e.g., water temperature) occur in the vicinity of the point that makes some species incapable of surviving on the other side and the dispersal barrier hypothesis that posits that species-range limits are clustered because ocean circulation in the vicinity of the point limits the dispersal of individuals beyond the point.

Approach: We are monitoring the spatial patterns of settlement for more than ten species over a stretch of coastline spanning more than $250 \mathrm{~km}$. These studies use a variety of settlement substrates that entice larvae of different species to settle. We are also beginning experimental studies of the performance of individuals of these species (growth rates, survivorship, and reproductive output) in the vicinity of and beyond their range boundary. To support these biological studies and to help interpret the findings, we are also measuring a suite of physical parameters at each study site [water temperature, air temperature, nutrients $\left(\mathrm{PO}_{4}\right.$, $\mathrm{NO}_{2}, \mathrm{NO}_{3}$, and $\mathrm{SiO}_{4}$ ), and chlorophyll].

Results to Date: We now have nearly an entire year's worth of settlement data for three species of hermit crabs [Pagurus samuelis, Pagurus granosemanis, and Pagurus hirsutiusculus (northern and southern subspecies)] and two bivalves (Septifer bifurcatus and Mytillus spp.). All of the transplant studies were not started until the summer, the peak of settlement for most of the species involved, so no data are yet available. The recruitment studies, however, are yielding very interesting results. There is a relatively abrupt change in recruitment in the vicinity of Pt. Conception. There is a relatively wide region (approximately 
$30 \mathrm{~km}$ ) from Pt. Conception to Pt. Arguello, where abrupt changes in recruitment occur. Species that are recruiting heavily north or south of this region, show abrupt drops in recruitment rates. In addition, species without a range boundary near Pt. Conception show a region of very low recruitment centered on Pt. Conception. These findings are consistent with barriers to larval dispersal playing an important role in the location and stability of this biogeographic boundary, although the short-term nature of these results make any conclusions premature at this point.

\section{GLOBAL-WARMING TRENDS AND \\ INTERNAL CLIMATE OSCILLATIONS: DETECTION AND MODELING \\ GHIL, MICHAEL, and NEELIN, DAVID \\ UNIVERSITY OF CALIFORNIA, LOS ANGELES \\ FY 199583 \\ FY 1994105}

Objectives: To decompose the existing multidecadal records of surface temperature, sea-level pressure, and sea-level height into their principal components with respect to spatial and temporal variability and to relate these dominant statistical features to biogeochemical, physical, and dynamic mechanisms, natural or connected with humanity's activities.

Approach: The detection of a systematic warming trend in global surface temperatures is rendered difficult by the presence of substantial variability on time scales both shorter and longer than the one of interest. Greenhouse trace gases have increased rapidly during the past century [e.g., carbon dioxide $\left(\mathrm{CO}_{2}\right)$ has increased by about $25 \%$ ]. Documented temperature change over the same time interval is only about $0.5^{\circ} \mathrm{C}$; this is both smaller than climate models would suggest and marred by nonnegligible uncertainties caused by temperature variability on other time scales and the possible effect of unrelated climatic mechanisms.

Advanced tools for the statistical analysis of climatic oscillations, and of their spatial patterns, have been developed in connection with paleoclimatic studies and with studies of low-frequency atmospheric variability. Singular-spectrum analysis (SSA) is a generalization of spectral analysis that uses dataadaptive filters and is particularly well-suited for the study of nonlinear, slightly irregular oscillations. Its adaptive prefiltering properties yield fruitful combinations with other spectral techniques, such as the maximum-entropy method and the multitaper method. Results to Date: A 10-year oscillation is observed, but no trend for the spatial empirical orthogonal function (EOF) associated with mean U.S. temperatures, while the EOF representing an East-West seesaw exhibits both a trend and a 23-year oscillation. This result suggests the presence of different modes of inter- decadal behavior for the Northern Hemisphere (NH) as a whole than for the two major NH ocean basins separately.

Applying SSA-MEM to the Southern Oscillation Index shows the method to have valuable skill out to 36 months. SSA-MEM prediction was applied to the global IPCC consensus time series and to the longer, but local, CET record. They foreshadow slight cooling of global surface-air temperatures in the late 1990s before subsequent further warming. Multichannel SSA (M-SSA) has proven useful in studying the nonlinear interaction of multiple time and space scales in climate phenomena. Subharmonics and frequency locking arise when internal frequencies interact with each other and with the annual cycle. With COADS data for seasurface temperatures (SSTs) and zonal winds, a dominant quasiquadrennial peak could be separated from a secondary quasi-biennial peak.

A bifurcation study of a simple ocean model forced by prescribed surface temperatures and salinity fluxes has yielded insight into the dynamics of the ceans' thermohaline circulation (THC). The continuous transition via increasingly asymmetric two-cell circulations can be understood as a function of Rayleigh number and the strength of the salt-flux forcing. When the salt-flux strength is sufficiently large, the system undergoes a Hopf bifurcation leading to sustained, long-period oscillations. This bifurcation is robust to changes of the spatial structure of the salt flux.

Coupling a nonlinear, thermodynamic sea-ice model with a zonally averaged ocean model was found to produce interdecadal oscillations. The mechanism involves a cycle of melting and freezing of sea ice and associated density effects on the THC and provides a consistent demonstration that THC-sea-ice interactions can introduce new interdecadal phenomena not present in the ocean alone. Thermal insulation by sea ice was found to stabilize the THC, while the freshwater flux associated with freezing or melting increases the effective inertia of the system; the latter tends to slow down THC response to long-time-scale external forcing.

\section{GLOBAL WARMING AND ECOSYSTEM CARBON STORAGE: COMBINING AN ELEVATIONAL TRANSECT STUDY WITH AN EXPERIMENTAL INVESTIGATION OF WARMING-INDUCED FEEDBACKS TO CLIMATE HARTE, JOHN \\ UNIVERSITY OF CALIFORNIA, BERKELEY FY 199570 \\ FY 199465}

Objectives: To quantify and understand the effects of climate change on the carbon cycle of a montane 
meadow ecosystem and, hence, to better understand $\mathrm{CO}_{2}$-mediated feedbacks to climate by means of an experimental warming manipulation and correlation analysis of natural climatic gradients.

Appreach: The study sites are ungrazed montane meadows near the Rocky Mountain Biological Laboratory (RMBL), Gunnison County, Colo. All sites are located in an open meadow with a mixed shrub/ forb/graminoid plant assemblage, including the shrub Artemisia tridentata (sagebrush) and the grass Festuca thurberi (fescue). Ten 3- by 10-m plots are laid out side-by-side along a north-south line. Each plot is on an incline, and is divided into a drier upper zone and a moister lower one. Every other plot is warmed with electric heaters suspended $2.5 \mathrm{~m}$ above the ground. Three other experimental sites were established approximately $200 \mathrm{~m}$ in elevation apart. At each of the three elevational sites, snowmelt date will be accelerated by snow removal and about $25 \%$ of summer rainfall will be diverted. This combination was chosen to closely mimic the microclimate effects at the warming meadow. Our prior work at the warming meadow has shown that soil moisture and date of snowmelt are far more explanatory than soil temperature for many ecosystem responses.

Carbon stocks in standing aboveground biomass are measured throughout the summer, and soil carbon stocks (roots and soil organic matter separately) are sampled at the beginning and end of the growing season in representative soil cores taken from each plot. The organic matter content of the oven-dried, $2 \mathrm{~mm}$-sieved soil is measured by loss of weight on combustion. Laboratory incubations are used to measure site-to-site differences in the dependence of soil respiration on temperature and moisture. Other measurements relevant to carbon dynamics are also taken, including soil moisture and temperature, microbial and mesofaunal biomass, nitrogen stocks in soil and vegetation, inorganic soil nitrogen content, and $\mathrm{C}: \mathrm{N}$ ratios in soil and plant biomass.

Results to Date: The heaters advance snowmelt by 1 to 2 weeks. The drier upper zone averages about $1.5^{\circ} \mathrm{C}$ warmer in the heated plots during the growing season; soil-water content as a percent of soil dry weight is typically 2 to $5 \%$ lower. The overall trend appears to show a loss of heated-plot soil carbon because of, we hypothesize, a decrease in photosynthetic production resulting from increased drought stress.

Automatic data loggers were installed at the elevational transect sites in July 1996; the initial data from these is not yet available. Lower site 1996 snowmelt date preceded that of the middle site by approximately 2 weeks, while upper site snowmelt lagged that of the middle by about 1 week. Soil organic matter is greatest at the middle site, and declines going both up and down the mountain. This is at odds with the increasing (decreasing) trend with altitude (temperature) that would be expected. Standing litter stock, however, exhibits a monotonic decline with elevation, indicating decreasing aboveground production with altitude. Laboratory incubations indicate greater soil respiration with altitude for any given temperaturemoisture combination.

\section{EFFECTS OF CLIMATIC CHANGES ON ECOLOGICAL COMMUNITIES IN FRAGMENTED HABITAT HASTINGS, ALAN \\ UNIVERSITY OF CALIFORNIA, DAVIS \\ FY 199561 \\ FY 19940}

Objectives: To determine the effects of global environmental change on species in communities whose habitat will undergo further fragmentation and experience reduction in quality of individual habitat patches. Approach: A general framework will be developed to describe communities (interacting species) in fragmented habitats. The goal will be to produce models that are mechanistic, yet can be parameterized simply and yield both quantitative and qualitative predictions. We will then use these models to look at the response of these communities to changes in the size and fragmentation of habitat patches. The models will be used both to determine the level of information required concerning changes in habitat and to determine changes in population levels, which are needed to make predictions of the response of the ecological communities. The final step will be to relate these general predictions to specific examples from the western United States.

Results to Date: Our work demonstrated that density dependence in competitive exclusion has a very small effect on coexistence. However, density dependence in extinction led to greatly reduced coexistence compared with density independent extinction, especially in the cellular automata. These results suggest that densitydependent interactions and extinction may play a large role in competitive coexistence.

We have developed some predator-prey models to look at the effects of alteration of movement. Oscillation in species' populations is altered considerably by the presence of dispersal in both species. As one aspect of global change for species living in fragmented habitats is the likelihood that dispersal rates between suitable habitats would change. One outcome is the possibility of extinction. More likely, however, is the situation in which the solution becomes unstable, producing a large number of predator-prey cycles (which can still lead to extinction). 


\section{SCALING UP FROM LEAF TO STANDS: COUPLING ECOPHYSIOLOGICAL MODELS \\ WITH REMOTE SENSING IN POPULUS, ABIES, AND PSEUDOTSUGA \\ HINCKLEY, THOMAS M. \\ UNIVERSITY OF WASHINGTON \\ FY 199534 \\ FY $1994 \quad 0$}

Objectives: To evaluate AVIRIS data as means of making physiologically relevant measurements at the canopy scale, particularly relating to the carbon and water cycles of two very different forest communities. Approach: The AVIRIS images and ground-based data will be used to estimate canopy biochemical and architectural affects on remotely sensed data. At Wind River, we have installed instruments in three trees, with one large Pseudotsuga menziesii monitored very intensively. The data are collected at 1- and 15-minute time intervals. At the Cedar River Watershed, we have a large dataset that we plan to synthesize with a canopy-reflectance and -productivity model that uses our measurements of canopy reflectance, light extinction coefficients, LAI, and other physiological measurements.

Results to Date: Airborne Visible/Infrared Imaging Spectrometer (AVIRIS) images were collected in October 1995 of the clonal Populus hybrid plantation. Differences across clone type and age were clearly evident from the images, and our task was to evaluate the canopy characteristics contributing to that distinction. Leaf area index (LAI) was also measured with different methods in each of the clone types at the plantation. Leaves were destructively harvested from upper and lower crown positions for the three clones types and analyzed in the laboratory for the following: leaf spectra, percent water, chlorophyll content, leaf thickness, and specific leaf area. Initial results suggest that, at the leaf scale, leaf reflectance was not significantly different among the three Populus clone types. Regarding the October image, ground-based pigment analyses show no significant differences in chlorophyll content per unit surface area across all clone types. However, the AVIRIS data show differences across clone types in the spectral region of chlorophyll reflectance. Our LAI measurements from the litter traps and ceptometer measurements approached the same values for all three clone types $(\sim 4.3)$.

\footnotetext{
HIGH-RESOLUTION ANALYSIS OF SALINITY CHANGE AND WETLAND DEVELOPMENT IN THE SAN FRANCISCO ESTUARY: 1000 BP TO THE PRESENT INGRAM, B. LYNN, and BYRNE, ROGER UNIVERSITY OF CALIFORNIA, BERKELEY FY 199572 FY 19940
}

Objectives: To analyze the Petaluma Marsh core, including carbon isotopic analyses of organic material separated from the core, and to take cores from two new marsh sites, Rush Ranch and Brown's Island for pollen and carbon-isotopic analyses.

Approach: Eight short cores were taken at Rush Ranch Marsh in Suisun Bay in February 1996. The cores were returned to the lab and stored in a cold room. All cores were X-radiographed to provide a record of core stratigraphy prior to sampling, and the $\mathrm{X}$-radiographs were digitized. Cores were then split and logged for their visual characteristics: Munsell color and general stratigraphy. They were analyzed for water content, loss on ignition, pollen, microfossils (foraminifera and ostracodes), and carbon isotopic distribution. Four samples were taken for radiocarbon analysis at Lawrence Livermore National Laboratory. Results to Date: Radiocarbon dating of the Rush Ranch samples gave ages of $840 \pm 60,1840 \pm 50$, $2390 \pm 50$, and $2400 \pm 60$ years, indicating that the sedimentation rate at the core site has varied significantly during the past 2700 years. Pollen studies indicate that the floristic composition of the marsh has changed dramatically during the past 500 years and especially so during the past 50 years. The record shows very clearly that the marsh has become more saline during the past 100 years. We assume that this primarily relects the diversion of freshwater out of the Sacramento River into the California Aqueduct, but the pollen evidence suggests that the increase in salinity may have begun earlier than that project, perhaps because of climatic shifts involving reduced winter precipitation in the watershed. Samples prepared from the lower section of the Rush Ranch Core indicate that the Salicornia was important for some time prior to $600 \mathrm{BP}$. In other words, there have been extended periods during the past several thousand years during which the marsh was as least as brackish as it is today. Presumably, this observation reflects changes in freshwater inflow via the Sacramento River.

No carbonate shells (macro or microfossils) were found in sediment cored at Rush Ranch. The carbon isotopic composition of plant material was used to provide information as to the changing proportions of $\mathrm{C} 3$ and $\mathrm{C} 4$ plants at the study sites. In the coarse fraction, the $\delta^{13} \mathrm{C}$ value showed a systematic decrease from the top of the core $(-16 \%)$ to $95 \mathrm{~cm}(-27 \%)$ with a slight increase from $95 \mathrm{~cm}$ to $115 \mathrm{~cm}(-25 \%)$. The fine fraction followed the same general pattern, but there is an offset between the coarse and fine fraction in the upper $40 \mathrm{~cm}$, with the fine fraction being 2 to $5 \%$ lighter. In the Petaluma core sediments, the $\delta^{13} \mathrm{C}$ values for the coarse fraction increased between $-22.6 \%$ at the top of the core, to $-16 \%$ at $111 \mathrm{~cm}$. The $\delta^{13} \mathrm{C}$ value decreased from $-16 \%$ at $111 \mathrm{~cm}$ to $-24 \%$ 
at $187 \mathrm{~cm}$. The fine fraction showed a very similar pattern.

\section{AN ECONOMIC ANALYSIS OF ADAPTATIONS TO GLOBAL WARMING AND POLICIES TO MITIGATE IT IN THE SACRAMENTO REGION JOHNSTON, ROBERT A. UNIVERSITY OF CALIFORNIA, DAVIS} FY 199532

FY 19940

Objectives: To investigate the social, economic, and environmental impacts of adaptive responses to warming and of mitigative policies, to design tax and other policies to reduce the adverse distributional consequences of the adaptive responses and mitigative policies, to evaluate the social impacts of these equity policies, and to develop greenhouse-gas projection models within Tranus.

Approach: We will run simulations with the economic model and develop one or more scenarios for adaptive and mitigative responses in this region (e.g., carbon taxes, immigration of lower-income households, and travel pricing). We will then test one or more redistributive policies, such as free transit passes for lower-income households.

Results to Date: We have completed the simulations with the economic model. The scenarios examined so far for the year 2015 are: future trend, beltways with high-occupancy vehicle (HOV) lanes, HOV lanes, and light-rail transit with parking charges. We may be able to run additional scenarios, depending on funding. We have measures for aggregate regional traveler surplus, surplus for three household income classes, emissions, agricultural-land conversion, and habitat change. We are now building the greenhouse-gas model.

\section{RELATIONS BETWEEN CATASTROPHIC CHANGES IN THE BENTHIC ECOSYSTEM AND LATE QUATERNARY GLOBAL CLIMATE CHANGE, SANTA BARBARA BASIN \\ KENNETT, JAMES P., and BEHL, RICHARD J. UNIVERSITY OF CALIFORNIA, SANTA BARBARA FY 199564$$
\text { FY } 19940
$$

Objectives: To characterize the ecological changes in the benthic environment of the Santa Barbara Basin associated with switches from laminated to bioturbated (nonlaminated) sediments, determining whether changes in sediment lamination strength are sensitively related to other proxies of bottom-water oxygen concentration by comparison with other proxies sensitive to changing oxygenation.
Approach: We are documenting changes in benthic foraminiferal assemblages; benthic macrofauna, including fossils and their behavioral traces; and surface water temperature by planktonic foraminiferal assemblages and oxygen isotopic composition. The biota, as represented by the fossil macro and microfaunal assemblages, have sensitively responded to variation in temperature and the oxygen concentration of the marginal marine environment. We are determining the rate of ecologic change by constructing a reliable, high-resolution chronology.

High stratigraphic resolution is being employed especially during the rapid transitions between laminated and nonlaminated sediments. Because the sedimentation rates of Hole $893 \mathrm{~A}$ are greater than $130 \mathrm{~cm} / 100 \mathrm{yrs}$, it is possible to obtain stratigraphic (chronological) resolution of 10 to 20 yrs and better.

Results to Date: We have discovered a series of 19 ecologic upheavals in the marginal marine environment off central/southern California during the past 80,000 years and established that these ecologic changes are tied to well-documented climatic and atmospheric perturbations that are almost certainly of global extent. Our work clearly demonstrates that the initiation of the climatic warmings occurred within only a few decades. Within this brief time interval, ocean surface temperatures warmed as much as $8^{\circ} \mathrm{C}$, and benthic and planktonic ecosystems responded just as rapidly. We have found that the speed and magnitude of the temperature changes were equal to or greater than those documented in the Greenland ice sheet, an environment suspected to be anomalously sensitive to climate change. The Santa Barbara Basin repeatedly switched from well oxygenated to very low oxygen levels in less than 50 years with an associated ecologic change.

We have also recently discovered similar, climaterelated ventilation changes in cored sequences (Ocean Drilling Program Leg 167) of the nearby Tanner and Santa Monica Basins, supporting our earlier suggestion that these changes were widespread and probably synchronous along the northeast Pacific margin. These results suggest sensitivity of broad areas of the ocean-atmosphere-biosphere system to short-term climate change.

\section{INCORPORATING LEARNING INTO AN INTEGRATED CLIMATE-ECONOMY MODEL KOLSTAD, CHARLES D. UNIVERSITY OF CALIFORNIA, SANTA BARBARA \\ FY 199548 \\ FY 199454}

Objectives: To enhance integrated-assessment capabilities by incorporating uncertainty and learning into 
dynamic models of economic growth, $\mathrm{CO}_{2}$ accumulation, and climate change.

Approach: The core of the research program is in finding efficient ways of solving dynamic programs with four to eight states. We have taken two tacks in solving this problem. One is to efficiently adapt the neural-network function approximation to solving the Bellman equation. We have written efficient computer code for solving this and adapted it to a stochastic version of Nordhaus's DICE model of climate change. Since a goal is to improve methods, it is also important to compare various methods for solving dynamic programs. To do this, we have established a small test problem, based on integrated assessment. We are also testing several existing algorithms and are comparing their performance to our neural-network algorithm.

Results to Date: We have been able to implement the neural network algorithm, adapting our MatLab software to deal with the algorithm in an efficient manner in application to our integrated-assessment model. We have also begun programming the comparative analysis of different algorithms. We are now able to solve integrated-assessment models involving learning that simply could not be solved before. Prior to this work, learning was confined to simple representations, such as one-shot learning or exogenous arrival of information. Bayesian learning in an integrated assessment context could not be examined empirically. Our work is also significant in providing researchers in other areas with new methods for solving dynamic resource problems specified as dynamic program.

\section{IMPACTS OF GLOBAL CHANGE ON $\mathrm{CO}_{2}$ SOURCE AND SINK ACTIVITY, $\mathrm{H}_{2} \mathrm{O}$ FLUX, FUNCTION, AND STRUCTURE OF SEMIARID SHRUBLAND ECOSYSTEMS OECHEL, WALTER C. SAN DIEGO STATE UNIVERSITY FY 199591 \\ FY 19940}

Objectives: To test and document the performance characteristics of the MedFACE system, including $\mathrm{CO}_{2}$ control and effect of implementation of the air barrier system on microclimate and energy balance; to document the materials, supplies, construction details, and control algorithms of the system; to determine baseline information before $\mathrm{CO}_{2}$ treatment is initiated; to initiate elevated- $\mathrm{CO}_{2}$ treatment; to determine effects of elevated $\mathrm{CO}_{2}$ and drought in a regenerating chaparral stand on carbon flux, water use, water-use efficiency, photosynthesis, leaf chemistry, growth and productivity, stand composition, belowground productivity, and belowground microbial, microfaunal, and floral populations; and to compare the results of the effect of elevated $\mathrm{CO}_{2}(550 \mathrm{ppm})$ and increased drought from the MedFACE systems to results obtained with the $\mathrm{CO}_{2} \mathrm{LT}$ null-balance greenhouse system as well as with the control FACE ring.

Approach: The field experiment is being carried out with the recently constructed Free-Air $\mathrm{CO}_{2}$ Enrichment (FACE) system and results are being compared to those obtained from null-balance $\mathrm{CO}_{2}$-controlled, naturally lighted and temperature-controlled $\left(\mathrm{CO}_{2} \mathrm{LT}\right)$ greenhouses maintained at six $\mathrm{CO}_{2}$ concentrations from $250 \mathrm{ppm}$ to $750 \mathrm{ppm}$ in $100 \mathrm{ppm}$ increments at San Diego State University's Sky Oaks Biological Field Station in northeastern San Diego County. Both systems have been deployed on the site of a 30-ha controlled burn that took place in July 1992. The FACE system consists of a ring $15 \mathrm{~m}$ in diameter, resulting in $177 \mathrm{~m} 2$ of resprouting chaparral being exposed to a 550-ppm $\mathrm{CO}_{2}$ concentration. With wind direction, an infrared gas analyzer, computer, and data acquisition and control systems, ambient air and $\mathrm{CO}_{2}$ are mixed in the inner ring by a 10,000-CFM blower and releised at various points along the ring by pneumatically controlled valves. The null-balance greenhouses are 2-m cubes and are run continuously, tracking ambient temperature and relative humidity at their assigned treatment level of $\mathrm{CO}_{2}$. Carbon-flux values are calculated every $2 \mathrm{~min}$. Comparisons of the individual plants and the ecosystem response to elevated $\mathrm{CO}_{2}$ treatments will be made among the different treatment methods. Parameters being measured include whole ecosystem carbon flux; carbon, nitrogen, and phosphorus concentrations of soil and plant tissue; $\mathrm{ACi}$ curves; RuBP levels and activity; transpiration; soil moisture; growth rates; and leaf area.

Results to Date: We were not able to reduce the amount of $\mathrm{CO}_{2}$ required by using a dual-array setup without roughly doubling the amount (and cost) of structure required. The hardware and software continue to be updated and improved. We modified the $\mathrm{CO}_{2}$ injection system by adding a "boost" solenoid valve which dumped a flow of about $1000 \mathrm{~L}$ min-1, giving a total capacity of about $2500 \mathrm{~L}$ min-1. This setup worked well, but on occasion, small $\mathrm{CO}_{2}$ crystals or liquid would form in the $\mathrm{CO}_{2}$ supply line, and these would damage the mass flow controllers (MFCs). We completely redesigned the $\mathrm{CO}_{2}$ flow-control system to one consisting of eight solenoids connected in parallel, each controlling $\mathrm{CO}_{2}$ flow through an orifice so that each succeeding orifice provides twice the flow of the preceding one. This binary flow divider has proven to be very reliable and has a much higher dynamic range (256:1) than the mass flow controllers (10:1).

$\mathrm{CO}_{2}$ treatment was initiated in December 1995 with the null-balance chambers and January 1996 for the FACE experiment. In the null-balance experiment, the most dramatic affect was very little growth in chambers treated with subambient $\mathrm{CO}_{2}$ while the 650 - 
and 750-ppm treatments resulted in nearly a two-fold increase in growth compared to the 350- to 550-ppm treatments. Plants treated with $550 \mathrm{ppm}$ in the FACE experiment exhibited a significantly lower amount of water stress compared to control plants. Presumably, the lower stress can be attributed to an increase in water-use efficiency under elevated $\mathrm{CO}_{2}$. However, there was no relationship between predawn water potentials and $\mathrm{CO}_{2}$ concentration in the null-balance experiment, and the overall mean water potential was similar to that for plants in the FACE experiment.

\section{ECOLOGICAL CONSEQUENCES OF THERMOKARST DEVELOPMENT IN BOREAL FORESTS \\ OSTERKAMP, T. E.; VIERECK, L. A.; CURLEY, J. S.; DOYLE, A. P.; BOONE, R. D.; and SHUR, Y. L. \\ UNIVERSITY OF ALASKA \\ FY 199579 \\ FY 19940}

Objectives: To acquire an understanding of how the boreal forest ecosystem responds to the thawing of ice-rich permafrost and development of thermokarst terrain.

Approach: Our strategy is to select a suite of research sites in boreal forests where thermokarst is in varying stages of development as a result of natural processes and human activities. Measurements are being made of the physical, mechanical, hydrological, chemical, and biological effects of thermokarst on the forest. This will allow us to investigate the ecological consequences of thermokarst at different periods in its formation and to reconstruct the immediate, intermediate, and longterm effects on the ecosystem.

Results to Date: Our site selections were completed: the Bonanza Creek LTER area and on the University of Alaska campus in Fairbanks with a secondary site at Mentasta. We installed automatic temperature loggers at all the sites. Of the two plots at the Bonanza Creek LTER site, one is a 200-year-old black spruce stand on an island of the Tanana River that is not presently underlain by permafrost; and the other is a 150-yearold black spruce stand underlain by permafrost and a very shallow active layer of 47 to $58 \mathrm{~cm}$. At the island site, the maximum soil temperatures during the growing season are conspicuously warmer than those at the other site.

At Mentasta, we determined that the volume of excess ice in the permafrost that thawed exceeded $5 \mathrm{~m}$, obtained tree cores and sections of dead trees, and obtained soil samples and organic material from up to $3 \mathrm{~m}$ deep in the permafrost. Current subsidence (deflation) of the ground surface indicates that the amount of excess ice in the permafrost can exceed $5 \mathrm{~m}$. The radiocarbon age of a wood sample taken from about the 3-m depth is $2690 \pm 70 \mathrm{BP}$, much younger than we believed it would be. Preliminary analyses of tree cores and cross sections indicate that wildfire is not common in this site and not a cause of the thermal degradation at the site. Tipped trees at the edge of active thermokarst can be used to age the time of thawing of the underlying permafrost as well as to determine the rate of lateral advance of a thawing front. The black spruce trees growing on permafrost do not appear to be sensitive to fluctuations in climate unless there is active subsidence resulting in tipping of the trees.

The campus thermokarst site is in a relatively undisturbed arboretum on the campus. Temperature measurements in a pipe driven to the 6-m depth indicate that the permafrost table must be at least several meters deeper. Mature white spruce grow densely here, and Equisetum and deep beds of sphagnum moss cover the ground. Soil samples were obtained at 30 sites. Bulk organic and mineral soils were collected for respiration and net mineralization assays. We also cored the nearby forest floor and 0 to $10 \mathrm{~cm}$ of mineral soil for precise gravimetric comparisons of water retention and total carbon and nitrogen. Organic matter accumulation and moisture does not appear to be significantly different across this spruce landscape where thawing affects landscape relief. Mineral soil moisture is affected by topography as expected. Radical topographic effects, such as water entrapment in thaw pits and hanging forest floors, have a strong effect on moisture and temperature regimes and therefore have large biological consequences.

\section{ROLE OF THE OCEAN IN CLIMATE VARIABILITY: OCEAN VENTILATION PROCESSES, THERMOHALINE VARIABILITY, AND FEEDBACKS OF THE OCEAN TO THE ICE AND TO THE ATMOSPHERE \\ SARACHIK, E. S., and YIN, F. L. UNIVERSITY OF WASHINGTON FY 199567 FY 199485}

Objectives: To identify and to understand mechanisms of ocean internal variability on decadal to millennial time scales and mechanisms of feedback of the ocean to the atmosphere and to the ice.

Approach: Both isopycnal (Hu's GFDL) and z-coordinate (GFDL MOM) coarse-resolution OGCMs are used. The atmospheric GCM is the NCAR CCM3, and the sea-ice model is a cavitating-fluid ice model developed by Flato and Hibler. Various idealizations based on observations were made to isolate the essential physical processes in studying particular mechanisms.

Results to Date: A coarse-resolution simulation of global ocean circulation with the isopycnal model has 
been carried out. The model geometry, surface forcing, and horizontal resolution are identical to those of a z-grid model with isopycnal-depth diffusion mixing parameterization. The vertical profiles of the globally averaged climatological potential temperature and salinity simulated by the isopycnal model are much improved over those by the z-grid model. The chronic warm bias of the thermocline and the cool bias of the abyss in z-grid model are significantly reduced in the isopycnal model, and the observed salinity minimum at the intermediate depth is well represented.

A simulation of upper-ocean variability during 1992 to 1993 has been performed with the isopycnal model and compared with that simulated by a z-grid model and with observations. The sea-surface height simulated by isopycnal and z-grid models are both in good agreement with that of TOPEX/POSEIDON. While the simulation of upper-ocean seasonal temperature anomalies by the isopycnal model agrees well with XBT data, the results of the z-grid model are less satisfactory. Because of differences in surface mixed layer formulations, temperature anomaly in the z-grid model does not penetrate as deeply as observed.

A coupled ocean-sea-ice model has been developed, and experiments with this model have been conducted. The model was found to be inherently unstable because of thermodynamic coupling when there is little external freshwater input. The presence of sea ice requires an upward ocean heat flux, provided by a warmer temperature at the subsurface than the freezing temperature at the surface. This unstable thermal structure leads to convective overturning and results in interdecadal oscillations. A freshwater transport through surface plays a crucial role in stabilizing this system.

We also investigated the response of air-sea momentum and heat fluxes of CCM3 to SST perturbations, documenting surface momentum and latent heat changes in response to a south-north Atlantic "dipole" SST perturbation in $\mathrm{CCM} 3$ resembling that derived from decadal variability in observational data.

\section{INVESTIGATION OF THE IMPACT OF CLIMATIC CHANGE ON THE \\ ECOSYSTEM AT HIGH LATITUDES \\ STAMNES, K., and ZHANG, T. UNIVERSITY OF ALASKA \\ FY 199569 \\ FY 199431}

Objectives: To develop a coupled atmosphere/land surface/permafrost (ALSP) model to improve our physical understanding of the transfer of momentum, heat, and water between the atmosphere and the land surface in Arctic regions.

Approach: Thermal regimes of the active layer and permafrost on the North Slope of Alaska will be investigated by analyzing observational data and by numerical modeling. We will use the ALSP model to investigate the effect of climatic factors on thermal processes of the active layer and permafrost at Barrow, Alaska.

Results to Date: The North Slope of Alaska can be divided into three major climatic zones: The Arctic Foothills Zone includes the stations and sites from Galbraith to Sagwon and has a higher elevation than the other zones and is farther from the ocean. The Arctic Inland Zone is located south of the Arctic Coastal zone and extends about $100 \mathrm{~km}$ along the Haul Road inland from the Arctic Coast. The Arctic Coastal Zone includes areas that extend inland about $20 \mathrm{~km}$ from the ocean. Data collected for more than six years on the thermal regimes of the active layer and permafrost in these three zones were analyzed and numerically modeled. Results indicated that, within about $120 \mathrm{~km}$ from the Arctic Coast, the mean annual air temperature was nearly constant at approximately $-12.4 \pm 0.4^{\circ} \mathrm{C}$, while the mean annual permafrost surface temperature varied from $-9.1^{\circ} \mathrm{C}$ at West Dock to about $-5.0^{\circ} \mathrm{C}$ at Happy Valley, a more than $4^{\circ} \mathrm{C}$ increase. Obviously, changes in mean annual air temperature alone cannot explain the variations in the thermal regimes of the active layer and permafrost on the North Slope of Alaska. The analysis and modeling found no single factor that can explain the permafrost temperature increase from the coast to inland. Contributing factors might include relatively short and cool summers, hard wind-packed snow, poorly developed vegetation, and flat ground surface along the Arctic Coast; longer and warmer summers, less hard wind-packed snow, well-developed vegetation, and rough ground surface inland; a warmer winter in the Toolik Lake area; and higher elevation near the Brooks Range.

Modeling results with ALSP gave good agreements between the measured and calculated values. Results from sensitivity analyses indicate that among the variable climate factors, air temperature is the most important single factor controlling the soil temperatures, while seasonal snow cover and soil moisture are also important but secondary factors. The existence of a thin depth hoar layer within the seasonal snow cover is crucial to its insulating effect, while snow thickness becomes a secondary factor. Thawing index and soil moisture conditions are the most important factors influencing active-layer thawing processes. Freezing index and seasonal snow cover influence the development of the active layer, but their effects are very limited. 


\section{GLOBE: EDUCATIONAL OUTREACH ON PROCESSES INVOLVING GLOBAL ENVIRONMENTAL CHANGE SUCHANEK, THOMAS H. UNIVERSITY OF CALIFORNIA, DAVIS FY 199437 FY 19930}

Objectives: To conduct a series of teacher training workshops, to provide potential regional infrastructure to accomplish GLOBE (Global Learning and Observations to Benefit the Environment) goals on a national level, to provide computer-networking facilities that can serve as a centralized clearinghouse for GLOBE data storage and acquisition, and to provide a highquality classroom curriculum on Global Systems Science education (in collaboration with the Lawrence Hall of Science) for the implementation of the GLOBE Initiative.

Approach: We will establish a teacher-training workshop process that will detect common goals and problems, identify potential solutions to problems, provide effective communication between trainers and teachers, design and field-test the educational units suggested in the GLOBE program, aid in the implementation of the GLOBE data-collection process in the classroom, develop solutions to difficulties with hardware and software, and help teachers trouble-shoot difficulties encountered in the classroom. An efficient and effective administrative infrastructure will be established to provide long-term stability in the GLOBE program. As another part of its educational outreach component, the Western Region of NIGEC (WESTGEC) (along with NSF) is supporting the development and implementation of a Global System Science (GSS) curriculum by the Lawrence Hall of Science at U.C. Berkeley. This program is designed to produce educational guides to be incorporated into high school students' existing classroom programs with the intent of increasing awareness about global processes and problems facing the environment. We envision using the high-quality GSS curriculum to implement GLOBE in high-school classrooms throughout the United States.

Results to Date: In July 1996 we hosted another GLOBE Workshop at U.C. Davis with 50 teachers attending. We also anticipate hosting additional GLOBE workshops in the fall of 1996 as well as the spring and summer of 1997.

\author{
SCALING UP FROM LEAVES TO STANDS: \\ COUPLING ECOPHYSIOLOGICAL MODELS \\ WITH REMOTE SENSING \\ USTIN, SUSAN L., and ROBERTS, DAR A. \\ UNIVERSITY OF CALIFORNIA, DAVIS and \\ UNIVERSITY OF CALIFORNIA, \\ SANTA BARBARA \\ FY 199572 \\ FY 19940
}

Objectives: To evaluate how reflected light that is measured at the scale of single leaves scales upwards to the branch and stand level, to evaluate the role of architecture in modifying that signal, to develop connections between remotely sensed data and canopy properties that can be used in ecosystem models of carbon flux, and to modify and test a watershed-scale ecosystem model for a northwest conifer forest.

Approach: We are using experimental and observational approaches to address how reflected light measured at the scale of single leaves scales upwards to the branch and stand level, how architecture modifies that signal, and how remotely sensed data and canopy properties can be used in ecosystem models of carbon flux. We are using model simulations and comparisons to field measurements to address the modification and testing of a watershed-scale ecosystem model for a northwest conifer forest. Our study is being conducted at three forested sites in Washington that are expected to exhibit different carbon budgets and are regionally important: clonal Populus stands near Wallula, old-growth and second-growth silver fir (Abies amabilis) stands in the Cedar River Watershed and at the old-growth mixed Douglas Fir/Hemlock at the Wind River Canopy Crane site.

Results to Date: Above-canopy spectral reflectance was measured at Wallula above two architecturally distinct Populus clones, a Trichocarpa deltoides hybrid with more horizontal leaves and a $T$. deltoides-T. nigra hybrid with more vertically oriented leaves. At the same time, leaves were destructively sampled at different levels of the crown and analyzed to quantify the spectral properties and leaf chemical composition. Spectral measurements at smaller scales were compared to apparent surface reflectance retrieved from Airborne Visible/Infrared Imaging Spectrometer (AVIRIS) data. Comparison indicated that, at the leaf scale, TXD and DXN clones are nearly identical, while at branch scales or larger, reflectance was significantly higher in the TXD clone. Initial estimates of leaf-area index (LAI) were also made with litter traps, hemispherical photographs, and a sun-fleck ceptometer.

Destructive samples, above-canopy reflectance, and vertical profiles of diffuse visible/NIR light were measured at all three sites. Vertical profiles were collected at the Crane Site, and horizontal transects of 
canopy spectra were also made. At Wallula, vertical light profiles were measured in 3-year-old stands of both clones. At all three sites, spectral measurements were complemented by hemispherical photographs and visible-light measurements to obtain measures of canopy structure. At each study site, we are using AVIRIS to map canopy liquid-water content, quantify canopy composition and texture, and map the distribution of water vapor across the landscape. We have begun development of a GIS database for the Wind River Valley to apply a dynamic watershed hydrologic model that links surface and subsurface processes with canopy physiology and energy budgets. The model uses AVIRIS data inputs to characterize surface conditions.

A new wavelet approach was applied to an analysis of Thematic Mapper (TM) images for the Pacific Northwest to study landscape patterns in forest structure and thereby to measure carbon stores. Simple methods like NDVI cannot determine the extent of regrowth of clearcut forests, and estimates of forest condition from forest logging records are subject to large errors. Our preliminary results indicate that the wavelet approach is promising for this application.

\section{COMPARISONS OF OBSERVED AND MODEL CLOUDINESS AND MODEL CLIMATE SENSITIVITY \\ WEARE, BRYAN C. UNIVERSITY OF CALIFORNIA, DAVIS FY 199556 \\ FY 199470}

Objectives: To identify how well models represent observed variations in cloudiness, to identify the reasons for success or lack thereof, and to elucidate how agreement or lack of agreement with observations is likely to be related to model sensitivity to climatic forcing.

Approach: Very large global data sets of cloudiness, radiation, and related variables will be analyzed. The cloud observations are from the International Satellite Cloud Climatology Project (ISCCP), and additional meteorological data are from the European Centre for Medium-Range Weather Forecasting.

The 30 models taking part in the Atmospheric Model Intercomparison Project (AMIP) will be used, 29 of which have cloud-formation mechanisms. All of these models have been run for a standard 10-year period with identical observed sea-surface temperature (SST) and sea-ice boundary conditions. Available from AMIP are total cloud amount, zonal averages of clouds at standard pressure levels, and the net solar and infrared radiation at both the top and bottom of the atmosphere.

Results to Date: Total cloud amounts from 29 AMIP models have been analyzed and compared with the ISCCP observations. The comparisons include annual global, zonal, and regional means; root-mean-square differences; smoothed seasonal cycles; and ENSO events. The results illustrate that no one model is best or worst in the three categories of evaluation, although qualitative differences certainly do exist. However, a careful analysis of the physical parameterizations in the five best and worst models does not identify a feature that clearly distinguishes the two groups

Monthly means of total solar and infrared fluxes at the top of the atmosphere for both cloudy and clear subregions have been analyzed from the Earth Radiation Budget Satellite (ERBS) for January 1985 through December 1988. The analysis indicates that the ERBS clear-sky infrared fluxes may be contaminated by undetected clouds, giving rise to uncertainties of up to about $10 \mathrm{Wm}-2$, which would have a substantial impact on the near balance of the average solar and infrared cloud radiative forcing.

The vertical structure of the zonally averaged cloudiness in observations and 25 AMIP models shows that all of those models have much more high cloudiness than is evident from the observations. However, many models display realistic meridional and seasonal variations. Low cloudiness in the AMIP models tends to be comparable to the few available ground-based observations. On the other hand, seasonal variability of low clouds is less well produced than for high clouds. 



\section{OCEAN SCIENCES RESEARCH}

Ocean sciences research is intended to foster an understanding of ocean-atmosphere-climate interactions and the ocean carbon cycle. This activity includes a Global Survey of $\mathrm{CO}_{2}$ in the Oceans, which is measuring the distribution of inorganic carbon throughout the world's oceans, and the Ocean Margins Program, which is quantifying biogeochemical processes and fluxes of carbon at the land-ocean interface.

\section{Program Manager}

Curtis R. Olsen

Environmental Sciences Division

Department of Energy, ER-74

19901 Germantown Road

Germantown, MD 20874-1290

(301) 903-5329

Internet: curtis.olsen@oer.doe.gov

\section{CARBON TRANSPORT IN THE BOTTOM BOUNDARY LAYER \\ AGRAWAL, YOGESH C. SEQUOIA SCIENTIFIC, INC. \\ FY 1996200 \\ FY 19950 \\ FY $1994 \quad 0$}

Keywords: particulate carbon, transport of carbon in the oceans

Objectives: To measure the cross-shelf transport of particulate carbon in the Ocean Margins Program field experimental area.

Approach: A laser-based particle analyzer and fluorometers and BASS-Tripod systems will be used to measure benthic fluxes of carbon particles.

Results to Date: New Project.

\section{SURFICIAL BIOTURBATION AND RAPID REMINERALIZATION PROCESSES IN THE CAPE HATTERAS SHELF AND SLOPE ALLER, ROBERT; COCHRAN, J. KIRK; LEE, CINDY; and ALLER, JOSEPHINE RF OF SUNY STONY BROOK \\ FY 1996130 \\ FY 1995140 \\ FY 199497}

Keywords: remineralization of carbon, Cape Hatteras, Ocean Margins Program

Objectives: To quantify and model benthic remineralization of biogenic debris and processes governing sediment-water exchange of dissolved and particulate constituents ( $\mathrm{C}, \mathrm{N}, \mathrm{Si}$, and $\mathrm{Mn}$ ) in the shelf and slope region of the Ocean Margins Program study area near Cape Hatteras.
Approach: To evaluate the shelf-break region as a major source and processing region for transfer of material from the shelf to the slope, this project seeks to quantify and model: (1) the net fluxes of reactive biogenic debris (planktonic $\mathrm{C}, \mathrm{Si}$, and $\mathrm{CaCO}_{3}$ ) to the sea floor at a range of sites; (2) the resulting net sedimentary remineralization rates $\left(\mathrm{N},{ }^{*} \mathrm{CO}_{2}, \mathrm{Mn}\right)$; and (3) the associated benthic community activities that partially control remineralization, including bioturbation, benthic shell deposition, and microbial abundance, activities, and biomass production. The project emphasis is on fluxes of sedimentary material and processes with characteristic reaction or transport time scales of several weeks to months, corresponding to and integrating over major nonsteady-state seasonal water-column events, such as plankton blooms or storms.

Methods are centered on solid-phase reactive or natural tracer constituents in surface sediments ( 0 to $5 \mathrm{~cm}$ ), such as chloropigments and 234Th, for which decomposition kinetics can be accurately inferred and that allow diagenetic-model conversion of measured sedimentary inventories into mass-flux estimates. The direct measurement of production rates of dissolved constituents within sediments will also be emphasized. Results to Date: Multiple indicators of benthic remineralization rates and associated infaunal community activities were examined in the shelf-break/slope region during July and August 1996. Samples were collected at depths of approximately 200,300, and $400 \mathrm{~m}$ along three primary transects (at $36^{\circ} 20^{\prime} \mathrm{N}$, $35^{\circ} 50^{\prime} \mathrm{N}$, and $35^{\circ} 25^{\prime} \mathrm{N}$ and $74^{\circ} 50^{\prime} \mathrm{W}$ ) by remote coring (HAPS, MK5 Box Corer, and Multi-Corer). Visual observation of recovered cores showed highly 
burrowed sediments as previously reported in this area. Preliminary laboratory work indicates extensive sediment reworking by an abundant benthos and high rates of carbon remineralization. Excess 234Th distributions in cores extend to depths of 3 to $5 \mathrm{~cm}$, indicative of extensive bioturbation. 234Th inventories are characteristic of high particle flux to the sea floor. The radiochemical profiles will be used to calculate particle mixing coefficients $(\mathrm{Db})$ and to model remineralization rates. Future analysis of sedimentary $\mathrm{Chl}$-a will further constrain mixing rates by macrofauna. Short-term incubation of sediments at in situ temperature $(0$ to $5 \mathrm{~cm}$ and 5 to $10 \mathrm{~cm}$ ) show high $* \mathrm{CO}_{2}$ production fluxes, ranging from 19.6 to $5.6,15.6$ to 5.2 , and 26.4 to $7.0 \mathrm{mmol} * \mathrm{CO}_{2} \mathrm{~m}-2^{\mathrm{d}-\mathrm{I}}$ at the northern, central, and southern transects, respectively. ${ }^{*} \mathrm{CO}_{2}$ production fluxes are generally greater in the shallower (about 200-m) stations along each transect. $\mathrm{C} / \mathrm{N}$ ratios from sediment incubations, equivalent to about 6.0 , indicate that the source of remineralized carbon is a fresh, labile carbon pool. Preliminary identification of the macrobenthic community shows that polychaetes $(0.3$ to $1.0 \mathrm{~mm})$ and nematodes $(0.3 \mathrm{~mm})$ numerically dominate. An extensive molluscan death assemblage exists at stations within the central transect.

\section{WHITECAPS AND AIR-SEA EXCHANGE $\mathrm{OF} \mathrm{CO}_{2}$ ASHER, WILLIAM PACIFIC NORTHWEST LABORATORY FY $1996465^{*}$ FY 1995500 FY 1994702}

*Includes consolidated funding for the research of D. W. Denbo and E. D. Skyllingstad.

Keywords: air-sea transfer velocity, satellite measurements, whitecaps

Objectives: To develop a method for estimating air-sea transfer velocity from satellite measurements of fractional-area whitecap coverage.

Approach: Laboratory experiments will be conducted in specially constructed whitecap simulation tanks to obtain the data necessary for parameterizing the effects of bubble-plume-mediated transfer processes on air-sea transfer velocities. This parameterization will be used to develop a model for estimating transfer velocities from remote measurements of whitecap coverage and wind speed. The field experiments necessary for testing and verifying the whitecap gas-exchange model will be conducted. Controlled experiments will be conducted to acquire the data necessary for the development of a model for estimating whitecap coverage from satellite measurements of passive-microwavebrightness temperature. Field experiments will be conducted to obtain data for verification of model.
Results to Date: With data collected in the whitecapsimulation tanks, a model for predicting the transfer velocity of any gas from solubility, diffusivity, and fractional-area-bubble-plume coverage was developed. The laboratory model was adapted for use in the ocean with a preliminary set of oceanic gas-transfer measurements. A comprehensive field experiment was planned to provide further verification of the gas-transfer model. Data were collected during the October 1993 Wave Basin Experiment (WABEX-93) that allowed the development of a useable relation for estimating whitecap coverage from brightness temperature. The model was adapted for use under oceanic conditions. Field experiments were planned to verify the model.

\section{SOURCES AND TURNOVER TIMES OF DISSOLVED, COLLOIDAL, AND \\ PARTICULATE ORGANIC CARBON IN THE MID-ATLANTIC BIGHT AND CHESAPEAKE BAY \\ BAUER, JAMES E., and DRUFFEL, ELLEN R. M. VIRGINIA INSTITUTE OF MARINE SCIENCES and UNIVERSITY OF CALIFORNIA, IRVINE \\ FY 1996126 \\ FY 1995119 \\ FY 199489}

Keywords: dissolved organic carbon fluxes, colloidal organic carbon fluxes, particulate organic carbon fluxes, sources, natural isotopes, residence times

Objectives: To evaluate the important sources of terrestrial and marine organic carbon to the MidAtlantic Bight and to assess their role(s) in the marine and global organic-carbon budgets.

Approach: This project will examine and evaluate the sizes, sources, turnover times, and fluxes of dissolved, colloidal, and particulate organic carbon (DOC, COC, and $\mathrm{POC}$, respectively) and dissolved inorganic carbon (DIC) pools in waters of the Mid-Atlantic Bight (MAB). On each of four transects across the MAB shelf and slope conducted at two times of the year, samples will be collected for determinations of carbon and nitrogen content and ${ }^{14} \mathrm{C}$ and $\delta^{13} \mathrm{C}$ isotopic compositions of bulk DOC and POC as well as the colloidal components of the $\mathrm{DOC}$ pool. We will obtain estimates of organic-carbon turnover produced by both the buildup and transport of autochthonously (from shelf primary production) and allochthonously (from estuarine-terrestrial and atmospheric inputs) derived material along and across the MAB shelf as well as that produced by short-term in situ processes. We will also evaluate the short-term (bimonthly) variability in these parameters as source indicators of organic matter carried in waters of the Chesapeake Bay outflow to the MAB shelf. This work will be closely coupled to that of other OMP PIs who will measure natural carbon 
isotopic abundances of selected individual biomarker molecules in the DOC and POC pools. These data will be compared to existing data sets for $\mathrm{DOC}$ and $\mathrm{POC}$ from the North Atlantic (Sargasso Sea) to evaluate the magnitude of organic-carbon sequestration and aging within the interior ocean and the similarities and disparities in organic-carbon cycling between the ocean's margins and interior.

Results to Date: This project has documented the existence of organic carbon of both terrestrial and marine origin in coastal waters that contributes to the total flux of carbon to the ocean's interior. Much of the geochemically young organic matter in continental shelf waters is used before making it to more seaward regions overlying the continental slope. It has also been shown that, taken as a whole, the ocean margin in the Mid-Atlantic Bight region of the North Atlantic is a source of geochemically old carbon to the interior North Atlantic. Thus, the MAB is a transient reservoir of young carbon fixed from atmospheric carbon dioxide.

\section{MECHANISMS OF DISSOLVED ORGANIC CARBON CYCLING IN AN OCEAN MARGIN BENNER, RONALD \\ UNIVERSITY OF TEXAS AT AUSTIN \\ FY 199635 \\ FY 199571 \\ FY 1994101}

Keywords: dissolved organic matter, carbohydrates, carbon cycle

Objectives: To determine the sources and mechanisms of carbohydrate production and consumption in ocean waters.

Approach: Dissolved carbohydrates will be measured in seawater samples after hydrolysis with sulfuric acid. Aldoses will be separated by anion exchange chromatography and quantified by pulsed amperometric detection. The molecular composition of aldoses and amino sugars will be used to determine sources and mechanisms of carbohydrate production. Laboratory incubations with selected cultures will be used to verify compositional trends.

Results to Date: This project just starting, but previous DOE funding was used to develop the methods for the determination of aldoses by anion exchange chromatography and pulsed amperometric detection.

\section{CARBON CYCLING ON THE CONTINENTAL MARGIN: EVIDENCE FROM SEDIMENT CARBON-14 AND NUTRIENT ELEMENTS BENNINGER, LARRY \\ UNIVERSITY OF NORTH CAROLINA \\ FY 199650 \\ FY $1995 \quad 50$ \\ FY 1994119}

Keywords: carbon cycling, sediment, carbon burial, accumulation rates

Objectives: To trace modern organic carbon in the sedimentary environment and to determine the rate of carbon burial in the sediments of the Hatteras margin. Approach: Near-surface sediments will be analysed for evidence of modern carbon deposition (excess 210-Pb, $\mathrm{Pu}$, and bomb 14-C), and longer cores will be analyzed for carbon burial rate. Sediment samples have been collected from surface vessels (grabs and a variety of cores) and from the Sea Link submersible (push cores). Overall, sample coverage has been from the estuaries (Chesapeake Bay and the Neuse River, N.C.) to the continental rise, emphasizing the continental shelf and slope. To characterize modern organic carbon, we have also collected biota from surface vessels (Sargassum, plankton) and from the submersible (benthos).

Results to Date: Sediment inventories of excess $210-\mathrm{Pb}$ and $\mathrm{Pu}$ on the continental shelf show that deposition of modern particles at shelf depths is not negligible. The data imply that shelf sediments contribute significantly to the remineralization of primary production in shelf waters. Modeling of excess $210-\mathrm{Pb}$ and $\mathrm{Pu}$ from slope sediments yields sediment accumulation rates of less than $100 \mathrm{~cm} / \mathrm{ky}$, not more than $500 \mathrm{~cm} / \mathrm{ky}$ as reported previously for a slope site near Cape Hatteras. Over rugged sea floor topography at slope depths (460 to $900 \mathrm{~m}$ ), modern sediment accumulation is concentrated at ridge tops; little modern sediment accumulates on adjacent valley walls or valley floors.

\section{DEPOSITION AND FATE OF MODERN ORGANIC CARBON IN SLOPE SEDIMENTS NEAR CAPE HATTERAS \\ BLAIR, NEAL, and DEMASTER, DAVID NORTH CAROLINA STATE UNIVERSITY FY 1996250 FY 1995250 FY $1994 \quad 0$}

Keywords: carbon in continental slope sediments Objectives: To verify if organic carbon deposition is focused onto the continental slope offshore of Cape Hatteras, N.C.; and if it is, to determine the fate of the carbon.

Approach: The accumulation rate of organic carbon will be estimated via carbon-14 geochronometric methods on sedimentary organic matter. The oxidation rate of the organic matter will be determined by a combination of incubation and flux studies as well as pore-water chemical measurements. Carbon-14 (in sedimentary organic matter, benthic animals, and diagenetic products) will be used to detect the presence of organic matter recently derived from atmospheric 
$\mathrm{CO}_{2}$. Samples have been collected via surface ship coring and submersible during the summer of 1996.

Results to Date: Carbon-14 measurements have been made on the organic fraction of kasten cores collected along a shore-perpendicular transect at $36^{\circ} 20^{\prime} \mathrm{N}$ in the northern part of the study area. The sediment accumulation rates along this transect show a maximum at $740 \mathrm{~m}$ water depth, and they are significantly slower than those in the southern part of the study area. We also have begun our work on determining the carbon-14 activity of benthic fauna to evaluate if there is preferential selection for young/labile organic matter by deposit-feeding infauna. Carbon-14 measurements from a bottom-dwelling suspension feeder (an acorn barnacle collected at $33^{\circ} \mathrm{N}$ latitude and $850 \mathrm{~m}$ water depth) indicate that the animal is primarily feeding on bomb-enriched organic matter. We have collected various infauna from our coring and submersible activities and are preparing them for accelerator dating at Lawrence Livermore National Laboratory.

\section{DETERMINATION OF OCEAN/ ATMOSPHERE CARBON DIOXIDE FLUX WITHIN THE OMP SURVEY AREA CHIPMAN, DAVID W., and TAKAHASHI, TARO LAMONT-DOHERTY EARTH OBSERVATORY FY 1996115 \\ FY 1995149 \\ FY $1994 \quad 0$}

Keywords: carbon dioxide flux, dissolved oxygen in surface waters, partial pressure of carbon dioxide in surface waters

Objectives: To measure the partial pressure of carbon dioxide and the concentration of dissolved oxygen in the surface waters of the continental shelf within the Ocean Margins Program survey area (Cape Hatteras to Cape Charles) and between that area and Georges Bank throughout the 1996 to 1997 OMP field program, and to calculate the direction and magnitude of the annual net ocean/atmosphere carbon dioxide flux within these areas.

Approach: Semicontinuous underway measurements of partial pressure of carbon dioxide (as well as atmospheric carbon dioxide concentration in pumped air samples) and concentration of oxygen will be made in pumped, uncontaminated surface water on all of the buoy deployment and recovery, longshore survey, and process-oriented cruises of the Ocean Margins Program with two of the LDEO automated IR-based analysis systems for carbon dioxide and pulsed polarographic oxygen sensors for oxygen concentration. During the bouy deployment and recovery cruises, discrete seawater samples for carbon dioxide and oxygen analysis will be taken throughout the water column in the vicinity of all of the moored gas sensors.
Results to Date: Carbon dioxide partial pressure measurements (but not oxygen concentrations) have been made in the surface waters of the Cape Hatteras-Cape Charles survey area during one scoping cruise (May 1993, R/V Gyre) and along the continental shelf between Cape Hatteras and Georges Bank during another (April to May 1994, R/V Columbus Iselin). The results of these two cruises have been summarized. All our proposed work for the OMP 1996 field season has been successfully completed with mooring deployment and recovery cruises in February to May and June to October aboard the R/V Oceanus and longshore survey and process cruises in March (R/V Endeavor) and July to August (R/V Seward Johnson). The data from these cruises are being processed and will be presented in the annual progress report.

\section{SEDIMENT MOTION AND BOTTOM- BOUNDARY-LAYER DYNAMICS OVER THE MID-ATLANTIC BIGHT SHELF AND UPPER SLOPE \\ CHURCHILL, JAMES H., and WILLIAMS, ALBERT J. \\ WOODS HOLE OCEANOGRAPHIC INSTITUTION \\ FY 1996100 \\ FY 1995100 \\ FY 1994229}

Keywords: benthic boundary layer, suspended sediments, turbidity, sediment transport

Objectives: To investigate the dynamics of particulate organic carbon transport over the inner Hatteras Shelf; to examine circulation over the Hatteras Shelf, particularly the export of Mid-Atlantic Bight (MAB) shelf water to the Gulf Stream; and to plan and prepare for the 1996-1997 Ocean Margins Program (OMP) field project.

Approach: Instrumented bottom tripods were deployed at two near-shore locations. The first was at the $20-\mathrm{m}$ isobath, roughly $4 \mathrm{~km}$ east of Beaufort Inlet. In place from 30 November through 8 December 1993, the tripod was equipped with triple-axis acoustic-traveltime current meters and optical-backscatterance sensors (OBS) at heights of 0.6, 1.0, 1.6, 2.6, and $4.6 \mathrm{~m}$ above the bottom. The second was at the $20-\mathrm{m}$ isobath east of Duck, N.C. Directly seaward, a companion tripod was deployed at the $25-\mathrm{m}$ isobath. The tripods were within an array of moorings set out as part of the NSF-funded Coastal Ocean Processes (COOP) project. Supplementing the suite of sensors (current meters and pressure and OBS sensors) at the 20-m tripod was an array of eight fiberoptic fluorometers. The tripod was deployed on 23 July 1994. It was "turned around" with data transfer on 24 August, and was recovered on 5 November 1995. 
Results to Date: Analysis of the data from the tripod deployed off Beaufort Inlet has focused on the OBS measurements, which were converted to suspendedparticulate concentrations (SPC) through calibration with sediment from the deployment site. Results showed that the OBS sensors could resolve nearbottom suspended-matter concentrations (a concern prior to deployment). The high-frequency $(4-\mathrm{Hz})$ data revealed a general tendency for SPC to increase with near-bottom speed, but a clear speed-SPC relationship was lacking. The low-passed, filtered time series exhibited coupled SPC and speed fluctuations, mostly associated with the semidiurnal tide. However, high near-bottom speeds in an early December storm were not accompanied by large SPC, possibly because of vigorous vertical mixing and dilution. Suspendedparticulate fluxes exhibited large fluctuations with a mean directed to the northeast (onshore). Measurements collected off Duck indicated a well-defined relationship between organic matter concentration and water mass within the near-shore zone. High chlorophyll concentrations were detected in waters of the thermocline and in the cold band, whereas a saline intrusion of slope and/or Gulf Stream water was found to be nearly devoid of organic matter. We are currently exploring the organic-matter/water-mass relationship further with shipboard hydrographic and fluorometer data collected during the COOP study. Our examination of regional circulation over the Carolina shelf indicated that MAB shelf water tends to exit the Hatteras Shelf in a narrow band $(40 \mathrm{~km}$ wide) near Diamond Shoals. Dominating this export is the adjustment of the SAB-MAB shelf-water front as it moves seaward and shoreward across the shelf. When the front progresses shoreward, a jet of MAB shelf water, roughly $40 \mathrm{~cm} / \mathrm{s}$ in magnitude and directed seaward, forms at its boundary.

\section{CAPE HATTERAS $\mathrm{PCO}_{2}$ AND NUTRIENT FLUXES FROM MOORINGS DEGRANDPRE, MICHAEL D. \\ UNIVERSITY OF MONTANA and WOODS HOLE OCEANOGRAPHIC INSTITUTION \\ FY 1996105 \\ FY 1995177 \\ FY 199486}

Keywords: coastal carbon cycle, $\mathrm{CO}_{2}$ sensors, moorings, nitrate sensors

Objectives: To study and improve our understanding of the $\mathrm{CO}_{2}$ cycle in a coastal region (Cape Hatteras, N.C.) by using mooring-based measurements of $\mathrm{CO}_{2}$ and nitrate.

Approach: The study requires both ship- and mooring-based measurements to characterize the complex biogeochemical processes in the coastal region off Cape Hatteras, NC. Our role is to study $\mathrm{CO}_{2}$ and nutrient $\left(\mathrm{NO}_{3}\right)$ cycling with mooring-based sensors. The $\mathrm{CO}_{2}$ sensors, called the Submersible Autonomous Moored Instruments for $\mathrm{CO}_{2}\left(\mathrm{SAMI}-\mathrm{CO}_{2}\right)$ and the nitrate sensors, called the nitrate OsmoAnalyzer, were developed for the project. Our approach is to deploy SAMI- $\mathrm{CO}_{2} \mathrm{~s}$ and nitrate OsmoAnalyzers on moorings within a control volume off Cape Hatteras. The proposed work should greatly improve our ability to predict surface seawater $\mathrm{pCO}_{2}$ and air-sea fluxes and will help us estimate perturbations in the ocean carbon cycle from climate change. This research will also take us one step further toward the goal of an automated Earth Observing System, where ocean-atmosphere observations of $\mathrm{pCO}_{2}$ and the flux of $\mathrm{CO}_{2}$ are monitored in real time along with other indicators of the biological state of the ocean (ocean color, fluorescence, oxygen, beam carbon attenuation, etc.).

Results to Date: Six new SAMI- $\mathrm{CO}_{2} \mathrm{~s}$ and three new nitrate OsmoAnalyzers were built for the field program. The instruments were deployed in February 1996 and were recovered in May 1996. The instruments were then redeployed in June 1996 and will be recovered in October 1996. The 3-month time-series data from the first deployment have been downloaded and are currently being analyzed. Preliminary evaluation of the data indicates that the mooring measurements match well with periodic ship-based measurements in the area. We are currently compiling other ship and mooring-based measurements for evaluation of the $\mathrm{CO}_{2}$ and nitrate time-series.

\section{DEVELOPMENT OF OCEANIC DEEP-CONVECTION MODELS FOR CLIMATE RESEARCH}

DENBO, D. W., and SKYLLINGSTAD, E. D. BATTELLE MARINE SCIENCES LABORATORY

$$
\begin{array}{lr}
\text { FY } 1996 & * \\
\text { FY } 1995 & 242 \\
\text { FY } 1994211
\end{array}
$$

*Funding consolidated with that of William Asher. Keywords: deep-convection parameterizations, ocean models, thermobaric instability, heat flux, latent heat flux, shear, wind stress

Objectives: To produce empirical relationships between the forcing and deep convection that will provide a basis for developing improved deep-convection parameterizations used in ocean models applied to climate studies.

Approach: Using a combination of idealized forcing scenarios with idealized temperature-salinity (T-S) profiles, we will determine the relationship among the forcing, T-S structure, and model response. The T-S structure will be characterized by the density gradient and potential for thermobaric instability (variation of fluid compressibility). The idealized forcing will consist of combinations of steady heat flux, latent heat 
flux, shear, and wind stress. The idealized T-S profiles will be constructed to represent a range of density gradients and of strengths of the thermobaric instability. The analysis of the model results will include deep-convection onset time, rate of mixed-layer growth, and the size and number $/ \mathrm{km}^{2}$ of plumes. The model runs will be conducted in a domain with $40^{3}$ points and 50-m grid spacing. Results will be checked for several forcing and T-S profile combinations with a higher grid resolution and larger domain. The robustness of the relationships determined above will be tested with the idealized forcing scenarios with several actual T-S profiles from the Greenland, Labrador, and Weddell seas, the major deep-convection regions. These profiles will be classified with the density gradient and potential for thermobaric instability.

Results to Date: A sensitivity study was conducted to look at the relationship among heat flux, latent heat flux, wind stress, shear, and perturbations of the surface mixed-layer temperature and salinity and the onset time and strength of deep-penetrative convection. The onset time of convection was found to be inversely proportional to the heat flux and linearly proportional to changes of the surface mixed-layer temperature and salinity. This result is consistent with the onset times being primarily determined by changes in the density of the mixed layer and not strongly related to the rate of density change. Therefore, high-frequency forcing of ocean general circulation models could be unnecessary for production of the correct timing of deep convection; however, errors in the mixed-layer temperature and salinity can strongly affect the timing.

\section{QUALITY CONTROL OF OCEANIC CARBON DIOXIDE MEASUREMENTS: PREPARATION AND DISTRIBUTION OF REFERENCE MATERIALS \\ DICKSON, ANDREW G. SCRIPPS INSTITUTION OF OCEANOGRAPHY FY $1996 \quad 55$ \\ FY 1995205 \\ FY 1994248}

Keywords: carbon dioxide, reference materials, total dissolved inorganic carbon, total alkalinity

Objectives: To develop and provide reference materials for carbon dioxide in sea water; coordinate and develop standard measurement procedures; provide quality-control guidance for measurements; and develop an automated, ultraprecise $\mathrm{pH}$ method to monitor the long-term stability of reference materials. Approach: Reference materials (RMs) for total dissolved inorganic carbon and for total alkalinity are prepared from natural seawater and bottled in $500-\mathrm{mL}$ borosilicate-glass bottles. These RMs are then certified and distributed for use by scientists studying the oceanic carbon dioxide system (in particular those funded by DOE for the Global Carbon Dioxide Survey). In addition, collaborative studies and a Handbook of Methods are being used to standardize the analytical practices of scientists involved in oceanic carbon dioxide studies.

Results to Date: We bottled eight batches (4926 bottles) of sea water for use as RMs, and distributed 4123 bottles to 27 different laboratories in 17 countries. (Note that support of this RM program is also provided by the National Science Foundation. More than three quarters of these RMs were used by U.S. investigators funded by DOE, NSF, and NOAA. These RMs have helped assure the quality of data both for the oceanic carbon dioxide survey and for studies in the coastal ocean.

The RMs have been certified for total dissolved inorganic carbon by extraction/manometry at the Scripps Institution of Oceanography. Development of a certification procedure for alkalinity has been an important area of work, and such a procedure is now being finalized. An international interlaboratory study was held at the Scripps Institution of Oceanography in June 1994 to compare approaches to the measurement of the partial pressure of carbon dioxide in sea water. This exercise (which was cosponsored by NSF) involved 13 groups from 8 countries. Each group brought its equipment to the study, and the equipment was operated in carefully designed side-by-side comparisons. Another international interlaboratory study was initiated for the measurement of the amount of carbon-13 present in the total dissolved inorganic carbon in sea water. Samples of reference material were sent to each of the 12 laboratories interested in this exercise.

Version 2 of the DOE Handbook of Methods for the Analysis of the Various Parameters of the Carbon Dioxide System in Sea Water was completed. This was published by CDIAC at Oak Ridge (ORNL/ CDIAC-74) and has been very widely distributed; it is also being distributed as a component of the JGOFS measurement protocols.

\section{SOURCES AND TURNOVER TIMES OF DISSOLVED, COLLOIDAL, AND \\ PARTICULATE ORGANIC CARBON IN THE MID-ATLANTIC BIGHT AND CHESAPEAKE BAY \\ DRUFFEL, ELLEN R. M. \\ UNIVERSITY OF CALIFORNIA, IRVINE FY 199690 \\ FY 199572 \\ FY 19940}

Keywords: isotopes, carbon sources, carbon transformations 
Objectives: To evaluate the important sources of terrestrial and marine organic carbon to the MidAtlantic Bight and to assess their role(s) in the marine and global organic carbon budgets.

Approach: This project will examine and evaluate the sizes, sources, turnover times, and fluxes of dissolved, colloidal and particulate organic carbon (DOC, COC, and $\mathrm{POC}$ ) and dissolved inorganic carbon (DIC) pools in waters of the Mid-Atlantic Bight (MAB). On each of four transects across the MAB shelf and slope conducted at two times of the year, samples will be collected for determinations of carbon and nitrogen content and ${ }^{14} \mathrm{C}$ and ${ }^{13} \mathrm{C}$ isotopic compositions of bulk $\mathrm{DOC}$ and $\mathrm{POC}$ as well as the colloidal components of the DOC pool. We will obtain estimates of organic carbon turnover caused by both the buildup and transport of autochthonously (from shelf primary production) and allochthonously (from estuarineterrestrial and atmospheric inputs) derived material along and across the MAB shelf as well as that produced by short-term in situ processes. We will also evaluate in a more rigorous manner the short-term (bimonthly) variability in these parameters as source indicators of organic matter carried in waters of the Chesapeake Bay outflow to the MAB shelf. This work will be closely coupled to that of other OMP researchers who will measure natural-carbon isotopic abundances of selected, individual, biomarker molecules in the DOC and POC pools. These data will be compared to existing data sets for $\mathrm{DOC}$ and $\mathrm{POC}$ from the North Atlantic (Sargasso Sea) to evaluate the magnitude of organic-carbon sequestration and aging within the interior ocean and the similarities and disparities in organic-carbon cycling between the ocean's margins and interior.

Results to Date: This project has documented the existence of organic carbon of both terrestrial and marine origin in coastal waters that contribute to the total flux of carbon to the ocean's interior. Much of the geochemically young organic matter in continentalshelf waters is used before making it to more-seaward regions overlying the continental slope. It has also been shown that, taken as a whole, the ocean margin in the MAB is a source of geochemically old carbon to the interior North Atlantic. Thus, the MAB is a transient reservoir of young carbon fixed from atmospheric carbon dioxide.

\section{COASTAL PRODUCTIVITY FALKOWSKI, PAUL BROOKHAVEN NATIONAL LABORATORY FY 1996200 FY 1995179 FY 1994194}

Keywords: sources of photosynthetically fixed carbon, sinks of photosynthetically fixed carbon
Objectives: To determine the sources and sinks of photosynthetically fixed carbon on continental margins in response to anthropogenic, energy-related activities. Approach: Carbon export from the continental shelves appears to be relatively small, averaging about $10 \%$ of the annual primary production. This flux could be greatly enhanced by cultural eutrophication along the eastern seaboard of the continental United States. Therefore, the potential advective fluxes will be analyzed to determine the processes that govern the production and fate of phytoplankton in continentalshelf environments and to focus on how oxidative processes are controlled in relation to long-term anthropogenic perturbations. The approach is multidisciplinary, involving shipboard observations, remote sensing, and moored instruments. Field and laboratory studies are under way to provide basic information on the effects of light, energy, and nutrients on phytoplankton growth and distribution.

Results to Date: The oxidation of organic carbon on the continental margin off the northeast United States has been found to approximately balance inputs; hence, the shelf does not appear to be a major net sink for carbon. This situation could rapidly change if anoxic conditions were to prevail as a result of coastal eutrophication. In that case, the shelf could become a net source of $\mathrm{CO}_{2}$ to the atmosphere.

\section{MOLECULAR ECOLOGY OF PHYTOPLANKTON PHOTOSYNTHESIS FALKOWSKI, PAUL \\ BROOKHAVEN NATIONAL LABORATORY \\ FY 1996150 \\ FY 1995267 \\ FY 1994330}

Keywords: phytoplankton, photosynthesis, ecology, photoluminescence

Objectives: To understand key molecular processes that determine photosynthetic rates or limitations in natural, marine, phytoplankton communities.

Approach: The approach is based on biophysical and molecular biological experimental research in the laboratcry and the development of techniques that can be transferred to the field.

Results to Date: In the application and interpretation of chlorophyll variable-fluorescence signatures, we have developed a mathematical model of variable fluorescence based on the fast-repetition-rate technique and have compared the results of that technique with alternative methods for estimating fluorescence parameters. The fluorescence-based measurements were compared with independent measurements of energy storage with photoacoustic techniques. The results provide a robust basis for the interpretation of fieldbased measurements conducted in conjunction with the 
Ocean Margins Program and the Ocean $\mathrm{CO}_{2} \mathrm{Bio}-$ sequestration project in the eastern Equatorial Pacific.

Because thermoluminescence profiles potentially contain information about the energies of activation of photosynthetic charge separation and the pathways of charge recombination in response to environmental stress, we constructed an instrument capable of measuring both thermoluminescence and delayed light emission at very low chlorophyll concentrations and used it to investigate the thermoluminescence-glow curves in marine phytoplankton. These are the first such measurements made on marine phytoplankton, and the initial results demonstrate a clear departure from the model biophysical constructs that have been derived from studies of higher plants and simple green algae.

Finally, we investigated the induction of specific proteases that appear under environmental stress conditions and are correlated with catastrophic cell death. The proteases appear to be novel and are normally silenced in growing cells. We are investigating the possibility that these are retroviral genes that have been incorporated into the eucaryotic genome and subsequently controlled via host selection.

\section{COASTAL TRANSPORT AND DIFFUSION FLAGG, CHARLES N. \\ BROOKHAVEN NATIONAL LABORATORY \\ FY 1996200 \\ FY 1995223 \\ FY 1994208}

Keywords: transport of continental shelf waters, baroclinic-pressure fields, ocean currents, primary and secondary production, carbon flux in the ocean

Objectives: To estimate the along-shore and offshore transport of continental shelf waters and their dissolved and particulate constituents, to characterize the genesis of the low-frequency (subsynoptic) baroclinicpressure field in the southern Mid-Atlantic Bight and its role in the production of the low-frequency baroclinic currents, and to define the three-dimensional character of primary and secondary production in the southern Mid-Atlantic Bight on synoptic time scales for the purpose of understanding the biologically mediated flux of carbon.

Approach: Twenty-seven moorings will be deployed for a year, and four to five hydrographic cruises are planned. Very-low-frequency temperature, salinity, and current data will be gathered from the moorings together with hydrographic survey results from the process cruises to look at the balance of terms in the momentum- and mass-conservation equations and thus better understand the factors controlling the seasonal mean currents. We also plan to study the kinematics and dynamics of frontal eddies and filaments with the intent of quantifying the cross-frontal transport that results from these processes by satellite-directed sampling with synoptic hydrographic surveys and TOYO high-speed sampling. An important element of the program stems from the use of a 3-D moored array of sensors plus Lagrangian studies within the array, which should make it possible to unravel the convolution of biological and physical processes over small to intermediate scales and to study directly the impact that local physics have on biological processes. This kind of study should also make it possible to define the spatial structure of the phytoplankton and zooplankton fields.

Results to Date: Three short "scoping" hydrographic cruises have been made to the southern Mid-Atlantic Bight during the lead-up to the Ocean Margins Program. On all three cruises, we have collected hydrographic and acoustic-Doppler-current-profiler (ADCP) data. The hydrographic and $A D C P$ data have been processed and entered into their respective databases, and data have been shared with collaborating investigators. Four moorings were also deployed as part of an effort to verify the feasibility of deploying oxygen sensors. Included on the moorings were a bottommounted ADCP and four SeaCats, the data from which have all been processed. Lastly, an ocean margins central data facility has been established that can handle CTD and bottle hydrographic data, time-series data from moored instruments, and space-time-series data from shipboard ADCPs.

\section{OMP DATA MANAGEMENT FLAGG, CHARLES $\mathrm{N}$. BROOKHAVEN NATIONAL LABORATORY \\ FY 199650 \\ FY 19950 \\ FY 19940}

Keywords: data management

Objectives: To serve as the storehouse for the field experimental data collected in 1996.

Approach: Data collected and processed at Brookhaven National Laboratory will be archived at the Carbon Dioxide Information Analysis Center.

Results to Date: Data collection and processing are in process.

\section{$\mathrm{CO}_{2}$ MEASUREMENTS ALONG THE WOCE SECTIONS IN THE PACIFIC AND INDIAN OCEANS \\ GOYET, CATHERINE \\ WOODS HOLE OCEANOGRAPHIC \\ INSTITUTION \\ FY 1996100 \\ FY 1995250 \\ FY 1994257}

Keywords: Indian Ocean, total $\mathrm{CO}_{2}$, total alkalinity 
Objectives: To measure and contribute accurate data on the carbon chemistry of seawater to a global oceanic database for numerical modeling.

Approach: Three carbon properties (total $\mathrm{CO}_{2}$, total alkalinity, and $\mathrm{pCO}_{2}$ ) are measured along WOCE cruises in the Pacific, Atlantic, and Indian Oceans. Total $\mathrm{CO}_{2}$ and total alkalinity are measured throughout the water column. The upper $1000 \mathrm{~m}$ of the ocean is more densely sampled than the deep ocean. The measurement rate is approximately $\mathbf{4 0}$ samples for each parameter (total $\mathrm{CO}_{2}$ and total alkalinity) per day. The partial pressure of $\mathrm{CO}_{2}$ in surface seawater is continually monitored along the cruise track. Aboard ship, total $\mathrm{CO}_{2}$ is measured with an extraction coulometric system, and total alkalinity analyses are performed with an automated potentiometric system.

Results to Date: During WOCE II (August 29, 1995, to October 16, 1995, in the North Indian Ocean), more than 3000 samples were collected for total $\mathrm{CO}_{2}$ and more than 3000 samples for total-alkalinity measurements on board. These samples were collected at 158 stations (including test stations). In addition, 80 samples were collected for C. D. Keeling. As expected from the physical properties at the time of measurements, $\mathrm{TCO}_{2}$ in surface seawater was significantly lower across the Bay of Bengal than across the Arabian Sea.

\section{SUPPORT FOR RESEARCH SHIPS OF THE UNIVERSITY-NATIONAL OCEANOGRAPHIC LABORATORY SYSTEM (UNOLS) TO CONDUCT THE DOE OCEAN MARGINS FIELD EXPERIMENT \\ HEINRICHS, DONALD F. NATIONAL SCIENCE FOUNDATION FY 19961105 \\ FY 19950 \\ FY 19940}

Keywords: technical support, research ships Objectives: Ocean margins research. Rent research vessels from the National Science Foundation for Ocean Margins research.

Approach: A vessel will be rented from the National Science Foundation for ocean-margins research.

Results to Date: The field effort for the Ocean Margins Program has been completed.

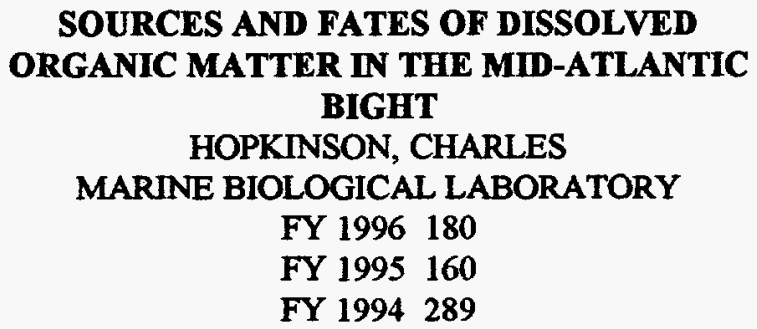

SOURCES AND FATES OF DISSOLVED ORGANIC MATTER IN THE MID-ATLANTIC BIGHT

HOPKINSON, CHARLES

MARINE BIOLOGICAL LABORATORY

FY 1996180

FY 1995160

FY 1994289

Keywords: ocean, continental shelf, nutrients, carbon, nitrogen, net metabolism

Objectives: To identify and determine the relative importance of various sources of dissolved organic matter to the continental shelf, to measure and model the use of the various dissolved organic matter (DOM) sources, and to estimate the net carbon balance for the Middle Atlantic Bight.

Approach: Identification of the various organic matter sources will be determined with a multitracer approach with emphasis on dissolved organic carbon (DOC) and dissolved organic nitrogen (DON) concentration, particulate organic carbon (POC) and particulate organic nitrogen (PON) concentration, and corresponding $\mathrm{C} / \mathrm{N}$ ratios. Study of the uses of the various organic matter sources will rely mainly on laboratory decomposition studies. Decomposition experiments will be carried out to measure the rate of removal of DOM (including $\mathrm{O}_{2}$ consumption, $\mathrm{TCO}_{2}$ production, and bacterial production) and the stoichiometry of remineralization. Information on the degree of chemical reduction of DOM will be used to predict organicmatter oxidation and microbial growth. The net carbon balance for the system, which is the difference between system production and respiration $(P-R)$, will be estimated by determining the deviation from conservative mixing of biogeochemical properties and CNP stoichiometry.

Results to Date: Dissolved organic carbon (DOC), nitrogen (DON), phosphorus (DOP), inorganic nitrogen, and inorganic phosphorus were measured from the Georges Bank region during spring 1993 and summer 1994. Concentrations of DOC in surface waters ranged from 65 to $92 \mathrm{M} \mathrm{C}$ and decreased with depth to $50 \mathrm{M}$ in shelf-slope water at a depth of $1500 \mathrm{~m}$. DON and DOP concentrations showed a similar pattern with depth, being higher in surface water and lower in deep water ( 5 to $3 \mathrm{M} \mathrm{N}$ and 0.17 to $0.02 \mathrm{M} \mathrm{P}$ ). There was an inverse relation between inorganic nutrient concentrations and DON and DOP. While DOC concentrations were elevated in regions of highest primary production, there was little evidence of corresponding elevations for DON and DOP. Slopes of regression lines relating total dissolved $\mathrm{N}$ and $\mathrm{P}$ to inorganic $N$ and $P$ indicated that 19 and $15 \%$ of remineralized $N$ and $P$ that accumulates in deeper water is derived from the export and decomposition of dissolved organic material (DOM). While overall inorganic $\mathbf{N}$ and $\mathbf{P}$ dynamics followed Redfield stoichiometry (C:N:P = 106:16:1), the $C: N: P$ stoichiometry of dissolved organic matter deviated substantially. Near-surface stoichiometry of DOM was $400: 1$ to $800: 1$ for DOC:DOP, $11: 1$ to $15: 1$ for DOC:DON, and 24:1 to 55:1 for DON:DOP. Relationships among DOC, DON, and DOP indicated that $N$ and $P$ were preferentially remineralized relative to 
carbon, resulting in deeper-water $\mathrm{C}: \mathrm{N}: \mathrm{P}$ ratios for DOM that deviated even further from Redfield. Decomposition studies revealed that the DOC decomposition rate was related to the initial DOC concentration. The higher the DOC concentration, the more quickly it was mineralized. DOC first-order decay coefficients ranged from undetectable for deep-slope water with an initial DOC concentration of $50 \mathrm{M}$ to $0.0024 \mathrm{~d}^{-1}$ for water collected from the Georges Bank shoals with an initial DOC concentration of $92 \mathrm{M}$. DON and DOP decomposition occurred concomitantly with DOC remineralization. Loss rates ranged from undetectable to $0.00064 d^{-1}$ for DON and from undetectable to $0.0023 \mathrm{~d}^{-1}$ for DOP. During DOM decomposition, we noted increases in the $C: N, C: P$, and $N: P$ ratios, which indicated preferential remineralization of $N$ and $P$ relative to $\mathrm{C}$. Vertical concentration gradients and slow DOM turnover times relative to water residence times in the Georges Bank continental shelf region indicate the potential for substantial export of DOM to deeper water. DOM export to depth and preferential remineralization of $\mathrm{N}$ and $\mathrm{P}$ relative to $\mathrm{C}$ may be important mechanisms for pumping carbon to depth in the ocean.

\section{SEA-FLOOR CYCLING OF ORGANIC MATTER IN THE CONTINENTAL MARGIN OF THE MID-ATLANTIC BIGHT JAHNKE, RICHARD \\ SKIDAWAY INSTITUTE OF OCEANOGRAPHY FY 1996111 \\ FY 199595 \\ FY 1994123}

Keywords: carbon flux in the ocean, remineralization, primary production

Objectives: To provide a minimum estimate of the carbon export from the Mid-Atlantic Bight continental shelf by quantitatively determining the organic carbon input, remineralization, and burial on the slope and rise regions of the sea floor adjacent to the North Carolina continental margin.

Approach: Benthic flux chambers will be deployed on the sea floor to directly determine the benthic exchange rates of oxygen, nutrients, and carbon. This information will be combined with information from sediment cores to quantify the fluxes of carbon to and from the sediments at selected slope and rise sites.

Results to Date: Results to date suggest that carbon inputs in the slope and rise regions immediately north of Cape Hatteras are among the highest in the world. Calculations suggest that $1.2 \times 10^{11} \mathrm{~mol} \mathrm{C} / \mathrm{y}$ may be remineralized on the Mid-Atlantic Bight continental slope.

\section{CARBON-14 ANALYSIS IN SUPPORT OF OCEAN MARGINS PROGRAMS \\ KASHGARIAN, MICHAELE \\ LAWRENCE LIVERMORE NATIONAL LABORATORY \\ FY 199671 \\ FY 19950 \\ FY 19940}

Keywords: carbon sources, turnover rates, radiocarbon, carbon-14, isotopes

Objectives: To use natural and bomb radiocarbon as tracers for particulate- and dissolved-organic-carbon dynamics.

Approach: Newly produced biomass in surface waters is imprinted with cosmic-ray-produced ${ }^{14} \mathrm{C}$ present in the dissolved inorganic carbon of the ocean mixed layer and, in recent docades, with an additional burden of bomb-produced radiocarbon from nuclear-weapons tests. Measurements of ${ }^{14} \mathrm{C}$ in dissolved and particulate organic carbon, or in specific sizes of particulates or classes of chemical compounds, show the apparent elapsed time since these molecules were present as primary production. These data provide information on the mixing of carbon from different (atmospheric, terrestrial, or marine) sources.

Results to Date: Radiocarbon measurements have helped to determine that different size classes of dissolved organic carbon (DOC) have different origins: large molecules (molecular weight greater than 10,000 ) are of relatively recent origin, while smaller ones are much older and may represent refractory fragments. Measurements have also been carried out on ultrasmall samples (tens of micrograms), consisting of specific classes of chemical compounds, and even individual compounds, extracted from sediments by Tim Eglington (WHOI) and colleagues as part of OMP's SEEP Program, to further elucidate sources and cycling of sedimentary carbon.

\section{OCEAN- $\mathrm{CO}_{2}$ MEASUREMENTS FOR THE WOCE HYDROGRAPHIC SURVEY KEELING, CHARLES \\ SCRIPPS INSTITUTION OF OCEANOGRAPHY FY 1996190 \\ FY 1995209 \\ FY 1994207}

Keywords: carbon in sea water, manometric assay Objectives: To provide reference dissolved inorganic carbon (DIC) concentrations measured on shore with a standard method for replicate seawater samples also measured for DIC at sea by coulometry.

Approach: The concentration of DIC in seawater has been determined by gas extraction followed by manometric assay on water samples collected during the WOCE Hydrographic Survey of the Pacific, Atlantic, and Indian oceans. Titration alkalinity (ALK) has been 
determined potentiometrically. Our DIC measurements provide a check on the performance of coulometric analyzers used for at-sea measurements of DIC by the $\mathrm{DOE} \mathrm{CO} \mathrm{C}_{2}$ Science Team and how well these analyzers are being tested for quality assurance with certified reference seawater solutions. The ${ }^{13} \mathrm{C} /{ }^{12} \mathrm{C}$ isotopic ratio of dissolved inorganic carbon in the samples has also been measured as an aid in determining the causes of variations in DIC, especially to provide an additional parameter which responds differently to temperature and organic processes than DIC does. The combined data will help establish the rate of uptake of industrial carbon dioxide by the oceans and the extent to which this uptake mitigates the buildup of carbon dioxide in the atmosphere.

Results to Date: Reference DIC values for seawater samples have been measured and reported to the Science Team for the $\mathrm{CO}_{2}$ in the Oceans program. During calendar 1995, replicate samples were collected from nine legs of the WOCE Indian Ocean expedition and from two legs in the Atlantic Ocean. In 1996, one set of samples from a WOCE Southern Ocean leg was collected. In addition to the 1200 replicate samples and reference solutions from 20 batches measured during the first three years of the project, an additional 190 samples have been measured to this date in 1996, including certification measurements for two additional batches of reference solutions. The shipboard coulometric systems tend to produce DIC concentration results lower than the shore-laboratory results by two to three micromoles per kilogram ( $\mu \mathrm{mol} / \mathrm{kg}$ ), even though shipboard results for the reference solutions consistently agree within $1 \mu \mathrm{mol} / \mathrm{kg}$ with the values certified by shore-laboratory analyses. The reason for this discrepancy is not yet understood. However, for 9 of the 21 cruise legs investigated by mid-1995, the average difference between shipboard and laboratory measurements is not significantly different than zero at the $95 \%$ confidence limit.

\section{MICROBIAL PROCESSES IN COASTAL MARINE SYSTEMS KEMP, PAUL F.

Keywords: population dynamics of marine bacteria, microbial growth, transformation of oceanic organic carbon

Objectives: To understand the detailed, taxon-specific population dynamics of marine bacteria in coastal ccean systems and to assess how environmental factors control microbial growth and how those controls influence the biological transformation of organic carbon in the coastal ocean.
Approach: The technical approach taken is based on fluorescently labeled, oligonucleotide hybridization probes. Probes are complementary to 16S rRNA sequences and are labeled by conjugation with a fluorochrome, Texas Red. When probes are hybridized to $16 \mathrm{~S}$ rRNA in preserved, intact cells, the resulting cell fluorescence is linearly proportional to rRNA content. This has been verified in both laboratory and field tests at BNL. Probe sequences can be selected that are characteristic of specific phylogenetic groups, ranging from universal probes to kingdom level. One can use specific probes to measure the rRNA content of one species within a mixed bacterial assemblage independently of other cooccurring species. Because cells can be examined individually, the frequency distribution of rRNA content among cells also can be determined. This practice provides an extremely useful indicator of population response to environmental change.

Results to Date: Growth-rate experiments with 28 marine bacterial isolates, originally obtained from a variety of sampling locations and representing various phylogenetic groups differing in physiological characteristics, indicate that a single mathematical relationship between rRNA content and growth can be applied to bacteria collected in the field, regardless of whether their species compositions were known.

On each of three cruises, IRNA content was measured in three to six cross-shelf transects. Biomass production was measured with thymidine- and leucineincorporation methods. Data show a strong spatial pattern in rRNA content, thymidine incorporation, and leucine incorporation. Preliminary results from subsequent cruises indicate that a multiparameter approach to measuring bacterial activity is valuable because the different measurements show similarly strong, but not identical, spatial patterns and significant correlations with different hydrographic and biological variables.

Data on rRNA distributions across the shelf indicate that the bacterial rRNA content, DNA synthesis, and protein synthesis change over scales of meters vertically and over a few kilometers horizontally. This variability necessitates stations spaced no more than $5 \mathrm{~km}$ apart and closely spaced sampling depths. There are too few data to assess the appropriate temporal scale, but as a first cut, the pre- and post-stratification water column must be examined.

Samples of the bacterial DNA collected in Cape Hatteras shelf waters were used to identify a suite of bacterial species abundant there. With PCR, bacterial 16S rRNA was amplified from these samples and has been cloned to create a $16 \mathrm{~S}$ gene library; 216 clones were obtained and are being screened for unique 16S rRNA signatures.

With five oligonucleotide probes that have approximately the same level of taxonomic specificity and 
additive probe fluorescence, detection limits are now down to less than $0.3 \mathrm{fg}$ rRNA cell-1, approximately two orders of magnitude lower than the rRNA content of an $E$. coli cell in exponential growth. At that level, rRNA is measurable in ca. 50 to $70 \%$ of cells from coastal waters. An image-analysis system that is much more light sensitive than was previously used and that allows time-integrated fluorescence measurements has considerably improved the current detection limits for rRNA in natural bacterial populations and greatly speeds sample analysis.

\section{FAST-REPETITION-RATE (FRR) \\ FLUOROMETER FOR MAKING IN SITU MEASUREMENTS OF PRIMARY PRODUCTIVITY \\ KOLBER, ZBIGNIEW \\ BROOKHAVEN NATIONAL LABORATORY \\ FY 199642 \\ FY 1995179 \\ FY 1994312}

Keywords: photosynthesis, primary production, fast-repetition-rate fluorometer

Objectives: To develop instrumentation and methods for measuring primary production with spatial and temporal resolution matching, or exceeding, the spatial and temporal scales of the water-mass structure on the North Atlantic continental shelf.

Approach: Fast-repetition-rate (FRR) fluorometry makes use of the relationship between fluorescence yield and photochemistry to estimate photosynthetic parameters, such as effective absorption cross-section, yield of primary photochemistry, and turnover time of photosynthesis. These parameters are then used to calculate the photosynthetic rates from the kinetics of light absorption and the electron flow between photosystem II and photosystem I. Because of the noninvasive character of FRR measurements and the high rate of data collection, the FRR method allows high-resolution measurements of primary production over large spatial and temporal scales. In situ FRR sampling (with a submersible FRR fluorometer) will be coordinated with onboard FRR measurements of the CTD/Rosette water samples (with two bench-top instruments). The resulting data set will be merged with dissolved gasses, nutrients, and (where applicable) with ${ }^{14} \mathrm{C}$ data. Alternatively, the submersible instrument will be used in a TOYO mode of operation to provide a high-speed 3-D photosynthetic signature of the water masses. The second bench-top instrument will be used in the continuous-mapping mode of subsurface photosynthetic parameters.

Results to Date: A novel method for measuring primary production was developed.

\section{DATA-MANAGEMENT SUPPORT FOR DOE'S GLOBAL SURVEY OF $\mathrm{CO}_{2}$ IN THE OCEANS \\ KOZYR, ALEX; BODEN, THOMAS; and CUSHMAN, ROBERT \\ OAK RIDGE NATIONAL LABORATORY \\ FY 1996150 \\ FY 1995150 \\ FY 1994145}

Keywords: oceans, ocean chemistry, carbonate chemistry, global change, greenhouse effect, climate change, global warming, data management, World Ocean Circulation Experiment, Joint Global Ocean Flux Study

Objectives: To acquire, inspect, document, archive, and distribute DOE-sponsored ocean-carbon measurements collected during or derived from the Joint Global Ocean Flux Study (JGOFS) aboard World Ocean Circulation Experiment (WOCE) cruises.

Approach: With help from researchers at LamontDoherty Earth Observatory (LDEO) and DOE's $\mathrm{CO}_{2}$ Survey Science Team, CDIAC's data-management plan will ensure timely submission of DOE-sponsored oceanographic data from the WOCE and JGOFS programs to CDIAC and subsequent archiving, quality control, documentation, and distribution. The plan adheres to WOCE data-sharing policies, and it facilitates incorporation of $\mathrm{CO}_{2}$ measurements into the larger WOCE and JGOFS data-management efforts. Data review is a coordinated effort among CDIAC, LDEO, and the contributing scientist. Data documentation prepared by CDIAC is reviewed and approved by the contributing scientist(s) before distribution.

These data documents or numeric data packages (NDPs) prepared by CDIAC consist of written documentation and machine-readable files of $\mathrm{CO}_{2}$-related measurements and derivations (i.e., total $\mathrm{CO}_{2}$, discrete $\mathrm{pCO}_{2}$, underway surface $\mathrm{pCO}_{2}$, and total alkalinity), hydrographic measurements, and nutrient measurements from WOCE surveys. The NDPs will be distributed by CDIAC free of charge via a variety of media, including 9-track magnetic tape, CD-ROM, IBMformatted floppy diskettes, 8-mm tapes, quarter-inch tape cartridges, and CDIAC's anonymous file transfer protocol (FTP) area and WWW home page over the Internet.

Results to Date: To date, $\mathrm{CDIAC}$ has received $\mathrm{CO}_{2}$ related measurements and derivations (total $\mathrm{CO}_{2}$, discrete $\mathrm{pCO}_{2}$, underway surface $\mathrm{pCO}_{2}$, and total alkalinity) from thirty-four WOCE cruises (Atlantic Ocean Sections A1E, A5, A9, A12/A21, A10, and A15; Pacific Ocean Sections S4P, P6E, P6C, P6W, P10, P13, P14C, P14Na, P14Nb, P16C, P17C, P17N, P17S/P16S, P16A/P17A, P17E/P19S, P19C, P21E, $P 21 \mathrm{~W}$, and P31; and Indian Ocean Sections I8S/19S, I9N, I8N/15E, I3, I7N, I1, I10, I5W/I4, and I2). CDIAC has received shore-based $\mathrm{CO}_{2}$-related analyses 
from five WOCE cruises (A9, A1E, P17S/P16S, P17C, and $\mathrm{P} 16 \mathrm{C}$ ). All data sets are being quality controlled. Seven data sets have been fully documented and are available from CDIAC:

- WOCE A12/A21 Sections in the South Atlantic Ocean, Weddell Sea, and Drake Passage (NDP045)

- WOCE A9 Section in the Atlantic Ocean (NDP051)

- WOCE Section AlE in the North Atlantic Ocean (NDP056)

- WOCE Sections P17S/P16S in the Pacific Ocean (NDP054)

- WOCE Section P16C in the Pacific Ocean (NDP060)

- WOCE Section P17C in the Pacific Ocean (NDP062)

- WOCE Section S4P in the South Pacific Ocean (NDP063)

CDIAC has received two datasets from NOAA/PMEL: surface underway $\mathrm{CO}_{2}$ measurements in Pacific and Indian Oceans during 1986 to 1989 and $\mathrm{CO}_{2}$ measurements in the Southwest Pacific Ocean during the NOAA/PMEL CGC-90 cruise. These datasets have been quality controlled and documented (NDP-047 and NDP-052). CDIAC has also developed a WWW home page that highlights and summarizes CDIAC's datamanagement support for the DOE Global Survey of $\mathrm{CO}_{2}$ in the Oceans (http://cdiac.esd.ornl.gov/oceans/ home.html).

\section{CARBON TRANSPORT IN THE BOTTOM} BOUNDARY LAYER

LOHRENZ, STEVEN, and ASPER, VERNON

UNIVERSITY OF SOUTHERN MISSISSIPPI

$\begin{array}{lr}\text { FY } 1996 & 50 \\ \text { FY } 1995 & 0 \\ \text { FY } 1994 & 135\end{array}$

Keywords: chlorophyll, fluorescence, carbon, sediment transport, benthic boundary layer, resuspension, carbon flux in the ocean

Objectives: To characterize distributions of chlorophyll fluorescence in relation to physical processes in the benthic boundary layer.

Approach: Multisensor fluorescence measurements will be made of photosynthetic pigments off Cape Hatteras.

Results to Date: Fluorescence variations in the benthic boundary layer have been initially characterized in the bottom $5 \mathrm{~m}$ of the water column at a $20-\mathrm{m}$ site near Duck, N.C., from July 24 to August 22, 1994. Estimates of pigment concentrations were variable with high maxima (greater than $100 \mathrm{mg} \mathrm{m}-3$ ). In some cases, variations were associated with wind and wave activity and possibly related to resuspension. Fluxes of chlorophyll and associated particulate organic carbon
(POC) were estimated as the product of concentration and current velocity. Episodic events contributed significantly to the flux of chlorophyll and associated POC at the site. The net cumulative flux over the bottom $4.4 \mathrm{~m}$ for the entire deployment period was $0.18 \mathrm{~kg}$ chlorophyll $\mathrm{m}^{-2}$ directed onshore and to the south. The results illustrate the potential for large lateral transport of organic matter on the shelf.

\section{MEASUREMENT OF DIC, ALKALINITY, AND pH ON WOCE/HP CRUISES MacKENZIE, FRED T., and WINN, CHRISTOPHER D. UNIVERSITY OF HAWAII FY 1996150 FY 1995175 FY 1994168}

Keywords: ocean, atmosphere, carbon cycle, WOCE Objectives: To contribute to the successful completion of the DOE-sponsored Global $\mathrm{CO}_{2}$ Survey of the Oceans.

Approach: Assistance will be provided in the collection and processing of survey measurements on the WOCE/HP program cruises.

Results to Date: We have collected carbon measurements on four WOCE cruises. These cruises include: P14 from the Aleutian Islands to Tarawa in the North Pacific, P31 from Tahiti to Samoa in the South Pacific, I8 from Sri Lanka to Western Australia in the Indian Ocean, and 17 from the Mauritius Islands to Oman in the Indian Ocean. We are also collecting measurements on 12 from Singapore to Mombassa in the Indian Ocean, which will complete the field work in the Indian Ocean.

In addition to the collection of the data sets described above, we are in the process of reducing the data collected on these cruises. We have completed the workup of the data from P14. A preliminary copy of these data has been passed to CDIAC. We have also processed all of the data from the underway system that Catherine Goyet provided for WOCE legs P17 and P14. When quality flags are assigned to this data set, we will transmit it to CDIAC. We have also completed the preliminary workup of the data from WOCE P31. These data have been included in the report to the State Department, and a copy of this data set has been forwarded to CDIAC.

In addition to data collection and work-up, we have used the survey data to advance our understanding of the carbon cycle in the oceans. The data that we collected on P14 have been used to develop some theories about the carbon cycle in the North Pacific. These data have been included in a recent article that has been submitted for publication in Deep-Sea Research. 


\section{ORGANIC CARBON BURIAL IN THE CAPE HATTERAS OCEAN MARGIN: RELATIONSHIP WITH MINERAL SURFACES \\ MAYER, LAWRENCE \\ UNIVERSITY OF MAINE \\ FX 199683 \\ FY 1995100 \\ FY 1994104}

Keywords: organic carbon, Chesapeake Bay

Objectives: To assess the role of mineral surface area in controlling organic carbon burial and to determine control(s) on replacement of terrigenous carbon by marine carbon in the coastal zone.

Approach: Control of organic carbon burial by mineral-surface adsorption will be assessed in the Hatteras region by measuring organic carbon-mineral surface-area relationships in sediments collected from each of the sedimentological provinces. Previous work has shown that organic carbon burial may exceed that predicted by this control in slope or estuarine regions, a possibility that can be tested by this surface-area normalization. The extent of replacement of adsorbed coatings of terrigenous organic carbon by marine organic matter will be tested by measurements of organic carbon, surface area, stable isotopes, and markers of marine organic-matter input in estuarine sediments. These measurements will be supplemented by experimental work examining the role of various environmental parameters in effecting this replacement.

Results to Date: The first year of this project has concentrated on obtaining samples for analysis and setting up for the experimental phase of the project. We have obtained sediment samples from the continental slope near Cape Hatteras and throughout Chesapeake Bay. We have instituted a seasonal sampling program for suspended particulate matter (SPM) from the Susquehanna River and are about half-way through this series. Preliminary results show the riverine SPM to have OC:SFA ratios above the monolayer-equivalent (ME) level described by Mayer. Slope samples include many examples of sediment at and above this ME level. A mesocosm experiment to examine the replacement of terrigenous with marine organic matter on mineral surfaces is being prepared. To date, we have grown and harvested a large amount of cultured phytoplankton with intensive ${ }^{13} \mathrm{C}$ labeling. We have obtained and established populations of a polychaete, Abarenicola marina, that will be used to test for macrofaunal influence on the replacement reaction.

\section{CARBON DIOXIDE IN THE OCEANS \\ MILLERO, FRANK J. \\ UNIVERSITY OF MIAMI \\ FY 1996100 \\ FY 1995200 \\ FY 1994194}

Keywords: carbon dioxide, Indian Ocean

Objectives: To conduct $\mathrm{CO}_{2}$ measurements in the oceans.

Approach: The input of $\mathrm{CO}_{2}$ in the oceans will be described.

Results to Date: During the past year, we have been measuring the components of the carbonate system as part of the Joint Global Ocean Flux Study (JGOFS) of the World Ocean Circulation Experiment (WOCE). The carbonate system was studied by measuring the partial pressure of $\mathrm{CO}_{2}$ in the atmosphere and surface waters, and the total alkalinity (TA) and total carbon dioxide $\left(\mathrm{TCO}_{2}\right)$ on all the waters collected during the cruise (surface to deep). During the past year, we have measured the carbonate parameters in the Indian Ocean (Leg I3) from Freemantle, Australia, to Port Louis, Mauritius. More than 3000 measurements were made on the cruise. Measurement of TA and $\mathrm{TCO}_{2}$ were made on 65 samples of certified reference material (CRM batch 23 and 30). The results' reproducibility was $\pm 1.3 \mathrm{mmol} \mathrm{kg}$ in TA and $\pm 1.2 \mathrm{mmol} \mathrm{kg-1} \mathrm{in}$ $\mathrm{TCO}_{2}$, and 0.004 in $\mathrm{pH}$ with the titration system. Measurements on 74 CRMs of $\mathrm{TCO}_{2}$ by coulometry gave a reproducibility of $\pm 0.9 \mathrm{mmol} \mathrm{kg}^{-1}$ and agree with the standard value to $\pm 2 \mathrm{mmol}^{-1}$. We also provide a titration system for all the other carbonate chemists making measurements in the Indian Ocean. During these cruises, more than 800 measurements were made on CRMs with these systems. The standard error of all the measurements was $\pm 4 \mathrm{mmol} \mathrm{kg}{ }^{-1}$ in TA, $\pm 5 \mathrm{mmol} \mathrm{kg}^{-1}$ in $\mathrm{TCO}_{2}$, and \pm 0.007 in $\mathrm{pH}$. These measurements of the carbonate system in the Indian Ocean will provide a benchmark to examine the changes in the $\mathrm{CO}_{2}$ system in the future and be useful in validating ocean atmospheric models for the changes in the carbonate system in the future.

\section{HYDROGRAPHY AND REMOTE SENSING PIETRAFESA, LEONARD J.;

Keywords: sediment dynamics, benthic boundary layer, ocean circulation, hydrography

Objectives: To conduct water-property sampling during the OMP mooring cruises from a shipboard CTD/Rosette system; to acquire and process satellite-derived, sea-surface data (thermal and visible) for 
the OMP coastal area; and to provide real-time distribution and supporting analyses.

Approach: Sampling of seawater properties will be done by means of a CTD/Rosette sampler outfitted with additional sensors for measuring oxygen, fluorescence, light transmission, underwater irradiance, and $\mathrm{pH}$ and with an altimeter. The Rosette discrete-depth water sampling will be for those physical, biological, and chemical analyses that can be taken sufficiently rapidly (for example, nutrients, oxygen, salinity, chlorophyll, particulate organic carbon, total suspended matter, $\mathrm{pCO}_{2}, \mathrm{pH}$, and phytoplankton).

North Carolina State University is currently operating a PC-based HPRT AVHRR collection system that consists of a receiving system, a workstation for image ingestion and processing, and suitable massstorage devices for archiving imagery. This state-of-the-art package gives us the ability to process satellite visible, thermal, and color imagery collected by polar-orbiting satellites. The observed parameters are: contemporary sea-surface temperature (SST) and sea-surface reflected and upwelling radiance that can be processed to a vegetation or suspended-sediment index, as well as historical ocean-color data collected via the Coastal Zone Color Scanner from 1978 to 1986. Real-time SST fields will be made available to researchers and will be archived to aid in the analyses. Results to Date: We have outfitted the CTD/Rosette system. We have arranged with NOAA to share a cruise with them (SABRE) on the Oregon II to supplement the hydrography for the initial period. The SST historical archive 1985 to the present (visible data: 1993 to the present) is already available for analysis.

\section{ASSESSMENT OF PRIMARY PRODUCTION AND OPTICAL VARIABILITY IN SHELF AND SLOPE WATERS NEAR CAPE HATTERAS \\ REDALJE, DONALD, and LOHRENZ, STEVEN UNIVERSITY OF SOUTHERN MISSISSIPPI \\ FY 1996116 \\ FY 1995143

FY 19940

Keywords: primary production, shelf waters, Ocean Margins Program

Objectives: To investigate the spatial and temporal variability in spectral absorption and attenuation, in photosynthesis-irradiance parameters, and in primary production in shelf and slope waters off of Cape Hatteras, N.C.

Approach: We are characterizing the inherent optical properties (i.e., spectral absorption and transmission of irradiance) of the various water masses and bio-optical provinces found in the study region. In addition, we are using the measured optical properties to determine the contribution of individual types of seawater constituents (i.e., dissolved matter, pigments, detritus, and water) to the total absorption and attenuation of irradiance. We will use this information to refine and evaluate various algorithms used to estimate the environmental concentrations of particles and chlorophyll in coastal waters. We are also measuring the spectral irradiance and radiance fields in our coastal water study region. In addition, we are measuring primary production using simulated in situ incubations and through the determination of photosynthesisirradiance (P-I) parameters and the use of current P-Ibased production models. We are also measuring the carbon-specific growth rates of the phytoplankton community and for pigment-taxonomic groups present in the study region. Production and irradiance measurements will then be used to help use evaluate and refine production models for coastal waters.

Results to Date: To date, we have acquired the necessary instruments and developed the protocols that will be used to help us meet the objectives of the project. We have conducted one research cruise in the coastal waters off Cape Hatteras (March 11-21, 1996) and are in the process of preparing for a second cruise to the study site (July 17-27, 1996). During the first cruise, we were able to survey the study region for surface variability in optical and biological properties and found significant spatial variability in our measured parameters. We also followed a particular parcel of water and made periodic measurements of the optical and biological properties every 3 to 4 days. Again, we found within this parcel of water a temporal variability in the parameters measured. Analyses of many of the samples obtained during this first cruise are still in progress, so we cannot make any complete assessments of results at this time.

\section{VARIABILITY OF CARBON-SYSTEM PARAMETERS IN COASTAL WATERS OF THE MID-ATLANTIC BIGHT OFF NEW JERSEY: A LINK TO THE OCEAN MARGINS PROGRAM \\ REIMERS, CLARE, and BOEHME, SUSAN RUTGERS, THE STATE UNIVERSITY OF NEW JERSEY FY 199680 FY 19950 FY 19940}

Keywords: coastal ocean, carbon fluxes in the ocean Objectives: To gain an understanding of the processes determining the exchange of carbon dioxide between the coastal ocean and the atmosphere by conducting and modeling a monthly time-series of geochemical measurements along a $32-\mathrm{km}$ transect of the New Jersey inner continental shelf.

Approach: Monthly measurements of $\mathrm{TCO}_{2}$, alkalinity, dissolved oxygen, DOC, nutrients, salinity, and temperature are being made in the surface and bottom 
waters of seven stations with water depths of 7 to $32 \mathrm{~m}$. A discrete sampler to make direct fugacity $\left(\mathrm{fCO}_{2}\right)$ measurements at a subset of stations is used to verify calculated $\mathrm{fCO}_{2}$ values and to establish the atmospheric $\mathrm{fCO}_{2}$ signal. The $\mathrm{fCO}_{2}$ determinations are being used in conjunction with the atmospheric measurements and continuous wind speed data collected at Rutgers Marine Field Station to calculate air-sea $\mathrm{CO}_{2}$ fluxes at each of the seven stations and an estimate of the average yearly flux.

During 1997, monthly transect cruises will be augmented with 2 to 3 across-shelf cruises that will employ an underway system for measuring $\mathrm{fCO}_{2}$. Continuous oxygen data from SEABIRD Seacat 25 CTDs installed at two permanent observatories on the shelf (LEO-15) and linked to the shore through a buried fiberoptic cable will also be analyzed.

Results to Date: $\mathrm{fCO}_{2}$ calculated from surface water samples collected on 19 cruises between April 1994 and September 1995 range from 220 to $660 \mathrm{~atm}$. Fluxes calculated from local wind speed and $\mathrm{fCO}_{2}$ data suggest the inner shelf acts as a source of atmospheric $\mathrm{CO}_{2}$ from June to October, but these fluxes are offset completely by large fluxes into the surface waters during the late winter to early spring.

\section{EXPORT AND MOLECULAR-LEVEL CHARACTERIZATION OF DISSOLVED AND PARTICULATE ORGANIC MATTER FROM THE MID-ATLANTIC BIGHT \\ REPETA, DANIEL; EGLINTON, TIMOTHY; and CHEN, ROBERT \\ WOODS HOLE OCEANOGRAPHIC INSTITUTION and \\ UNIVERSITY OF MASSACHUSETTS \\ FY 1996280 \\ FY 1995260 \\ FY 1994378}

Keywords: Bight particulate organic matter, seawater, Mid-Atlantic

Objectives: To quantify the along-shelf production, transport, and annual export of dissolved organic carbon (DOC) from the Mid-Atlantic Bight (MAB) and to characterize dissolved organic matter (DOM) at the molecular level to identify the sources and relative amounts of reactive and nonreactive DOM.

Approach: Carbon budgets will be constructed from data collected on two spring cruises (1994 and 1996) and a summer cruise (1996). We sample five to six transects per survey, each running perpendicular to the coast to just seaward of the shelf/slope break ( 20 to $2000 \mathrm{~m}$ ). Transects are evenly spaced between Cape Cod and Cape Hatteras, and stations are occupied every 5 to 10 miles. Vertical sampling resolution is 5 to $10 \mathrm{~m}$ on the shelf and 100 to $300 \mathrm{~m}$ in deep water. We integrate continuous synoptic measurements of dissolved fluorescence and vertical profiles made with a CTD fluorometer (set to dissolved fluorescence) with discrete measurements of POC, PON, and DOC. These data are calibrated and smoothed, and the flux is modeled with physical oceanographic and water-masstransport data.

Molecular characterizations are made on the colloidal fraction sampled by ultrafiltration to identify major sources and potential reactivity of DOM. Samples are analyzed for $\mathrm{C} / \mathrm{N} / \mathrm{S}$ ratio by elemental analyzer, the composition of major biochemicals (carbohydrates, lipids, and proteins) by NMR and CNMR, and biomarkers (including stable isotopes) by GC/MS and GC-IR/MS. Results from these analyses are used to define end-member DOM characteristics and to infer DOM properties throughout the MAB. We combine these data with optical (fluorescence), hydrographic (temperature and salinity), and concentration (DOC) measurements to calculate the relative contributions of different sources and the quality of the organic matter from each.

Results to Date: We have developed analytical methods for isolating and identifying dissolved polysaccharides in seawater, including appropriate methods for desalting DOM, size fractionation by gel-permeation chromatography, and 2-D NMR. Results indicate that total dissolved carbon in surface water increases threefold between Cape Cod and Cape Hatteras, with the increase correlating well with a freshening of surface water because of riverine discharges onto the shelf. The DOM composition, however, does not change between samples, suggesting that the added DOM is not terrestrial in origin, although it appears tied to the input of fresh water. The DOM we have characterized from the shelf is nearly indistinguishable from open-ocean DOM with major sugars like galactose, glucose, xylose, fructose, mannose, rhamnose, and arabinose that are highly acetylated (monosaccharide/acetate ratio of $5 / 2$ ), suggesting a common structure and source. Culture experiments show that the DOM can be directly biosynthesized by marine algae or bacteria and is very resistent to degradation.

\section{THE PRODUCTION OF COLLOIDS IN THE BENTHIC BOUNDARY LAYER AND PARTICLE-PARTICLE INTERACTIONS \\ SANTSCHI, PETER; HONEYMAN, BRUCE; and BASKARAN, MAHALINGAM \\ TEXAS A\&M UNIVERSITY, GALVESTON, and COLORADO SCHOOL OF MINES \\ FY 1996100 \\ FY 199584 \\ FY 1994262}

Keywords: colloids, thorium isotopes, turnover rates, benthic boundary layer, organic particulate matter 
Objectives: To gain a better understanding of (1) the pool size of organic matter in the water column, in particular as it pertains to the region of the benthic boundary layer (BBL); (2) the ages and turnover rates of organic matter in the water column, with special emphasis on contrasting the BBL with the rest of the water column; (3) the sources of organic matter in the water column, with special emphasis on the BBL and water circulation in the Middle Atlantic Bight; and (4) the isotopes as tracers of scavenging of particulate matter in the water column, with special emphasis on boundary-exchange mechanisms.

Approach: Hydrographic surveys will be carried out with CTD, fluorometry, and transmissometry casts; sediment trap samples and large-volume water samples will be collected for radiochemical, chemical, and biochemical analyses; and samples will be analyzed in the lab.

Results to Date: Field experiments were carried out to determine the boundary stress and friction velocity at $800 \mathrm{~m}$ water depth at Cape Hatteras, and lab experiments were conducted to study the diffusion and resuspension of colloids as a function of shear stress. In some cases (such as the calibration experiments described in Guo and Santschi and the biomarker analysis of MAB samples), further sample and data analysis was necessary and was completed during this funding year.

\section{GLOBAL SURVEY OF CARBON DIOXIDE IN THE OCEAN \\ SARMIENTO, JORGE, and KEY, ROBERT PRINCETON UNIVERSITY \\ FY 1996110 \\ FY 1995200 \\ FY 1994198}

Keywords: total alkalinity, total $\mathrm{CO}_{2}, \mathrm{pCO}_{2}$, Indian Ocean

Objectives: To obtain total carbon dioxide $\left(\mathrm{TCO}_{2}\right)$ and total alkalinity (TA) measurements throughout the water column and underway $\mathrm{CO}_{2}$ partial pressure $\left(\mathrm{pCO}_{2}\right)$ measurements in the surface water and atmosphere along WOCE/WHP legs in the Pacific and Indian Oceans.

Approach: $\mathrm{TCO}_{2}$ and TA measurements are made in full accordance with accepted $\mathrm{DOE}$ procedures given in the DOE $\mathrm{CO}_{2}$ handbook. $\mathrm{TCO}_{2}$ is measured with a commercial coulometer and automated samplestripping system (SOMMA) for WOCE/WHP legs P10, I9N, and I10. TA is measured with a closed-cell titration system for WOCE/WHP legs P16A/P17A, $\mathrm{P} 10,19 \mathrm{~N}$, and $\mathrm{I10}$. Underway $\mathrm{pCO}_{2}$ measurements are made for WOCE/WHP legs P17E/P19S, P10, and all nine of the WOCE Indian Ocean Survey legs with an automated system with a nondispersive infrared analyzer and disk equilibrator system.
Results to Date: In November 1995 we completed WOCE leg 110 with nearly 1000 discrete TA and $\mathrm{TCO}_{2}$ samples analyzed; underway $\mathrm{pCO}_{2}$ measurements were made along the entire cruise track. At the end of leg I2, the underway $\mathrm{pCO}_{2}$ system was returned to Princeton from Cape Town, South Africa, for postcruise calibration of the gases and sensors.

In total, the Princeton underway $\mathrm{pCO}_{2}$ system made nearly 250,000 measurements of surface water and atmospheric $\mathrm{CO}_{2}$. The overall data quality for the entire expedition is excellent. The postcruise calibrations on the underway system have been completed, and the $\mathrm{CO}_{2}$ data have been calculated to the $\mathrm{xCO}_{2}$ stage. Final calculations require accurate sea-surface temperature and salinity data. These data were recorded by the Knorr's IMET system, but are still in the process of an extensive cleanup and quality-control check as well as calibration to the surface CTD values from all the legs. We have begun quality control and calibration on the $\mathrm{TCO}_{2}$ and TA samples collected on legs $19 \mathrm{~N}$ and I10, but significant work remains because postcruise calibration of the instruments is still under way.

\section{HIGH-RESOLUTION SAMPLING OF CARBON FLUX THROUGH BACTERIVOROUS AND HERBIVOROUS PROTISTS IN CONTINENTAL SHELF WATERS OFF CAPE HATTERAS SHERR, BARRY \\ OREGON STATE UNIVERSITY FY 1996127 \\ FY $1995 \quad 72$ \\ FY 199478}

Keywords: bacteria, protists, food webs, carbon flow, continental shelf, microbes, Mid-Atlantic Bight

Objectives: To determine the spatial and temporal patterns of (1) grazing mortality caused by heterotrophic protists, for bacteria in continental shelf waters off Cape Hatteras, N.C., and (2) number of active bacterial cells with respect to top-down controls (grazing mortality) and bottom-up controls (phytoplankton standing stocks and rates of primary production).

Approach: Protist grazing rates will be determined via rate of uptake of fluorescently labeled bacteria. The number of active bacteria will be determined by the number of bacteria able to reduce a fluorogenic redox compound (CTC) in the electron-transport system and by the number of bacteria that have visible nucleoids (DNA-containing regions). These data will be combined with data from other projects focused on the biology of the continental shelf to construct a model of carbon flow through shelf food webs.

Results to Date: Two OMP field cruises were completed. We have finished the initial analysis of the data 
collected in the first cruise (3/96) and are now working on samples collected during the second cruise (7/96). Preliminary analysis of the data from the 3/96 cruise suggests that the number of active bacteria (metabolically active with visible nucleoids) was highest at nearshore stations and was positively related to standing stock of phytoplankton. Rates of bacterivory were also highest at nearshore stations and lowest at shelfslope stations. The proportion of metabolically active bacteria was higher ( 20 to $50 \%$ active) among particle-attached bacteria than that among freely suspended bacteria ( 2 to $15 \%$ active).

\section{OCEAN-SURFACE-LAYER DYNAMICS SKYLLINGSTAD, ERIC, and DENBO, DONALD PACIFIC NORTHWEST NATIONAL LABORATORY

*Funding consolidated with that of William Asher. Keywords: surface-layer dynamics, Pacific warm pool, El Niño/Southern Oscillation, sea-surface temperature, mixed-layer entrainment, shear-generated turbulence, thermocline

Objectives: To determine, by numerical experiments on surface-layer dynamics, if a more detailed treatment of surface-layer processes will substantially change climate projections based on simple mixed-layer models.

Approach: Key mixing processes in the ocean surface layer will be simulated with a 3-D ocean large-eddysimulation model. Areas of the world ocean that are thought to play a significant role in ocean-atmosphere coupling will be emphasized. The model will be compared to observed turbulent-flux estimates whenever possible.

Results to Date: Data acquired during the Tropical Ocean Global Atmosphere COARE program were used to examine the role of turbulent mixing in the climatically important Pacific warm pool. The warm pool is an important component in the $\mathrm{El} \mathrm{Nino/Southern}$ Oscillation climate system. Determining what factors control the sea-surface temperature (SST) in this region is important for accurate prediction of the coupled climate. We used the large-eddy-simulation model to reproduce the turbulent boundary layer and showed that the SST is controlled by the surface heat flux and the exchange of water between the mixed layer and the thermocline. Momentum transierred to the water by westerly wind bursts is important for mixed-layer entrainment because of shear-generated turbulence at the top of the thermocline. We found that exchange rates at the mixed-layer base were as high as $300 \mathrm{~W} / \mathrm{m}^{2}$ when strong winds were present.

\author{
NATIONAL OCEAN SCIENCE \\ PARTNERSHIPS: IMPROVING SCIENTIFIC, \\ TECHNOLOGICAL, AND EDUCATIONAL \\ LINKAGES AMONG ACADEMIC \\ INSTITUTIONS, MARINE INDUSTRIES, \\ AND FEDERAL AGENCIES \\ SPINRAD, RICHARD W. \\ CONSORTIUM FOR OCEANOGRAPHIC \\ RESEARCH AND EDUCATION \\ FY 199650 \\ FY 199550 \\ FY 19940
}

Keywords: oceanography, program management

Objectives: To develop recommendations and implementation plans for new partnership opportunities in ocean science (including coastal research) consistent with the recommendations of the Ocean Studies Board of the National Academy of Science.

Approach: The key issues in the oceanographic research community are identified and then couched in terms of opportunities for developing new partnerships among rcsearchers in government, academia, and industry. The findings are presented to the executive and legislative bodies of government with an emphasis on definition of how to perform the research better, rather than on what research should be performed.

Results to Date: Four working groups (with participants from government agencies, academia, and industry) were convened. They focused on the role of oceanographic research in the areas of national security, economic development, quality of life, and education and communication. A clear pattern emerged in terms of the need to focus on partnership development in terms of data, resources, and education. Specific opportunities were defined for improving access to and quality of oceanographic data. Partnerships that would allow access to classified data holdings were also identified. Partnership opportunities specific to data were identified for formalizing long-term observational programs and for quality assurance of oceanographic data entered into globally accessible databases. One partnership opportunity identified was personnelexchange programs and internships. Because much of the expertise in the oceanographic community is widely, but thinly, dispersed there, a mechanism is needed for allowing (and encouraging) researchers from labs in government, academia, and the private sector to participate in flexible exchange programs easily and without penalty. These results are being synthesized into a comprehensive plan of action for the oceanographic community. 


\section{MECHANISMS OF DISSOLVED ORGANIC CARBON CYCLING IN AN OCEAN MARGIN STROM, SUZANNE, and DAGG, MICHAEL WESTERN WASHINGTON UNIVERSITY and LUMCON \\ FY 199635 \\ FY 1995107 \\ FY 1994113}

Keywords: dissolved organic carbon, phytoplankton, protozoa, copepods, carbohydrates

Objectives: To measure the amounts and types of dissolved organic carbon produced by marine planktonic organisms, particularly phytoplankton, protozoa, and copepods.

Approach: Laboratory experiments using simplified versions of natural planktonic communities have been conducted. Experiments are performed in artificial seawater to minimize background DOC concentrations. Production of DOC and dissolved carbohydrates is measured directly, bacterial growth is also measured as an indicator of DOC production.

Results to Date: DOC production by grazers, especially protozoans, was found to greatly outweigh phytoplankton DOC production. Phytoplankton DOC production averaged 3 to $7 \%$ of algal carbon content per day. Grazer DOC production averaged 16 to $37 \%$ of algal carbon content during an ingestion event. DOC was relatively carbohydrate-rich; dissolved carbohydrates comprised 22 to $30 \%$ of total DOC. Extrapolating to the ocean, grazer DOC production should be 4 to 6 times greater than algal $\mathrm{DOC}$ production under typical euphotic zone conditions.

\section{MEASUREMENTS OF TOTAL $\mathrm{CO}_{2}$ CONCENTRATION, PARTIAL PRESSURE OF $\mathrm{CO}_{2}$, AND ALKALINITY IN SEAWATER DURING WOCE EXPEDITIONS IN THE SOUTH PACIFIC OCEAN TAKAHASHI, TARO \\ LAMONT-DOHERTY EARTH OBSERVATORY FY 1996290 \\ FY 199583 \\ FY 1994178}

Keywords: Pacific Ocean, total $\mathrm{CO}_{2}$ Objectives: To determine the total concentration and partial pressure of $\mathrm{CO}_{2}$ and alkalinity in seawater during WOCE expeditions in the South Pacific Ocean (WOCE lines P16S, P17S and E, and P19).

Approach: The concentration of total $\mathrm{CO}_{2}$ in discrete samples from throughout the water column is determined with a semiautomated coulometric-analysis system designed and constructed at LDEO. A gaschromatograph-based system, also designed and constructed at LDEO, has been used to measure the partial pressure of $\mathrm{CO}_{2}$ in the same water samples. Alkalinity concentrations in these same water samples have been computed from the two measured values in conjunction with measurements of salinity and the concentration of the nutrient salts made by others.

Results to Date: All three north-south sections $\left(17^{\circ} \mathrm{S}\right.$ to $62^{\circ} \mathrm{S}$ at $150.5^{\circ} \mathrm{W}, 6^{\circ} \mathrm{S}$ to $63^{\circ} \mathrm{S}$ at $135^{\circ} \mathrm{W}$, and $13^{\circ} \mathrm{N}$ to $68^{\circ} \mathrm{S}$ at $88^{\circ} \mathrm{W}$ ) show strong subsurface maxima for total $\mathrm{CO}_{2}$ concentration to the north of approximately $48^{\circ} \mathrm{S}\left(150.5^{\circ}\right.$ and $\left.135^{\circ} \mathrm{W}\right)$ or $56^{\circ} \mathrm{S}$ $\left(88^{\circ} \mathrm{W}\right.$ ). The east-west section along $53^{\circ} \mathrm{S}$ (line P17E) shows a similar maximum in total $\mathrm{CO}_{2}$ at a depth of approximately $3000 \mathrm{~m}$ and a maximum in partial pressure of $\mathrm{CO}_{2}$ at approximately $1700 \mathrm{~m}$. Both of these maxima strengthen to the east, having their highest values on the continental slope of South America. This high-total- $\mathrm{CO}_{2}$ water appears to be related to similar water observed during an earlier expedition at much shallower depths within the Drake Passage, where it shoals to the south to depths of $200 \mathrm{~m}$ or less. In the midlatitudes, the surface waters east of about $130^{\circ} \mathrm{W}$ are sources to the atmosphere, while those waters west of this longitude are sinks for atmospheric $\mathrm{CO}_{2}$. On the average, these two areas appear to cancel each other, resulting in the temperate subtropical South Pacific Ocean being a neutral reservoir for atmospheric $\mathrm{CO}_{2}$ during the season of these expeditions (austral summer).

\section{CONTRIBUTION OF ZOOPLANKTON TO THE BIOMASS, COMPOSITION, AND FATE OF LIVING AND DETRITAL POC ON THE HATTERAS OCEAN MARGIN \\ VERITY, PETER, and PAFFENHOFER, G.-A. SKIDAWAY INSTITUTE OF OCEANOGRAPHY FY 1996293 \\ FY 1995200 \\ FY 1994274}

Keywords: zooplankton, detritus, phytoplankton, MidAtlantic Bight

Objectives: To determine the contribution of protoand metazooplankton as pools, consumers, and producers of POC.

Approach: We are combining distributional studies with time-series incubations, using sophisticated technology, including imaging cytometry and optical counters, to determine the carbon biomass of phytoand zooplankton, herbivory by zooplankton, and related processes. Additionally, we have developed a method to quantify detrital carbon, which is often a larger fraction of total POC than is plankton carton. An essential component of the OMP field program is to determine the production and fate of $P O C$ at Cape Hatteras.

Results to Date: We developed and tested an optical zooplankton counter and fully enhanced our colorimage-analysis system. We evaluated a solid-phase enzyme-linked immunospot assay to quantify predation 
by metazoan zooplankton on protozoans and improved methods to determine ingestion and growth rates of salps (and accompanying pellet-production rates) under conditions that very closely resemble their environment. The image-analyzer data provided insights on basic ecosystem parameters relevant to carbon flux from the continental ocean to the deep ocean. We are currently quantifying samples and analyzing the data collected during three cruises conducted in 1996.

\section{OCEAN MARGINS PROGRAM: COORDINATION VERITY, PETER SKIDAWAY INSTITUTE OF OCEANOGRAPHY FY 199640 \\ FY 199567 \\ FY $1994 \quad 0$}

Keywords: Ocean Margins Program, program management, planning, meetings

Objectives: To provide coordination services for the Ocean Margins Program (OMP), including preparation, quality control, and formatting of OMP documents; setup, maintenance, and improvement of OMP electronic bulletin boards; and planning and implementation of the annual OMP meetings of principal investigators.

Approach: During the planning, articulation, and justification of the OMP, numerous coordination services are provided. These services include proposal reviews, technical-merit panel reviews, programmatic reviews, steering-committee meetings, principalinvestigator meetings, cruise logistical arrangements, document preparation and publication, computergraphics services, and facilitation of electronic communication.

Results to Date: New Research Summaries documents were produced. Two electronic bulletin boards were set up: one for all OMP PIs and a separate one for OMP program managers and steering-committee members. A master address list was established, continuously updated, and distributed to the OMP community. An annual PI meeting was hosted in Savannah, Georgia, and partially supported by SkIO, which is located on the adjacent coast. The attendees were primarily OMP PIs, with a few program managers from other federal agencies with related marine interests. Coordination services included convention details (meeting rooms, A/ $\mathrm{V}$ equipment, break services, poster arrangements, etc.); organized social functions (receptions and dinners); and transportation services among hotels, functions, and Skidaway Institute of Oceanography.
INORGANIC CARBON MEASUREMENTS FOR THE WORLD OCEAN CIRCULATION EXPERIMENT-WORLD HYDROGRAPHIC PROGRAM

WALLACE, DOUGLAS W. R., and JOHNSON, K. M.

BROOKHAVEN NATIONAL LABORATORY

FY 1996500

FY 1995613

FY 1994659

Keywords: transient tracers, World Ocean Circulation Experiment, CFCs, dissolved inorganic carbon Objectives: To collect, report, and interpret a global data set for dissolved inorganic carbon in the oceans. Approach: Water samples are being analyzed at sea during research cruises of the World Ocean Circulation Experiment (WOCE). Samples are analyzed for at least two parameters of the inorganic carbon system in seawater. The resulting data set is being interpreted with inverse-mode calculations to determine the meridional transport of $\mathrm{CO}_{2}$ by the ocean circulation. This analysis seeks to identify and quantify global sources and sinks of $\mathrm{CO}_{2}$ for the atmosphere. Increases in the anthropogenic $\mathrm{CO}_{2}$ content of the ocean are being quantified through comparisons with historical data and correlations with transient tracers, such as CFCs. BNL scientists will provide technical assistance; instrument calibration services; and new, automated analytical instrumentation to national and international scientific investigators involved with oceanic $\mathrm{CO}_{2}$ data collection. Coordination and guidance of DOE's Global Survey of $\mathrm{CO}_{2}$ in the Oceans will be provided.

Results to Date: A vast data set of unprecedented extent and accuracy has been collected from the Pacific, South Atlantic, and Indian oceans in collaboration with other investigators involved in DOE's Global Survey of $\mathrm{CO}_{2}$ in the Oceans. This data set, acquired since 1990 , will be at least five times larger than the combined data set collected during the past 25 years. Most recently, BNL scientists provided the equipment for measurements of total dissolved inorganic carbon by several teams of investigators during a 14-month expedition to the Indian Ocean. The data are being archived at the Carbon Dioxide Information Analysis Center for use by other investigators and carbon-cycle modelers. Analysis of data collected from the South Atlantic Ocean with a new, multiparameterregression technique has demonstrated that the uptake of anthropogenic $\mathrm{CO}_{2}$ by the oceans can be identified and quantified from repeated ocean measurements made on approximately decadal intervals.

Inventories of anthropogenic $\mathrm{CO}_{z}$ in the oceans have been quantified and compared to distributions and inventories of transient tracers. Inverse model calculations have shown that there is a large, net, 
southwards transport of dissolved $\mathrm{CO}_{2}$ within the South Atlantic Ocean at approx. $19^{\circ} \mathrm{S}$. This implies that the Arctic Ocean and the North Atlantic Ocean form a large, net sink for atmospheric $\mathrm{CO}_{2}$. These interpretations of ocean data, together with atmospheric $\mathrm{CO}_{2}$ data and models, will constrain the magnitude of terrestrial $\mathrm{CO}_{2}$ sinks, and help identify feedbacks that might affect future atmospheric $\mathrm{CO}_{2}$.

\section{MARINE BIOGEOCHEMISTRY \\ WALLACE, DOUGLAS W. R., and LAROCHE, JULIE \\ BROOKHAVEN NATIONAL LABORATORY \\ FY 1996278 \\ FY 1995249 \\ FY 1994194}

Keywords: coastal biogeochemistry, carbon cycle

Objectives: To develop an understanding of the fundamental processes controlling coastal biogeochemical processes (particularly the carbon cycle) over continental margins to assess the potential impacts of anthropogenic (and natural) perturbations.

Approach: The program uses biological and chemical data measured during research cruises, as well as from moored instruments and laboratory experiments, to infer mechanisms and rates of key carbon-cycling processes, such as air-sea gas exchange, biocalcification, photosynthesis, and respiration. Parameters that will be measured include inorganic carbon (particulate and dissolved), nutrients, and dissolved oxygen. Particular attention will be focused on developing new approaches to studying the environmental regulation of calcification by marine algae. In the near-term, measurements will be made as part of the Ocean Margins Program field experiment over the continental shelf and slope north of Cape Hatteras.

Results to Date: Measurements have been made during a series of cruises to the Cape Hatteras region, as well as during one along-shore cruise from Cape Cod to Cape Hatteras. Oxygen and $\mathrm{CO}_{2}$ data have been used to infer rates of primary productivity and watermass origins as well as to calibrate in situ dissolvedgas sensors. The chemical data have been combined with hydrographic data in an online database and made available to other investigators. Investigations have begun into the use of multiple-linear-regression approaches for the estimation of parameters that cannot be measured directly in situ from moored instruments (e.g., total dissolved inorganic carbon) using proxy (predictor) variables that can be measured in situ (e.g., temperature, salinity, oxygen, and $\mathrm{pCO}_{2}$ ). These relationships will be used during the Ocean Margins Program field experiment to examine variations in $\mathrm{CO}_{2}$ over the continental shelf on diurnal to seasonal time scales.

\section{STUDIES OF CURRENT AND BOTTOM BOUNDARY LAYERS IN THE OMP REGION \\ WEATHERLY, GEORGES \\ FLORIDA STATE UNIVERSITY \\ FY 1996375 \\ FY 1995775 \\ FY 199480}

Keywords: current meters, water budget, salt budget, bottom boundary layer, moored sensing systems

Objectives: To make time-series measurements in the water column to aid in estimating the water and salt budget in the OMP region and to conduct computermodel studies of, as well as make actual observations of, the bottom boundary layer (BBL) on the continental slope of the OMP region.

Approach: Moored arrays of instruments are being deployed to measure currents, temperature, and salinity in the OMP region to collect sufficient data to estimate the mass and salt budgets of the OMP region. The Sandia Ocean Model System (SOMS) primitive equation model is being used to run computer simulations of the BBL on the continental slope in the OMP region. This model has proven to be useful for studying the BBL in other regions of the ocean. An instrument is being deployed to make actual measurements in the BBL.

Results to Date: Forty moorings were emplaced in the OMP region. Two of these moorings were recovered prematurely because of boat traffic; all but two of the remaining 38 were recovered in a subsequent cruise in May 1996. The data recovered is currently being processed. On a third cruise in June 1996, 31 moorings were redeployed in the OMP region. These mooring are scheduled to be recovered in October 1996.

The SOMS model has been thoroughly checked and updated to simulate the OMP slope BBL. Its simulations indicate that cross-isobath variations (e.g., bottom slope changes and density fronts) lead to BBL detachment and consequent export of $\mathrm{BBL}$ waters to the ocean interior.

\section{MEASUREMENTS OF SURFACE-OCEAN \\ CARBON DIOXIDE PARTIAL PRESSURE DURING WOCE WEISS, RAY F. \\ SCRIPPS INSTITUTION OF OCEANOGRAPHY \\ FY 199640 \\ FY 199581 \\ FY 199475}

Keywords: oceans, nitrous oxide, WOCE, oceanic uptake of carbon dioxide, oceanic uptake of atmospheric nitrous oxide

Objectives: To assess the spatial and temporal roles of the surface oceans with respect to the uptake and 
release of atmospheric carbon dioxide and nitrous oxide by underway measurements aboard WOCE vessels of the partial pressures of these gases in the ocean and in the atmosphere.

Approach: Shipboard measurements of $\mathrm{pCO}_{2}$ and $\mathrm{pN}_{2} \mathrm{O}$ in surface waters and in the atmosphere are being made on all nine legs of the 1994 to 1996 U.S. WOCE Indian Ocean Expedition. The measurements are made with an automated, high-precision, gaschromatographic system. $\mathrm{CO}_{2}$ is measured by flame ionization detection after quantitative reduction to methane with nickel and palladium catalysts, and $\mathrm{N}_{2} \mathrm{O}$ is measured by electron-capture detection. Surface waters are measured with a two-stage equilibration chamber by equilibrating the measured gas phase with continuously pumped seawater. The system operates continuously, measuring both the surface ocean and the atmosphere twice each hour.

Results to Date: Eight legs of the U.S. WOCE Indian Ocean Expedition have been completed, and seven legs have undergone preliminary, shore-based data processing. Although these new observations are still not adequate to resolve in detail both the spatial and seasonal characteristics of $\mathrm{CO}_{2}$ and $\mathrm{N}_{2} \mathrm{O}$ in these vast regions, the relationships that were observed are consistent with expectations based on variations in temperature and biological productivity and with the few available prior measurements.

For $\mathrm{CO}_{2}$, the northern limb of the South Indian subtropical gyre is generally supersaturated, while the cooler southern limb is generally undersaturated. The greatest undersaturations were found in the southwestern Indian Ocean during the austral winter, while the greatest supersaturations were found in the Arabian Sea during the boreal summer. Measurements in the Bay of Bengal showed general undersaturations, especially toward the north and east. Unlike the results from the Pacific and Atlantic oceans, these Indian Ocean measurements do not show a pronounced equatorial $\mathrm{CO}_{2}$ maximum.

For $\mathrm{N}_{2} \mathrm{O}$, the Indian Ocean is either near saturation or supersaturated everywhere. The highest supersaturations are found in the Arabian Sea, in the western equatorial region, and in coastal waters. The regions south of the polar front are also generally supersaturated. As with $\mathrm{CO}_{2}$, the Indian Ocean differs from the other major oceans in that there is no equatorial $\mathrm{N}_{2} \mathrm{O}$ maximum extending across the width of the ocean.

\section{MOORED SENSING SYSTEMS WIRICK, CREIGHTON D. BROOKHAVEN NATIONAL LABORATORY \\ FY 1996270 \\ FY 1995224 \\ FY 1994147}

Keywords: moored sensing systems

Objectives: To use moored sensors to measure dissolved oxygen to investigate the carbon cycle on the continental margin near Cape Hatteras during the integrated, multidisciplinary Oceans Margin program field experiment.

Approach: Previously developed instruments will be deployed as part of the OMP field experiment.

Results to Date: Data collection is ongoing. 


\title{
PROGRAM ON ECOSYSTEM RESEARCH
}

The Program on Ecosystem Research identifies the processes that allow land ecosystems to adjust to global environmental changes and allows this understanding to be used to protect and restore ecosystems.

\author{
Program Manager \\ Jerry W. Elwood \\ Environmental Sciences Division \\ Department of Energy, ER-74 \\ 19901 Germantown Road \\ Germantown, MD 20874-1290 \\ (301) 903-4583 \\ Internet: jerry.elwood@oer.doe.gov
}

\section{RESPONSES OF SOIL MICROORGANISMS AND MICROBIALLY MEDIATED PROCESSES DURING SUCCESSION TO ELEVATED $\mathrm{CO}_{2}$ IN A MEDITERRANEAN-TYPE ECOSYSTEM ALLEN, MICHAEL SAN DIEGO STATE UNIVERSITY FY 1996187 \\ FY 1995198 \\ FY 19940}

Keywords: elevated $\mathrm{CO}_{2}$, mycorrhizae, plant symbionts, soil food webs, soil microbes

Objectives: To determine whether increased $\mathrm{CO}_{2}$ and other global-change processes will alter the belowground resource acquisition by plants during succession by changing microbial composition and dynamics. Approach: The amounts and rates of two microbial processes (mycorrhizal and nitrogenase activity) will be contrasted experimentally in high vs ambient $\mathrm{CO}_{2}$ environments. Our initial work on mycorrhizae has been to describe the seasonal and spatial dynamics and determining rates of mycorrhizal formation and testing those against a model of mycorrhizal development. We evaluate different sampling and statistical analysis strategies than generally undertaken because soil microbial components are constructed at much different spatial scales than those of plants. We also assess the response of mycorrhizal fungi to elevated $\mathrm{CO}_{2}$ and elevated $\mathrm{CO}_{2}$ in growth chambers and in field fumigation studies. These analyses occur simultaneously with efforts to assess the diversity of mycorrhizal fungi with both direct isolation and molecular techniques. The ITS region of the ribosomal DNA of mycorrhizal fungi was amplified with PCR for studying fungal-diversity changes. To study nitrogen dynamics in this system, we are measuring the natural abundance $d 15 \mathrm{~N}$ and, to determine nitrogenase diversity, an alternate probe with a highly conserved region of the nifH gene.

Results to Date: There are no chamber effects for microbial structure that could lead to spurious results. Microbial activity at the plant-soil interface was increased by elevated $\mathrm{CO}_{2}$, indicating that rhizosphere microorganisms are carbon-limited. Microbes stimulated used simple carbohydrates and amino acids; those that declined used phenolic compounds. Under lownutrient conditions, mycorrhizal fungi responded positively to increased $\mathrm{CO}_{2}$. Transport organs, intraand extraradical hyphae, and arbuscules were significantly increased. Under elevated $\mathrm{CO}_{2}$ and increased soil nutrients, arbuscules were reduced. There was a greater mass of nonmycorrhizal fungi and bacteria. Many invertebrate taxa increased under elevated $\mathrm{CO}_{2}$, especially with increased nutrients. These appear to be consuming the decomposer and pathogenic microbes but not the mycorrhizal fungi. We have not yet detected a change in mycorrhizal fungal species with a combination of traditional morphological and newly developed molecular techniques. There was no fixation in the Ceanothus with ambient $\mathrm{CO}_{2}$. The lack of differences between plants and soils also suggests that about 2 to $5 \mathrm{~kg} / \mathrm{ha}$ of nitrogen are deposited, primarily as dryfall. The added nitrogen also complicates the mycorrhizal dynamics and native $\mathrm{N}_{2}$-fixing organisms and their activity, particularly when coupled with elevated $\mathrm{CO}_{2}$. 


\section{GENERALIZING RESULTS FROM THE WALKER BRANCH WATERSHED \\ THROUGHFALL DISPLACEMENT EXPERIMENT WITH A HIERARCHIC MODEL OF FOREST-ECOSYSTEM MASS AND ENERGY EXCHANGE AMTHOR, JEFF \\ LAWRENCE LIVERMORE NATIONAL LABORATORY \\ FY 1996150 \\ FY 199523 \\ FY $1994 \quad 0$}

Keywords: forest ccosystems, mathematical modeling, mass fluxes, energy fluxes, water stress, photosynthesis, respiration

Objectives: To test and improve a comprehensive, explanatory model of forest ecosystem mass and energy exchange with results from the large-scale, multiyear Walker Branch Watershed Throughfall Displacement Experiment.

Approach: A mechanistic model of forest ecosystem mass and energy exchange is being parameterized for a deciduous-forest site near ORNL, the Walker Branch Watershed Throughfall Displacement Experiment (WBW TDE). Model simulations (with a 1-hour model time step) are conducted for the site with measured and hypothetical environmental conditions (solar radiation input, air temperature, humidity, wind speed above the canopy, $\mathrm{CO}_{2}$ concentration, and precipitation amount) at the forest site. Detailed comparisons are being made between measured and modeled soil and plant responses to natural variability in seasonal and annual precipitation, and to human manipulations involving $30 \%$ throughfall amounts in a deciduous forest. The measurement-model comparisons are used to test the model. Model shortcomings exposed by this set of model tests will be addressed with further modeldevelopment work. The tested and improved model will be used to extrapolate results from the WBW TDE in both space and time. The model will also be used to help explain results obtained in the WBW TDE and thus to develop general relationships about terrestrial ecosystem responses to the environment that may be applicable to other ecosystems.

Results to Date: In these early months of the project, effort focused on interfacing environmental measurements (input data) at the WDW TDE site to the forest ecosystem model. In turn, this has contributed an additional dimension to quality control of data collection in the WDW TDE. The model was shown to be sensitive to interannual variability in environmental conditions at the site. The forest-model photosynthesis and stomatal-conductance submodels were improved and simplified, based on recent leaf-level physiological research and data synthesis.

\author{
PLANT NITROGEN BUDGETS UNDER \\ ELEVATED CARBON DIOXIDE LEVELS: \\ REGULATION BY NITROGEN ABSORPTION \\ AND ASSIMILATION \\ BLOOM, ARNOLD \\ UNIVERSITY OF CALIFORNIA, DAVIS \\ FY 19960 \\ FY 1995175 \\ FY 19940
}

Keywords: elevated $\mathrm{CO}_{2}$, nitrates, nitrous oxide, photoassimilation

Objectives: To investigate the effects of enriched $\mathrm{CO}_{2}$ on the uptake of nitrogen from soil by plants and on the $\mathrm{CO}_{2}$ sequent use of nitrogen by the plants.

Approach: A series of growth chamber experiments is examining the functional response of plants grown under constant, low levels of ammonium and/or nitrate nitrogen, high light, and arbitrary $\mathrm{CO}_{2}$ levels. These experiments should provide evidence as to whether and how $\mathrm{CO}_{2}$ enrichment influences the relative rates of shoot carbon fixation, nitrate photoassimilation, transpiration, nitrous oxide emissions, root respiration, ammonium and nitrate absorption by roots, root proton pumping, and nitrate assimilation by roots. The ratio of the stable isotope ${ }^{15} \mathrm{~N}$ to ${ }^{14} \mathrm{~N}$ in the reduced-nitrogen fraction of a plant indicates the plant's relative reliance upon ammonium and nitrate as nitrogen sources. This measure will be applied to several species grown in the field under normal and elevated $\mathrm{CO}_{2}$.

Results to Date: A growth chamber experiment on wheat showed that elevated $\mathrm{CO}_{2}$ inhibits $\mathrm{NO}_{2}^{-}$and $\mathrm{NO}_{3} \cdot$ photoassimilation. Many plant responses to elevated $\mathrm{CO}_{2}$ seemed to derive from these effects.

\section{MOLECULAR AND PROCESS ADJUSTMENTS OF NITROGEN CYCLING IN THE SHRUB-STEPPE BOLTON, HARVEY \\ PACIFIC NORTHWEST NATIONAL LABORATORY \\ FY 1996194 \\ FY 1995196 \\ FY 1994196}

Keywords: nitrogen, gene probes

Objectives: To develop an understanding of how the nitrogen cycle of semiarid shrub-steppe ecosystems will adjust to changes in temperature and moisture at the molecular (i.e., gene) and process (i.e., ecosystem) levels.

Approach: Temperature and moisture are varied through elevational gradients in the field and in the laboratory to determine how nitrogen-cycling processes and the genes adjust to these changes. We focus on the microbially catalyzed input $\left(\mathrm{N}_{2}\right.$ fixation) and loss $\left(\mathrm{N}_{2} \mathrm{O}\right.$ from nitrification) of nitrogen from the shrub-steppe with both process-level measurements and molecular 
probes to detect changes in the abundance (amount of a specific gene) and expression (amount of RNA) of genes responsible for these microbially catalyzed processes. Rates of nitrogen fixation via acetylene reduction, $\mathrm{N}_{2} \mathrm{O}$ evolution via static chamber, and soil nitrogen pools via extraction are quantified.

Results to Date: Soil nitrogen and carbon properties varied over a 650 -m elevational gradient. Soil underneath Pseudoroegneria spicata had a larger nitrification potential and a more efficient microbial biomass than cryptogamic-crust soil. Microbial biomass decreased as a function of elevation, and decreased more dramatically in crust soil.

An electroelution technique was developed to separate humics from soil DNA and RNA extracts and applied to PCR detection of $16 \mathrm{~S}$ genes and rRNA of ammonia oxidizers from soil. The PCR technique found ammonia-oxidizer populations in forest, semiarid and temperate grasslands, and agricultural soils did not change after 3 weeks when amended with ammonium. Ammonia-oxidizer most probable number (MPN) counts were low and increased after 3 weeks to near PCR levels. The PCR results suggest that ammonia oxidizers did not increase during the 3-week incubation. The enhancement in MPN suggests that ammonia oxidizers were more culturable after 3 weeks. Therefore, the MPN technique may in-accurately estimate the ammonia-oxidizer population and growth.

\section{TEMPERATURE ADJUSTMENTS IN SUGAR MAPLE: IMPLICATIONS FOR FOREST SUCCESSION IN A WARMER CLIMATE GUNDERSON, CARLA A.; NORBY, RICHARD J.; O'NEILL, ELIZABETH G.; POST, W. M.; TUSKAN, GERALD A.; and GUNTER, LEE E. OAK RIDGE NATIONAL LABORATORY FY 1996193 FY 1995150 FY 1994195}

Keywords: sugar maple, acclimation, adaptation, photosynthesis, respiration

Objectives: To evaluate the physiologically and genetically controlled homeostatic adjustments of sugar maple and other forest species to temperature variation at different time scales, integrate the adjustment processes across levels of organization, and evaluate the implications of these adjustments for forest successional responses to global climate change. Approach: The photosynthetic and respiratory responses to temperature of sugar maple and selected woody species are being evaluated in the laboratory to determine the capacity for temperature acclimation in relation to other species characteristics, including physiological and ecological differences. Sugar-maple microcosms maintained under different temperature regimes are also being evaluated for adjustments in bacterial populations and system-level processes, such as nutrient loss in response to temperature stress. Field studies will complement laboratory results, and will examine the consequences of temperature increases superimposed upon seasonal temperature changes. Genetic characterization of sugar maple populations from the northern, central, and southern portions of the range are being conducted to link genetic variability [in randomly amplified polymorphic DNA (RAPD) markers and isozymes] with population differences in temperature response. Data obtained from laboratory studies will be used in forest succession models, in combination with other adjustment scenarios, to evaluate predictions of global climate change effects on forest species' ranges, and to evaluate the importance of mechanistic understanding of temperature adjustments in predictions of ecosystem response.

Results to Date: Physiological acclimation of photosynthesis to elevated temperature was found in sugar maple seedlings from both northern and southern sources, although photosynthesis remained high over a broad range of temperatures in all cases. Respiration was more sensitive to short-term temperature increases in northern sources, but acclimation to higher growth temperature occurred in both populations. The occurrence of acclimation alters the prediction of carbon gain based on short-term measurements and must, therefore, be considered in models of plant response to climate change.

Soil respiration in intact sugar-maple microcosms increased with a $4^{\circ}$ temperature elevation $\left(3^{\circ}\right.$ soil temperature), but calcium and nitrate losses from the microcosms, indicators of ecosystem stress, have not differed between ambient and elevated temperature treatments. Population-level genetic characterization of sugar maple has indicated high overall levels of RAPD band variation among individuals within the species and low differentiation among regions, consistent with the limited regional differences observed in aoclimation potential. The potential for photosynthetic acclimation has also been demonstrated in eastern cottonwood, such that optimum temperature for photosynthesis changed according to growth-temperature regime.

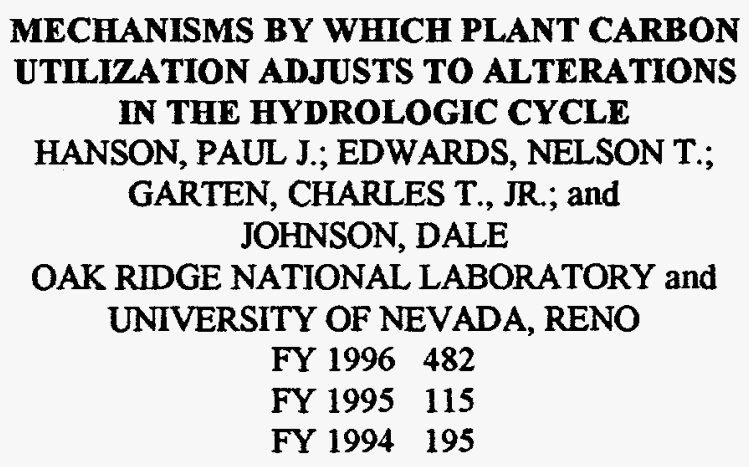


Keywords: net ecosystem production, nutrients Objectives: To identify physiological and growth adjustments of overstory trees vs understory shrubs and herbs to determine the buffering capacity of large vs small stature vegetation, and to study the impact of variable precipitation inputs on soil-nutrient availability for plant growth and function.

Approach: The manipulation of soil moisture is being implemented by a gravity-driven transfer of throughfall precipitation from one treatment plot to another. Throughfall is intercepted in approximately 2000 subcanopy troughs suspended above the forest floor of the dry plots and transferred across an ambient plot for subsequent distribution onto the wet treatment plot. The experimental system is able to produce statistically significant differences in soil water content in years having both extremely dry and extremely wet conditions. Furthermore, comparisons of pre- and postinstallation soil-temperature measurements have documented the ability of the experimental design to produce these changes without changing the microclimate of the forest understory. The results of this work will be integrated within existing models to provide information on the effects of chronic regional precipitation changes on the stability, diversity, and function of biological organisms within forest communities.

Results to Date: We have shown that overstory, or canopy, trees show minimal responses to experimentally altered precipitation levels whereas understory saplings showed reduced and increased growth on the dry and wet plots, respectively. No mortality was observed during a wet year, but exceptionally dry conditions resulted in $C$. florida mortality of 4,9 , and $21 \%$ for the wet, ambient, and dry treatments, respectively. Combined growth responses indicate that the small-stature vegetation of an upland oak stand will be more sensitive to changing precipitation patterns than established, dominant canopy trees. Observed growth data and model simulations suggest that seasonal differences in rainfall inputs will have a greater impact on plant productivity and net ecosystem production than an identical increase or decrease in precipitation distributed throughout the annual cycle.

\section{INTERACTIVE EFFECTS OF ELEVATED $\mathrm{CO}_{2}$, DROUGHT, AND HIGH TEMPERATURE ON PLANT WATER-USE EFFICIENCY HSIAO, THEODORE, and PAW U, K. T. UNIVERSITY OF CALIFORNIA, DAVIS FY 19960 \\ FY 1995175 \\ FY $1994 \quad 0$}

Keywords: elevated carbon dioxide, drought, water deficit, water stress, water-use efficiency, photosynthesis, transpiration
Objectives: To develop a fundamentally based wateruse-efficiency framework for single leaves of most plant species in relation to $\mathrm{CO}_{2}$ levels and water and high-temperature stresses and to scale up the concept to canopy water-use efficiency through simulation and model verification.

Approach: A number of crop species are grown under different levels of $\mathrm{CO}_{2}$ and temperature in controlledenvironment chambers, and their biomass and water use and selected environmental parameters are measured. The constancy and variability in the ratio of intercellular $\mathrm{CO}_{2}$ of their leaves and ambient $\mathrm{CO}_{2}$ concentrations under a wide range of conditions are evaluated. Together, these data are used to formulate the water-use-efficiency framework at the single-leaf or plant level. Field experiments are conducted on a large field of maize with and without water stress. Canopy photosynthesis and evapotranspiration are measured by Bowen ratio/energy balance, and a range of micrometeorological data are collected to test and further validate a previously published higher-order closure model for canopies. Several other models are also used to scale up from single-leaf to canopy processes. Water-use efficiency calculated with the models are checked against the field experimental data.

Results to Date: The ratio of intercellular $\mathrm{CO}_{2}$ to ambient $\mathrm{CO}_{2}$ has been found to remain nearly constant for a wide range of conditions in the several species studied so far, simplifying the task of predicting wateruse efficiency of single leaves. The efficiency of water use for biomass production was enhanced by elevated $\mathrm{CO}_{2}$, as expected. The extent of the enhancement predicted by the water-use-efficiency framework is very close to the measured values in several experiments. Field experiments for two seasons showed that biomass water-use efficiency of maize was relatively constant when normalized for the evaporative demand and temperature and was not significantly affected by water stress. A coupled stomatal-control and photosynthesis model was formulated and integrated with a layeredcanopy higher-order closure model. This model has been compared with some of the more conventional models for the estimation of canopy photosynthesis and water use in simulation runs. Extensive field data on profiles of temperature, water vapor, wind, and $\mathrm{CO}_{2}$ as well as canopy $\mathrm{CO}_{2}$ assimilation and evapotranspiration have been or are being collected. Model testing with these results are in progress. 


\section{BIOLOGICAL MECHANISMS BY WHICH \\ WHOLE-PLANT WATER USE RESPONDS TO ALTERATIONS IN THE HYDROLOGIC CYCLE \\ HUSTON, MICHAEL; WULLSCHLEGER, STANLEY; and COOPER, LEE \\ OAK RIDGE NATIONAL LABORATORY \\ FY 1996195 \\ FY 1995124 \\ FY 1994195}

Keywords: evapotranspiration, water use

Objectives: To evaluate the effects of climate and topographic position on the water flux and storage of trees ranging in size from seedlings to canopy, to evaluate the effects of climate and topography on the growth, shade tolerance, and survival of forest tree seedlings, and to evaluate the use of stable isotopes of carbon and oxygen as integrators of plant water use. Approach: Sapflow is being measured on red maple trees over a broad range of sizes. Water storage capacity in large trees is being estimated with time-domain reflectometry. Growth, mortality, and physiology are being measured on seedlings of sugar maple, tulip poplar, and white oak planted across three topographic positions (top, middle, and lower slope) and the three treatments (wet, control, and dry) on the throughfall displacement experiment site. Seedlings of sugar maple and tulip poplar were grown under controlled conditions of light and water in shade houses for a single growing season. Abscised leaves of red maple, white oak, chestnut oak, dogwood, and tulip poplar were collected and analyzed to determine the ratios of the stable isotopes of oxygen and carbon.

Results to Date: Short-term variation in soil water availability, related to topographic position, seasonal precipitation, and net radiation, have a significant effect on the whole-plant water use of saplings and canopy trees of red maple. Seedling mortality is significantly correlated with topographic position and water treatment, with strong year-to-year variation related to growing season precipitation and significant differences among species. Interannual variation in both $\delta^{18} \mathrm{O}$ and $\delta^{13} \mathrm{C}$ were greater than treatment effects for most species. Red maple showed the strongest treatment effects, with significant treatment differences in either carbon or oxygen isotopic ratios, or both.

\section{BELOWGROUND TREE AND \\ MYCORRHIZAL RESPONSES TO ALTERED HYDROLOGIC INPUTS \\ JOSLIN, JOHN, and O'NEILL, ELIZABETH \\ OAK RIDGE NATIONAL LABORATORY \\ FY 1996193 \\ FY 1995115 \\ FY 1994195}

Keywords: fine roots, water availability, mycorrhizae, minirhizotron

Objectives: To test for the occurrence of altered partitioning of carbohydrates and increased density of rooting in deeper horizons at the stand level.

Approach: At the Walker Branch Watershed Throughfall Displacement Experiment, changes in the fine root biomass over three depth increments will be measured through repeated soil core sampling prior to treatment initiation and after 3 years of treatment. Ingrowth cores are being used to provide a measure of net root-biomass carbon accumulation in cores of identical volume, soil material, and density, where the initial root volume is zero. Minirhizotrons installed at upper and lower slope positions are similarly being used as an index of the rate of root production. This approach is particularly useful for characterizing the seasonal timing of growth, while at the same time it is capable of detecting differences in growth rates across small-depth-interval increments. Water-availability effects upon the mycorrhizal symbiosis will be assessed by quantifying colonization in fine roots recovered from the in-growth cores.

Results to Date: Results from root-biomass sampling indicate that the density of fine-root biomass is randomly distributed across the entire experimental area. Approximately $75 \%$ of the fine roots are in the upper $30 \mathrm{~cm}$, with $16 \%$ and $9 \%$ in the $30-$ to $60-\mathrm{cm}$ and $60-$ to $90-\mathrm{cm}$ depth increments, respectively. The rootshoot hypothesis has received no support to date. In fact, whereas foliar litter production has shown no treatment effect, total net root production (minirhizotron observations) reflects a strong trend toward reduced fine-root growth in the dry treatment with the ambient treatment intermediate. Treatment differences in the phenology of root growth were apparent on the upper slope position. Minirhizotron data indicate that the production of new root tips (number) and net production of new root material (length) both declined in the dry treatment following a dry period and virtually ceased there for the rest of the growing season. Root growth continued in the ambient and wet treatments through September. However, 2 years of ingrowth core data (a dry plus a wet year) indicate no difference in root production with slope position.

FOREST-ATMOSPHERE CARBON
TRANSFER AND STORAGE: INTERACTING
EFFECTS OF ELEVATED CO AND O$_{3}$
ON ASPEN FOREST ECOSYSTEMS
KARNOSKY, DAVID; PREGITZER, KURT S;
and KUBISKE, MARK
MICHIGAN TECHNOLOGICAL UNIVERSITY
FY 1996 150
FY 1995239
FY $1994 \quad 0$


Keywords: aspen, free-air carbon dioxide enrichment, ecophysiological growth model

Objectives: To examine the interacting effects of elevated $\mathrm{CO}_{2}$ and $\mathrm{O}_{3}$ on a regenerating aspen (Populus tremuloides) forest ecosystem with a free-air carbon dioxide enrichment (FACE) facility at Rhinelander, Wis., and to parameterize and test an ecophysiological model of aspen growth and development to scale individual tree responses to the ecosystem level.

Approach: Three replicate FACE rings are being established for a factorial combination of treatments $\left(\mathrm{CO}_{2}, \mathrm{O}_{3}, \mathrm{CO}_{2}+\mathrm{O}_{3}\right.$, and control) in a randomized block design. Rooted cuttings from eight clones previously characterized for $\mathrm{O}_{3}$ and $\mathrm{CO}_{2}$ sensitivities will be planted $0.5 \mathrm{~m}$ apart in one-half of each FACE ring. The other half will be planted with full-sibling seedlings from wild, unselected $P$. tremuloides. The close spacing will simulate a naturally regenerating aspen forest. Fumigations with elevated $\mathrm{CO}_{2}$ $(550 \mathrm{ppm})$ and $\mathrm{O}_{3}(50 \mathrm{ppb}$ over background during photosynthetically active daylight hours to a target of $80 \mathrm{ppm}-\mathrm{h} \cdot \mathrm{O}_{3}$ seasonal exposure) will be conducted over the approximately 100-day growing season. Measurements of growth (height, diameter, biomass, leaf area, root production, and root mortality), physiological processes, and status (photosynthesis, respiration, stomatal conductance, and chlorophyll content), plant nutrient status (nitrogen), foliar biochemistry (carbohydrates, phenolic glycosides, and antioxidants), litter quality and decomposition rates, and soil respiration will all be made throughout each growing season. Results to Date: No results were reported.

\section{GENETIC ANALYSIS OF INTERACTING TROPHIC LEVELS IN A STRESSED PINYON-JUNIPER COMMUNITY: A MODEL FOR COMMUNITY ADAPTATION DURING GLOBAL CLIMATE CHANGE \\ KEIM, P.; WHITHAM, T. G.; COBB, N. S.; and GEHRING, C. A. NORTHERN ARIZONA UNIVERSITY \\ FY 1996198 \\ FY 1995200 \\ FY 1994194}

Keywords: Sunset Crater, pinyon trees, juniper trees, mycorrhizal mutualists

Objectives: To use recently created volcanic environmental locations at Sunset Crater National Monument to understand the effect of global climate change on the pinyon-juniper community by contrasting the community and its components at hot, dry locations with those at adjacent, moister, cooler locations.

Approach: We are combining traditional and experimental ecology with molecular genetic analysis to understand the effects of climate change on the pinyon-juniper woodland. We have censused and ma- nipulated insect populations during the past 13 years to examine herbivore impacts on plants. DNA analysis of pinyon pine has been used to understand gene flow and genetic mechanisms of adaptation to a drier climate. Mycorrhizal mutualists have been characterized by DNA analysis, and these are now being coupled with microscopic identification methods. Mycorrhizal censuses with molecular and morphological analysis have been performed at various experimental locations and across years.

Results to Date: Plants growing in the stressed environment have reduced reproductive capacity, increased herbivory, and higher levels of mycorrhizal colonization. The physiological basis for herbivore resistance appears to be resin production. Resistant trees also support more mycorrhizal mutualists and, thus, enhance their nutrient-absorption capacity. In general, trees growing at the drier cinder site have double the ectomycorrhizal densities than those in the adjacent soil sites. This increase in mutualists appears to be an important compensation needed to survive the harsher environment. Because pinyon pines represent the dominant community member, this shift in energy allocation to mycorrhizae (i.e., mycorrhizae consume 10 to $60 \%$ of a plant's photosynthate) represents a major change in energy allocation for the whole community. Based on an analysis of 300 species of arthropods associated with pinyon pine, overall species richness declines by $50 \%$, and abundance of these species is reduced twelve-fold, despite the fact that environmental stress results in otherwise rare species reaching outbreak levels. These results indicate that global climate change may directly or indirectly reduce overall species diversity and abundance of nonoutbreak herbivore species. Although pinyon pines have only had a few hundred years to respond to this altered environment, genetic differentiation and evolution have occurred. DNA marker analysis of pinyon pine has identified genetic differences between cinder and soil pinyon populations. Our initial success was with chloroplast DNA markers that have introgressed from xeric-adapted pinyon species. These results suggest that interspecific gene flow may allow more rapid adaptation to climate change than would be predicted from single-taxon models.

GENETIC ANALYSIS IN A STRESSED
PINYON-JUNIPER COMMUNITY:
RESPONSES OF SOIL MICROFLORA TO
RAPID AND RECENT ENVIRONMENTAL
CHANGES
KUSKE, CHERYL
LOS ALAMOS NATIONAL LABORATORY
FY 1996194
FY 1995193
FY 1994196


Keywords: soil microbiology, 16S ribosomal RNA, molecular typing, microbial community, ERIC, RFLP, Pseudomonas

Objectives: To develop molecular and microbial culture techniques to describe soil microbial communities; to use these techniques to identify key microbial groups that are important to the adjustment of terrestrial plants to climate changes; and to monitor their population dynamics over time.

Approach: A natural comparative experiment will be used to identify differences in the soil microbial communities between an environmentally stressed and an adjacent unstressed pinyon-juniper woodland. The stressed area is a volcanic cinder field where pinyon and juniper have colonized the hotter, dryer cinder field. These trees show many signs of stress, including decreased plant growth and productivity, and increased susceptibility to herbivorous insects. We are comparing several components of the soil microbial communities associated with pinyon roots (rhizospheres) and interspaces (between the widely spaced trees) to identify changes in microbial abundance, diversity, and species composition that have accompanied the trees as they have colonized the extreme environment at the cinder field. We examine abundance and diversity of different functional components of the cultivable (heterotrophic bacteria, Pseudomonas sp., saprophytic fungi, and humate- and chitin-utilizing actinomycetes) and nonculturable soil microbial community with microbial plating studies, 16S RFLP, ERIC fingerprinting and sequencing, microscopic-biomass measurements, direct DNA analysis of the nonculturable bacterial community, and chemical and physical analysis of the soil samples.

Results to Date: Beneficial Pseudomonas sp. were more abundant in pinyon rhizospheres on the stressed cinder field than on the unstressed soil site. They were also more abundant in tree rhizospheres of pinyon stressed by insect feeding, indicating they may be important in natural ecosystems to help plants tolerate stress from environmental conditions or insect herbivory. Of the Pseudomonas isolates, $\mathbf{9 5 \%}$ fell into three new species. Molecular population typing indicated that $75 \%$ of the isolates from soil rhizospheres and more than $50 \%$ of the isolates from the cinder rhizospheres were specific for their respective sites, indicating Pseudomonas populations diverged considerably as they colonized trees on the cinders. Population dynamics of fungi, bacteria, and actinomycetes follow different patterns in colonizing the stressed cinder site. Abundance and diversity of heterotrophic fungi was similar in tree rhizospheres at either site, indicating their populations were maintained in the stressed environment. Bacterial/actinomycete abundance and diversity were lower on trees on cinders than in the unstressed site. Molecular analysis identified four new bacterial kingdoms that are abundant in soils from our study site and other geographic regions.

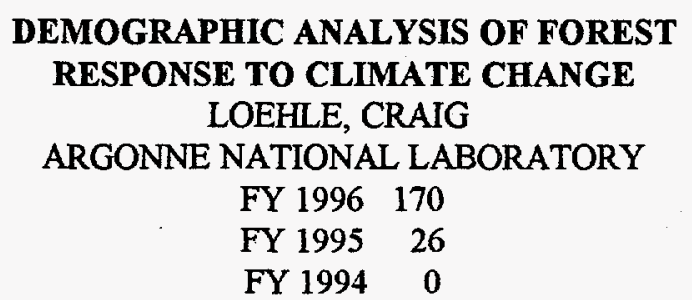

Keywords: forest growth, diebacks

Objectives: To conduct computer modeling of the responses of forests to climate change.

Approach: An extant model, SORTIE, will be used to predict forest response to climate change. Constraints governing trade-offs between parameters (e.g., growth rate vs. shade tolerance) will be examined to constrain model parameters to more realistic values. Testing the models against self-thinning, size distribution, and longevity measures will provide a rigorous independent assessment of demographic and transient responses. Testing SORTIE against historical stand diebacks will challenge the model under these crucial conditions. The analysis of U.S. Forest Service plotremeasurement data will provide a critical test of the geographic-scale variations in tree growth rates, particularly whether trees are limited by moisture and temperature at their range boundaries or are simply outgrown by competitors. Model experiments will address the problem of vegetation inertia. The inclusion of restrictions on seed rain, age of sexual maturity, stump sprouting, and mortality is expected to significantly alter predictions compared with past models, in particular increasing inertia of stand structure in response to climate change.

Results to Date: A key component of this study is the tree-mortality function. Longer-lived trees may have a greater tolerance of short-term climatic fluctuations than short-lived species. Literature on tree-mortality functions currently in use is being reviewed. In addition, a tree-ring data set is being obtained to allow new models to be developed and tested. Stump and root sprouting have never been incorporated into a growth model. Sprouts grow faster than seedlings and enable many species to persist in the face of disturbances such as fire. A rationale and a preliminary model for sprouting have been written, and a table on the sprouting ability of eastern species has been compiled. 


\author{
MOLECULAR AND PHYSIOLOGICAL \\ ANALYSIS OF DOWN-REGULATION OF \\ PHOTOSYNTHESIS \\ LONG, STEPHEN; LAROCHE, JULIE; \\ and HENDREY, GEORGE \\ UNIVERSITY OF ESSEX and BROOKHAVEN \\ NATIONAL LABORATORY \\ FY 1996192 \\ FY 1995194 \\ FY 1994194
}

Keywords: photosynthesis, down-regulation, photosynthetic proteins, ribonucleic acid

Objectives: To elucidate a molecular basis for the down-regulation associated with long-term exposure to elevated $\mathrm{CO}_{2}$.

Approach: Increased photosynthetic $\mathrm{CO}_{2}$ uptake and primary production are initially observed when plants are exposed to elevated $\mathrm{CO}_{2}$. In the long term, this gain may be counteracted by a decrease in photosynthetic capacity, termed down-regulation.

Results to Date: In sour orange trees, no differences were found in concentrations of photosynthetic proteins between elevated and ambient treatments. No down-regulation was observed in elevated $\mathrm{CO}_{2}$ samples. Increased amounts of a leaf glycoprotein (GP24) occurred in elevated $\mathrm{CO}_{2}$ but in old leaves only. The function of this protein is not known, but in other species of orange it is associated with chilling- and frost-tolerance.

The proteins were characterized from Scirpus olneyii grown for 8 years under either ambient or elevated $\mathrm{CO}_{2}$ on a tidal marsh on the Chesapeake Bay. In this system, there has been no physiological evidence of down-regulation of photosynthesis; this contrasts with the many controlled environment laboratory studies that have shown a loss of photosynthetic capacity. One-dimensional polyacrylamide gel electrophoresis and Western blotting of soluble and thylakoid membrane proteins from $S$. olneyii showed that Rubisco and Rubisco activase decreased slightly as a proportion of total protein in photosynthetic tissue grown under elevated and ambient $\mathrm{CO}_{2}$ concentrations.

D1, a photosystem-II core protein, showed a $39 \%$ increase under elevated $\mathrm{CO}_{2}$ and the light-harvesting chlorophyll-binding protein LHCII showed a $16 \%$ increase. These results provide the first evidence of increased capacity for photosynthetic electron transport and regeneration of the acceptor for assimilating $\mathrm{CO}_{2}$ under long-term growth in elevated $\mathrm{CO}_{2}$.

\section{MECHANISMS CONTROLLING THE MYCORRHIZAL SYMBIOSIS UNDER A CHANGING GLOBAL ENVIRONMENT \\ MILLER, R. MICHAEL, and JASTROW, JUIE D. ARGONNE NATIONAL LABORATORY \\ FY 1996195 \\ FY 1995194 \\ FY 1994197}

Keywords: carbohydrate allocation, carbon allocation, mycorrhizae, nutrient acquisition, nutrient allocation Objectives: To determine whether a primary mechanism of control of the mycorrhizal symbiosis is the balance between photosynthate supply to the roots and the host's need for nutrients.

Approach: Experiments for determining carbon gain are conducted for facultative (Fermilab) and obligate (Kansas) mycorrhizal ecotypes of Andropogon gerardi. For each ecotype, we collect data from five sequential harvests of $A$. gerardi plants $(30,45,60,75$, and 90 days). At each harvest, above- and belowground biomass and shoot surface area are quantified. Roots are measured for their length and surface area, as well as mycorrhizal colonized length. Fungal structures, including intraradical hyphae, coils, arbuscules, and vesicles, are quantified, and their metabolic activity is determined by measuring succinate dehydrogenase activity. The amount of extraradical hyphae of the mycorrhizal fungus is quantified. These measures allow for determination of internal vs. external allocation of the mycorrhizal fungus. Shoot and root tissue phosphorus and nitrogen and carbohydrate pools, especially aldo and keto sugars, are also determined.

Results to Date: Mycorrhizal and nonmycorrhizal prairie peninsula $A$. gerardi have similar biomasses, even though mycorrhizal plants have greater tissue concentrations of phosphorus. Increases in tissue nitrogen concentrations were not found to occur with mycorrhizae. These findings suggest that the relationship between prairie peninsula big bluestem and the mycorrhizal fungus is facultative. In contrast, our findings for $A$. gerardi from the Konza prairie suggested that relationship is obligate. For the obligate ecotype, we found a strong positive relationship between shoot-tissue nitrogen concentration and net photosynthesis (A) but none between $A$ and tissue phosphorus concentration. The relationship for either tissue phosphorus or tissue nitrogen with A was not affected by the presence or absence of mycorrhizae. However, a significant mycorrhizal effect was present in the relationship of the ratio of phosphorus to nitrogen in the shoot-tissue (P:N) with $A$. The values for $A$ were higher for mycorrhizal plants than nonmycorrhizal plants at equivalent shoot tissue $\mathrm{P}: \mathrm{N}$ ratios. Shoot $P: N$ ratio also has a similar effect on net carbon gain, with mycorrhizal plants having higher carbon gains than nonmycorrhizal plants at equivalent shoot $\mathrm{P}: \mathrm{N}$ 
ratios. Net carbon gain of $A$. gerardi followed the trend of net photosynthesis, with mycorrhizal plants fixing more carbon than nonmycorrhizal plants at equivalent shoot P:N ratios. Two potential mechanisms appear to be taking place. When nitrogen is adequate in relation to phosphorus ( $\mathrm{P}: \mathrm{N}$ is about 0.10 ), mycorrhizal plants appear to have greater leaf surface area. When nitrogen becomes limiting ( $P: N>0.12$ ), the amount of mycorrhizal fungus increases, and sink strength increases. Our data indicate that the mycorrhizal fungus is a major component of the host's carbon sink strength and is associated with specific kinds of carbohydrates that accumulate in mycorrhizal and nonmycorrhizal shoots and roots.

\section{MEASUREMENTS OF ECOSYSTEM RESPONSE IN WALKER BRANCH WATERSHED \\ MULHOLLAND, PATRICK, and HUSTON, MICHAEL \\ OAK RIDGE NATIONAL LABORATORY \\ FY 1996195 \\ FY 1995131 \\ FY 1994170}

Keywords: forest ecosystems, streams, watersheds, hydrology, biogeochemistry

Objectives: To quantify intra- and interannual variation in hydrology; soil and stream water chemistry; and forest biomass, productivity, and species composition in response to variations in climate and to changes in the deposition of chemicals from the atmosphere. Approach: Measurements will be made of precipitation inputs and stream outputs of water and chemicals; the chemical content of soils; and forest biomass, productivity, and species composition to quantify the response of forest and stream ecosystems to variations in climate and to changes in the deposition of chemicals from the atmosphere, many of which are the result of energy-related activities. The measurements are also used to develop and test biogeochemical and ecological models, to help interpret results from experimental studies of climate change being conducted on the watershed, and to support DOE's environmental restoration activities on the Oak Ridge Reservation. The Walker Branch measurements represent one of the few long-term, combined records of hydrology, biogeochemistry, and ecology available for forest ecosystems in the eastern United States. These measurements provide the long-term environmental characterization necessary for interpreting the results of past, present, and future DOE-funded research in Walker Branch Watershed.

Results to Date: Although the total atmospheric deposition of sulfur to the forest is declining, atmospheric deposition of nitrogen is increasing. Stream output of sulfur is highly dependent on runoff and represents 30 to $70 \%$ of inputs, whereas stream output of nitrogen is very low ( $<5 \%$ of inputs) and relatively independent of runoff. The difference between annual precipitation and annual runoff (an indirect measure of catchment evapotranspiration) has been relatively constant over the past 25 years, but is positively related to growing season precipitation. There has been a reduction in exchangeable $\mathrm{Ca}$ and $\mathrm{Mg}$ in the upper soil horizons that appears to be the result of long-term leaching processes. Stream nutrient ( $\mathrm{N}$ and $\mathrm{P}$ ) concentrations show significant seasonal variation as a result of pulses of heterotrophic and autotrophic nutrient immobilization following the input of leaves in autumn and the increase in available light in spring prior to canopy closure. Significantly drier conditions during the 1980 s, including a severe multiyear drought, resulted in significant increases in mortality of some species of trees (e.g., tulip poplar) but had little effect on others (e.g., oaks), compared to the wetter 1970s. Long-term equilibria between vegetation and soil $\mathrm{C}: \mathrm{N}$ ratios are related to topographic position and disturbance history.

\section{IMPACT OF GLOBAL CLIMATE CHANGE ON ECOSYSTEM-LEVEL INTERACTIONS AMONG SYMPATRIC PLANTS FROM ALL THREE PHOTOSYNTHETIC PATHWAYS NOBEL, PARK \\ UNIVERSITY OF CALIFORNIA, LOS ANGELES FY 19960 FY 1995171 FY 19940}

Keywords: Sonoran Desert, photosynthetic pathways Objectives: To determine biochemical and physiological responses to environmental factors for codominant species in the Sonoran Desert from all three photosynthetic pathways, the C3 Encelia farinosa, the C4 Pleuraphis rigida, and the CAM Agave deserti, which are being used to predict global-climate-change effects on plant interactions at the ecosystem level.

Approach: The project is divided into five parts: $\mathrm{CO}_{2}$ uptake, which has been measured for all three species under a variety of conditions; field manipulations, which have been established at the University of California's Philip L. Boyd Deep Canyon Desert Research Center as well as at the University of Riverside Agricultural Research Station; carboxylation enzymes, whose measurement techniques and initial results have boen determined for $A$. deserti and $E$. farinosa; carbon partitioning, which has recently been initiated for $E$. farinosa; and prediction of $\mathrm{CO}_{2}$ uptake under various environmental conditions, which will be made using an environmental productivity index (EPI) that is the product of water, temperature, and photosynthetic-photon-flux (PPF) indices established for each species. 
Results to Date: The water index for $A$. deserti was always greater than for the other two species. During drought, $A$. deserti had the lowest optimal temperature for the temperature index, and $P$. rigida had the highest. The PPF index required the highest PPF levels for $A$. deserti and the lowest for $P$. rigida. $\mathrm{CO}_{2}$ uptake predicted using EPI compared well with $\mathrm{CO}_{2}$ uptake measured in the field for each species. Net $\mathrm{CO}_{2}$ uptake for $E$. farinosa was always higher under doubled $\mathrm{CO}_{2}$, but it decreased through the light period, whereas the change of $\mathrm{CO}_{2}$ uptake for plants under ambient $\mathrm{CO}_{2}$ was relatively minimal; both sucrose and starch contents increased through the light period and were higher for the doubled $\mathrm{CO}_{2}$ concentration. Net $\mathrm{CO}_{2}$ uptake for $A$. deserti was $50 \%$ enhanced after longterm exposure to a doubled $\mathrm{CO}_{2}$ concentration; such enhancement was generally greater when other environmental conditions were less than optimum. Pleuraphis rigida was the most sensitive to rainfall; its vegetative growth and carboxylase activities coincided with seasonal changes in rainfall. For E. Farinosa, the initial slopes of assimilation vs intercellular $\mathrm{CO}_{2}$ concentration curves were similar for sun and shade plants at low PPF but higher for sun plants as the PPF was increased.

\section{ENVIRONMENTAL, GENETIC, AND ECOPHYSIOLOGICAL VARIATION OF WESTERN AND UTAH JUNIPER AND THEIR HYBRIDS: A MODEL SYSTEM FOR VEGETATION RESPONSE TO CLIMATE CHANGE \\ NOWAK, ROBERT, and TAUSCH, ROBIN \\ UNIVERSITY OF NEVADA, RENO, and \\ U.S. FOREST SERVICE \\ FY 1996147 \\ FY 1995144 \\ FY $1994 \quad 0$}

Keywords: RFLP, drought, photosynthesis, water-use efficiency

Objectives: To determine relationships between the environment and both the genetic and ecophysiological characteristics for two model species, Juniperus osteosperma and $J$. occidentalis, over their modern elevational and spatial distribution.

Approach: Field-study plots were established to incorporate both local and regional environmental variation. Because water is the primary factor that limits growth and productivity in the Great Basin, characterization of the physical environment focused on drought severity. Moisture deficit was calculated using data from weather stations across the Great Basin. A second technique that uses the hydrogen and oxygen isotopic ratio of plant cellulose was also used. Genetic characteristics of juniper was based primarily on restriction-fragment-length-polymorphism (RFLP) techniques. Ecophysiological data included measurements of leaf gas exchange (photosynthesis, transpiration, and stomatal conductance), leaf water potential, carbon-isotope composition, and leaf growth rates. Vegetation cover data by individual species were collected to characterize the plant community.

Results to Date: Populations of western juniper tended to have the greatest amount of genetic variability. Genetic variation in Utah juniper increases dramatically along the western portion of the Great Basin. Changes in leaf photosynthesis do not appear to be directly influenced by the climatic environment. Leaf conductance and plant genotype appear to be more important factors affecting photosynthesis, with leaf nitrogen playing a less important role. Leaf growth is sensitive to drought for Utah juniper, but elevational differences in growth rates tended to be small. For western juniper, leaf growth was generally greater at low elevations than at high, and the effects of drought were more prominent in spring rather than summer. Species density was relatively stable among plots at different elevations, but species composition varied widely across the Great Basin. Regardless of the species or subspecies of juniper present, juniper occurs over the same elevational range (1400 to $2800 \mathrm{~m}$ ). Particular assemblages of understory species appear to have an affinity for their own overstory juniper species or subspecies, but environment does have an effect on the vegetation assemblages.

\section{CURRENT AND FUTURE CARBON BUDGETS FOR TROPICAL RAIN FORESTS: \\ A CROSS-SCALE ANALYSIS OBERBAUER, STEVEN F. FLORIDA INTERNATIONAL UNIVERSITY FY 1996405 FY 19950 FY $1994 \quad 0$}

Keywords: rain forests, carbon budgets, carbon exchange, whole-tree response functions, belowground carbon

Objectives: To directly estimate forest-level carbon exchange of a lowland tropical rain forest in Costa Rica through eddy-covariance studies carried out under varied climatic conditions, to measure net primary production and its spatial and temporal variation within this tropical-rain-forest landscape, to determine whole-tree response functions to climatic variation for the major functional types of tropical-rain-forest canopy trees, to assess belowground carbon pools and dynamics of the forest, and to develop and test a topdown process model of tropical-rain-forest productivity.

Approach: Whole-forest gas exchange will be measured with eddy correlation; net primary productivity and carbon stocks will be measured across the major 
edaphic gradients at La Selva, Costa Rica; and wholetree climatic responses will be measured. Data will be used to parameterize and test a top-down process model of forest productivity.

Results to Date: New project.

\section{RESPONSE OF MEDITERRANEAN-TYPE ECOSYSTEMS TO ELEVATED \\ ATMOSPHERIC $\mathrm{CO}_{2}$ AND ASSOCIATED CLIMATE CHANGE \\ OECHEL, WALTER \\ SAN DIEGO STATE UNIVERSITY \\ FY 1996200 \\ FY 1995200 \\ FY 19940}

Keywords: homeostatic adjustment, acclimation, mediterranean-type ecosystem, chaparral

Objectives: To evaluate the patterns of homeostatic adjustment to elevated $\mathrm{CO}_{2}$ and drought in chaparral; to evaluate the mechanisms and controls on homeostatic adjustment including biological (biochemical, physiological, morphological, and ecosystem) and physical (resource availability) factors; and to develop the understanding necessary to predict the likely effects of elevated atmospheric $\mathrm{CO}_{2}$ and variations in soilmoisture availability on chaparral water use, potential water yield, productivity, fuel accumulation, fire frequency, species competition, and stand composition. Approach: The $\mathrm{CO}_{2}$-controlled, ambient light- and temperature-controlled $\left(\mathrm{CO}_{2} \mathrm{LT}\right)$ null-balance chambers currently running in the chaparral at $\mathrm{CO}_{2}$ concentrations of from 250 to $750 \mathrm{ppm} \mathrm{CO}$ are used. Results from these highly controlled manipulations are validated against Free Air $\mathrm{CO}_{2}$ Enrichment (FACE) manipulations in an area adjacent to the $\mathrm{CO}_{2} \mathrm{LT}$ nullbalance greenhouses. These relatively short-term results (years to decades) are being compared to longterm results from Mediterranean-type ecosystems (MTEs) surrounding natural $\mathrm{CO}_{2}$ springs in northern Italy, especially near Laiatico, Italy. This interdisciplinary research investigates biochemical, physiological, growth, and ecosystem responses, homeostatic adjustment at these organizational levels to variations in $\mathrm{CO}_{2}$ concentration and moisture availability. Interactions among these factors and levels are also explored. Above- and belowground processes are being studied.

Results to Date: After 1 year of continuous treatment, water use is decreased at elevated $\mathrm{CO}_{2}$, and soil mositure content is increased in the field. In $\mathrm{CO}_{2}$ springs at Laiatico, water use and stress are similar at elevated $\mathrm{CO}_{2}$. Water-use efficiency increases at elevated $\mathrm{CO}_{2}$. However leaf-area index (LAI) also increases at elevated $\mathrm{CO}_{2}$. The increase in LAI offsets some to all of the water savings from elevated $\mathrm{CO}_{2}$. Net primary productivity increases with increasing atmospheric $\mathrm{CO}_{2}$. Net ecosystem $\mathrm{CO}_{2}$ flux increases (sequestering increases) with increasing atmospheric $\mathrm{CO}_{2}$ from preindustrial $\mathrm{CO}_{2}$ levels to double current ambient levels. Homeostatic adjustment (acclimation) occurs. However, the extent of homeostatic adjustment appears to vary by water stress, source-sink status, and phenologic state. Such adjustment occurs at several levels, including biochemical, physiological, plant, allocation, growth, population, and ecosystem processes.

\section{MECHANISMS OF MYCORRHIZAL CONSTRAINT OF FOREST ECOSYSTEM RESPONSE TO GLOBAL CLIMATE CHANGE \\ O'NEILL, ELIZABETH; TSCHAPLINSKI, TIMOTHY; O'NEILL, ROBERT; and GUNDERSON, CARLA \\ OAK RIDGE NATIONAL LABORATORY \\ FY 1996193 \\ FY 1995150 \\ FY 1994195}

Keywords: mycorrhizae, elevated $\mathrm{CO}_{2}$, drought, loblolly pine

Objective: To determine whether mycorrhizal fungal species that are symbiotic with forest trees (1) are functionally redundant, (2) can be manipulated by changing the environment of the host tree, and (3) feed back on host-tree physiology to affect the response of the host to changing climatic factors.

Approach: Loblolly pines were grown in split-root pots, inoculated with either like or unlike species of mycorrhizal fungi and exposed to two levels of atmospheric $\mathrm{CO}_{2}$ and two levels of water availability to assess the degree of mycorrhizal redundancy. Seedling biomass, photosynthesis, and root and needle biochemistry were measured after one growing season. Currently, a new cohort of pine seedlings is being grown in forest soil and the diversity of mycorrhizal species on seedling roots will be quantified after exposure of the seedlings to one of four combinations of $\mathrm{CO}_{2}$ concentration and water availability. To test for feedback effects, mycorrhizal communities will be assembled from fungal isolates with known responses to $\mathrm{CO}_{2}$ and drought and inoculated on aseptically grown loblolly pine seedlings. Growth and biochemistry of these seedlings will be measured to determine whether the artificially assembled mycorrhizal communities result in different responses of the host plant to environmental conditions.

Results to Date: Pines that are symbiotic with multiple species of mycorrhizal fungi do not respond to environmental conditions in the same way as those colonized by a single spocies, even when the overall extent of colonization is similar (i.e., mycorrhizae appear not to be functionally redundant). Shoot growth in plants 
inoculated with the same mycorrhizal species in both sides of the split system was enhanced 25 to $36 \%$ by $\mathrm{CO}_{2}$ enrichment. No effects of $\mathrm{CO}_{2}$ enrichment or water availability were seen in plants inoculated with multiple fungal species. Photosynthetic rates were higher overall in seedlings grown at elevated $\mathrm{CO}_{2}$. Root concentrations of most monosaccharides and organic acids were little changed by mycorrhizal treatment, $\mathrm{CO}_{2}$ enrichment, or water availability. Concentrations of one sugar alcohol increased with $\mathrm{CO}_{2}$ enrichment, with the difference between the ambient and $\mathrm{CO}_{2}$-enriched treatments most pronounced in seedlings inoculated with multiple fungal species. In the current experiment, pines growing under ambient $\mathrm{CO}_{2}$ and moisture conditions for one year have developed vigorous root systems with an estimated 5 to 10 indigenous mycorrhizal species present. Exposure of these seedlings to elevated $\mathrm{CO}_{2}$ and reduced water will be initiated in late spring 1996 to determine whether mycorrhizal communities on roots change in response to the new environmental conditions.

\section{DYNAMICS OF NORTHEASTERN FORESTS IN A CHANGING CLIMATE \\ PACALA, S. W.; LEVIN, S. A.; CANHAM, C. D.; and BAZZAZ, F. A. \\ PRINCETON UNIVERSITY, NEW YORK \\ BOTANICAL GARDEN CARY ARBORETUM, and HARVARD UNIVERSITY

$\begin{array}{ll}\text { FY } 1996 & 198 \\ \text { FY } 1995 & 194 \\ \text { FY } 1994 & 194\end{array}$

Keywords: scaling rules, biodiversity, impact of animals on forests

Objectives: To understand the scaling rules in forests that translate individual performance into community and ecosystem dynamics, the tradeoffs among species that maintain the biodiversity of forest trees, the relationships between forest biodiversity and a forested ecosystem's response to global climate change, the impact of animals on the dynamics of forest trees and on ecosystem function, and the impact of heterogeneous soils and topography on forest dynamics.

Approach: Forest-gap models make system-level forecasts by predicting the fate of each tree (dispersal, birth, death, growth, resource uptake, etc.), whereas global models of vegetation predict total carbon pools at a larger scale. To bridge the gap between these two types of models, we are deriving equations that relate the large-scale properties to the individual-based simulators. We are examining the distribution of modeled species in parameter space and then characterizing the manifold (surface) that best describes the modeled species. The manifold is an embodiment of the trade-offs present in the model that controls biodiversity and forest dynamics. It reveals how much strategic diversity there is within the uniform functional groups present in global models. We are using the models to forecast the response of the forest to climate change in two cases, the first containing the measured species and the second containing only a single average type. The differences between these cases is a measure of the importance of biodiversity to system-level response to climate change. Finally, an extensive series of field observations and experiments in New England are being used to study natural enemies, water, nutrients, and climate.

Results to Date: We have shown that our individual-based forest model (SORTIE) may be expressed as a nonlinear point process and have discovered how to derive equations that govern the mean biomass of each species across a large landscape or across a large ensemble of separate runs. After estimating the manifold in parameter space containing our measured species, we developed a version of SORTIE in which a large number of species were drawn from across this manifold. This model allowed us to complete the observed strategies of forest species with all possible intermediate strategies and showed that the measured strategic diversity in our forest could allow the coexistence of more than 50 species for more than 10,000 years. The tradeoffs among species defined by the manifold indicate that the assumptions used to parameterize a significant fraction of global-change models are seriously flawed. We have used the SORTIE model to examine increases in total basal area caused by a doubling of $\mathrm{CO}_{2}$ with (1) the observed diversity of species and (2) only a single, average species present, the difference between the runs being taken as a measure of the importance of biodiversity. Fifty years after an instantaneous $\mathrm{CO}_{2}$ doubling, increases in basal area in the diverse-species runs were approximately twice as large as those in the averagespecies runs.

We have completed 2 years of measurements of herbivory by white-tailed deer and of seed predation by small mammals that monitored natural levels of herbivory and seed predation, levels of seed production, and mammal abundance. One interesting preliminary result is that white-tailed deer preferentially browse saplings with high-tissue-nitrogen content, which is correlated with the local $\mathrm{N}$-mineralization rate and thus with the litter chemistry of canopy trees. We have also initiated extensive measurements of fluxes, sapling performance, and seedlings artificially planted across a range of topography and geologic parent material. One interesting preliminary finding is that the abundance of calcium oxide in the parent material of noncalcareous soils apparently affects the local distribution of trees and that some species enhance the local availability of calcium and others diminish it. 
INTERACTION OF BIODIVERSITY, $\mathrm{CO}_{2}$, AND NITROGEN ON ECOSYSTEM FUNCTIONING REICH, PETER B.

UNIVERSITY OF MINNESOTA

FY 1996505

FY $1995 \quad 0$

FY $1994 \quad 0$

Keywords: biodiversity, soil nitrogen availability, grassland ecosystems

Objectives: To obtain information on the interactive effects of species richness, elevated carbon dioxide, and nitrogen deposition on grassland ecosystems.

Approach: A long-term study will be conducted of a grassland ecosystem in which plant diversity, soil nitrogen availability, and atmospheric $\mathrm{CO}_{2}$ concentration are directly controlled in a replicated full-factorial experiment. The main experiment will have 240 plots planted to various combinations of $1,4,9$, or 16 perennial prairie plant species randomly chosen from 16 common species in four plant functional groups (C3 grasses, C4 grasses, C3 legumes, and C3 nonlegume forbs). The 240 plots will be partitioned into six 29-m rings, three of which will be exposed to ambient $\mathrm{CO}_{2}$ and three to elevated $\mathrm{CO}_{2}$ using the established FACE technology. The 40 plots per ring will be partitioned into five replicates of each species richness treatment at both low (ambient soil nitrogen level) and high soil nitrogen levels (ambient $+5 \mathrm{~g}$ per $\mathrm{m}^{2}$ per year). An additional 48 plots are also included (spread across the six rings) to test for functional group vs species-richness effects. Data will be collected on numerous ecosystem, community, and population processes and parameters in all 288 plots, including aboveground net primary productivity and plant standing crop, leaf-area index, canopy light interception, belowground productivity, root standing crop, plant species abundance, soil and plant canopy $\mathrm{CO}_{2}$ and water-vapor flux rates, soil moisture, tissue carbon and nitrogen levels, changes in soil total carbon, total nitrogen stores, and dynamics of soil nitrogen and carbon in the rooting zone.

Results to Date: New project.

\section{BIOCHEMICAL MECHANISMS OF DROUGHT TOLERANCE OF FOUR DECIDUOUS SPECIES: IMPLICATIONS FOR FOREST SUCCESSION UNDER DROUGHT TSCHAPLINSKI, TIMOTHY, and GUNDERSON, CARLA \\ OAK RIDGE NATIONAL LABORATORY \\ FY 1996193 \\ FY 1995256 \\ FY 1994195}

Keywords: drought tolerance, osmotic potential, leaf water potential, stomatal conductance

Objectives: To assess the degree and biochemical bases of drought tolerance and osmotic adjustment of several deciduous species in a mature forest, growing under ambient throughfall, ambient minus $30 \%$ and ambient plus $30 \%$ throughfall, as supplied by the Throughfall Displacement Experiment.

Approach: Mid-crown branches of deciduous species that span the canopy of the Throughfall Displacement Experiment are collected periodically during the growing season for trees of all three throughfall levels, including ambient (control), dry (30\% less than ambient), and wet ( $30 \%$ more than ambient). The species sampled include understory dogwood, red maple, sugar maple, and black gum (an intermediate) and overstory red maple, white oak, and chestnut oak. A pressure cylinder is used to determine leaf water potential. A vapor-pressure osmometer is routinely used to track the osmotic potential at full turgor of different species to specifically dry events. Osmotic potential at full turgor is used to assess the degree of drought tolerance and evidence of osmotic adjustment to drought. Leaves are analyzed for solutes that constitute osmotic potential, including soluble carbohydrates, organic acids, amino acids, phenolic compounds, and inorganic ions.

Results to Date: During wet years, species vary in drought tolerance (as assessed by osmotic potential at full turgor) with American beech, dogwood, black gum, and sugar maple least tolerant; red maple intermediate; and white oak and chestnut oak most tolerant. Dogwood and chestnut oak trees are the only species to consistently demonstrate osmotic adjustment to drought, indicating that it is a species-specific response and not restricted solely to species considered tolerant. In contrast with red maple, where canopy position has no effect on osmotic potential at full turgor, understory chestnut oak seedlings have higher values (less negative) than do overstory trees and display no evidence of osmotic adjustment. A large fraction of the adjustment to drought in dogwood and chestnut oak trees could be accounted for by carbohydrates, organic acids, and cations, but the relative contribution of any given class of solutes varies with species and time.

During dry years, overstory trees have similar low osmotic potential at full turgor in all treatments in Aug./Sept., but species that are considered less drought tolerant have greater year-to- year variation in osmotic potential at full turgor. In contrast with overstory trees, treatment differences in osmotic potential at full turgor are evident for all understory species, including red maple, dogwood, beech, and chestnut oak. In contrast with a wet year, understory red maple and chestnut oak seedlings display osmotic adjustment in a dry growing season. Interannual variation in drought induces greater drought responses among species than do the treatments, which tend to establish transient differences dependent on the precipitation pattern. 


\section{ANIMAL MODEL FOR GENETIC/EVOLUTIONARY RESPONSES TO GLOBAL CLIMATE CHANGE \\ WATT, WARD B. \\ STANFORD UNIVERSITY

$\begin{array}{rr}\text { FY } 1996 & 92 \\ \text { FY } 1995 & 172 \\ \text { FY } 1994 & 0\end{array}$

Keywords: bioenergetics, thermal stability, fecundity, thermal depression of population

Objectives: To evaluate the role of genetically mediated thermal constraints on energy processing mechanisms, inherent in all thermally sensitive organisms, in limiting the nature and/or the speed of evolving adaptive responses to potential global change.

Approach: We use an insect model system that has three potential roles in the interactions of global change with ecosystem function: as an early-warning system, as an important interactor, and as an exemplar for parallel thermal sensitivities in many other plants and ectothermic animals. We study genetic variation in enzymes of energy processing in this test system, focusing on a series of genetic variants in one enzyme (phosphoglucose isomerase, PGI), found in all natural populations of these insects. We also study intrinsic thermal constraints on the flexibility of metabolic response to thermal change, which appear to mean that evolution of such organisms to compensate in other ways for the above genetically mediated tradeoff is not a realistic possibility over the projected time scales of potential global thermal change. To accomplish these tasks, we use DNA sequencing; functional study of enzyme genotypes' kinetic and stability parameters; isotope tracer, HPLC, and related metabolic techniques; and computer models.

Results to Date: Advances have been made in the in vivo assay of energy-rich metabolites and used to test, at the metabolic level, the causes of animal thermoregulation and, hence, animal sensitivity to environmental thermal change. We have developed computerized HPLC analysis of adenylates from insect flight muscle during the last few years, improving its throughput by a factor of 3 , incorporating the 24-hour use of a refrigerated autosampler. We have tamed the highly variable Fiske and SubbaRow citrate-molybdate assay for inorganic phosphate. With a free-flightchamber assay for insect flight, we have used these improved metabolic methods to show that the narrowness of animal thermoregulation is a response to strong constraint on coadjustment of the temperature coefficients of reactions within integrated energy metabolism, implying that animals cannot easily evolve changed thermal specificity in response to rapid habitat change. A major reorganization of metabolism would need more time than the time scale of expected global change would allow.
We studied PGI in the alpine species Colias meadii. In ordinary electrophoresis, it seems to share electromorph alleles with the lowland species at both PGI and phosphoglucomutase, PGM, genes. However, high-resolution electrophoresis finds differences between PGI electromorphs of meadii and those of the lowland species. We improved our assay of enzyme thermal stability, and used it to address the question whether taxa in colder habitats have PGI variants of lower thermal stability, rendering them even more vulnerable to rapid global thermal change. C. meadii genotypes do indeed differ in stability among themselves and are, in general, lower in stability than those of lowland Colias. We have studied the basic kinetics of the common $C$. meadii genotypes and found that there is major heterozygote, genotype $2 / 3$, advantage in substrate binding affinity, $K_{m}$. Moreover, the more stable $2 / 2$ homozygote has a twofold disadvantage in $\mathrm{K}_{\mathrm{m}}$ vs the less stable 3/3 homozygote. As the genotypes do not differ greatly in $V_{\max }$, genotypic $V_{\max } / K_{m}$ ratios are dominated by the $K_{m}$ differences, leading to heterozygote advantage in this ratio. Therefore the kinetics/ stability tradeoff does apply to PGI of this distinct species. The functional differences successfully predict PGI heterozygote advantage in male mating success of C. meadii in the wild. Thus selection maintaining the polymorphism in this alpine species is parallel to that working on the lowland ones, and the global-change bind of reproductive effectiveness vs thermal resistance probably does apply with even stronger force to the more thermally sensitive alpine species.

We have begun a direct-manipulation experiment to translocate our study insects from cool habitats to high-air-temperature habitats, and monitor their genotype-specific responses in terms of changes in survival vs female fecundity. More insects must still be run to complete the design, but so far, the most thermally labile, but kinetically effective, genotypes do show increased mortality in this imposed heat stress, and the thermally most stable genotypes, while resisting this mortality factor as expected, do not recoup their reproductive disadvantage.

\section{CHANGES IN THE FLUX OF CARBON BETWEEN PLANTS AND SOIL MICROORGANISMS AT ELEVATED $\mathrm{CO}_{2}$ : PHYSIOLOGICAL PROCESSES WITH ECOSYSTEM-LEVEL IMPLICATIONS ZAK, DONALD, and PREGITZER, KURT UNIVERSITY OF MICHIGAN and MICHIGAN TECHNOLOGICAL UNIVERSITY \\ FY 1996225 \\ FY 1995120 \\ FY 1994135}

Keywords: trees, soil microorganisms, carbon cycling, nitrogen cycling 
Objectives: To quantifying the physiological mechanisms leading to increased belowground plant production, microbial biomass, and rates of nitrogen cycling under elevated atmospheric carbon dioxide.

Approach: We have grown trembling aspen (Populus tremuloides) under experimental conditions of atmospheric carbon dioxide (ambient and twice ambient) and soil nitrogen availability (low and high). The atmospheric carbon dioxide concentration was manipulated with the use of open-topped chambers and we used different native soils to modify soil nitrogen availability. After 2 years of growth under experimental conditions, we labeled plants with ${ }^{14} \mathrm{C}$ and ${ }^{15} \mathrm{~N}$ to trace the flow of carbon and nitrogen between plants and soil microorganisms.

Results to Date: Our results indicate that increased photosynthesis in the lower canopy leaves of trembling aspen growing under elevated atmospheric carbon dioxide is linked to increased rates of root production. After one year of growth under experimental conditions, atmospheric carbon dioxide did not significantly alter the biomass, activity, or community composition of soil microorganisms. Moreover, gross rates of nitrogen mineralization and microbial immobilization did not differ in the soil of plants grown under ambient and twice-ambient atmospheric carbon dioxide. In short, we found no evidence to suggest that increased belowground plant production under elevated carbon dioxide will slow the rate of nitrogen cycling in soil. Presently, we are analyzing the results of our ${ }^{14} \mathrm{C}$ and ${ }^{15} \mathrm{~N}$ labeling experiment.

\author{
GENETIC CONTROL MECHANISMS FOR \\ ADAPTIVE STRATEGIES TO ARID \\ ECOSYSTEMS \\ ZEIGER, EDUARDO \\ UNIVERSITY OF CALIFORNIA, LOS ANGELES \\ FY 1996150 \\ FY 1995155 \\ FY $1994 \quad 0$
}

Keywords: plant adaptations, stomatal conductance, elevated carbon dioxide, genetic control

Objectives: To detect and characterize physiological and genetic processes of plant adaptations to climate change, with emphasis on temperature and elevated carbon dioxide.

Approach: A historical series of Gossypium barbadense bred for heat resistance was used to relate genetically determined increases in stomatal conductance with enhanced heat resistance.

Results to Date: Natural populations of G. barbadense were found to have a range of stomatal conductances and carbon-isotope discrimination that can be related to prevailing temperatures in their original habitats. These findings indicate that the stomatal response to temperature could play a role in plant adaptations to their environment. Introgressed, nearly homozygous genotypes from a cross between $G$. hirsutum and $G$. barbadense were used to define a broad range of variation in stomatal conductance. RAPD markers for each genotype are available, and their potential use to screen for genetic variation of the stomatal response to temperature in natural populations is being assessed. A guard-cell DNA library has been completed. A putative molecular sensor of ambient carbon dioxide concentrations in guard cells is being investigated. 


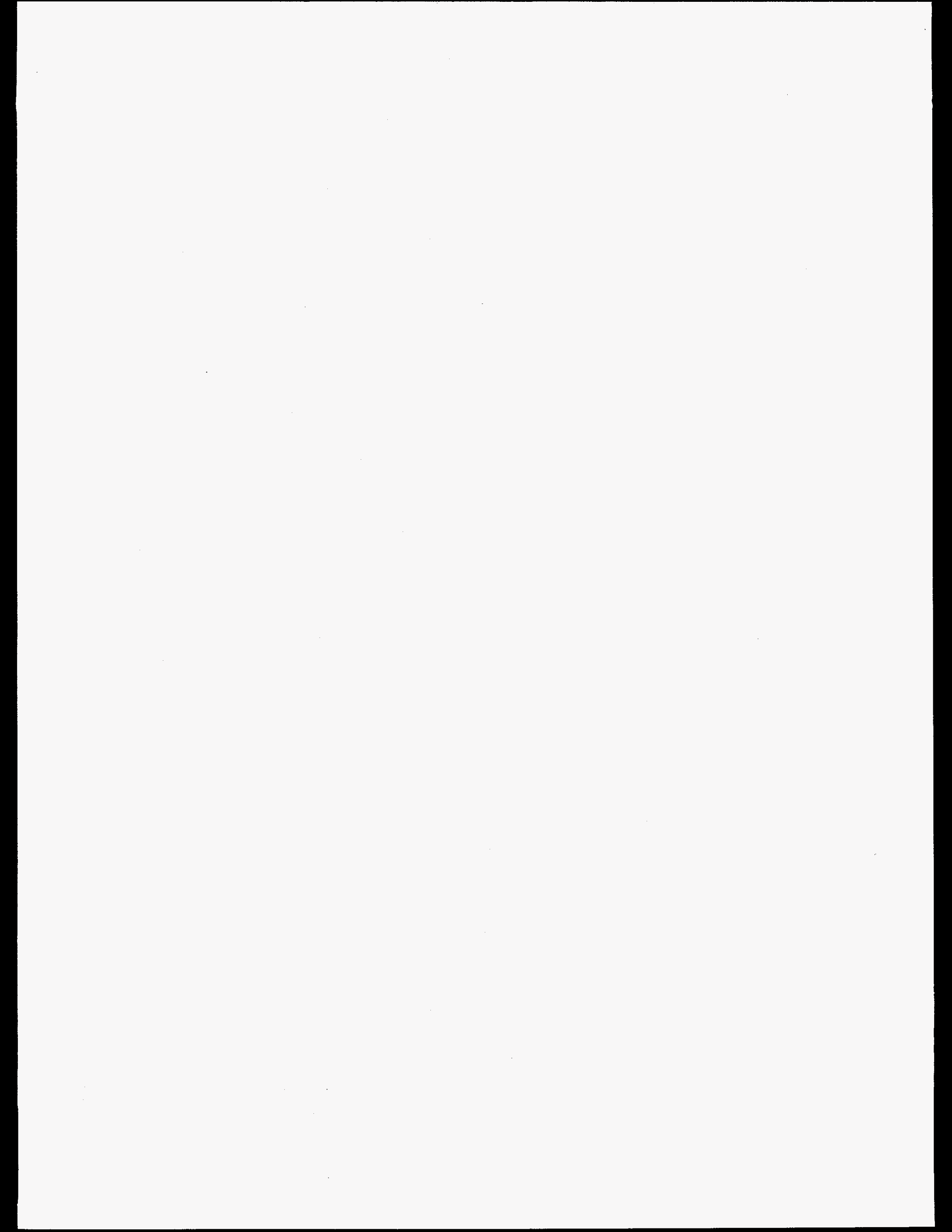




\title{
TERRESTRIAL CARBON PROCESSES
}

Research on terrestrial carbon processes investigates the global carbon cycle and the role that vegetation plays in that cycle. The carbon-cycle portion measures, models, and estimates changes in the physical, chemical, and biological processes controlling sources and sinks of atmospheric carbon dioxide, with an emphasis on the carbon processes of terrestrial ecosystems. The vegetation portion experiments on the response of terrestrial vegetation to excess $\mathrm{CO}_{2}$ and altered climate variables; the research augments studies of ecosystem function and response.

\author{
Program Manager \\ Roger C. Dahlman \\ Environmental Sciences Division \\ Department of Energy, ER-74 \\ 19901 Germantown Road \\ Germantown, MD 20874-1290 \\ (301) 903-4951 \\ Internet: roger.dahlman@oer.doe.gov
}

\section{EXPERIMENTAL AND THEORETICAL STUDIES OF THE ANNUAL EXCHANGE OF CARBON DIOXIDE AND ENERGY BY A TEMPERATE FOREST ECOSYSTEM \\ BALDOCCHI, DENNIS, and AMTHOR, JEFF \\ NATIONAL OCEANIC AND ATMOSPHERIC ADMINISTRATION and LAWRENCE \\ LIVERMORE NATIONAL LABORATORY \\ FY 1996250 \\ FY 199570 \\ FY 1994105}

Keywords: carbon dioxide exchange, temperate forests, micrometeorology, physiological ecology, drought, evaporation, AmeriFlux

Objectives: To quantify experimentally and theoretically the net annual fluxes of carbon dioxide and energy between a temperate broad-leaved forest and the atmosphere.

Approach: We are using the eddy-covariance method to measure $\mathrm{CO}_{2}$, water-vapor, and energy-flux densities on a near-continuous basis over a temperate, broadleaved forest ecosystem near Oak Ridge, Tennessee. This method allows us to measure mass and energy fluxes on hourly, daily, seasonal, yearly, and interannual time scales.

We are using the NOAA/ATDD CANOAK and the LLNL. Earth System models to integrate carbon and water-vapor fluxes of leaves and soil to the canopy scale. The models link mechanistic and physiological submodels for photosynthesis, respiration, and stomatal conductance with micrometeorological submodels that compute driving environmental variables at the surface of the leaf. The models are being used to help interpret field measurements and to integrate flux measurements in time and space.

Results to Date: Flux-covariance measurements of carbon dioxide and water vapor started on October 24, 1994, over the Walker Branch Watershed forest. In the interim, we have acquired more than 2 years of nearly continuous data.

The daily carbon dioxide fluxes of a temperate forest experience strong seasonality. During the leafless period, respiration by the boles and soil ranged between 0 and $3 \mathrm{gC} \mathrm{m}^{-2}$ day $^{-1}$. At leaf-out, the forest experienced a rapid transition from a system losing carbon to one gaining carbon (on a daily basis). Peak rates of net carbon assimilation occurred around day 150 and reached about $12 \mathrm{gC} \mathrm{m}^{-2} \mathrm{day}^{-1}$. Afterward, maximum daily carbon uptake gradually declined until leaf senescence. Then the forest system lost carbon dioxide again.

The long-term measurements are letting us explore the impact of growing season on annual carbon fluxes. The 1996 growing season was delayed by about 20 days, compared to the 1995 season. This delay seems to be causing the forest to take up about $90 \mathrm{gC} \mathrm{m}^{-2}$ less in 1996 as opposed to 1995 .

The long-term record is allowing us to examine episodic events. Forests in the southeastern United States experienced a prolonged dry spell and abovenormal temperatures during the 1995 growing season. When the soil profile was moist, net ecosystem $\mathrm{CO}_{2}$ exchange rates were a strong function of the amount of available sunlight and its diffuse-to-total fraction. During the early stages of the drought, high air temperatures limited canopy carbon dioxide fluxes, as leaf. 
soil, and bole respiration rates began to outpace photosynthesis. Later in the drought, the limited availability of soil moisture restricted the forest's ability to acquire carbon and transpire water.

With regard to modeling, accurate estimates of net ecosystem carbon and water exchange were attained when the forest was simulated as well-watered with standard model parameters. During the drought, only a multiplicative parameter of the stomatal-conductance model had to be adjusted with a water-stress index to obtain acceptable agreement between measured and calculated carbon and water fluxes; model parameters for leaf photosynthesis were held constant in accordance with physiological data. For this application, a water-stress index was defined as the ratio between cumulative precipitation and potential evaporation (after full leaf expansion).

\section{SYSTEM CARBON FLUXES AND SEQUESTRATION IN PONDEROSA PINE AT VARIED NITROGEN AND WATER AVAILABILITIES: A LONG-TERM FIELD EXPERIMENT AT ELEVATED $\mathrm{CO}_{2}$ BALL, J. TIMOTHY, and JOHNSON, DALE W. DESERT RESEARCH INSTITUTE \\ FY 1996300 \\ FY 1995300 \\ FY 19940}

Keywords: elevated $\mathrm{CO}_{2}$, soil nitrogen, forest carbon sequestration, ponderosa pine

Objectives: To determine in a forest system the extent to which soil nitrogen availability interacts with elevated $\mathrm{CO}_{2}$ to influence patterns and processes of ecosystem carbon sequestration.

Approach: To assess both the response of ponderosa pine systems to rising atmospheric $\mathrm{CO}_{2}$ and the carbon sequestration potential of this system, we have, for the past 4 years, been conducting in Placerville, California, a field experiment with open-top chambers that crosses three $\mathrm{CO}_{2}$ concentrations (ambient, $+175 \mathrm{ppm}$, and $+350 \mathrm{ppm}$ ) with three nitrogen treatments (background, $+10 \mathrm{gN} \mathrm{m}^{-2} \mathrm{y}^{-1}$, and $+20 \mathrm{gN} \mathrm{m}^{-2} \mathrm{y}^{-1}$ ). This experiment will be extended to cross two $\mathrm{CO}_{2}$ levels (ambient and $+350 \mathrm{ppm}$ ) with out low and middle nitrogen treatments (background and $+10 \mathrm{gN} \mathrm{m}^{-2} \mathrm{y}^{-1}$ ) and with two levels of water availability (plentiful and limiting).

Results to Date: The highest nitrogen treatment enhanced biomass by a factor of 1.8 in the first growing season but since has relaxed to a factor of 1.2. Nitrogen enhancement varied in parallel in the $\mathrm{CO}_{2}$ treatments, showing little evidence of multiplicative interaction. Growth enhancement because of $\mathrm{CO}_{2}$ began at a factor of 1.15 , increased to 1.5 as canopies closed, and has since relaxed to 1.2 with little evidence of resource interaction. Process measurements at several scales help explain why increased use-efficiencies at lower scales have not changed overall ecosystem $\mathrm{C} / \mathrm{N}$ relationships.

\section{PRESENT AND FUTURE CARBON BALANCE OF RUSSIA'S NORTHERN ECOSYSTEMS CHAPIN, F. STUART, III \\ UNIVERSITY OF CALIFORNIA, BERKELEY \\ FY 1996123 \\ FY 1995162 \\ FY 19940}

Keywords: Russia, tundra, boreal forests

Objectives: To document the seasonal pattern of $\mathrm{CO}_{2}$ flux in the major Siberian ecosystem types, to determine the environmental and biological correlates of $\mathrm{CO}_{2}$ flux, and to relate winter soil respiration to the soil energy budget.

Approach: Our approach is to measure $\mathrm{CO}_{2}$ flux with chamber and aerodynamic methods.

Results to Date: Ecosystem $\mathrm{CO}_{2}$ flux is in approximate annual balance in five major Siberian ecosystems, with net carbon gain in summer approximately balanced by winter respiration. Disturbance causes an increase in summer carbon gain and winter respiratory loss, leading to more pronounced seasonal amplitude of flux. Increasing disturbance could account for the increasing amplitude of atmospheric $\mathrm{CO}_{2}$ observed at high latitudes.

\section{THE EFFECT OF RISING ATMOSPHERIC $\mathrm{CO}_{2}$ CONCENTRATION ON CARBON ACCUMULATION AND NUTRIENT CYCLING IN TERRESTRIAL ECOSYSTEMS DRAKE, BERT \\ SMITHSONIAN INSTITUTION \\ FY 1996433 \\ FY 1995445 \\ FY $1994 \quad 50$}

Keywords: carbon sequestration, nutrient cycling, terrestrial carbon, photosynthesis, respiration, methane Objectives: To determine the effects of nutrient limitations on stimulation of carbon storage by elevated $\mathrm{CO}_{2}$ in a wetland on Chesapeake Bay and ascrub-oak ecosystem on Merritt Island Wildlife Refuge, Kennedy Space Center, Florida.

Approach: Stands of vegetation are surrounded by open-top chambers in which plants are exposed to one of two levels of atmospheric $\mathrm{CO}_{2}$ : normal ambient or elevated by $350 \mathrm{ppm} \mathrm{CO}_{2}$. Treatments continue 24 hours a day throughout the growing season, which is year-round for the Florida scrub-oak site and from late April to early November for the Maryland wetland site. The response of the following plant and ecosystem processes are determined periodically: photosynthesis, respiration, transpiration, tissue composition, and 
biochemistry for individual leaves; allocation of biomass to above- and belowground structures; pools and fluxes of carbon and nitrogen; concentration of mineral nutrients in soil; soil microbiology; insect/ plant interactions; soil respiration; and ecosystem water and carbon balance. Isotopic analyses of plants and soil will be used to locate pools of added carbon resulting from the elevated $\mathrm{CO}_{2}$ treatments.

Results to Date: Elevated- $\mathrm{CO}_{2}$-stimulated carbon accumulation have been observed for 9 years of elevated- $\mathrm{CO}_{2}$ treatment in the Chesapeake Bay wetland and 3 years of a pilot study in the scrub-oak ecosystem. This increased carbon accumulation resulted both from a stimulation of photosynthesis per unit leaf area and an inhibition of specific dark respiration $(\mathrm{g} / \mathrm{g})$. Although photosynthesis was stimulated an average of $50 \%$ in the wetland ecosystem, this occurred in conjunction with acclimation in the photosynthetic apparatus, which resulted in reduction of the amount of Rubisco in the leaves of elevated- $\mathrm{CO}_{2}$-treated plants of approximately $40 \%$. This loss of protein resulted in a decrease of leaf nitrogen concentration of approximately $20 \%$, and this influenced the plant/insect interactions on the wetland ecosystem. An important discovery is that the effect of elevated $\mathrm{CO}_{2}$ on dark respiration occurs through two, separate, independent effects: a direct inhibition of the respiratory activity of certain key respiratory enzymes of the electron-transport system and a reduction of activity associated with reduced amounts of the enzymes. The enzymes found to be sensitive to elevated $\mathrm{CO}_{2}$ include cytochromec-oxidase and succinate dehydrogenase. This effect is observed to occur in a very large fraction of the ecosystem respiration. Fine-root growth is stimulated in both the wetland and scrub-oak ecosystems; and in the scrub-oak community, elevated $\mathrm{CO}_{2}$ leads to an improved soil-water balance by reducing evapotranspiration. This effect is mediated by stomatal closure and by the fact that elevated $\mathrm{CO}_{2}$ did not significantly increase the leaf-area index over that for plants exposed to normal ambient $\mathrm{CO}_{2}$ concentration.

CMEAL: $\mathrm{CO}_{2}$ MODELS/EXPERIMENTS ACTIVITY FOR IMPROVED LINKS FIELD, CHRISTOPHER, and BALL, J. TIMOTHY CARNEGIE INSTITUTION OF WASHINGTON and DESERT RESEARCH INSTITUTE FY 1996150

FY 1995150

FY 19940

Keywords: elevated carbon dioxide, biogeochemistry models, metadata

Objectives: To test the ability of ecosystem models to correctly simulate ecosystem-scale responses to increased $\mathrm{CO}_{2}$.
Approach: The approach has two components. One emphasizes the analysis of experimental data to synthesize broad patterns across experiments. The other involves model sensitivity studies, comparing a range of models to see which processes are important and how different indirect effects change in importance with changes in environmental conditions.

Results to Date: We have completed the first phase of the metadata analysis and the first phase of the model intercomparisons. We have identified a number of important mismatches between the assumptions in the models and the results of the experiments. We have also identified a number of issues where the models and experiments are working on nonoverlapping time scales. Some of the most interesting results involve the importance of specific indirect mechanisms, including nutrient retranslocation and changes in stomatal conductance.

FOREST SOIL CARBON DYNAMICS

GARTEN, CHARLES, Jr. ; POST, MAC; COOPER, LEE; and HANSON, PAUL

OAK RIDGE NATIONAL LABORATORY

FY 1996239

FY 1995150

FY 19940

Keywords: forest soils, terrestrial carbon processes Objectives: To better understand forest soil carbon dynamics and thereby improve models used to predict the role of forest soils as a source or sink for increasing concentrations of atmospheric carbon dioxide and to determine how the turnover times of different soil carbon pools vary with forest ecosystem properties and climate in the southern Appalachian Mountains.

Approach: Six forest communities with different soil properties are being studied along an elevation gradient in the southern Appalachian Mountains. The sites include coniferous and deciduous forests over a range of soil properties, temperature, and moisture regimes. Natural carbon inputs in leaf litter fall have been supplemented with glycine that is highly enriched in a minor carbon isotope (carbon-13) or replaced with organic matter that is uniquely labeled with carbon-13. The carbon-13 tracer can be followed through time to evaluate the fate of carbon inputs and to estimate the turnover rates of different soil-carbon pools. Physicalseparation methods that lead to separate and distinguishable forms of soil organic matter are being tested to determine their adequacy for describing the fate of carbon inputs to forest soils.

Results to Date: Study sites were established and characterized during the first year of work. The carbon concentration in the silt-clay fraction increased from 1 to $2 \%$ at low-elevation sites to 5 to $8 \%$ at the highelevation sites. 
Autumn litter fall varied from 13.4 to $18.2 \mathrm{mg}$ dry matter $/ \mathrm{cm}^{2}$. Root mass varied from 0.4 to $13.7 \mathrm{mg} / \mathrm{g}$ soil. There were strong site differences in the quality of aboveground litter-fall inputs; the $\mathrm{C} / \mathrm{N}$ ratio of litter fall varied from 34.3 to 83.0. Carbon-13 abundance in litter fall decreased from $-29.10 \%$ at low elevations to $-27.63 \%$ at high elevations. High-elevation soils are more acidic and exhibit higher rates of net nitrogen mineralization potential than low-elevation soils. There is a strong elevation gradient in organic matter mass and carbon content. Organic-matter carbon increases from $56 \mathrm{mg} / \mathrm{cm}^{2}$ at low elevations to $270 \mathrm{mg} / \mathrm{cm}^{2}$ at high elevations. Climate data indicate a $6^{\circ} \mathrm{C}$ difference in soil temperature between low- and high-elevation sites. High-elevation soils are also wetter. Our preliminary estimates for organic-matter carbon-turnover times range from 3 to 15 years.

\section{FOREST-ATMOSPHERE CARBON TRANSFER AND STORAGE-I (FACTS-I) HENDREY, GEORGE \\ BROOKHAVEN NATIONAL LABORATORY \\ FY 19962575 \\ FY 19951680 \\ FY 19941169}

Keywords: forest-atmosphere exchange of $\mathrm{CO}_{2}$, FACE, down-regulation, loblolly pine

Objectives: To quantify processes regulating forest carbon balance at scales of integration ranging from the molecular to the forest ecosystem and to provide integrated, verified models of processes regulating carbon exchange between the forest and the atmosphere, and carbon storage within the forest ecosystem. Approach: Six plots in a 14-year-old loblolly pine plantation have been equipped for free-air carbon dioxide enrichment (FACE) experiments. The plots are $30 \mathrm{~m}$ in diameter, and the stand of trees is currently $12 \mathrm{~m}$ tall. Three of the FACE-equipped plots will receive continuous fumigation with $\mathrm{CO}_{2}$ at $200 \mathrm{ppm}$ above the present ambient concentration. Three other plots, the control plots, will not be enriched but are otherwise treated identically to the enriched plots. Enrichment will occur 24 hours a day throughout the year. This research will quantify processes regulating carbon sequestration under current ambient atmospheric $\mathrm{CO}_{2}$ concentrations and at the elevated concentrations expected to prevail in the next century. The models will provide a means for linking process-level information to forest-stand, regional, and global scales. Results to Date: This project began in the Spring of 1995. A site for the FACTS-I FACE facility was selected in the Duke Forest, in North Carolina, and extensive surveys of vegetation and soils were carried out to facilitate selection of the experimental plots. Engineering work was carried out on the FACE Forest Prototype system in the same forest location to provide a final design for the FACE equipment. In FY 1996, the FACTS-I facility was constructed and tested; the full experiment began at dawn on August 27, 1996. We have initiated measurements of tree physiology at subcellular to whole-tree levels, conducted micrometeorological measurements to quantify carbon fluxes, and continued the development of a set of models describing processes regulating the fluxes of carbon between a forest ecosystem and the atmosphere.

\section{CARBON DIOXIDE FLUX MEASUREMENT NETWORK \\ HOLLINGER, DAVID \\ USDA FOREST SERVICE \\ FY 199698 \\ FY 19950 \\ FY 19940}

Keywords: AmeriFlux, eddy flux, science team, program management

Objectives: To establish a flux network science team and science team chair to ensure comparability among established and planned $\mathrm{CO}_{2}$ flux-measurement sites in the United States and to transform the independent stations into a flux network.

Approach: The Science Team will provide information, guidance, quality assurance and control, and data exchange to help develop a network of flux-measurement stations. The Science Team will help in site selection, hardware and software selection, calibration, intercomparison, data archiving, and synthesizing network results.

Results to Date: Meetings have been held with representatives from candidate sites to decide on network organization and standards.

\section{CONTROLS ON SOIL CARBON RETENTION UNDER ELEVATED ATMOSPHERIC $\mathrm{CO}_{2}$ JASTROW, JULIE D.; MILLER, R. MICHAEL; BOUTTON, THOMAS W.; and RICE, CHARLES W. \\ ARGONNE NATIONAL LABORATORY, TEXAS A\&M UNIVERSITY, and KANSAS STATE UNIVERSITY \\ FY 1996200 \\ FY 1995200 \\ FY 19940}

Keywords: terrestrial carbon processes, elevated- $\mathrm{CO}_{2}$, soil carbon dynamics, soil organic matter, tallgrass prairie

Objectives: To better understand and quantify the processes involved in soil carbon storage and turnover, which are essential for determining the potentialcarbon-sink strength of terrestrial ecosystems.

Approach: We are using physical and biological fractionation techniques to identify and quantify measurable soil carbon pools that may be used to better 
parameterize simulation models of soil-organic-matter dynamics and to determine whether the dynamics of these measurable carbon pools are modified under elevated atmospheric concentrations of $\mathrm{CO}_{2}$. This study will both investigate the potential effects of elevated atmospheric $\mathrm{CO}_{2}$ on soil-carbon dynamics and help to validate and improve the estimates of carbon pools and fluxes used in simulation models of soil organic matter, allowing extrapolation of results to the broader scales necessary to predict the role of terrestrial ecosystems in the global carbon cycle.

Results to Date: Soil carbon accrual in reconstructed tallgrass prairie occurred in the heavy fraction (more than $1.85 \mathrm{~g} \mathrm{~cm}^{-3}$ ) rather than in the light fraction (1.85 $\mathrm{g} \mathrm{cm}^{-3}$ or less) released by dispersion of macroaggregates with relatively low ultrasonic energy. This information suggests that carbon was accumulating as organic cores of undispersed stable microaggregates within macroaggregates or as microbially produced mineral-associated complexes. The natural abundance of stable carbon isotopes following a change in the photosynthetic pathway of vegetation was used to estimate input rates and turnover of organic carbon in soil aggregates. The turnover time of old carbon was 412 years for microaggregates but averaged 140 years in macroaggregates, indicating that old carbon in microaggregates may be both biochemically recalcitrant and physically protected. Net inputs of new carbon generally increased with aggregate size. However, net carbon inputs to microaggregates were essentially equal to those for small macroaggregates, suggesting that microaggregate formation and degradation may be more dynamic than expected and that significant intermediate-term carbon accrual may be occurring in microaggregates as well as in macroaggregates. Physical and biological fractionations of soils from the elevated-CO $\mathrm{CO}_{2}$ field experiment at Kansas State University have been initiated.

\section{A STUDY OF THE ABUNDANCE AND ${ }^{13} \mathrm{C} /{ }^{12} \mathrm{C}$ RATIO OF ATMOSPHERIC CARBON DIOXIDE TO ADVANCE THE SCIENTIFIC UNDERSTANDING OF TERRESTRIAL PROCESSES REGULATING THE GLOBAL CARBON CYCLE KEELING, CHARLES D. \\ SCRIPPS INSTITUTION OF OCEANOGRAPHY FY 1996290 \\ FY 1995290 \\ FY 1994276}

Keywords: $\mathrm{CO}_{2}$ exchange, terrestrial carbon, carbon-13/carbon-12 ratio, carbon cycle

Objectives: Establish quantitative relationships and mechanisms that affect secular trends and interannual variations in atmospheric $\mathrm{CO}_{2}$, including prediction of the role of the terrestrial carbon cycle.
Approach: The concentration and ${ }^{13} \mathrm{C} /{ }^{12} \mathrm{C}$ ratio of atmospheric $\mathrm{CO}_{2}$ will be measured at an array of stations and at rural vegetated sites.

Results to Date: Measurements made on air collected at our array of 10 stations from the Arctic to the South Pole indicate that the concentration of atmospheric $\mathrm{CO}_{2}$ has been rising for 3 years at the rate expected from rises in earlier decades. Integrated over the record period since 1989 , the $\mathrm{CO}_{2}$ concentration appears, however, to reflect about four billion tons of carbon unexpectedly removed from the atmosphere between 1989 and 1993, when the rate of rise was unusually slow. This four billion tons has not since been returned to the atmosphere. Isotopic measurements indicate that this removal was mostly caused by the terrestrial biosphere. We propose that it reflects an increase in net primary production of vegetation only partially compensated for by increases in respiration of $\mathrm{CO}_{2}$. Recent comparison of our data with remotely sensed data of the Earth's greenness suggests that much of the removal has occurred in the latitude band from about 50 to $70^{\circ} \mathrm{N}$, with some additional uptake occurring in the tropics. A 20 to $30 \%$ increase in amplitude of the seasonal cycle of $\mathrm{CO}_{2}$ at our high latitude stations and an advance in the timing of the $\mathrm{CO}_{2}$ drawdown since the early 1970s suggests that the growing season has lengthened at high latitudes, consistent with observed warming in the spring at these latitudes.

\section{SOIL CARBON STORAGE AND $\mathrm{N}_{2} \mathrm{O}$ EMISSIONS FROM WHEAT \\ AGROECOSYSTEMS AS AFFECTED BY \\ FREE-AIR $\mathrm{CO}_{2}$ ENRICHMENT (FACE) AND NITROGEN TREATMENTS \\ LEAVITT, S. W.; THOMPSON, T.; MATTHIAS, A.; RAUSCHKOLB, R.; and CHO, $\mathrm{H}$. UNIVERSITY OF ARIZONA \\ FY 1995490 \\ FY 1994440 \\ FY $1994 \quad 0$}

Keywords: wheat, carbon exchange, soil carbon sequestration, agroecosystems, FACE

Objectives: To determine the influence of elevated $\mathrm{CO}_{2}$ concentrations on wheat carbon-exchange processes, biomass accumulation, and yield; on soil carbon exchange and sequestering; and on $\mathrm{N}_{2} \mathrm{O}$ emissions and to determine the interactive effects of soil nitrogen content with the different $\mathrm{CO}_{2}$ levels on the wheat agroecosystem.

Approach: Wheat will be maintained in an open field with four control and $\mathrm{CO}_{2}$-enriched plots, where the enriched plots will receive free-air $\mathrm{CO}_{2}$ enrichment (FACE) at $200 \mathrm{ppm}$ above ambient by means of a computer-controlled delivery system. For the two growing seasons of experimentation, two soil-nutrientnitrogen levels will be imposed by applying ample and 
very low levels of fertilizer with a split-plot design. Biomass accumulation will be monitored in the core experiment through above- and below-ground plant sampling during the growing season. Plant and soil carbon-exchange rates, as well as soil organic carbon content, will also be measured. Soil nutrient and water status with depth will be monitored for comparison among plot pairs, for identifying potential remedial actions within or between seasons, and for establishing whether any other nutrient may have become limiting. Specific, sensitive experiments will use the low ${ }^{13} \mathrm{C} /{ }^{12} \mathrm{C}$ ratios of the tank $\mathrm{CO}_{2}$ as a tracer to quantify the flow of carbon from plants into the soil organic carbon pools. A pure ${ }^{13} \mathrm{CO}_{2}$ tracer applied within the control plots will allow similar tracing of carbon accumulation under parallel control conditions without raising $\mathrm{CO}_{2}$ concentrations. The difference in tracer accumulation between control and enriched plots will represent real increases in carbon sequestration under high $\mathrm{CO}_{2}$. Tracer ${ }^{15} \mathrm{~N}$ applied to subplots will permit determination of fertilizer nitrogen recovered in plants and soils, and $\mathrm{N}_{2} \mathrm{O}$ from soils will be measured in subplots to determine magnification or reduction effects of enriched $\mathrm{CO}_{2}$ treatments on $\mathrm{N}_{2} \mathrm{O}$ emission from wheat agroecosystems. Fluxes, limiting factors, and synergisms in response to $\mathrm{CO}_{2}$ enrichment will be studied. Results to Date: No results were reported.

\section{TEMPERATURE AND $\mathrm{CO}_{2}$ INTERACTIONS IN TREES \\ NORBY, RICHARD J.; O'NEILL, ELIZABETH G.; WULLSCHLEGER, STAN D.; EDWARDS, NELSON T.; GUNDERSON, CARLA A.; and GARTEN, CHARLES T. OAK RIDGE NATIONAL LABORATORY \\ FY 1996654 \\ FY 1995550 \\ FY 1994428}

Keywords: terrestrial carbon processes, sugar maple, red maple, elevated $\mathrm{CO}_{2}$, open-top chambers

Objectives: To describe the processes involved in carbon cycling and sequestration by forests from the perspectives of tree growth and physiology, soil processes, decomposition, and biotic interactions.

Approach: The effects of elevated $\mathrm{CO}_{2}$ and elevated mean air temperature on growth and physiological processes in red maple and sugar maple are being measured in trees that were planted in 12 open-top chambers and maintained at ambient or elevated $\mathrm{CO}_{2}$ combined with ambient or elevated air temperature. The responses of processes that control or influence carbon sequestration in ecosystems are being determined, including fine root production and turnover; nitrogen mineralization; nitrogen and carbonate leaching; $\mathrm{CO}_{2}$ efflux from soil; ${ }^{13} \mathrm{C}$ sequestration and flux; herbivore consumption of leaves; and tissue quality and decomposition of leaves, fine roots, and wood. The differences in response of red maple and sugar maple will be evaluated in relation to the different natural ranges of the two species and their different ecological amplitudes.

Results to Date: Autumn leaf senescence was delayed in response to elevated temperature; spring bud burst was delayed in elevated temperature after an unusually warm winter, but was accelerated after a cold winter. There were no phenological adjustments in response to elevated $\mathrm{CO}_{2}$. Elevated $\mathrm{CO}_{2}$ increased aboveground growth of both species, and elevated temperature depressed growth, with a larger negative effect in sugar maple. Photosynthetic enhancement by elevated $\mathrm{CO}_{2}$ was sustained for two years. The effects of high temperature on aboveground growth were not completely explained by photosynthesis and appear to be more related to developmental patterns. Respiration of red maple roots was significantly lower in elevated temperature, probably reflecting decreased root mass. Nitrate concentration in soil water was significantly higher in elevated temperature, which probably reflects the lower root mass and decreased nitrogen uptake, since there have been no indications of changes in nitrogen mineralization or soil microbial activity. Nevertheless, foliar nitrogen content per unit leaf area increased in elevated temperature and was not affected by $\mathrm{CO}_{2}$ concentration.

\section{COLLABORATION: EFFECTS OF ELEVATED \\ $\mathrm{CO}_{2}$ ON ROOT DYNAMICS AND ROOT FUNCTION IN A MOJAVE DESERT ECOSYSTEM \\ NOWAK, ROBERT S. UNIVERSITY OF NEVADA, RENO FY 1996182 FY 19950 FY 19940}

Keywords: fine-root productivity, root function Objectives: To determine the effects of elevated atmospheric $\mathrm{CO}_{2}$ on belowground fine-root productivity, fine-root turnover, and root function in an an intact desert ecosystem.

Approach: Fine-root dynamics and function will be measured in the Desert FACE Experiment.

Results to Date: New project.

PROCESSES AFFECTING CARBON FLUXES
OF GRASSLAND ECOSYSTEMS UNDER
ELEVATED CO
OWENSBY, CLENTON; HAM, JAY; RICE,
CHARLES; and KNAPP, ALAN
KANSAS STATE UNIVERSITY
FY 1996255
FY 1995265
FY $1994 \quad 50$


Keywords: tallgrass prairie, $\mathrm{CO}_{2}$ flux, water vapor flux, elevated $\mathrm{CO}_{2}$, terrestrial carbon

Objectives: To determine the impact of elevated $\mathrm{CO}_{2}$ on carbon and water-vapor fluxes of a tallgrass prairie ecosystem under varying water status and to determine the impact of long-term $\mathrm{CO}_{2}$ fumigation on nutrient cycling and carbon storage in soil.

Approach: Research will be conducted on an intact grassland with open-top chambers of sufficient size to allow for direct measurement of above- and belowground processes affecting the carbon cycle. Continued fumigation of our sites with high $\mathrm{CO}_{2}$ will ensure that changes in carbon pools can be measured against the substantial carbon background in grassland soils. By manipulating water availability in a separate set of fumigation chambers, we will explicitly test our hypothesis that $\mathrm{CO}_{2}$-induced stomatal closure, which decreases water use and creates more favorable plantwater relations in $\mathrm{CO}_{2}$-enriched plots, will be most important during periodic drying cycles. Because previous research at this site has shown that increased soil carbon storage under elevated $\mathrm{CO}_{2}$ is affected by nitrogen availability, the impact of elevated $\mathrm{CO}_{2}$ on carbon storage and nitrogen cycling in a grassland ecosystem will be determined. We will chemically, physically, and biologically fraction the soil organic carbon and nitrogen pools of plots exposed to elevated $\mathrm{CO}_{2}$ for 8 years. ${ }^{15} \mathrm{~N}$ will be added to trace nitrogen in the different soil nitrogen fractions to provide data for modeling of soil organic matter pools. We will measure carboxylation-enzyme concentration in ambient and double ambient $\mathrm{CO}_{2}$ environments. Measurements will be used to assist in the development and verification of both local and mesoscale models of $\mathrm{CO}_{2}$ effects on grassland ecosystems.

Results to Date: Net carbon exchange has been greater under elevated $\mathrm{CO}_{2}$, and water-vapor fluxes have been lower. The result has been increased biomass production under elevated $\mathrm{CO}_{2}$ when water stress has been present.

Long-term incubations of soils indicate increased carbon sequestered in the elevated $\mathrm{CO}_{2}$ plots. Regrowth potential is increased under elevated $\mathrm{CO}_{2}$. Soil water levels have been greater under elevated $\mathrm{CO}_{2}$.

\section{MEASUREMENT OF THE POOL SIZES AND FLUX RATES OF SOIL ORGANIC FRACTIONS REQUIRED FOR MODELING AND DECISION MAKING PAUL, ELDOR A. MICHIGAN STATE UNIVERSITY FY $1996 \quad 80$ FY 19950 FY 1994164}

Keywords: soil organic matter, soil management
Objectives: To use tracers $\left({ }^{13} \mathrm{C}\right.$ and $\left.{ }^{14} \mathrm{C}\right)$ and wellmanaged long-term sites to determine the kinetics of soil organic matter relative to global change.

Approach: The signal from a free atmosphere carbon enrichment (FACE) site in Arizona and a $\mathrm{CO}_{2}$ site enclosed in chambers in California will be used to extend our knowledge of the active and slow pools as well as to carbon date the resistant pools. We will analyze our corn-belt sites to determine the effects of soil texture (sand, silt, and clay) and soil aggregates on the ${ }^{13} \mathrm{C}$ signal. The $\mathrm{C} 3-\mathrm{C} 4$ plant switch in continuous corn plots on a previously forested site provides the necessary tracer to follow the ${ }^{13} \mathrm{C}$ kinetics through the soil organic matter.

There are two isotopes of carbon, ${ }^{14} \mathrm{C}$ and ${ }^{13} \mathrm{C}$, that can be used to measure soil organic matter dynamics. ${ }^{14} \mathrm{C}$ is used in carbon dating together with acid hydrolysis to measure the dynamics of the old resistant fraction. ${ }^{13} \mathrm{C}$, either from a $\mathrm{C} 4-\mathrm{C} 3$ plant switch or from enhanced $\mathrm{CO}_{2}$ experiments, provides the short-ierm tracer. These techniques, together with extended incubation, provide information on the active and slow pool. The use of well-managed long-term field sites provides a tracer signal for field verification and modeling. It also provides information on plant-residue inputs and management feedbacks. Modeling of the tracer carbon will improve our predictive capabilities. Results to Date: We have obtained excellent ${ }^{13} \mathrm{C}$ measurements from ten field sites. These results show a large site effect on soil-organic-matter (SOM) dynamics. Lacustrine sites with a high clay content show a large, old, soil organic matter pool and little corn rooting at depth. Roots are of greater significance in well-drained, fine-textured soils. ${ }^{14} \mathrm{C}$ dating is giving some problems with unknown contamination, but acid hydrolysis is giving very meaningful data on the size of old resistant pools. Laboratory incubation uses microbial enzymes to analytically determine the active and slow pool. We are finding the active pool to represent a small fraction of organic matter present for a few months. The slow pool was determined by $\mathrm{CO}_{2}$ evolution and curve fitting to represent carbon persisting in the soil for 10 to 90 years, depending on the site. This value is highly correlated with the turnover time of noncorn carbon determined with ${ }^{13} \mathrm{C}$ in field experiments. ${ }^{13} \mathrm{C}$ can differentiate corn from noncorn carbon where corn has been continuously grown on a previous C3 site.

JOINT CMEAL/VEMAP WORKSHOP
PETERSON, BRUCE
MARINE BIOLOGICAL LABORATORY
FY 199625
FY $1995 \quad 25$
FY $1994 \quad 0$


Keywords: $\mathrm{CO}_{2}$ fertilization, nutrient cycling, wholeecosystem modeling, plant physiology

Objectives: To improve our understanding of the interaction between nutrient cycling and whole-plant physiological responses to elevated $\mathrm{CO}_{2}$.

Approach: We will hold a workshop that will bring together groups involved in $\mathrm{CO}_{2}$ fertilization experiments, whole-ecosystem modeling, and whole-ecosystem flux measurements. Invited participants will include groups associated with the VEMAP and CMEAL projects. First, we will examine the mechanisms identified in $\mathrm{CO}_{2}$ studies at the plant level and their incorporation into models. Second, we will evaluate the use of whole-system measurements in testing models.

Results to Date: No results were reported.

\section{EFFECTS OF ELEVATED $\mathrm{CO}_{2}$ ON ROOT DYNAMICS AND ROOT FUNCTION IN A MOJAVE DESERT ECOSYSTEM PHILLIPS, DONALD L.

Keywords: fine-root dynamics, root function Objective: To determine the effects of elevated atmospheric $\mathrm{CO}_{2}$ on belowground fine-root productivity, fine-root turnover, and root function in an intact desert ecosystem.

Approach: Fine-root dynamics and function will be measured in the Desert FACE Experiment.

Results to Date: New project.

GLOBAL CARBON CYCLE STUDIES
POST, WILFRED; KING, ANTHONY; DRAKE,
JOHN; and WULLSCHLEGER, STAN
OAK RIDGE NATIONAL LABORATORY
FY $1996 \quad 650$
FY 1995600
FY 19941164

Keywords: terrestrial ecosystem carbon, biogeochemical cycles, carbon sequestration

Objectives: To develop methods for evaluating the significance of terrestrial carbon processes in the past and future evolution of the global carbon cycle and for carbon-sequestration analysis in relation to potential policy options.

Approach: We have assembled appropriate global databases, developed an integrated framework, and coded a global terrestrial model that provides the basis for a global terrestrial-carbon-modeling framework. This model framework is modular in construction so that various components may be substituted with new ones, as required, to evaluate the global-scale influ- ences of research findings and new hypotheses. We have developed an improved photosynthesis component that includes standard photosynthesis equations, and a standard representation of ecosystem nitrogen cycling consistent with the soil-carbon-dynamics component.

We will use this modeling framework, in collaboration with experimentalists, to explore the impact of terrestrial-ecosystem responses to $\mathrm{CO}_{2}$ and climate variation on the global carbon cycle. In addition to providing a method of spatial and temporal extrapolation/interpolation to explore the global-carbon-cycle implications of various hypotheses, the modeling framework can also provide a means of cross-checking any proposed formulations. A global model of seasonal uptake and release of carbon coupled with an atmospheric transport model must be consistent with observed spatial and temporal patterns of seasonal changes in $\mathrm{CO}_{2}$ concentration. Carbon iso-topes can also be introduced, especially the critical discrimination during photosynthesis, and the extended model evaluated against spatial and temporal patterns of ${ }^{13} \mathrm{CO}_{2}$ in the atmosphere.

Results to Date: We have implemented a global model that contains simplified representations of plant and soil processes. In this model, the net primary production (NPP) of each grid cell is computed with a modification of the Miami model. Foley found that NPP predicted with the empirically based Miami model is highly correlated to global patterns of NPP predicted by a highly process-based model of NPP called DEMETER. NPP is partitioned among four plant parts (leaf, branch, stem, and root) with an adaptation of the model of Goudriaan and Ketner. Decomposition of litter and soil carbon is computed with an implementation of the Rothamsted model that produces output identical to the code obtained from Jenkinson. At equilibrium under current climate conditions, this model predicts an NPP of $56 \mathrm{PgC} \mathrm{yr}^{-1}$ vegetation carbon of $724 \mathrm{Pg}$, and active soil carbon of $1288 \mathrm{Pg}$ for a total ecosystem carbon of $2012 \mathrm{Pg}$. We investigated the response of NPP and carbon storage in vegetation and soil to a doubling of atmospheric $\mathrm{CO}_{2}$ and changes in climate projected by five atmospheric GCMs.

CARBON STORAGE AND CYCLING, SOIL
MICROBIOLOGY, AND WATER QUALITY IN
CO ${ }_{2}$-ENRICHED AGRO-ECOSYSTEMS
ROGERS, HUGO
ARS-USDANATIONAL SOIL DYNAMICS
LABORATORY
FY 199650
FY $1995 \mathbf{5 0}$
FY $1994 \quad 0$

Keywords: terrestrial carbon, carbon cycle, water quality, soil microbiology 
Objectives: To examine carbon and nitrogen dynamics (in relation to water quality) and soil microbiology in two $\mathrm{CO}_{2}$-enriched crop systems: grain sorghum (C4) and soybean (C3).

Approach: Atmospheric $\mathrm{CO}_{2}$ increase has important implications for terrestrial carbon processes. Plant growth is typically stimulated by elevated $\mathrm{CO}_{2}$ because $\mathrm{CO}_{2}$ is the substrate of photosynthesis and because water use efficiency increases. Enhanced growth, above and below the ground, immediately leads to hypotheses of changes in soil and rhizosphere microbiology (which mediate carbon flow within the soil) and alterations in water quality (i.e., more nutrient capture). To date, however, research linking belowground processes and carbon dynamics under increasing atmospheric $\mathrm{CO}_{2}$ has been limited. A 5-year study of belowground responses of the two crop species is under way. An open-top chamber system was installed on an outdoor soil bin ( $7 \mathrm{~m}$ by $76 \mathrm{~m}$ by $2 \mathrm{~m}$ deep) filled, in 1966, with Blanton loamy sand (loamy, silicious, thermic Grossarenic Paleudults) supported on a tile and gravel drainage basin. Sorghum bicolor (L.) Moench and Glycine max (L.) Merr. are being grown. These species offer contrasting characteristics with respect to photosynthetic pathways, responses to $\mathrm{CO}_{2}$, rooting patterns, nitrogen fixation, decomposition rates, and impact on soil carbon and nitrogen cycling; both are prominent the world over, including the Third World.

Results to Date: Elevated $\mathrm{CO}_{2}$ (for both species) had little effect on energy concentration, did not affect specific growth cost, but caused substantial increases in plant energy and growth per unit ground area. Transpiration was significantly reduced for both species. Early results indicate less nitrate movement to groundwater and, thus, better groundwater quality. Significant effects of $\mathrm{CO}_{2}$ on monthly assays of soil microbial activity, although infrequent, indicate higher activity under elevated $\mathrm{CO}_{2}$ during the growing season. Stable isotopic tracing $\left({ }^{13} \mathrm{C}\right.$ in $\mathrm{CO}_{2}$ supply source) has been demonstrated to be a feasible approach in the plant/soil systems being studied. Preliminary results suggest that mechanisms of soil carbon storage are different for the two species.

\section{SIMULATIONS OF THE CARBON CYCLE IN THE OCEAN \\ SARMIENTO, JORGE \\ PRINCETON UNIVERSITY \\ FY 1996149 \\ FY 1995345 \\ FY 1994344}

Keywords: ocean carbon

Objectives: To develop 3-D ocean and coupled oceanatmosphere models of the ocean carbon cycle, to develop a seasonal ecosystem model that couples nitrogen and carbon cycling, to perform seasonal simulations of carbon cycling, and to calculate anthropogenic carbon uptake by the ocean on basin and global scales.

Approach: Climate and seasonal variability change ocean dynamics, nutrient fluxes to the photic zone, and sea-air heat and water fluxes. The effect of these changes on the ocean carbon cycle and on anthropogenic carbon uptake by the ocean are examined with 3-D seasonal ocean models and coupled ocean-atmosphere models. Two approaches are used to account for the influence of biology on the carbon cycle. The first entails the development of a prognostic ecosystem model to produce realistic simulations of the response of ocean biota to changing nutrient inputs. The second is based on simulations in which a nutrient-restoring approach is used to predict new production.

Results to Date: Coupled ocean-atmosphere GCM greenhouse warming simulations suggest that changes in ocean circulation from atmospheric warming will dramatically decrease the rate at which oceans absorb anthropogenic carbon. The net southward oceanic transport of carbon across the Equator predicted by the ocean model is too small ( $0.3 \mathrm{PgC} / \mathrm{year})$ to support the large interhemispheric ocean transport of more than $0.9 \mathrm{PgC} / y$ ear that has been suggested by other investigators based on atmospheric GCMs. The ocean-model results are confirmed by analysis of observations and suggest that the interhemispheric transport in the atmosphere may be larger than previously thought. Simulations of the change in ocean $\delta^{13} \mathrm{C}$, coupled with estimates of fossil fuel emissions, atmospheric $\mathrm{CO}_{2}$, and $\delta^{13} \mathrm{C}$ and of the net change in the terrestrial biosphere ( $+0.3 \mathrm{PgC} /$ year), suggest that the size of the terrestrial biosphere that exchanges carbon with the atmosphere on a 20-year time scale is of the order of $2000 \mathrm{PgC}$.

\section{FOREST RESPONSE TO $\mathrm{CO}_{2}$ ENRICHMENT: PHYSIOLOGY AND ECOLOGY OF LOBLOLLY PINE \\ STRAIN, BOYD, and THOMAS, RICHARD \\ DUKE UNIVERSITY and WEST VIRGINIA UNIVERSITY \\ FY $1996 \quad 50$ \\ FY 1995407 \\ FY 1994436}

Keywords: loblolly pine, photosynthesis, stomatal conductance, water-use efficiency

Objectives: To examine the direct physiological effects of $\mathrm{CO}_{2}$ on plants with an emphasis on investigating the long-term effects of elevated concentrations of $\mathrm{CO}_{2}$ on loblolly pine.

Approach: Trees were grown for 4 years in open-top chambers in the field to examine physiological controls on plant response to $\mathrm{CO}_{2}$ enrichment. Emphasis was 
placed on feedback effects of the plant source-sink carbon balance on $\mathrm{CO}_{2}$ assimilation rate and plant growth. Measurements were made of net photosynthesis, transpiration, stomatal conductance, tissue nitrogen, carbon, and phosphorus contents, biomass produced in leaves, stems and roots, photosynthetic enzyme (rubisco) activities and concentrations, chlorophyll concentration, carbohydrate concentrations, leaf ultra structure, and wood anatomy and density. These data are being used in collaboration with James Reynolds and his GePSi model of plant growth and SERECO to examine ecosystem to improve modeling capabilities in a forest ecosystem.

Results to Date: Elevated $\mathrm{CO}_{2}$ increased net photosynthesis (60 to $130 \%$ in summer and 14 to $44 \%$ in winter), slightly decreased stomatal conductance and transpiration sufficiently to increase instantaneous water-use-efficiency, increased nitrogen and phosphorus to carbon ratios, decreased chlorophyll concentrations, decreased rubisco concentration and activity level, and affected leaf ultrastructure and wood anatomy.

Total plant biomass increased in response to elevated $\mathrm{CO}_{2}$. The absolute difference in tree mass between ambient and elevated-CO $\mathrm{CO}_{2}$-grown trees was $0.024 \mathrm{~kg}, 0.63 \mathrm{~kg}, 4.37 \mathrm{~kg}$, and $11.39 \mathrm{~kg}$ after one, two, three, and four seasons, respectively. After four growing seasons, elevated- $\mathrm{CO}_{2}$ trees were $10 \%$ taller, had $39 \%$ more leaf area, and had $90 \%$ more biomass than ambient $\mathrm{CO}_{2}$ trees. Allometric analyses of biomass allocation patterns demonstrated size-dependent shifts in allocation but not direct effects of elevated $\mathrm{CO}_{2}$ on partitioning of biomass. Diurnal oscillations in nitrogen and carbohydrate concentrations were associated with declines in net photosynthesis, but seasonal and annual increases in total plant growth indicated that morphological and physiological adjustments ameliorated the short-term differences observed. The bottom line is that loblolly pine may be a significant and increasing sink for atmospheric $\mathrm{CO}_{2}$ in the future.

\section{SCIENCE AND TECHNOLOGY REVIEWS OF FACE \\ STRAIN, BOYD R. DUKE UNIVERSITY \\ FY 199650 \\ FY 19950 \\ FY 19940}

Keywords: workshops and conferences

Objectives: To support the International FACE Science and Protocol Meeting.

Approach: A moeting will be held for FACE researchers to review issues, research approaches, facilities, and techniques.

Results to Date: New project.
INTERANNUAL VARIATIONS IN THE RATE

OF CARBON STORAGE BY A MIDLATITUDE FOREST

WOFSY, STEVEN, and GOULDEN, MICHAEL HARVARD UNIVERSITY

FY 1996160

FY 1995155

FY 19940

Keywords: net ecosystem exchange, interannual variations, AmeriFlux

Objectives: To measure the net uptake of $\mathrm{CO}_{2}$ by midlatitude forests, to define the mechanisms responsible for interannual variations in the net carbon exchange, to define the factors responsible for differences in the net carbon exchange between midlatitude deciduous forests and boreal/subboreal evergreen forests, and to establish the application of long-term eddy-correlation flux measurements in understanding the global carbon cycle.

Approach: We have made long-term measurements of net exchange of $\mathrm{CO}_{2}, \mathrm{H}_{2} \mathrm{O}$, heat, and momentum with the eddy-correlation method to measure mass and energy fluxes every hour for 6years. We have undertaken field experiments to define systematic errors in this method with independent means to measure components of the flux (e.g.,soil and bole chambers). Results to Date: The large areas of midlatitude deciduous forests are capable of removing significant amounts of $\mathrm{CO}_{2}$ from the atmosphere, 2 to 2.5 tonnes $\mathrm{C}$ per ha per year at our site. Carbon uptake in deciduous forests responds most strongly to temperatures in spring and winter, with warmer temperatures in those seasons leading to enhanced rates for carbon storage, which is contrary to expectations from ecosystem models and opposite to the response in boreal/ subboreal evergreen forests. The eddy-correlation flux method can define interannual variations in net carbon uptake to better than \pm 0.2 tonnes $C$ per ha per year. 


\section{ENVIRONMENTAL- REMEDIATION RESEARCH}





\section{ENVIRONMENTAL-REMEDIATION RESEARCH EXCLUDING SUBSURFACE SCIENCE}

The Environmental Remediation research portfolio is focused on developing an understanding of the fundamental physical, chemical, geological, and biological processes that must be marshaled for the development and advancement of new, effective, and efficient processes for the remediation and restoration of the nation's nuclear-weapon-production sites. A primary effort is a comprehensive research program in bioremediation that integrates the full range of fundamental scientific disciplines necessary to advance this emerging technology from one of hit-or-miss to one of sustained, cost-effective utililty. Operation of the Environmental Molecular Sciences Laboratory, the only national collaborative-user facility dedicated to the Department's environmental missions, is a key part of the Office 's commitment to environmental remediation.

\section{Program Managers}

Paul Bayer

Environmental Sciences Division

Department of Energy, ER-74

19901 Germantown Road

Germantown, MD 20874-1290

(301) 903-5324

Internet: paul.bayer@oer.doe.gov

D. Joy Grimes

Environmental Sciences Division

Department of Energy, ER-74

19901 Germantown Road

Germantown, MD 20874-1290
John C. Houghton

Environmental Sciences Division

Department of Energy, ER-74

19901 Germantown Road

Germantown, MD 20874-1290

(301) 903-8288

Internet: john.houghton@oer.doe.gov

Curtis R. Olsen

Environmental Sciences Division

Department of Energy, ER-74

19901 Germantown Road

Germantown, MD 20874-1290

(301) 903-5329

Internet: curtis.olson@oer.doe.gov

\section{NABIR PROGRAM OFFICE \\ BENSON, SALLY M. \\ LAWRENCE BERKELEY NATIONAL \\ LABORATORY \\ FY 1996225 \\ FY 19950 \\ FY $1994 \quad 0$}

Keywords: NABIR program, administrative support, newsletters

Objectives: To provide logistical, technical, and other support to the NABIR program in cooperation with two other national laboratories.

Approach: A web site, newsletters, conference planning, proceedings volumes, fliers, etc. will be developed and maintained.

Results to Date: New project.

\section{UTILIZATION OF GEMS FOR BIOREMEDIATION}

BURLAGE, ROBERT and SAYLER, GARY

OAK RIDGE NATIONAL LABORATORY and

THE UNIVERSITY OF TENNESSEE

FY 1996280

FY 1995340

FY 19940

Keywords: bioremediation, polyaromatic hydrocarbons Objectives: To study gene expression in situ in the presence of polyaromatic hydrocarbons (PAH).

Approach: A genetically engineered bacterium has been created that emits bioluminescence when naphthalene is bioavailable. A culture of this bacteria will be inoculated in soil that has been contaminated with naphthalene, phenanthrene, and anthracene. Bioluminescence will be detected through fiberoptic cables that are situated within the contaminated zone. Sensitive 
photomultipliers will read the signal on a continuous basis. Other important parameters (moisture content, temperature, oxygen concentration, etc.) will also be monitored. The experiment will continue for several months and will be accompanied by extensive controls. Results to Date: Regulatory approvals for this work have been obtained. All components of the experiment (fermentation facilities, soil and chemicals, photodetectors, etc.) have been identified and obtained. Readiness review has been scheduled.

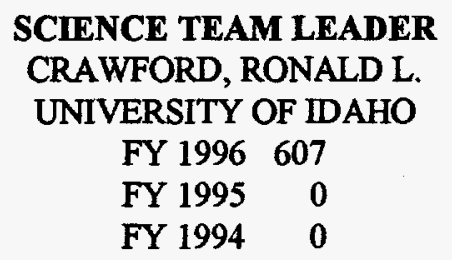

Keywords: administrative support, science team Objective: To develop scientific direction for research; assist in identifying research opportunities and directions (e.g., hold workshops and attend relevant meetings and colloquia); provide coordination among the investigators and with other NABIR elements; communicate research findings to relevant audiences; identify targets of opportunity and encouraging research directed at those targets; and conduct the research presented in the application and approved for funding. Approach: The leader has interests in biotransformation and biodegradation, acceleration, and biomolecular science and engineering. He is professor of microbiology at the University of Idaho, where he directs a research team in environmental biotechnology and is codirector of UI's Center for Hazardous Waste Remediation Research and the director of the University's Institute for Molecular and Agricultural Genetic Engineering. From 1987 to 1990 , he was head of the Department of Bacteriology and Biochemistry. Previously, he was professor of microbiology at the Gray Freshwater Biological Institute at the University of Minnesota, where he conducted research on environmental microbiology and hazardous-waste treatment. Results to Date: New project.

\section{EMSL INTERIM OPERATIONS DUNNING, THOM \\ PACIFIC NORTHWEST NATIONAL LABORATORY \\ FY 1996560 \\ FY 19950 \\ FY 19940}

Keywords: facility operations, Environmental Molecular Sciences Laboratory

Objectives: To provide the Environmental Molecular Sciences Laboratory (EMSL) with funding to support the availability of specific equipment and instrumentation to conduct collaborative science.
Approach: Funding was provided to the EMSL for operations and maintenance (including labor) to support the availability of the infrared spectroscopy facility (instrumentation for clusters and surfaces and associated pumps); an oxide-molecular-beam epitaxyforce-sensitive microscope, and a suite of ultrahighfield surface science and analysis capabilities; the 750- $\mathrm{MHz}$ nuclear-magnetic-resonance spectrometer, and the ONYX computer system for data analysis of liquid-state structures; and system administration to allow the high-performance computer system (192processor IBM RS/6000 SP2) to be made available 6 months earlier than planned.

Results to Date: EMSL researchers and external users of the equipment and instrumentation were able to conduct research with this equipment in the performance of Environmental Management Science Program research, as well as collaborative research.

\section{SUMMER EXPLORATORY WORKSHOP: APPLYING THE COLLABORATORY TO IMPROVE THE UNDERGRADUATE SCIENCE LABORATORY DUNNING, THOM \\ PACIFIC NORTHWEST NATIONAL LABORATORY \\ FY 199623 \\ FY 19950 \\ FY 19940}

Keywords: workshops, Environmental Molecular Sciences Laboratory

Objectives: To enable the Environmental Molecular Sciences Laboratory (EMSL) to develop, host, and participate in a 4-day workshop with eight northwestern colleges and universities and two other educational institutions to develop mechanisms to engage faculty in EMSL research projects and apply the collaboratory approach to classroom instruction, curriculum development, and undergraduate research experiences.

Approach: Electronic laboratories without walls, or collaboratories, can enable geographically distant people from government and industrial labs, colleges, and universities to work closely together, providing electronic access to remote expertise, information, and research facilities. Recognizing the powerful potential of the collaboratory concept, researchers at Pacific Northwest National Laboratory (PNNL) are developing an extensive suite of computer-based tools to support research programs and facilitate collaborative use of the Environmental Molecular Sciences Laboratory (EMSL). In an effort to extend access to EMSL's scientific expertise and technical resources, PNNL joined with others to form a consortium that would thoroughly evaluate the collaboratory as a means to enhance undergraduate education and research. The 
Collaboratory for Undergraduate Research and Education consortium includes representatives from the University of Washington, Evergreen State College, Portland State University, Reed College, Heritage College, Bellevue Community College, University of Montana, University of Wyoming, and the EMSL.

Results to Date: On October 17-19, 1996, a workshop funded under the DOE and National Science Foundation joint agreement in education was held at PNNL to develop a model for innovative higher-education improvement. Workshop participants included 16 faculty members from eight western colleges and universities, a representative of the Northwest Academic Computing Consortium, EMSL scientists, and specialists from PNNL's Science Education Programs. Workshop activities included hands-on training with EMSL's collaboratory capabilities, analysis of opportunities for curriculum enhancement, faculty development and research activities, and development of a framework for a joint DOE-NSF education proposal. A wealth of insight was gained, and many educationapplication scenarios were identified. In addition, several groups committed to immediately start developing example projects with the EMSL collaboratory tools.

\section{NABIR PROGRAM OFFICE GEHRS, CARL

Keywords: administrative support

Objectives: To provide logistical, technical, and other support to the NABIR program in cooperation with two other national laboratories.

Approach: A web site, newsletters, conference planning, proceedings volumes, fliers, etc. will be developed and maintained.

Results to Date: New project.

\section{BROAD RESEARCH PROGRAM ON THE SCIENCES OF COMPLEXITY GOLDBERG, ELLEN \\ SANTA FE INSTITUTE \\ FY 1996120 \\ FY 19950

FY 19940

Keywords: complex systems, adaptive computation Objective: To provide core support for the Santa Fe Institute.

Approach: The research consists of (1) modeling and theory of complex systems, and particularly complex adaptive systems, built around investigation of the concept of simple rules leading to complex behavior; (2) specific applications of that modeling and theory to research in adaptive computation; (3) applications of the modeling and theory, as well as the new tools developed from the Institute's research in adaptive computation, to science in general; and (4) applications of the modeling and theory, as well as emerging scientific insights, to understanding and modeling real-world problems.

Results to Date: New project.

\section{AUTOTROPHIC BIOFILMS FOR REMOVING CONTAMINANTS FROM INDUSTRIAL WASTEWATER HILL, W. R. \\ OAK RIDGE NATIONAL LABORATORY FY 1996102 FY 19950 FY $1994 \quad 0$}

Keywords: wastewater treatment, biofilms Objective: To develop naturally occurring autotrophic biofilms for use in removing organic and inorganic contaminants from dilute wastewater.

Approach: An integral part of this effort will be the development of novel, real-time sensors for dissolved contaminants; the sensors will be used in conjunction with the biofilm cleanup systems. Environmental and constitutive conditions affecting the capacity of autotrophic biofilms to sorb and actively take up metals and PAHs in industrial wastewaters will initially be quantified in small-scale mesocosms. Information gained in the mesocosm studies will be extrapolated and tested in large-scale artificial-stream channels that approximate realistic industrial applications. Optical and biotechnological sensors for organic contaminants (e.g., PAHs), and heavy metals (e.g., chromium and nickel) will be constructed and used to quantify contaminant removal by the biofilms. Development of these sensors will be of considerable benefit to industry regardless of the specific cleanup technology. Technological development of naturally occurring autotrophic biofilms has the potential to provide industry with a low-cost, effective means of reducing the concentrations of mixed environmental contaminants and recovering materials that can be recycled back into the production process.

Results to Date: New project.

INTEGRATION OF LIFE-CYCLE MODELING
WITH INDUSTRIAL-PRODUCT AND
PROCESS DESIGN
HUMPHREYS, KENNETH
PACIFIC NORTHWEST NATIONAL
LABORATORY
FY 199650
FY 19950
FY 19940


Keywords: life-cycle analysis, environmental impacts, energy efficiency

Objectives: To encourage and enhance the use of lifecycle assessment (LCA) in the evaluation products and processes in terms of their associated energy and environmental impacts.

Approach: Based on research needs clearly articulated by U.S. industry, the usefulness of existing life-cycle tools and techniques will be evaluated within the chemical, aluminum, steel, and automotive industries and basic methodological improvements will be defined that are needed to allow these tools and techniques to be applied most advantageously during industrial-product and process design. An existing LCA software tool will be integrated with an off-theshelf industrial-design tool (e.g., process model or CAD system) and, in partnership with the pulp and paper industry, an approach will be developed for integrating uncertainty analysis into LCA.

Results to Date: New project.

\section{SURFACE CHEMISTRY OF SUBSURFACE BASALT \\ INGRAM, JANI C. \\ IDAHO NATIONAL ENGINEERING \\ LABORATORY \\ FY 1996200 \\ FY $1995 \quad 0$ \\ FY $1994 \quad 0$}

Keywords: sorption, degradation, chlorinated organic compounds, basalt

Objectives: To identify mechanisms that control the sorption and degradation chemistry of chlorinated organic compounds in the subsurface.

Approach: Static secondary ion mass spectrometry (SIMS) will be used as the primary investigation tool along with Raman spectroscopy, scanning electron microscopy/wavelength dispersive spectroscopy (SEM/WDS), petrographic microscopy, and X-ray photoelectron spectroscopy (XPS). Direct information will be provided on the chemical reactivity of the basalt surface, which is critical to gaining insight into the mechanisms by which chlorinated organics are sequestered and/or degraded at the basalt surface.

Results to Date: Current efforts are centered on chemical characterization of the basalt surfaces with SIMS, SEM/WDS, and petrographic microscopy. Sorption experiments of trichloroethylene on the basalts have been planned. Some mineral separations have been performed on the basalts: plagioclase and olivine have been accomplished. Method development for detection of chlorinated organics on silicate surfaces by Raman spectroscopy is currently in progress.
ENHANCEMENT OF LIFE-CYCLE ANALYSIS WITH ECONOMIC INPUT-OUTPUT TECHNIQUES AND APPLICATION TO CHEMICAL AND FOREST-PRODUCT INDUSTRIES

LAVE, LESTER B.

CARNEGIE-MELLON UNIVERSITY FY 1996250

FY 19950

FY 19940

Keywords: life-cycle analysis

Objectives: To develop the ability to include environmental consequences in such decisions as the choice of alternative materials, designs, and manufacturing processes.

Approach: A life-cycle-analysis (LCA) tool will be used to analyze environmental impacts of industrial activities throughout the economy of a country. The LCA tool will include both detailed product- and process-specific and economy-wide environmental analyses. This new tool will be used to estimate the life-cycle implications for products and processes in forest-products and chemical companies.

Results to Date: New project.

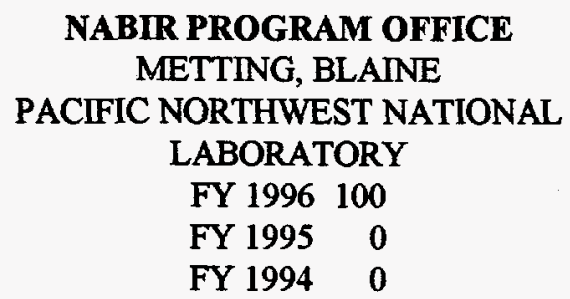

Keywords: administrative support

Objectives: To provide logistical, technical, and other support to the NABIR program, in cooperation with two other national laboratories.

Approach: A web site, newsletters, conference planning, proceedings volumes, fliers, etc. will be developed and maintained.

Results to Date: New project.

\author{
SOIL REMEDIATION STRATEGIES AND \\ ENDPOINTS: QUANTIFYING THE \\ AVAILABILITY OF AGED HYDROCARBONS \\ TO BIOATTENTUATION \\ PEYTON, BRENT \\ PACIFIC NORTHWEST NATIONAL \\ LABORATORY \\ FY 1996165 \\ FY 19950 \\ FY 19940
}

Keywords: biodegradation, mesoscale modeling

Objective: To quantify the fundamental chemical, physical, and microbiological processes that control the availability of hydrocarbons to biodegradation by microorganisms (bioavailability) and then to integrate 
these processes into a mesoscale model that can be used to assess the overall effects of these processes.

Approach: Cost-effective solutions are needed to determine whether, and how, to clean up low-levels of hazardous petroleum hydrocarbons in soils at active and former industrial sites. This research is expected to provide data on the fate and transport of low-level hydrocarbon contamination, and will help determine whether intrinsic or "no-action" solutions are effective for soils contaminated with low-levels of hydrocarbons.

Microscale measurements will be conducted to determine the physicochemical factors and microbial parameters affecting desorption rates and bioavailability of hydrocarbon contaminants in aged soils, where some fraction of a contaminant may be firmly sequestered in small pores within soil particles and aggregates or embedded or complexed in natural organic matter. Improved conceptual and numerical microscale models will be developed of the physicochemical factors and the microbial parameters controlling sequestration. The microscale models will be incorporated into a mesoscale model to more accurately assess the competitive and interrelated nature of contaminant release and biodegradation rates. And the mesoscale model will be confirmed by comparing the model results with those obtained from larger-scale, longterm experiments with aged soils and known microbial populations in soil columns.

Results to Date: New project.

\section{CONTROLLING MIGRATION AND BIODEGRADATION OF NAPLS IN FRACTURED ROCKS OF THE VADOSE ZONE \\ PRUESS, KARSTEN \\ LAWRENCE BERKELEY NATIONAL \\ LABORATORY \\ FY 1996175 \\ FY 19950 \\ FY 19940}

Keywords: nonaqueous-phase-liquid contamination, fractured rock, migration of contaminants, biodegradation

Objectives: To provide a scientific basis for field investigations, site characterization, and remedial action for nonaqueous-phase-liquid (NAPL) contamination in fractured rock.

Approach: Flow-visualization experiments in transparent replicas of natural rock fractures will be conducted to reveal infiltration characteristics of water and NAPLs. Biological activity will be introduced to examine effects on flow behavior from changing fluidand solid-phase properties. Quantitative experiments will probe the role of fracture topography and orientation and of fluid properties on flow behavior in fractured rock and phase partitioning of NAPL contami- nants. The fluid-dynamics investigations set the stage for the biodegradation studies because they determine the contaminant source term. The extent of biotransformation of organic contaminants originating from NAPLs will be measured in a fractured rock core. "Geocosms" will be used to validate stable isotope measurements as a potential field monitoring tool to characterize biotransformation processes.

Results to Date: Samples were obtained for microbiological work at the Test Area North (TAN) site at the Idaho National Engineering Laboratory (INEL). Fracture samples for abiotic flow studies were obtained from the TAN site, from Box Canyon, and from the USGS core library. A number of two- and three-phase flow-visualization experiments were conducted in transparent replicas of natural fractures and in semitransparent rock-replica combinations. Liquid flow was observed to proceed along localized flow channels in an intermittent manner, resulting from an interplay of capillary and gravity forces; this was confirmed through visualization experiments in various parallelplate assemblies. Sampling and culturing techniques were developed, and distributions of microbial organisms on basalt surfaces were measured. Large differences were found between fractures in vesicular and dense basalts. Design studies for a long-term geocosm experiment were performed. Analytical methods were developed and employed to measure isotopic ratios of organic liquids. These techniques will be used in the geocosm experiments to identify physical and microbial isotope fractionation processes. High-resolution simulations of water seepage in heterogeneous fractures were performed. These reproduced many of the features seen in the experiments, such as flow fingering, bypassing, and ponding but not the pervasive flow intermittency. An approximate invariance of seepage under simultaneous space and time scaling was theoretically derived and confirmed through numericalsimulation experiments.

\section{GAS TRANSPORT IN SOILS AND ITS RELATION TO RADON AVAILABILITY: HOT-SPOT IDENTIFICATION AND FLOW CHARACTERISTICS NEAR STRUCTURES REIMER, MICHAEL, and MORSE, GEROME COLORADO SCHOOL OF MINES FY 19960 \\ FY 1995140 \\ FY 1994150}

Keywords: radon, radon transport, soil gas Objectives: To provide empirical information related to developing a method to predict areas of the United States where homes are located that have the potential for the highest indoor radon concentrations.

Approach: Ground-tnuth measurement will be used to evaluate the confirmation of predictive parameters for 
estimating indoor radon concentrations. A series of measurements will focus on soil-gas concentrations and indoor radon measurements. Indoor measurements will use a variety of techniques, including short-term, long-term, continuous, and grab samples. In addition, cooperative studies with other researchers on the disturbance of a pristine site during construction will be evaluated to determine if predictive factors are valid for pre- and postconstruction activities.

Results to Date: Findings to date on in-home measurement indicate and confirm results of previous research that there is a significant difference in indoor radon concentrations related to the measurement protocol and specific device used. This finding indicates that action levels may be more realistic and informative if linked to the measurement protocol. Measurements indicate that even small indoor air exchanges in the basement during furnance cycling can have a significant effect on the indoor radon concentration. Further, open-air exchange with basement air, such as with using outdoor air for combustion, reduces the pressure differential between the structure and outside.

\section{ENVIRONMENTAL BIOTECH '96 SUPPORT RUFFIN, MORRIE

Keywords: conferences and workshops Objectives: To expand a successful biotechnology conference to include bioremediation objectives.

Approach: An extant, successful biotechnology conference will be expanded to include topics and presentations relative to bioremediation.

Results to Date: The workshop was held, and a session on bioremediation was included. Several papers were given relevant to the NABIR program. Department of Energy Assistant Secretary Grumbly gave a keynote address.

\section{FIELD RELEASE OF GENETICALLY ENGINEERED BIOLUMINESCENT REPORTER BACTERIA FOR PAH BIOREMEDIATION IN SUBSURFACE SOIL SAYLER, GARY, and DRAKE, JAMES THE UNIVERSITY OF TENNESSEE \\ FY 1996327 \\ FY 1995300 \\ FY 19940}

Keywords:polyaromatic hydrocarbons, bioremediation, bioluminescence, biodegradation, GEM release, Pseudomonas fluorescens
Objectives: To test in situ the PAH-biodegradation efficacy of Pseudomonas fluorescens in subsurface soil, to evaluate bioluminescence-reporting capability in environmental monitoring, and to develop a model ecological framework for the introduction of a GEM in a subsurface environment.

Approach: Soil microcosms will be investigated, advanced fiberoptic and optoelectronic bioluminescent biosensors will be developed, intermediate-scale bioremediation tests will be conducted in lysimeters, and essential documentation for regulatory compliance will be prepared.

Results to Date: The GEM was successfully introduced into and maintained in the subsurface with simultaneous biodegradation activity, efficient detection and real-time, online measurement of bioluminescence by biosensors was accomplished, and regulatory approval was received from the EPA for release of GEM under the TSCA statute.

\section{INVESTIGATION OF RADON, THORON, AND THEIR PROGENY NEAR THE EARTH'S SURFACE \\ SCHERY, STEPHEN, and WASIOLEK, PIOTR \\ NEW MEXICO INSTITUTE OF MINING AND TECHNOLOGY \\ FY 1996188 \\ FY 1995186 \\ FY 1994174}

Keywords: dry deposition, radon, thoron, decay products

Objectives: To improve understanding of the physical processes controlling concentrations of radon, thoron, and their progeny in the atmospheric environment and the dose delivered by them.

Approach: Experimental measurements will be made of the activity-weighted size distribution of radon and thoron progeny outdoors. The deposition velocity will also be measured for ultrafine particles with radondecay products as natural tracers. Models will be developed to interpret the data.

Results to Date: Work in 1996 concentrated on optimizing the sampling parameters of the relaxededdy accumulator (REA) and actual measurement of the atmospheric flux density for ultrafine particles (i.e., in the size range of 0.5 to $5 \mathrm{~nm}$ ). To optimize the collection efficiency of the REA system, 30 measurements of the activity-weighted size distribution of radon progeny were made at a field site near the New Mexico Tech campus. Activity median diameter (AMD) for the ultrafine fraction was $2.2 \mathrm{~nm}$, and activity geometric mean diameter (AGMD) was $1.6 \mathrm{~nm}$. Based on these results, the REA system was adjusted to achieve the highest collection efficiency for the ultrafine particles. A 635-wire-mesh screen with a 
flow rate of $268 \mathrm{Lpm}$ was selected, yielding a collection efficiency for $1.6-\mathrm{nm}$ particles of $78 \%$. Sampling $4 \mathrm{~m}$ above ground, 42 measurements of the ultrafine flux density were made at a site located $30 \mathrm{~km}$ west from Socorro. The average meteorological conditions during sampling were characterized by low relative humidity of $16 \%$, moderate wind speed, and air temperature of $21^{\circ} \mathrm{C}$. To achieve required alphacounting statistics, the sampling time was between 04:00 and 11:00 MST. A correction for zero mean vertical wind was applied to each sample. Preliminary results indicate increasing surface deposition with increasing horizontal wind. For stable nighttime conditions, the estimated deposition velocity was $1.3 \pm 0.4 \mathrm{~cm} / \mathrm{s}$, although this number is subject to a future correction for transient redistribution of the atmospheric radon profile. Experimental work continues to increase the precision of measurements and to obtain flux density data for a wider range of meteorological conditions.

\section{EXPERIMENTAL AND THEORETICAL INVESTIGATIONS OF RADON AVAILABILITY, MIGRATION, AND ENTRY SEXTRO, RICHARD G. LAWRENCE BERKELEY NATIONAL LABORATORY \\ FY $1996 \quad 90$ \\ FY 1995348 \\ FY 1994360}

Keywords: radon availability, radon migration, radon entry

Objectives: To phase down the experimental facility in the California Coastal Range.

Approach: Operations at the California Coastal Range facility will be closed out.

Results to Date: Phase-down in process.

\section{MICROBIAL GENOME SEQUENCING SMITH, DOUGLAS ROBERT GENOME THERAPEUTICS CORPORATION FY 1996400 \\ FY 1995415 \\ FY $1994 \quad 0$}

Keywords: genome sequences, microbial organisms, fluorescence sequencing techniques

Objectives: To decipher the complete genome sequences of microbial organisms that may be useful in energy production or bioremediation.

Approach: The sequencing is being done by a combination of computer-assisted multiplex and automated-fluorescence (ABI) sequencing techniques. The strategy is to generate approximately 8 -fold random coverage by a whole-genome shotgun phase followed by primer-directed gap-filling and finishing to produce a contiguous genome sequence. The first two genomes targeted for completion are the $1.7 \mathrm{Mb}$ genome the archeon Methanobacterium thermoautotrophicum $(\mathrm{H})$, a well-studied thermophilic methanogen that may be useful for methane production and detoxification of biowaste and the 4.1-Mb genome of Clostridium acetobutylicum (ATCC 824), a solventproducing organism with potential industrial applications for the production of acetone, butanol, and ethanol from plant byproducts.

Results to Date: No results were reported.

\section{PCB DESTRUCTION AND MOBILITY REDUCTION BY BIOSTABILIZATION SMITH, JOHN R. ALUMINUM COMPANY OF AMERICA FY 1996384 \\ FY 19950 \\ FY 19940}

Keywords: polychlorinated biphenyls, biostabilization, bioremediation

Objectives: To achieve a greater understanding of the bioavailability of hydrophobic organic compounds [primarily polychlorinated biphenyls (PCBs)] under field conditions, to investigate the reduction in mobility of these compounds, and to determine the potential for intrinsic bioremediation of these compounds.

Approach: The land-treatment units will be monitored; and the extent of PCB desorption, biodegradation, and PCB mobility will be determined. The solubility and release rates of PCBs in soil/sludge mixtures will be determined. Appropriate models will be developed that identify the key physiochemical and microbial parameters that control the fate of residual PCBs. And PCB reductions will be correlated with reductions in toxicity to terrestrial organisms. The results of this effort will help determine the extent of intrinsic PCB biodegradation and will contribute to determinations of the endpoints for risk-based cleanup of PCBs and other "how clean is clean" discussions. The findings should contribute to approaches for contaminated-soil cleanup activities at former industrial sites (i.e., brownfields) where hydrophobic organic compounds were once used.

Results to Date: New project

\section{COLLOQUIUM ON "THE MICROBLAL WORLD: FOUNDATION OF THE BIOSPHERE" \\ STALEY, JAMES T. AMERICAN SOCIETY FOR MICROBIOLOGY FY 199625 \\ FY 19950 \\ FY 19940}

Keywords: workshops and conferences 
Objectives: To allow the American Society of Microbiology to convene a colloquim of leading scientists to determine how to proceed to address the urgent task of understanding microbial diversity.

Approach: The American Society of Microbiology (ASM) convened the colloquim in November 1995 and invited approximately 50 scientists to address issues such as: how microbial diversity should be measured, how taxonomic studies of poorly known phyla should be encouraged, what environments (in addition to marine and terrestrial) needed to be studied, what new technologies could be developed to enhance the study of microbial diversity, and how microbiologists could coordinate their research activities with botanists and zoologists.

Results to Date: The ASM prepared a white paper for review by the participants of the colloquim, the Board of Governors of the American Academy of Microbiology, and a panel of outside reviewers. The report will be published by ASM and disseminated to the microbiological research community, government scientific advisors, leaders in industry, and directors of appropriate federal agencies.

\section{INTRINSIC BIOREMEDIATION OF GAS-CONDENSATE HYDROCARBONS: DEVELOPMENT OF A SCIENTIFIC BASIS TO SUPPORT REGULATORY DECISIONS AT HYDROCARBON-CONTAMINATED SITES SUBLETTE, KERRY L. UNIVERSITY OF TULSA FY 1996399 \\ FY 19950 \\ FY $1994 \quad 0$}

Keywords: bioremediation by microorganisms, hydrocarbons, biodegradation

Objectives: To determine whether, and at what rate, intrinsic bioremediation of hydrocarbons by indigenous microorganisms occurs by conducting laboratory and field studies of anaerobic biodegradation of benzene, toluene, ethylbenzene, and xylene (BTEX) and of other gas-condensate hydrocarbons.

Approach: Groundwater, soils, and soil gas from two active natural gas production sites in Fort Lupton, Colorado, will be sampled and analyzed. The mechanisms, rates, and extent of natural attenuation at the sites will be investigated through laboratory and field studies. Either a predictive model of intrinsic bioremediation of hydrocarbons will be developed, or an existing computational model will be modified. The results will be used to help determine whether intrinsic bioremediation accompanied by appropriate site monitoring is a viable option for the remediation of hydrocarbon-contaminated sites.

Results to Date: New project.

\section{RADON RELEASE AND MOBILITY IN THE SUBSURFACE ENVIRONMENT THOMAS, DONALD \\ UNIVERSITY OF HAWAII \\ FY 199625 \\ FY 199581 \\ FY 199473}

Keywords: soil-gas transport, soil volatiles, advection, radon transport

Objectives: To document and model the processes by which radon and other soil volatiles move through the soil column in response to changes in soil physical characteristics and environmental variables.

Approach: The concentrations of radon in soil gases are documented at time intervals that are consistent with both changes in soil physical characteristics (e.g., air-filled porosity, density, permeability, and moisture content) as well as with normal changes in environmental variables (barometric pressure changes, rainfall, and wind speed). We also make detailed studies of the soil physical characteristics (porosity and permeability) as functions of moisture content under field and laboratory conditions and artificially control these conditions over a broad range to test the changes in soil characteristics as the conditions change. We use an adaptation of an existing model (LEACHM) that characterizes moisture and solute transport in the soil to describe volatile phase transport (including radon) in the subsurface soils.

Results to Date: Flux measurements of radon loss to a surface flux chamber have indicated that this method may only be able to accurately measure diffusional transport of soil volatiles to the surface. Under conditions of high soil permeability, advective transport processes make substantial contributions to volatile loss; hence, the flux-chamber method may substantially underestimate the rates of volatile transport to the atmosphere.

We have demonstrated a method of estimating gas conductivity of a soil with a modification of the method used for measuring saturated soil hydraulic conductivity. We have demonstrated the impact of soil moisture content on the gas conductivity of highly structured soils. Although more work remains to be done on the numerical model for highly permeable soils, the present work has enabled us to accurately describe observed concentration gradients of radon in the soil and to model temporal variations in radon activity in response to changes in soil moisture and barometric pressure. 


\section{HIGH-THROUGHPUT DNA SEQUENCING AND CHARACTERIZATION OF DIVERSE MICROBIAL GENOMES VENTER, J. CRAIG \\ THE INSTITUTE FOR GENOMIC RESEARCH FY 1996425 \\ FY 1995391 \\ FY $1994 \quad 0$}

Keywords: microbial genome sequencing, highthroughput DNA sequencing, archaeal genomics Objectives: To characterize the genomes of diverse microorganisms and to use the resulting whole-genome information to further understand microbial phylogeny, physiology, structural biology, and ecology.

Approach: This project will be cofunded by the Health Effects and Life Sciences Research Division of DOE. Libraries will be constructed of genomic DNA with random 1.5- to 4.5-kb DNA fragments as inserts placed into plasmid vectors and random $15-$ to $20-\mathrm{kb}$ DNA fragments as inserts placed into lambda vectors. Both ends of a sufficiently large number of randomly selected clones will be sequenced to determine the entire genomic sequence by covering the genome 6- to 9-fold. The genomic sequence will be assembled from the collection of random sequence fragments, and the remaining gaps will be closed by PCR and primer walking on the lambda library templates. The complete set of genes will be identified by comparing derived protein sequences with the content of amino-acid- and nucleotide-sequence databases.

Results to Date: The complete genome sequence of the extreme thermophilic archaeon Methanococcus jannaschii has been determined, and its genomic content has been analyzed. Random sequencing of a sufficiently large number of small-insert-size plasmid clones and 1000 large-insert-size lambda clones from genomic DNA of the sulfur-reducing archaeon Archaeoglobus fulgidus has been finished.

\section{AUTOMATED MULTIPLEX MICROBIAL- GENOME SEQUENCING WEISS, ROBERT B. UNIVERSITY OF UTAH \\ FY 1996589 \\ FY 1995571 \\ FY 1994250}

Keywords: Pyrococcus furiosus, automated hybridization, enzyme-linked fluorescence

Objectives: To use automated hybridization and imaging chambers designed for fluorescent multiplexed mapping and sequencing as the core technology for rapid, cost-effective sequencing of the genomes of $P$. furiosus and other microbes.

Approach: Novel laboratory methods and instrumentation are being developed into an integrated system for directed sequencing, emphasizing the use of "multiplexing" both in sequencing and mapping. Membrane-based techniques allow for batch processing of DNA samples in high-throughput automated systems. Spatially localized enzyme-linked fluorescent detection of DNA is carried out by automated hybridization/imaging chambers.

Results to Date: We have begun production sequencing of the $P$. furiosus genome. Plasmid libraries containing 2875 clones ( $6 x$ coverage) have been constructed in 21 multiplex vectors. These clones have been individually sized. Plasmid inserts are stably maintained by our vector system, which limits copy number to one until induction of runaway plasmid replication. Cosmid libraries containing 500 clones ( $8 x$ coverage) have been constructed in four multiplex vectors. Our directed-sequencing strategy uses a series of synthetic Gamma Delta transposons with common sequencing primers and unique multiplex identifiers. Groups of ten inserts in different vectors are taken through the process of transposition and mapping. Transposon insertions are obtained, insertion sites are mapped, and a minimal spanning set is chosen to give sequence coverage of each insert. Currently, 149 inserts have entered the transposition pipeline (estimated coverage about $1.4 \mathrm{Mb}$ ), and 91 inserts have exited. The status of these 91 inserts is: $0.76-\mathrm{Mb}$ minset coverage; $0.20 \mathrm{Mb}$ in analysis; $0.44 \mathrm{Mb}$ on membranes.

Double-stranded DNA is isolated from pooled cultures of inserts for both end- and transposonsequencing. Sequence ladders are blotted onto nylon membranes by direct-transfer electrophoresis. Membranes are placed in probe chambers for automatic repeated hybridization and imaging. Two such chambers have been configured with double drums for large capacity (three sequencing membranes), and two more are being modified in this way. Nine end-sequence membranes, containing a total of 5750 sequence tracks, have been produced and are currently being probed. Minimal sets from 79 inserts have been sequenced, resulting in $405 \mathrm{~kb}$ in consensus sequence. Similarity searches of our sequences against the public databases have detected approximately 120 matches to known sequences, allowing probable identification of many genes.

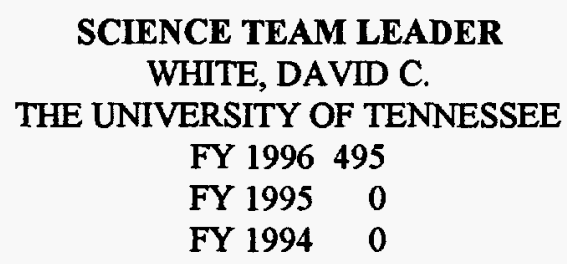

Keywords: NABIR, program management Objectives: To contribute scientific leadership to the NABIR program. 
Approach: We will develop scientific direction for the program science element, assist in identifying research opportunities and directions (e.g., hold workshops and attend relevant meetings and colloquia), provide coordination among the investigators in the program element and with other NABIR elements, communicate research findings to relevant audiences, identify targets of opportunity and encourage research directed at those targets, and conduct research.

Results to Date: New project.

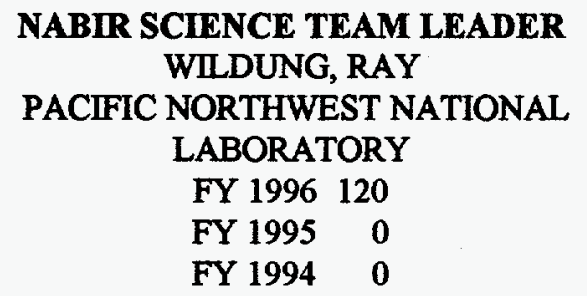

Keywords: NABIR, program management Objectives: To contribute scientific leadership to the NABIR program.

Approach: We will develop scientific direction for the program science element, assist in identifying research opportunities and directions (e.g., hold workshops and attend relevant meetings and colloquia), provide coordination among the investigators in the program element and with other NABIR elements, communicate research findings to relevant audiences, identify targets of opportunity and encourage research directed at those targets, and conduct research.

Results to Date: New project.

\section{PARTIAL SPONSORSHIP OF INTERNATIONAL SYMPOSIUM ON ENVIRONMENTAL BIOTECHNOLOGY WISE, DONALD L. NORTHEASTERN UNIVERSITY FY 199615 FY 19950 FY 19940}

Keywords: workshops and conferences
Objective: To provide partial funding for the third biennial meeting of the International Society for Environmental Biotechnology.

Approach: This third biennial meeting of the International Society for Environmental Biotechnology (ISEB) was planned to present over 100 state-of-the-art technical papers by practicing technologists in the broad field of environmental biotechnology. The focused nature of this ISEB meeting afforded substantial directed time for professional interchange. Papers were accepted from universities and research institutions in Australia, Belgium, Canada, Denmark, Egypt, France, Germany, Japan, Malaysia, Northern Ireland, Philippines, the former Soviet Union, Thailand, the United Kingdom, and the United States.

Results to Date: A successful scientific meeting with several different scientific sessions was held July 15 to 20, 1996 at Northeastern University in Boston, Massachusetts. Procedings of the meeting, including all 160 papers, will be published in a dedicated issue of Resources, Conservation, and Recycling, and in Biodegradation.

\section{HIGH-THROUGHPUT DNA SEQUENCING AND CHARACTERIZATION OF DIVERSE MICROBLAL GENOMES WOESE, CARL R. UNIVERSITY OF ILLINOIS FY $1996 \quad 61$ \\ FY 1995100 \\ FY 19940}

Keywords: DNA sequencing, characterization of microbial genomes

Objectives: To contribute to the sequencing of two to three prokaryotic genomes.

Approach: The genomes of Methanococcus jannaschii, Archaeoglobus fulgidus, and a prokaryote to be determined will be sequenced and made publicly available.

Results to Date: The genome of Methanococcus jannaschii is complete and is about to be published. 
The Subsurface Science Program seeks to understand the physical, chemical, and biological processes controlling the fate of complex chemical mixtures released to terrestrial subsurface environments; it also supports other, associated research in the deep terrestrial biosphere.

\author{
Program Manager \\ Frank J. Wobber \\ Environmental Sciences Division \\ Department of Energy, ER-74 \\ 19901 Germantown Road \\ Germantown, MD 20874-1290 \\ (301) 903-5549
}

Internet: frank.wobber@oer.doe.gov

THERMODYNAMIC AND KINETIC
PROPERTIES OF CHEMICAL SPECIES
PRESENT IN MIXED
ORGANIC-RADIONUCLIDE WASTES
AL MAHAMID, ILHAM
LAWRENCE BERKELEY NATIONAL
LABORATORY
FY $1996 \quad 75$
FY 1995213
FY 1994145

Keywords: plutonium, nitrilotriacetic acid, ethylenediaminetetraacetic acid, complexation, migration of radionuclides

Objectives: To study the behavior of plutonium in the presence of nitrilotriacetic acid (NTA) and ethylenediaminetetraacetic acid (EDTA), which have been identified as contaminants in the mixed organicradionuclide waste at DOE sites.

Approach: The study focuses on the reactions of plutonium with organic facilitators such as NTA and EDTA. If an interaction between a radionuclide and a complexing agent stabilizes the radionuclide in a soluble or insoluble form, the migration of the radionuclide in the geosphere may be enhanced or reduced. Therefore, it is important to understand the thermodynamics and kinetics of multi-species reactions. The plutonium-to-ligand-ratios are varied over a large range to cover possible site conditions where a large excess of NTA and EDTA may be present.

Results to Date: We studied the complexation behavior of the different oxidation states of plutonium [Pu(III), $\mathrm{Pu}(\mathrm{IV}), \mathrm{Pu}(\mathrm{V})$, and $\mathrm{Pu}(\mathrm{VI})]$ in the presence of NTA and EDTA at various concentrations of plutonium and at several $\mathrm{Pu} /$ chelate ratios. We found that $\mathrm{Pu}(\mathrm{IV})$ is the predominant oxidation state at $\mathrm{pH} 5$ to 8. $\mathrm{Pu}(\mathrm{III})$ was oxidized, and $\mathrm{Pu}(\mathrm{V})$ and $\mathrm{Pu}(\mathrm{VI})$ were reduced. The presence of NTA and EDTA in plutonium solution stabilizes plutonium in soluble form and therefore enhances its potential migration in the subsurface.

\author{
TRANSPORT OF SUBSURFACE BACTERIA \\ IN POROUS MEDIA \\ ARNOLD, ROBERT G.; BAYGENTS, JAMES C.; \\ and OGDEN, KIMBERLY L. \\ UNIVERSITY OF ARIZONA \\ FY 1996100 \\ FY 199598 \\ FY 199497
}

Keywords: biocolloids, porous media, sticking coefficient, iron oxide

Objectives: To study theoretical shortcomings in the area of biocolloid transport through porous media, including the effects of iron and aluminum in the sediment, the electrophoretic mobility of the bacteria, and the chemical properties of the mobile phase.

Approach: The transport properties of specific bacterial isolates obtained from sediments and groundwater of the Oyster site will be determined with MARK minicolumns, and the information obtained will be forwarded to subprogram collaborators to aid in designing the field experiment. For the more promising isolates, longer column experiments are performed to determine the adsorption and desorption characteristics of the strains; look for the effects of preferred binding sites; and estimate the numbers of bacteria to be injected for optimal monitoring, precision, etc.

The effects of phosphate, iron oxide, aluminum, and growth media characteristics on bacterial transport and adhesion will be investigated. Quantification of iron and phosphate effects on cell transport and adhesion will make the results of our field test transfer 
able to other environmental settings. Capillary electrophoresis studies will be performed to elucidate the respective roles of surface charge and other factors on cell/sediment affinity. How bacterial transport varies with sediment type at the Oyster site will be determined by obtaining 100 different facies samples from the Oyster site to serve as collector materials for MARK-column experiments.

Results to Date: MARK transport experiments involving bacterial isolates from the Oyster site were conducted with artificial groundwater and Oyster sediments. The organisms exhibited order-of-magnitude differences in their affinities for Oyster sediments, indicating that results of the field experiment should be highly dependent on the selection of microorganisms. Microbe-collector affinity was a function of depth within the MARK columns, suggesting that cellcollector affinity is a distributed function even in a monoclonal population of bacteria. Capillary electrophoresis data show that electrophoretic mobility and surface-charge density are also distributed (bimodal) functions in some pure bacterial strains. There are related implications for bacterial-transport modeling inasmuch as residual bacteria should become considerably more mobile at points removed from the point of injection.

For PL2W21 and PL2W31, MARK experiments were performed with borosilicate glass beads, quartz, and iron-coated quartz and artificial groundwater. PL2W31 exhibited considerably less affinity for the sediment than for glass, quartz, or iron-coated quartz.

Experiments to determine the effects of groundwater chemistry on bacterial transport found that phosphate plays an important role in cell affinity for iron-bearing sediments. Results suggest that phosphate is primarily responsible for surface reactions that determine the influence of iron oxide content on the affinity of cells for the collector surface.

\section{DOE SUBSURFACE MICROBIAL CULTURE COLLECTION AT FLORIDA STATE UNIVERSITY \\ BALKWILL, DAVID \\ FLORIDA STATE UNIVERSITY \\ FY 1996298 \\ FY 1995217 \\ FY 1994276}

Keywords: culture collection, subsurface microorganisms, microbial phylogeny, databases, biodegradation, bioremediation

Objectives: To partially characterize and maintain a permanent resource collection of microbial cultures isolated from terrestrial subsurface environments and to maintain databases describing the cultures and the samples from which they came.
Approach: Strains of aerobic (or facultatively anaerobic) microorganisms from subsurface environments are preserved by freezing and storage at ultralow temperatures. Cultural, morphological, and selected physiological characteristics of each culture are determined and recorded in digitized databases. Physical, chemical, and other characteristics of the subsurface materials from which the cultures were isolated are also recorded. Cultures, databases, and data search/sort services are made available to DOE-funded and other scientists on request.

Selected groups of cultures are more fully characterized by analysis of $16 \mathrm{~S}$ ribosomal RNA gene sequences to determine their phylogenetic relatedness to each other and to previously described microbial forms (information used to assess the diversity and novelty of the collection). Selected cultures are also screened for useful metabolic capabilities (e.g., the ability to biodegrade aromatic compounds).

Three related research efforts are supported by subcontracts: characterization and maintenance of a permanent collection of strictly anaerobic subsurface microorganisms, characterization of selected subsurface cultures by analysis of cell lipid profiles (MIDI) and/or phospholipid fatty acids (PLFAs), and characterization of ribosomal RNA operons in subsurface bacteria.

Results to Date: 2,150 cultures were provided to nine DOE and four non-DOE scientists on request; 1,725 new cultures were added to the collections and partially characterized; and databases describing the cultures and the samples from which they came were updated with 31,000 new data points.

Phylogenetic analysis of $16 \mathrm{~S}$ ribosomal RNA gene sequences showed that many strains fall within previously described genera, but probably are novel species. (Genera in which strains have been placed include: Streptomyces, Bacillus, Arthrobacter, Staphylococcus, Rothia, Micrococcus, Pseudomonas, Comamonas, Sphingomonas, Acinetobacter, and Zoogloea.) Phylogenetic and biochemical (MIDI and PLFA) analyses showed that, while the collection contains groups of closely related strains, few are exact duplicates. Screening for useful biodegradative properties indicated that $15 \%$ of 400 strains examined have such properties and should be investigated for possible uses in bioremediation. Some strains have proven to be quite novel. One anaerobic strain was found to be the first and only strictly anaerobic member of genus Bacillus and to have unique metabolic traits. Another isolate was closely related to Bacillus simplex but could biosorb cobalt, cadmium, nickel, and strontium. 

Approach: Interdisciplinary biogeochemical studies of coupled microbiology and geochemistry processes are performed in batches, in columns, and at the intermediate scale to investigate how chelate sorption-desorption, chelate aqueous speciation, and chelate-enhanced mineral solubility affect chelate biotransformations and radionuclide behavior. The research focuses on cobalt (II, III) and Pu (IV, V, VI) and the chelating agents nitrilotriacetate (NTA) and ethylenediaminetetraacetic acid (EDTA).

Individual processes are quantified to support the coupled studies (e.g., enzymatic activity for and biodegradation rates of specific metal-chelate complexes; levels of chelate biodegradation products in solution; chelate sorption-desorption rates and identification of sorbed complexes). Chelate-degrading microorganisms and/or consortia, subsurface sediments, and surrogate minerals are used. Experiments are conducted as a function of microbial populations and growth conditions, chelated radionuclide aqueous speciation, the surface chemistry of dominant subsurface sorbents, and environmental factors (e.g., $\mathrm{pH}$ and native cations, such as $\mathrm{Ca}, \mathrm{Al}$, and $\mathrm{Fe}$ ), which influence both microbial metabolism and aqueous speciation. Mechanistic models that couple (1) mixed equilibrium-kinetic abiotic heterogeneous reactions, (2) Monod kinetics describing biotic processes, and (3) flow in porous media are used to develop research hypotheses, interpret results, and predict microbial activity and radionuclide behavior.

Results to Date: NTA adsorbed to gibbsite was not biodegraded by Chelatobacter heintzii at $\mathrm{pH} 6,7$, and 8. An equilibrium-kinetic mechanistic model showed that NTA desorption limited biodegradation. NTA desorption was $\mathrm{pH}$ dependent and slow because bidentate and binuclear surface complexes were formed. ${ }^{60} \mathrm{CoNTA}$ degradation did not affect ${ }^{60} \mathrm{Co}$ sorption to gibbsite. The ${ }^{60} \mathrm{CoNTA}$ was not bioaccumulated inside or biosorbed outside $C$. heintzii. Sorption will therefore decrease NTA bioremediation in gibbsitic subsurface systems and microorganisms such as $C$. heintzii will not alter ${ }^{60} \mathrm{Co}$ transport.

NTA monooxygenase degraded CoNTA and NiNTA, which were recalcitrant to whole cells. Cellular transport of these complexes was slow. These results suggest that the ex situ enzyme or modified cellular NTA transport are useful bioremediation strategies for recalcitrant NTA complexes.

BNC1 degraded various metal-EDTA complexes but not CoEDTA. Cobalt toxicity did not limit degradation. CoEDTA was not transported into BNC1, while cell extracts quickly degraded CoEDTA. The results suggest that modification of cellular uptake would be a useful bioremediation strategy for ${ }^{60}$ CoEDTA.
EDTA desorption from FeOOH (goethite) was rapid and $\mathrm{pH}$ dependant because of the formation of monodentate surface complexes. EDTA desorption and biodegradation rates are comparable, suggesting significant coupled interactions are possible among sorption-degradation-transport processes under laboratory and field conditions.

Mechanistic modeling of metal-NTA and metal-EDTA adsorption reactions with equilibrium and kinetic models (coupled to aqueous speciationsolubility reactions) describe adsorption processes under kinetic or equilibrium control. Results suggest major differences in the surface bonding of metal-NTA and -EDTA complexes, with Fe and Al oxides common in subsurface sediments. This modeling of adsorption coupled to microbiological and transport processes will enable quantitative evaluation of chelated radionuclide transport and chelate bioremediation strategies in future intermediate-scale and field-site experiments.

\section{SUBSURFACE MICROBIAL CULTURE COLLECTION, WESTERN BRANCH BOONE, DAVID R. OREGON GRADUATE INSTITUTE FY 199684 \\ FY 199580 \\ FY 19940}

Keywords: anaerobic bacteria, thermophilic bacteria, iron-reducing bacteria, manganese-reducing bacteria Objectives: To collect and characterize cultures of anaerobes from the deep terrestrial subsurface.

Approach: Anaerobic strains are isolated from the deep terrestrial subsurface, processed, stored frozen in liquid nitrogen, and provided to other program scientists as requested; selected strains were characterized. Results to Date: The characterization of anaerobic, thermophilic, iron- and manganese-reducing strains isolated from the Taylorsville Triassic Basin led to the description of a new species of bacterium, Bacillus infernus ("the Bacillus from hell," named for the deep subsurface source of the type strain). This species is the first anaerobic species of Bacillus, and its report has required a redefinition of this genus.

Two other groups of anaerobic subsurface isolates have been identified as likely representing new species of bacteria. One is a group of thermophilic sulfate reducers, also isolated from the Taylorsville Triassic Basin. These bacteria appear to belong in the genus Desulfotomaculum, but they differ physiologically and phylogenetically from previously described species. The other is a group of mesophilic iron and manganese reducers isolated from samples obtained from a drilling site at Cerro Negro in New Mexico. 


\author{
ENVIRONMENTAL PROPERTIES \\ CONTROLLING MICROBIOLOGICAL \\ HETEROGENEITY \\ BROCKMAN, FRED, and \\ MURRAY, CHRISTOPHER \\ PACIFIC NORTHWEST NATIONAL \\ LABORATORY \\ FY 1996600 \\ FY 1995500 \\ FY 19940
}

Keywords: microbiological heterogeneity, geostatistic models, biogeochemistry, moisture flux

Objectives: To provide geostatistical models of the spatial heterogeneity and distribution of microbial activity in subsurface sediments and the geological and geochemical factors controlling this distribution.

Approach: In this study, the distribution of microbial properties in the semiarid western United States and high-precipitation regions of the northeastern United States are being analyzed with geostatistics, a form of spatial statistics that provides quantitative models of the spatial auto- and cross-correlation between variables. The specific approach is to sample and analyze geologically similar sediments (in terms of grain size and mineralogy) at several sites marked by varying degrees of precipitation and recharge. Sites that have been examined thus far have approximately $10 \mathrm{~m}$, $4 \mathrm{~mm}$ ( 2 sites), and $25 \mathrm{~cm}$ of annual recharge. At each site, spatial continuity is examined by measuring microbiological, physical, and chemical properties of regularly spaced samples in boreholes, and geostatistics is used to quantify the spatial variability of those properties. Those physical and chemical properties that are spatially correlated with the distribution of the microbiological properties are identified. Sampling is performed at multiple scales centered at a single point to examine the variability of microbial properties for various sample sizes. How nutrient additions affect the variability of microbial properties for various sample sizes is examined.

Results to Date: The spatial continuity of microbiological properties is normally less than that of the physical and chemical properties of the same samples. There was pronounced anisotropy of some variables, with greater continuity of properties in horizontal transects that were parallel to the sedimentary beds. Spatial cross-correlation of physical, chemical, and microbiological variables (aerobic mineralization with sulfate, moisture, and nitrate) was detected at the 10-m-recharge site, probably related to physical and chemical conditions that favored long-term survival of dormant microorganisms. Microorganisms capable of growth or activity in the studied subsurface environments display a patchy distribution at distances of centimeters. The scale of averaging for microbial activity decreases as recharge increases.
MICROBIAL ECOLOGY OF THE

SUBSURFACE: IMPLICATIONS OF

TEMPERATURE, PRESSURE, AND

ELECTRON FLOW

COLWELL, FREDERICK

IDAHO NATIONAL ENGINEERING

LABORATORY

FY 1996335

FY 1995200

FY 1994258

Keywords: microbial ecology, contaminant degradation

Objectives: To determine the ecology of subsurface microbial communities based on their preferred electron donors and acceptors and on the geochemical, hydrological, and thermal conditions of the environment in which they exist. During FY 1996, efforts shifted to consideration of DOE waste sites (primarily at the Idaho National Engineering Laboratory) and the microbial communities there that may be able to degrade contaminants.

Approach: Samples will be acquired from pristine environments in which the geology determines whether microorganisms are present because of transport or long-term survival. The research will integrate prior knowledge of subsurface microbial ecology to determine microbial-community diversity, distribution, and activity in contaminated subsurface environments, and cores will be acquired from waste-impacted aquifers and vadose zones. Techniques for sampling geologically diverse environments and quality assurance methods to obtain defensible subsurface samples will be used in this research. Laboratory efforts will include growth of microorganisms from remote subsurface habitats and use of instruments for growing bacteria from high-temperature/high-pressure zones in the subsurface.

Results to Date: The presence of sulfur-oxidizing microorganisms was determined in anaerobic subsurface sedimentary rocks, thereby demonstrating the potential for complete cycling of sulfur in the subsurface. The presence of $\mathrm{Fe}$ (III) reducers and fermenters was determined in deep sandstones that only as recently as 5 million years ago cooled to temperatures that would permit microbial recolonization. Carbonuse patterns were assessed for microbial communities derived from SSP research sites where microbial, chemical and physical heterogeneities of subsurface strata are being studied. The pressure-temperature growth optima was determined for a subsurface bacterium from the Taylorsville Triassic Basin to understand subsurface survival in the context of known temperatures and pressures for that environment. Techniques for hard-rock microbial core sampling were developed for the Subsurface Science Program, leading to the acquisition of essential microbiological, 
chemical, and hydrological data to augment cleanup of subsurface contaminants at $\mathrm{DOE}$ sites.

\section{MULTISCALE HETEROGENEITY, PARAMETER ESTIMATION, MODELS, AND SCALEUP \\ CUSHMAN , JOHN \\ PURDUE UNIVERSITY \\ FY 1996125 \\ FY 1995168 \\ FY 1994154}

Keywords: multiscale heterogeneity, subsurface contaminants

Objectives: To develop multiscale simulations and parameter-estimation methods to predict and help control the evolution of contaminants in a physically, chemically, and biologically heterogeneous subsurface environment.

Approach: Stochastic-perturbation theory, adaptive parameter-estimation methodology, and Monte Carlo techniques will be used to construct and verify simulation and design tools.

Results to Date: We have used first- and second-order stochastic perturbation theory, Kalman-like estimators, and FFT to develop nonlocal Eulerian simulators and parameter estimation tools for the mean concentration (and its various spatial moments) of reactive, degradable chemicals. Specifically, we have studied chemicals that undergo first-order nonequilibrium reactions with first-order degradation in a physically, chemically, and biologically heterogeneous environment. Most recently we have used the Monte Carlo method in conjunction with a random field generator to test the validity of the theories.

Higher-order velocity covariances are important as the conductivity heterogeneity increases. Contrary to the common belief, local-scale dispersion is important to consider. The theories break down as the integral scale for the conductivity increases. Triplet correlation functions may be important, depending on the degree of heterogeneity. If the porosity is considered correlated to the conductivity, then its inclusion in the stochastic theories may be more important than higherorder velocity-covariance corrections. Our simulations suggest that monitoring programs need to be designed to effectively predict the success of containment and remediation strategies.

\section{SUBSURFACE TRANSPORT OF BACTERIA WITH ALTERED ADHESION CHARACTERISTICS DEFLAUN, MARY F. ENVIROGEN, INC. FY 1996223 \\ FY 1995125 FY 19940}

Keywords: bacteria, adhesion, bacterial transport, bioaugmentation, aquifers, bioremediation

Objectives: To examine the effect of adhesion on bacterial transport in aquifers and to determine the predictive nature of laboratory microcosms in a fieldtransport experiment.

Approach: Bacteria adhere to solid surfaces by a number of mechanisms which may limit their penetration through soil and sediments. This research examines the role of adhesion in bacterial transport in aquifers by comparing the transport of a naturaladhesion variant to that of the adhesive wild-type bacterial strain. In addition to developing a stableadhesion variant and comparing its transport properties to those of the wild-type strain in bench-scale studies, the cell-surface characteristics that would account for the altered-adhesion phenotype will be determined. Field-transport experiments will be used to determine how natural heterogeneity affects the enhanced transport achieved in bench-scale sedimentcolumn and aquifer studies.

Results to Date: Envirogen researchers are currently preparing for the first bacterial injection experiment to take place at the Oyster site in June. The strain selected for the first injection (PL2W31) is a relatively low-adhesion organism that is readily transported through aquifer sediments in bench-scale experiments. Strains that have been characterized for subsequent injections include those with adhesion and/or membrane characteristics that are significantly different from PL2W31. Several hundred isolates representing sediment-associated and groundwater bacteria from the Oyster aquifer have been characterized, and a database has been developed. These strains are currently being archived in the Subsurface Microbial Culture Collection (SMCC). These strains have been characterized by DNA fingerprinting by AP-PCR (Arbitrarily Primed Polymerase Chain Reaction), metabolic and antibioticresistance profiles, percent adhesion, and morphology. A comparison of these characteristics in strains isolated from groundwater and those isolated from sediment indicate significantly different populations. This baseline data set will be used to assess changes in the indigenous population in the aquifer following the injection experiment. The results from the bacterial transport experiments at Oyster will be applied to sediments with similar physical/chemical characteristics at Dover Air Force Base in Dover, Delaware. The investigator is also working with a research team that is extending these and other research results to the Test Area North (TAN) contaminated area at the Idaho National Engineering Laboratory (INEL). Activities at this site include assessing different remediation strategies for chlorinated hydrocarbon contamination of a fractured bedrock aquifer including biostimulation and bioaugmentation. 


\section{ADHESIVE PROPERTIES OF BACTERIA AND THEIR TRANSPORT AND COLONIZATION IN THE SUBSURFACE ENVIRONMENT FLETCHER, MADILYN UNIVERSITY OF MARYLAND \\ FY 1996107 \\ FY 1995141 \\ FY 1994136}

Keywords: bacterial transport, adhesion, porous media, biogeochemistry, Pseudomonas

Objectives: To identify surface characteristics of bacteria that determine their adhesion to surfaces and affect their transport through porous media.

Approach: By transposon mutagenesis, mutants of Pseudomonas species from the subsurface will be produced that are altered in surface adhesives. The alterations in adhesives of selected mutants will be determined by chemical and physical analysis of cell surface polymers. The effect of adhesiveness on transport and long-term distribution and colonization of bacteria in porous media will be evaluated by evaluating transport and retention of adhesion mutants in laboratory columns containing subsurface sediments.

Results to Date: Adhesion mutants have been obtained from a strain of $P$. fluorescens and from pseudomonad subsurface isolates from the DOE Savannah River Site (Aiken, S.C.) and the GEMHEX program at the Hanford Reservation, Washington. The subsurface strains were identified by $16 \mathrm{~S}$ rRNA sequence data obtained by the Subsurface Microbial Culture Collection. We have also analyzed outer-membrane proteins and lipopolysaccharide (LPS) in a range of transport variants obtained from Envirogen, Inc. Laboratory column experiments with silica sand and sediments from the site at Oyster, Va., demonstrate that cell adhesiveness correlates with poor transport through porous media. Analysis of two transport mutants indicate that alterations in LPS resulted in altered adhesiveness. Thus far, our results have found no indications that other cell-surface components, such as proteins or extracellular polysaccharide, are involved in adhesion to sediment surfaces. Column-transport experiments and adhesion assays on pseudomonads demonstrated that transport and adhesion were altered by starvation of the bacteria and by solution biochemistry. The effect of benzoate utilization by a $P$. cepacia strain on its attachment properties is being evaluated in conjunction with $E$. Murphy and T. Ginn who are using this bacterial strain in intermediate-scale transport experiments at PNNL.

\section{BIODEGRADATION OF SELECTED ORGANIC COMPOUNDS AND COMPLEXING AGENTS OF RADIONUCLIDES AND METALS \\ FRANCIS, AROKIASAMY \\ BROOKHAVEN NATIONAL LABORATORY \\ FY 1996150 \\ FY 1995261 \\ FY 1994263}

Keywords: biodegradation, complexing agents, radionuclides, toxic metals, microbial stabilization Objectives: To determine the mechanisms of microbial degradation of organic complexing agents that mobilize radionuclides and toxic metals at contaminated sites.

Approach: The biodegradation of naturally occurring complexing agents (i.e., citric acid) and selected organic compounds commonly used in radionuclide and metal extractions will be determined under aerobic and anaerobic conditions. The mechanisms of biotransformation of radionulcide/metal-citrate complexes under aerobic, denitrifying, fermentative, ironreducing, and sulfate-reducing conditions will be investigated. In addition, the cometabolic degradation of the compounds in the presence of other carbon sources present in mixed wastes will be investigated. The effects of iron, and manganese oxide minerals, and environmental variables [such as $\mathrm{Eh}, \mathrm{pH}$, temperature, moisture, nutrients, and alternate electron acceptors (nitrate and sulfate)] on the rate and extent of degradation will be elucidated. In situ bioremediation of contaminated sites containing organic complexing agents can be achieved by the addition of suitable microbial cultures, nutrients, and manipulation of the environmental conditions.

Results to Date: The bidentate complexes of $\mathrm{Ca}$, $\mathrm{Fe}(\mathrm{III}), \mathrm{Ni}$, and $\mathrm{Zn}$ were readily biodegraded, whereas the tridentate complexes of $\mathrm{Cd}$ and $\mathrm{Cu}$ and the binuclear complex of $U$ were recalcitrant. Uptake studies with ${ }^{14} \mathrm{C}$-labeled citric acid showed that biodegradation of uranium citrate was limited by the transport inside the cell, whereas the tridentate complexes, cadmium and copper citrate, were neither transported inside the cell nor metabolized by the bacteria.

Speciation of uranium in cultures of Clostridium spp. by XANES at the NSLS and by XPS showed that soluble U(VI) was reduced to insoluble U(IV). Reduction of uranium occurred only in the presence of growing or resting cells. Organic-acid metabolites, the extracellular components of the culture medium, and heat-killed cells failed to reduce uranium under anaerobic conditions.

A novel process (U. S. Patent No. 5,292,456) has been developed to clean up and reclaim uranium and toxic-metal-contaminated soils. Citric acid is used to extract metals and radionuclides from solid wastes 
through the formation of water-soluble metal citrate complexes. The extract containing radionuclide/metal complexes is then subjected to aerobic microbial degradation followed by photodegradation. The metal citrate complexes, with the exception of uranium, undergo biodegradation and are recovered in a concentrated form with the bacterial biomass. Undegraded uranium citrate is easily separated from the rest of the metals after biodegradation. On subsequent exposure to light, the uranium citrate complex undergoes rapid photodegradation and uranium precipitates out as a stable oxide. Uranium-contaminated soils from RMI in Ashtabula and Fernald, Ohio, and sludge from the Y-12 Plant at Oak Ridge, Tennessee, have been successfully treated with this process. The process also removes lead from municipal solid waste incinerator ash.

\section{SUBSURFACE ANAEROBIC BIOGEOCHEMICAL PROCESSES \\ FREDRICKSON, JAMES; BROCKMAN, FRED; and STEVENS, TODD \\ PACIFIC NORTHWEST NATIONAL LABORATORY \\ FY 1996958 \\ FY 1995945 \\ FY 1994776}

Keywords: biogeochemistry, anaerobic processes, metal reduction, sulfate reduction, microbial ecology, basalt, phylogeny, 16S Rrna

Objectives: To determine the role of coupled microbiological, geochemical, and transport processes on the rate and extent of microbial reduction of $\mathrm{Fe}$ (III) oxyhydroxide minerals; to develop an understanding of the physical and geochemical factors controlling microbial-community structure and function at and adjacent to interfaces between low- and high-permeability rocks and sediments in the subsurface; and to probe the biogeochemical processes and community structure of subsurface lithotrophic microbial ecosystems.

Approach: Geochemical interactions between iron and phosphorus are being investigated because phosphorus is strongly bound to iron oxides and can be a potentially important factor limiting growth of dissimilatory iron-reducing bacteria (DIRB) in anaerobic sediments. Experiments in well-defined systems allow for detailed investigations of the geochemical factors controlling this process. Lipid phosphate is being used as a measure of microbial biomass to quantify growth parameters necessary for coupled geochemical microbial transport modeling of biogeochemical reactions involving microbial metal reduction. Experiments are also being conducted to investigate the rate and extent of microbial reduction of iron associated with natural subsurface sediments from the Hanford Redox Manip- ulation field site. Field investigations will be conducted at one or more, shallow iron-rich Oyster and Milford sites, where surface-derived detrital and soluble organic carbon may drive microbial iron reduction.

Results to Date: Saturation of iron oxide surfaces by Fe(II) severely restricted the rate and extent of microbial reduction of Fe(III) oxides. Therefore, sorption, complexation, and precipitation reactions involving $\mathrm{Fe}$ (II) will have a major impact on the rate and extent of the microbial reduction of iron oxides that occurs in anaerobic sediments. Lipid-phosphate is highly correlated $\left(r^{2}=0.96\right)$ with direct microscopic counts and can be quantitatively extracted from iron-oxide-coated silica sand. Therefore, lipid-P will be an excellent measure of biomass in transport experiments.

Investigations at the Cerro Negro, N.M., field site revealed that microbial activity was maximum at shale-sandstone interfaces because of the coincidence of electron donor (organic carbon) from the shale and soluble electron acceptor (sulfate) at these interfaces. Geochemical and physical properties have imposed conditions that have limited bacterial transport yet have promoted the long-term maintenance of organic carbon and a low-biomass but active anaerobic microbial population at these interfaces.

Investigations of the Columbia River basalt (CRB) aquifers beneath the Hanford Site indicate that they contain anaerobic microbial communities that function independently of photosynthesis. They rely on abiotically generated $\mathrm{H}_{2}$ for energy. Hydrogenotrophic anaerobic bacteria were numerically dominant to heterotrophic bacteria, and among the $\mathrm{H}_{2}$-based anaerobes, the populations of acetogens were 10 to 100 times higher than those of Fe(III) reducers, sulfate reducers, or methanogens. Laboratory experiments demonstrated that $\mathrm{H}_{2}$ is evolved from basalt-water interactions at room temperature and pressure and is probably the source of the high concentration of $\mathrm{H}_{2}$ (micromolar) in the CRB groundwaters.

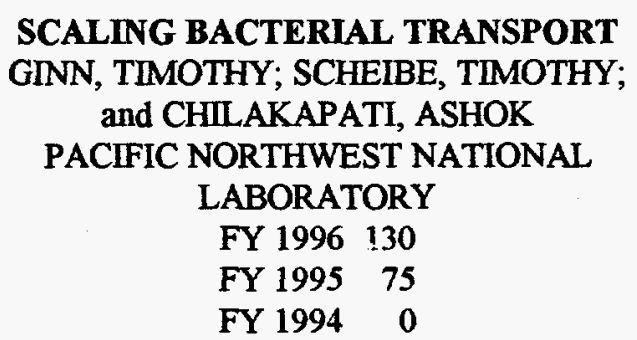

Keywords: bacterial transport, scaling theory, data integration, mathematical modeling

Objectives: To mathematically quantify and scale coupled processes involved in the subsurface transport of microbes to provide the predictive means to integrate microbiological, geochemical, and hydrogeological data for the design and interpretation of in situ microbial-transport experiments in the field. 
Approach: Hydrogeological (nonuniform convection, dispersion, and exclusion), microbiological (degradation, growth, and decay), and biogeochemical (attachment, adhesion, and detachment) processes will be examined to determine how information derived at small scales can be used to predict average behavior at the field scale. Mathematical models of bacterial transport processes will be joined with realistic 3-D representations of heterogeneous geohydrologic and geochemical controlling properties to provide a simulation framework for assessing experimental observations. A high-resolution 3-D aquifer model will be used that combines in situ data sets (core samples, borehole/surface geophysics, and core geologic interpretations) with ex situ statistical descriptions (outcrop studies) to give an accurate representation of hydrogeological and geochemical properties of the aquifer that are heterogeneous on multiple scales. Bacteria-solid interaction processes will be combined with hydrogeologic equations of flow and transport, and the coupled processes will be simulated in the high-resolution 3-D aquifer. These deterministic simulations will be used both to interpret data from field experiments and as the basis for upscaling studies. Scaling of the average system behavior will be accomplished through Monte Carlo simulations with the high-resolution aquifer model and through stochastic-analytic (nonlocal) techniques.

Results to Date: Data on aquifer flow properties and hydrofacies structure have been incorporated in the aquifer model. The expanded model is a high-resolution 3-D representation of the aquifer-transport properties at the field site. Outcrop and borehole data have been integrated into a consistent multiscale framework for representing the spatial variability of hydrogeologic properties at the centimeter cross-bedding scale, and the meter hydrofacies scale.

A Monte Carlo study of the aquifer flow field was undertaken to model tracer (inert bromide and sorbing sulphate) behavior under different experimental control strategies (injection rates, hydraulic control). The model predictions brought to light several issues that impacted tracer experimental design, including the likely existence and impacts of high-conductivity preferential flow pathways and the expected width, direction, and speed of movement of the tracer plume. With the new fully conditioned aquifer model, a second Monte Carlo effort was undertaken. This numerical simulation program incorporates laboratoryderived representations of bacterial attachment/ detachment processes within the flow field established by the planned hydraulic controls.

Data collection efforts are ongoing, and multipleinjection experiments are planned, so a strategy was developed for integrating new data streams into the model framework.
Data from column experiments in bacterial transport completed by collaborators at the University of Arizona were used to develop new preliminary first-order reversible kinetic models for bacterial attachment-detachment for strain PL2W31, selected for injection in the planned field experiment. Previously, mathematical models of bacterial transport processes (convection, dispersion, and attachment) were derived and used to aid collaborators at the University of Arizona in the design of column experiments to assess bacterial attachment mechanisms and rates.

\section{SUBSURFACE SCIENCE GEOLOGIC DATA INTERPRETATION AND GEOTECNICAL SUPPORT GRIFFIN, W. TIMOTHY GOLDER ASSOCIATES, INC. FY 1996300 \\ FY 1995300 \\ FY 1994397}

Keywords: surveys, safety, administrative support Objectives: To provide geological, geotechnical, regulatory, health and safety, and logistical support to SSP field sampling programs in order to facilitate the acquisition of subsurface samples for microbiological research.

Approach: Support (e.g., preparation and filing of NEPA documentation, drilling specifications, identification of suppliers of specialized services) will be provided for SSP field sampling programs.

Results to Date: Support was provided to a number of field-sampling programs for the SSP during 1996, including sampling programs in Winona and Washtucna, Wash., and Oyster, Va. The NEPA categorical exclusion request packages for the Winona and Washtucna sites were compiled and submitted. The drilling specifications for the two sites were developed, bids were solicited from drilling companies in the region, and the best-qualified company was subcontracted to conduct the drilling and sampling. A supplier of specialized core liners capable of surviving autoclave temperatures was identified, and the liners were purchased for the sampling project. Field geologists from Golder supervised and documented all drilling- and sampling-support operations. At the Oyster site, the proposed sandpit excavation site was inspected, and an excavation plan that would meet OSHA safety requirements was prepared. Surveying and excavation services were specified and subcontracted. Golder also assisted in data collection at the site. Future sampling activities at the site are being planned, including final site reclamation. 


\section{PHYSIOLOGICAL STATE AND BIOCHEMICAL POTENTIAL OF DEEP \\ SUBSURFACE MICROORGANISMS GRIFFITHS, ROBERT P. \\ OREGON STATE UNIVERSITY \\ FY 199649 \\ FY 1995143 \\ FY 1994126}

Keywords: microbial ecology

Objectives: To study whole cores and sediment samples to determine the physiological state of in situ microbial communities.

Approach: The physiological state of deep subsurface microorganisms will be investigated. Field (core) samples will be collected, and techniques will be optimized for evaluating the physiology and biochemical potential of deep subsurface microorganisms. The feasibility of measuring phosphatase activity and glucose uptake in deep sediments will be demonstrated. These same techniques will then be applied to cores collected from the deep subsurface.

Results to Date: Results using whole cores show increases in microbial activity within the first hour of incubation and demonstrate that microbial activity can be measured within a few hours after a core is recovered. The research also shows a four-fold range in activities in different size fractions of crushed rock, suggesting that particle size needs to be standardized. Analysis of additional samples collected from deep geological units at the Cerro Negro, N.M., drilling site demonstrate that the relative microbial activities observed with both phosphatase activity and glucose uptake rates show essentially the same pattern as observed by other investigators using different techniques.

\section{MICROBIAL METHANE CYCLE IN SUBSURFACE SEDIMENTS: ITS ROLE IN MICROBIAL SURVIVAL \\ GROSSMAN, ETHAN; AMMERMAN, JAMES; and SUFLITA, JOSEPH \\ TEXAS A\&M UNIVERSITY and \\ UNIVERSITY OF OKLAHOMA \\ FY 19960 \\ FY 1995148 \\ FY 1994130}

Keywords: sulfate reduction, pyrite oxidation, microbial survival, geochemistry, aquifers

Objectives: To determine the factors controlling microbial activity and survival in the subsurface; specifically, to determine whether microbial communities in aquitards and in aquifer microenvironments provide electron donors and/or acceptors that enhance microbial survival in aquifers.

Approach: Eight boreholes drilled in the Eocene Yegua formation form a $\mathrm{T}$, with well clusters parallel and perpendicular to dip direction. Boreholes range in depth from 8 to $31 \mathrm{~m}$, with screened intervals ranging from 6 to $31 \mathrm{~m}$. Sediments are collected aseptically and assayed for a variety of microorganisms and metabolic capabilities, including viable aerobic and anaerobic heterotrophs, sulfate-reducing bacteria (SRB), SRB activity, and iron and sulfur oxidizers. In addition, DNA is being recovered and amplified by polymerase chain reaction, and experiments are being performed to evaluate the biodegradability of naturally occurring carbon sources amended to subsurface sediments. Sediment geochemistry [organic-carbon content, ${ }^{13} \mathrm{C} /{ }^{12} \mathrm{C}$, sulfur chemistry, ${ }^{34} \mathrm{~S} /{ }^{32} \mathrm{~S}$, and bioavailable $\mathrm{Fe}(\mathrm{III})]$ provides information on the interrelationships among microorganisms and their environment. Slug tests and groundwater analyses (major ions, $\mathrm{pH}$, dissolved oxygen, $\mathrm{H}_{2} \mathrm{~S}, \mathrm{Fe},{ }^{34} \mathrm{~S} /{ }^{32} \mathrm{~S},{ }^{13} \mathrm{C} /{ }^{12} \mathrm{C},{ }^{14} \mathrm{C}$, and tritium) help define chemical environments, flow patterns, and groundwater ages.

Results to Date: Pyrite oxidation in shallow sediments (about 6 to $8 \mathrm{~m}$ ) is indicated by low pH $(<5)$, high iron oxide and sulfate concentrations, and $10{ }^{34} \mathrm{~S} /{ }^{32} \mathrm{~S}$ ratios in groundwater sulfate. Sulfur- and iron-oxidizing bacteria enumerated from unsaturated-zone sediments have concentrations of up to 104 cells per gram of sediment and are able to use pyrite as a sole energy source. Imaging of radiolabeled-sulfide production from intact cores indicates high spatial variability in sulfate-reducing activity, even at the centimeter scale. Greater in situ rates of sulfate reduction (SR) are measured near clay-sand contacts and lignite clasts, suggesting that activity is limited by availability of electron donors. ${ }^{35} \mathrm{SO}_{4}$ reduction assays indicate high SR rates in shallow ( 0 to $8 \mathrm{~m}$ ) and deep (17 to $28 \mathrm{~m}$ ) sediments. Concurrent SR and sulfur-oxidizing activity at shallow depth is strong evidence for internal sulfur cycling in an aerobic aquifer.

Deeper sandy aquifers ( 17 to $30 \mathrm{~m}$ ) exhibit high SR activity and SRB numbers (104 cells $/ \mathrm{mL})$. Sulfate is completely consumed in one month in unamended incubations of these sediments, even though they are low in organic matter. Calculations of vertical sulfate transport rates suggest that shallow sulfide oxidation provides sulfate for deeper SR. Sulfur cycling may provide a survival mechanism for subsurface bacteria.

COMPARATIVE ANALYSES OF SUBSURFACE BACTERIAL COMMUNITY STRUCTURE: CORRELATIONS AMONG COMMUNITY COMPOSITION, ORIGIN, AND ENVIRONMENTAL PARAMETERS HOLBEN, WILLIAM E. UNIVERSITY OF MONTANA FY 1996259 FY 1995191 FY 1994121 
Keywords: bacterial transport, microbial ecology Objectives: To refine and apply laboratory methods for extraction of DNA from field samples and to use profile analysis of DNA variability to investigate and differentiate bacterial communities without lengthy and often inconclusive culturing and isolating individual species.

Approach: In situ microbial communities from aseptic subsurface microbiology cores will be analyzed, and results will be correlated with geochemical and hydrologic properties. New stable isotope labeling and detection techniques will be used to study the activity and transport of microbial communities in the subsurface by determining and comparing the stableisotope composition of bacteria, solid-sediment matrix, and groundwater (pore-water solutes). The methods will be extended to track the transport of bacteria in the subsurface based on differences in isotopic composition, with a focus on detecting isotopically labeled, injected bacteria.

Results to Date: Biochemical and molecular biological methods for DNA extraction, purification, and analysis have been developed to evaluate the bacterial community structure in varied subsurface environments. A bacterial tracking method that uses stable isotopes has been applied to track bacterial transport.

\section{EFFECT OF PHYSICAL AND CHEMICAL HETEROGENEITIES IN A POROUS MEDIUM ON THE TRANSPORT OF BACTERIA HORNBERGER, GEORGE M. UNIVERSITY OF VIRGINIA FY 199697 FY 199598 FY 1994130}

Keywords: porous media, bacterial transport Objectives: To determine how physical, chemical, and mineralogical heterogeneities in an unconsolidated sand aquifer impact the transport of bacteria through porous media.

Approach: The effect of heterogeneities in grain size, iron coatings on the mineral grains, and mineralogy of the grains on the transport of bacteria will be examined with well-characterized geological explosures in a pit that is representative of the geology found within an experimental flow field. Natural heterogeneities will be analyzed in reference to their control of the transport of bacteria. This work will form part of an integrated approach that uses a combination of field characterization, laboratory experimentation, and data analysis in which physical and chemical heterogeneities are considered.

Results to Date: The physical and chemical heterogeneities in near-surface sediments are being characterized at the field scale, and the importance of the characteristics of the sediments on parameters affect- ing bacterial transport is being examined. Information on the spatial structure of the data collected in the field is being used to infer the expected uncertainties in the prediction of bacterial transport in the field. Preliminary tracer studies (to simulate bacterial transport and to evaluate the influence of chemical heterogeneities on bacterial transport) have been completed; the information gained is being used to refine research models at the study site and as a precursor to additional core experiments by other participants that are aimed at quantifying differences in bacterial transport by geological facies.

\section{MICROBIAL AND CHEMICAL CONTROLS ON COCONTAMINANT FATE AND TRANSPORT \\ JARDINE, PHILIP; BROOKS, SCOTT; and GORBY, YURI \\ OAK RIDGE NATIONAL LABORATORY and \\ PACIFIC NORTHWEST NATIONAL LABORATORY \\ FY 1996350 \\ FY 1995301 \\ FY 1994155}

Keywords: biogeochemical dynamics, microbial reduction, radionuclides, organochelates, cocontaminant transport, kinetic modeling

Objectives: To determine how bacteria can be used effectively to immobilize high-priority radionuclides and metals, such as uranium (U) and cobalt $(\mathrm{Co})$, that are present in contaminated groundwater systems.

Approach: The reactions (e.g., adsorption, oxidation, and reduction) that govern the fate of contaminants as they move through subsurface soils and aquifer minerals will be studied by investigating the mechanisms and rates by which metal-reducing bacteria produce the less mobile, reduced forms of the chelated radionuclides from their more mobile, oxidized forms. By systematically varying relevant environmental parameters (e.g., $\mathrm{pH}$, fluid residence time in flowing systems, and dominant mineral phase), we will quantify the conditions that allow metal-reducing bacteria to immobilize contaminant metals and radionuclides. The geochemical reaction mechanisms that result in the formation of the mobile, oxidized forms of the complexed radionuclides will also be studied as will the factors that allow these reactions to occur and allow the oxidized forms to persist in reducing environments. Our unifying experiments will study the competing, interactive microbial and geochemical processes that dictate the fate and transport of contaminant metals and radionuclides.

Results to Date: We have found that: Metal-reducing bacteria can convert Co(III)EDTA to Co(II)EDTA and can effectively compete with geochemical oxidation reactions. Microbial reduction of Mn-oxides promotes 
the formation of $\mathrm{Mn}$ (II) that can effectively dissociate Co(II)EDTA, thereby stabilizing $\mathrm{Co}-60$ on mineral surfaces and enhancing in situ remedial strategies. The common subsurface mineral oxides of manganese and iron oxidize Co(II)EDTA to Co(III)EDTA; the extent of the oxidation reaction depends on $\mathrm{pH}$, dissolved oxygen, and residence time in the column. The rate and extent of Co-EDTA geochemical oxidation is limited by surface-reduction products that reversibly poison the redox reaction. The mechanisms of these reactions are being independently confirmed with $\mathrm{X}$-ray absorption spectroscopy. An analytical technique was developed to separate and quantify $\mathrm{Co}$ (In)EDTA and CO(III)EDTA in pore water with ion chromatography.

\section{ORIGINS OF SUBSURFACE MICROORGANISMS: RELATING LABORATORY MICROCOSM STUDIES TO A GEOLOGIC TIME SCALE \\ KIEFT, THOMAS; PHILLIPS, FRED; and AMY, PENNY \\ NEW MEXICO INSTITUTE OF MINING AND TECHNOLOGY and UNIVERSITY OF NEVADA, LAS VEGAS \\ FY 199671 \\ FY 1995200 \\ FY 1994185}

Keywords: microbial ecology, microbial survival, groundwater age, groundwater flow

Objectives: To determine the potential for microorganisms to survive (i.e., to maintain metabolic capability) in subsurface environments through geologic time (thousands to millions of years) and to characterize the changes in physiology and community structure that occur during long-term survival.

Approach: We are determining the potential for microbial survival in subsurface environments with field and laboratory experiments. In a collaborative field experiment at Cerro Negro, New Mexico, we have tested the alternative hypotheses that microbial communities in shales and sandstones of the San Juan Basin are derived from original Cretaceous marine sediment communities or that they were transported from the surface more recently. Methods include microbiological and hydrological characterization.

This study provides an understanding of the origins of subsurface microorganisms, their potential for transport, and their influence on subsurface biogeochemistry of the subsurface. In another project, we are determining the physiological status and community structure of microorganisms that have been geohydrologically isolated in buried soils (paleosols) for up to 1 million years. Besides demonstrating million-yearold viable microbial communities buried under unsatu- rated conditions, the study quantifies changes in community structure over geologic time.

Results to Date: Microbial communities in shale and sandstone near Cerro Negro appear to be derived from Cretaceous populations in unfractured shales and from recently transported populations in fractures and in sandstones. Our groundwater-age data $(20,000$ to 30,000 years) support the transport hypothesis.

Our paleosol chronosequence study, comprising a sequence of buried soils ranging from modern to 1 million years old, showed that microbiological parameters follow consistent trends with sediment age. Microbial numbers, diversity, and activity decline with age. Membrane phospholipid fatty acid (PLFA), diglyceride fatty acid (DGFA), and rRNA probe data show that the proportion of live cells declines with age. Viable microbes were detected in the oldest paleosol. The finding of viable microbial communities in geohydrologically isolated unsaturated subsurface environments extends our concepts of microbial subsurface survival, suggests unusual survival characteristics of potential use in introduced organisms, and suggests a potential for metabolic activity in other ancient sediments. In laboratory microcosms, subsurface microorganisms show high potential for survival. Survival is better in porous media than in liquid suspension. Survival is enhanced by saturated conditions and in paleosols. PLFA data show profiles typical of physiologically stressed microorganisms. These data show conditions that favor subsurface microbial survival and may be applicable to bioremediation efforts.

\section{COLLOID MOBILIZATION FROM AQUIFER SEDIMENTS IN THE SUBSURFACE LIANG, LIYUAN, and GU, BAOHUA OAK RIDGE NATIONAL LABORATORY \\ FY 1996150 \\ FY 199585 \\ FY 1994553}

Keywords: colloids, iron oxide, organic ligands Objectives: To determine the fate and transport of the uranyl ion as a result of iron oxide dissolution and mobilization in the presence of citrate under both biotic and abiotic geochemical environments.

Approach: In a subsurface media containing ironoxide-citrate-uranyl microbes, the transport of uranyl is hypothesized to be controlled in the following manner: (1) at low uranyl and citrate concentrations, where surface sites are available for complexation, uranyl is largely cotransported by sorption onto mobilized oxide particles; (2) a high citrate concentration increases uranyl transport as dissolved species (uranyl-citrate complexes); and (3) microbially induced dissolution of iron oxide causes surface-active ferrous species, which will reduce U(IV) to U(III), and precipi- 
tation of U(III) oxide on the solid. Laboratory experiments are being conducted to determine the dissolution kinetics of ferrihydrite (iron oxyhydroxide present at field sites) at $\mathrm{pH} 4$ and 6 in the presence of oxalate, citrate, and ascorbate. Competitive sorption and displacement of natural organic matter (NOM) subcomponents are being investigated for charge-reversal effects on the colloidal stability of iron oxide and the transport of the colloids.

Results to Date: Organic compounds, such as oxalate, citrate, and ascorbate, can dissolve iron oxide with a rate on the order of hours to days. NOM-containing functional groups, such as carboxyls and hydroxyls, can enhance the dissolution in the subsurface as observed in field experiments. The implications of this work are that (1) iron oxide colloids can be potentially released in situ when the conditions are favorable for the dissolution of the cementitious iron oxide and/or the charge reversals on particles and (2) the contaminants associated with the iron oxide, such as uranyl, will be affected in terms of the distribution in aqueous and surface phases. Laboratory batch and column studies showed that, in a binary organic system (phthalate, polyacrylate, and NOM subcomponents), the strong-binding organic components competed for surface sites and displaced the weak-binding organic compounds. The redistribution of the organic compounds resulted in the release of iron oxide colloids because of the increased colloidal stability caused by the strongly bound organic compounds. The work will benefit bioremediation for better delivery of nutrients and biocolloids (such as engineered microbes) to the subsurface, and for more accurate prediction of colloid-facilitated contaminant transport.

\section{BIOGEOCHEMICAL HETEROGENEITY IN THE SUBSURFACE LONG, PHILIP PACIFIC NORTHWEST NATIONAL LABORATORY \\ FY 1996443}

FY 1995225

FY 1994410

Keywords: biogeochemistry, subsurface microbiology, bioremediation, microbial transport

Objectives: To identify the critical biogeochemical heterogeneities that may limit the survival and transport of microorganisms in natural subsurface systems that typify the subsurface materials in which DOE will need to carry out in situ bioremediation.

Approach: Research is focused on establishing the relationship between physical/chemical heterogeneity and microbial distribution and function in the subsurface including (1) establishing the relationship between permeability and microbial distribution/ activity in shallow sandy sediments through applica- tion of ultra sensitive thermal imaging technology, (2) relating heterogeneities in permeability and grain size parameters to key enzyme activity and phospholipid fatty acid measurements in sediments with both sharp and gradational boundaries between sandstone and shale, and (3) defining the spatial distribution of anaerobic bacterial activity vis a vis grain size and heterogeneities in detrital organic matter with new autoradiography techniques developed by the University of Oklahoma. These three approaches to biogeochemical heterogeneity will contribute to an ability to predict how microbial activity can be expected to vary in the subsurface and to defining situations in which relatively minor changes in subsurface conditions can facilitate intrinsic bioremediation.

Results to Date: Fresh exposures of sandy aquifer material at the Oyster Pit were imaged with the ultrasensitive infrared (IR) camera. Initial interpretation of the images suggests a good correlation between individual sedimentary beds and centimeter-scale permeability distribution. Permeability contrasts at this scale apparently will need to be accounted for in modeling bacterial transport and in situ bioremediation. The $\mathbf{R}$ camera has also been used to measure the volume of sediment interrogated by the air minipermeameter. The volume of sediment actually interrogated appears to be smaller by about $50 \%$ than earlier estimates, indicating that the resolution of the air-permeameter measurements is significantly better than previously thought.

Analysis of shales and sandstones from field studies indicate that subsurface bacteria require porethroat diameters greater than $\mathbf{0 . 2}$ microns to maintain activity but that viable bacteria can be maintained in poorly permeable rocks, such as shales, with restrictive pore-throat diameters. Results also suggest that microorganisms recolonize medium- to fine-grained sediments at rates greater than $4 \mathrm{~m} /$ million years and that fractures may play a key role in microbial transport in fine-grained rocks. Overall, field studies indicate that physical properties of low-permeability, fine-grained subsurface materials will have to be altered (e.g., by hydrofracturing to increase permeablility) to complete in situ bioremediation within a reasonable time.

Preliminary results from analysis of autoradiography of sulfate-reducing bacteria (SRB) performed by Krumholz and Suflita at University of Oklahoma on Cerro Negro samples indicates that SRB are irregularly distributed in both coarse- and fine-grained samples, but are more abundant in coarse samples such as sandstones, where colonies occur every 2 to $5 \mathrm{~cm}$. There is not a strong correlation between SRB colonies and details of the sediment fabric suggesting that colony location may be controlled by population dynamics rather than specific environmental factors or nutrient distributions. 


\section{CHARACTERIZING THE EFFECT OF SUBSURFACE HETEROGENEITY ON BACTERIAL BEHAVIOR AND FLUID TRANSPORT WITH GEOPHYSICAL METHODS \\ MAJER, ERNEST L. \\ LAWRENCE BERKELEY NATIONAL LABORATORY \\ FY 1996250 \\ FY 1995282 \\ FY 1994586}

Keywords: bioremediation, hydrogeology, seismic imaging

Objectives: To identify the fundamental scales of physical heterogeneity controlling subsurface bacterial transport and complexity through the development and application of high-resolution geophysical methods.

Approach: High-resolution (cm scale) seismic and electrical imaging in a wide range of geologic conditions (dispersive to fast-path environments) and scales (laboratory to field dimensions) is being conducted in conjunction with controlled transport and bacterial injection experiments to relate the variation in geologic properties (lithology, grain size, porosity, fracture content, etc.) to variations in subsurface properties that may control subsurface microbial behavior (permeability, mineralogy, fluid content, nutrient flux and concentration, etc.). The geophysical methods are used for extrapolating point measurements (core) to volumetric estimates of physical and chemical properties at DOE sites where there has been information gathered on the microbiological, physical, and chemical properties. The approach uses controlled meter-scale field sites and supplementary laboratory- and intermediate-scale information to characterize those physical properties that affect fluid flow and chemical transport and that can be imaged with in situ methods.

Results to Date: At the field scale, high-resolution cross-hole seismic and radar surveys have been performed to infer lithology and moisture distribution. The results have been used to model the fluid-transport properties. Resolution of sedimentary sequences at the centimeter scale has been achieved. Radar and crosshole seismic measurements have been carried out at experimental bacterial-transport sites to define heterogeneity and lithologic control in preparation of tracerand bacterial-injection experiments. At the laboratory scale, block- and core-scale experiments have been performed on core samples to correlate $\mathrm{P}$ - and S-wave amplitudes, velocities, and gamma-ray attenuation with moisture content and bulk-grain-size variation, at the millimeter level of resolution. The results are being correlated with the bacterial properties at the same scale.

Field and laboratory results both find strong correlations between seismic attenuation and grain size. Radar is being used to map the water table and variations in lithology. Both radar and seismic measurements map the spatial variation in lithologic heterogeneity (layer thickness, interbedding, lensing, etc.) and are good indicators of the scale of spatial heterogeneity of the subsurface geology.

\section{ROLE OF STARVATION GENES IN THE SURVIVAL OF DEEP-SUBSURFACE MICROBIAL COMMUNITIES MATIN, ABDUL STANFORD UNIVERSITY \\ FY 199642 \\ FY 1995302 \\ FY 1994250}

Keywords: starvation genes, stress proteins, subsurface bacteria

Objectives: To determine (by evaluating stress proteins) whether subsurface bacteria can compete with similar surface bacteria under conditions that would prevail in the subsurface.

Approach: The research involves a blend of techniques: genetic manipulation, oligotrophic cultivation, assessment of the condition and activity of the individual bacterial cell, and in situ microbial ecology. The investigators are using microstat cultivation in groundwater as the simulated subsurface habitat and assessing the numbers and individual physiologic state of bacterial cells that can colonize groundwater-bathed surfaces. The suitability of such cultivation and assessment will be tested in the long term by placing the device into the subsurface at a field site. A subsurface isolate is being used that can (1) colonize the microstat and (2) express stress proteins that can be identified as such and compared with those synthesized by a similar organism from surface soil. Three derivatives of the subsurface isolate, each defective in the production of one stress protein, are to be used; the relative competitive success of surface soil vs subsurface isolates and of wild-type vs its defective derivatives in the microstat will be evaluated.

Results to Date: Progress has been made in understanding long-term microbial survival. The results are relevant to determination of survival qualities and mechanisms in subsurface bacteria.

$$
\begin{gathered}
\text { PORE-WATER CHEMISTRY } \\
\text { MCKINLEY, JAMES } \\
\text { PACIFIC NORTHWEST NATIONAL } \\
\text { LABORATORY } \\
\text { FY } 1996200 \\
\text { FY } 1995175 \\
\text { FY } 1994222
\end{gathered}
$$

Keywords: biogeochemistry, nutrient limitation, microbial ecology, sulfate reduction, pore-water chemistry 
Objectives: To determine the relationship between microbial community function and associated aquifer geochemistry (i.e., biogeochemistry) by investigating the geochemical controls on microbial community structure and activity and to assess the success of aseptic microbial sampling through design and implementation of chemical tracers.

Approach: At Cerro Negro (CN), N.M., it was hypothesized that organic carbon (electron donor) was supplied from carbon-rich shales to adjacent sandstone aquifers containing abundant sulfate (electron acceptor). A passive, multilevel sampler was deployed across a sandstone/shale boundary to measure compositional gradients of microbially sensitive components [e.g., $\mathrm{pH}$, anionic electron acceptors, organic acids, dissolved gases, and stable isotopes (C, O, H, and S)]. A sandstone substrate was used to determine in situ populations via colonization. The sample interval was $25 \mathrm{~cm}$ over a total interval of $20 \mathrm{~m}$ (80 samples); sampling extended from January to May 1996.

The geochemistry of Columbia River basalt (CRB) aquifers was used to investigate the hypothesis that bacterial communities derive energy from abiotic hydrogen produced by basalt/groundwater interaction. The dependence of microbial-community function on aquifer geochemistry was investigated by comparison of community structure with the concentrations of electron acceptors, dissolved gases, and organic carbon; the effects of the microbial community on system geochemistry were evaluated by examining the systematics of stable-carbon isotope signatures and the concentrations of metabolic byproducts.

At the TAN site, multilevel sampling of chemical and microbial heterogeneity will be used to determine the distribution of TCE in fractured basalt aquifers and to assess the response of in situ microbial communities to the presence of TCE.

Results to Date: Aqueous and solid-phase geochemical measurements at $\mathrm{CN}$ (taken at a relatively coarse sample interval) were consistent with the hypothesis of cross-stratigraphic electron donor/acceptor limitation of microbial populations; sulfate-reducing bacteria (SRB) were at maximum numbers at the sandstoneshale interface, and organic carbon concentrations and SO4 concentrations were higher in shales and sandstones, respectively. The geochemistry and flux of dissolved nutrients across the lithologic boundary have apparently permitted the long-term survival of anaerobic populations at this interface, where the ratio of electron donors to acceptors is optimal.

Anaerobic bacterial communities in CRB appear to derive energy from nonphotosynthetic energy in the form of abiotic $\mathrm{H}_{2}$. The concentration of $\mathrm{SO}_{4}$ determines whether SRB or methanogenic bacteria (MB) are numerically dominant. Experimental interaction of ferrous silicate-bearing rocks (e.g., CRB) with water confirmed the generation of $\mathrm{H}_{2}$ by this chemical system. Systematic variations in ${ }^{13} \mathrm{C} /{ }^{12} \mathrm{C}$ indicated that methanogenesis via the $\mathrm{CO}_{2}$ reduction/ $\mathrm{H}_{2}$ oxidation pathway was predominant throughout the aquifer, supporting the hypothesis that hydrogenotrophic bacteria were primary producers within this ecosystem. The CRB aquifers represent the first-known photosynthesis-independent ecosystem and are a plausible model for early life on Earth.

\section{GEOLOGICAL SUPPORT FOR MICROBIAL ORIGINS SUBPROGRAM MEYER, WILLIAM T. \\ ARGONNE NATIONAL LABORATORY \\ FY 19960 \\ FY 1995216 \\ FY 1994271}

Keywords: geological models, bacterial survival, bacterial transport

Objectives: To investigate the suitability of geological, geochemical, and hydrological environments for the collection of microbiological samples and to assist in the selection of sample sites for the Microbial Origins Subprogram of the Subsurface Science Program.

Approach: Geological scenarios were developed to define the geological, geochemical, and hydrological conditions that will meet the goals of the Microbial Origins Subprogram. Prospective field sites were identified and rated with respect to their potential to provide meaningful aseptic samples for microbiological analysis. At the Naval Oil Shale Reserve 3 (NOSR3) in the Piceance Basin, Colorado, large reserves of natural gas are found in the LateCretaceous-age Mesa Verde Group and the Tertiary Wasatch sands. Temperatures in the Mesa Verde Group during deep burial in early Eocene time probably exceded $250^{\circ} \mathrm{F}$ and would have been high enough to sterilize the rock. During the past 10 million years, downcutting of the Colorado River system has removed as much as $5000 \mathrm{ft}$ of overburden, significantly reducing the temperature. The thermal history of the basin allows the concept of recolonization of sterilized rock units in the Mesa Verde Group and the Wasatch Formation to be tested by means of a single drill hole at NOSR3.

Results to Date: Aseptic sampling at NOSR3 was successfully concluded in December 1994. Cores were recovered from depths of 2808 to 2828,6543 to 6551 , and 6862 to $6877 \mathrm{ft}$. The cores represent environments that have been heated and sterilized in the geological past and have since cooled to temperatures at which bacteria can survive, representing a test of the ability of subsurface bacteria to migrate and recolonize a formerly sterilized environment. The aseptic samples were provided to principal investigators in the Microbial Origins Subprogram. Preliminary data developed 
at Argonne from temperature logs and existing bottom-of-hole data indicate temperatures of about 43,86 , and $89^{\circ} \mathrm{C}$ for the three cores, in order of increasing depth.

\section{COMPARATIVE EVOLUTION OF THE recA GENE OF SURFACE AND DEEP- SUBSURFACE MICROORGANISMS MILLER, ROBERT V. OKLAHOMA STATE UNIVERSITY FY $1996 \quad 57$ FY 1995128 FY 1994120}

Keywords: microbial ecology, genetics

Objectives: To determine the evolutionary rates of subsurface microorganisms and the use of evolutionary divergences to establish isolation of subsurface microorganisms from surface environments.

Approach: The sequence diversity of the recA gene, which is central to the process of generalized homologous recombination and DNA repair, will be assessed. Specific bacterial species were selected from the Subsurface Science Culture Collection in consultation with other investigators. A surface strain of the same species was then studied and serves as a reference strain.Restriction fragment length polymorphism (RFLP) analysis was completed on the strains to determine population variation, followed by GC-clamp PCR. Mismatch cleavage pattern will be used to determine $300 \mathrm{bp}$ regions of identity with the reference strain. DNA sequencing was done with PCR generated fragments.

Results to Date: The data set has been analyzed for constancy of the molecular clock with the relative-rate test and will be analyzed to estimate divergence of surface and subsurface populations.

\section{FACTORS AFFECTING THE TRANSPORT OF BACTERIA IN POROUS MEDIA MILLS, AARON; HERMAN, JANET; and HORNBERGER, GEORGE UNIVERSITY OF VIRGINIA \\ FY 1996188 \\ FY 1995190 \\ FY 1994168}

Keywords: bacterial transport, tracers, bioremediation Objectives: To examine the effect of microbiological, chemical, and hydrological factors on the advective transport of bacterial cells through unconsolidated porous media.

Approach: Cell characteristics will be determined for populations of bacteria isolated from sediment and water samples from a pristine coastal aquifer in Virginia. An organism will be isolated from groundwater at the field site and tested (in this and other labs) for its mobility (in short-column adhesion assays, 13\% recovery) and resistance to a variety of antimicrobial compounds. DAPI staining of bacterial cells will be studied for use as a tracer in field injections. Nonreactive-, reactive-, and bacterial-tracer experiments will be conducted on aquifer material collected from the Oyster experimental field site.

Results to Date: No significant differences were found for adhesion of attached and unattached populations of isolates in small sand columns. Hydrophobicity and electrophoretic mobility did not differ significantly for the two groups. Isolates were tested for resistance to antibiotics (ampicillin, erythromycin, gentamycin, nalidixic acid, streptomycin, kanamycin, rifampin, and tetracycline). The percentage of (attached) isolates resistant to antibiotics ranged from $50 \%$ for gentamycin to $6 \%$ for tetracycline; in general resistance was higher for unattached organisms. Approximately $19 \%$ were resistant to four or more of the antibiotics tested. The results suggest that the attached and unattached populations are similar, and that they are subsets of one population with individuals moving from one attached location to another. These results indicate that isolates from either the sediment or the groundwater could serve as suitable candidates for injection. The isolate (PL2W31) is resistant to at least $50 \mathrm{gL}^{-1}$ of nalidixic acid but sensitive to the other seven compounds examined. It is slightly hydrophobic and has a neutral to very slightly negative surface charge. Fattyacid profiles indicate the organism is closely related to the genus Micrococcus; the organism will be used for the initial injection studies at the Oyster site in 1996. Stained cells were much less adhesive than unstained cells in Oyster sand, suggesting that the use of stained cells could yield an overestimate of bacterial transport in field experiments, but that stained cells would provide a traceable signal. Equilibrium sorption isotherms determined for several bacteria on different fractions of the sand indicated a higher sorption to that material with higher amounts of iron and aluminum coatings as well as differences among the strains tested. Columns containing homogeneously packed aquifer material showed greater retardation of the reactive tracer $\left(\mathrm{SO}_{4}{ }^{2-}\right)$ with respect to the conservative tracer $(\mathrm{Br})$ than a similar comparison done in cores from the aquifer with the chemical and physical heterogeneities intact. Bacterial-tracer experiments in cores with intact chemical and physical heterogeneities also showed greater recovery of cells and less retardation than in homogeneously packed columns of Oyster sand. The results of the experiments suggest that use of intact cores as opposed to repacked porous media more accurately simulates transport of bacteria in heterogeneous media found in field situations. 


\section{COCONTAMINANT REACTION CHEMISTRY AT INTERFACES MORRIS, DAVID; CONRADSON, STEVEN; and BERG, JOHN \\ LOS ALAMOS NATIONAL LABORATORY FY 1996200 \\ FY 1995222 \\ FY 1994185}

Keywords: biogeochemistry, clay minerals, radionuclides, organic ligands, sorption

Objectives: To provide comprehensive, molecular-level data on the chemical nature of radionuclide surface complexes on clay minerals and metal oxides derived from surface and shallow subsurface soils and on the role of organic complexing agents and microbial communities in modifying these interactions.

Approach: Surface-sensitive, molecular-level spectroscopic probes will be applied to characterize the surface complexes formed in binary (radionuclide plus surface) and ternary (radionuclide plus organic ligand plus surface) cocontaminant systems. The hypothesis that complexing organic cocontaminants (e.g., citrate or oxalate) will modify the sorption behavior and surface complexation mechanisms of uranium on subsurface solids will be examined.

A very powerful combined approach of spectroscopic characterization and thermodynamic modeling of surface complexation has been developed and is being tested in collaboration with investigators at PNNL. This approach provides a convergent description of surface complexation phenomena and leads to greater insight into the mechanisms and structures of surface complexes.

Results to Date: The sorption and surface complexation of uranyl, its hydrolysis products, and its complexes with oxalate and citrate have been studied on a reference smectite ( $\left.\mathrm{SWy}^{-1}\right)$ and kaolinite $(\mathrm{Kga}-1)$ and on a smectitic material derived from a soil $\left(\mathrm{LK}^{-1}\right)$. We used spectroscopy and modeling to constrain the description of the surface complexation in these systems in the absence of the organic complexant. We have confirmed the presence and relative abundances of cation-exchange complexes $\left(\mathrm{UO}_{2} \mathrm{X}_{2}\right)$ vs inner-sphere complexes on the amphoteric metal-oxide-like ( $\mathrm{SO}^{-} \mathrm{UO}^{2+} ; \mathrm{S}=\mathrm{Al}, \mathrm{Si}$ ) crystallite edge sites. We have also detected more hydrolyzed edge-site complexes $\left(\mathrm{SiO}-\left(\mathrm{UO}_{2}\right)_{3}(\mathrm{OH})_{5}\right)$ consistent with the modeling predictions and the solution speciation. Spectroscopic results for uranyl sorption to the soil smectite $\left(\mathrm{LK}^{-1}\right)$ provide a means to distinguish between two equally probable modeling predictions and demonstrate that edge-site complexation to both aluminol and silanol sites exists. These results indicate that edge-site complexation contributes more to uranyl sorption on the soil smectite than on the reference smectite as a result of particle size and morphology. Investigation into the uranyl-oxalate-SWy $\mathrm{y}^{-1}$ ternary system reveal that sorption is strongly suppressed at low and intermediate $\mathrm{pH}$ because of competition for uranyl by the ligand. At high $\mathrm{pH}$, sorption is observed and presumed to be the result of outer-sphere complexation of a uranyl-oxalate complex.

\section{INTERMEDIATE-SCALE INVESTIGATIONS OF MICROBIAL HETEROGENEITY MURPHY, ELLYN, and GINN, TIMOTHY PACIFIC NORTHWEST NATIONAL LABORATORY \\ FY 1996400 \\ FY 1995400 \\ FY 1994388}

Keywords: physical heterogeneity, chemical heterogeneity, reactive transport, biogeochemistry, bacterial transport, scaling

Objectives: To develop a fundamental understanding of how coupled physical and chemical heterogeneities affect microbial distribution and activity in subsurface environments and how these factors control intrinsic and manipulated bioremediation.

Approach: Interactive mechanisms (physical, chemical, and microbiological) that control the distribution and structure of subsurface microbial communities in heterogeneous porous media under controlled laboratory conditions in intermediate-scale flow cells (1-mlong, 2-D flow cells) will be identified and tested. Intermediate-scale experiments will simulate complex physical heterogeneities that approximate sedimentary formations commonly found in the eastern coastal plain (e.g., the Savannah River Site and Dover Air Force Base) and western alluvial sites (e.g., the Hanford Site). A heterogeneous hydraulic conductivity (K) field will be generated by packing the flow cell with randomly located low-K inclusions (silt-size) imbedded in a high-K sand matrix. For conditions ranging from flow and transport processes in physically heterogeneous systems and processes to more complex, coupled chemical and biological processes in natural systems will be tested. Experimental results are evaluated with high-resolution modeling (RAFT) to quantify these complicated biogeochemical processes. Results to Date: The physical heterogeneities created interfaces where slow transport from the low-K inclusion maintained combined oxygen and substrate availability adjacent to the inclusion while oxygen was rapidly depleted in the high-K sand. Therefore, microbial activity was greatest near these interfaces because of pore-scale mixing. Pore-scale mixing in heterogeneous systems reduced the effect of microbial metabolic lag, which, in turn, effectively increased the degradation activity in a fast-moving contaminant plume. Endogenous respiration, however, maintained low oxygen concentrations in the flow cell well after 
the disappearance of substrate. Both endogenous respiration and metabolic lag have been incorporated into the dual-Monod formulation of the reaction kinetics that is used in the RAFT modeling.

Microbial-reaction kinetics determined in batch experiments worked well to describe processes in the intermediate-scale flow cell. All of the biomass growth that occurred during the flow-cell experiment could be accounted for in the aqueous stream. A possible explanation is that the microorganisms may be transported by a growth process in which progeny are dispersed into the aqueous stream by cell division. These results led to the incorporation of dual-phase (aqueous and attached) microbial degradation kinetics into RAFT to improve predictive capabilities.

The basic scaling approach developed by this project (stochastic convective reaction, SCR) has been extended to include coupled physical and chemical heterogeneity effects on bioreactive transport. Experimental methods for creating isolated chemical heterogeneity (in the form of organic inclusions) in the flowcell experiments are currently being evaluated.

\section{MICROBIAL TRANSPORT/SURVIVAL IN SUBSURFACE SYSTEMS MURPHY, ELLYN \\ PACIFIC NORTHWEST NATIONAL LABORATORY \\ FY 1996450 \\ FY 1995450 \\ FY 1994388}

Keywords: biogeochemistry, bacterial transport, geochemistry, respiration rates, environmental tracers Objectives: To provide a quantitative understanding of the geochemical interactions that influence microbial survival during transport in the subsurface.

Approach: We use a combination of geochemical tools (natural stable and cosmogenic isotopes, groundwater chemistry, reaction-path geochemical models, physically based transport models) and microbiological tools [molecular analyses such as community- and organism-level phospholipid fatty acid (PLFA), microbial identification (MIDI), phylogenetic analyses] to quantify microbial/geochemical interactions affecting survival, activity, and transport in natural field settings, both saturated and unsaturated.

For example, we combined data on the geochemistry of electron donor/acceptor pools, equilibrium geochemical reactions, fractionation of stable isotopes (e.g., carbon, sulfur, and iron), and groundwater ages to calculate population in situ respiration rates of aerobic heterotrophs, $\mathrm{MnO}^{2-}=\mathrm{Fe}$ (III)-, and $\mathrm{SO}_{4}{ }^{2-}-$ reducing bacteria along a well-defined groundwater flow path. These natural in situ rates indicate dominant populations within the microbial community that can be stimulated during intrinsic bioremediation activities.

In unsaturated systems, the transport and survival of indigenous microorganisms were evaluated at the semiarid Hanford Site in Washington State. In semiarid regions, recharge occurs primarily in low-elevation drainages. These areas are the primary vectors by which microorganisms enter the unconfined aquifer at the Hanford Site. Microorganisms undergo physiological changes which facilitate survival and transport through the deep unsaturated zones found in the western United States. Movement through the unsaturated zone is also a selection process, which ultimately determines the structure of the microbial community available for intrinsic bioremediation in the unconfined aquifer at Hanford. Natural environmental tracers deposited from the atmosphere were used to determine the recharge rates (effective transport fluxes) through the unsaturated zone. Molecular techniques were used to evaluate and compare the microbial-community structure along unsaturated flow paths characterized by high and low transport fluxes.

Results to Date: In saturated groundwater systems, in situ respiration rates were determined for individual populations within a diverse microbial community consisting of aerobic heterotrophs and manganese oxide-, iron-, and sulfate-reducers. The activity of different populations of microbes dominated at different points along sections of the flow path, and the low in situ rates were consistent with natural subsurface environments.

Iron isotope measurements on groundwater and crystalline and amorphous phases of iron oxides showed that the lighter iron isotope $\left({ }^{54} \mathrm{Fe}\right)$ was consistently depleted in the groundwater samples. The mechanism resulting in the heavier isotopic signal in groundwater may result from a microbially mediated process (e.g., Thiobacillus) that preferentially oxidizes isotopically light dissolved ferrous iron to iron oxides. These results suggest that, if fractionation occurs during dissimilatory iron reduction (iron oxides converted to soluble ferrous iron), it is masked by the larger fractionation occurring during the iron oxidation portion of the cycle. Iron isotopes will provide a valuable tool for understanding the complex iron cycle in subsurface systems.

In unsaturated sediments, the transport of microorganisms is maximized at zones of high recharge in the semiarid West. Natural environmental tracers indicated that the ages of pore waters in the unsaturated zone ranged from 13,000 to 30,000 years at the low-recharge sites to tens to hundreds of years at the high-recharge sites. Because microorganisms are transported by water films, these geochemical tracers provide a minimum time over which the microorganisms could have been transported. 
Drilling and sampling in the Palouse region of eastern Washington State was completed in May 1996. These sediments represent a continuous chronosequence of aeolian-deposited sediments ranging in age from recently deposited sediments at the surface to sediments deposited one million years ago at depth. Detailed analyses of the sediment and pore water ages and characterization of the microbial community will provide insight into isolation age and survival mechanisms used by microorganisms over long time frames in arid environments.

\section{EVOLUTION OF A METABOLIC GENE IN SUBSURFACE MICROORGANISMS NICHOLS, BRIAN \\ UNIVERSITY OF ILLINOIS, CHICAGO CAMPUS FY 19960 \\ FY 1995150 \\ FY 1994142}

Keywords: evolution, nucleotide sequences, $\operatorname{trp} E$, Arthrobacter

Objectives: To determine nucleotide sequence of $t r p E$ gene from subsurface isolates of Arthrobacter sp. to compare relationships with surface isolates.

Approach: The trpE sequences will be amplified from chromosomal DNA preparations by PCR, and nucleotide sequences will be determined from amplified products. The nucleotide sequences will be aligned and analyzed for relationships with McClade and PAUP analysis.

Results to Date: Sequences of the entire $\operatorname{trpE}$ gene have been obtained from Arthrobacter globiformis ATCC 8010, and partial sequences have been obtained from 18 Arthrobacter isolates from the GEMHEX experimental site. The relationships are under current analysis and are being compared to similar results from 16S rRNA sequences and $r e c A$ sequences.

\section{MICROBIAL COMMUNITIES IN SUBSURFACE ENVIRONMENTS: DIVERSITY, ORIGIN, AND \\ IN SITU METABOLIC ACTIVITIES NIERZWICKI-BAUER, SANDRA RENSSELAER POLYTECHNIC INSTITUTE FY 199698 FY 1995175 FY 1994162}

Keywords: molecular biology, microbial ecology, evolution, origins of subsurface microbes

Objectives: To develop and use molecular techniques to assess the influence of environmental conditions on the distribution, evolution, and metabolic activities of subsurface microorganisms by means of 16S-rRNAtargeted oligonucleotide probes, polymerase chain reaction (PCR) amplification of gene sequences, and sequencing techniques.
Approach: Target sequences for selected microbial types and groups have been identified by analysis of rRNA sequence data for subsurface microorganisms and other microorganisms in the rRNA databases. Hybridization probes for these target sequences are produced and used to classify isolated strains of subsurface microbes into phylogenetic groups.

Results to Date: A method to extract total community DNA from GEMHEX lacustrine sediments was developed. The 16S rRNA genes from extracted community DNA were PCR amplified and cloned and are being sequenced. Isolates obtained from sediments at the GEMHEX borehole have been phylogenetically classified with 16S rRNA group- and species-specific targeted probes. Microbial-diversity estimates were compared based on in situ

hybridizations with 16S rRNA targeted probes, and 16S rRNA sequencing has been conducted. Seventeen isolates analyzed by these approaches revealed complete agreement between the two methods.

Efforts to correlate geological information with microbial population studies within the GEMHEX interval has indicated the presence of distinct microbial communities within different lithological units having unique environmental conditions (e.g., varying $\mathrm{pH}, \mathrm{Eh}$, and solution chemistry). A good correlation among PFLA data, in situ phylogenetic identification, and geological characteristics was observed.

Pseudomonad isolates from the SRS screened for the presence of group I self-splicing introns indicate that 13 of 50 isolates may possess introns. Current studies involve RFLP analyses and sequencing.

\section{GEOCHEMICAL CONSTRAINTS ON THE LONGEVITY AND ENVIRONMENTAL HABITAT OF SUBSURFACE MICROBIAL COMMUNITIES ONSTOTT, T. C. PRINCETON UNIVERSITY FY 1996250 FY 1995240 FY 1994143}

Keywords: geomicrobiology, hydrogeology Objectives: To acquire samples bearing microorganisms from the deep subsurface and to characterize those samples by petrographic and geochemical methods to constrain the origins of the microbial communities.

Approach: Microbial sampling of the deep subsurface will be undertaken in the Taylorsville Basin, San Juan Basin, and Piceance Basin.

Results to Date: Microbial sampling of Taylorsville Basin was completed in April 1992, and studies of microbiota are ongoing. We have completed observations with respect to the thermal history of the basin and a fully coupled thermal/fluid flow history. Using 
solute-transport models, we are completing estimates of the migration rates in these transient models to determine the origins of the microbial populations recovered from $2.8 \mathrm{~km}$ beneath the surface. Modeling of the petrophysical logs and borehole temperature data by a steady-state fully coupled 2-D heat transport model indicates that the groundwater age of the microbial sampling is approximately 80 million years. Analyses of the petrography, stable isotope chemistry, and fluid inclusions indicate that formation temperatures exceeded the maximum survival temperature for bacteria 210 to 190 million years ago. Fluid-inclusion analyses and transient-fluid-flow models indicate that the bacteria migrated into the sampling zone during the period of uplift and erosion of the basin, soon after 190 million years ago. Microbial sampling of the San Juan Basin was completed in October 1994 and studies of the microbiota are ongoing. We completed our research on the delineation of the thermal, salinity, and diagenetic history of the microbial site. A multilevel water sampler (MLS) was extracted from the borehole in May 1996, and the water samples are being geochemically, isotopically, and microbially analyzed. Analyses of the petrography and stable isotope chemistry indicate that the formation did not exceed temperatures for microbial survival, but that the original marine depositional environment was replaced by a freshwater meteoric environment during uplift in the Miocene. Analyses of the sulfur isotopes of pyrite have revealed the early presence of microbial sulfate reduction. Analyses of sulfur isotopes in groundwater indicates that sulfate reduction is ongoing today.

Microbial sampling of the Piceance Basin was completed in 1994. We have completed initial geochemical and microbial sampling of groundwater and natural gas condensate for microbiota and geochemistry. Studies of the microbiota are ongoing. We have completed our delineation of the thermal, salinity, and fluid-flow history of the sample strata. Analyses of the petrography, stable isotopes, fluid inclusions, and vitrinite reflectance indicate that the microbial sampling zones were heated to temperatures exceeding those for microbial survival. The presence of bacteria in one of these zones and their absence in the other reflects microbial transport during uplift and cooling of the basins during the past 5 million years. Numerical simulations of the fluid flow in the basin are providing constraints on the groundwater ages that will be tested by cosmogenic isotope analyses. Analyses of groundwater samples indicate that the microbial communities in Piceance Basin are stratified with sulfate reducers dominating the top $500 \mathrm{~m}$ and metal reducers dominating the depths between 500 and $2000 \mathrm{~m}$.

\author{
SPATIAL HETEROGENEITY OF \\ SUBSURFACE MICROBES \\ PALUMBO, ANTHONY; PHELPS, TOMMY; \\ and WILSON, GLENN \\ OAK RIDGE NATIONAL LABORATORY and \\ THE UNIVERSITY OF TENNESSEE \\ FY 1996495 \\ FY 1995341 \\ FY 1994291
}

Keywords: subsurface heterogeneity, biogeochemical dynamics, field scale

Objectives: To evaluate the effect of subsurface heterogeneity on potential remediation activities under different hydrogeological conditions and to facilitate research in contaminant transport in subsurface environments. Comparison will be made between highrecharge regions of the eastern coastal plain and the low-recharge regions of western United States.

Approach: Ground-penetrating radar will be used to geophysically characterize the sites. The permeability and grain-size distribution of the subsurface sediments will be geologically characterized. The organic and inorganic nutrients of subsurface sediments will be geochemically characterized. The biomass, activity, microbial-community structure, lipid biomarker, and genetic DNA of subsurface sediments will be microbiologically characterized. And geochemical and microbiological properties will be correlated by using geostatistical techniques and multiple-constraint models.

Results to Date: Studies of subsurface heterogeneity at the Oyster Site and the Abbott's Pit Site in Virginia demonstrated that (1) variations in sample volume $(0.1$ to $100 \mathrm{~g})$ and fine-scale $(\sim 5 \mathrm{~cm})$ sampling were relatively insensitive to variations in microbiological and chemical properties at these sites; (2) variability of microbiological and chemical properties increased significantly at scales greater than $1 \mathrm{~m}$; (3) water availability, inorganic nutrients (such as phosphate), and mineralogical compositions affected microbial diversity and activity (although microbial activities appeared to affect the distribution of iron and nitrate in sediment profiles); and (4) a multiple-constraint model helped us to relate microbiological heterogeneity to the distribution of physical and geochemical properties in the subsurface more accurately than using conventional regressional models. A modified geostatistical model has enhanced our capability to predict microbial variability within meters of distance. These results contrast significantly with studies at the White Bluff Site in Washington State, where microbial abundance and activity are low and discontinuous because of the low water recharge into the subsurface. Results of the heterogeneity study provide critical information for implementing DOE bioremediation programs under different hydrogeological conditions. Our experience 
and knowledge of small-scale heterogeneity and its relationship to larger scales in uncontaminated sites give us confidence and expertise to characterize microbial variability and to understand the transport of contaminants and the delivery of nutrients for in situ bioremediation at contaminated sites.

\section{CORE SUPPORT OF THE WATER SCIENCE AND TECHNOLOGY BOARD PARKER, STEPHEN D. NATIONAL ACADEMY OF SCIENCES FY 199680 \\ FY 199525 \\ FY 199425}

Keywords: hydrology, hydrogeology, bioremediation Objectives: To provide independent evaluations of activities in the hydrologic sciences, analyses of groundwater movement and the hydrologic cycle, and water-quality- and water-resources-management investigations.

Approach: The Board conducts independent scientific and technical reviews and objective assessments of the state-of-the-art related to surface-water and groundwater environments. The Board assists DOE and other agencies by providing scientific advice on issues in the fields of hydrology and hydrogeology. DOE provides core support to the Board, which assists in completing studies in hydrology and related fields.

Results to Date: The Board completed many important studies, including one on Opportunities in the Hydrologic Sciences, which defined long-term research needs in hydrology. The Board expects to update this work with a series of seminars and/or colloquia that will refine and update the Board's analysis of future research. The Board reports on bioremediation and groundwater-cleanup alternatives that have been used in planning future OHER research (NABIR). Besides informal written reports in response to requests from the DOE, the Board provides DOE with a scientific contact point on national surface and groundwater research.

\section{MICROBIAL ECOLOGY OF DEEP MICROBIOLOGY RESEARCH SITES PHELPS, TOMMY \\ OAK RIDGE NATIONAL LABORATORY}

FY 1996255

FY 1995223

FY 1994281

Keywords: subsurface science, microbial ecology, microbiology, bioremediation

Objectives: To develop and substantiate a conceptual understanding of geological and biological factors facilitating bacterial activity and survival in subsurface sediments, including gaining a basic understanding of subsurface biological/chemical/physical processes, transferring increased understanding to further the application and success of bioremediation at DOE sites, linking our increased understanding with need of $\mathrm{EM}$, and transferring knowledge and developed technologies to field-scale remediation.

Approach: Groundwater and sediment chemistry, hydraulic characteristics, and geologic interpretations provided by collaborators is combined with multiple measures of microbial activity, and biostimulation experiments, biomass determinations, microbial functional group enumerations, activity measurements, and nutrient-limitation experiments to develop an understanding of nutrient and energy flux in varied subsurface environments. Linking geological and biological results and interpretations are used to communicate a cross-disciplinary understanding through peerreviewed publications. This approach has been successfully employed to constrain microbial activities and generation times in aquifer flow paths, enrich suspected but previously unobserved novel types of metalreducing microorganisms with unusual metabolic potentials, compare and contrast physical and nutrient constraints on microbial populations in varied subsurface habitats, and transfer novel biostimulation technologies to EM.

Results to Date: Substrate pools of $10 \mu \mathrm{g} / \mathrm{L}$ were found to retain microbial communities larger than $100,000 / \mathrm{g}$. Average microbial doubling times in aquifers were estimated to be on the order of centuries. Even though growth was slow, microbial metabolism displayed major impacts on groundwater chemistry and bioremedial potential. Results revealed that nutrient flux often limited microbial growth and toxicant removal.

Geologically sequestered environments revealed novel thermophilic microbes that produce magnetic iron oxides. These nanometer-sized, octahedral-shaped crystals of magnetite or maghemite may be useful in bioremediation or biotechnology applications. During early biosphere evolution, thermophilic metal-reducing respiratory microorganisms may have complemented the formation of banded iron. The nanometer-sized reduced metal oxides also dechlorinate chlorocarbons. Furthermore, these microorganisms can reduce iron, manganese, cobalt, uranium, chromium, and other metals, suggesting potential roles for these microorganisms in remediating mixed wastes.

\author{
THERMOPHILIC EXPLORATORY \\ RESEARCH \\ PHELPS, TOMMY \\ OAK RIDGE NATIONAL LABORATORY \\ FY 19960 \\ FY 199530 \\ FY 199430
}


Keywords: subsurface science, microbial ecology, bioremediation, microbiology

Objectives: To investigate mechanisms of microbial survival in sequestered subsurface environments subjected to thermal stresses of 50 to $80^{\circ} \mathrm{C}$ and hundreds of atmospheres of pressure.

Approach: Bioreactors will be developed to emulate the high temperatures and pressures characteristic of subsurface formations several kilometers beneath the land surface. Replicate bioreactors could be used to test variables affecting survival of microbial cultures derived from sequestered thermal environments. By examining growth, survival, activity, and microbiallipid changes over time as affected by substrates, electron donors and acceptors, or mineral phases, we could gain insight on microbial survival in sequestered environments.

Results to Date: The inert bioreactor systems (titanium vessels with quartz linings) are capable of maintaining sterile, anaerobic, thermal, and hydrostatically stable conditions at 110 atmospheres pressure and at $65^{\circ} \mathrm{C}$ for periods of months at a time. In all conditions, more than $99 \%$ of the cells die within 2 months at temperatures of $65^{\circ} \mathrm{C}$ and a pressure of $100 \mathrm{~atm}$. In the absence of mineral phases from the environment and with the cells suspended in buffer devoid of nutrients, less than $0.01 \%$ of the cells survive after 2 months. In contrast, low concentrations of nutrients ( $10 \mathrm{mg} / \mathrm{L}$ ) enabled the survival of $0.15 \%$ of the microorganisms versus $1.5 \%$ survival when high concentrations ( $1000 \mathrm{mg} / \mathrm{L})$ of organics were abundantly available. The presence of mineral solids from the sequestered environment substantially improved microbial survival, but the best survival was at room temperature, where more than $1 \%$ of the cells survived after 3 months of storage. These results suggest that temperature and pressure challenge the integrity of bacterial survival over long periods of time. While environmentally derived solid-matrix minerals prolong survival, the challenges imposed by temperature and pressure are dramatic.

\section{RADIONUCLIDE SPECLATION IN GROUNDWATER SYSTEMS REED, DONALD

Keywords: actinides, microbes, nitrilotriacetic acid, biodegradation, radiotoxicity

Objectives: To investigate the interactions between microbiological systems and plutonium-organic complexes to identify key processes leading to the mobilization and/or immobilization of actinides in subsurface environments.
Approach: The model system (C. heintzii, nitrilotriacetic acid, and plutonium) is being investigated to determine the effects of plutonium on the biodegradation of nitrilotriacetic acid and the effects of the microbiological activity on the fate and speciation of the plutonium present in solution. The effects of plutonium on the viability of $C$. heintzii (i.e., radiotoxicity) will be established by a combination of gamma irradiation studies and isotope-specific plutonium studies, where the number of colony-forming units are established as a function of plutonium concentration, plutonium activity, and absorbed gamma dose. A series of biodegradation experiments will establish the effects of plutonium on the rate of degradation and the effects of biodegradation on the fate of plutonium. A model specific to our Pu-NTA-C. heintzii system will be developed; fundamental data (e.g., mass balance, relationships between cell growth and oxygen availability, and biomass correlations) will be amassed.

Results to Date: Studies of the Pu-NTA-C. heintzii system show that the radiotoxicity of plutonium is primarily radiolytic in nature, but bioassociation of the plutonium is also a necessary step for toxicity. A strong bioassociation between plutonium and $C$. heintzii was produced by two mechanisms: a fast association observed at low [Pu] because of biouptake/ sorption and a slower process caused by destabilization of the NTA complex in solution and subsequent plutonium polymer formation.

Only free NTA was readily degradable. NTA present as a Pu-NTA complex was not directly degradable.

An assessment of data for modeling the degradation of NTA by $C$. heintzii showed that there was inadequate data on biomass, mass balance, and kinetics to model the degradation process. Significant improvements in the modeling of the degradation of NTA by $C$. heintzii were made after (1) obtaining data on mass balance between mineralization rates, NTA loss, and ammonia production; (2) obtaining accurate biomass data; (3) direct monitoring of NTA degradation rather than mineralization rates to obtain the kinetic data; and (4) a quantification of oxygenavailability limitations.

$$
\begin{gathered}
\text { MODELING OF COUPLED PROCESSES IN } \\
\text { SUBSURFACE TRANSPORT OF REACTIVE } \\
\text { CONTAMINANTS } \\
\text { RITTMANN, BRUCE, and VALOCCH, ALBERT } \\
\text { NORTHWESTERN UNIVERSITY and } \\
\text { UNIVERSITY OF ILLINOIS } \\
\text { FY } 1996164 \\
\text { FY } 19950 \\
\text { FY } 1994 \quad 0
\end{gathered}
$$

Keywords: reactive transport, biodegradation, adsorption, complexation, cocontaminants 
Objectives: To develop and use a 2-D transport model that can handle spatial heterogeneity and the complex chemical and microbial reactions present when radionuclides and strong organic chelators are cocontaminants in a subsurface setting.

Approach: At the University of Illinois, a code called FEREACT is being developed to model transport with spatial heterogeneity and relatively simple reactions. At Northwestern University, a code called CCBATCH is focusing on the complex chemical and microbial reactions and batch experiments used to elucidate them. Both codes are being developed so they can ultimately be combined.

Results to Date: A 2-D multiple-component reactive transport code named FEREACT has been developed and tested. A preliminary analysis of the impact of chemical heterogeneity upon transport-model uncertainty has been completed with Monte-Carlo simulations with FEREACT. The batch code CCBATCH has been developed and tested for simultaneous chemical and microbiological reactions important for the transport of organically complexed radionuclides. It has been applied to simulate degradation of NTA in metal-NTA experiments conducted at PNNL and to simulate degradation of citrate in metal-citrate experiments conducted at BNL. A cocontaminant-chemistry workshop was held on integrating biogeochemical modeling with bench- and intermediate-scale experimentation.

\section{IMPACT OF MEASUREMENT INSTRUMENT AND CONCEPTUAL MODEL ON ANALYSIS OF SUBSURFACE HETEROGENEITY SILLIMAN, STEPHEN, and KULPA, CHARLES UNIVERSITY OF NOTRE DAME FY 1996191 FY 1995122 FY 199499}

Keywords: microbial transport, instrumentation, groundwater experiments

Objectives: To conduct controlled laboratory and numerical experiments whereby the impact of physical heterogeneity on the prediction of chemical and microbial transport can be demonstrated and characterized with high-density sampling strategies in combination with existing deterministic and stochastic theories of groundwater flow and transport. Short-term objectives include testing of a newly developed sampling strategy and the design of a microbial-transport experiment in heterogeneous medium.

Approach: A combination of laboratory flow cells, numerical modeling, and theoretical analysis will be used to identify the effects that selection of instrument and conceptual model have on characterizing physical heterogeneity. Both controlled laboratory experiments on 2- and 3-D heterogeneous porous media and numer- ical simulation will be used. Measurements include dense sampling networks for microbial and chemical concentration, sampling of outflows for microbes and chemical concentration, and dense sampling networks for pressure. Conservative chemicals and bacteria are used as tracers. These experiments will be interpreted in terms of (1) modifications of our field techniques, (2) assessment of prediction uncertainties in established data-analysis techniques, (3) assesssment of the increase in prediction uncertainty as a function of declining sampling density, and (4) comparison of the cause of prediction uncertainty in chemical vs microbial transport.

Results to Date: Laboratory results demonstrated the strong influence of contacts between sediments with different pore sizes on the retention of particles. Further, retention of particles was reduced when the dimension of the flow system was increased from two to three dimensions. A tremendous uncertainty was found inherent in characterizing hydraulic gradients from limited data. Two measurement strategies were developed for characterizing local pore-water velocities in our laboratory flow cells. These strategies are currently being applied to characterization of the statistical description of the velocity distribution in a 2-D, random-field medium.

\section{EFFECTS OF CORRELATED PHYSICAL AND CHEMICAL HETEROGENEITIES ON COCONTAMINANT TRANSPORT \\ SMITH, ROBERT, and SCHAFER, ANNETTE IDAHO NATIONAL ENGINEERING LABORATORY \\ FY 1996600 \\ FY 1995475 \\ FY 1994556}

Keywords: biogeochemistry, spatial heterogeneity, bioremediation, sorption, uranium, trichloroethylene Objectives: To determine the interrelationships between geological features and the spatial variability of permeability and chemical reactivity and to evaluate the role of correlated hydrological and geochemical heterogeneities in controlling (1) the subsurface distribution of microbially active zones; (2) the field-scale transport of bacteria, electron donors/acceptors, and nutrients; and (3) the fate and transport of cocontaminant mixtures.

Approach: Field and laboratory investigations of correlated physical and chemical heterogeneities will be conducted at a field site near Oyster, Virginia. The results of this research will include a geostatistical representation of the physical and chemical properties that control the subsurface transport of bacteria and the fate and transport of cocontaminant mixtures. The role of physical and chemical heterogeneity in the distribution of biologically active zones will be investigated at 
the trichloroethylene-(TCE) and radionuclide contaminated Test Area North (TAN) site at the Idaho National Engineering Laboratory. Although the scale of heterogeneity at the TAN site is much larger than at the Oyster site, the sites are similar in that thin, low-permeability, high-reactivity zones separate thick, high-permeability, low-reactivity zones. By conducting field investigations at the Oyster site (correlation lengths of centimeters to meters) and the TAN site (correlation lêngths of tens to hundreds of meters), the effects of scale of heterogeneity on transport of cocontaminant mixtures, electron donors/acceptors, and nutrients and the distributions of subsurface microbial activity can be evaluated.

Results to Date: A preliminary geostatistical model of physical and chemical heterogeneity has been developed and used in the design and interpretation of the results of the tracer and bacterial transport experiments conducted at the Oyster site. The geostatistical model includes measured cross-correlations between chemical reactivity [as quantified by the adsorption of uranium(VI)] and permeability that are consistent with relationships derived from theoretical considerations. In addition, the relative significance of intrinsic biodegradation of TCE at TAN on the establishment of remediation goals has been conducted. These research results have been transferred to the INEL site remediation program (EM-40) in support of natural-attenuation treatability studies required by the TAN Record of Decision (ROD). Additional, ongoing tech transfer meetings with the site-remediation group has resulted in the transfer of Subsurface Science Program aseptic coring and sampling technology to the site-remediation group in support of the natural attenuation and bioremediation treatability studies required by the TAN ROD.

\section{RELEASE OF OXIDE-BOUND METALS BY NATURALLY OCCURRING AND CONTAMINANT-DERIVED ORGANIC COMPOUNDS STONE, ALAN \\ JOHNS HOPKINS UNIVERSITY \\ FY 19960 \\ FY 199593 \\ FY 199491}

Keywords: ethylenediaminetetraacetic acid, nitrilotriacetic acid, cobalt, manganese

Objectives: To determine how organic compound structure affects the ability of naturally occurring and contaminant-derived compounds to reduce toxic metal contaminants, form complexes with toxic metal contaminants, and adsorb onto mineral surfaces.

Approach: Capillary electrophoresis will be used to examine the reaction of EDTA, NTA, IDA, and their breakdown products with contaminant metal species
[Co (II/III)] and naturally occurring metal species [Mn (II/III)]. $\mathrm{CoOOH}(\mathrm{s})$ and $\mathrm{MnOOH}(\mathrm{s})$ have been synthesized; ligand-assisted and reductive dissolution is monitored upon addition of the candidate organic compound. A number of authentic standards for breakdown products are available commercially. Other breakdown products, and various complexes with $+\mathrm{II}$ and + III metal species, have been synthesized in our laboratory. Results to Date: As expected, addition of EDTA to $\mathrm{CoOOH}$ suspensions yields dissolved Co(III)EDTA complexes. To our surprise, reduced cobalt complexes [e.g., Co(II)EDTA] and EDTA oxidation products (e.g., ED3A) are also produced. Reaction of NTA with $\mathrm{CoOOH}$ suspensions also yields $\mathrm{Co}$ (II//II) complexes and NTA oxidation products (e.g., IDA). MnOOH (manganite) reacts with EDTA to yield dissolved Mn(II) complexes and EDTA oxidation products; no dissolved Mn(II) complexes are formed. Numerous experiments examining the stoichiometry of $\mathrm{CoOOH}$ and $\mathrm{MnOOH}$ dissolution reactions at varying $\mathrm{pH}$ and organic-compound concentrations have been completed.

Addition of IDA to $\mathrm{CoOOH}$ suspensions yields two distinct stereoisomers of Co(III)(IDA)2. The ratio of these two stereoisomers depends upon the dimensions of the crystalline $\mathrm{COOOH}$ particles.

\section{CYCLING OF ORGANIC MATTER BY ANAEROBIC MICROORGANISMS IN CONSOLDATED SUBTERRANEAN FORMATIONS \\ SUFLITA, JOSEPH, and KRUMHOLZ, LEE UNIVERSITY OF OKLAHOMA \\ FY 1996120 \\ FY 199561 \\ FY 19940}

Keywords: cretaceous, sulfate reducing bacteria, anaerobic bacteria, Cerro Negro

Objectives: To characterize in situ sulfate-reduction activities within intact cores from the Cerro Negro drilling project with the silver-foil technique, to determine the source of organic matter used for the reduction of sulfate with the use of microcosms, and to isolate pure cultures of sulfate-reducing bacteria from the most active sediments.

Approach: Our initial experiments focus on a localization of sulfate-reducing microorganisms on a 2-D scale within the geological material. We use $35 \mathrm{~S}$ sulfate incubated on the surface of a core face that has been overlain with silver-oxide-coated silver foil. Silver sulfide can then be quantitated on a 2-D scale by autoradiographic analysis. We have previously demonstrated that sulfate-reduction activities are high in only a few subsurface samples, such as sandstone materials that are adjacent to and below shales. This relationship will be investigated by supplying organic matter from 
the shales to the particular sandstone samples in microcosms. Organic extracts will ultimately be characterized to determine the source of organic matter for these microorganisms. Concomitant experiments will determine whether pure cultures of sulfate-reducing bacteria can be isolated from the most active sedimentary rocks.

Results to Date: Shale and sandstone cores from the Cerro Negro site exhibited sulfate-reducing activity over the 4- to 6-week incubation period. Several cores, notably sandstones from near sand-shale interfaces were by far the most active. These interfacial cores exhibited 100- to 1000-fold greater levels of sulfatereducing activity as compared to adjacent shales and noninterfacial sandstones. Experiments showed that the electron donor for sulfate reduction in sandstones could be obtained from the shale deposits. These rock samples were also acetogenic, and acetate was shown to be produced at levels proportional to sulfide levels. Acetogenic bacteria capable of growing with $\mathrm{H}_{2}$ and $\mathrm{CO}_{2}$ were subsequently isolated from these samples. These experiments lead us to believe that organic-rich shales can slowly deliver organic matter to highly active microbial communities living at the sandstone-shale interfaces.

\section{HYDRAULIC PROPERTIES IN SHALLOW MARINE AQUIFERS SCALED BY DEPOSITIONAL ARCHITECTURE SWIFT, DONALD J. P., and NIEDORODA, ALAN W. OLD DOMINION UNIVERSITY \\ FY 1996126 \\ FY 1995126 \\ FY 19940}

Keywords: bioremediation, hydrologic properties, Atlantic Coastal Plain

Objectives: To provide geological and geostatistical characterization of DOE sites in the middle Atlantic coastal plain and to link mathematical descriptions of the depositional fabric with mathematical descriptions of the resulting hydrologic properties across a range of physical scales to predict the distribution of these properties in sedimentary aquifers.

Approach: Aquifers at selected coastal plain sites will be observed by means of detailed field sampling, geophysical imaging, and laboratory analysis. Geostatistical descriptions of physical heterogeneity within the aquifers will be developed at successive spatial scales. Geostatistical descriptions will be used to formulate simulations of the observed sedimentary fabric base of the physics of sedimentation. The simulated fabrics will be subjected to simulated patterns of flow. Results to Date: Atlantic coastal plain aquifers exhibit characteristic patterns at a substratal scale, at a stratal scale, at a facies scale, and at a sequence-stratigraphic scale. These patterns control the hydrologic properties of the sediment and the resultant groundwater flow and they are predictable. Prediction requires direct examination of surface exposures, simulations of the fabric of the sedimentary deposit, and conditioning of the model through repeated comparisons of observations with simulations. Analysis of the Oyster and Mappsville sites on the Virginia Eastern Shore shows that the sands and gravelly sands of these sites were deposited on the shoreface of a coastwise-prograding spit. The deposits constitute a single shoreface facies, but are readily resolved into a Shelly gravelly subfacies, a cross-stratified sand subfacies, and a horizontally stratified sand subfacies. These subfacies exhibit consistent hydrologic properties with permeability decreasing from 1 (moderately permeable) to 3 (highly permeable), but the correlation between depositional unit and hydrologic property is considerably strengthened if the subfacies are resolved into their respective stratal units (strata, cross-strata, bed sets, and crossstrata sets). The next larger scale of organization at the Oyster and Mappsville sites is the spit-growth increment, bounded below by the spit base, and laterally by erosional surfaces. These geometry of boundaries, which markedly affect flow anisotropy, can be observed only by geophysical imaging techniques.

Initial studies indicate that storm-dominated shoreface sedimentation can be simulated by computing bed-preservation potentials, based on probability frequency distributions of maximum wave height. The storm and tidal current climate must likewise be evaluated so bed-load deposits (cross-stratified and gravelly facies) will be adequately represented in the simulations of the resulting sedimentary fabric.

\section{MICROBIALLY MEDIATED MINERAL DISSOLUTION IN SUBSURFACE ENVIRONMENTS \\ ULLMAN, WILLIAM, and KIRCHMAN, DAVID UNIVERSITY OF DELAWARE \\ FY 1996107 \\ FY 1995107 \\ FY $1994 \quad 0$}

Keywords: deep microbiology, mineral dissolution, feldspars, groundwater, biogeochemistry

Objectives: To determine how bacteria and their metabolites influence the rates of mineral/water exchange in the groundwater/aquifer environment. Approach: Mixed assemblages of bacteria collected from a field site will be experimented with, cultured bacteria will be studied at a range of naturally occurring temperatures, isotopic strategies will be investigated to determine mineral dissolution rates in future field studies, and empirical and mechanistic models coupling microbial metabolism and mineral/water exchange will be developed. 
Results to Date: Experiments concerning the temperature dependence of ligand-promoted feldspar dissolution were completed. They indicated that those common ligands that form strong and stable complexes with aluminum in solution are able to catalyze feldspar-mineral dissolution at low temperatures ( 5 to $30^{\circ} \mathrm{C}$ ). Additional experiments concerning the temperature dependence of ligand excretion by bacteria, where growth was limited by the lack of inorganic nutrients, and the effect of these excretion products on feldspar dissolution were also completed. They confirmed that mineral-dissolution rates from bacteria may be higher at lower temperatures because of an accumulation of dissolution-enhancing excretion products in solution. A reanalysis of a series of experiments using natural assemblages of bacteria indicated that dissolution-enhancing compounds are formed under anoxic and nutrient-unlimited conditions in the laboratory.

Two new lines of experiments have been initiated. The first involves the role of naturally occurring complex organic molecules in feldspar dissolution. Dissolved humic material was collected from natural waters and concentrated to be used in a series of batch dissolution experiments. The second involves the use of a novel analytical approach to separate and quantify the amounts and stability of aluminum organic complexes. This work is directed toward the determination of useful thermodynamic constants concerning ligands commonly found in nature and associated with microbial processes in nature.

\section{OHER AND WALCOFF: A PARTNERSHIP FOR TECHNICAL AND ADMINISTRATIVE SUPPORT \\ WALCOFF, CAROL \\ WALCOFF AND ASSOCIATES, INC. \\ FY 199629 \\ FY 199530 \\ FY 199450}

No abstract available.

\section{SIGNATURE LIPID BIOMARKERS FOR IN SITU MICROBIAL BIOMASS, COMMU- NITY STRUCTURE, AND \\ NUTRITIONAL STATUS OF DEEP-SUBSURFACE MICROBIOTA \\ WHITE, DAVID, and RINGELBERG, DAVID THE UNIVERSITY OF TENNESSEE \\ FY 1996135 \\ FY 199588 \\ FY 199488}

Keywords: microbial ecology, phospholipid fatty acids Objectives: To examine the indigenous microbiota in deep subsurface sediments by application of the signature-lipid-biomarker tochnology providing insight into the microbial ecology of this vast ecosystem.

Approach: Through the application of signature-lipidbiomarker technology (SLB), it is anticipated that the following objectives can be obtained: (1) to provide a comprehensive characterization of subsurface microbial ecology through the SLB analysis of SSP samples as a timely, cost-effective, and quantitative means by which to provide a baseline for correlations with collaborative geochemical, geophysical, and biological studies; (2) to correlate results from the SLB analysis of differing sites to develop fundamental trends in subsurface microbial ecology; and (3) to correlate results from the SLB analyses to those obtained from DNA molecular techniques and community-level physiological profile analyses.

Results to Date: Membrane lipid changes were determined in two subsurface isolates, Pseudomonas sp. and Arthrobacter sp., over 1-year and microcosm observations were related to those observed in situ. The lipid content of novel strains of subsurface Desulfotomaculum sp. were described. None-viable microbial biomass was related to viable microbial biomass in sediment cores recovered from a New Mexico borehole showing a significant positive correlation with depth that supported the thermal-aureole hypothesis. Evidence was found of community-compositional differences directly related to sediment age in support of the chronosequence study. Microbial heterogeneity at two sites on the East Coast was described, showing spatial heterogeneity (viable microbial biomass and community composition) to exist on scales ranging from kilometers down to centimeters.

\section{ENVIRONMENTAL SCIENCE RESEARCH CENTER WILDUNG, RAYMOND PACIFIC NORTHWEST NATIONAL LABORATORY FY 19961395 FY 19952020 FY 19942583}

Keywords: geochemistry, microbiology, remediation, mixed wastes

Objectives: To provide the DOE sites with new knowledge, tools, and approaches for defining, scaling, and manipulating coupled biogeochemical and hydraulic processes that control contaminant transport and the efficacy of in situ remediation of mixed contaminants in subsurface environments.

Approach: Advanced intermediate- and field-scale approaches will be used to bridge the gap between mechanistic studies targeting biogeochemical reactions (e.g., sorption and biotransformation) and the resolution of problems at DOE sites, where spatial and temporal heterogeneities in microbial, geochemical, 
and geologic properties have scale-dependent effects on contaminant behavior and remediation strategies. Intermediate-scale research (2-D, meter-size flow cells) is directed toward understanding the coupled biogeochemical and transport processes that control contaminant distribution and partitioning under controlled conditions of physical heterogeneity. Field research builds on this knowledge to develop new tools and approaches for predicting contaminant behavior in natural environments and for in situ remediation based on the dynamic interactions of coupled subsurface processes. These studies are under way in a number of different hydrogeologic regimes nationally (INELTAN, Dover Air Force Base, Hanford-Redox, and Paducah).

Results to Date: Interactive, nonlinear geochemical reactions were shown to regulate the behavior of Co(II)EDTA complexes in intermediate-scale flow systems containing iron oxide inclusions of varying dimensions, providing new insights into the biogeochemical processes and geologic features that must be characterized to predict contaminant transport in the field.

Microbial distribution and function in the subsurface were determined to be significantly related to the spatial continuity of physical properties, sedimentpore throat diameter, and biogeochemical dynamics across sedimentary interfaces.

A novel concept for a permeable in situ barrier for redox-sensitive species (e.g., chromium, uranium, and technetium) was transferred to the DOE Office of Environmental Management and is being demonstrated in the field. Innovative new approaches were developed to ensure that fundamental research results are available to DOE and industry.

\section{SUBSURFACE BEHAVIOR OF ORGANICALLY COMPLEXED RADIONUCLIDES ZACHARA, JOHN

Keywords: radionuclides, complexation, biogeochemistry, microbiology

Objectives: To establish how surface chemical processes meter the activity and function of metal-reducing bacteria in subsurface materials and how such metering may change in direction, magnitude, and rate in presence of water flow which controls nutrient, substrate, and contaminant flux.
Approach: Field observations and intermediate-scale experiments in meter-sized flow cells will be used (1) to determine the biogeochemical reaction mechanisms controlling the retention and stability of organically complexed radionuclides in subsurface materials, emphasizing competitive complexation, adsorption, and dissolution reactions; (2) to investigate the role of metal-oxidizing and reducing-bacteria on the surface chemical properties of subsurface materials and their reactivity toward organically complexed and polyvalent radionuclides; (3) to develop linked chemical and microbiologic models (for research and transfer to EM) of cocontaminant speciation, reaction, transformation, and biodegradation for experimental interpretation, and field simulation and prediction; and (4) to evaluate the scaling of biogeochemical reaction networks mediated by microorganisms through intermediate-scale experiments of the reactive transport of contaminant mixtures through mineralogically and microbiologically heterogeneous porous media.

Results to Date: A statistical survey that set project and programmatic priorities was performed on contaminant mixtures at DOE sites. A comprehensive soil and subsurface-sediment collection was assembled and serves as a resource for OHER and EM investigators.

Research showed that the complexation of radionuclides by organic ligands enhanced subsurface migration rates by reducing the sorptivity $\left(\mathrm{K}_{\mathrm{d}}\right)$ of the radionuclide for mineral surfaces. However, $\mathrm{Fe}^{3+}$ and $\mathrm{Al}^{3+}$, liberated by mineral dissolution, promoted dissociation of the complex and release of the radionuclides over long contact times.

Iron-reducing bacteria reduced from 20 to $50 \%$ of the Fe(III) oxides in subsurface materials under anoxic conditions. Bacterial reduction was metered by morphologic and surface chemical features of the Fe(III) oxides. Microbiologically solubilized Fe(II) displaced radionuclides from mobile organic complexes, allowing for sorption of the displaced radio-nuclide by mineral phases. Abiotic- and microbiologic-driven dissociation reactions counteract the mobilizing potential of complexing agents, enhancing sorption with transport distance.

Solutions of ${ }^{60} \mathrm{Co}$ (II)EDTA were followed through intermediate-scale flow cells containing subsurface sand with "band-like" Fe(III) oxide inclusions to test scaling hypoth-eses. The experiments, backed by kinetic- and equilibrium-geochemical-transport modeling, showed that the length scale of the Fe(III) oxide zones was a critical determinant of reactivity. Important insights were provided on the types of geologic features that require characterization to predict radionuclide transport in the field. 

APPENDIXES 



\section{APPENDIX A: ADDRESSES OF PRINCIPAL INVESTIGATORS}

Ackerman, Thomas

Department of Meteorology

503 Walker Bldg.

The Pennsylvania State University

University Park, PA 16802

ackerman@essc.psu.edu

http://www.met.psu.edu/

Addis, Robert $P$.

Westinghouse Savannah River Company

P.O. Box 616

Aiken, SC 29802

robert.addis@srs.gov

Agrawal, Yogesh C.

Sequoia Scientific, Inc.

P.O. Box 592

Mercer Island, WA 98040

agrawal@sequoiasci.com

Ahearne, John F.

Sigma Xi, The Scientific Research Society

99 Alexander Drive

P.O. Box 13975

Research Triangle Park, NC 27709

u7666ja@vml.hqadmin.doe.gov

Ahmad, Zia

Science and Data Systems, Inc.

16509 Copperstrip Lane

Silver Spring, MD 20906

ahmad@ozone.stx.com

Al Mahamid, Ilham

Lawrence Berkeley National Laboratory

1 Cyclotron Road; MS 70A-1150

Berkeley, CA 94720

almahamid@1bl.gov

Albert, Brian

USDOE Environmental Measurements Laboratory

201 Varick Street

New York, NY 10014-3621

balbert@eml.doe.gov

Albrecht, Bruce

The Pennsylvania State University

Department of Meteorology

505 Walker Bldg.

State College, PA 16802

bia@psuvm.psu.edu
Allen, Michael

San Diego State University

Department of Biology

San Diego, CA 92182-4614

mallen@sciences.sdsu.edu

Aller, Josephine

State University of New York

Stony Brook, NY 11794-5000

jaller@ccmail.sunysb.edu

Aller, Robert

State University of New York

Marine Sciences Research Center

Stony Brook, NY 11794-5000

raller@ccmail.sunysb.edu

Ammerman, James

Texas A\&M University

Department of Oceanography

College Station, TX 77843

jammer@astra.tamu.edu

Amthor, Jeff

Lawrence Livermore National Laboratory

P.O. Box 808; L-256

Livermore, CA 94550-0099

amthorl@llnl.gov

Amy, Penny

University of Nevada

Department of Biological Science

Las Vegas, NV 89154

amy@ccmail.nevada.edu

Anderson, John $\mathbf{R}$.

University of Wisconsin

Space Science and Engineering Center

225 W Dayton St.

Madison, WI 53706

anderson@ssec.wisc.edu

http://scrap.ssec.wisc.edu:80/ anderson

Anderson, Susan

Lawrence Berkeley National Laboratory

1 Cyclotron Road

Berkeley, CA 94720

slanders@mh1.lbl.gov 
Arakawa, Akio

University of California

Department of Atmospheric Sciences

405 Hilgard Avenue

Los Angeles, CA 90024

aar@atmos.ucla.edu

Arnold, Robert G.

University of Arizona

Department of Chemical and Environmental Engineering

Tucson, AZ 85721

arnold@bigdog.engr.arizona.edu

Asher, William

Pacific Northwest National Laboratory

JISAO

Box 354235

University of Washington

Seattle, WA 98195-4325

asher@atmos.washington.edu

Asper, Vernon

University of Southern Mississippi

Institute of Marine Sciences

Bldg. 1103

Stennis Space Center, MS 39529

vasper@whale.st.usm.edu

http://www.coam.usm.edu/cms/

Atherton, Cynthia

Lawrence Livermore National Laboratory

7000 East Avenue

Livermore, CA 94551-9900

cyndi@tropos.llnl.gov

Bader, David C.

Pacific Northwest National Laboratory

901 D St., SW; Suite 900

Washington, DC 20024

dc_bader@pnl.gov

Baer, Ferdinand

University of Maryland

Department of Meteorology

College Park, MD 20742

baer@atmos.umd.edu

http://www.metolab3.umd.edu/meteorology.html

Baerwald, Thomas J.

Committee on Environment and Natural Resources

The National Science Foundation

4201 Wilson Blvd., $705 \mathrm{~N}$

Arlington, Virginia 22230

tbaerwal@nsf.gov
Baldocchi, Dennis

National Oceanic and Atmospheric Administration

Atmospheric Turbulence and Diffusion Division

P.O. Box 2456

Oak Ridge, TN 37831

baldocchi@atdd.noaa.gov

http://www.atdd.noaa.gov

Balkwill, David

Florida State University

Department of Biological Science

312 Nuclear Research Bldg.

Tallahassee, FL 32306-3043

balkwill@bio.fsu.edu

Ball, J. Timothy

Desert Research Institute

Biological Sciences Center

P.O. Box 60220

Reno, NV 89506-0220

tball@maxey.unr.edu

http://www.dri.edu

Banta, Robert $M$

NOAA Environmental Research Laboratories

R/E/ET2

325 Broadway

Boulder, CO 80303-3328

rbanta@etl.noaa.gov

Barber, Robert W.

USDOE

Office of Environment, Safety, and Health

Office of Field Support

EH-53, MS 5042

Germantown, MD 20874

Robert.Barber@hq.doe.gov

Barchet, W. Richard

Pacific Northwest National Laboratory

P.O. Box 999

Richland, WA 99352

wr_barchet@pnl.gov

http://www.pnl.gov:2080/

Barnard, James

Pacific Northwest National Laboratory

P.O. Box 999, MSIN K9-30

Richland, WA 99352

jc_barnard@pnl.gov

http://www.pnl.gov:2080/ 
Barnett, Tim

Scripps Institution of Oceanography

Climate Research Division, M/S 0224

La Jolla, CA 92014

tbarnett@ucsd.edu

http://meteora.ucsd.edu/crd.html

Barnette, Daniel

Sandia National Laboratories

MS 1111, Org 1421

P.O. Box 5800

Albuquerque, NM 87185-1111

dwbarne@cs.sandia.gov

Barr, Sumner

Los Alamos National Laboratory

MS F665

Los Alamos, NM 87545

barr@kokopelli.lanl.gov

http://ees-www.lanl.gov/EES5

Baskaran, Mahalingam

Texas A\&M University at Galveston

5007 Avenue U

Galveston, TX 77551

baskaranm@tamug.tamu.edu

Bauer, James E.

Virginia Institute of Marine Sciences

P.O. Box 1346

Gloucester Point, VA 23062

bauer@vims.edu

$\mathrm{http} / / \mathrm{www} . v i m s . e d u$

Baygents, James C.

University of Arizona

Department of Chemical and Environmental Engineering

Tucson, AZ 85721

jcb@maxwell.che.arizona.edu

Bayne, Stewart

Oak Ridge Institute for Science and Education

P.O. Box 117

Oak Ridge, TN 37831

baynes@orau.gov

Bazzaz, F. A.

Harvard University

Department of Organismic \& Evolutionary Biology

16 Divinity Avenue

Cambridge, MA 02138

f_bazzaz@nocmsmgw.harvard.edu
Belzer, David

Pacific Northwest National Laboratory

P.O. Box 999, MS K8-17

Richland, WA 99352

db_belzer@pnl.gov

Benkovitz, Carmen M.

Brookhaven National Laboratory

DAS/ECD, Bldg. 426, P.O. Box 5000

Upton, NY 11973-5000

cmb@bnl.gov

http://www.ecd.bnl.gov/

Benner, Ronald

University of Texas at Austin

Marine Science Institute

750 Channelview Drive

Port Aransas, TX 78373

benner@utmsi.zo.utexas.edu

Benninger, Larry

University of North Carolina

Department of Geology

CB\#3315 Mitchell Hall

Chapel Hill, NC 27599-3315

lbenning@email.unc.edu

Benson, Sally M.

Lawrence Berkeley National Laboratory

Earth Sciences

1 Cyclotron Road

Berkeley, CA 94720

mbenson@ibl.gov

Berg, John

Los Alamos National Laboratory

MS E510

Los Alamos, NM 87545

jberg@lanl.gov

Berkowitz, Carl M.

Pacific Northwest National Laboratory

P.O. Box 999, MSIN K9-30

Richland, WA 99352

cm berkowitz@pnl.gov

http://w3.pnl.gov:2080/atmos_sciences

Bian, Xindi

Pacific Northwest National Laboratory

P.O. Box 999

Richland, WA 99352

x_bian@pnl.gov

http://www.pnl.gov:2080/ 
Bisson, Scott E.

Sandia National Laboratories

P.O. Box 969; MS 9057

Livermore, CA 94551-0969

sebisso@sandia.gov

Bjornstad, David

Oak Ridge National Laboratory

P.O. Box 2008

Bldg. 4500 North, MS 6205

Oak Ridge, TN 37831

dub@ornl.gov

Blair, Neal

North Carolina State University

Marine, Earth \& Atmospheric Sciences

Raleigh, NC 27695-8208

neal_blair@ncsu.edu

Bleck, Rainer

University of Miami

4600 Rickenbacker Causeway

Miami, FL 33149

rbleck@rsmas.miami.edu

http://www.rsmas.miami.edu

Bloom, Arnold

University of California

Department of Vegetable Crops

University of California

Davis, CA 95616

ajbloom@ucdavis.edu

Boden, Thomas

Oak Ridge National Laboratory

Carbon Dioxide Information Analysis Center

Bldg. 1000; MS 6335

Oak Ridge, TN 37831-6335

tab@ornl.gov

http://cdiac.esd.ornl.gov

Boehme, Susan

Rutgers, The State University of New Jersey

Institute of Marine and Coastal Sciences

New Brunswick, NJ 08903-0231

boehme@ahab.rutgers.edu

Bohm, Emanuele

North Carolina State University

P.O. Box 8208

Raleigh, NC 27695

bohm@meadsp.nrrc.ncsu.edu
Bolton, Harvey

Pacific Northwest National Laboratory

900 Battelle Blvd., MS K4-06

Richland, WA 99352

h_bolton@pnl.gov

Boone, David R.

Oregon Graduate Institute

P.O. Box 91000

Portland, OR 97291-1000

boone@ese.ogi.edu

http://www.ese.ogi.edu/ese_docs/boone.html

Bossert, James

Los Alamos National Laboratory

P.O. Box 1663, MS D401

Los Alamos, NM 87545

bossert@lanl.gov

Boutton, Thomas W.

Texas A\&M University

Department of Rangeland Ecology and Management

College Station, TX 77843-2126

boutton@acs.tamu.edu

Bradley, Raymond S.

University of Massachusetts

Department of Geosciences

Amherst, MA 01003-5820

tbradley@climatel.geo.umass.edu

Brasseur, Guy

National Center for Atmospheric Research

P.O. Box 3000

Boulder, $\mathrm{CO} 80307$

brasseur@ucar.edu

Brewer, Alan

NOAA Environmental Technology Laboratory

325 Broadway

Boulder, CO 80303-3328

alan.brewer@noaa.gov

Brockman, Fred

Pacific Northwest National Laboratory

P.O. Box 999; MS K4-06

Richland, WA 99352

fj_brockman@pnl.gov

Brooks, Scott

Oak Ridge National Laboratory

Environmental Sciences Division

P.O. Box 2008, MS 6038

Oak Ridge, TN 37831-6038

3sb@ornl.gov 
Bryan, Kirk

Princeton University

AOS Program, Sayre Hall

Princeton, NJ 08542

kbryan@splash.princeton.edu

http://www.aos.princeton.edu

Burlage, Robert

Oak Ridge National Laboratory

Environmental Sciences Division

P.O. Box 2008, MS-6036

Oak Ridge, TN 37831

rvx@ornl.gov

Cahalan, Robert

NASA Goddard Space Flight Center, Code 913

Greenbelt, MD 20771

cahalan@clouds.gsfc.nasa.gov

http://climate.gsfc.nasa.gov/ cahalan/Home.html

Cairns, Brian

NASA Goddard Institute for Space Studies

2880 Broadway

New York, NY 10025

crbxc@giss.nasa.gov

Canham, C. D.

New York Botanical Garden Cary Arboretum

Institute of Ecosystem Studies

Box AB

Millbrook, NY 12454

Carlson, Barbara

NASA Goddard Institute for Space Studies

2880 Broadway

New York, NY 10025

pabec@giss.nasa.gov

Carmichael, Gregory

University of Iowa

125D Chem Bldg

Iowa City, IA 52240

gcarmich@icaen.uiowa.edu

http://www.cgrer.uiowa.edu

Cess, Robert D.

State University of New York

Marine Sciences Research Center

Stony Brook, NY 11794-5000

cess@atmsci.msrc.sunysb.edu

Cessi, Paola

Scripps Institution of Oceanography

UCSD-0230

La Jolla, CA 92093-0230

pcessi@ucsd.edu
Chapin, F. Stuart, III

University of California

Department of Integrative Biology

Berkeley, CA 94720-3140

fschapin@garnet.berkeley.edu

Chen, Robert

University of Massachusetts, Boston

Environmental Sciences Program

100 Morrissay Blvd.

Dorchester, MA 02125

chenr@umbsky.cc.umb.edu

Chervin, Robert

National Center for Atmospheric Research

P.O. Box 3000

Boulder, CO 80307-3000

chervin@ucar.edu

Chilakapati, Ashok

Pacific Northwest National Laboratory

P.O. Box 999, MS K9-36

Richland, WA 99352

a_chilakapati@pnl.gov

Chin, Hung-neng

Lawrence Livermore National Laboratory

7000 East Avenue

Livermore, CA 94551-9900

chin2@llnl.gov

Chipman, David W.

Lamont-Doherty Earth Observatory

Rt. 9W

Palisades, NY 10964

chipman@Ideo.columbia.edu

Cho, $\mathrm{H}$.

University of Arizona

Department of Soils, Water, and Environmental Science

Tucson, AZ 85721

http://www.arl.arizona.edu/ispe/

Churchill, James $\mathrm{H}$.

Woods Hole Oceanographic Institution

Woods Hole, MA 02543

jchurchill@cliff.whoi.edu

Churnside, James

CIRES/NOAA

Environmental Technology Laboratory

325 Broadway

Boulder, CO 80303-3228

jchurnside@etl.noaa.gov

http://www.etl.noaa.gov 
Clark, William C.

Harvard University

John F. Kennedy School of Government

79 John F. Kennedy Street

Cambridge, MA 02138

william_clark@harvard.edu

Clough, Shepard A.

Atmospheric \& Environmental Research, Inc.

840 Memorial Drive

Cambridge, MA 02139

clough@aer.com

Cobb, N. S.

Northern Arizona University

Department of Biological Sciences

Flagstaff, AZ 86011-5640

cobb@nau.edu

Cochran, J. Kirk

State University of New York

Marine Sciences Research Center

Stony Brook, NY 11794-5000

james.cochran.1@sunysb.edu

Cohn, Stephen

National Center for Atmospheric Research

P.O. Box 3000

Boulder, CO 80307-3000

cohn@ucar.edu

Colwell, Frederick

Idaho National Engineering Laboratory

Biotechnologies

P.O. Box 1625

Idaho Falls, ID 83415-2203

fxc@inel.gov

Connell, Peter S.

Lawrence Livermore National Laboratory

7000 East Ave., MS L240

Livermore, CA 94550-9900

connell@leland.llnl.gov

Conradson, Steven

Los Alamos National Laboratory

MS D429

Los Alamos, NM 87545

conradson@lanl.gov

Constantin, Milton J.

Oak Ridge Institute for Science and Education

P.O. Box 117

Oak Ridge, TN 37831-0117

constanm@orau.gov
Cooper, Lee

Oak Ridge National Laboratory

ESD, ORNL

Oak Ridge, TN 37831-6038

jg9@ornl.gov

Cotton, William

Colorado State University

Department of Atmospheric Science

Fort Collins, CO 80523

cotton@isis.atmos.colostate.edu

Coulter, Bryan

Oak Ridge Institute for Science and Education

P.O. Box 117

Oak Ridge, TN 37831-0117

coulterb@orau.gov

Coulter, Richard

Argonne National Laboratory

Environmental Research Division, Bldg. 203

9700 South Cass Avenue

Argonne, IL 60439

rl_coulter@anl.gov

Crawford, Ronald L.

University of Idaho

Institute for Molecular and Agricultural Genetic Engineering

Food Research Center 103

Moscow, ID 83844-1052

crawford@uidaho.edu

Cress, Ted S.

Pacific Northwest National Laboratory

P.O. Box 999, Battelle Boulevard

Richland, WA 99352

ts_cress@pnl.gov

Cummings, Ronald

Georgia State University

School of Policy Studies

University Plaza

Atlanta, GA 30303

prcrgc@langate.gsu.edu

http://prcweb.gsu.edu/enveco/enveco.html

Curry, J. A.

University of Colorado

Campus Box 429

Boulder, CO 80309

judith.curry@colorado.edu 
Cushman, John

Purdue University

1395 Math

West Lafayette, IN 1395-47907

75674.1670@compuserve.com

Cushman, Robert

Oak Ridge National Laboratory

Carbon Dioxide Information Analysis Center

Bldg. 1000, MS 6335

Oak Ridge, TN 37831-6335

rma@ornl.gov

http://cdiac.esd.ornl.gov

Dagg, Michael

LUMCON

8124 Highway 56

Chauvin, LA 70344

mdagg@coco.lumcon.edu

Dannevik, William

Lawrence Livermore National Laboratory

7000 East Avenue

Livermore, CA 94551-9900

dannevik1@1lnl.gov

Daum, Peter H.

Brookhaven National Laboratory

DAS/ECD, Bldg. 426, P.O. Box 5000

Upton, NY 11973-5000

daum2@bnl.gov

http://www.ecd.bnl.gov/

Davidovits, Paul

Boston College

Department of Chemistry

Chestnut Hill, MA 02167-3835

davidovits@hermes.bc.edu

Davis, Anthony

NASA Goddard Space Flight Center, Code 913

Greenbelt, MD 20771

davis@climate.gsfc.nasa.gov

http://climate.gsfc.nasa.gov/ davis/Home.html

Dean, Sumner

Los Alamos National Laboratory

MS F663

Los Alamos, NM 87545

shd@lanl.gov

Deflaun, Mary F.

Envirogen, Inc., Princeton Research Center

4100 Quakerbridge Road

Lawrenceville, NJ 08648-4702

74024.3076@compuserve.com
Degrandpre, Michael D.

University of Montana

Department of Chemistry, Chem-Pharm Bldg.

Missoula, MT 59812

mdegrand@selway.umt.edu

Del Genio, Anthony

NASA Goddard Institute for Space Studies

2880 Broadway

New York, NY 11704

pdadd@giss.nasa.gov

Deluisi, John

National Oceanic and Atmospheric Administration

Air Resources Laboratory

325 Broadway

Boulder, CO 80303-3328

deluisi@srrb.noaa.gov

Demaster, David

North Carolina State University

Marine, Earth, and Atmospheric Sciences

Raleigh, NC 27695-8208

dave_demaster@ncsu.edu

Denbo, D. W.

Battelle Marine Sciences Laboratory

1529 West Sequim Bay Road

Sequim, WA 98382

dwd@mystery.pnl.gov

http://www.msl.pnl.gov:2080/dwd/

Diaz, Henry F.

NOAA Environmental Research Laboratories

325 Broadway

Boulder, CO 80303

hfd@cdc.noaa.gov

http://www.cdc.noaa.gov/ hfd/

Dickinson, Robert E.

University of Arizona

Institute of Atmospheric Physics

PAS Bldg. \#81

Tucson, AZ 85721

robted@stratus.atmo.arizona.edu

http://www.atmo.arizona.edu:8080/home.shtml

Dickson, Andrew G.

Scripps Institution of Oceanography

9500 Gilman Drive

La Jolla, CA 92093-0902

adickson@ucsd.edu 
Dietz, Russell N.

Brookhaven National Laboratory

DAS/ECD, Bldg. 426

P.O. Box 5000

Upton, NY 11973-5000

mmarsch@bnluxl.bnl.gov

http://www.ecd.bnl.gov/

Doran, J. Christopher

Pacific Northwest National Laboratory

P.O. Box 999, MSIN K9-30

Richland, WA 99352

jc_doran@pnl.gov

http://www.pnl.gov:2080/

Doskey, Paul

Argonne National Laboratory

Environmental Research Division, Bldg. 203

9700 South Cass Avenue

Argonne, Il 60439

paul_doskey@qmgate.anl.gov

Dowlatabadi, Hadi

Carnegie Mellon University

Department of Engineering and Public Policy

5000 Forbes Avenue

Pittsburgh, PA 15213-3890

hadi@cmu.edu

Drake, Bert

Smithsonian Institution

P.O. Box 28

Edgewater, MD 21037

drake@serc.si.edu

Drake, James

The University of Tennessee

411 Dabney Hall

Knoxville, TN 37919

drake@utk.edu

Drake, John B.

Oak Ridge National Laboratory

Mathematical Sciences Section

P.O. Box 2008, Bldg. 6012

Oak Ridge, TN 37831-6367

drakejb@ornl.gov

Druffel, Ellen R. M.

University of California, Irvine

Department of Earth System Science

PSRF-207

Irvine, CA 92717-3100

edruffel@uci.edu
Dudhia, Jim

National Center for Atmospheric Research

P.O. Box 3000

Boulder, CO 80307-3000

dudhia@ucar.edu

Dukowicz, John K.

Los Alamos National Laboratory

T-3, MS B216

Los Alamos, NM 87545

duk@lanl.gov

http://gnarly.lanl.gov/Pop/Pop.html

Dunlap, Kelly

DOE/OSTI

P.O. Box 62

Oak Ridge, TN 37831

kelly.dunlap@ccmail.osti.gov

http://www.doe.gov./html/osti/ostipg.html

http://www.doe.gov/waisgate/er.html

Dunning, Thom

Pacific Northwest National Laboratory

P.O. Box 999

Richland, WA 99352

th_dunning@pnl.gov

Easter, Richard

Pacific Northwest National Laboratory

P.O. Box 999, MSIN K9-30

Richland, WA 99352

rc_easter@pnl.gov

http://www.pnl.gov:2080/

Easterling, David

NOAA/NESDIS/National Climatic Data Center

151 Patton Ave.

Asheville, NC 28801

deaster1@ncdc.noaa.gov

Eberhard, Wynn

NOAA Environmental Technology Laboratory

R/E/ET2

325 Broadway

Boulder, CO 80303-3328

weberhard@etl.noaa.gov

Edgerton, Sylvia

Argonne National Laboratory

Environmental Research Division, Bldg. 203

9700 South Cass Avenue

Argonne, IL 60439-4843

edgerton@oerhp01.er.doe.gov 
Edmonds, James E.

Pacific Northwest National Laboratory

D Street, S.W., Suite 900

Washington, DC 20024-2115

ja_edmonds@pnl.gov

Edwards, Nelson T.

Oak Ridge National Laboratory

P.O. Box 2008, Bldg. 1059

Oak Ridge, TN 37831-6422

nte@ornl.gov

Eglinton, Timothy

Woods Hole Oceanographic Institution

360 Woods Hole Road

Woods Hole, MA 02543-1543

teglinton@whoi.edu

Ehrlich, Paul R.

Stanford University

Department of Biological Sciences Studies

Stanford, CA 94305-5020

pre@leland.stanford.edu

Ellingson, Robert

University of Maryland

Department of Meteorology

College Park, MD 20742

bobe@atmos.umd.edu

Emanuel, Kerry

Massachusetts Institute of Technology

Rm 54-1620

77 Massachusetts Ave.

Cambridge, MA 02139

emanuel@texmex.mit.edu

http://cirrus.mit.edu/CMPOhome.html

Emery, W. J.

University of Colorado

Campus Box 429

Boulder, CO 80309

william.emery@colorado.edu

Erickson, David

National Center for Atmospheric Research

P.O. Box 3000

Boulder, CO 80307-3000

erickson@ucar.edu

Ermak, Donald

Lawrence Livermore National Laboratory

7000 East Avenue

Livermore, CA 94551-9900

ermak1@llnl.gov
Evans, J. L.

The Pennsylvania State University

503 Walker Bldg.

University Park, PA 16802

evans@essc.psu.edu

Fairall, C. W.

NOAA Environmental Technology Laboratory

325 Broadway

Boulder, CO 80303-3228

christopher.w.fairall@noaa.gov

Falkowski, Paul

Brookhaven National Laboratory

Department of Applied Science

P.O. Box 5000

Upton, NY 11973-5000

falkowsk@bnlux1.bnl.govl

http://www.oasd.bnl.gov/ oasd/

Farrell, Brian

Harvard University

Department of Earth and Planetary Sciences

Pierce Hall,

29 Oxford St.

Cambridge, MA 02139

farrell@psyche.harvard.edu

Fast, Jerome

Pacific Northwest National Laboratory

P.O. Box 999, MSIN K9-30

Richland, WA 99352

jd_fast@pnl.gov

http://www.pnl.gov:2080/

Fein, Jay S.

National Science Foundation

4201 Wilson Blvd., $775 \mathrm{~S}$

Arlington, VA 22230

jfein@nsf.gov

Ferrare, Richard

Hughes STX

NASA Goddard Space Flight Center, Code 912

Greenbelt, MD 20771

ferrare@agnes.gsfc.nasa.gov

http://rsd.gsfc.nasa.gov/912

Field, Christopher

Carnegie Institution of Washington

Department of Plant Biology

290 Panama Street

Stanford, CA 94305

chris@jasper.stanford.edu 
Finlayson-Pitts, Barbara

University of California

Department of Chemistry

Irvine, CA 92697-2025

bjfinlay@uci.edu

Flagg, Charles N.

Brookhaven National Laboratory

Department of Applied Science, Bldg. 318

P.O. Box 5000

Upton, NY 11973-5000

flagg@bnl.gov

url: www.oasd.bnl.gov/ oasd/

Fletcher, Madilyn

University of Maryland

701 E. Pratt St., Suite 236

Baltimore, MD 20212

fletcher@umbi.umd.edu

Formento, John

Argonne National Laboratory

9700 S. Cass Ave

Argonne, IL 60439

formento@dis.anl.gov

Foster, Ian

Argonne National Laboratory

Mathematics and Computer Science Division

Bldg 221

9700 S. Cass Ave

Argonne, IL 60439

itf@mcs.anl.gov

http://www.mcs.anl.gov/chammp

Francis, Arokiasamy

Brookhaven National Laboratory

Department of Applied Science, Bldg. 318

P.O. Box 5000

Upton, NY 11973-5000

francisl@bnl.gov

Frank, W. M.

The Pennsylvania State University

503 Walker Bldg.

University Park, PA 16802

wmf3@psu.edu

Frederick, John

University of Chicago

5734 S. Ellis

Chicago, IL 60637

frederic@rainbow.uchicago.edu
Fredrickson, James

Pacific Northwest National Laboratory

MS K4-06

P.O. Box 999

Richland, WA 99352

jk_fredrickson@pnl.gov

Gabriel, Philip

Colorado State University

Department of Atmospheric Science

Fort Collins, CO 80523-1371

gabriel@herschel.atmos.colostate.edu

Gaffney, Jeffrey

Argonne National Laboratory

Bldg. 203

9700 South Cass Avenue

Argonne, IL 60439

jeff_gaffney@qmgate.anl.gov

Gage, Kenneth

NOAA/Environmental Research Laboratories

325 Broadway

Boulder, CO 80303-3228

kgage@al.noaa.gov

http://www.al.noaa.gov

Gao, Weigang

Argonne National Laboratory

Environmental Research Division, BIdg. 203

9700 South Cass Avenue

Argonne, Il 60439

weigang_gao@qmgate.anl.gov

Garten, Jr., Charles

Oak Ridge National Laboratory

P.O. Box 2008, MS 6038

Oak Ridge, TN 37830-6038

ctg@ornl.gov

Gates, Lawrence

Lawrence Livermore National Laboratory

7000 East Avenue

Livermore, CA 94551-9900

gates5@llnl.gov

Gautier, Catherine

University of California

ICESS - Ellison Hall

Santa Barbara, CA 93106

gautier@icess.ucsb.edu 
Gehring, C. A.

Northern Arizona University

Department of Biological Sciences

Flagstaff, AZ 86011-5640

cag@nau.edu

Gehrs, Carl

Oak Ridge National Laboratory

Center for Biotechnology

Oak Ridge, TN 37830

gehrscw@ornl.gov

Ghan, Steven

Pacific Northwest National Laboratory

P.O. Box 999

Richland, WA 99352

sj_ghan@pnl.gov

http://www.pnl.gov.2080/

Ginn, Timothy

Pacific Northwest National Laboratory

P.O. Box 999; MS K9-36

Richland, WA 99352

tr_ginn@pnl.gov

http://etd.pnl.gov:2080/IGGroup/oystertime.html

Girvin, Don C.

Pacific Northwest National Laboratory

900 Battelle Blvd

Mailstop K3-61

Richland, WA 99352

dc girvin@pnl.gov

Glantz, Michael

National Center for Atmospheric Research

P.O. Box 3000

Boulder, CO 80307-3000

glantz@ucar.edu

Goldberg, Ellen

Santa Fe Institute

1399 Hyde Park Road

Santa Fe, NM 87501

barbara@santafe.edu

Goldsmith, John E. M.

Sandia National Laboratories

P.O. Box 969; MS 9057

Livermore, CA 94551-0969

jgold@ca.sandia.gov

Gorby, Yuri

Pacific Northwest National Laboratory

P.O. Box 999

Richland, WA 99352

ya_gorby@ccmail.pnl.gov
Goulden, Michael

Harvard University

20 Oxford St.

Cambridge, MA 02138

mlg@io.harvard.edu

http://www-as.harvard.edu

Goulder, Lawrence H.

Stanford University

Department of Economics

Landau Economics Bldg., Room 328,

Stanford, CA 94305-6072

goulder@leland.stanford.edu

Goyet, Catherine

Woods Hole Oceanographic Institution

MS 25, 360

Woods Hole Road

Woods Hole, MA 02543-1541

cathy@co2.whoi.edu

Gray, William M.

Colorado State University

205A Atmospheric Science

Ft. Collins, CO 80523-1371

barb@tutt.atmos.colostate.edu

Greening, Lorna

Lawrence Berkeley National Laboratory

Bldg. 90, Room 4000

Berkeley, CA 94720

lagaps@dante.lbl.gov

Griffin, W. Timothy

Golder Associates Inc.

136 S. Illinois Ave., Suite 200

Oak Ridge, TN 37830

tgriffin@golder.com

Griffiths, Robert P.

Oregon State University

Department of Forest Science

Forest Science Laboratory 020

Corvallis, OR 97331-7501

griff@fsl.orst.edu

Grossman, Allen

Lawrence Livermore National Laboratory

1-104, Atmospheric Sciences Division

7000 East Ave.

Livermore, CA 94551-9900

grossman1@llnl.gov

http://www.llnl.gov/lnl/04atmos.html 
Grossman, Ethan

Texas A\&M University

Department of Geology and Geophysics

College Station, TX 77843-3115

e-grossman@tamu.edu

Gu, Baohua

Oak Ridge National Laboratory

MS-6036

P.O. Box 2008

Oak Ridge, TN 37831-6036

b26@ornl.gov

Gudiksen, Paul

Lawrence Livermore National Laboratory

7000 East Avenue

Livermore, CA 94551-9900

gudiksen1@lln1.gov

Gunderson, Carla A.

Oak Ridge National Laboratory

Bldg 1059; MS 6422

P.O.Box 2008

Oak Ridge, TN 37831-6422

cgu@ornl.gov

Gunter, Lee E.

Oak Ridge National Laboratory

Bldg 1506; MS 6034

P.O. Box 2008

Oak Ridge, TN 37831-6034

ygl@ornl.gov

Gutowski, William

Iowa State University

3021 Agronomy

Ames, IA 50010

gutowski@iastate.edu

Hack, James

National Center for Atmospheric Research

P.O. Box 3000

Boulder, CO 80307-3000

jhack@ncar.ucar.edu

Hales, Jeremy

Envair/Argonne National Lab

$4811 \mathrm{~W} 18$ th Avenue

Kennewick, WA 99337

jake@odysseus.owt.com

Hall, Michael J.

National Oceanic and Atmospheric Administration

1100 Wayne Avenue, Suite 1210

Silver Spring, MD 20910-5803

michael.hall@noaa.gov
Ham, Jay

Kansas State University

Department of Agronomy

Throckmorton Hall

Manhattan, KS 66506-5501

snafu@ksu.ksu.edu

Hameed, Sultan

State University of New York

Stony Brook, NY 11794-5000

hameed@atmsci.msrc.sunysb.edu

Hammitt, James

Harvard University

718 Huntington Ave.

Boston, MA 02115

jkh@hsph.harvard.edu

http://128.103.75.55/Organizations/hcra/hcra.html

Han, Yong

University of Colorado, CIRES/NOAA

Environmental Technology Laboratory

325 Broadway

Boulder, CO 80303-3228

yyh@etl.noaa.gov

http://www.etl.noaa.gov

Hansen, D. Alan

Electric Power Research Institute

3412 Hillview Avenue

Palo Alto, CA 94304

ahansen@msm.epri.com

Hanson, Paul J.

Oak Ridge National Laboratory

P.O. Box 2008

Bldg. 1059

Oak Ridge, TN 37831-6422

pjx@ornl.gov

http://www.esd.ornl.gov/programs/WBW/

TDEAAAAA.HTM

Harrison, Lee

State University of New York

Atmospheric Sciences Research Center

100 Fuller Road,

Albany, NY 12205-5741

lee@solsun 1.asrc.albany.edu

Heath, Donald

Research Support Instruments, Inc.

5500 Central Avenue, Suite 202

Boulder, CO 80301

dheath@csn.net 
Heinrichs, Donald F.

National Science Foundation

Division of Ocean Sciences, Room 725N

4201 Wilson Boulevard

Arlington, VA 22230

dheinric@nsf.gov

Henderson, David

DOE/OSTI

P.O. Box 62

Oak Ridge, TN 37831

david.henderson@ccmail.osti.gov

http://www.doe.gov./html/osti/ostipg.html

Hendrey, George

Brookhaven National Laboratory

Bldg. 318, Department of Applied Science

P.O. Box 5000

Upton, NY 11973-5000

hendrey@bnl.gov

Herman, Janet

University of Virginia

Department of Environmental Sciences

Charlottesville, VA 22903

jsh5w@virginia.edu

http://atlantic.evsc.virginia.edu/ alm7d/pirch

Hibler, Lyle

Pacific Northwest National Laboratory

Marine Sciences Laboratory

1529 West Sequim Bay Rd.

Sequim, WA 98382-9099

If_hibler@pnl.gov

Hicks, Bruce

NOAA Air Resources Laboratory

1315 East West Highway

Silver Spring, MD 20910

hicks@arlrisc.ssmc.noaa.gov

Hill, Walter $\mathbf{R}$.

Oak Ridge National Laboratory

P.O. Box 2008

Oak Ridge, TN 37831-6351

h6t@ornl.gov

Hoffert, Martin I.

New York University

Department of Physics, Earth Systems Group

26-36 Stuyvesant Street

New York, NY 10003

hoffert@is2.nyu.edu
Holben, William E.

University of Montana

Division of Biological Sciences

Missoula, MT 59812

bholben@selway.umt.edu

http://www.umt.edu/biology/dbs/holben.htm

Hollinger, David

USDA Forest Service

271 Mast Rd.

Durham, NH 03824

davidh@christa.unh.edu

http://www.esd.ornl.gov:80/programs/NIGEC

Honeyman, Bruce

Colorado School of Mines

Department of Environmental Sci. \& Engineering

Golden, CO 80401

bhoneyma@slate.mines.edu

Hood, Lon L.

University of Arizona

Space Sciences Bldg.

Tucson, AZ 85721-0092

lon@lpl.arizona.edu

Hopkins, Tom S.

North Carolina State University

P.O. Box 8208

Raleigh, NC 27695

hopkins@meavax.nrrc.ncsu.edu

Hopkinson, Charles

Marine Biological Laboratory

Woods Hole, MA 02543

chopkins@lupine.mbl.edu

Hornberger, George $\mathbf{M}$.

University of Virginia

Department of Environmental Sciences

Charlottesville, VA 22903

gmh3k@virginia.edu

http://atlantic.evsc.virginia.edu/ alm7d/pirch

Howarth, Richard

University of California

Environmental Studies Program

214 College Eight Academic Bldg.

Santa Cruz, CA 95064

howarth@cats.ucsc.edu

Hsiao, Theodore

University of California

Department of Land, Air, and Water Resources

Davis, CA 95616

tchsiao@ucdavis.edu 
Humphreys, Kenneth

Pacific Northwest National Laboratory

P.O. Box 999; MS K8-17

Richland, WA 99352

kk_humphreys@pnl.gov

Huston, Michael

Oak Ridge National Laboratory

Environmental Sciences Division

Oak Ridge, TN 37831-6335

mhu@ornl.gov

http://www.esd.ornl.gov/programs/WBW/

TDEAAAAA.HTM

Ingram, Jani $\mathbf{C}$.

Idaho National Engineering Laboratory

MS 2430

Idaho Falls, ID 83415-2107

uoa@inel.gov

Intrieri, Janet

NOAA Environmental Technology Laboratory

325 Broadway, R/E/ET2

Boulder, CO 80303-3328

janet.m.intrieri@noaa.gov

Jacoby, Henry

Massachusetts Institute of Technology

Sloan School of Management, E52-444

Cambridge, MA 02142

hjacoby@mit.edu

http://web.mit.edu/globalchange/www/

Jaffe, Adam

Brandeis University

Waltham, MA 02254

jaffe@binah.cc.brandeis.edu

Jahnke, Richard

Skidaway Institute of Oceanography

10 Ocean Science Circle

Savannah, GA 31411

rick@skio.peachnet.edu

Jain, Atul

University of Illinois

105 South Gregory Ave.

Urbana, IL 61801

jain@uiatma.atmos.uiuc.edu

Jardine, Philip

Oak Ridge National Laboratory

Environmental Sciences Division

P.O. Box 2008; MS 6038

Oak Ridge, TN 37831-6038

ipj@ornl.gov
Jastrow, Julie D.

Argonne National Laboratory

Environmental Research Division

Bldg. 203

9700 South Cass Avenue

Argonne, IL 60439-4843

jdjastrow@anl.gov

Jayne, John T.

Aerodyne Research, Inc.

45 Manning Road

Billerica, MA 01821

jayne@aerodyne.com

Jensen, Tommy

Colorado State University

Department of Atmospheric Science

Fort Collins, CO 80523

jensen@neptune.atmos.colostate.edu

Johnson, Dale

University of Nevada, Reno

7010 Dandini Blvd.

Reno, NV 89512

dwj@maxey.dri.edu

http://www.dri.edu

Johnson, Donald

University of Wisconsin

Space Science and Engineering Center

1225 W. Dayton St.

Madison, WI 53706

donj@ssec.wisc.edu

http://ssec.wisc.edu

Johnson, Kenneth $M$.

Brookhaven National Laboratory

Bldg. 318, Department of Applied Science

P.O. Box 5000

Upton, NY 11973-5000

Jones, Philip D.

University of East Anglia

Climatic Research Unit

Norwich, UK NR4 7TJ

p.jones@uea.ac.uk

http://www.cru.uea.ac.uk/

Jones, Sonja

Oak Ridge National Laboratory

Carbon Dioxide Information Analysis Center

Bldg. 1000, MS 6335

Oak Ridge, TN 37831-6335

cdp@ornl.gov

http://cdiac.esd.ornl.gov 
Joslin, John

Oak Ridge National Laboratory

Bldg. 1059

P.O. Box 2008

Oak Ridge, TN 37831-6422

joslin@mhs-tva.attmail.com

http://www.esd.ornl.gov/programs/WBW/

TDEAAAAA.HTM

Kahn, James R.

Oak Ridge National Laboratory

P.O. Box 2008, MS-6205

Oak Ridge, TN 37831-6205

kahnjr@ornl.gov

Kaiser, Dale

Oak Ridge National Laboratory

Carbon Dioxide Information Analysis Center

Bldg. 1000, MS-6335

Oak Ridge, TN 37831-6335

d9k@ornl.gov

Karl, Thomas

NOAA/NESDIS/National Climatic Data Center

151 Patton Ave.

Asheville, NC 28801

tkarl@ncdc.noaa.gov

http://www.ncdc.noaa.gov

Karnosky, David

Michigan Technological University

School of Forestry and Wood Products

107 Widmaier

Houghton, MI 49931-1295

karnosky@mtu.edu

http://forestry.mtu.edu/

Kashgarian, Michaele

Lawrence Livermore National Laboratory

7000 East Avenue

Livermore, CA 94551-9900

kashgarian@1lnl.gov

Keeling, Charles

Scripps Institution of Oceanography

9500 Gilman Drive

La Jolla, CA 92093-0220

cdkeeling@ucsd.edu

Keim, $P$.

Northern Arizona University

Department of Biological Sciences

Flagstaff, AZ 86011-5640

keim@nau.edu
Kemp, Paul F.

Brookhaven National Laboratory

Oceanographic and Atmospheric Sciences Division

Bldg. 318

P.O. Box 5000

Upton, NY 11973-5000

kemp@bnluxl.bnl.gov

Kennedy, Donald

Stanford University

Institute for International Studies

Stanford, CA 94305-6055

donald.kennedy@forsythe.stanford.edu

Key, Robert

Princeton University

Geology Department

Guyot Hall

Princeton, NJ 08544

key@geo.princeton.edu

Khalil, M. A. K.

Portland State University

P.O. Box 751

Portland, OR 97207-0751

aslam@atmos.phy.pdx.edu

http://global.phy.pdx.edu

\section{Kieft, Thomas}

New Mexico Institute of Mining and Technology

Department of Biology

Socorro, NM 87801

tkieft@nmt.edu

http://www.nmt.edw/-biology/keift/keiftfrontpage.

html

Kiehl, Jeffrey

National Center for Atmospheric Research

P.O. Box 3000

Boulder, CO 80307-3000

jtkon@ncar.ucar.edu

Kim, K.-Y.

Texas A\&M University

Department of Meteorology, MS 3150

College Station, TX 77843-3150

kykim@csrp.tamu.edu

King, Anthony

Oak Ridge National Laboratory

Environmental Sciences Division

P.O. Box 2008

Oak Ridge, TN 37831-6335

awk@ornl.gov 
Kinnison, Douglas E.

Lawrence Livermore National Laboratory

7000 East Ave., MS L240

Livermore, CA $94551-9900$

dkin@cal-bears.llnl.gov

Kirchman, David

University of Delaware, College of Marine Studies

700 Pilottown Road

Lewes, DE 19958-1298

kirchman@udel.edu

Kleinman, Lawrence

Brookhaven National Laboratory

DAS/ECD, Bldg. 426

P.O. Box 5000

Upton, NY 11973-5000

kleinman@bnlcl6.bnl.gov

http://www.ecd.bnl.gov/

Kliewer, Kenneth L.

Oak Ridge National Laboratory

P.O. Box 2008

Oak Ridge, TN 37831

kliewerkl@ornl.gov

Knapp, Alan

Kansas State University

Department of Biology

Manhattan, KS 66506

aknapp\%lter-konza.konza.ksu.edu@ksuvm.ksu.edu

Knuth, Ronald

USDOE Environmental Measurements Laboratory

201 Varick Street

New York, NY 10014-3621

http://www.eml.doe.gov/

Kolb, Charles E.

Aerodyne Research, Inc.

45 Manning Road

Billerica, MA 01821

kolb@aerodyne.com

Kolber, Zbigniew

Brookhaven National Laboratory

Bldg. 318

P.O. Box 5000

Upton, NY 11973-5000

kolber@bnlcl6.bnl.gov

Kolstad, Charles

University of California

Department of Economics

Santa Barbara, CA 93106-9210

kolstad@econ.ucsb.edu
Koracin, Darko

Desert Research Institute

Atmospheric Sciences Center

P.O. Box 60220

Reno, NV 89506

darko@sage.dri.edu

http://www.dri.edu

Kozyr, Alex

Oak Ridge National Laboratory

Carbon Dioxide Information Analysis Center

Bldg. 1000, MS 6335

Oak Ridge, TN 37831-6335

alex@alex.esd.ornl.gov

http://cdiac.esd.ornl.gov

Kreidenweis, Sonia

Colorado State University

Department of Atmospheric Science

Fort Collins, CO 80523

soniak@aerosol.atmos.colostate.edu

Kromidas, Lambros

USDOE Environmental Measurements Laboratory

201 Varick Street

New York, NY 10014-3621

http://www.eml.doe.gov/

Kropfli, Robert A.

National Oceanic and Atmospheric Administration

R/E/ET6, 325 Broadway

Boulder, CO 80303

rkropfli@etl.noaa.gov

http://www.etl.noaa.gov

Krueger, Steven

University of Utah

Department of Meteorology

Salt Lake City, UT 84112-1183

krueger@ncar.ucar.edu

Krumbolz, Lee

University of Oklahoma

Department of Botany and Microbiology

770 Van Vleet Oval

Norman, OK 73019

krumholz@uoknor.edu

Kubiske, Mark

Michigan Technological University

Houghton, MI 49931-1295

mkubiske@mtu.edu 
Kukla, George

Lamont-Doherty Earth Observatory

P.O. Box 1000,

Palisades, NY 10964

kukla@ldeo.columbia.edu

Kulpa, Charles

University of Notre Dame

Notre Dame, IN 46556

charles.f.kulpa.1@nd.edu

http://www.nd.edw/ cbpc

Kuo, Ying-Hwa (Bill)

National Center for Atmospheric Research

P.O. Box 3000

Boulder, CO 80307-3000

kuo@ucar.edu

Kuske, Cheryl

Los Alamos National Laboratory

Life Sciences Division; M888

Los Alamos, NM 87545

kuske@lanl.gov

Lacis, Andrew

NASA Goddard Institute for Space Studies

2880 Broadway

New York, NY 10025

ccaal@giss.nasa.gov

http://www.giss.nasa.gov

Lala, Gar

State University of New York

100 Fuller Road,

Albany, NY 12205-5741

lala@sundog.asrc.albany.edu

Laroche, Julie

Brookhaven National Laboratory

Department of Applied Science, Bldg. 318

P.O. Box 5000

Upton, NY 11973-5000

laroche@bnlux1.bnl.gov

Larsen, Richard J.

USDOE Environmental Measurements Laboratory

201 Varick Street

New York, NY 10014-4811

larsenr@eml.doe.gov

Laulainen, Nels

Pacific Northwest National Laboratory

P.O. Box 999, MSIN K9-30

Richland, WA 99352

ns_laulainen@pnl.gov

http://www.pnl.gov:2080/
Lave, Lester B.

Carnegie Mellon University

Graduate School of Industrial Administration

5000 Forbes Ave.

Pittsburgh, PA 15213

1101+@andrew.cmu.edu

Leavitt, S. W.

University of Arizona

West Stadium, Room 218

Tucson, AZ 85721

sleavitt@ltrr.arizona.edu

Lee, Cindy

State University of New York

Marine Sciences Research Center

Stony Brook, NY 11794-5000

cindy.lee@sunysb.edu

Lee, Hsi-Na

USDOE Environmental Measurements Laboratory

201 Varick Street

New York, NY 10014-4811

hnlee@eml.doe.gov

Lee, In Young

Argonne National Laboratory

Environmental Research Division, Bldg. 203

9700 South Cass Avenue

Argonne, IL 60439

in_young_lee@qmgate.anl.gov

Lee, J. T.

Los Alamos National Laboratory

MS D401

Los Alamos, NM 87545

jtlee@lanl.gov

Lee, Jai Hoon

Brookhaven National Laboratory

DAS/ECD, Bldg. 815

P.O. Box 5000

Upton, NY 11973-5000

srs2@bnl.gov

http://www.ecd.bnl.gov/

Lee, Yin-nan

Brookhaven National Laboratory

DAS/ECD, Bldg. 426

P.O. Box 5000

Upton, NY 11973-5000

ynlee@bnl.gov

http://www.ecd.bnl.gov/ 
Leifer, Robert

USDOE Environmental Measurements Laboratory

210 Varick Street

New York, NY 10014-3621

rleifer@eml.doe.gov

http://www.eml.doe.gov/

Lempert, Robert J.

Rand Corporation

P.O. Box 2138

1700 Main Street

Santa Monica, CA 90407-2138

robert_lempert@rand.org

Leung, L. Ruby

Pacific Northwest National Laboratory

P.O. Box 999

Richland, WA 99352

1_leung@pnl.gov

http://www.pnl.gov:2080/

Levin, S. A.

Princeton University

Department of Ecology and Evolutionary Biology

Princeton, NJ 08544-1003

simon@eno.princeton.edu

Liang, Liyuan

Oak Ridge National Laboratory

P.O.Box 2008

Oak Ridge, TN $37831-6038$

lul@ornl.gov

Liebetrau, Albert

Pacific Northwest National Laboratory

P.O. Box 999, MS K5-12

Richland, WA 99352

am_liebetrau@pnl.gov

Lindzen, Richard

Massachusetts Institute of Technology

RM 54-1720

Cambridge, MA 02139

Liou, K. N.

University of Utah

Department of Meteorology

Salt Lake City, UT 84112

knliou@climate.met.utah.edu

Loehle, Craig

Argonne National Laboratory

Environmental Research Division, Bldg. 203

9700 South Cass Avenue

Argonne, IL 60439

craig_loehle@qmgate.anl.gov
Logan, Jennifer

Harvard University

Pierce Hall

29 Oxford St.

Cambridge, MA 02138-2901

jal@io.harvard.edu

Lohrenz, Steven

University of Southern Mississippi

Institute of Marine Science

Bldg. 1103, Room 102

Stennis Space Center, MS 39529

slohrenz@whale.st.usm.edu

http://www.coam.usm.edu/cms

Long, Philip

Pacific Northwest National Laboratory

P.O. Box 999; MS K9-48

Richland, WA 99352

pe_long@pnl.gov

http://www.pnl.gov:2080/

Long, Stephen

University of Essex

Department of Biology

Colchester, U.K. CO4 3SQ

steve@essex.ac.uk

http://www.essex.ac.uk/bcs/staff/longa.html

Louis, Jean-Francois

Atmospheric and Environmental Research, Inc.

840 Memorial Dr.

Cambridge, MA 02139

jfl@aer.com

MacKenzie, Fred T.

University of Hawaii

1000 Pope Road

Honolulu, HI 96822

fredm@soest.hawaii.edu

Madronich, Sasha

National Center for Atmospheric Research

P.O. Box 3000

Boulder, CO 80307-3000

sasha@ucar.edu

Mahen, Edward C., Jr.

Advanced Research Projects Agency

3701 North Fairfax Drive

Arlington, VA 22203-1714 
Majer, Ernest L.

Lawrence Berkeley National Laboratory

Earth Sciences Division, MS 90-1116

One Cyclotron Road

Berkeley, CA 94720

elmajer@lbl.gov

http://www-esd.lbl.gov

Malone, Robert

Los Alamos National Laboratory

ACL, MS B287

Los Alamos, NM 87545

rcm@lanl.gov

http://www.acl.lanl.gov/GrandChal/GCM/

CHAMMP.html

Margolin, Len G.

Los Alamos National Laboratory

XHM, MS F663

Los Alamos, NM 87545

len@lanl.gov

Marley, Nancy A.

Argonne National Laboratory

Environmental Research Division, Bldg. 203

9700 South Cass Avenue

Argonne, IL 60439

jeff_gaffney@qmgate.anl.gov

Marotzke, Joachim

Massachusetts Institute of Technology

RM 54-1718

Cambridge, MA 02139

jochem@sound.mit.edu

Marshak, Alexander

NASA Goddard Space Flight Center, Code 913

Greenbelt, MD 20771

marshak@climate.gsfc.nasa.gov

http://climate.gsfc.nasa.gov/ marshak/Home.html

Matin, Abdul

Stanford University

Department of Microbiology and Immunology

Fairchild Bldg., D317

Stanford, CA 94305-5402

a.matin@Forsythe.stanford.edu

Matrosov, Sergey Y.

National Oceanic and Atmospheric Administration R/E/ET6

325 Broadway

Boulder, CO 80303

smatrosov@etl.noaa.gov
Matthias, A.

University of Arizona

Shantz, Room 529

Tucson, AZ 85721

allan-matthias@ns.arizona.edu

Mayer, Lawrence

University of Maine

Darling Center

Walpole, ME 04573

Imayer@maine.maine.edu

McCord, Raymond

Oak Ridge National Laboratory

Bldg. 1507, MS 6407

Oak Ridge, TN 37831-6407

rqm@ornl.gov

McElroy, Michael

Harvard University

Department of Earth and Planetary Sciences

Pierce Hall, 20 Oxford St.

Cambridge, MA 02138

mbm@io.harvard.edu

http://www-as.harvard.edu/index.html

McIntosh, Robert

University of Massachusetts

Knowles Engineering Bldg., Rm 113

Amherst, MA 01003

mcintosh@ecs.umass.edu

http://www.ecs.umass.edu/ece/labs/mirsl.html

McKinley, James

Pacific Northwest National Laboratory

MSIN K3-61

P.O. Box 999

Richland, WA 99352

jp_mckinley@pnl.gov

McMurry, Peter H.

University of Minnesota

Department of Mechanical Engineering

111 Church St., SE

Minneapolis, MN 55455

mcmurry@me.umn.edu

Mechoso, Carlos R.

University of California

Department of Atmospheric Sciences

7127 Math Sciences Bldg.

405 Hilgard Avenue

Los Angeles, CA 90095-1565

mechoso@atmos.ucla.edu 
Meehl, Gerald A.

National Center for Atmospheric Research

P.O. Box 3000

Boulder, CO 80307-3000

meehl@ncar.ucar.edu

Melfi, S. Harvey

University of Maryland, Baltimore County

Department of Physics

Baltimore, MD 21228

melfi@umbc.edu

Mendelsohn, Robert

Yale University

School of Forestry and Environmental Studies

360 Prospect St.

New Haven, CT 06511

mendel@yale.edu

Metting, Blaine

Pacific Northwest National Laboratory

P.O. Box 999 / MS P7-54

Richland, WA 99352

fb_metting@pnl.gov

http://www.pnl.gov:2080/

Meyer, William T.

Argonne National Laboratory

Environmental Research Division

Bldg. 203

9700 South Cass Avenue

Argonne, Il 60439

wtmeyer@anl.gov

Michalsky, Joseph

State University of New York

100 Fuller Road

Albany, NY 12205-5741

joe@hazy.asrc.albany.edu

Miller, R. Michael

Argonne National Laboratory

Environmental Research Division, Bldg. 203

9700 South Cass Avenue

Argonne, IL 60439-4843

rmmiller@anl.gov

Miller, Robert V.

Oklahoma State University

Department of Microbiology and Molecular Genetics

307 Life Sciences East

Stillwater, OK 74078

rum67@okway.okstate.edu
Millero, Frank J.

University of Miami

4600 Rickenbacker Causeway

Miami, FL 33149-1098

fmillero@rsmas.miami.edu

http://www.rsmas.miami.edu

Mills, Aaron

University of Virginia

Department of Environmental Sciences

Clark Hall

Charlottesville, VA 22903

alm7d@virginia.edu

Minnis, Patrick

NASA Langley Research Center

MS 420

Hampton, VA 23681-0001

p.minnis@larc.nasa.gov

Mirin, Arthur

Lawrence Livermore National Laboratory

7000 East Avenue

Livermore, CA 94551-9900

mirin@llnl.gov

Mishchenko, Michael

NASA Goddard Institute for Space Studies

2880 Broadway

New York, NY 10025

crmim@giss.nasa.gov

http://www.giss.nasa.gov

Mitchell, David L.

Desert Research Institute

Atmospheric Sciences Center

P.O. Box 60220

Reno, NV 89506

mitch@sage.dri.edu

Morris, David

Los Alamos National Laboratory

MS J586

Los Alamos, NM 87545

demorris@lanl.gov

Morse, Gerome

Colorado School of Mines

Department of Physics

1500 Illinois Street

Golden, CO 80401

gmorse@physics.mines.edu 
Moss, Richard

Pacific Northwest National Laboratory

P.O. Box 999; MS K8-17

Richland, WA 99352

rh_moss@pnl.gov

Moyers, Jarvis

National Science Foundation

Division of Atmospheric Science

4201 Wilson Blvd., $775 \mathrm{~S}$

Arlington, VA 22230

jmoyers@nsf.gov

Mulholland, Patrick

Oak Ridge National Laboratory

Environmental Sciences Division

P.O. Box 2008

Oak Ridge, TN 37831-6036

jdn@ornl.gov

http://www.esd.ornl.gov/programs/WBW

Muller, Richard A.

Lawrence Berkeley National Laboratory

50-232 LBL

Berkeley, CA 94720

ramuller@LBL.gov

Murcray, Frank

University of Denver

Department of Physics

University Park

Denver, CO 80208-0202

murcray@ram.phys.du.edu

Murphy, Ellyn

Pacific Northwest National Laboratory

MS K3-61

P.O. Box 999

Richland, WA 99352

em_murphy@pnl.gov

http://etd.pnl.gov:2080/IGGroup/ellyn.html

Murray, Christopher

Pacific Northwest National Laboratory

P.O. Box 999, Mailstop K9-48

Richland, WA 99352

cj_marray@pnl.gov

Murray, William L.

National Oceanic and Atmospheric Administration 1100 Wayne Avenue, Suite 1210

Silver Spring, MD 20910-5603

murray@ogp.noaa.gov
Nachtigal, Noel

Oak Ridge National Laboratory

P.O. Box 2008, Bldg. 6012

Oak Ridge, TN 37831-6367

santa@msr.epm.ornl.gov

Neff, William D.

NOAAVEnvironmental Research Laboratories

325 Broadway

Boulder, CO 80303-3328

wneff@etl.noaa.gov

http://www.etl.noaa.gov

Nelson, Tommy

Oak Ridge National Laboratory

Carbon Dioxide Information Analysis Center

Bldg. 1000; MS-6335

Oak Ridge, TN $37831-6335$

trn@ornl.gov

http://cdiac.esd.ornl.gov

Newman, Leonard

Brookhaven National Laboratory

DAS/ECD, Bldg. 426

P.O. Box 5000

Upton, NY 11973-5000

newman@bnluxl.bnl.gov

http://www.ecd.bnl.gov/

Nichols, Brian

University of Illinois at Chicago

Department of Biological Sciences

900 S. Ashland Ave

Chicago, IL 60607

brian.p.nichols@uic.edu

Niedoroda, Alan W.

Woodward Clyde Consultants

3676 Hartsville Rd.

Tallahassee, FL 32303

awniedo0@wcc.com

Nierzwicki-Bauer, Sandra

Rensselaer Polytechnic Institute

Department of Biology, Science Center IW14

Troy, NY 12180-3590

nierzs@rpi.edu

Nobel, Park

University of California

Department of Biology

Los Angeles, CA 90095

psnobel@lbes.medsch.ucla.edu

http://www.lifesci.ucla.edu/bio/Faculty/Nobel/

Research/RESEARCH.HTM 
Norby, Richard J.

Oak Ridge National Laboratory

Bldg 1059; MS-6422

P.O. Box 2008

Oak Ridge, TN 37831-6422

rjn@ornl.gov

Nordhaus, William D.

Yale University

Department of Economics

P.O. Box 2125, Yale Station

New Haven, CT 06520-2125

william.nordhaus@yale.edu

North, Gerald

Texas A\&M University

Department of Meteorology

College Station, TX 77843-3150

north@csrp.tamu.edu

Novakov, Tihomir

Lawrence Berkeley National Laboratory

1 Cyclotron Rd.; MS-73

Berkeley, CA 94720

tnovakov@lbl.gov

Nowak, Robert $\mathrm{S}$.

University of Nevada, Reno

Department of Environment and Resource

Sciences/MS 199

Reno, NV 89557

nowak@scs.unr.edu

O'Neill, Elizabeth

Oak Ridge National Laboratory

Bldg. 1059

P.O. Box 2008

Oak Ridge, TN 37831-6422

ego@ornl.gov

O'Neill, Robert

Oak Ridge National Laboratory

Bldg 1505

P.O. Box 2008

Oak Ridge, TN 37831-6038

rvo@ornl.gov

Oberbauer, Steven F.

Florida International University

Department of Biological Sciences

OE 235

Miami, FL 33199

oberbauc@scrums.fiu.edu
Oechel, Walter

San Diego State University

Department of Biology

San Diego, CA 92182

oechel@sunstroke.sdsu.edu

http://sonofsun.sdsu.edu./GCRG/

Ogden, Kimberly L.

University of Arizona

Dept. of Chemical and Environmental Engineering

Tucson, AZ 85721

ogden@bigdog.engr.arizona.edu

Olson, Richard

Oak Ridge National Laboratory

Bldg. 1507, MS 6407

Oak Ridge, TN 37831-6407

rjo@ornl.gov

Onstott, T. C.

Princeton University

Department of Geological and Geophysical Sciences

Princeton, NJ 08544

tullis@wargon.princeton.edu

Owensby, Clenton

Kansas State University

Department of Agronomy, Throckmorton Hall

Manhattan, KS 66506-5501

owensby@ksu.ksu.edu

Pacala, S. W.

Princeton University

Department of Ecology and Evolutionary Biology

Princeton, NJ 08544-1003

steve@eno.princeton.edu

Paffenhofer, G.-A.

Skidaway Institute of Oceanography

10 Ocean Science Circle

Savannah, GA 31411

paffenhofer@skio.peachnet.edu

Palumbo, Anthony Vito

Oak Ridge National Laboratory

P.O. Box 2008

Oak Ridge, TN 37831

palumboav@ornl.gov

Paluszkiewicz, Theresa

Pacific Northwest National Laboratory

Marine Sciences Laboratory

1529 West Sequim Bay Rd.

Sequim, WA 98382-9099

tp@circe.pnl.gov 
Parker, Stephen D.

National Academy of Sciences

Water Science and Technology Board

2101 Constitution Avenue, NW, HA 462

Washington DC 20418

sdparker@nas.edu

Parson, Edward A.

Harvard University

John F. Kennedy School of Government

79 John F. Kennedy Street

Cambridge, MA 02138

tparson@ksgrsch.harvard.edu

Parsons, David

National Center for Atmospheric Research

P.O. Box 3000

Boulder, CO 80307-3000

parsons@ucar.edu

Paul, Eldor A.

Michigan State University

512 Plant and Soil Science Bldg.

East Lansing, MI 48824-1325

eapaul@msu.edu

Paw U, K. T.

University of California

Department of Land, Air, and Water Resources

Davis, CA 95616

ktpawu@ucdavis.edu

Pendleton, Linwood

University of Southern California

Department of Economics

Los Angeles, CA 90089-1253

linwoodp@rcf.usc.edu

Penner, Joyce

Lawrence Livermore National Laboratory

7000 East Avenue

Livermore, CA 94551-9900

penner1@1lnl.gov

Person, Mark

University of Minnesota

Department of Geology and Geophysics

Minneapolis, MN 55455

mperson@darcy.geo.umn.edu

http://www.geo.umn.edu/docs/dept.html

Peterson, Bruce

Marine Biological Laboratory

7 MBL St.

Woods Hole, MA 02543

peterson@lupine.mbl.edu
Peyton, Brent M.

Pacific Northwest National Laboratory

P.O. Box 999; MS P7-41

Richland, WA 99352

bm_peyton@pnl.gov

Phelps, Tommy

Oak Ridge National Laboratory

Bldg 1505, MS 6038

P.O. Box 2008

Oak Ridge, TN 37830

tkp@ornl.gov

http://www.esd.ornl.gov:80/sections/ebs/mi/

Phillips, Donald L.

Environmental Protection Agency

Environmental Research Laboratory

200 SW 35th Street

Corvallis, OR 97333

phillips.donald@epamail.epa.gov

Phillips, Fred

New Mexico Institute of Mining and Technology

Department of Earth and Environmental Science

Socorro, NM 87801

phillips@nmt.edu

Piacsek, Steve

Naval Research Laboratory, Code 7322

Stennis Space Center, MS 39529

piacsek@nrlssc.navy.mil

Pietrafesa, Leonard J.

North Carolina State University

Department of Marine, Earth, and Atmospheric

Sciences

P.O. Box 8208

Raleigh, NC 27695

leonard_pietrafesa@ncsu.edu

Platt, Charles Martin Richard

Commonwealth Scientific and Industrial Research

Organisation

Private Bag 1

Aspendale, VIC 3195

Australia

martin.platt@dar.csiro.au

Post, Wilfred M.

Oak Ridge National Laboratory

Environmental Sciences Division

P.O. Box 2008

Oak Ridge, TN 37830-6335

wmp@ornl.gov 
Potra, Florian

University of Iowa

Department of Mathematics

Iowa City, IA 52240

potra@cgrer.uiowa.edu

Potter, Gerald

Lawrence Livermore National Laboratory

7000 East Avenue

Livermore, CA 94551-9900

potter@oliver.llnl.gov

Pratt, Robert

Pacific Northwest National Laboratory

P.O. Box 999

Richland, WA 99352

rg_pratt@pnl.gov

Pregitzer, Kurt S.

Michigan Technological University

School of Forestry and Wood Products

Ann Arbor, MI 49931

kspregit@mtu.edu

Prell, Warren

Department of Geological Sciences

Brown University

Providence, RI 02912-1846

warren_prell@brown.edu

Preller, Ruth

Naval Research Laboratory

Stennis Space Center, MS 39529

preller@nrlssc.navy.mil

Prinn, Ronald

Massachusetts Institute of Technology, 54-1312

Cambridge, MA 02139

rprinn@mit,edu

http://web.mit.edu/globalchange/www/

Pruess, Karsten

Lawrence Berkeley National Laboratory

1 Cyclotron Road

Berkeley, CA 94720

k_pruess@1bl.gov

Rabitz, Hersch

Princeton University

13 Holly Lane

Lawrenceville, NJ 08648

hrabitz@chemvax.princeton.edu
Ramanathan, V.

Scripps Institution of Oceanography

9500 Gilman Drive

La Jolla, CA 92093-0221

ram@fiji.ucsd.edu

Ramaswamy, V.

Princeton University

Geophysics Fluid Dynamics Laboratory

Princeton, NJ 08542

vr@gfdl.gov

Randall, Cora

LASP, University of Colorado

Boulder, CO 80309-0392

cora.randall@lasp.colorado.edu

Randall, David

Colorado State University

Department of Atmospheric Science

Ft. Collins, CO 80523-1371

randall@redfish.atmos.colostate.edu

http://www.atmos.colostate.edu

Rasmussen, R. A.

Oregon Graduate Institute

P.O. Box 91000

Portland, OR 97291-1000

rrasmus@ese.ogi.edu

Rauschkolb, $\mathbf{R}$

University of Arizona

Maricopa Agriculture Center

Forbes, Room 25

Tucson, AZ 85721

roy-rauschkolb@ns.arizona.edu

Reck, Ruth

Argonne National Laboratory

Environmental Research Division, Bldg. 203

9700 South Cass Avenue

Argonne, IL 60439

ruth_reck@qmgate.anl.gov

Redalje, Donald

University of Southern Mississippi

Bldg. 1103, Room 102

Stennis Space Center, MS 39529

dredalje@falcon.st.usm.edu

http://www.coam.usm.edu/cms 
Reed, Donald

Argonne National Laboratory

Chemical Technology Division

Bldg. 205

9700 S. Cass Ave

Argonne, IL 60439

reedd@cmt.anl.gov

Reich, Peter B.

University of Minnesota

115 GRN H

1530 N. Cleveland Ave.

St. Paul, MN 55108

preich@mercury.forestry.umn.edu

Reimer, Michael

Colorado School of Mines

Institute for Resource and Environmental Geosciences

1500 Illinois Street

Golden, CO 80401

mreimer@mines.edu

Reimers, Clare

Rutgers, The State University of New Jersey

Institute of Marine and Coastal Sciences

New Brunswick, NJ 08903-0231

reimers@ahab.rutgers.edu

Reinsel, Gregory

University of Wisconsin, Madison

1210 W. Dayton

Madison, WI 53706

reinsel@stat.wisc.edu

Repeta, Daniel

Woods Hole Oceanographic Institution

360 Woods Hole Road

Woods Hole, MA 02543-1543

drepeta@whoi.edu

Revercomb, Henry E.

University of Wisconsin

Space Science and Engineering Center

1225 West Dayton Street

Madison, WT 53706

hankr@ssec.wisc.edu

http://www.ssec.wisc.edu/

Rice, Charles

Kansas State University

Department of Agronomy, Throckmorton Hall

Manhattan, KS 66506-5501

crice@1ter-konza.konza.ksu.edu
Richards, Kenneth

Indiana University

Public And Environmental Affairs

Bloomington, IN 47401

kenricha@indiana.edu

Riley, James

University of Washington

Department of Mechanical Engineering, FU-10

Seattle, WA 98195

rileyj@u.washington.edu

Ringelberg, David

The University of Tennessee

Center for Environmental Biotechnology

10515 Research Drive, Suite 300

Knoxville, TN 37932-2575

ringelbe@utkux.utk.edu

Rittmann, Bruce

Northwestern University

Department of Civil Enginnering

2145 Sheridan Road

Evanston, IL 60208-3109

b-rittmann@nwu.edu

Roberts, Dorothy

Oak Ridge Institute for Science and Education

P.O. Box 117

Oak Ridge, TN 37831-0117

robertsd@orau.gov

Rodhe, Henning

Stockholm University

Department of Meteorology, S-106 91

Stockholm, Sweden

rodhe@misu.suse

Rogers, Hugo

USDA ARS National Soil Dynamics Laboratory

P.O. Box 3439

Auburn, AL 36831-3439

hrogers@ag.auburn.edu

Roiz, Peter

USDOE Environmental Measurements Laboratory

201 Varick Street

New York, NY 10014-3621

http://www.eml.doe.gov/ 
Roop, Joseph M.

Pacific Northwest National Laboratory

P.O. Box 999; MS K8-17

Richland, WA 99352

joe.roop@pnl.gov

Rooth, Claes

University of Miami

4600 Rickenbacker Causeway

Miami, FL 33149

crooth@rsmas.miami.edu

http://www.rsmas.miami.edu

Ruby, Leung

Pacific Northwest National Laboratory

P.O. Box

Richland, WA 99352

1ly@arcus.pnl.gov

http://www.pnl.gov:2080/

Ruffin, Morrie

Biotechnology Industry Organization

Suite 1100

1625 K St., NW

Washington D.C. 20006

bio@bio.org

Rusch, David

LASP, University of Colorado

Boulder, CO 80309-0392

david.rusch@lasp.colorado.edu

Sands, Ronald

Pacific Northwest National Laboratory

901 D Street SW, Suite 900

Washington, DC 20024

rd_sands@pnl.gov

Santschi, Peter

Texas A\&M University at Galveston

5007 Avenue U

Galveston, TX 77551

santschi@tamug.tamu.edu

Sarmiento, Jorge

Princeton University

POAS, Sayre Hall, Forrestal Campus

Princeton, NJ 08544-0710

jls@splash.princeton.edu

Sassen, Kenneth

University of Utah

Department of Meteorology

Salt Lake City, UT 84112-1183

ksassen@atmos.met.utah.edu
Sathaye, Jayant

Lawrence Berkeley National Laboratory

MS $90-4000$

1 Cyclotron Road

Berkeley, CA 94720

jasathaye@lbl.gov

Sayler, Gary

The University of Tennessee

10515 Research Dr., Suite 100

Knoxville, TN 37932

sayler@utk.edu

Schafer, Annette

Idaho National Engineering Laboratory

Biotechnologies Department

P.O. Box 1625

Idaho Falls, ID 83415-2107

nsa@inel.gov

Scheibe, Timothy

Pacific Northwest National Laboratory

MS K9-36, PO Box 999

Richland, WA 99352

td_scheibe@pnl.gov

Schery, Stephen

New Mexico Tech

Department of Physics

Socorro, NM 87801

schery@mailhost.nmt.edu

Schipper, Lee

Lawrence Berkeley National Laboratory

Bldg. 90

Berkeley, CA 94720

ljsocd@dante.lbl.gov

Schlesinger, Michael

University of Illinois

Department of Atmospheric Sciences

105 S. Gregory Avenue

Urbana, IL 61801

schlesin@uiatma.atmos.uiuc.edu

http://crga.atmos.uiuc.edu/

Schneider, Hans $\mathbf{R}$.

Harvard University

Pierce Hall

29 Oxford St.

Cambridge, MA 02138-2901

hrs@io.harvard.edu 
Schneider, Niklas

Scripps Institution of Oceanography

9500 Gilman Drive

La Jolla, CA 92093-0221

Schneider, Stephen

Stanford University

Institute for International Studies

Stanford, CA 94305-6055

stephen.schneider@forsythe.stanford.edu

Schwartz, Stephen E.

Brookhaven National Laboratory

DAS/ECD, Bldg. 426

P.O. Box 5000

Upton, NY 11973-5000

ses@bnl.gov

http://www.ecd.bnl.gov/

Scott, Michael

Pacific Northwest National Laboratory

P.O. Box 999, MS K8-17

Richland, WA 99352

mj_scott@pnl.gov

Sedjo, Roger

Resources for the Future

1616 P Street NW

Washington, DC 20036

sedjo@rff.org

Semeraro, Dave

Oak Ridge National Laboratory

P.O. Box 2008

Oak Ridge, TN 37831-6367

semeraro@msr.epm.ornl.gov

Semtner, Albert

Naval Postgraduate School

833 Dyer Road

Monterey, CA 93943

sbert@ucar.edu

http://vislab-www.nps.navy.mil/ - braccio

Senum, Gunnar I.

Brookhaven National Laboratory

DAS/ECD, Bldg. 426, P.O. Box 5000

Upton, NY 11973-5000

gsenum@bnl.gov

http://www.ecd.bnl.gov/

Sextro, Richard G.

Lawrence Berkeley National Laboratory

MS 90-3058

Berkeley, CA 94720

rgsextro@lbl.gov
Sharpe, Steven

Pacific Northwest National Laboratory

P.O. Box 999

Richland, Washington 99352

sw_sharpe@pnl.gov

Shaw, Joseph

University of Colorado, CIRES/NOAA

Environmental Technology Laboratory

325 Broadway

Boulder, CO 80303-3228

jshaw@etl.noaa.gov

Shaw, William J.

Pacific Northwest National Laboratory

P.O. Box 999, MS K9-30

Richland, WA 99352

wj_shaw@pnl.gov

Sherr, Barry

College of Oceanic and Atmospheric Sciences

Oregon State University

104 Ocean Admin Bldg

Corvallis, OR 97331-5503

sherrb@ucs.orst.edu

Shorter, Jeffrey

Mission Research Corporation

1 Tara Blvd., \#302

Nashua, NH 03062

shorter@mrcnh.com

http://www.mrcnh.com/sen_anal/

Shugart, Lee

Oak Ridge National Laboratory

P.O. Box 2008

Oak Ridge, TN 37831-6036

19s@ornl.gov

Silliman, Stephen

University of Notre Dame

Civil Eng. and Geol. Sciences

Notre Dame, IN 46556

silliman@thiem.ce.nd.edu

http://www.nd.edu/ silliman/

Simpson, David

Resources for the Future

1616 P Street NW

Washington, DC 20036

simpson@rff.org 
Skyllingstad, Eric

Pacific Northwest National Laboratory

Marine Sciences Laboratory

1529 West Sequim Bay Rd.

Sequim, WA 98382-9099

ed_skyllingstad@pnl.gov

http://www.pnl.gov:2080/

Slinn, George

Cascade Scientific Research Corporation

$1515 \mathrm{NW}$ 13th St:

Corvallis, OR 97330

slinnwgn@aip.org

Smith, Douglas Robert

Genome Therapeutics Corporation

100 Beaver St

Waltham, MA 02154-8440

smith@cric.com

Smith, Gregory P.

SRI International

333 Ravenswood Ave., PS047

Menlo Park, CA 94025

smith@mplvax.sri.com

Smith, John R.

Aluminum Company of America Technical Center 100 Technical Drive

Alcoa Center, PA 15069

Smith, Richard D.

Los Alamos National Laboratory

T-3, MS B216

Los Alamos, NM 87545

rds@acl.lanl.gov

http://gnarly.lanl.gov/Pop/Pop.html

Smith, Robert

Idaho National Engineering Laboratory

P.O. Box 1625

Idaho Falls, ID 83415-2107

rqs@inel.gov

Smith, William

University of Wisconsin

1225 West Dayton, Rm. 251

Madison, WI 53706

bills@ssec.wisc.edu

Sohngen, Brent

Ohio State University

Department of Agricultural Economics

2120 Fyffe Rd.

Columbus, $\mathrm{OH}$ 43210-1067

sohngen.1@osu.edu
Solomon, Stanley

LASP, University of Colorado

Boulder, CO 80309-0392

stanley.solomon@lasp.colorado.edu

Somerville, Richard C. J.

Scripps Institution of Oceanography

9500 Gilman Drive, Dept. 0224

La Jolla, CA 92093-0224

rsomerville@ucsd.edu

http://meteora.ucsd.edu/

Sowle, David H.

Mission Research Corporation

1 Tara Blvd., \#302

Nashua, NH 03062

http://www.mrenh.com/sen_anal/

Spejewski, Eugene

Oak Ridge Institute for Science and Education

P.O. Box 117

Oak Ridge, TN 37831-0117

spejewse@orau.gov

Spinhirne, James

NASA Goddard Space Flight Center, Code 912

Greenbelt, MD 20771

jspin@virl.gsfc.nasa.gov

http://virl.gsfc.nasa.gov/mpl.html

Spinrad, Richard W.

Consortium for Oceanographic Res. and Education

Suite 800

1755 Massachusetts Avenue NW

Washington, DC 20036-2102

rspinrad@brook.edu

Springston, Stephen $\mathbf{R}$.

Brookhaven National Laboratory

DAS/ECD, Bldg. 815

P.O. Box 5000

Upton, NY 11973-5000

srs2@bnl.gov

http://www.ecd.bnl.gov/

Stacy, Walter

National Center for Atmospheric Research

P.O. Box 3000

Boulder, CO 80307-3000

stacy@ucar.edu

Staley, James $T$.

University of Washington

G-311 Health Sciences Center; MS SC-42

Seattle, WA 98195

jtstaley@u.washington.edu 
Stamnes, Knut

University of Alaska

Geophysical Institute

P. O. Box 757320

Fairbanks, AK 99775

knut@kaja.gi.alaska.edu

Starr, David

NASA Goddard Space Flight Center, Code 913

Greenbelt, MD 20771

starr@climate.gsfc.nasa.gov

http://rsd.gsfc.nasa.gov/913

Stavins, Robert

Harvard University

John F. Kennedy School of Government

79 John F. Kennedy Street

Cambridge, MA 02138

rstavins@ksg1.harvard.edu

Stephens, Graeme

Colorado State University

Department of Atmospheric Science

Fort Collins, CO 80523-1371

stephens@langley.atmos.colostate.edu

http://www.atmos.colostate.edu/

Stephens, Pamela

National Science Foundation

4201 Wilson Blvd., Rm.775

Arlington, VA 22230

pstephen@nsf.gov

Stevens, Todd

Pacific Northwest National Laboratory

P.O. Box 999

Richland, WA 99352

to_stevens@pnl.gov

Stokes, Gerald $M$.

Pacific Northwest National Laboratory

P.O. Box 999

Richland, WA 99352

gm_stokes@pnl.gov

Stone, Alan

Johns Hopkins University

3400 North Charles Street

Baltimore, MD 21218

dog_zats@jhuvms.hcf.jhu.edu

Stone, Peter

Massachusetts Institute of Technology

RM 54-1718

Cambridge, MA 02139

phstone@mit.edu
Stooksbury, Billie

Oak Ridge Institute for Science and Education

P.O. Box 117

Oak Ridge, TN 37831-0117

stooksbb@orau.gov

Strain, Boyd

Duke University

Department of Botany

P.O. Box 90340

Durham, NC 27708-0340

bstrain@acpub.duke.edu

Streets, David

Argonne National Laboratory

9700 S. Cass Ave., DIS/900

Argonne, IL 60439

dstreets@anl.gov

Streit, Gerald

Los Alamos National Laboratory

MS F604

Los Alamos, NM 87545

ges@lanl.gov

http://coyote.lanl.gov/tsa4/tsa4home.html

Strom, Suzanne

Western Washington University

1900 Shannon Point Rd.

Anacortes, WA 98221

stroms@henson.cc.wwu.edu

Sublette, Kerry L.

University of Tulsa

Department of Chemical Engineering

600 South College Avenue

Tulsa, OK 74104

che_kls@centum.utulsa.edu

Suflita, Joseph

University of Oklahoma

Department of Botany and Microbiology

770 Van Vleet Oval

Norman, OK 73019

msuflita@aardvark.ucs.uoknor.edu

Swift, Donald J. P.

Old Dominion University

Department of Oceanography

Norfolk, VA 23529

djs100f@ludwick.ocean.odu.edu

Swift, Norman

USDOE, Chicago Operations Office

9800 Cass Ave.

Argonne, IL 60439 
Takahashi, Taro

Lamont-Doherty Earth Observatory

P.O. Box 1000

Palisades, NY 10964-8000

taka@1deo.columbia.edu

Tang, Ignatius $\mathbf{N}$.

Brookhaven National Laboratory

DAS/ECD, Bldg. 815

P.O. Box 5000

Upton, NY 11973-5000

tang1@bnl.gov

http://www.ecd.bnl.gov/

Tausch, Robin

USDA Forest Service

920 Valley Rd.

Reno, NV 89512

Taylor, Karl

Lawrence Livermore National Laboratory

7000 East Avenue

Livermore, CA 94551-9900

taylor13@llnl.gov

Thomas, Donald

University of Hawaii

Hawaii Institute of Geophysics

2525 Correa Rd.

Honolulu, HI 96822

dthomas@soest.hawaii.edu

Thomas, Richard

West Virginia University,

Brooks Hall

P.O. Box 6057

Morgantown, WV 26506-6057

rthomas@wvnvm.wvnet.edu

Thompson, $\mathrm{T}$.

University of Arizona

Tucson, AZ 85721

Thompson@ag.arizona.edu

Tiao, George

University of Chicago

Chicago, IL 60637

get@gsbget.uchicago.edu

Tie, Xuexi

National Center for Atmospheric Research

P.O. Box 3000

Boulder, CO 80307-3000

xxtie@ucar.edu
Tilley, J. S.

University of Colorado

Boulder, CO 80309

Toon, O. B.

NASA-Ames, MS 245-3

Moffett Field, CA 94035-1000

toon@sky.arc.nasa.gov

http://cloudl.arc.nasa.gov/espo/success/

Tschaplinski, Timothy

Oak Ridge National Laboratory

P.O. Box 2008

Oak Ridge, TN 37831-6422

t2t@ornl.gov

http://www.esd.ornl.gov/programs/wbw/

Turner, Robert

Oak Ridge National Laboratory

Bldg. 1505, MS 6038

Oak Ridge, TN 37831-6038

rtt@ornl.gov

Tuskan, Gerald

Oak Ridge National Laboratory

P.O. Box 2008

Oak Ridge, TN 37831-6422

gtk@ornl.gov

Uliman, William

University of Delaware

College of Marine Studies

700 Pilottown Road

Lewes, DE 19958-1298

ullman@UDel.edu

http://www.udel.edu/cms

Valero, Francisco

University of California, San Diego

3209 IGPP-II

9500 Gilman Dr.

La Jolla, CA 92093

fvalero@ucsd.edu

Valocchi, Albert

University of Illinois

205 North Matthews Avenue

Urbana, IL 61801

valocchi@cern.ce.uiuc.edu

Varanasi, Prosad

State University of New York

Marine Sciences Research Center

149 Dana Hall

Stony Brook, NY 11794

pvaranasi@ccmail.sunysb.edu 
Venter, J. Craig

The Institute for Genomic Research

9712 Medical Center Drive

Rockville, MD 20850

jcventer@tigr.org

http://www.tigr.org

Verity, Peter

Skidaway Institute of Oceanography

10 Ocean Science Circle

Savannah, GA 31411

peter@skio.peachnet.edu

Vitko, John

Sandia National Laboratories

P.O. Box 969

Livermore, CA 94551

john_vitko@sandia.gov

Volker, Mohnen

State University of New York

Department of Atmospheric Science

1400 Washington Avenue

Albany, NY 12222

vam@atmos.albany.edu

Vorosmarty, Charles

University of New Hampshire

Morse Hall

Durham, NH 03824

charles.vorosmarty@unh.edu

http://pathfinder-www.sr.unh.edu/

Voyles, Jimmy W.

Pacific Northwest National Laboratory

P.O. Box 999

Richland, WA 99352

jw_voyles@pni.gov

Walcoff, Carol

Walcoff and Associates, Inc.

12015 Jackson Lee Highway

Suite 500

Fairfax, VA 22033

Wallace, Douglas W. R.

Brookhaven National Laboratory

Oceanographic and Atmospheric Sciences Division

Bldg. 318

P.O. 5000

Upton, NY 11973

wallace@bnl.gov

http://www.oasd.bnl.gov/ oasd/ $1 /$
Wang, Wei-chyung

State University of New York

Atmospheric Sciences Research Center

100 Fuller Rd.

Albany, NY 12205

wang@climate.asrc.albany.edu

Washington, Warren $\mathbf{M}$.

National Center for Atmospheric Research

P.O. Box 3000

Boulder, CO 80307-3000

wmw@ncar.ucar.edu

Wasiolek, Piotr

New Mexico Tech

Department of Physics

Socorro, NM 87801

piotr@mailhost.nmt.edu

Watt, Ward B.

Stanford University

Department of Biological Sciences

Stanford, CA 94305-5020

ward.watt@forsythe.stanford.edu

Weatherhead, Elizabeth

University of Colorado, CIRES

NOAA R/E/ARx1

325 Broadway

Boulder, CO 80303

betsy@srrb.noaa.gov

Weatherly, Georges

Florida State University

Oceanography Department (3048)

Tallahassee, FL 32306-3048

weatherly@ocean.fsu.edu

Webb, Thompson, III

Department of Geological Sciences

Brown University

Providence, RI 02912-1846

thompson_webb_iii@brown.edu

Webster, Peter J.

University of Colorado

Campus Box 311

Boulder, CO 80309

peter.webster@colorado.edu

Wei, Zongying

Research Support Instruments, Inc.

5500 Central Avenue, Suite 202

Boulder, CO 80301

zwei@csn.org 
Weinstein-Lloyd, Judith

State University of New York/Old Westbury

Chemistry/Physics Department

P.O. Box 210

Old Westbury, NY 11568

jlloyd@bnl.gov

Weiss, Ray F.

Scripps Institution of Oceanography

University of California, San Diego

La Jolla, CA 92093-0220

rfweiss@ucsd.edu

Weiss, Robert B.

University of Utah

Department of Human Genetics

2100 Eccles Institute of Human Genetics

Salt Lake City, UT 84112

bob.weiss@genetics.utah.edu

Wesely, Marvin

Argonne National Laboratory

Environmental Research Division, Bldg. 203

9700 South Cass Avenue

Argonne, IL 60439

mlwesely@anl.gov

Westwater, Edgeworth

NOAA/Environmental Research Laboratories

325 Broadway

Boulder, CO 80303-3228

ewestwater@etl.noaa.gov

Weyant, John P.

Stanford University

Department of Engineering-Economic Systems

Terman Engineering Bldg., Room 406

Stanford, CA 94305-4022

john.weyant@forsythe.stanford.edu

http://www-leland.stanford.edw/ -weyant/

White, Andrew

Los Alamos National Laboratory

Advanced Computing Laboratory

Los Alamos, NM 87545

abw@lanl.gov

White, David C.

The University of Tennessee

Center for Environmental Biotechnology

10515 Research Drive, Suite 300

Knoxville, TN 37932-2575

milipids@aol.com

http://www.ra.utk.edu/ceb/home.htm
Whiteman, C. David

Pacific Northwest National Laboratory

P.O. Box 999, MSIN K9-30

Richland, WA 99352

cd whiteman@pnl.gov

http://www.pnl.gov:2080/

Whiteman, David

NASA Goddard Space Flight Center, Code 924

Greenbelt, MD 20771

dave@eibl.gsfc.nasa.gov

http://rsd.gsfc.nasa.gov/924

Whitham, T. G.

Northern Arizona University

Department of Biological Sciences

Flagstaff, AZ 86011-5640

tgw@nau.edu

Wigley, T. M. L.

National Center for Atmospheric Research

P.O. Box 3000

Boulder, CO 80307-3000

wigley@ncar.ucar.edu

Wigmosta, Mark

Pacific Northwest National Laboratory

P.O. Box 999

Richland, WA 99352

ms_wigmosta@pnl.gov

http://www.pnl.gov:2080/

Wilcoxen, Peter

University of Texas

Department of Economics

Austin, TX 78712

wilcoxen@eco.utexas.edu

Wildung, Ray

Pacific Northwest National Laboratory

P.O. Box 999; MS P7-54

Richland, WA 99352

re_wildung@pnl.gov

http://www.pnl.gov:2080/

Williams, Albert J.

Woods Hole Oceanographic Institution

Woods Hole, MA 02543

awilliams@whoi.edu

Williamson, David

National Center for Atmospheric Research

P.O. Box 3000

Boulder, CO 80307-3000

wmson@ucar.edu 
Winn, Christopher D.

University of Hawaii

1000 Pope Road

Honolulu, HI 96822

cwinn@ucsd.edu

Wirick, Creighton D.

Brookhaven National Laboratory

P.O. Box 5000

Upton, NY 11973-5000

http://www.oasd.bnl.gov/ oasd/

Wiscombe, Warren

NASA Goddard Space Flight Center, Code 913

Greenbelt, MD 20771

wiscombe@climate.gsfc.nasa.gov

http://www.gsfc.nasa.gov/GSFC_homepage.html

Wise, Donald L.

Northeastern University

342 Snell Engineering Center

360 Huntington Avenue

Boston, MA 02115

Woese, Carl R.

University of Illinois

131 Burrill Hall

407 S. Goodwin Avenue

Urbana, IL 61801

carl@ninja.life.uiuc.edu

Wofsy, Steven

Harvard University

29 Oxford St.

Cambridge, MA 02138

scw@io.harvard.edu

http://www-as.harvard.edu/people/SteveWofsy.html

Worley, Pat

Oak Ridge National Laboratory

P.O. Box 2008

Oak Ridge, TN 37831-6367

worley@msr.epm.ornl.gov

Worsnop, Douglas R.

Aerodyne Research, Inc.

45 Manning Road

Billerica, MA 08121-3976

worsnop@aerodyne.com

Wuebbles, Donald J.

University of Illinois

Department of Atmospheric Science

105 South Gregory Street

Urbana, IL 61801-3070

wuebbles@uiatma.atmos.uiuc.edu
Wullschleger, Stanley D.

Oak Ridge National Laboratory

Bldg. 1059

P.O. Box 2008

Oak Ridge, TN 37831-6422

w5d@ornl.gov

Young, G. S.

The Pennsylvania State University

503 Walker Bldg.

University Park, PA 16802

young@ems.psu.edu

Young, William

Scripps Institution of Oceanography

University of California, San Diego

La Jolla, CA 92093-0230

wryoung@ucsd.edu

Zachara, John

Pacific Northwest National Laboratory

P.O. Box 999; K3-61

Richland, WA 99352

jm_zachara@pnl.gov

Zak, Donald

University of Michigan

School of Natural Resources \& Environment

Ann Arbor, MI 48109-1115

drzak@umich.edu

Zeiger, Eduardo

University of California

Department of Biology

Los Angeles, CA 90024-1606

zeiger@lbes.medsch.ucla.edu

Zhang, Guang J.

Scripps Institution of Oceanography

9500 Gilman Drive

La Jolla, CA 92093-0221

gzhang@ucsd.edu

Zhong, Sharon S.

Pacific Northwest National Laboratory

P.O. Box 999; MSIN K9-30

Richland, WA 99352

szhong@rossby.pnl.gov

http://www.pnl.gov:2080/

Zivkovic, Marina

Atmospheric and Environmental Research, Inc.

840 Memorial Drive

Cambridge, MA 02139-3794

marina@aer.com 


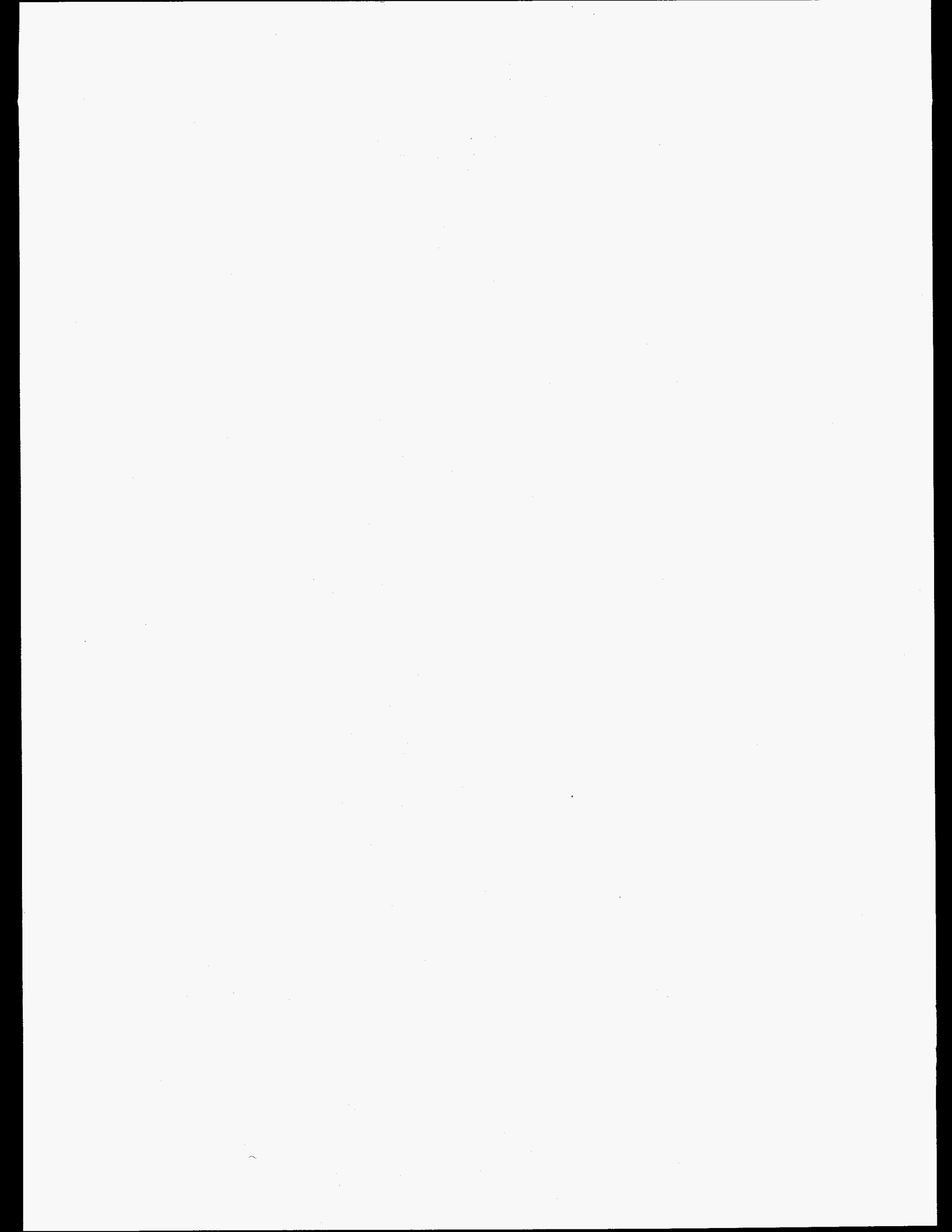




\section{APPENDIX B: ACRONYMS}

1-D

2-D

3-D

4-D

Ac

ACC

ACM

ACP

ACP

ADCP

AEC

AEEI

AER

AERI

AGCM

AIM

ALE

ALK

AM

AMD

AMIP

AMS

AMWSR

ANL

AODC

AOS

AP-PCR

APBL

ARCS

ARCSS

ARESE

ARM

ARMA

ARS

ASA

ASCOT

ASL

ASM

ASTEX

ATCC

ATDD

ATMOS

ATPEX

AVHRR

AVIRIS

AXCTD

BARFEX

BASE

BASS

BATS

BAU

BBL

BBSS
One-dimensional

Two-dimensional

Three-dimensional

Four-dimensional

Altocumulus

Antarctic Circumpolar Current

Advanced climate model

Atmospheric Chemistry Program

Anvil cirrus parameterization

Acoustic-Doppler current profiler

Atomic Energy Commission

Autonomous energy-efficiency index

Atmospheric \& Environmental Research, Inc.

Atmospheric emitted radiance instrument

Atmospheric general circulation model

Approximate inertial manifold

Arbitrary Lagrangian-Eularian

Alkalinity

Arbuscular mycorrhizal

Activity median diameter

Atmospheric Model Intercomparison Project

American Meteorological Society

Advanced microwave scanning radiometer

Argonne National Laboratory

Acridine orange direct count

Aerosol observation system

Arbitrarily primed polymerase chain reaction

Atmospheric planetary boundary layer

Atmospheric radiation and cloud station

Arctic System Science Program

ARM Enhanced Shortwave Experiment

Atmospheric Radiation Measurement (Program)

Autoregressive moving-average

Agricultural Research Service

Anthropogenic sulfate aerosol

Atmospheric Studies in Complex Terrain

Atmospheric surface layer

American Society of Microbiology

Atlantic Stratocumulus Transition Experiment

American Type Culture Collection

Atmospheric Turbulence and Diffusion Division

Atmospheric Trace Molecule Spectroscopy Experiment

ARM Tropical Pacific Experiment

Advanced very-high-resolution radiometer

Airborne visible infrared imaging spectrometer

Autonomous expendable CTD profiler

Boardman Regional Flux Experiment

Broadband ATM Satellite Experiment

Benthic acoustic stress sensor

Biosphere-atmosphere transfer scheme

Business as usual

Bottom boundary layer

Balloon-borne sounding system 


\begin{tabular}{|c|c|}
\hline BEM & Boundary-element method \\
\hline BNL & Brookhaven National Laboratory \\
\hline BOA & Bottom of the atmosphere \\
\hline BOREAS & Boreal Ecosystems Atmosphere Study \\
\hline BTEX & Benzene, toluene, ethylbenzene, and xylene \\
\hline BVE & Barotropic vorticity equation \\
\hline CaBS & California Basins Study \\
\hline $\mathrm{CAC}$ & Committee on Atmospheric Chemistry \\
\hline CAGEX & CERES/ARM/GEWEX Experiment \\
\hline CAM & Crassulacean acid metabolism \\
\hline CAMRAQ & Consortium for Advanced Modeling of Regional Air Quality \\
\hline CAP & Coupled Arctic Program \\
\hline CARDS & Climatological aerological reference data set \\
\hline CART & Clouds and Radiation Testbed \\
\hline CAS & Chinese Academy of Sciences \\
\hline $\mathrm{CCCO}$ & Committee on Climatic Change and the Ocean \\
\hline CCD & Charged coupled device \\
\hline $\mathrm{CCM}$ & Community climate model \\
\hline $\mathrm{CCN}$ & Cloud condensation nuclei \\
\hline $\operatorname{CCS}$ & Center for Computational Sciences \\
\hline CD-ROM & Compact disk-read only memory \\
\hline CDIAC & Carbon Dioxide Information Analysis Center \\
\hline CDMS & Climate data management system \\
\hline CE & Office of Conservation and Renewable Energy \\
\hline CEA & Cause-and-effect analysis \\
\hline CEES & Committee on Earth and Environmental Sciences \\
\hline CEM & Cumulus ensemble model \\
\hline CENR & Committee on Environment and Natural Resources \\
\hline CEPEX & Central Equatorial Pacific Experiment \\
\hline CFC & Chlorofluorocarbon \\
\hline CGE & Computable general equilibrium \\
\hline CHAMMP & Computer Hardware, Advanced Mathematics, and Model Physics \\
\hline CHARTS & Code for High-Resolution Atmospheric Radiative Transfer \\
\hline CIESIN & Consortium for International Earth Science Information Network \\
\hline CIS & Commonwealth of Independent States \\
\hline CLASP & Coupled land-atmosphere simulation program \\
\hline CLIMAP & Climate Long-Range Investigation Mapping and Prediction \\
\hline CLOT & Carbon layer open tubular \\
\hline CMA & China Meteorological Administration \\
\hline CMDL & Climate Modeling and Diagnostics Laboratory \\
\hline CMEAL & $\mathrm{CO}_{2}$ Models/Experiments Activity for Improved Links \\
\hline CMP & Computer model package \\
\hline CMS & Comprehensive modeling systems \\
\hline CNC & Condensation nuclei counter \\
\hline COADS & Comprehensive Ocean-Atmosphere Data Set \\
\hline COARE & Coupled Ocean Atmosphere Response Experiment \\
\hline $\mathrm{COC}$ & Colloidal organic carbon \\
\hline COHMAP & Cooperative Holocene Mapping Project \\
\hline COOP & Coastal ocean processes \\
\hline $\mathrm{CP}$ & Circulation patterns \\
\hline CPMS & Climatological Prediction by Model Statistics \\
\hline CPRS & Cloud-profiling radar system \\
\hline CPU & Central processing unit \\
\hline CRADA & Cooperative Research and Demonstration Agreement \\
\hline CRB & Columbia River basalt \\
\hline CRF & Cloud radiative forcing \\
\hline
\end{tabular}




\begin{tabular}{|c|c|}
\hline CRM & Certified reference material \\
\hline CRT & Chemical radiative transport \\
\hline CSIRO & Commonwealth Scientific and Industrial Research Organisation \\
\hline CSMP & Climate System Modeling Project \\
\hline CST & CHAMMP Science Team \\
\hline CSU & Colorado State University \\
\hline CT & Total dissolved inorganic carbon \\
\hline CTD & Continuous-temperature-depth \\
\hline CTM & Chemical transport model \\
\hline DAPI & 4,6-diamidino-2-phenylindole \\
\hline DAS & Data-assimilation system \\
\hline DDA & Discrete-displacement method \\
\hline DDI & Data and dimensions interface \\
\hline DDMP & Domain-decomposition-message-passing \\
\hline DEM & Digital elevation model \\
\hline DGFA & Diglyceride fatty acids \\
\hline DIA & Direct-interaction approximation \\
\hline DIAL & Differential absorption lidars \\
\hline DIC & Dissolved inorganic carbon \\
\hline DIRB & Dissimilatory iron-reducing bacteria \\
\hline DMCC & DOE Meteorological Coordinating Committee \\
\hline DMS & Dimethyl sulfide \\
\hline DMSO & Dimethyl sulfoxide \\
\hline $\mathrm{DMSO}_{2}$ & Dimethyl sulfone \\
\hline DNA & Deoxyribonucleic acid \\
\hline DNS & Direct numerical simulation \\
\hline DOC & Dissolved organic carbon \\
\hline DOD & Department of Defense \\
\hline DOE & Department of Energy \\
\hline DOE-CH & USDOE, Chicago Operations \\
\hline DOM & Dissolved organic matter \\
\hline DON & Dissolved organic nitrogen \\
\hline DOP & Dissolved organic phosphorus \\
\hline DOY & Day of the year \\
\hline DRIFTS & Diffuse reflectance infrared spectroscopy \\
\hline DRS & Data retrieval and storage \\
\hline DSIT & Data and Science Integration Team \\
\hline DSM & Demand-side management \\
\hline DTR & Diurnal temperature range \\
\hline EAC & East Asia climate \\
\hline EBM & Energy-balance model \\
\hline ECLIPS & Experimental Cloud Lidar Pilot Study \\
\hline ECMWF & European Centre for Medium-Range Weather Forecasts \\
\hline EDI & Equivalent-damage index \\
\hline EDTA & Ethylenediaminetetraacetic acid \\
\hline EM & Environmental Management \\
\hline EMF & Energy Modeling Forum \\
\hline EML & Environmental Measurements Laboratory \\
\hline EMSL & Environmental Molecular Sciences Laboratory \\
\hline ENSO & El Niño/Southern Oscillation \\
\hline EOF & Empirical orthogonal function \\
\hline EOP & Experiment Operations Plan \\
\hline EPA & Environmental Protection Agency \\
\hline EPI & Environmental productivity index \\
\hline EPRI & Electric Power Research Institute \\
\hline ER & Office of Energy Research \\
\hline
\end{tabular}




\begin{tabular}{|c|c|}
\hline ERBE & Earth Radiation Budget Experiment \\
\hline ERBS & Earth Radiation Budget Satellite \\
\hline ERDA & Energy Research and Development Administration \\
\hline ESD & Environmental Sciences Division \\
\hline EST & Experiment Support Team \\
\hline ETEX & European Tracer Experiment \\
\hline ETH-Z & Eidgenossische Technische Hochschule - Zurich \\
\hline ETL & Environmental Technology Laboratory \\
\hline FACE & Free-air carbon dioxide enrichment \\
\hline FANGIO & Feedback Analysis for GCM Intercomparison and Observations \\
\hline FAO & U.N. Food and Agriculture Organization \\
\hline FARS & Facility for Atmospheric Remote Sensing \\
\hline FAST & Fourier amplitude-sensitivity test \\
\hline FCCSET & Federal Coordinating Council for Science, Engineering, and Technology \\
\hline FCT & Flux-corrected transport \\
\hline FDDA & Four-dimension data assimilation \\
\hline FE & Office of Fossil Energy \\
\hline FEM & Finite-element methods \\
\hline FFcer & Forest-floor $\mathrm{CO}_{2}$ exchange rates \\
\hline FFT & Fast Fourier transform \\
\hline FIA & Forest inventory and analysis \\
\hline FID & Flame ionization detector \\
\hline FIFE & First ISLSCP Field Experiment \\
\hline FIRE & First ISCCP Regional Experiment \\
\hline FOV & Field of view \\
\hline FRR & Fast repetition rate \\
\hline FSSP & Forward-scattering spectrometer probe \\
\hline FTIR & Fourier transform infrared \\
\hline FTP & File-transfer protocol \\
\hline FTS & Fourier transform spectrometer \\
\hline FY & Fiscal year \\
\hline GAMS & General algebraic modeling system \\
\hline GATE & Global Atmospheric Research Program \\
\hline GC-FID & Gas chromatography with flame-ionization detection \\
\hline GC-MS & Gas chromatography and mass spectrometry \\
\hline GCDIS & Global Change Data and Information System \\
\hline GCEP & Global Change Education Program \\
\hline GChM & Global chemistry model \\
\hline GCIP & GEWEX Continental-Scale International Project \\
\hline GCM & General circulation model \\
\hline GCRP & Global Change Research Program \\
\hline GCSS & GEWEX Cloud System Study \\
\hline GCTE & Global Change and Terrestrial Ecosystems (Project) \\
\hline GDD & Growing-degree day \\
\hline GDP & Gross domestic product \\
\hline GEIA & Global Emissions Inventory Activity \\
\hline GEMHEX & Geochemical, Microbiological, and Hydrological Experiment \\
\hline GEOSECS & Geochemical Ocean Sections Study \\
\hline GEWEX & Global Energy and Water Experiment \\
\hline GFDL & Geophysical Fluid Dynamics Laboratory \\
\hline GGI & Greenhouse-gas indicies \\
\hline GHCN & Global Historical Climatology Network \\
\hline GHG & Greenhouse gases \\
\hline GIS & Geographic information system \\
\hline GISMO & Graphic-information-system modeling \\
\hline GISS & Goddard Institute for Space Studies \\
\hline
\end{tabular}




\begin{tabular}{|c|c|}
\hline $\begin{array}{l}\text { GLOBE } \\
\text { GOES }\end{array}$ & $\begin{array}{l}\text { Global Learning and Observations to Benefit the Environment } \\
\text { Geostationary Orbiting Environmental Satellite; Geostationary Operational } \\
\text { Environmental Satellite }\end{array}$ \\
\hline GPS & Global positioning system \\
\hline GrADS & Gridded Analysis Data System \\
\hline GRASS & Geographic Resource Analysis Support System \\
\hline GRIB & Gridded binary \\
\hline GSFC & Goddard Space Flight Center \\
\hline GSS & Global-Systems Science \\
\hline GTS & Global Telecommunications System \\
\hline GWP & Global warming potential \\
\hline GWR & Gravity-wave retardation \\
\hline HAPEX & Hydrologic Atmospheric Pilot Experiment \\
\hline HOPE & Hamburg ocean primitive equation \\
\hline HOV & High-occupancy vehicle \\
\hline HPCCI & High-Performance Computing and Communications Initiative \\
\hline HPCRC & High-Performance Computing Research Center \\
\hline HPLC & High-pressure liquid chromatography \\
\hline HPRT & High-resolution picture transmission \\
\hline HSCT & High-speed civil transport \\
\hline I/O & Input/output \\
\hline I-S & Isentropic-sigma \\
\hline IA & Integrated assessment \\
\hline IASC & International Arctic Science Committee \\
\hline IBP & International Biophysical Program \\
\hline IC & Interaction coefficient \\
\hline ICL & Integrated cloud liquid \\
\hline ICRCCM & InterComparison of Radiation Codes in Climate Models (Program) \\
\hline IDASS & Integrated data-assimilation and sounding system \\
\hline IDP & Instrument Development Program \\
\hline IEM & Institute of Experimental Meteorology \\
\hline IFA & Inner flux array \\
\hline IGAC & International Global Atmospheric Chemistry \\
\hline IGBP & International Geosphere-Biosphere Program \\
\hline IIASA & International Institute for Applied Systems Analysis \\
\hline IM & Ice nuclei \\
\hline INEL & Idaho National Engineering Laboratory \\
\hline IO & Indian Ocean \\
\hline IOP & Intensive operational period \\
\hline IPCC & Intergovernmental Panel on Climate Change \\
\hline IPCC/RSWG/EIS & Response Strategies Working Group, Energy and Industry Subgroup \\
\hline IR & Infrared \\
\hline IRGA & Infrared gas analyzer \\
\hline ISCCP & International Satellite Cloud Climatology Project \\
\hline ISEB & International Society for Environmental Biotechnology \\
\hline ISLSCP & International Satellite Land-Surface Climatology Project \\
\hline ISS & Integrated sounding system \\
\hline ISTAP & International Space Technology Assessment Program \\
\hline ITCZ & Intertropical convergence zone \\
\hline IWC & Ice-water content \\
\hline IWP & Ice-water path \\
\hline JFD & Joint frequency distributions \\
\hline JGOFS & Joint Global Ocean Flux Study \\
\hline JJA & June-July-August \\
\hline LAI & Leaf-area index \\
\hline LAID & Land-atmosphere interactions dynamics \\
\hline
\end{tabular}




\begin{tabular}{|c|c|}
\hline LANL & Los Alamos National Laboratory \\
\hline LAPS & Large Aggregate Profiling System \\
\hline LBL (lbl) & Line by line \\
\hline LCA & Life-cycle assessment (or analysis) \\
\hline LCL & Lifting condensation level \\
\hline LDEO & Lamont-Doherty Earth Observatory \\
\hline LES & Large-eddy simulation \\
\hline LIDAR & Laser-radar \\
\hline LIRAD & Lidar/radiometer \\
\hline LLJ & Low-level jet \\
\hline LLNL & Lawrence Livermore National Laboratory \\
\hline LPDA & Laser-phased Doppler anemometer \\
\hline LPG & Liquid propane gas \\
\hline LPS & Lipopolysaccharide \\
\hline LRA & Lagrangian renormalized approximation \\
\hline LSG & Large-scale geostrophic \\
\hline LTER & Long-term ecological research \\
\hline LWC & Liquid water content \\
\hline LWP & Liquid-water path \\
\hline MAB & Mid-Atlantic Bight \\
\hline MAC & Maricopa Agriculture Center \\
\hline MAP & Mesoscale Alpine Programme \\
\hline MAPR & Multiple-antenna wind profiler \\
\hline MARI & Mexico City Air Quality Research Initiative \\
\hline MAST & Monterey Area Ship Trails \\
\hline MB & Methanogenic bacteria \\
\hline MCC & Mesoscale convective complex \\
\hline MCP & Mixed-complementarity problems \\
\hline MCS & Mesoscale convective system \\
\hline MCTEX & Maritime Continent Thunderstorm Experiment \\
\hline MDSRS & Morphology-dependent simulated Raman scattering \\
\hline ME & Monolayer equivalent \\
\hline MedFACE & Mediterranean free-air carbon dioxide enrichment \\
\hline MFRSR & Multifilter rotating shadowband radiometer \\
\hline MICOM & Miami isopycnic-coordinate ocean model \\
\hline MIDI & Microbial identification \\
\hline MIMD & Multiple-instruction, multiple-data \\
\hline MINK & Missouri-Iowa-Nebraska-Kansas \\
\hline MIRSL & Microwave Remote Sensing Laboratory \\
\hline MIT & Massachusetts Institute of Technology \\
\hline MJO & Madden-Julian oscillation \\
\hline MM5 & Mesoscale model \\
\hline MMS & Modular modeling system \\
\hline MOM & Modular ocean model \\
\hline MPC & Massively parallel computer \\
\hline MPI & Max Planck Institute \\
\hline MPIR & Multispectral pushbroom imaging radiometer \\
\hline MPL & Micropulse lidar \\
\hline MPN & Most-probable number \\
\hline MSA & Methane sulfonic acid \\
\hline MSL & Mean sea level \\
\hline MSU & Microwave sounding unit \\
\hline MTE & Mediterranean-type ecosystem \\
\hline MWR & Microwave radiometer \\
\hline MWSR & Microwave water substance radiometer \\
\hline NABIR & Natural and accelerated bioremediation \\
\hline
\end{tabular}




\begin{tabular}{|c|c|}
\hline NADW & North Atlantic deep water \\
\hline NAPL & Nonaqueous phase liquid \\
\hline NARE & North Atlantic Regional Experiment \\
\hline NARSTO & North American Research Strategy for Tropospheric Ozone \\
\hline NAS & National Academy of Sciences \\
\hline NASA & National Aeronautics and Space Administration \\
\hline NATO & North Atlantic Treaty Organization \\
\hline NBT & Nitro blue tetrazolium chloride \\
\hline NCAR & National Center for Atmospheric Research \\
\hline NCDC & National Climatic Data Center \\
\hline NCE & Net carbon exchange \\
\hline NCEP & National Centers for Environmental Prediction \\
\hline NDP & Numeric data package \\
\hline NDVI & Normalized-difference vegetation index \\
\hline NE & Office of Nuclear Energy \\
\hline NEE & Net ecosystem exchange \\
\hline NEP & Net environmental productivity \\
\hline NEPA & National Environmental Policy Act \\
\hline NES & National Energy Strategy \\
\hline nf-EBM & Noise-forced energy-balance model \\
\hline NIGEC & National Institute of Global Environmental Change \\
\hline NIST & National Institute of Standards and Technology \\
\hline NMC & National Meteorological Center \\
\hline NMHC & Nonmethane hydrocarbons \\
\hline NMOC & Nonmethane organic compounds \\
\hline NMR & Nuclear magnetic resonance \\
\hline NOAA & National Oceanic and Atmospheric Administration \\
\hline NOM & Natural organic matter \\
\hline NOSR & National Oil Shale Reserve; Naval Oil Shale Reserve \\
\hline NPP & Net primary productivity \\
\hline NRL & Naval Research Laboratory \\
\hline NRP & National Research Program \\
\hline NRPGC & National Research Program for Global Change \\
\hline NS & Navier-Stokes \\
\hline NSA & Northern Slope of Alaska \\
\hline NSF & National Science Foundation \\
\hline NSLS & National Synchrotron Light Source \\
\hline nss & Non-sea-salt \\
\hline NSTC & National Science and Technology Council \\
\hline NTA & Nitrilotriacetic acid \\
\hline NTMB & Neotropical migrant breeding birds \\
\hline NWS & National Weather Service \\
\hline NYU & New York University \\
\hline OBS & Optical-backscatterance sensor \\
\hline OECD & Organization for Economic Cooperation and Development \\
\hline OGCM & Ocean general circulation model \\
\hline OHER & Office of Health and Environmental Research \\
\hline OLR & Outgoing longwave radiation \\
\hline OMB & Office of Management and Budget \\
\hline OMP & Ocean Margins Program \\
\hline OMWOG & Ocean Measurements Working Group \\
\hline OPC & Optical particle counter \\
\hline ORISE & Oak Ridge Institute for Science and Engineering \\
\hline ORNL & Oak Ridge National Laboratory \\
\hline OSB & Ocean Studies Board \\
\hline OSC & Office of Scientific Computing (DOE) \\
\hline
\end{tabular}




\begin{tabular}{|c|c|}
\hline OSHA & Occupational Safety and Health Administration \\
\hline OSSE & Observing Simulation System Experiment; Observation System Simulation Experiment \\
\hline OSU & Oregon State University \\
\hline P-I & Photosynthesis-irradiance \\
\hline PAN & Peroxyacetyl nitrate \\
\hline PAR & Photosynthetically active radiation \\
\hline PBL & Planetary boundary layer \\
\hline PCB & Polychlorinated biphenyl \\
\hline PCCM & Parallel Community Climate Model \\
\hline PCMDI & Program for Climate Model Diagnosis and Intercomparison \\
\hline PCR & Polymerase chain reaction \\
\hline PDA & Phased-doppler anemometer \\
\hline PDE & Partial differential equation \\
\hline PDF & Probability distribution function \\
\hline PDL & Polarization-diversity lidar \\
\hline PDS & Photothermal deflection spectroscopy \\
\hline PE & Office of Policy, Planning, and Analysis \\
\hline PEMEX & Mexican National Oil Company \\
\hline PEPCase & Phosphenol-pyruvate carboxylase \\
\hline PER & Program on Ecosystem Research \\
\hline PFS & Parallel file system \\
\hline PFT & Perfluorinated tracer \\
\hline PGI & Phosphoglucose isomerase \\
\hline PGM & Phosphoglucomutase \\
\hline PGPR & Plant-growth-promoting rhizobacteria \\
\hline PHA & Pulse-height analysis \\
\hline PI & Principal investigator \\
\hline PIC & Pulse ionization chamber \\
\hline PIC & Particle in cell \\
\hline PLFA & Phospholipid fatty acids \\
\hline PMEL & Pacific Marine Environmental Laboratory \\
\hline PMIP & Paleoclimate Modeling Intercomparison Project \\
\hline PNL & Pacific Northwest Laboratory \\
\hline PNNL & Pacific Northwest National Laboratory \\
\hline POC & Particulate organic carbon \\
\hline POCM & Parallel ocean-climate model \\
\hline POES & Polar Orbiting Environmental Satellite \\
\hline POM & Particulate organic matter \\
\hline PON & Particulate organic nitrogen \\
\hline POP & Parallel ocean program \\
\hline PPF & Photosynthetic photon flux \\
\hline PRC & People's Republic of China \\
\hline PROBE & Pilot Radiation Observation Experiment \\
\hline PSC & Polar stratospheric clouds \\
\hline PSU & Pennsylvania State University \\
\hline PV & Potential vorticity \\
\hline PWV & Precipitable water vapor \\
\hline QASSAC & Quality Control and Science Activity Center \\
\hline QBO & Quasibiennial Oscillation \\
\hline QC/SAC & Quality Control/Science Activities Center \\
\hline QLN & Quantitative Links Network \\
\hline QME & Quality-Measurement Experiment \\
\hline QTO & Quasitriennial Oscillation \\
\hline R\&D & Research and development \\
\hline RAMS & Regional Atmospheric Modeling System \\
\hline RANDAB & Radionuclide Database \\
\hline
\end{tabular}


Random amplified polymorphic DNA

Radio acoustic sounding system

RCM

RCRA

RCRE

RCS

REA

$\operatorname{ReCM}$

RFLP

RFP

RGI

RISC

RM

RMCW

RMS

RNA

ROD

RRTM

RSR

RSS

RT

Rubisco

SAB

SAGE

SAMI

SAQ

SAT

SAVE

SBIR

SBUV

SCAR

SCM

SCR

SDSC

SEEP

SEM

SERDP

SGCR

SGM

SGP

SHEBA

$\mathrm{SiB}$

SIMD

SIMS

SIO

SIP

SL

SLB

SLP

SLT

SMCC

SOAR

SOC

SOCEX

SOM

SOMMA

Radiative-convective model

Resource Conservation and Recovery Act

Required collaborative research experience

Remote cloud sensing

Relaxed-eddy accumulator

Regional climate model

Restriction fragment length polymorphism

Rocky Flats Plant

Regional-global interactions

Reduced instruction set chip

Reference material

Random-modulated continuous wave

Root mean square

Ribonucleic acid

Record of decision

Rapid radiative transfer model

Rotating shadowband radiometer

Rotating shadowband spectroradiometer

Radiative transfer

Ribulose-1,5-bisphosphate carboxylase

South Atlantic Bight

Stratospheric Aerosol and Gas Experiment

Submersible autonomous moored instruments

Subcommittee on Air Quality

Surface air temperature

South Atlantic Ventilation Experiment

Small Business Innovation Research

Satellite-based ultraviolet

Scientific Committee on Antarctic Research

Single-column model

Stochastic-convective reaction

San Diego Supercomputer Center

Shelf-Edge Exchange Processes (in the Middle Atlantic Bight)

Scanning electron microscope

Strategic Environmental Research and Development Program

Subcommittee on Global Change Research

Second-generation model

Southern Great Plains

Surface Heat Balance of the Arctic

Simple-Biosphere

Single-instruction, multiple-data

Secondary-ion mass spectrometer

Scripps Institution of Oceanography

Soil inoculum potential

Semi-Lagrangian

Signature lipid biomarker

Sea-level pressure

Semi-Lagrangian transport

Subsurface Microbial Culture Collection

State-of-the-art report

Soil organic carbon

Southern Ocean Cloud Experiment

Soil organic matter

Single-operator multiparameter metabolic analyzer 
SORTI

SOS

SPC

SPECTRE

SPM

SPOT

SR

SRB

SRL

SSA

SSBUV

SSM/I

SSP

SST

STARS

STBL

STTR

SWE

SWIR

T-S

TA

TAN

TCE

TDE

TECO

TF

TFP

TGG

THC

TM

TOA

TOF

TOGA

TOMS

TOPEX

TOZ

TRMM

TSCA

TTO

TUNES

TUV

TWP

UARS

UAV

UC

UCAR

UCLA

UCNC

UCR

UNCED

URL

USDA

USGCRP

USGS

UW
Solar-radiance-transmission interferometer

Southern Oxidants Study

Suspended particulate concentration

Spectral Radiance Experiment

Suspended particulate matter

Satellite pour l'Observation de la Terre; Systeme Probatoire pour l'Observation de la

Terre

Sulfate reducers

Sulfate-reducing bacteria

Scanning Raman lidar

Singular spectrum analysis

Surface- and satellite-based ultraviolet

Special sensor microwave/imager

Subsurface Science Program

Sea-surface temperature

Study of Transport and Chemical Reactions in the Stratosphere

Stratus-topped marine boundary layer

Small Business Technology Transfer

Shallow-water equations

Short-wavelength infrared

Temperature-salinity

Total alkalinity

Test Area North

Trichloroethylene

Throughfall Displacement Experiment

Terrestrial ecosystems

Transfer function

Total-factor productivity

Temperature-gradient greenhouses

Thermohaline circulation

Thematic Mapper

Top of the atmosphere

Theoretical orthogonal function

Tropical Ocean Global Atmosphere

Total Ozone Measuring Satellite

Topography Ocean Experiment

Total ozone

Tropical Rainfall Measuring Mission

Toxic Substances Control Act

Transient Tracers in the Ocean

Thomas Washington's Unexpected Nautical Extravaganza, South-North Expedition

Tropospheric ultraviolet-visible

Tropical Western Pacific

Upper Atmosphere Research Satellite

Unmanned aerial vehicles

University of California

University Corporation for Atmospheric Research

University of California at Los Angeles

Ultrafine-condensation-nucleus counter

University of California at Riverside

United Nations Conference on the Environment and Development

Uniform resource locator

United States Department of Agriculture

U.S. Global Change Research Program

United States Geological Survey

University of Wisconsin 
VAM

VBATS

VCS

VEMAP

VORTEX

WABEX

WAIS

WBW

WCRP

WESTGEC

WG

WGGC

WGNE

WHP

WILASS

WMO

WOCE

WOCE/HP

WPL

WSI

WST

WUE

WWW

XANES

XPS

ZSI
Vesicular-arbuscular microrrhizae

Vectorized biosphere-atmosphere transfer scheme

Visualization and Computation System

Vegetation/Ecosystem Modeling and Analysis Project

Verification of the origins of Rotation in Tornadoes Experiment

Wave Basin Experiment

Wide-area information service

Walker Branch Watershed

World Climate Research Program

Western Region of NIGEC

Working group

Working Group on Global Change

Working Group for Numerical Experimentation

World Hydrographic Program

Water Infiltration in Layered Subfreezing Snow (Project)

World Meteorological Organization

World Ocean Circulation Experiment

World Ocean Circulation Experiment Hydrographic Program

NOAA Wave Propagation Laboratory

Whole-sky imager

Whitecap simulation tank

Water-use efficiency

World-Wide Web

$\mathrm{X}$-ray absorption near-edge structure

X-ray photoelectron spectroscopy

Zenith sky intensities 


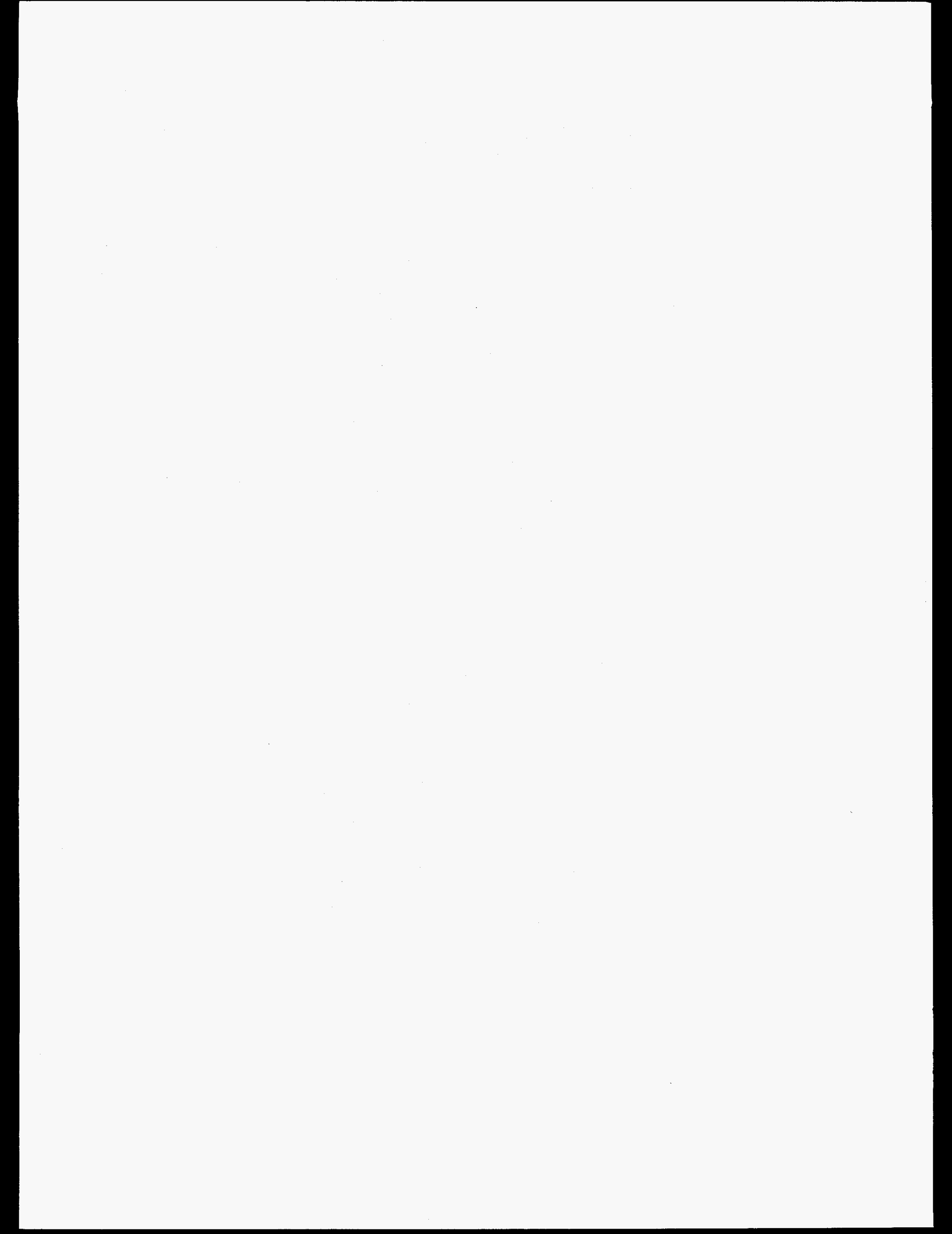


INDEXES 


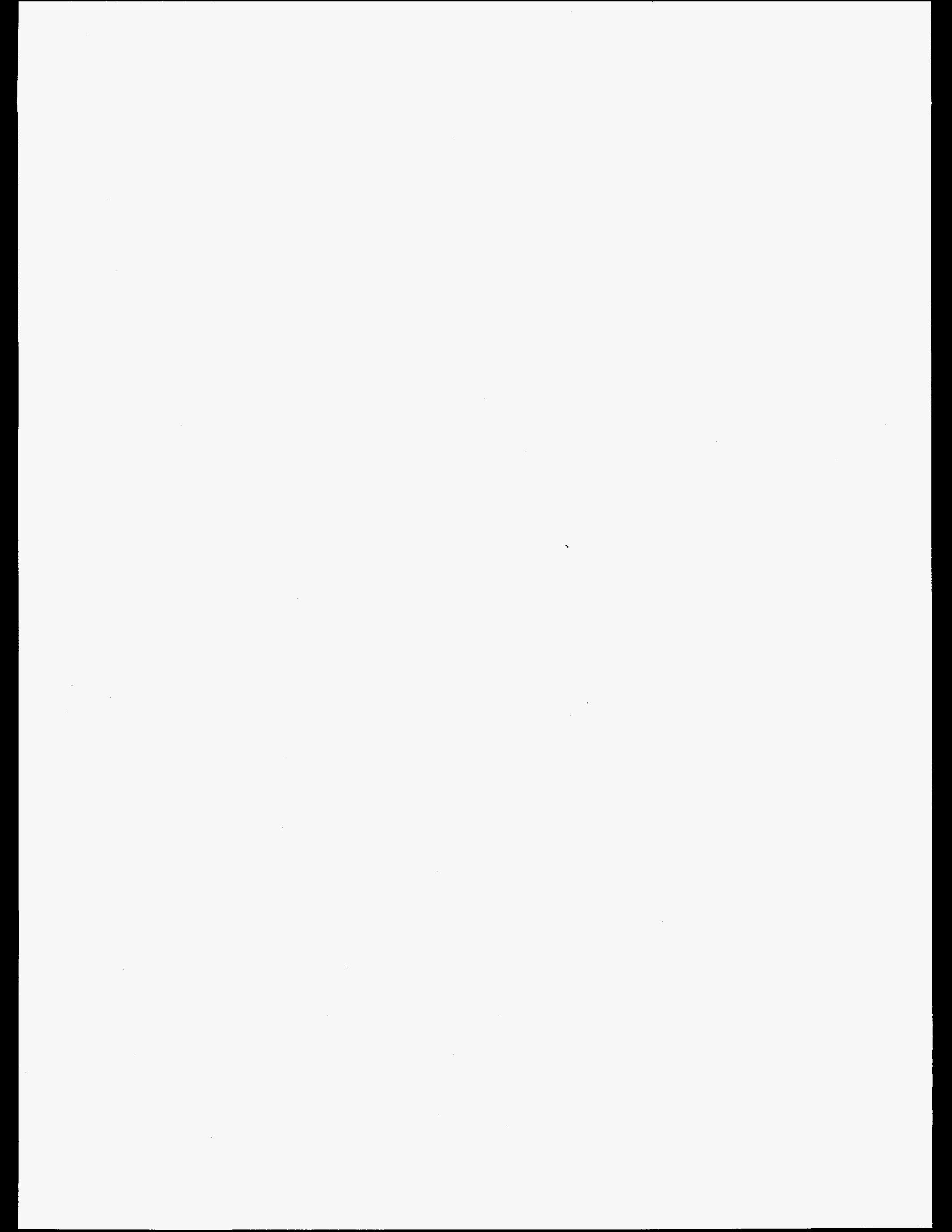


Ackerman, Thomas

Addis, Robert P.

Agrawal, Yogesh C.

Ahearne, John F.

Ahmad, Zia

Al Mahamid, Ilham

Albert, Brian

Albrecht, Bruce

Allen, Michael

Aller, Josephine

Aller, Robert

Ammerman, James

Amthor, Jeff

Amy, Penny

Anderson, John R.

Anderson, Susan

Arakawa, Akio

Arnold, Robert G.

Asher, William

Asper, Vernon

Atherton, Cynthia

Bader, David C.

Baer, Ferdinand

Baerwald, Thomas J.

Baldocchi, Dennis

Balkwill, David

Ball, J. Timothy

Banta, Robert M.

Barber, Robert

Barchet, W. Richard

Barnard, James

Barnett, Tim P.

Barnette, Daniel

Barr, Sumner

Baskaran, Mahalingam

Bauer, James E.

Baygents, James C.

Bayne, Stewart

Bazzaz, F. A.

Belzer, David

Benkovitz, Carmen M.

Benner, Ronald

Benninger, Larry

Benson, Sally $\mathrm{M}$.

Berg, John

Berkowitz, Carl M.

Bian, Xindi

Bisson, Scott E.

Bjornstad, David

Blair, Neal

Bleck, Rainer

Bloom, Arnold

Boden, Thomas
Boehme, Susan

Bohm, Emanuele

Bolton, Harvey

Boone, David R.

190,229

Bossert, James

Boutton, Thomas W.

Bradley, Raymond S.

Brasseur, Guy

Brewer, Alan

Brockman, Fred

Brooks, Scott

Bryan, Kirk

Burlage, Robert

Cahalan, Robert

Cairns, Brian

Canham, C. D.

Carlson, Barbara

Carmichael, Gregory

Cess, Robert D.

Cessi, Paola

Chapin, F. Stuart, III

Chen, Robert

Chervin, Robert

Chilakapati, Ashok

Chin, Hung-Neng

Chipman, David W.

Cho, $\mathrm{H}$.

Churchill, James H.

Cluurnside, James

Clark, William C.

Clough, Shepard A.

Cobb, N. S.

Cochran, J. Kirk

Cohn, Stephen

Colwell, Frederick

Connell, Peter S.

Conradson, Steven

Constantin, Milton J.

Cooper, Lee

Cotton, William

Coulter, Bryan

Coulter, Richard

Crawford, Ronald L.

Cress, Ted S.

Cummings, Ronald

Curry, J. A.

Cushman, John

Cushman, Robert

Dagg, Michael

Dannevik, William

Daum, Peter H.

Davidovits, Paul

Davis, Anthony
230

40,52

208

68

40

10

231, 234

237

78

217

30,33

15

200

15

41

4, 68

79

206

182

79

234

4

170

209

170

11

105

4, 69

194

167

19

231

60

243

93

62, 193, 207

5,14

96

5,41

218

6

97

8,29

232

$70,95,98,178$

185

80

42

42,65

30 
Dean, Sumner

Deflaun, Mary F.

Degrandpre, Michael D.

Del Genio, Anthony

Deluisi, John

Demaster, David

Denbo, D. W.

Diaz, Henry F.

Dickinson, Robert E.

Dickson, Andrew G.

Dietz, Russell N.

Doran, J. Christopher

Doskey, Paul

Dowlatabadi, Hadi

Drake, Bert

Drake, James

Drake, John B.

Druffel, Ellen R. M.

Dudhia, Jim

Dukowicz, John K.

Dunlap, Kelly

Dunning, Thom

Easter, Richard

Easterling, David

Eberhard, Wynn

Edgerton, Sylvia

Edmonds, James E.

Edwards, Nelson T.

Eglinton, Timothy

Ehrlich, Paul R.

Ellingson, Robert

Emanuel, Kerry

Emery, W. J.

Erickson, David

Ermak, Donald

Evans, J. L.

Fairall, C. W.

Falkowski, Paul

Farrell, Brian

Fast, Jerome

Fein, Jay S.

Ferrare, Richard

Field, Christopher

Finlayson-Pitts, Barbara

Flagg, Charles N.

Fletcher, Madilyn

Formento, John

Foster, Ian

Francis, Arokiasamy

Frank, W. M.

Frederick, John

Fredrickson, James

Gabriel, Philip

Gaffney, Jeffrey

Gage, Kenneth

Gao, Weigang

$9,13,15$

43

169

171,184

68

82

172

44

$9,44,45$

45,64

98

206

222

82,212

168,172

19

90

70

218

46

70

10

70

$98,99,107$

191, 210

182

99

10,17

83

29

40

46

30

12

173

83

46

71

17

207

47

174

233

47

83

233

30

63

234

26

48

11

41,64
Garten, Charles, Jr.

Gates, Lawrence

Gautier, Catherine

Gehring, C. A.

Gehrs, Carl

Ghan, Steven

Ginn, Timothy

Girvin, Don C.

Glantz, Michael

Goldberg, Ellen

Goldsmith, John E. M.

Gorby, Yuri

Goulden, Michael

Goulder, Lawrence H.

Goyet, Catherine

Gray, William M.

Greening, Lorna

Griffin, W. Timothy

Griffiths, Robert P.

Grossman, Allen

Grossman, Ethan

Gu, Baohua

Gudiksen, Paul

Gunderson, Carla A.

Gunter, Lee E.

Gutowski, William

Hack, James

Hales, Jeremy

Hall, Michael J.

Ham, Jay

Hameed, Sultan

Hammitt, James

Han, Yong

Hansen, D. Alan

Hanson, Paul J.

Harrison, Lee

Heath, Donald

Heinrichs, Donald F.

Henderson, David

Hendrey, George

Herman, Janet

Hibler, Lyle

Hicks, Bruce

Hill, Walter R.

Hoffert, Martin I.

Holben, William E.

Hollinger, David

Honeyman, Bruce

Hood, Lon L.

Hopkins, Tom S.

Hopkinson, Charles

Hornberger, George M.

Howarth, Richard

Hsiao, Theodore

Humphreys, Kenneth

Huston, Michael
$191,207,210$

13,33

194

219

12,54

234,243

229

108

219

13

237

214

99

174

13

106

234

236

48,71

236

238

46

$191,199,201,210$

191

84

14,84

48

85

210

68

100

11

49

191,207

18,51

196, 208

242

62,219

101

236

208

182

237,242

101

192

219

193, 197 
Ingram, Jani $\mathbf{C}$.

Intrieri, Janet

Jacoby, Henry

Jaffe, Adam

Jahnke, Richard

Jain, Atul

Jardine, Philip

Jastrow, Julie D.

Jayne, John T.

Jensen, Tommy

Johnson, Dale

Johnson, Donald

Johnson, K. M.

Jones, Philip D.

Jones, Sonja

Joslin, John

Kahn, James R.

Kaiser, Dale

Karl, Thomas

Karnosky, David

Kashgarian, Michaele

Keeling, Charles

Keim, P.

Kemp, Paul F.

Kennedy, Donald

Key, Robert

Khalil, M. A. K.

Kieft, Thomas

Kiehl, Jeffrey

Kim, K.-Y.

King, Anthony

Kinnison, Douglas E.

Kirchman, David

Kleinman, Lawrence

Kliewer, Kenneth

Knapp, Alan

Knuth, Ronald

Kolb, Charles E.

Kolber, Zbigniew

Kolstad, Charles

Koracin, Darko

Kozyr, Alex

Kreidenweis, Sonia

Kromidas, Lambros

Kropfli, Robert A.

Krueger, Steven

Krumholz, Lee

Kubiske, Mark

Kukla, George

Kulpa, Charles

Kuo, Ying-Hwa (Bill)

Kuske, Cheryl

Lacis, Andrew

Lala, Gar

Laroche, Julie

Larsen, Richard J.
42,65

178

102

18

178

187,196 51
Laulainen, Nels

Lave, Lester B.

Leavitt, S. W.

209

Lee, Cindy

Lee, H. N.

Lee, In Young

Lee, J. T.

Lee, Jai Hoon

Lee, Yin-Nan

Leifer, Robert

Lempert, Robert J.

Leung, L. Ruby

Levin, S. A.

Liang, Liyuan

Liebetrau, Albert

Lindzen, Richard

Liou, K. N.

Loehle, Craig

Logan, Jennifer

Lohrenz, Steven

Long, Philip

Long, Stephen

Louis, Jean-Francois

MacKenzie, Fred T.

Madronich, Sasha

Mahen, Edward C., Jr.

Majer, Ernest L.

Malone, Robert

Margolin, Len G.

Marley, Nancy

Marotzke, Joachim

Marshak, Alexander

Matin, Abdul

Matrosov, Sergey Y.

Matthias, A.

Mayer, Lawrence

McCord, Raymond

McElroy, Michael

McIntosh, Robert

McKinley, James

McMurry, Peter H.

Mechoso, Carlos R.

Meehl, Gerald A.

Melfi, S. Harvey

Mendelsohn, Robert

Metting, Blaine

Meyer, William T.

Michalsky, Joseph

Miller, R. Michael

Miller, Robert V.

Millero, Frank J.

Mills, Aaron

Minnis, Patrick

Mirin, Arthur

Mishchenko, Michael

Mitchell, David L.
196,208 
Morris, David

Morse, Gerome

Moss, Richard

Moyers, Jarvis

Mulholland, Patrick

Muller, Richard A.

Murcray, Frank

Murphy, Ellyn

Murray, Christopher

Murray, William L.

Nachtigal, Noel

Neff, William D.

Nelson, Tommy

Newman, Leonard

Nichols, Brian

Niedoroda, Alan W.

Nierzwicki-Bauer, Sandra

Nobel, Park

Norby, Richard J.

Nordhaus, William D.

North, Gerald

Novakov, Tihomir

Nowak, Robert S.

O'Neill, Elizabeth

O'Neill, Robert V.

Oberbauer, Steven F.

Oechel, Walter

Ogden, Kimberly L.

Olson, Richard

Onstott, T. C.

Owensby, Clenton

Pacala, S. W.

Paffenhofer, G.-A.

Palumbo, Anthony V.

Paluszkiewicz, Theresa

Parker, Stephen D.

Parson, Edward A.

Parsons, David

Paul, Eldor A.

Paw U, K. T.

Pendleton, Linwood

Penner, Joyce

Person, Mark

Peterson, Bruce

Peyton, Brent

Phelps, Tommy

Phillips, Donald L.

Phillips, Fred

Piacsek, Steve

Pietrafesa, Leonard J.

Platt, Charles Martin Richard

Post, Wilfred M.

Potra, Florian

Potter, Gerald

Pratt, Robert

Pregitzer, Kurt S.
198,210

$191,193,199,210$

199

198

199

227

98

245

210

200

185

246

88

247

105

19

211

192

103

$20,37,74$

84

211

220

246,247

212

238

89

180

20,26

$191,207,212$

91,92

105

193,202
Prell, Warren

Preller, Ruth

Prinn, Ronald

101

Pruess, Karsten 221

Rabitz, Hersch $\quad 59$

Ramanathan, V.

Ramaswamy, V.

Randall, David

$22,73,85,89$

Rasmussen, R. A.

72

Rauschkolb, R.

209

Reck, Ruth

57

Redalje, Donald $\quad 181$

Reed, Donald 248

Reich, Peter B. 201

Reimer, Michael 221

Reimers, Clare $\quad 181$

Reinsel, Gregory 63

Repeta, Daniel $\quad 182$

Revercomb, Henry E. 22

Rice, Charles

Richards, Kenneth

208,210

Riley, James

105

Ringelberg, David $\quad 252$

Rittmann, Bruce 248

Roberts, Dorothy $\quad 96$

Rodhe, Henning 23

Rogers, Hugo 212

Roiz, Peter 53

Roop, Joseph M. $\quad 105$

Rooth, Claes $\quad 78$

Ruby, Leung 12

Ruffin, Morrie $\quad 222$

Rusch, David $\quad \mathbf{5 8}$

Sands, Ronald $\quad 105,107$

Santschi, Peter 182

Sarmiento, Jorge $\quad 183,213$

Sassen, Kenneth $\quad 15,23$

Sathaye, Jayant 106

Sayler, Gary $\quad 217,222$

Schafer, Annette 249

Scheibe, Timothy 234

Schery, Stephen 222

Schipper, Lee $\quad 106$

Schlesinger, Michael 73

Schneider, Hans R. $\quad 55$

Schneider, Niklas 21

Schneider, Stephen 99

Schwartz, Stephen E. 23, 24, 58

Scott, Michael 105, 107

Sedjo, Roger 107

Semeraro, Dave $\quad 82$

Semtner, Albert $\quad 90$

Senum, Gunnar I. $\quad 44$

Sextro, Richard G. 223

Sharpe, Steven 24 
Shaw, Joseph

Shaw, William J.

Sherr, Barry

Shorter, Jeffrey

Shugart, Lee

Silliman, Stephen

Simpson, David

Skyllingstad, Eric

Slinn, George

Smith, Douglas Robert

Smith, Gregory P.

Smith, John R.

Smith, Richard D.

Smith, Robert

Smith, William

Sohngen, Brent

Solomon, Stanley

Somerville, Richard C. J.

Sowle, David H.

Spejewski, Eugene

Spinhirne, James

Spinrad, Richard W.

Springston, Stephen R.

Stacy, Walter

Staley, James T.

Stamnes, Knut

Starr, David

Stavins, Robert

Stephens, Graeme

Stephens, Pamela

Stevens, Todd

Stokes, Gerald M.

Stone, Alan

Stone, Peter

Stooksbury, Billie

Strain, Boyd

Streets, David

Streit, Gerald

Strom, Suzanne

Sublette, Kerry L.

Suflita, Joseph

Swift, Donald J. P.

Swif, Norman

Takahashi, Taro

Tang, Ignatius $\mathbf{N}$.

Tausch, Robin

Taylor, Karl

Thomas, Donald

Thomas, Richard

Thompson, $T$.

Tiao, George

Tie, Xuexi

Tilley, J. S.

Toon, O. B.

Tschaplinski, Timothy

Turner, Robert

Tuskan, Gerald
$59,88,171,184$

93, 96

170,185
Ullman, William

Valero, Francisco

Valocchi, Albert

248

Varanasi, Prosad

28

Venter, J. Craig

225

Verity, Peter

Vitko, John

185,186

Volker, Mohnen

35

50

Vorosmarty, Charles

Voyles, Jimmy W.

84

28

Walcoff, Carol

252

Wallace, Douglas W. R.

Wang, Wei-Chyung

Washington, Warren $M$.

Wasiolek, Piotr

Watt, Ward B.

Weatherhead, Elizabeth

Weatherly, Georges

Webb, Thompson, III

Webster, P. J.

Wei, Zongying

Weinstein-Lloyd, Judith

Weiss, Ray F.

Weiss, Robert B.

Wesely, Marvin

Westwater, Edgeworth

Weyant, John P.

White, Andrew

White, David C.

Whiteman, C. David

Whiteman, David

Whitham, T. G.

Wigley, T. M. L.

Wigmosta, Mark

Wilcoxen, Peter

Wildung, Ray

Williams, Albert J.

Williamson, David

Wilson, Glenn

Winn, Christopher D.

Wirick, Creighton D.

Wiscombe, Warren

Wise, Donald L.

Woese, Carl R.

Wofsy, Steven

Worley, Pat

Worsnop, Douglas R.

Wuebbles, Donald J.

Wullschleger, Stanley D.

Young, G. S.

Young, William

Zachara, John

Zak, Donald

Zeiger, Eduardo

Zhang, Guang J.

Zhong, Sharon S.

Zivkovic, Marina
186,187

$28,62,74$

75

222

202

63

187

76

29

49

63

187

225

$41,63,64$

11

108

91

225,252

$12,45,64,66$

17

194

71

54

106

226,252

170

84

246

179

188

30

226

226

214

82

42,65

100,108

193,210

30

79

253

202

203

21

12,66 91 


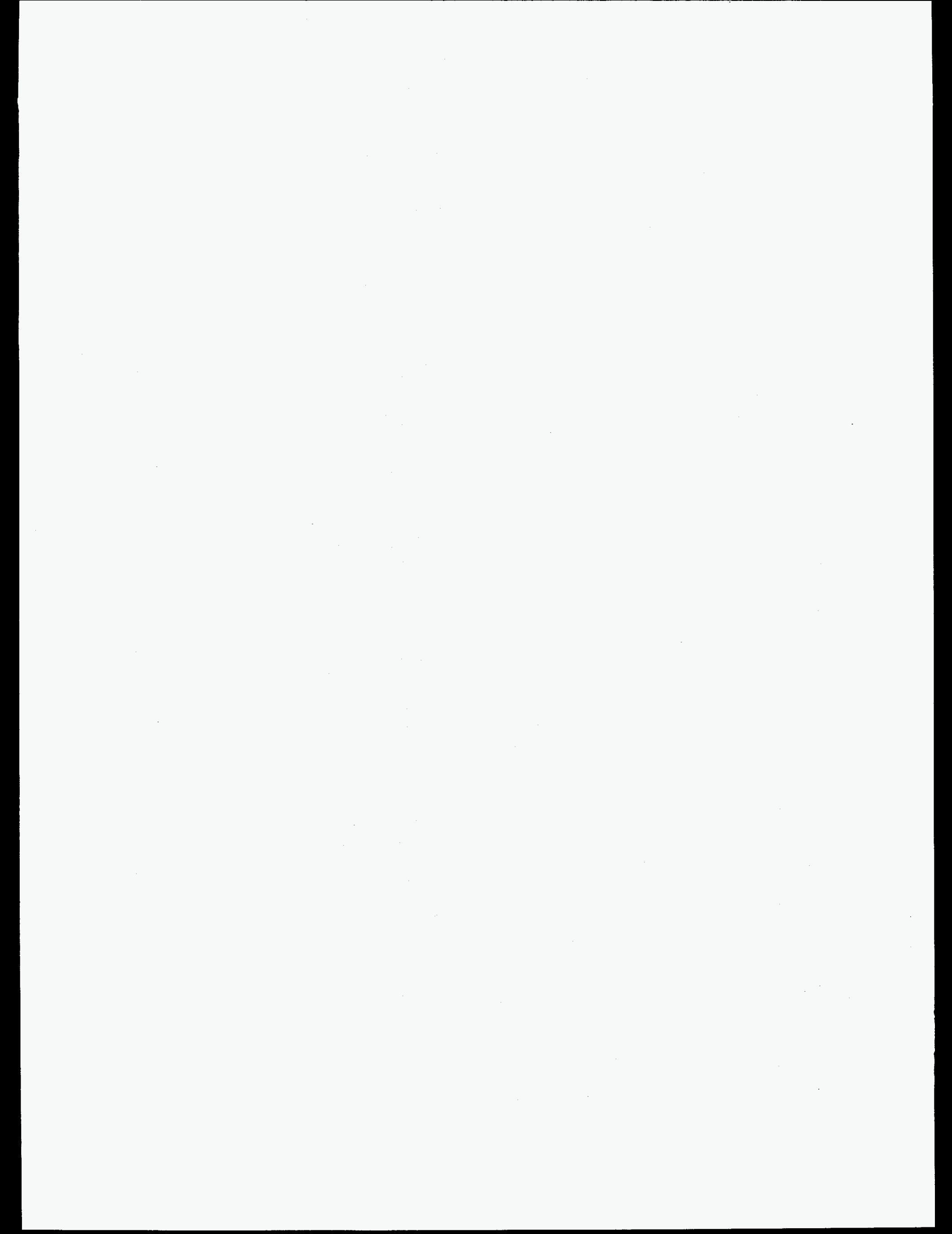


Advanced Research Projects Agency

Aerodyne Research, Inc.

Aluminum Company of America

American Society for Microbiology

Argonne National Laboratory

$5,41,45,47,48$, $57,63,64,70,83,108$, $195,196,208,241,248$

ARS-USDA/National Soil Dynamics Laboratory 212

Atmospheric and Environmental

Research, Inc.

Battelle Marine Sciences Laboratory

$4,16,69,91$

171

222

Biotechnology Industry Organization

42,65

Brandeis University

107

Brookhaven National Laboratory

$23,24,42,44$, $52,58,61,173,174,177,178$, $186,187,188,196,208,233$

Brown University

Carnegie Institution of Washington

207

Carnegie-Mellon University

Cascade Scientific Research Corporation

98,220

60

Colorado School of Mines

Colorado State University

182,221

$5,13,14,22$, $26,34,73,85,89$

Committee on Environment and

Natural Resources

68

Commonwealth Scientific and

Industrial Research Organisation

20,26

Consortium for Oceanographic

Research and Education

184

Desert Research Institute

Duke University

$18,206,207$

213, 214

Electric Power Research Institute

Envair

Envirogen, Inc.

49

48

232

212

198

Florida Interational Univery

Florida State University

Genome Therapeutics Corporation

$187,228,229$

Geophysics Fluid Dynamics Laboratory

223

Georgia State University

14

97

Golder Associates Inc.

Harvard University

Hughes STX, NASA Goddard

Space Flight Center

235

$55,83,100,103$,

$105,107,200,214$

Idaho National Engineering

Laboratory

Indiana University

Institute for Genomic Research, The

Iowa State University

Johns Hopkins University

$220,231,249$

105

225

84

250
Kansas State University

208,210

Lamont-Doherty Earth Observatory

$72,170,185$

LASP, University of Colorado

58

Lawrence Berkeley National Laboratory $57,62,72$, $106,217,221,223,227,240$

Lawrence Livermore National Laboratory 4, 20, 37, $46,48,60,71,74,80,91,92,176,190,205$

Los Alamos National Laboratory $39,40,52$, $81,86,87,90,91,194,243$

LUMCON

185

Marine Biological Laboratory

175,211

Massachusetts Institute of

Technology

$83,86,91,101$

Michigan State University

211

Michigan Technological University

193,202

Mission Research Corporation

34,59

NASA

33,34

NASA

33,34

NASA Goddard Institute for Space Studies 9, 13, 15

NASA Goddard Space Flight Center 25, 30

NASA-Ames

National Academy of Sciences

National Center for Atmospheric

Research

$14,19,40,55,75,79,84,108$

National Oceanic and Atmospheric

Administration $\quad 11,12,16,43,73,85,205$

National Science Foundation $\quad 56,71,73,175$

Naval Postgraduate School

90

Naval Research Laboratory

89

New Mexico Institute of Mining and

Technology

222,238

New York Botanical Garden Cary Arboretum 200

New York University

NOAA Air Resources Laboratory

101

NOAAVEvironmental Research Laboratories $\quad 57$

NOAA Environmental Technology Laboratory 10

NOAA/NESDIS/National Climatic Data Center 70

North Carolina State University $\quad 169,180$

Northeastern University $\quad 226$

Northern Arizona University $\quad 194$

Northwestern University 248

Oak Ridge Institute for Science and Education

$39,61,93,96$

Oak Ridge National Laboratory

$62,70,82,86$, $95,97,98,102,178,191,193,197$, $199,201,207,210,212,217$, $219,237,238,246,247$

17

Ohio State University

Oklahoma State University

104

Old Dominion University

Oregon Graduate Institute

Oregon State University
242

251

72,230

183,236 
Pacific Northwest National Laboratory $\quad 6,9,12$, $24,26,28,38,39,44,45,46$, $51,54,59,60,65,66,77,88$, $98,99,104,105,107,168$. $184,190,218,219,220,226$, $229,231,234,237,239,240$, $243,244,252,253$

Pennsylvania State University, The $\quad 3,30,84$

Portland State University

Princeton University

Purdue University

RAND Corporation $59,78,183,200,213,245$

72

Rensselaer Polytechnic Institute

Research Support Instruments, Inc.

Resources for the Future

RF of Stony Brook

Rutgers, The State University of New Jersey $\quad 181$

San Diego State University

189,199

Sandia National Laboratories $13,35,77$

Santa Fe Institute

219

Science and Data Systems, Inc.

Scripps Institution of

Oceanography $77,79,172,176,187,209$

Sequoia Scientific, Inc. 167

Sigma Xi, The Research Society
Skidaway Institute of Oceanography

Smithsonian Institution

SRI International

Stanford University

State University of New York at Stony Brook

State University of New York at

Albany

$18,28,50,51,62,74$

State University of New York/Old Westbury

$176,185,186$ 206

60 $99,108202,240$

$4,28,68$

Stockholm University

Texas A\&M University

$88,208,236$

Texas A\&M University at Galveston

UCAR/NCAR

University of Alaska

University of Arizona

University of California, Berkeley

University of California, Davis

University of California, Irvine

University of California, Los Angeles

University of California, San Diego

University of California, Santa Barbara

University of California, Santa Cruz

University of Chicago

63
$21,25,35$

$13,33,102$
University of Colorado

University of Delaware

$8,11,29,58,63$

University of Denver

251

University of East Anglia

19

University of Essex

University of Hawaii

University of Idaho

University of Illinois

University of Illinois, Chicago Campus

University of Iowa

University of Maine

196

179,224

218

University of Maryland

University of Maryland, Baltimore County

University of Massachusetts

University of Miami

University of Michigan

University of Minnesota

University of Montana

University of Nevada, Reno

University of New Hampshire

University of North Carolina

University of Notre Dame

University of Oklahoma

University of Southern California

University of Southern Mississippi

University of Tennessee, The 217, 222

University of Texas

University of Tulsa

University of Utah

University of Virginia

University of Washington

University of Wisconsin

USDA Forest Service

USDOE Chicago Operations Office

USDOE Environmental Measurements

Laboratory

USDOE Office of Field Support

USDOE Office of Scientific and

Technical Information

Virginia Institute of Marine Sciences

Walcoff and Associates, Inc.

West Virginia University

Western Washington University

Woods Hole Oceanographic

Institution

Yale University

$170,171,174,182$
$17,68,182$

$78,81,180$

$56,84,201$

171,236

238

$191,198,210$

84

169

249

236,250

103

179,181

106,169

224

$15,23,34,225$

237,242

38

$22,24,63,83,85$

198, 208

27

51,53

33

70

168

252

213

185

37

103, 104
University of Nevada, Las Vegas

Westinghouse Savannah River Company 


\section{SUBJECT INDEX}

16S rRNA

Absorption by clouds

Absorption, clear-sky

Acclimation

Actinides

Adaptation

Adaptive computation

Adhesion

Adjoint methods

Administrative support

Adsorption

Advection

Aerial observations

Aerosols

anthropogenic

characterization of

effects on clouds of

microphysics of

optical properties of

research on

scattering coefficient of

size distribution of

Agriculture

Agroecosystems

Air pollution

Air quality

Air-quality management

Air-quality modeling

Air-quality research tools

Air-sea transfer velocity

Air/water exchange coefficients

Airborne measurements

Albedo

Altocumulus

Ameriflux

AMIP

Anaerobic bacteria

Anaerobic processes

Aquifers

Archeal genomics

Arctic ice

Arctic stratus

Arthrobacter

ASCOT

Aspens

Atlantic Coastal Plain

Atmosphere

chemistry of

diffusion in
195,234

4,24

35

191,199

248

191

219

232,233

16

$56,70,71,96,104$,

$217,218,219,220,235$

248

224

35

$17,18,23,24,39,46$, $58,61,70,71,72,98$

74

53

20

14

57

43

53

56

107

209

103

$44,45,64$

49

49

49

168

42

38,39

58

15

205, 208, 214

73,91

230,250

234

232, 236

225

89

14

245

$37,40,41,44,52$

194

251

179

$37,38,39,45,48$,

$55,57,63,64,70$
Atmosphere (cont.)

dispersion in

tracing of

41

turbulence of

44

59

Atmosphere-ocean interactions $\quad 29,73$

Automated hybridization 225

Automatic differentiation $\quad 41$

Bacteria 183, 232

anaerobic $\quad 230,250$

population dynamics of marine $\quad 177$

survival of $\quad 241$

thermophilic 230

transport of

$232,233,234,237$,

$241,242,243,244$

$\begin{array}{lr}\text { Baroclinic-pressure fields } & 174 \\ \text { Baroclinic waves } & 83\end{array}$

Basalt 220, 234

Basin-plain/plateau circulation $\quad 66$

BATS 82

Bioaugmentation $\quad 232$

Biocolloids 227

Biodegradation $\quad 220,221,222,224$,

Biodiversity 107, 200, 201

Bioenergetics 202

Biofilms $\quad 219$

Biogeochemical cycles $\quad 212$

Biogeochemical dynamics 237, 246

Biogeochemistry $\quad 197,229,231,233,234,239$,

$240,243,244,249,251,253$

coastal

187

Bioluminescence 222

Bioremediation $\quad 217,222,223,228,229,232$, $239,240,242,247,248,249,251$

by microorganisms

224

Biosphere-atmosphere transfer scheme $\quad 82$

Biostabilization 223

Boreal forests 206

Boundary layers $\quad 45$

benthic $170,179,180,182$

bottom 187

chemistry of $\quad 59$

marine 12

meteorology of 64

parameterizations of 51

planetary $\quad 89$

structure of 51

Buildings $\quad 105$

Calibration $\quad 49$

Cape Hatteras $\quad 167$

Carbohydrates 169, 185

allocation of 196 


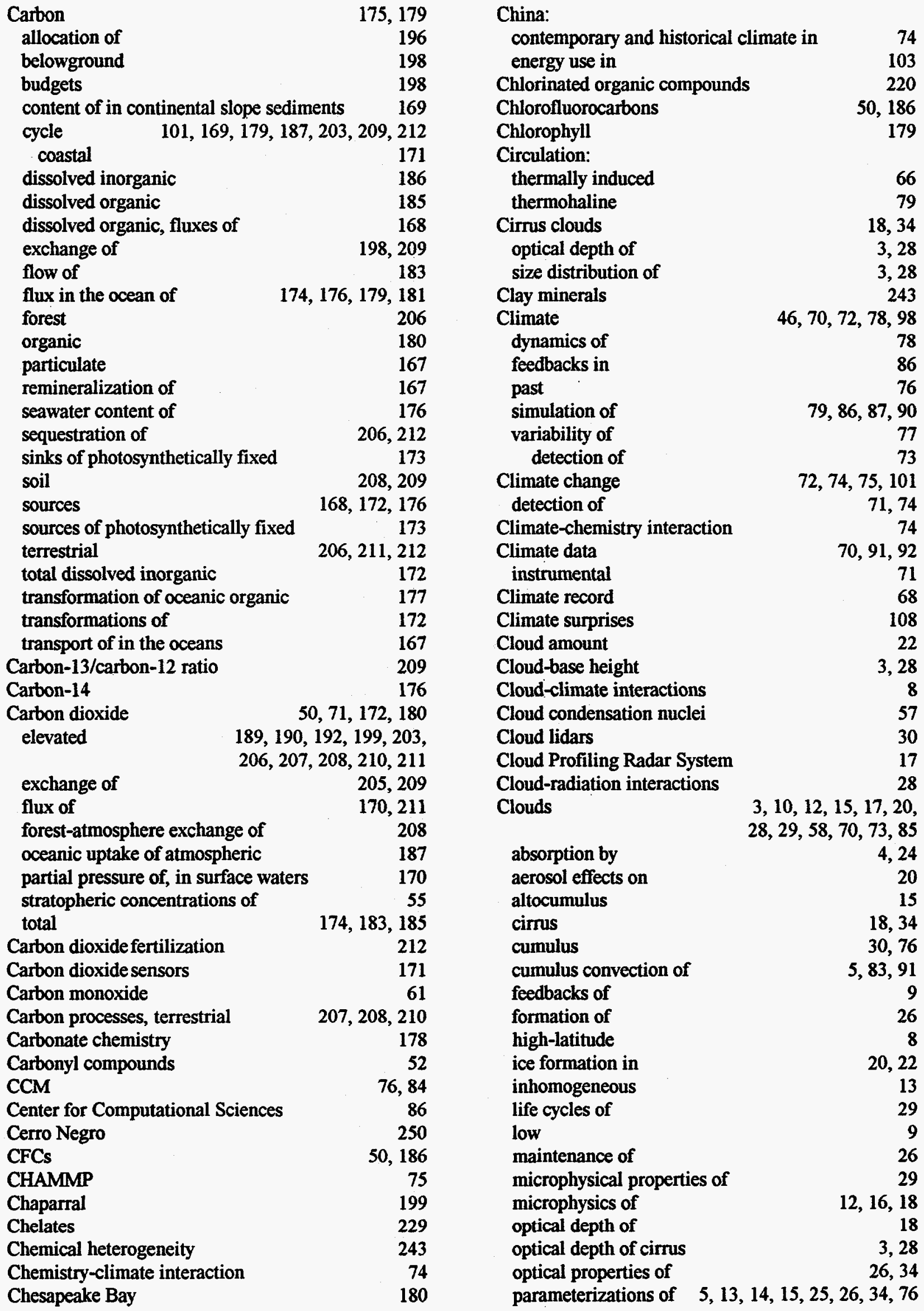


Clouds (cont.)

properties of

radiation parameterizations for

radiative processes of

radiative properties of

retrieval algorithms for

size distribution of cirrus

stratocumulus

structure of

water formation in

Cobalt

Cocontaminants, transport of

Colloidal organic carbon, fluxes of

Colloids

Complex systems

Complex terrain

meteorology of

Complexation

Complexing agents

Computational fluid dynamics

Computer resources

Computing, massively parallel

Contaminants:

migration of

sequestration of

Contamination, nonaqueous-phase liquid

Continental shelf

Continental shelf waters, transport of

Contingent valuation analysis

Convection

intense

subgrid-scale

Convective mixing

Cooling rate

Copepods

Cretaceous

Culture collection

Cumulus clouds

convection in

Current meters

Data

analysis of

assimilation of

4-D

display of

fusion of

homogeneity of

integration of

interpretation of

management of

satellite

Databases

Data centers

Data sets

Data systems, integrated

Decay products
26,33

3,28

13

18,34

23

3,28

76

30,33

22

$171,181,250$

237,248

168

182,238

219

$38,40,41,44,45,46$

$40,52,64,66$

$227,248,253$

233

38,78

91

$77,80,91$

221

231

221

175,183

174

97

79

13

83,91

88

69

185

250

228

30,76

$5,83,91$

187

28

40

$16,19,46$

46

34

16

70

234

60

$92,174,178$

34

$53,95,98,228$

95

95

28

222
Deep microbiology

Degradation

Diebacks of forests

40,52

Dispersion

186

185

$\begin{array}{ll}\text { fluxes of } & 168\end{array}$

Diurnal cycle $\quad 12$

DNA sequencing $\quad 226$

high-throughput 225

Down-regulation

196,208

Drought

Drought tolerance

$192,198,199,205$

Dry deposition

201

Dynamic programming

$52,64,222$

East Asian monsoon

102

Ecology

173,205

Economic growth

103

Ecosystems:

forest

190,197

grassland

201

health of

Mediterranean-type $\quad 199$

Eddy flux 208

EDTA 227, 250

Education $\quad 93$

graduate 93

El Nino/Southern Oscillation $\quad 184$

Elevated carbon dioxide

$189,190,192,199$, $203,206,207,208,210,211$

Emergency response

Emission controls

103

Emissions

natural

$45,98,107$

Empirical orthogonal functions

64

Energy balance

Energy demand

88

Energy efficiency

Energy emissions

Energy fluxes

Energy policy

Energy use

Environmental Molecular

Sciences Laboratory

Enzyme-linked fluorescence

ERIC

Ethylene-diaminetetraacetic acid

Evaporation

Evapotranspiration

107,220

42

190

101

103

218

225

195

227,250

205

193

Evolution

Exchange of carbon dioxide, forest-atmosphere

Extreme events

108

FACE 
Facility operations

Faculty and student grants

FANGIO

Fast-repetition-rate fluorometer

Fecundity

Feedback, cloud

Feldspars

Fellowships

Field instrumentation

Field scale

Fine roots

dynamics of productivity of

Fluorescence enzyme-linked

Fluorescence sequencing techniques

Fluxes

heterogeneous

inhomogeneous

latent heat

Flying platforms

Food webs

Forest-atmosphere exchange of carbon dioxide

Forest ecosystems

Forests:

growth of

impact of animals on

rainforests

soils of

temperate

Fourier transform spectrometer

Fractured rock

Free-air $\mathrm{CO}_{2}$ enrichment

Free radicals

GCSS

GEM release

Gene probes

Genetic control

Genetic damage

Genetic resources

Genetics

Genome sequences

Geochemistry

Geomicrobiology

Geostatistics

Glossaries

Grassland ecosystems

Gravity waves

Greenhouse gases

Groundwater

age of

experiments with

flow of

Halogens

Heat flux
$194,208,209$

242,245

$223,225,226$

$236,244,252$

245

231
Heterogeneous chemistry

Home pages $\quad 82$

Homeostatic adjustments $\quad 199$

Homogeneous nucleation $\quad 56$

Human society

102

Hydrocarbons

52,224

Hydrogeology

245,247

Hydrography

Hydrologic cycle

84,85

Hydrologic properties

251

Hydrology

India

Indian Ocean

Industrial leak detection

Industrial sector

$197,240,247$
106

$197,240,247$
106

$174,180,183$

44

105

Information analysis centers

Information dissemination

60,95

Information systems

Infrared radiation

Inorganic carbon:

dissolved

total dissolved

Instrument selection

61,249

Instrumentation

$97,98,99,100,101$,

Integrated assessment

$102,103,104,105,106,107$

Integrated sounding systems

Intel Paragon

Interannual variations

International Global Atmospheric Chemistry 56

Investment theory

IR-radiometer

Iron oxide

16,20

Iron-reducing bacteria

227,238

Irreversibilities

Isopycnic coordinates

Isotopes

natural

172,176

thorium

168

182

Joint Global Ocean Flux Study $\quad 178$

Juniper trees

Katabatic flow

Latent heat flux

Leaf water potential

Learning

Lidar

$3,10,11,20,23,28,30,57$

Lidar, micropulse

Lidars, Raman

$11,13,17$

Life-cycle analysis

Ligands, organic

238,243

Line-by-line calculations

Loblolly pine

Low-level jet

$199,208,213$

Management

Manganese

Manganese-reducing bacteria 
Manometric assay 176

Marine atmosphere

42

Marine bacteria, population dynamics of $\quad 177$

Mass fluxes

190

Massively parallel computing

Meetings

Mesoscale circulation

Mesoscale convection

Mesoscale eddies

Metadata

Metal reduction

Meteorological Coordinating Committee

Meteorological satellite sensors

Meteorology

valley

Methane

Mexico City

Mexico City Air-Quality

Study Project

Microbial organisms

communities of

ecology of

ecology of

$240,242,245,247,248,252$

genome sequencing of

genomic characterization of

growth of

heterogeneity of

phylogeny of

physiology of

soil

survival of

transport of

Microbial reduction

Microbial stabilization

Microbiology

soil

Micrometeorology

Micropulse lidar

Microwave radiometers

Mid-Atlantic Bight

Mineral dissolution

Minerals, clay

Minirhizotron

Mixed-layer dynamics

Mixed-layer entrainment

Mixed wastes

Mixing, atmosphericpotential vorticity

Modeling

air-quality

climate

integrated

kinetic

mathematical

mesoscale

ocean general circulation
$231,234,236,237,238$

226

177

$77,80,91$

39,186

59,66

5,30

78,80

207

234

39

34

45,73

40

$39,40,41,44,61$

37

$183,223,248$

195

7

229,231

228

229

189,203

236,238

$229,239,249$

237

233

$239,247,248,252,253$

195,212

64,205

25

11,16

$182,183,185$

251

243

193

79

184

252

86

40

49

$9,75,82,86,91,92$

237

190,234

46,220

77
$50,72,206$
Modeling (cont.)

paleoclimatic-sensitivity $\quad 76$

tropospheric ozone 41

whole-ecosystem $\quad 212$

Models 73

3-D chemical-transport $\quad 40$

atmospheric $\quad 77,86,87$

atmospheric-chemistry 51

biogeochemical 207

climate $\quad 85$

testing of $\quad 76$

cloud

$14,27,30$

cloud-resolving $\quad 15,18$

coupled atmosphere-ocean $\quad 73$

coupled climate $\quad 80,83$

DICE 104

ecophysiological growth 194

energy-balance $\quad 88$

forecast 46

general-circulation $\quad 5,12,15,28,30,62$,

$68,74,76,77,80,82,89$

generalized vertical coordinate $\quad 76$

geologic 241

geostatistic 231

GISS GCM 9

global ocean $\quad 78,79,90$

ice 89

integrated-assessment $\quad 99,102$

intercomparison of $\quad 54,91$

isentropic-sigma coordinate $\quad 85$

isopycnal-ocean $\quad 81$

land-surface $\quad 82$

longwave-radiation $\quad 10$

Miami Isopycnic-Coordinate Ocean $\quad 81$

MICOM 81

microphysical $\quad 58$

$\begin{array}{lr}\text { nonhydrostatic } & 19\end{array}$

numerical 85

ocean $\quad 77,78,85,86,87,89,90,171$

ocean-atmosphere 91

ocean general circulation $\quad 80$

overlapping-generations $\quad 101$

PCCM2

radiative transfer $\quad 4,26,27,69$

RAMS

regional climate $\quad 54$

sea-ice

90

single-column $\quad 19,25,83,91$

SOS

validation of

59

Moisture flux 231

Moisture profiles $\quad 24$

Molecular biology $\quad 245$

Molecular typing 195

Moored sensing systems $\quad 171,187,188$

Multifilter rotating-shadowband radiometer $\quad 18$ 
Multiscale heterogeneity

Mycorrhizae

Mycorrhizal mutualists

NABIR program

NARSTO

Net ecosystem

Net ecosystem production

Net metabolism

Newsletters

Nitrate sensors

Nitrates

organic

Nitriloacetic acid

Nitrogen cycling

Nitrogen oxides

Nitrogen, soil

Nitrous oxide

oceanic uptake and release of

North America

Nucleotide sequences

Numerical methods

Nutrient limitation

Nutrients

acquisition of

allocation of

cycling of

Ocean Margins Program

Oceanography

Oceans

carbon in

chemistry of

circulation of

coastal

currents in

interactions of with the atmosphere

mixed layer of

physics of

transport of carbon in the

turbulence in

Open-top chambers

Optical depth

Optical properties

Orbital inclination

Organic carbon

colloidal fluxes of

dissolved

fluxes of

transformation of oceanic

Organic compounds

Organic ligands

Organic nitrates

Organic particulate matter

Organic peroxides

Organochelates

Osmotic potential

Oxidants
232

$189,193,196,199$

194

$217,225,226$

48

214

192

175

95, 217

171

61,190

52

$227,248,250$

203

$47,52,61$

201, 206

187,190

187

72

245

87

240

175,192

196

196

206, 212

$167,181,186$

184

$175,178,179,187$

213

178

180

181

174

29,73

85

88

167

80

210

$3,18,28,46,50$

9

73

180

168

185

168

177

45,48

238,243

52

182

52

237

201

48
Oxygen dissolved in surface waters

170

Ozone

$18,37,39,47,49,50$,

$52,57,58,59,60,63$

atmospheric

43,62

change in

depletion of

48

formation of

57

measurements of

42

pollution by

49

profiles, Umkehr-retrieved

trends in

55

tropospheric

Pacific Ocean

21,184

warm pool

68

Paleoclimate reconstructions $\quad 74$

PANS

48

Parallel algorithms

82

Parallel computing

Parameterizations

anvil cirrus

cloud

cloud-radiation

deep convection

$5,13,14,15,25,26,34,76$

3,28

171

fine-mesh atmospheric

ocean-convection

radiation

14,28

subgrid-scale

77,80

Particulate carbon

167

Particulate organic carbon, fluxes of $\quad 168$

Particulate organic matter

182

$\mathrm{pCO}_{2}$

183

Perfluorocarbon tracers (PFTs)

44

Peroxides

61,63

Peroxyacetyl nitrates

48

PFTs

Pharmaceuticals

107

Phospholipid fatty acids

252

Photoassimilation

190

Photochemistry, tropospheric $\quad 42$

Photodissociation $\quad 55$

Photoluminescence $\quad 173$

Photolysis rates $\quad 55$

Photo-oxidants

52

Photosynthesis

$173,178,190,191$,

$192,196,198,206,213$

Photosynthetic pathways

197

196

234

Phylogeny

microbial

243

205

Physiological ecology

173,185

Phytoplankton

194

Pinyon trees

89

Planetary boundary layer

Planning

$6,44,47,61,186$ 
Plants:

adaptations by

physiology of

symbionts of

Plutonium

PMIP

Pollutant plumes

Pollutant transport

Pollution

air

ozone

Polyaromatic hydrocarbons

Polychlorinated biphenyls

Ponderosa pine

Population:

impacts of

marine bacteria, dynamics of

thermal depression of

Pore-water chemistry

Porous media

Postdoctoral fellowships

Precipitation chemistry

Precipitation statistics

Primary and secondary production

Primary production

Proceedings, conference

Profilometers:

aerosol

cloud

Program administration

Program coordination

Program management

Project management

Protists

Protozoa

Pseudomonas

Pseudomonas fluorscens

Pulse-height analysis

Pyrite oxidation

Pyrococcus furiosus

Quality assurance

Radar

Radiance

Radiation

atmospheric

shortwave

Radiative balance

Radiative effects

Radiative fluxes

Radiative forcing

Radiative heating

Radiative properties of clouds

Radiative transfer

Radioacoustic sounding systems
$227,233,237$

$176,178,181$

$6,63,68,184$, $186,208,225,226$

$27,28,47,60$

183

185

195,233

222

56

236

225

50

$16,17,23$

22

50,89

20

24

70

20

$15,34,35$

$23,24,46,48,61,71$

26

18

$24,26,30,69,84$
Radioactivity

$50,53,248$

Radiocarbon

Radiometry

Radionuclides

migration of

$11,16,18,19,20,52$

$229,233,237,243,253$

Radon

227

availability of

221, 222

entry by

measurements of

migration of

223

transport of

221,224

Rainforests

Raman lidars

$11,13,17$

RASS

59

Reaction kinetics

Reactive transport

243,248

Recreation

103

Recreational resources, valuation of

103

Red maple

210

Reference materials

Regional climate $\quad 74$

Regional climate model $\quad 54$

Remediation $\quad 252$

Remineralization $\quad 176$

Remineralization of carbon $\quad 167$

Remote sensing $\quad 3,5,16,23,26,30,89,168$ ground-based 28

Reproduction $\quad 62$

Research facilities 38

Research ships $\quad 175$

Residence times 168

Respiration 190, 191, 206

Respiration rates 244

Resuspension 179

RFLP 195, 198

Ribonucleic acid 196

Rice agriculture $\quad 72$

Rock, fractured 221

Root function $\quad 210,212$

Rotating-shadowband spectroradiometer $\quad 18$

Russia 206

Safety 235

SAGE satellite $\quad 58$

Salt budget $\quad 187$

Sampling: aircraft 53

environmental 53

Satellite measurements $\quad 168$

Scale dependence $\quad 30$

Scaling $\quad 30,243$

Scaling rules $\quad 200$

Scaling theory 234

Science team 208, 218

Sea salt $\quad 47$

Sea-surface temperature $\quad 21,184$

Seawater 182 
Sediments:

dynamics of

suspended

transport of

Seismic imaging

Sensing systems, moored

Sensitivity analysis

Sensors, carbon dioxide

Sequencing:

DNA

fluorescence

genome

microbial genome

nucleotide

Sequestration

soil carbon

Shear

Shelf waters

Shortwave irradiance

Shortwave radiation

Simulation, direct numerical

Site implementation

Site operations

Social sciences

Social welfare

Sodium chloride

Soil gas

Soils:

carbon dynamics of

carbon sequestration of

food webs in

forest

gas transport in

management of

microbes in

microbiology of

microorganisms in

nitrogen in

availability of

organic matter of

radionuclides in

volatiles in

Solar spectral radiometer

Sonoran Desert

Sorption

Spatial heterogeneity

Spectra

Spectral irradiance

Spectral radiance

Spectral transport

Spectral validation

Spectroradiometers

Starvation genes

Sticking coefficient

Stomatal conductance

Stratocumulus clouds
170,179

240

$171,187,188$

$41,59,60$

171

226,227

223

$223,225,226$

225

245

206

209

171

181

18

24

80

6

6,27

$97,99,104,106$

102

47

221

208

209

189

207

224

211

189

195,212

203

206

201

208, 211

237

224

19

197

$220,229,243,249$

249

24

18

34

83

4

$201,203,213$

76
Stratosphere

$\mathrm{CO}_{2}$ concentrations of

$40,48,50,53,58$

transport in

55

Stratosphere-troposphere exchange

Stratus-cloud mean particle size

Stratus-cloud mean particle-number density $\quad 3,28$

Streams

197

Stress proteins

240

Subsurface bacteria

240

Subsurface contaminants

232

Subsurface heterogeneity

246

Subsurface microorganisms

Subsurface science

Sugar maple

Sulfate-reducing bacteria

Sulfate reduction

Sulfates

Sulfur

Sulfur dioxide

Sulfuric acid

Sunset Crater

Surface boundary conditions

Surface-layer dynamics

Surface waters, oxygen dissolved in

Surveys

Synoptic influences

Tallgrass prairie

Technical reviews

Technical support

Technological change

Technology adoption, impact of

Technology innovation

Technology transfer

Teleconnection

Temperature:

anomaly trends in

atmospheric

profiles of

sea-surface

Temperature inversions

Temperature record

Tephra layers

Terrestrial carbon

Terrestrial carbon processes

Thermal stability

Thermally driven wind systems

Thermally forced flows

Thermally induced circulation

Thermobaric instability

Thermocline

Thermodynamics

Thermohaline circulation

Thorium isotopes

Thoron

Thunderstorms

Timber
$228,229,245$

227-253

191,210

250

$234,236,240$

$23,46,61$

98

61

56

194

9

184

170

235

208, 211

175

$97,99,104,106$

105

107

37

74

23

70

24

21, 184

64

68

73

206, 211, 212

$207,208,210$

202

64

57

66

171

184

61

79

182

222

12

104 
TOMS data

Total alkalinity

Total $\mathrm{CO}_{2}$

Total dissolved inorganic carbon

Toxic metals

Trace gases

Tracers

environmental

transient

water-soluble

Transformation of oceanic organic carbon

Transpiration

Transport

long-range

microbial

reactive

semi-Lagrangian

Trees

aspen

juniper

loblolly pine

pinyon

ponderusa pine

red maple

sugar maple

Trend analysis

Trichloroethylene

Troposphere

Tropospheric chemistry

Tropospheric ozone

Tropospheric photochemistry

$\operatorname{trp} E$

Tundra

Turbidity

Turbulence

atmospheric

shear-generated

Turnover rates

UAV

Ultrafine aerosols

Ultraviolet atmospheric radiance

Ultraviolet-B radiation

Ultraviolet radiation

Umkehr-retrieved ozone profiles
$172,174,183$

$174,183,185$

172

233

71

242

244

186

44

177

192

45,85

$229,239,249$

243,248

83

203

194

194

199, 208, 213

194

206

210

191,210

70

249

$40,47,48,50$

37

63

42

245

206

50,170

29

59

184

176,182

33-36

56

49

$41,43,50,52,62,63$

55,63

43
Uncertainty

Uncertainty analysis

U.S. Global Change

Research Program

Universities and colleges, minority

Unmanned aerospace vehicles

Uranium

Urban air sheds

Valuation of recreational resources

Variability, low-frequency

Visibility

Wastewater treatment

Water:

availability of $\quad 193$

budgets of 187

deficits of $\quad 192$

quality of $\quad 212$

use of 193

Water stress

190,192

Water-use efficiency

$192,198,213$

Water vapor

fluxes of

profiles of

structure of

$13,17,18,24,83,85,86,91$

211

Watersheds

33

Weather variability 72

Western Pacific warm pool 21

Wet scavenging $\quad 52$

Wheat 209

Whitecaps 168

Whole-tree response functions $\quad 198$

Wind profiling radar $\quad 59$

Wind stress $\quad 171$

Wind systems:

local

66

thermally driven

WOCE

64

Workshops and conferences $23,85,104,108$, $214,218,222,223,226$

World Ocean Circulation

Experiment

$178,179,186,187$

World Wide Web

Zooplankton

185 



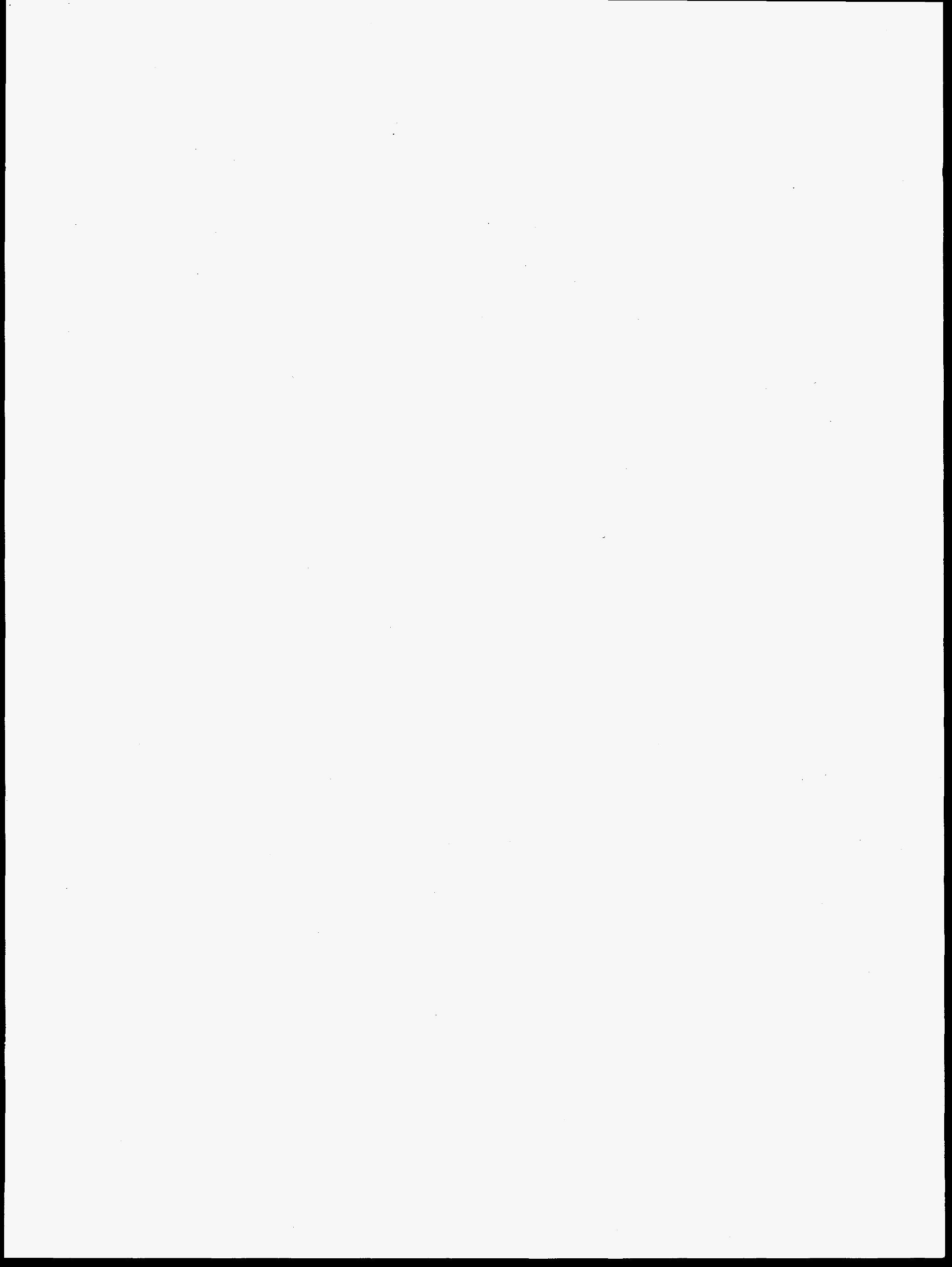


